\title{
Iscrizioni della città etrusca di Adria
}

Testi e contesti tra Arcaismo ed Ellenismo

Andrea Gaucci

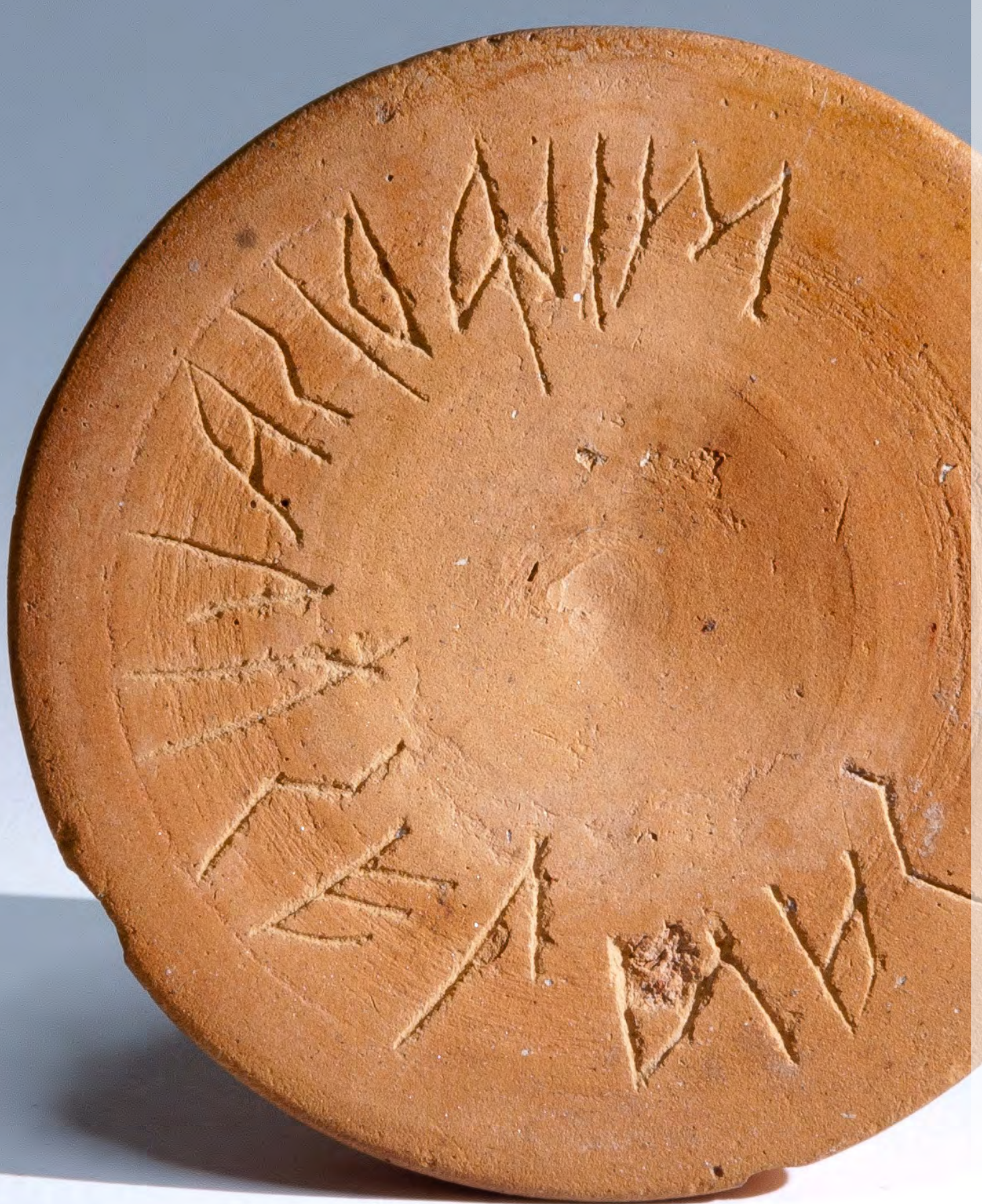


ALMA MATER STUDIORUM

UNIVERSITÁ DI BOLOGNA

DIPARTIMENTO DI STORIA CULTURE CIVILTÀ

\section{Archeologia}




\section{Collana DiSCi}

Il Dipartimento di Storia Culture Civiltà, attivo dal mese di ottobre 2012, si è costituito con l'aggregazione dei Dipartimenti di Archeologia, Storia Antica, Paleografia e Medievistica, Discipline Storiche Antropologiche e Geografiche e di parte del Dipartimento di Studi Linguistici e Orientali.

In considerazione delle sue dimensioni e della sua complessità culturale, il Dipartimento si è articolato in Sezioni allo scopo di comunicare con maggiore completezza ed efficacia le molte attività di ricerca e di didattica che si svolgono al suo interno. Le Sezioni sono: 1) Archeologia; 2) Storia antica; 3) Geografia; 4) Studi antropologici, orientali, storico-religiosi; 5) Medievistica; 6) Scienze del Moderno. Storia, Istituzioni, Pensiero politico.

Il Dipartimento ha inoltre deciso di procedere ad una riorganizzazione unitaria di tutta la sua editoria scientifica attraverso l'istituzione di una Collana di Dipartimento per opere monografiche e volumi miscellanei, intesa come Collana unitaria nella numerazione e nella linea grafica, ma con la possibilità di una distinzione interna che attraverso il colore consenta di identificare con immediatezza le Sezioni.

Nella nuova Collana del Dipartimento troveranno posto, dopo rigorosi referaggi esterni, i lavori dei colleghi, ma anche e soprattutto i lavori dei più giovani che si spera possano vedere in questo strumento una concreta occasione di crescita $\mathrm{e}$ di maturazione scientifica.

\section{Comitato editoriale}

Direttore: Andrea Augenti

Codirettori: Francesca Cenerini, Antonio Curci, Cristiana Facchini, Claudio Minca (Responsabili di Sezione)

\section{Comitato Scientifico}

\section{Archeologia}

Mauro Menichetti (Università degli Studi di Salerno)

Timothy Harrison (University of Toronto)

Annalisa Marzano (University of Reading)

\section{Storia Antica}

Arnaldo Marcone (Università degli Studi Roma Tre)

Denis Russet (École Pratique des Hautes Études, Paris)

\section{Geografia}

Michael Buzzelli (University of Western Ontario)

Dino Gavinelli (Università degli Studi di Milano)

\section{Studi antropologici, orientali, storico-religiosi}

Nazenie Garibian (“Matenadaran”, Scientific Research Institute of Ancient Manuscripts -Yerevan, Armenia)

Ruba Salih (School of Oriental and African Studies, University of London) 
Andrea Gaucci

\section{Iscrizioni della città etrusca di Adria}

\section{Testi e contesti tra Arcaismo ed Ellenismo}


Il saggio è stato sottoposto a blind peer review.

Bononia University Press

Via Ugo Foscolo 7, 40123 Bologna

tel. $(+39) 051232882$

fax $(+39) 051221019$

www.buponline.com

info@buponline.com

Quest'opera è pubblicata sotto licenza Creative Commons BY-NC-SA 4.0

ISSN 2284-3523

ISBN 978-88-6923-713-3

ISBN online 978-88-6923-714-0

DOI $10.30682 /$ disciarche28

In copertina: Iscrizione CIE 20822. Fotografia di Olivo Bondesan. Adria, Museo Archeologico Nazionale -

Direzione Regionale Musei Veneto. Su concessione del Ministero per i Beni e le attività culturali e per il turismo.

Impaginazione: DoppioClickArt - San Lazzaro (BO)

Prima edizione: marzo 2021 
Ai miei genitori, ai miei maestri, a tutto il personale del Museo Archeologico Nazionale di Adria 



\section{Sommario}

Prefazione

G. Sassatelli, E. Govi, S. Bonomi

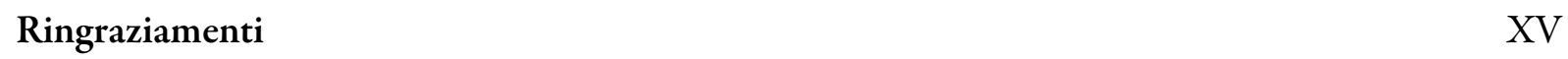

Introduzione 1

1. Quadro storico-archeologico di Adria preromana 1

1.2. Storia degli scavi 4

2. Le ragioni di una analisi 9

I. Storia degli studi e quantificazione delle iscrizioni etrusche 13

$\begin{array}{ll}\text { I.1. Il dibattito scientifico tra XIX e XX secolo } & 13\end{array}$

$\begin{array}{ll}\text { I.2. La quantificazione delle iscrizioni } & 20\end{array}$

I.2.1. Adria e l'Etruria padana tra la fine del VI e gli inizi del IV sec. a.C. 21

I.2.2. Adria e l'Etruria padana nel periodo ellenistico 26

II. Supporti, posizione, tecniche di scrittura 29

II.1. Supporti 29

II.1.1. Ceramica depurata etrusco-padana 29

$\begin{array}{ll}\text { II.1.2. Ceramica attica } & 31\end{array}$

II.1.3. Ceramica grezza $\quad 32$

$\begin{array}{ll}\text { II.1.4. Bucchero } & 33\end{array}$

II.1.5. Ceramica grigia $\quad 33$

II.1.6. Ceramica a v.n. di periodo ellenistico 34

II.1.7. Anfore greco-italiche 36

II.2. Posizione e impaginazione $\quad 37$

II.3. Tecniche di scrittura, ductus, legature $\quad 39$

III. Il sistema scrittorio $\quad 43$

III.1. Il quadro delle norme scrittorie tra VI e II sec. a.C. 43

III.2. La paleografia tra VI e II sec. a.C.

$\begin{array}{ll}\text { III.3. Puntuazione } & 73\end{array}$

$\begin{array}{ll}\text { III.4. Abbreviazioni e lettere in legatura } & 73\end{array}$

$\begin{array}{ll}\text { IV. Onomastica } & 79\end{array}$

$\begin{array}{ll}\text { IV.1. Il periodo tardo-arcaico } & 80\end{array}$

IV.2. V e IV sec. a.C. 
IV.3. Fine IV-II sec. a.C. $\quad 86$

IV.3.1. Le formule onomastiche $\quad 86$

$\begin{array}{lr}\text { IV.3.2. Classificazione delle iscrizioni con formule onomastiche } & 109\end{array}$

$\begin{array}{ll}\text { IV.3.3. I nomi familiari } & 114\end{array}$

$\begin{array}{ll}\text { V. Alfabetari, teonimi, lessico } & 123\end{array}$

$\begin{array}{ll}\text { V.1. Sequenze alfabetiche } & 123\end{array}$

V.2. $A l$, il "dono" 126

V.3. Teonimi e lessico del sacro 127

V.4. Altre voci di lessico (helis, $\theta i$, sesiu, tutis, usti/uzti) 130

$\begin{array}{ll}\text { VI. Epigrafia in contesto } & 135\end{array}$

$\begin{array}{ll}\text { VI.1. Abitato } & 135\end{array}$

VI.1.1. Gli scavi presso l'attuale Museo Archeologico Nazionale $\quad 142$

$\begin{array}{ll}\text { VI.1.2. Il cortile di Antonio Ornati } & 149\end{array}$

VI.1.3. I fondi Lodo e Calisti nella zona sud-orientale della città 151

VI.1.4. I reperti del Museo Domestico della Famiglia Bocchi 152

VI.2. Sepolcreti urbani 154

VI.2.1 Il settore meridionale $\quad 154$

VI.2.2 Il settore orientale $\quad 189$

$\begin{array}{ll}\text { VI.3. Il suburbio } & 195\end{array}$

Conclusioni. Oltre 400 anni di cultura epigrafica e società 207

1. La formazione della società etrusca nell'Arcaismo 208

2. Indizi di continuità culturale tra V e IV sec. a.C. 210

3. Una società in evoluzione tra III e II sec. a.C. 211

$\begin{array}{ll}\text { Bibliografia } & 217\end{array}$

Indice dei tituli di CIE IV, I, 1

$\begin{array}{ll}\text { Tavole } & 243\end{array}$ 


\section{PREFAZIONE}

Nel 2017 è stato pubblicato il fascicolo IV,1 del Corpus Inscriptionum Etruscarum dedicato ad Adria, esito di un lungo lavoro di ricerca di Andrea Gaucci. La prestigiosa iniziativa del Corpus, avviata nel 1893 sotto l'egida dell'Accademia delle Scienze di Berlino e successivamente anche sotto quella dell' Istituto Nazionale di Studi Etruschi e Italici, in tutti i 14 fascicoli fino ad ora pubblicati e variamente distribuiti all'interno di 3 volumi, ha sempre accolto solo iscrizioni dell'Etruria propria. Con questo fascicolo dedicato ad Adria, si è inaugurato un nuovo volume del Corpus (il IV volume che a sua volta sarà suddiviso in almeno 3 fascicoli) destinato ad accogliere le iscrizioni dell'Etruria Padana e dei territori vicini. Si tratta di una importante novità nella storia della disciplina, a conferma di una posizione ormai consolidata nei nostri studi che considera anche aree fino ad ora giudicate "periferiche" come parte integrante dell'Etruria e dei suoi processi storici.

Le norme e la consuetudine redazionale del Corpus impongono una drastica selezione delle informazioni contenute nelle singole schede, che di fatto sono limitate ad una oggettiva descrizione del dato epigrafico, con qualche apertura alle diverse letture, anch'esse solo presentate e non discusse. $\grave{E}$ un tratto che risponde alla impostazione ottocentesca e positivistica del Corpus, ma che si giustifica anche per ragioni di spazio editoriale vista la grande quantità dei documenti via via presi in considerazione. Nasce proprio da questo limite strutturale l'idea di un volume che raccolga in modo organico e completo tutta l'analisi che la ricca documentazione presentata consente, forzatamente esclusa dal fascicolo del Corpus appena pubblicato. Si tratta di una esigenza ancora più pressante nel caso di Adria dove la documentazione epigrafica, assai più consistente di quanto si potesse ipotizzare (oltre 1.000 documenti), nella quasi totalità è riferibile a contesti precisi e relativamente ben documentati (vari settori dell'abitato, necropoli, aree sacre, territorio circostante) ed è quindi accompagnata da una grande quantità di informazioni, ricavabili dallo studio archeologico di materiali e di contesti, che vanno ben oltre il dato epigrafico e linguistico. La grande quantità di questi dati extra-testuali da un lato favorisce una comprensione più piena dello stesso dato epigrafico, e dall'altro consente una valutazione storica di Adria e del suo territorio affatto nuova e ricca di conseguenze.

Alla acribia e al rigore filologico dimostrati nella raccolta e nella presentazione dei dati per il Corpus, Andrea Gaucci ha saputo accostare ora una notevole capacità di organizzare in modo solido ed 
efficace la analisi ed un ampio commento alla documentazione. Alla presentazione del quadro storicoarcheologico di Adria preromana, aggiornato, completo e assai bene articolato, e alla storia degli studi, entrambe utilissime e ricche di alcune importanti novità, seguono i capitoli più propriamente legati alle tematiche epigrafiche e linguistiche. Si parte dall'analisi dei supporti, suddivisi per tipi di produzione, con considerazioni nuove sulla posizione e sulla impaginazione delle singole iscrizioni, sui $d u c-$ tus e sulle legature. Segue una serrata analisi del sistema scrittorio e della varietà delle sue norme che nel quadro generale della epigrafia etrusca tra VI e II secolo a.C. sono ricche di implicazioni storiche (vedi oltre). Si passa poi all'onomastica, rigorosamente esaminata in una puntuale e dettagliata sequenza cronologica che ci consente di entrare in modo molto concreto nelle principali fasi della storia sociale della città e delle sue relazioni con i vicini ambiti territoriali e culturali. Con un taglio più contestuale e linguistico sono quindi affrontati gli alfabetari, i teonimi e alcune importanti questioni lessicali. Un ultimo capitolo, dedicato alla "Epigrafia in contesto" riprende quelle specificità della documentazione epigrafica di Adria cui si è fatto cenno affrontandone ed approfondendone tutti i principali aspetti. I singoli documenti epigrafici sono ricondotti al loro contesto e più precisamente all'abitato, all'interno del quale rientrano le aree sacre, alle necropoli e alle singole sepolture, al territorio circostante. La "rilettura" della documentazione epigrafica all'interno dei singoli contesti archeologici consente alcuni importanti passi avanti nella interpretazione e nella valutazione storica di entrambi. Le conclusioni raccolgono infine tutte queste considerazioni approdando ad un quadro storico della città assolutamente nuovo e del tutto inaspettato. Se ne indicano qui solo i punti salienti.

Sul piano della scrittura, anche a seguito di alcune importanti osservazioni di Giovanni Colonna, si era ormai consolidata l'idea di un legame molto stretto per non dire esclusivo di Adria con la cultura scrittoria etrusco-meridionale, in particolare orvietana. In realtà risultano utilizzate ad Adria entrambe le norme scrittorie con una significativa presenza di pratiche epigrafiche diverse e non rigidamente alternative, chiaro segnale di una mescolanza che non si presta a categorizzazioni troppo rigide e lascia intravedere uno spettro di aree etrusche interessate a questa precoce presenza in Adriatico molto più ampio e articolato di quello che si poteva pensare. È una mescolanza che trova conferma anche nell'onomastica, la quale a sua volta documenta un'ampia apertura verso tutta l'Etruria, senza distinzioni, ma anche verso ambiti culturali e territoriali diversi dall'Etruria, come il mondo venetico, retico e celtico, in linea del resto con le profonde connessioni che si evincono dalle produzioni artigianali, dai rapporti culturali e delle relazioni commerciali. Se ne ricava in buona sostanza l'idea di una città fortemente intrecciata non solo con tutta l'Etruria meridionale e settentrionale, e ovviamente con l'Etruria padana, ma anche con tutte le altre realtà dell'Italia settentrionale (Veneti, Reti, Celti golasecchiani).

Molto importanti anche le nuove acquisizioni sul piano del sacro, sia in rapporto alle divinità che rispetto al lessico. E soprattutto quelle che toccano da vicino la struttura sociale della sua popolazione con particolare riguardo alla possibile individuazione di una solida élite di cultura schiettamente etrusca, forse articolata in classi, aperta però alla inclusione di individui privi di tutti i diritti o di servi, specialmente se di origine alloctona. Ancora una volta un'Adria aperta e fortemente articolata al suo interno dove alla ricchezza e alla complessità dei corredi funerari fanno da riscontro le testimonianze epigrafiche ed onomastiche.

Non mancano tra l'altro alcune preziose testimonianze della città anche sul piano istituzionale se è giusta l'integrazione e l'interpretazione delle tre occorrenze del digramma śp inteso come abbreviazione di *śpura, con allusione alla comunità nel suo insieme, forse in una pratica di dono "pubblica". Molto importanti anche alcune considerazioni sulla tarda etruscità padana di area adriatica, consentite dalla documentazione epigrafica. All'interno di quella vivacità economica, produttiva e commerciale e culturale, che è una acquisizione relativamente recente ma ormai ben consolidata nei nostri studi, si intravedono comportamenti e processi articolati oltre che diversificati nel tempo. Le testimonianze epigrafiche evidenziano continuità e diversità all'interno di questo fenomeno. Tra III e II secolo a. C. ad Adria, come a Spina, si assiste ad un aumento considerevole delle testimonianze epigrafiche che lasciano intravedere un uso più ampio e generalizzato della scrittura. Questo fenomeno, che traduce una 
fedeltà alla tradizione scrittoria e alla cultura etrusca refrattaria a stimoli esterni, sembra rispondere all'esigenza culturale, ma anche ideologica e politica di difendere e salvaguardare la propria identità etrusca all'interno del più generale naufragio che l'aveva travolta in tutta l'area padana.

Ma la novità maggiore del libro è il riconoscimento pieno e ben motivato della etruscità di Adria. Per la sua posizione ai margini nord-orientali dell'Etruria padana e per la sua vicinanza al mondo veneto, in passato si era spesso dubitato di questa identità culturale insistendo più del dovuto su quei caratteri di mescolanza e di intreccio che la città indubbiamente presenta. È assolutamente vero che la città mostra grandi aperture verso altri ambiti, vicini (Veneti, Reti, Celti) e lontani (Greci e Italici), esattamente come Spina. Ma proprio come a Spina tutto questo si innesta su una forte e prevalente etruscità di fondo, linguistica, sociale e culturale, che trova ora una piena conferma dal dato epigrafico. Si tratta di una acquisizione molto importante. Fino ad ora infatti quando si ricorreva a quella bellissima sintesi di Tito Livio sugli Etruschi controllori di entrambi i mari dell'Italia, "quello di sotto" cioè il Tirreno, e "quello di sopra" cioè l'Adriatico (una supremazia sostanziata tra l'altro dai due thesauroi etruschi di Care e di Spina nel santuario di Delfi), eravamo per così dire costretti di fronte alla straordinaria efficacia di questo quadro ad ammettere la fragilità della sua motivazione. Livio infatti sottolinea che il nome "Adriatico" dato al "mare di sopra" è la conseguenza di una supremazia etrusca perché deriva da Adria "colonia etrusca". E su questa "Adria colonia etrusca" abbiamo dovuto fare tutti un po' di sforzi, quanto meno attenuando la perentorietà dell'asserzione liviana. Ora non è più necessario perché Livio sa esattamente quello dice e mostra ancora una volta una conoscenza profonda dei fenomeni storici di cui si occupa e che sintetizza con grande efficacia.

La comunità scientifica deve essere grata ad Andrea Gaucci per il lavoro svolto nella confezione del $C I E$ di Adria e per lo sforzo di condensare in questo volume le analisi e le riflessioni che da quello sono derivate, dimostrando come lo studio dell'epigrafia etrusca sia uno strumento imprescindibile per la ricostruzione storica. Questo libro costituisce anche un punto di svolta nella ricerca. Esso indica infatti una direzione, un modo di affrontare l'epigrafia che, sviluppando tutte le potenzialità di una lettura contestuale, approda ad analisi complesse che travalicano la lettura del documento valorizzando le specificità del contesto. Anche in questo il lavoro di Gaucci, che prende le mosse da una impostazione della Cattedra di Etruscologia e Archeologia Italica di Bologna, potrà dare un sostanziale contributo alla conoscenza e agli studi. Analisi sistematiche ed approfondite su siti e su aree territoriali in una prospettiva diacronica ampia portano sempre esiti davvero significativi per la ricerca.

Elisabetta Govi

Professore Ordinario di Etruscologia e Antichità Italiche Alma Mater Studiorum - Università di Bologna

Giuseppe Sassatelli

Professore Emerito di Etruscologia e Archeologia Italica Alma Mater Studiorum - Università di Bologna 

Questo lavoro di Andrea Gaucci è l'integrazione critica e interpretativa del suo monumentale volume dedicato alle iscrizioni di Adria del Corpus Inscriptionum Etruscarum, indispensabile per contestualizzare dal punto di vista archeologico e vestire di significato storico il consistente patrimonio epigrafico adriese.

Come il lettore avrà modo di apprezzare, si tratta di uno studio preciso e approfondito, frutto di anni di fatica e impegno, che ha il pregio di attirare ancora una volta su Adria l'attenzione che essa sempre merita per le sue peculiarità, utilizzando documenti straordinari attraverso i quali emergono direttamente dal passato remoto della città i nomi di uomini e donne che la abitarono.

Qui si ha la definitiva e inoppugnabile dimostrazione che Adria fu una polis etrusca, eliminando finalmente quei dubbi che sempre l'hanno confinata ai margini degli studi e degli interessi scientifici come un'entità indefinibile e nebulosa, né greca né etrusca né veneta, sulla quale le fonti storiche erano avare di notizie, certamente secondaria e trascurabile.

Negli anni in cui mi furono affidate sia la direzione del Museo Archeologico Nazionale di Adria sia la tutela della città e del territorio bassopolesano, dal 1989 al 2009, ho sofferto di questa marginalità perché nel riordinare progressivamente le collezioni museali e nell'affrontare numerose campagne di scavo mi ero ben presto convinta dell'etruscità dell'antico centro pur in una quadro multietnico e dell'eccezionale ricchezza e varietà della documentazione archeologica, sia preromana sia romana, che meritava di essere studiata e pubblicata. Ho avuto la fortuna di accogliere in Museo molti giovani studiosi coraggiosi che hanno affrontato con intraprendenza e passione ricerche lunghe e complesse su contesti e classi di materiali dalle quali sono scaturiti importanti articoli e monografie che hanno progressivamente fatto uscire Adria dalla zona d'ombra in cui era relegata. Il mio e il loro lavoro sono stati poi alla base dell'ordinamento scientifico del riallestimento organico del Museo conclusosi nel 2009. Molti anni sono passati nel frattempo, sono proseguiti scavi e studi che hanno affinato, modificato e approfondito le conoscenze.

Il presente studio di Andrea Gaucci, il più recente della serie sopra ricordata, illustra con chiarezza l'evoluzione e la complessità della società adriese così come desumibili dalla documentazione epigrafica, stabilendo importanti punti fermi e aprendo nuove questioni. 
Resterà ancora aperta una vecchia questione, ovvero se la fondazione di Adria sia da attribuire a iniziativa greca o etrusca: solo un rinvenimento fortunato, ammesso che accada, potrà dare una risposta certa. Se possiamo ritenere plausibile l'ipotesi che prospectors di origine greca abbiano per primi individuato il luogo come favorevole ai commerci, è invece certo - e lo dimostrano soprattutto gli scavi in via Ex Riformati - che solo agli Etruschi intorno alla metà del VI secolo a.C. può essere attribuito l'impianto urbanistico della città con la sua rete regolare di canali e con gli isolati costituiti da riporti su piattaforme e fascinate lignee e delimitati da palificate.

Di lingua etrusca doveva essere quindi il potere politico che governava la città portuale, ma quale sia stato il suo assetto iniziale e quale sia stata la sua evoluzione nel tempo rimangono a tutt'oggi materia di ipotesi. Non credo che si possa parlare di una vera e propria aristocrazia, semmai di un'oligarchia forse inizialmente in mano ai fondatori, che successivamente nella liquida e inclusiva società dello scalo si è aperta a homines novi, anche stranieri, ricchi e intraprendenti.

Sulla scorta di quanto ci fanno intravvedere i documenti epigrafici sulla società adriese occorre ora tornare al riesame dell'abbondante documentazione archeologica, soprattutto delle necropoli, che ancora attende di essere puntualmente esaminata e che richiederà altri anni di lavoro e altri giovani coraggiosi che vorranno affrontarlo.

Simonetta Bonomi

Soprintendente Archeologia Belle Arti e Paesaggio del Friuli Venezia Giulia 


\section{RINGRAZIAMENTI}

Il titolo scelto, Le iscrizioni della città etrusca di Adria, richiama in maniera neanche troppo velata il volume Le iscrizioni e i graffiti della città etrusca di Marzabotto a cura di Giuseppe Sassatelli. Nel presente lavoro su Adria, inteso come commento alle iscrizioni del fascicolo adriese del CIE, manca una completa analisi dei graffiti, ma medesima è l'attenzione verso l'inestricabile relazione tra i testi, brevi o lacunosi che siano, il loro medium materiale e il contesto di pertinenza, nella consapevolezza che solo mettendo a sistema questi aspetti si può arrivare a una soddisfacente e consapevole analisi. A Giuseppe Sassatelli e a Elisabetta Govi devo tanti ringraziamenti, troppi da ricordare tutti, ma qui mi preme ringraziare entrambi per avermi proposto la tesi magistrale sulle iscrizioni etrusche di Adria e portato al Museo. Quel giorno è nato un legame indissolubile con quel luogo. E ciò è merito di Simonetta Bonomi, che lo ha fatto diventare nei suoi anni di direzione un laboratorio dove anche uno studente acerbo è accettato e lasciato lavorare con piena liberalità, nella convinzione che solo la comunità e non il singolo individuo potrà portare nuovamente in luce il passato di una città di cui sappiamo ancora troppo poco. D'altra parte, dopo gli scavi e la riorganizzazione della raccolta tra gli anni Sessanta e Settanta del secolo scorso nei quali ebbe un ruolo fondamentale Umberto Dallemulle, proprio Simonetta, come funzionario, è stata protagonista di una nuova e ricca stagione di scavi, che ha portato al completo riallestimento dell'esposizione così come oggi lo vediamo (progetto ben documentato dalle tre Guide edite tra il 2006 e il 2010). A lei si devono in particolare gli scavi della necropoli tardo-arcaica di Ca' Cima e le molte altre di periodo ellenistico, nonché importanti indagini sull'abitato preromano, che hanno contribuito a conoscere in maniera più consapevole e approfondita l'insediamento preromano.

Dopo Simonetta, passata al ruolo di Soprintendente, altre Direttrici sono arrivate, Giovanna Gambacurta, Maria Cristina Vallicelli e adesso Alberta Facchi. A loro va la mia più grande riconoscenza, anzi tutto per aver continuato questa splendida tradizione di apertura e liberalità. Ringrazio anche la Direzione Musei del Veneto, che ha sempre favorito il mio lavoro e come ultimo atto ha concesso l'autorizzazione alla pubblicazione di documenti e fotografie dall'Archivio storico del Museo. Le immagini contenute nel volume sono infatti su concessione del Ministero per i Beni e le attività culturali e per il turismo. 
Al Museo si possono passare lunghe giornate di studio nei depositi, che di fatto sono un museo essi stessi, così ben ordinati grazie alla perizia e alla straordinaria cura che dedica loro Leonardo Di Simone, che dell' istituzione è pietra portante, assieme a tutto il personale, che sempre mi ha accolto con grande calore: Damiano Fabbri, Fabrizio Boscarato, Rosa Damiano, Giovanna Furlanetto, Romeo Giribuola, Luciana Nicoletto, Susanna Ruzza, Roberto Sanavio, Silvana Villani, Antonio Zandonà, Daniele Zanirato. Un pensiero particolare va a Olivo Bondesan, che troppo presto ci ha lasciati. Alla sua memoria dedico la copertina, che ospita una delle bellissime fotografie da lui realizzate con alta professionalità.

La mia riconoscenza va anche ad Adriano Maggiani, Luciano Agostiniani, Enrico Benelli, Anna Marinetti, Paolo Poccetti, Valentina Belfiore, Alberto Calderini, Riccardo Massarelli, Luca Rigobianco, che in maniera più o meno profonda, con i loro lavori e stimoli hanno contribuito a che avviassi e concludessi questo commento al fascicolo adriese del CIE.

Molte altre sarebbero le persone ancora da ringraziare e spero non me ne vogliano, ma questo libro è votato ad Adria e a tutte le comunità che la formano, non importa di quale tempo!

Con questo testo ho esaurito il mio debito verso l'epigrafia adriese, ma con la città etrusca è solo un arrivederci... o come scriverebbe Leonardo, un etrusco saluto. 


\section{INTRODUZIONE}

\section{Quadro storico-archeologico di Adria preromana}

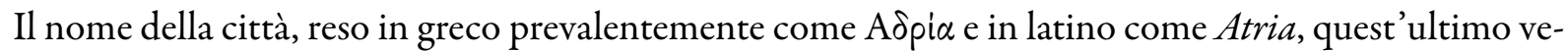
rosimilmente derivante dal nome etrusco ${ }^{1}$, è attestato per la prima volta da Ecateo di Mileto (FGrHist, $1 \mathrm{~F} 90=99 \mathrm{Nenci}$ ) alla fine del VI sec. a.C. Secondo Ecateo, la città avrebbe dato nome all'omonimo fiume (identificabile con il corso del Tartaro-Po) e alla parte più settentrionale del mare Adriatico ${ }^{2}$. Nelle fonti antiche la città viene spesso identificata come colonia etrusca, mentre solo Pompeo Trogo (apud Iust. XX 1, 9), probabilmente riprendendo da Filisto di Siracusa, la definisce greca ${ }^{3}$. G. Colonna ha più volte suggerito di vedere in Adria la colonia senza nome fondata dagli Egineti nel 519 a.C. presso gli Umbri (Strab. VIII 6, 16), ma non vi sono riscontri documentari in merito se non le iscrizioni greche riconosciute dal medesimo Colonna come opera di Eginetí.

Non sono note informazioni dirette riguardo il passaggio di Adria nell'orbita romana, definitivamente inserita con il processo istituzionale che, dopo l'estensione della cittadinanza latina nell'89 a.C. con la lex Pompeia de Transpadanis, arriva alla creazione del municipium, avvenuta nel 42 a.C. successivamente alla lex Roscia del 49 a.C. che concedeva la cittadinanza romana 5 . La città

${ }^{1} L V$, p. 633. Per una rassegna delle attestazioni del nome della città nelle fonti antiche, cfr. GiANGiULIO 1984, pp. 38-39.

2 Colonna 1974a, p. 13, nota 42.

3 Sulle fonti relative ad Adria, si rinvia alla rassegna in Giangiulio 1984, p. 39.

4 Colonna 1974a, pp. 5-10; Colonna 2003, p. 169. L. Braccesi invece colloca a Rimini questa colonia (BRACCESI 2006, p. 99), mentre M. Luni ha proposto Numana (Luni 2004, p. 56). Sulle iscrizioni greche, cfr. AntonetTi 2005, p. 123, nota 38, con riferimenti. Per successive posizioni di maggiore prudenza sull'ipotesi di una colonia egineta ad Adria, si vedano i riferimenti riportati in ibid., pp. 126-127, nota 54, e Colonna 2008, p. 46, nota 36. Dubbi sulla reale fondazione di una colonia di Egina in Adriatico sono espressi da F. Cordano e quindi ripresi da C. Antonetti (2005, pp. 126-127, con precedenti riferimenti).

5 Cfr. Buchi 1984, pp. 81-82. Per quanto concerne il III e il II sec. a.C., cfr. GaucCi 2016, pp. 195-196, 206-207, con riferimenti; per una sintesi, cfr. BONOMI 2003a, pp. 460-461. 
continuò comunque a godere di prestigio, se nel I sec. d.C. Plinio parla del «nobili portu oppidi Tuscorum Atriae» (Nat. hist. III 120)6.

Se questi sono i pochi dati desumibili a grandi linee dalle fonti antiche sulle vicende storiche e culturali di Adria nel periodo preromano, grazie a una maggior attenzione verso il dato archeologico e agli scavi condotti in particolare negli ultimi decenni, ormai la città è riconosciuta come etrusca per cultura e lingua almeno dagli ultimi decenni del VI sec. a.C. e fino al completo inserimento nell'orbita romana ${ }^{7}$.

Durante il VI sec. a.C., quando va collocata l'apertura da parte dei Focei della rotta verso l' Adrias, cioè il tratto di mare tra la foce del Po e l'Istria, se valido quanto riportato da Erodoto (I 103, 1) ${ }^{8}$, il sistema politico e insediativo della costa medio-alto adriatica subì un consistente riassetto. A nord, le più aggiornate ricerche riconoscono nell'antico corso dell'Adige il limite fra l'area veneta a nord e i territori a sud-ovest di pertinenza etrusca9 ${ }^{9}$. Mentre in Veneto l'asse politico-economico era quello plavense e il centro costiero proiettato sull'Adriatico era senza alcun dubbio Altino, a sud dell'Adige, nel Polesine fino a quel momento spopolato, si svilupparono insediamenti del tutto nuovi, cioè Adria lungo il fiume Tartaro e San Basilio sulla costa. Allo stato attuale degli studi, risulta difficile definire gli attori politici che determinarono la nascita di questi porti fluviali ${ }^{10}$. E possibile ritenere che Adria e San Basilio si siano formati come empori greci ${ }^{11}$, probabilmente su impulso foceo, con la funzione di drenare le risorse che giungevano nell'Adriatico settentrionale. Un modello plausibile potrebbe prevedere che Adria sia nata sotto la garanzia politica etrusca e che dovette rappresentare per i Greci una alternativa al commercio direttamente gestito dai Veneti tramite Altino, che catalizzava, sotto il controllo di Padova, le merci provenienti dalle rotte più orientali. D'altra parte, per gli Etruschi questo empórion rappresentava l'opportunità di affermarsi nuovamente (dopo Verucchio) e con nuova forza sulla costa adriatica, forse anche grazie al consenso di Este, che controllava le direttrici commerciali alpine. Appare diverso il caso di San Basilio, probabilmente un esperimento alternativo ad Adria ${ }^{12}$, su cui adesso si stanno concentrando nuove ricerche.

La fase più antica di Adria è documentata finora da pochi frammenti ceramici corinzi e grecoorientali ${ }^{13}$, da un livello insediativo di pieno VI sec. a.C. accertato negli scavi di via Ex Riformati (2015-2016), nonché da un ridotto numero di sepolture databili tra la metà e la seconda metà del VI sec. a.C., caratterizzate da corredi molto ridotti e con una connotazione culturale di carattere misto ${ }^{14}$. Negli oggetti di marca culturale etrusca, greca, retica, veneta destinati a questi corredi si

${ }^{6}$ Per ciò che concerne il periodo imperiale, cfr. Bonomi 2003a, pp. 460-461.

7 Si veda il quadro di sintesi offerto da SASSATELLI 2008a, pp. 81-85; più recentemente GaUCCi 2017.

8 Un sintetico quadro è offerto in GaUCCI 2017, con riferimenti. Sui materiali che documentano le rotte greche in adriatico, si rimanda a Colonna 2003, pp. 156-157, con riferimenti. Non va comunque sottovalutato il ruolo di Corinto e soprattutto di Corcira nell'attivazione di queste rotte (BRACCESI 2001, pp. 39-43, e ANTONELli 2008, pp. 165-167; per le fonti e in particolare il periodo tra VI e V sec. a.C., si veda INTRIERI 2010).

9 Capuis, Gambacurta 2015, p. 454.

10 Gli studiosi hanno portato il loro punto di vista, spesso diverso, relativamente agli attori politici che hanno determinato la nascita di Adria. L. Braccesi e poi A. Maggiani (Maggiani 2008, pp. 342, 344, con riferimenti), hanno identificato Este come il centro egemone emanatore, mentre M. Harari, G. Sassatelli, E. Govi hanno piuttosto visto lo sviluppo dell'emporio come una iniziativa congiunta di Greci ed Etruschi (HARARI 2001, p. 45; SASSATEli 2008a, pp. 81-85; Govi 2012, p. 144). Anche secondo G. Colonna l'impulso sarebbe venuto dagli èmporoi greci, fra i quali un ruolo preponderante lo ebbero i Focei, con il consenso degli indigeni (Colonna 2003, p. 159, a cui si rimanda anche per il legame tra i Focei e l'idronimo Tartaro).

11 L'empórion per sua natura richiedeva un rapporto tra il potere locale e lo straniero e inoltre aveva il suo fulcro in un santuario che tutelava l'insediamento (Gras 2010). Ad Adria, il santuario è probabilmente quello ipotizzato presso la località Tomba, per il quale si veda oltre.

12 Sassatelli 2008a, pp. 82-83.

13 VAllicelli 2002, con riferimenti.

14 Per l'abitato, Bonomi, Vallicelli, Balista 2020, pp. 194-195. Per la necropoli di Ca' Cima, Bonomi 2003b, pp. 141-142; da ultimo, si rimanda alle considerazioni in GAUCCI 2015, pp. 116-117. 
può ravvisare comunque il ruolo di centro di scambio che aveva l'insediamento. Anche nella vicina San Basilio abbiamo la medesima fisionomia. Pur nella perdita dei pochi dati di necropoli, si hanno anche qui ceramiche corinzie ed etrusco-corinzie, che suggeriscono come fin dallo scorcio del VII sec. a.C. i flussi di beni dall'Etruria meridionale (se non le persone), probabilmente tramite Verucchio (ormai venuto meno come caposaldo di controllo commerciale), arrivassero lungo la costa fin nel Polesine ${ }^{15}$, dove si erano quindi spostati nell'Arcaismo gli interessi degli Etruschi.

I dati archeologici indicano che nel periodo tardo-arcaico un radicale cambiamento trasformò Adria in una città caratterizzata da un intreccio ortogonale di canali e abitazioni in materiali deperibili. Le testimonianze epigrafiche in lingua greca hanno portato G. Colonna a individuare nella parte nord-orientale della città antica, nel luogo detto La Tomba, un santuario dove si offrivano dediche ai greci Apollo e Iride ${ }^{16}$. Questo doveva essere frequentato da Egineti (almeno un antroponimo è femminile ${ }^{17}$ ), ed Etruschi, per i quali S. Bruni ha ipotizzato la presenza di empóroi e artigiani fiesolani, oltre che probabilmente da altri distretti etrusco-settentrionali come quello aretino ${ }^{18}$, ma forse anche Veneti di origine patavina ${ }^{19}$. Adria in effetti si configura come un polo di attrazione unico nel panorama dell'Adriatico settentrionale. Infatti, agli aspetti già citati va aggiunta l'eccezionale (per il comparto padano) concentrazione di vasi figurati di matrice etrusco-tirrenica, che testimoniano il ruolo degli empóroi e forse di artigiani da Orvieto e Vulci ${ }^{20}$, città da cui proviene anche il vasellame bronzeo delle sepolture ${ }^{21}$ e alla cui influenza culturale è da rimandare il numero di kyathoi attici rinvenuti, significativo indicatore al pari della produzione figurata etrusca e che come questa non ha confronto in Etruria padana ${ }^{22}$. La città è inoltre terminal assieme a Gazzo Veronese di una circolazione atesina di prodotti transalpini ${ }^{23}$.

Se gli Egineti dettero impulso allo sviluppo dell'insediamento, e questo è indubbio, non è chiaro quanto profondo sia stato il loro coinvolgimento politico. Allo stato attuale della documentazione una più o meno diretta presenza greca nella città si osserva soprattutto dalle testimonianze del santuario $\mathrm{e}$ dei relativi aspetti cultuali che emergono dall'analisi della cultura materiale ${ }^{24}$. Sebbene si possa accettare l'idea di una comunità di Egineti stanziale ad Adria (ma quali le caratteristiche?), la città tardoarcaica è etrusca in tutti gli aspetti, dall'urbanistica al controllo del territorio, dalla cultura materiale alla ritualità funeraria, e non da ultimo sono da rimarcare gli stretti rapporti culturali, artigianali e commerciali con l'Etruria tirrenica ${ }^{25}$.

15 Colonna 2003, p. 158.

16 Colonna 1974a; ID. 2003, pp. 167-169; Antonetti 2005.

17 Antonetti 2005, p. 128. Si richiama anche l'ipotesi formulata da D. Baldassarra relativa a una connotazione femminile dell'antroponimo espresso dall' iscrizione greca di San Basilio (BALDASSARRA 2013, pp. 118-199, SP IP* 1).

18 BRUNi 2008, pp. 319-321.

19 BRUNi 2011.

20 Govi 2012.

21 Bonomi 2004, pp. 65-69.

22 Cfr. Tonglet 2018, in particolare p. 259.

${ }^{23}$ Per considerazioni di sintesi, cfr. Bonomi 2004, p. 65; G. Gambacurta, in Gambacurta, Ruta Serafini 2018, pp. 25 e 27, e Appendice.

24 Cfr. Gaucci 2012a, pp. 165-166, con riferimenti.

25 Si veda Sassatelli 2008a, pp. 82-85; Gaucci 2012a, pp. 165-166, con riferimenti; Govi 2012; BonOMI, VALLICELli, BALISTA 2020, p. 203. Vale la pena accennare alla spedizione cumana del 524 a.C. (Dion. Hal. VIII 3-4), più volte analizzata da G. Colonna (2003, p. 169; ID. 2005, p. 366, con precedenti riferimenti) e sulla quale è intervenuto anche G. SASSATElli (1999, pp. 84-87); questi studiosi hanno indicato negli Etruschi di Verucchio / Rimini, Bologna e Adria i protagonisti della vicenda assieme a Umbri e Dauni. Secondo Colonna, le implicazioni della spedizione, la cui storicità è sostanzialmente accettata dalla critica, sono molteplici (lo sviluppo del santuario di Cupra Marittima in ambito Piceno, la riorganizzazione del comparto etrusco-campano, il dono del trono al santuario di Olimpia e l'incremento della presenza greca in Adriatico) e un ruolo importante dietro questi fatti dovettero averlo gli Egineti, ma anche le città dell'Etruria interna, Chiusi e Orvieto, il cui peso nella riorganizzazione etrusca della Campania settentrionale e di quella padana è stato più volte sottolineato dalla critica. 
Adria controllava un territorio che si estendeva verso ovest almeno fino all'attuale città di Rovigo, cioè lungo la via di percorrenza lungo il Po che conduceva al fiume Mincio e ai percorsi alpini più occidentali. Le testimonianze archeologiche finora note mostrano un centro attivo fino alla prima metà del V sec. a.C., e poi una sostanziale cesura fino al tardo IV sec. a.C. I più recenti scavi portano a ritenere che l'interruzione dello sviluppo della città sia stata primariamente causata da importanti episodi alluvionali tra la fine del V e buona parte del IV sec. a.C. Proprio questi episodi avrebbero alzato il livello del terreno, mettendo fine alla fase umida che caratterizzò l'insediamento dalla fine del VI sec. a.C. e dando avvio con l'inizio del periodo ellenistico a una nuova fase di maggior stabilità idrogeologica ${ }^{26}$.

Dalla fine del IV sec. a.C., le necropoli testimoniano una continuità culturale etrusca della comunità cittadina fino al passaggio tra II e I sec. a.C., quando fu abbracciato il rituale funerario romano. Adria pare essere stata una città molto ricca ed economicamente florida, in stretti rapporti commerciali e politici con i Celti, in particolare i Cenomani dal II sec. a.C. ${ }^{27}$, e i Veneti. Grazie ad alcuni documenti epigrafici etruschi e latini di fine III-prima metà del II sec. a.C. rinvenuti in sepolture, si può ipotizzare che singoli individui di cultura latina (forse giunti dalla meridionale Rimini?), fossero già stanziati ad Adria in questo periodo. Ma la presenza culturale di Roma è tangibile sicuramente nel pieno II sec. a.C., quando è stata proposta di datare la ristrutturazione dell'abitato, che mantenne l'impianto urbano etrusco ma con la realizzazione di strade lastricate e cloache per interrare i canali che lambivano gli isolati fin dal periodo arcaico ${ }^{28}$.

\subsection{Storia degli scavi}

Notizie di rinvenimenti archeologici ad Adria si hanno già dal XVI sec. ${ }^{29}$. Relativamente all'età preromana, rimane famoso il rinvenimento nel XVII sec. dei bronzetti votivi presso la Chiesa di Santa Maria della Tomba ${ }^{30}$. Tuttavia è solo nel XVIII sec. prima con la collezione Silvestri ${ }^{31}$ e poi con l'attività di Ottavio Bocchi, che prendono avvio ricerche finalizzate espressamente alla conoscenza dell'abitato antico e alla raccolta di testimonianze che ne illustrassero il passato ${ }^{32}$. L'attività della famiglia Bocchi vide quindi con Francesco Girolamo, figlio di Ottavio, la fondazione di un Museo Domestico nel 1770, ben noto alla comunità scientifica dell'epoca; inoltre, assieme al fratello Stefano, questi avviò a partire dal 1803 una serie di indagini volte all'investigazione dell'abitato antico, all'epoca ritenuto etrusco, e prevalentemente svolte nel luogo detto "la Tomba" presso la chiesa summenzionata ${ }^{33}$ (Figg. 1-2, 6). Le indagini continuarono e a partire dal 1809 furono sovvenzionate con fondi pubblici. Le ricerche furono portate avanti anche dopo la scomparsa di F.G. nel 1810 e del fratello Stefano nell'aprile del 1818. Le attività di scavo continuarono fino alla sospensione dei finanziamenti pubblici attorno al $1830^{34}$. Come già sostenuto da G. Colonna ${ }^{35}$, le ricerche dei due fratelli Bocchi meritano un posto nella storia della disciplina etruscologica, in quanto questi volsero, prima dell'avvio di altre importanti imprese nell'Etruria meridionale, il loro interesse verso le vestigia di abitato, indizio di una profonda curiosità intellettuale e sensibilità culturale in un'epoca volta allo scavo sistematico delle necropoli, sicuramente più gratificante (sotto diversi punti di vista).

26 Bonomi, Vallicelli, Balista 2020, passim. Sulla rarefazione dell'evidenza archeologica nel IV sec. a.C., BonoMI 2000, p. 93.

27 Cfr. A. Ruta Serafini, in Gambacurta, Ruta Serafini 2018, pp. 64-71; Gaucci 2016, pp. $195-199$.

28 Bonomi, Robino 2007.

29 Giangivlio 1984, p. 40; Baldassarra 2013, pp. 1-4.

30 BRUNi 2011, p. 125, note 2-4.

31 Zerbinati 1994, pp. 234-244.

32 Giangivlio 1984, p. 40; Baldassarra 2013, pp. 5-9.

33 GiangiUlio 1984, pp. 40-41; WiEL-MARIN 2005, pp. 24-25, 37; BALDASSARRA 2013, pp. 9-15.

34 Baldassarra 2013, p. 15.

35 Colonna 2003, p. 162. 


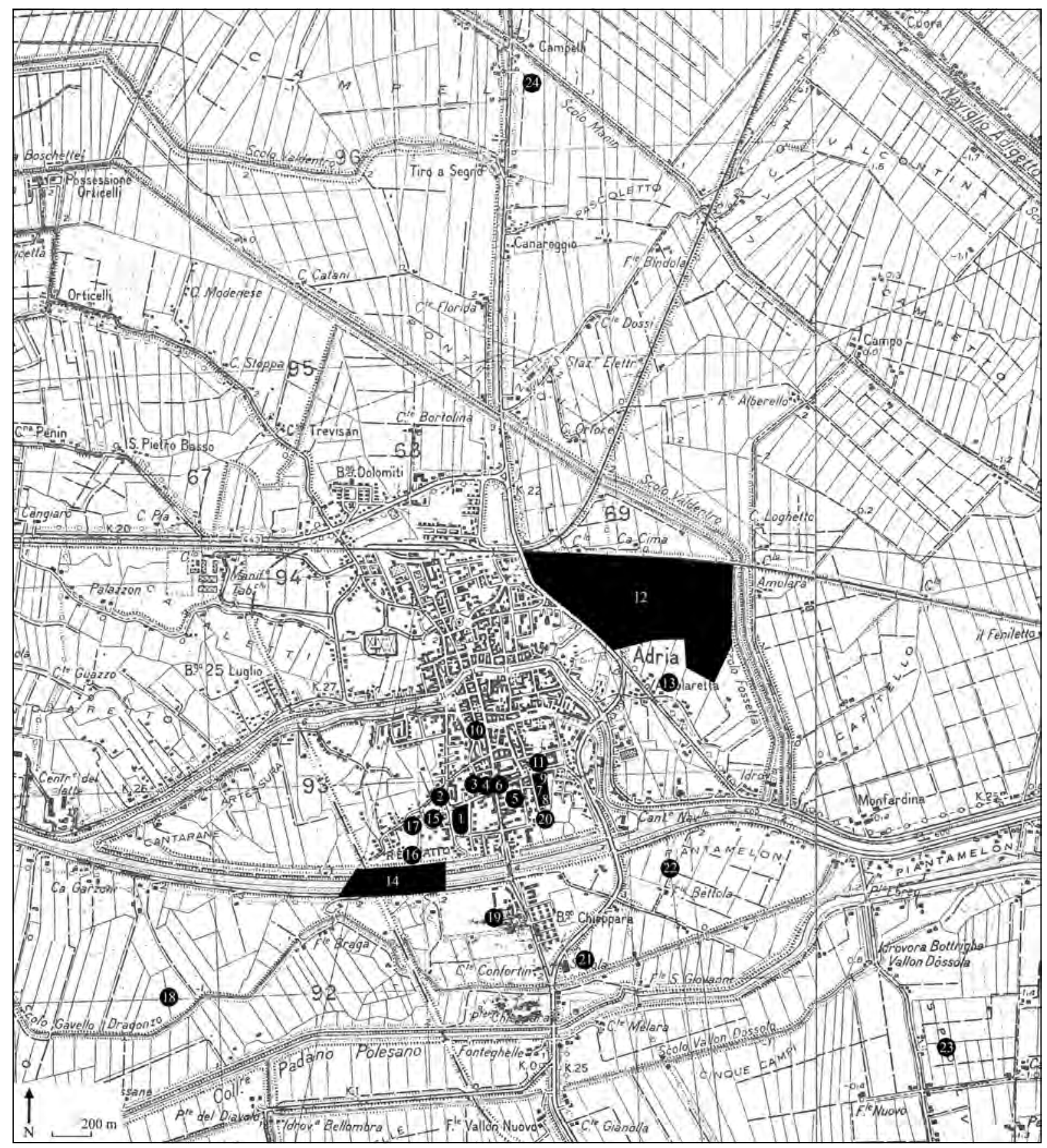

Fig. 1. Planimetria di Adria con indicazioni dei contesti di rinvenimento di iscrizioni e graffiti. Il cortile Ornati è collocato nella sua esatta posizione, come adesso ricostruito grazie al rinvenimento delle piante originali di F.A. Bocchi (Facchi, Vallicelli, Wiel-Marin c.s.).

Un'altra importante stagione di studi e ricerche si ha con il nipote di F.G., Francesco Antonio, che non solo approdò a un catalogo sistematico dei reperti del Museo Domestico ${ }^{36}$, ma grazie a finanziamenti pubblici poté eseguire scavi in alcuni rilevanti punti dell'area della città antica, cioè principalmente nel Pubblico Giardino (attualmente sede del Museo Archeologico), nel cortile Ornati e nel

36 WIEL-MARIN 2005, pp. 45-48. In particolare, si ricorda qui che dovevano esistere cataloghi, seppure parziali, dei materiali rinvenuti negli scavi dei primi decenni dell'Ottocento, non più conservati (ibid., pp. 44-45). 


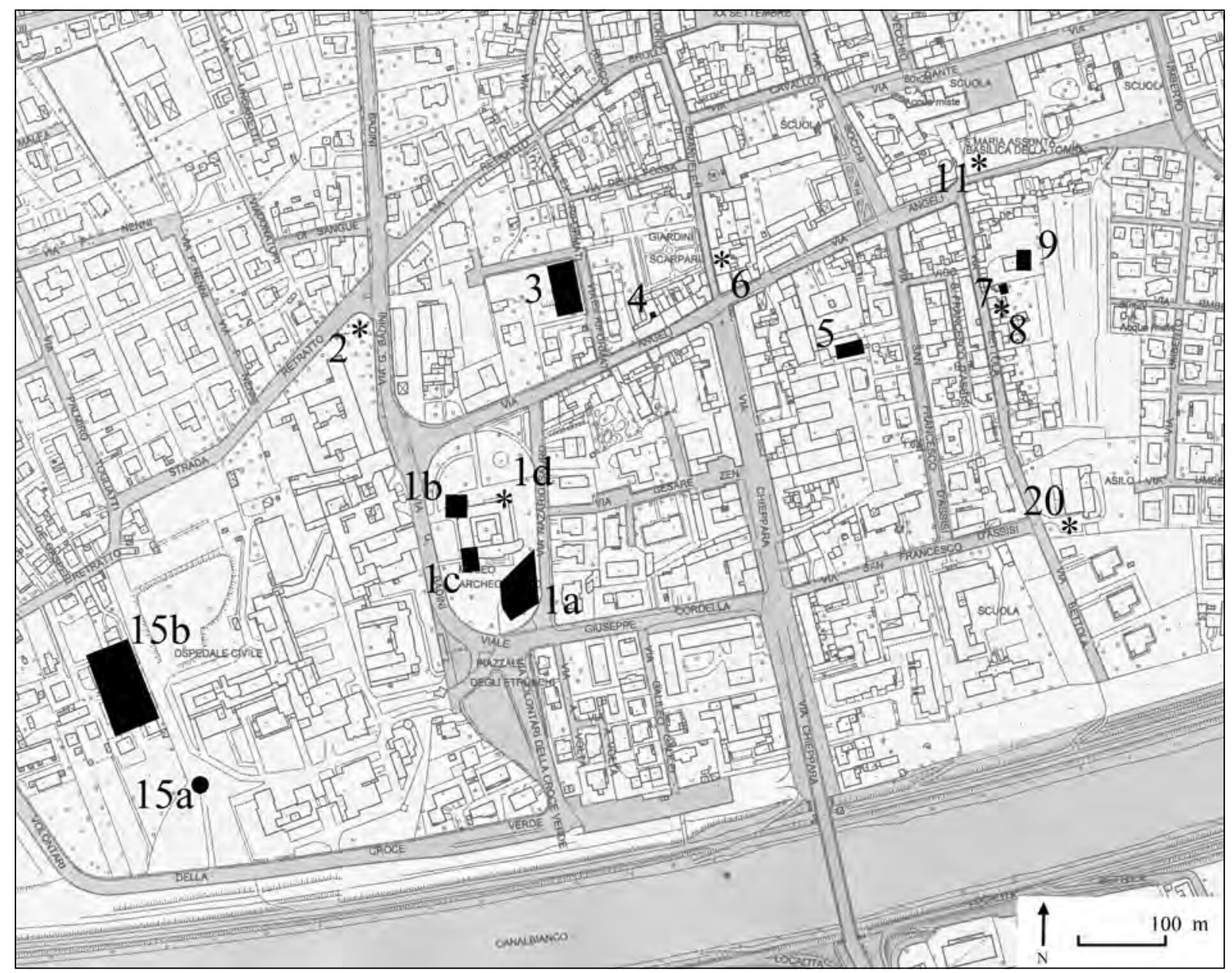

Fig. 2. Dettaglio della parte meridionale dell'abitato moderno con indicazioni dei contesti di rinvenimento di iscrizioni e graffiti. Il cortile Ornati è collocato nella sua esatta posizione, come adesso ricostruito grazie al rinvenimento delle piante originali di F.A. Bocchi (Facchi, Vallicelli, Wiel-Marin c.s.).

fondo Lodo alla Bettola ${ }^{37}$. I reperti recuperati confluirono nella raccolta civica, ordinata dallo studioso secondo i medesimi criteri di quella privata e nella quale era confluita già la collezione civica dovuta agli scavi di inizio secolo finanziati pubblicamente ${ }^{38}$. Contestualmente, nel 1878 fu edito, su iniziativa di Th. Mommsen, il volume di R. Schöne ${ }^{39}$, un catalogo ragionato del Domestico museo, tale era l'interesse internazionale sulla collezione.

I cataloghi manoscritti redatti da F.A. Bocchi e conservati nell'Archivio del Museo Archeologico Nazionale di Adria sono un riferimento fondamentale per le iscrizioni raccolte nelle collezioni del Museo Domestico della famiglia Bocchi e del Museo Civico. Proprio tali manoscritti infatti costituiscono ancora oggi i principali strumenti per poter analizzare gli oggetti delle due collezioni e i loro contesti ${ }^{40}$. Tutti i reperti vennero ordinati secondo classi di materiale formulate dallo studioso e distinte da una sequenza alfabetica primaria contraddistinta da lettere maiuscole (per le ceramiche di

37 Bocchi 1879, pp. 89-106, 212-224; ID. 1888. Si veda anche BonOmi 1993, pp. 75-85, dove si dedica particolare attenzione agli scavi nel Pubblico Giardino.

38 BALDASSARRA 2013, pp. 22-26.

39 SCHÖNE 1878.

40 In particolare, per i reperti del Museo Domestico si è fatto riferimento alla I stesura, che arriva fino alla fine del 1887, mentre la II stesura sembra essere una copia della I stesura nella versione del 1868 (tutte le necessarie informazioni sui cataloghi manoscritti sono presentate in WIEL-MARIN 2005, pp. 44-49, a cui si rimanda per approfondimenti). 
periodo preromano che qui interessano: A, Primo stile; B, Secondo stile; C, Varietà di stile incerto; D, Vasi a vernice nera o nerastra; F I, Vasi senza vernice a pasta gialliccia, rossiccia o bianchiccia; F II, Vasi senza vernice a pasta cinerea). Notevole interesse ebbero per il Bocchi i documenti epigrafici, distinti da una sequenza alfabetica secondaria con lettere minuscole che distinguono specifiche categorie all'interno di quelle primarie (per le ceramiche di periodo preromano che qui interessano: A.b., Primo stile con lettere graffite; C.e., Varietà con lettere graffite; D.f., Vasi neri con graffiture; F.i., Vasi senza vernice con graffiture; F.j, Vasi senza vernice con bolli ${ }^{41}$ ), e sempre accompagnati da un apografo. Proprio quest'ultima accortezza documentaria ha facilitato nella maggior parte dei casi il riconoscimento degli oggetti quando non più accompagnati dall'etichetta con il numero di inventario originale redatto dal Bocchi medesimo (in inchiostro nero per la collezione privata e in inchiostro rosso per la collezione civica $)^{42}$.

Sebbene gli interessi di scavo della famiglia Bocchi furono rivolti primariamente alla conoscenza dell'abitato antico, non va tralasciato che nel Museo Domestico confluirono molti materiali provenienti dai sepolcreti ellenistici di Adria e un significativo numero di vasi sempre da contesti funerari furono acquistati da F.A. Bocchi per il Museo Civico. La provenienza funeraria di questi oggetti è desumibile dallo stato di conservazione.

Nei primi anni del XX sec. si deve soprattutto a L. Conton la garanzia di una continuità con la tradizione bocchiana di scavo in abitato: in località Bettola arrivò a indagare gli strati tardoarcaici. Ma il medesimo Conton portò avanti anche indagini in contesti di necropoli di età classica e soprattutto ellenistica in località Retratto, Amolaretta e Cuora a sud di Adria, i primi condotti nel rispetto documentario dei contesti sepolcrali rinvenuti ${ }^{43}$. I materiali di questi scavi furono purtroppo dispersi in varie collezioni private e pubbliche ${ }^{44}$, fra cui alcuni oggetti oggi conservati nel Museo dell'Università di Padova e del Museo Archeologico di Este, e solo in minima parte sono recentemente riconfluiti nelle collezioni del Museo Archeologico di Adria. Negli anni 1904-1905 e 1908, anche A. Alfonsi scavò aree di abitato in località Amolaretta e Retratto ${ }^{45}$, e inoltre nel 1910 eseguì un saggio in profondità nell'orto del ginnasio "Carlo Bocchi", retrostante l'abside della chiesa di S. Maria Assunta della Tomba ${ }^{46}$, dove rinvenne strutture lignee associate a materiale riferibile a età tardo-arcaica.

Una nuova stagione di ricerche si ebbe a partire dal 1936, quando furono avviati nuovi scavi sotto la supervisione della Reale Soprintendenza alle Antichità del Veneto nel Prato dell'Ospedale ${ }^{47}$ (già chiamato Pubblico Giardino nel XIX sec., e oggi sede del Museo Archeologico Nazionale), in vicinanza di quelli già effettuati da F.A. Bocchi. Le indagini continuarono anche negli anni 1938-1939 ${ }^{48}$, in pa-

${ }^{41}$ Queste categorie epigrafiche sono distinte anche per tecnica di realizzazione. Fra quelle che in questa sede non sono prese in considerazione, si citano a titolo esemplificativo quelle con lettere dipinte appartenenti alla produzione attica (A.a, Primo stile con lettere dipinte; B.c, Secondo stile con lettere nero-cupe sul fondo nero; B.d, Secondo stile con lettere nere sul fondo giallo), ma anche i bolli, appartenenti a produzioni successive all'orizzonte cronologico in esame (D.g, Vasi neri con bolli; E.k, Vasi a vernice rossiccia o gialliccia con bolli; ecc.). Per un quadro d'insieme, si veda ibid., pp. 45-46.

${ }^{42}$ Molti dei documenti epigrafici registrati in questi Cataloghi non sono stati reperiti. Seppure F.A. Bocchi abbia prodotto un apografo per ogni reperto inventariato, per prudenza nel CIE IV, I, 1 sono stati editi unicamente i graffiti alfabetici e non alfabetici riconosciuti come appartenenti a queste collezioni e conservati attualmente in Museo e inventariati; le iscrizioni non rinvenute ma di cui si dispone dell'apografo dello studioso sono state invece edite, in quanto non è possibile per queste incorrere in errori di fraintendimento. Si puntualizza che ai graffiti alfabetici e non alfabetici privi di un contesto certo e che non è stato possibile ricondurre alle collezioni ottocentesche, non sono stati dedicati singoli tituli, ma sono comunque stati elencati in CIE IV, I, 1, p. 195.

43 Conton 1904 e 1908.

44 Mangani 1980, pp. 121-122, nota 3; Tamassia 1993, p. 8, nota 7.

45 Alfonsi 1908.

46 Conton 1910, pp. 196-198; SCARAni 1971, pp. 31-32, 9 - XIII; Giangiulio 1984, p. 42.

47 Dallemulle 1977, col. 170, nota 19, figg. 2-3, con riferimenti.

48 Battaglia 1957, p. 122; ID. 1958-1959, p. 341, fig. 129; SCARANi 1971, p. 29, n. 9 - III. 
rallelo con il recupero di 380 sepolture (247 comprese tra l'età tardo-arcaica e quella ellenistica, di cui quasi tutte di quest'ultimo periodo ${ }^{49} ; 133$ di età romana) rinvenute durante i lavori per la inalveazione di un ramo secondario del Canalbianco, allora nominato "Canale Mussolini"; tale impresa costituisce ancora oggi uno delle più rilevanti operazioni archeologiche effettuate ad Adria, sotto la direzione di Giulia Fogolari della Reale Soprintendenza alle Antichità del Veneto e con la supervisione di G.B. Scarpari ${ }^{50}$. Purtroppo, gli spostamenti e rimaneggiamenti subiti nel tempo da questo ricco contesto hanno portato spesso confusione nella composizione dei singoli corredi ${ }^{51}$. Una ricostruzione filologica di questi ultimi è possibile seppure in maniera limitata; infatti la documentazione di scavo conservata è piuttosto succinta e vaga nella descrizione delle singole sepolture e dei reperti a queste afferenti; inoltre le fotografie di scavo sono in numero molto ridotto. Solo per le prime 214 sepolture disponiamo di un elenco dei singoli reperti redatto dall'assistente di scavo A. Nicolussi ${ }^{52}$.

Dopo la seconda guerra mondiale, vi furono ulteriori indagini condotte dall'allora Soprintendenza alle Antichità del Veneto e prevalentemente rivolte ai contesti funerari ${ }^{53}$, che portarono nuovi dati sulle necropoli di età ellenistica. Nell' inverno 1956-1957 si indagò le aree funerarie di età ellenistica in località Retratto (proprietà Donà) ${ }^{54} \mathrm{e}$ in località Campelli a nord della città moderna ${ }^{55}$. Come per gli scavi di necropoli anteriori al conflitto mondiale, anche per questi non è sempre certa la coerenza dei corredi recuperati ${ }^{56}$. Solo a partire dalle indagini effettuate nel 1964 in località Piantamelon ${ }^{57}$, negli anni 1966, 1967, 1969 in località Ca' Garzoni ${ }^{58}$, e nel 1970 in località Ca' Cima ${ }^{59}$, si posseggono dati certi sulla consistenza dei corredi funerari rinvenuti. Non mancarono comunque indagini dell'abitato antico come nell' inverno 1954-1955, quando si condussero scavi nell'area del Pubblico Giardino ${ }^{60}$, in vicinanza a quelli già effettuati. Nel 1964 e nel 1967 si adottò una metodologia di indagine innovativa: furono infatti effettuati carotaggi nella zona dell'Ospedale civile e in località Bettola, che permisero di documentare la profondità del livello tardo-arcaico e classico dell' insediamento antico ${ }^{61}$.

Dopo circa vent'anni, una nuova e fortunata stagione di scoperte per la conoscenza dell'insediamento preromano è stata avviata nel 1990, con lo scavo della necropoli di via Spolverin in località Bottrighe $^{62}$. A partire dal 1993 ripetute indagine nell'area di Ca' Cima, a nord-est dell'abitato antico, hanno ampliato la conoscenza di questa area funeraria e in particolare portato in luce un significativo numero di sepolture comprese tra l'età arcaica e la prima metà del V sec. a.C.63 (oggetto di una mostra dal titolo "Etruschi Adriati") ${ }^{64}$, ma anche di età ellenistica e romana. Nel 1996 è stata indagata un'area

49 Per le sepolture di età tardo-arcaica, cfr. GAUCCI 2015, p. 115, con riferimenti.

50 Fogolari 1940, pp. 431-442; SCARAni 1971, pp. 33-34, 9-XXIV; SCARPari 1977. Cfr. anche Atria 1989, pp. 462-464, n. 400; Robino 2016, p. 91. Nella località chiamata Artessura, verosimilmente una zona periferica della necropoli del Canalbianco, nell'anno 1939 fu rinvenuta una sepoltura di età ellenistica (cfr. Atria 1989, pp. 460-461, n. 399).

51 Mangani 1980, p. 122, nota 3; Giangiulio 1984, p. 43; Robino 2016, pp. $92-94$.

52 Per la descrizione della documentazione di scavo di Canalbianco e in particolare di quella redatta dal Nicolussi, si veda Robino 2016, pp. 91-92 e fig. 1.

53 A queste ricerche vanno aggiunti corredi da sequestro in località Bellombra e Vallieri, riferiti al IV-III sec. a.C. (TAMASSIA 1993, p. 8, nota 7).

54 TAMassia 1993, pp. 10-11.

55 Bolognesi 1998-1999, pp. 245-247.

56 TAMASSIA 1993 , p. 8 , nota 7.

57 Fogolari, Scarfì 1970, p. 24; si veda anche la sintesi in Atria 1989, p. 470, n. 407.

58 Mangani 1982, p. 5; si veda anche la sintesi in Atria 1989, pp. 458-460, n. 398.

59 Dallemulle, Marzola 1977, pp. 3-4.

60 Fogolari 1955, n. 2479; EAD. 1956, p. 541. Cfr. Scarani 1971, p. 29, n. 9 - IV.

${ }^{61}$ SCARfì 1964, p. 396; Ead. 1968, pp. 49-50; Fogolari, SCarfì 1970, p. 24, nota 23.

62 Bonomi, Peretto, Tamassia 1993, pp. 91-96.

63 Per considerazioni preliminari sulla necropoli di Ca' Cima, in corso di pubblicazione, cfr. Bonomi 2003b, pp. 140145; EAD. 2003c, pp. 73-74; EAD. 2004, pp. 65-69; WIEL-MARIN 2005, pp. 86-87.

${ }^{64}$ Bonomi, Camerin, Tamassia 2002a. Una prima mostra fu organizzata nel 1996 dal titolo "Il banchetto nell'aldilà. Corredi funerari di Adria tra VI e V sec. a.C.”. 
di necropoli con sepolture di periodo ellenistico in località Piantamelon ${ }^{65}$. Infine, rinvenimenti più puntuali sempre di periodo ellenistico si ebbero durante lavori effettuati all'interno dell'Ospedale Civile nel $1993^{66}$. Parallelamente alle indagini di necropoli, resti dell'abitato preromano furono indagati, quasi un secolo dopo gli scavi del Conton, nel 1994 in via San Francesco ${ }^{67}$, e più recentemente nel 2000 in via Bettola ${ }^{68}$ e nel 2000-2001 e 2004 in via Ex Riformati ${ }^{69}$, importanti per lo studio delle fasi abitative preromane, dall'età arcaica (pieno VI sec. a.C.) fino a tutto il periodo ellenistico. A queste indagini si è fermata la ricognizione per l'edizione del fascicolo adriese del CIE.

Le attività di scavo ad Adria sono continuate negli anni recenti nella parte meridionale e occidentale della città moderna. Presso l'Ospedale intitolato a Santa Maria Regina degli Angeli è stato rinvenuta un'area funeraria di periodo ellenistico e romano $(2007)^{70}$. Il rinvenimento (2010-2011) di una porzione dell' impianto urbano romano impostato su quello preromano in questa zona occidentale permette di definire i limiti della città verso Occidente ${ }^{71}$. Gli scavi condotti nel 2007 in via Bettola e quindi nelle vie Amolaretta e Zaccagnini (2010-2011) ${ }^{72}$ nella zona orientale della città moderna, hanno portato al rinvenimento di aree funerarie databili tra il periodo tardo-arcaico e l'età ellenistica e romana. Non c'è dubbio che queste sepolture appartenessero alla necropoli orientale della città antica e che l'area di via Bettola rappresentasse il limite fra lo spazio urbano e quello sepolcrale. Negli ultimi anni (2015-2016) invece sono state condotte nuove indagini di notevole importanza in via Ex Riformati ${ }^{73}$, in continuità con lo scavo del 2004.

\section{Le ragioni di una analisi}

Gli studi sulle iscrizioni adriesi di periodo preromano sono un riflesso del carattere misto e di frontiera di questo importantissimo sito. Infatti, per circa un secolo i più eminenti studiosi, cimentandosi con questi documenti, ne hanno stabilito variamente la natura linguistica e culturale come "euganea", etrusca, venetica, greca (cfr. I). Tali posizioni, anche molto diverse fra loro, sono sicuramente in parte dovute alla difficoltà di interpretare questi lacerti di brevissimi testi, spesso graffiti su ceramiche ormai deteriorate dal tempo e che solo un sempre maggior affinamento degli studi paleografici e ceramologici negli ultimi decenni ha permesso di leggere in maniera via via più accurata e di datare con maggior precisione. Tuttavia è indubbio che proprio la posizione geografica di Adria, al confine moderno fra Veneto e Emilia-Romagna, cioè fra il territorio degli antichi Veneti e il territorio etrusco, ha fortemente influito sul dibattito, che aveva (e in alcuni casi, erroneamente, ha tuttora; cfr. I.1) alla base il riconoscimento stesso di questo luogo come veneto piuttosto che gravitante sull'Etruria padana.

Inoltre, va riconosciuto che cimentarsi con le iscrizioni e i graffiti di Adria preromana significa tener conto di una pluralità di culture epigrafiche che dal periodo tardo-arcaico fino alla romanizzazione si affiancarono a quella etrusca sicuramente dominante, all' interno di una città che fu prima di tutto uno dei principali porti dell'Adriatico.

Proprio in questa prospettiva di compresenza di più culture epigrafiche, è necessario puntualizzare che per la fase di VI-V sec. a.C. il significativo numero di iscrizioni in alfabeto e lingua greca individuate, a cui vanno aggiunti i graffiti commerciali di questo ambito culturale, è stato oggetto di un

65 Mosca, Puppo 2000, pp. 135-136.

66 Bellintani et al. 1995, pp. 41, 42-43, 52-54, 62.

67 Camerin, Tamassia 1998-1999, pp. 212-235; Bonomi, Camerin, Tamassia 2002b, pp. 201-207.

${ }^{68}$ Bonomi, Camerin, Tamassia 2002b, p. 201, nota 2.

69 Bonomi, Camerin, Tamassia 2002b, p. 201, nota 2; Bonomi, Robino 2007, pp. 88-89; Bonomi, GambaCURTA 2017, pp. 69-74.

70 BonOMi 2011, pp. 62-66.

71 Gambacurta, Bacci, Marcassa 2012, pp. 44-50; Bonomi, Gambacurta 2017, p. 70, nota 8.

72 Gambacurta et al. 2012, pp. 43-44.

73 Gennuso, Marchesini, Vallicelli 2018; Bonomi, Vallicelli, Balista 2020. 
recente catalogo edito da D. Baldassarra ${ }^{74}$. Per l'epoca ellenistica e fino alla romanizzazione è noto invece un numero non irrilevante di iscrizioni latine e di altre culture epigrafiche dell' Italia centro-settentrionale $^{75}$, che meriterebbero una analisi autonoma. Come puntualizzato nel De edendi rationibus ${ }^{76}$, all'interno del fascicolo adriese del CIE sono state inserite le iscrizioni non etrusche provenienti da contesti chiusi in associazione a iscrizioni etrusche e/o redatte su supporti di sicura produzione locale: 7 iscrizioni galliche in alfabeto leponzio databili tra II e I sec. a.C. (CIE 20198, 20578, 20580, 20581, 20590, 20765, 20915), 3 venetiche di II sec. a.C. (CIE 20911, 21014), 11 latine databili tra la fine del III e la prima metà del I sec. a.C. su vasellame di produzione locale (CIE 20335, 20339, 20500, 20813, 20831, 20950, 21003, 21021) e su anfore greco-italiche (CIE 20405, 20577, 20952) delle quali una verosimilmente redatta non ad Adria ed eccezionalmente databile entro la fine del IV e la prima metà del III sec. a.C. ${ }^{77}$; a questi documenti si aggiunge una iscrizione umbra dal territorio graffita su un vaso a v.n. forse locale della fine del II-I sec. a.C. $\left(\right.$ CIE 21031) ${ }^{78}$.

Adria risulta quindi non solo un centro di frontiera politica (tra Etruschi e Veneti), ma anche di frontiera culturale per il suo ruolo di porto, dove emergono aspetti di una cultura epigrafica etrusca molto complessa, che derivano da apporti provenienti da diversi ambiti territoriali, ma dove anche si interfacciano competenze scrittorie diversificate (etrusca/greca nella fase più antica; etrusca/latina/ celtica/venetica nella fase ellenistica).

Tale complessità rende certamente la città un laboratorio di ricerca privilegiato, unico nel suo genere, dove i più aggiornati modelli teorici relativi ai fenomeni di mobilità e permeabilità culturale potrebbero essere applicati in maniera ampia e articolata grazie a molti strumenti di indagine, fra cui sicuramente l'epigrafia è uno dei più significativi ed efficaci. È peraltro ormai riconosciuto dalla critica più aggiornata che il tentativo di applicare modelli desunti dall'ambito storico e antropologico si scontra con la realtà della ricerca archeologica, che dovrebbe essere sempre fortemente ancorata al datum e al contesto ${ }^{79}$. Anche in questo Adria si presenta come un caso paradigmatico: a fronte di una storia degli scavi molto complessa e che affonda le sue radici già del XVIII sec., si può inserire la maggior parte dei documenti epigrafici nel quadro (più o meno di dettaglio) dei relativi contesti archeologici.

Si ha dunque primariamente la possibilità di cimentarsi con la complessa documentazione epigrafica di una città portuale etrusca. Proprio la scelta di ribadire nuovamente quale doveva essere l'importanza della cultura etrusca (non solo epigrafica) in questo centro del Delta padano, ha portato a intitolare questo libro Iscrizioni della città etrusca di Adria. Così concepito, questo titolo vuole essere un tributo scientifico e culturale al volume Iscrizioni e graffiti della città etrusca di Marzabotto curato da G. Sassatelli nel 1994, che rimane un punto di riferimento imprescindibile per gli studi epigrafici relativi all'Etruria padana, nonché da un punto di vista metodologico ed editoriale per l'epigrafia etrusca in generale. Questa impostazione degli studi prettamente epigrafica e fortemente improntata sull'analisi dei documenti in contesto, già messa in atto per la più antica documentazione di Bologna dal medesimo Sassatelli ${ }^{80}$, è importante riferimento per il presente lavoro. Infatti, il "contesto", nelle sue diverse declinazioni, dal supporto ceramico del documento epigrafico al carattere specifico nel quale esso è

74 BAldassarra 2013. Rispetto al materiale edito da D. Baldassarra, nel fascicolo adriese del CIE sono stati nuovamente presentati soltanto quei graffiti non rigidamente attribuibili a un milieu alfabetico greco piuttosto che etrusco.

75 Rimangono ad esempio escluse alcune iscrizioni su anfora in alfabeto venetico e leponzio (cfr. ToNiOLO 2000, p. 103, n. 2, fig. 246), che potrebbero comunque essere eventualmente il prodotto di un milieu culturale locale minoritario rispetto a quello etrusco. A tale riguardo, vale la pena ricordare che tra le iscrizioni irreperibili c'è anche quella latina (M. AEMILI o M. AIMIL, secondo la documentazione di scavo) su ciotola a v.n. della tomba a cremazione 259 di Canalbianco, datata alla prima metà del III sec. a.C.

76 CIE IV, I, 1, p. VII.

77 REI LXXIX, pp. 346-348.

78 Ibid., pp. 348-350.

79 Per questo approccio critico, cfr. in particolare Stockhammer 2012; Pappa 2013.

${ }^{80}$ Cfr. Sassatelli 1981-1982; ID. 1985. 
stato rinvenuto, rappresenta un elemento imprescindibile con cui fare i conti in una analisi epigrafica che si possa ritenere soddisfacente.

Proprio per questo motivo è previsto un capitolo che riporti il documento epigrafico alla dimensione di reperto all' interno del proprio contesto e lo analizzi per il suo valore archeologico (cfr. VI).

Il lavoro ha tuttavia come obiettivo primario quello di proporsi come uno strumento di analisi al fascicolo CIE IV, I, 1. Tale esigenza nasce dalla natura stessa del Corpus, concepito come sola raccolta di testi epigrafici e del relativo apparato critico e bibliografico, ma che non ospita commenti di analisi e riflessioni interpretative. Si è dunque sentita la necessità di superare l'edizione dei dati (sebbene passaggio preliminare fondamentale) e approdare a una più profonda conoscenza della cultura epigrafica come fenomeno capace di fornire elementi di analisi della struttura sociale e culturale di un intero centro in una prospettiva diacronica.

L'analisi è limitata alla città di Adria e ai suoi insediamenti satelliti (pochi km di distanza), non comprendendo in maniera diretta il suo ampio territorio e in particolare i siti di Le Balone, San Cassiano e San Basilio. Sebbene Adria rappresenti un caso eccezionale per l'ambito padano, in quanto ha restituito una documentazione molto ricca sia per la città sia per gli insediamenti del suo territorio, la scelta effettuata è finalizzata a indagare nella maniera più efficace e senza eccessive dispersioni la cultura epigrafica adriese quale sistema autonomo. In ogni caso, l'assenza dei siti minori in questo lavoro non costituisce un impoverimento o una lacuna, per tre distinte ragioni: anzi tutto la trattazione prevede il ricorso quando opportuno alle iscrizioni e agli altri documenti epigrafici del territorio; il numero di questi, sebbene rilevante, risulta comunque notevolmente inferiore a quello del centro principale, circa 41 tituli (CIE 21031-21071) a fronte dei 883 di Adria e degli insediamenti satelliti; infine, le iscrizioni di Le Balone sono state già oggetto di più trattazioni ${ }^{81}$, mentre è in corso di stampa uno studio analitico di quella di San Cassiano da parte di A. Gobbi ${ }^{82}$.

L'analisi sviluppata nei seguenti capitoli si concentra sulle iscrizioni (comprensive dei digrammi), ma non sulle singole lettere e sui graffiti non alfabetici. Si affrontano le tecniche di scrittura e supporti (cfr. II), le norme scrittorie e la paleografia (cfr. III), l'onomastica e le relative formule (cfr. IV), il restante lessico (cfr. V), sempre in una prospettiva di ampio respiro dal periodo arcaico a quello ellenistico. Proprio la prospettiva diacronica è decisamente rilevante, perché Adria si presenta come l'unica città padana per la quale è possibile affrontare una analisi che vada dalla fase formativa della città etrusca nel VI sec. a.C. fino al pieno processo di romanizzazione della comunità a cavallo tra II e I. Le osservazioni che emergono dai capitoli menzionati sono la base per affrontare una più consapevole analisi dei documenti epigrafici in contesto, che dovranno in questo caso tenere conto di tutte le occorrenze, comprensive dei graffiti alfabetici e non alfabetici (cfr. VI).

\footnotetext{
81 Marinetti 1994; Gaucci 2012a, pp. 162-163.

82 Si ringrazia A. Gobbi per aver messo a disposizione con grande liberalità il manoscritto Iscrizione e sigla elaborato per l'edizione del contesto di scavo. Si ringrazia inoltre M. Harari e l'équipe di San Cassiano, in particolare E. Smoquina e M.T.A. Robino per tutto l'aiuto concesso nella fase di realizzazione del fascicolo CIE IV, I, 1.
} 



\section{STORIA DEGLI STUDI E QUANTIFICAZIONE DELLE ISCRIZIONI ETRUSCHE}

\section{I.1. Il dibattito scientifico tra XIX e XX secolo}

Le iscrizioni preromane di Adria sono state oggetto di interesse già durante l'Ottocento da parte degli esponenti della famiglia Bocchi ${ }^{1}$ e di altre famiglie della città, come quella dei Silvestrii ${ }^{2}$ e dei Grotto, che le acquisirono come parte delle loro collezioni. Ad esempio, si è a conoscenza che un alfabetario greco, ora disperso, faceva parte della collezione Grotto già dal 1738. Questo documento fu menzionato da $\mathrm{O}$. Bocchi, entrando così precocemente in letteratura (CIG IV, 8343)3. Apparteneva a questa importante famiglia anche una iscrizione recuperata in zona Retratto (cfr. infra). Entrambe sono menzionate nel volume di R. Schöne del $1878^{4}$.

In questa fase pionieristica degli studi, nella quale non era ancora acquisita in maniera definitiva l'identificazione dei vasi figurati come attici, che si deve principalmente all'opuscolo di L. Lanzi del $1806^{5}$, anche le iscrizioni dipinte su questi ultimi erano ritenute oggetto di interesse quali manifestazioni di una possibile identità locale antica della città. Non a caso è possibile ricostruire un carteggio del 1804 relativo a una iscrizione dipinta su due frammenti di un vaso attico a f.n. rinvenuto da Francesco Girolamo Bocchi negli scavi del settembre di quell'anno, immesso dallo scavatore nel circuito epistolare degli studiosi dell'epoca ${ }^{6}$. I carteggi di F.G. Bocchi presso l'Archivio Comunale di Adria Antica della Biblioteca Comunale di Adria ci restituiscono una lettera di L. Lanzi al conte Giovanni de Lazara datata 13 ottobre 1804, e una lettera di G. de Lazara a F.G. Bocchi in data 31 ottobre 1804, nelle quali si parla di una iscrizione, menzionata anche nella copia della lettera di L. Lanzi all'abate I.

\footnotetext{
1 Sulla famiglia Bocchi e il suo ruolo nella raccolta di antichità adriesi, si rinvia all'esauriente quadro offerto in WIELMarin 2005, pp. 21-36, e BALdassarra 2013, pp. 5-25.

2 Per il ruolo di questa famiglia, cfr. Zerbinati 1993 e 2003, pp. XXI-XLV.

3 Su questo alfabetario, si veda in particolare Colonna 1974a, pp. 6-8, n. 6, tav. I, b, e da ultimo BALdAssarra 2013 , pp. 130-132, con relativa bibliografia.

${ }^{4}$ SCHÖNE 1878, p. 146, nn. 616-617.

5 LANZI 1806.

${ }^{6}$ Questo carteggio è ricostruito in GAUCCI 2012a, pp. 151-152, trattando di una iscrizione recentemente attribuita non correttamente ad Adria da S. BRUNI (2001, p. 65, note 57-58; ID., in REE LXXIV, pp. 366-367, n. 110; ID. 2011 , p. 126, nota 9) e dimostrandone invece la provenienza dal territorio senese.
} 
Morelli, allora prefetto della Biblioteca Marciana di Venezia, in data 7 ottobre 1804, inviata da I. Filiasi a F.G. Bocchi ${ }^{7}$. Proprio il contenuto della lettera ha permesso di suppore che il disegno che il conte de Lazara spedì al Lanzi sia quello di un frammento di vaso figurato con iscrizione greca dipinta che va a integrare il disegno di un altro frammento del medesimo vaso già invia a L. Lanzi dall'abate I. Morelli ${ }^{8}$, riconoscibile, grazie a una nota nella busta riordinata da F.A. Bocchi, quali i reperti Inv. Museo Bocchi A.a. 2 e $2^{\prime 9}$, rinvenuti nel settembre del 1804 . La rete di relazioni disegnata dagli scambi di missive del Bocchi ci mostra dunque in maniera concreta come questi partecipasse al fervido dibattito che animava questo periodo ${ }^{10}$. Infatti già nel 1794 F.G. Bocchi era a conoscenza dell'opera di L. Lanzi sulla lingua etrusca $^{11}$, e i rapporti, indiretti, tra il Lanzi e il Bocchi sono già testimoniati in una lettera datata 22 aprile 1796 che Giuseppe Perli Remondini di Bassano inviò al Bocchi comunicandogli un giudizio del Lanzi su una iscrizione romana della quale il Bocchi aveva inviato un disegno ${ }^{12}$.

Non sembra però che le testimonianze epigrafiche abbiano catalizzato l'interesse dei Bocchi negli anni successivi al carteggio menzionato. Nella seconda edizione del suo saggio sulla lingua etrusca, edito nel 1825, L. Lanzi menzionò in effetti «alquante iscrizioni di questi popoli» (da intendere i Veneti), raccolte dal Bocchi ad Adria ${ }^{13}$, ma l'unica che ricorda è appunto l'alfabetario greco prima appartenente alla collezione Grotto ${ }^{14}$ (e ora disperso).

Pochi anni dopo, F.W.E. Gerhard, nella sua visita ad Adria nel 1832, oltre all'ovvia menzione delle ceramiche figurate, nulla dice sulle iscrizioni ${ }^{15}$. Successivamente, nell'importante lavoro del 1853 sulle iscrizioni c.d. "nord-etrusche", nel quale si pongono le basi per una distinzione linguistica della documentazione epigrafica dell' Italia settentrionale, Th. Mommsen citò l' iscrizione CIE 21027 rinvenuta tra Adria ed Este e appartenente alla collezione della famiglia Silvestri, affermando che «mit Recht, denke ich, und auch der Fundort spricht mehr gegen als fur die Vereinigung dieses Stuckes mit den euganeischen Inschriften ${ }^{16}$. L'iscrizione, che confluì poi nel Corpus Inscriptionum Italicarum di A. Fabretti (CII 39) assieme all'alfabetario greco della collezione Grotto (CII 41), era già entrata nel

\footnotetext{
${ }^{7}$ Rispettivamente $A C A A$, busta 363/7a.3 (copia di F.G. Bocchi); ACAA, busta 363/7a.4; ACAA, busta 363/7a.2. Le lettere fanno parte della busta corrispondente al tomo settimo dell'epistolario di F.G. Bocchi, con la dicitura "Lettere che trattano della storia d'Adria ed altre materie letterarie dirette a Francesco Girolamo Bocchi nobile adriese. Tomo settimo", ordinate da F.A. Bocchi (Tognon 2004, pp. 9-11, 21-22).

${ }^{8}$ Nella copia della lettera di L. Lanzi a I. Morelli, datata genericamente all'ottobre 1804 ( $A C A A$, busta 363/7a.2bis), si dice che il conte de Lazara recapitò a L. Lanzi il disegno di un secondo frammento del vaso con iscrizione, che conferma l'ipotesi formulata dall'abate, e infatti nella lettera di L. Lanzi a G. de Lazara datata 13 ottobre 1804 si ringrazia il conte per l'invio del disegno del secondo frammento (ACAA, busta 363/7a.4). Nella lettera a F.G. Bocchi del 31 ottobre 1804, G. de Lazara chiede al Bocchi una nuova copia del disegno (da inviargli unitamente alla lettera ricevuta da L. Lanzi), perché l'abate aveva trattenuto quella inviatagli $(A C A A$, busta 363/7a.4): «[...] e che io Le spedisco perché ne tragga / copia, rimandandomela poi unitam. ${ }^{\text {te }}$ a un nuovo di/segno de fram. ${ }^{\text {ti }}$ sud. ${ }^{\text {ti }}$ avendosi trattenuto il Lanzi quello / che mi ha prima spedito; $[\ldots] \gg$. Altre lettere trattano di questi medesimi disegni, indirizzate al fratello Tommaso Bocchi e ad altri eruditi del tempo (contenute in $A C A A$, busta $363 / 7 \mathrm{~b}$ ).

9 BAldassarra 2013, pp. 39-40, Adria FN 1, con bibliografia completa. R. SCHÖNE (1878, p. 54, n. 109, tav. XIII, 1, $\mathrm{a}-\mathrm{b})$, ricorda la data di rinvenimento, cioè i primi giorni di settembre del 1804, e inoltre che i frammenti erano già stati editi da M. Boni nel 1815, assieme al testo della lettera di L. Lanzi a G. de Lazara del 13 ottobre 1804, già ricordata (Boni 1815, pp. 42-48, Nota C - De Cimelio Bocchiano, nn. 24, 24a; si veda anche in LANZi 1806, p. 25). M. Boni edita la riproduzione di quelli che dobbiamo presupporre furono i disegni inviati da F.G. Bocchi a I. Morelli e G. de Lazara.

${ }_{10}$ Per l'attività epistolare di F.G. Bocchi con vari eruditi del tempo, cfr. PASTEGA 2011, in particolare p. 67 per l'anno 1804.

11 LANZi 1789. Tale conoscenza è nota sulla base dell'invio al Bocchi della copia di alcuni passi da parte di Giuseppe Gennari (ACAA, busta 359/5c.1).

12 Pastega 2011, p. 65.

13 LANZI 1824-1825², II, III, pp. 547-548.

${ }^{14}$ LANZI 1824-1825², II, III, p. 569, n. 13, p. 753.

15 F.W.E. GERHARD, in Bullist 1832, pp. 90, 205.

16 Mommsen 1853, p. 213, n. 35.
} 
dibattito sulle iscrizioni euganee grazie alla menzione (senza però la trascrizione del testo) che ne fece G. Furlanetto nel lavoro del 1847 a questo tema dedicato ${ }^{17}$, ripreso poi da G. Da Schio che invece non ne riconosceva l'appartenenza ai c.d. testi "euganei" ${ }^{18}$, dicitura di lunga tradizione erudita che è alla base dell'epigrafia venetica ${ }^{19}$. Queste poche attestazioni non furono riconosciute come etrusche per lingua e scrittura neanche da K.O. Müller e W. Deecke nel 1877 all'interno del quadro offerto sulle attestazioni della fascia adriatica ${ }^{20}$.

Ma proprio in quei medesimi anni i resoconti dei ritrovamenti effettuati da Francesco Antonio Bocchi in Notizie degli Scavi di Antichità degli anni 1877 e 1879, prevalentemente provenienti dai suoi scavi nell'abitato antico, avviarono una nuova stagione di condivisione delle scoperte delle più antiche testimonianze di Adria con la comunità scientifica. In questi contributi si può evidenziare la rilevante presenza di ceramica iscritta, definita dallo scavatore italica ed etrusca nel $1877^{21}$, generalmente italica e più dubitativamente umbra ed etrusca nel $1879^{22}$. Senza sminuire i meriti personali del Bocchi, non si può sottacere che l'impulso alla conduzione di scavi archeologici ad Adria derivava dagli interessi verso il periodo preromano dei dirigenti del Ministero della Pubblica Istruzione nell'assegnare i fondi, soprattutto di L. Pigorini, Capo-sezione della Direzione centrale degli Scavi e dei Musei del Regno. Questi, che in quel periodo stava elaborando la sua teoria sulla migrazione dei proto-italici dall'area danubiana quale spiegazione della formazione della civiltà villanoviana, aveva visitato nel 1877 la collezione dei Bocchi cogliendo le potenzialità del sito $^{23}$. L'opera del Bocchi fu dunque guidata anche scientificamente da autorevoli figure (come attestano anche le scrupolose lettere di istruzioni di G. Fiorelli, a capo della Direzione) e il dubbio del Bocchi nel riconoscimento di alcuni caratteri epigrafici come umbri assieme a quelli etruschi a livello della "stazione etrusca" nel 1879 evoca forse proprio un tentativo di cogliere gli indizi di quei popoli terramaricoli (italici, cioè umbri) che nella prospettiva del Pigorini avrebbero abitato il territorio prima degli Etruschi.

Il Bocchi quindi, Regio Ispettore degli Scavi e già prima membro della Commissione Conservatrice dei Monumenti e delle Opere d'Arte per la Provincia di Rovigo, era pienamente e consapevolmente inserito nel fervido dibattito scientifico che animava in quegli anni lo sviluppo delle scienze pre- e protostoriche e coglieva nelle iscrizioni rinvenute un indizio importante di conferma degli schemi teorici che soprattutto G. Chierici e L. Pigorini propugnavano.

Sempre i quei medesimi anni, per la precisione nel 1878 , uscì alle stampe la pubblicazione di R. Schöne, a lui affidata dal Reale Istituto Germanico su impulso di Th. Mommsen, che aveva compreso l'importanza della collezione privata dei Bocchi, visitando il museo nel 1867 per la redazione del CIL. Il catalogo fu stilato dallo Schöne nel 1868 e il lavoro consegnato nel 1870, quindi anteriormente alle campagne di scavo di F.A. Bocchi ${ }^{24}$. Nell'opera sono presentate molte iscrizioni e anche graffiti riconosciuti come greci, che susciteranno nel tempo un vivace dibattito sulla presenza della colonia siracusana nella città ${ }^{25}$. Già l'editore pone l'accento su queste iscrizioni greche, in particolare quelle votive, sottolineandone come dovettero essere necessariamente realizzate nella medesima Adria ${ }^{26}$.

${ }^{17}$ Furlanetto 1847, p. XLVI, n. XII.

18 DA Schio 1853, p. 44, nota 5.

$19 L V$, I, pp. 3-5.

20 «Die inschriften von Padua und Adria sind weder in Sprache noch in Schrift Tuskisch» (Müller, DeECKE 1877, I, p. 138, nota 53, con riferimento alle attestazioni note in $C I I)$.

${ }^{21}$ Воссні 1877, p. 198.

22 Воссні 1879, pp. 90, 96, 100, 214.

${ }^{23}$ FaCCHI 2018, pp. 116-120; VAllicelli 2018, pp. 125-127, 133. Per un quadro sullo sviluppo delle teorie di Pigorini, si veda Cupitò, PALtineri 2014.

24 SCHÖNE 1878, p. VI.

25 In particolare COLONNA 1974a e 2003, Antonetti 2005 e BALDASSARRA 2013; si richiama anche il contributo, ormai superato, di MAMBELLA 1984.

26 SCHÖNE 1878, p. XI. 
Nell'autunno del 1879 il museo adriese fu visitato da un altro autorevole studioso, E. Brizio ${ }^{27}$, all'epoca professore di Archeologia a Bologna, accompagnato dall'allievo G. Ghirardini. La visita stimolò il Brizio a un breve lavoro su Adria edito nel medesimo anno, nel quale toccò molti problemi. Lo studioso, di diversa impostazione scientifica e visione rispetto a Pigorini ${ }^{28}$, dette una sua personale lettura dei rinvenimenti del Bocchi, ritenendo che a una prima popolazione "indigena", su cui sospende il giudizio e che avrebbe convissuto con la colonia greca di V sec. a.C., sarebbe poi seguita una conquista etrusca. E. Brizio pose così l'accento sulle testimonianze greche, in accordo con Schöne e in netta contrapposizione con W. Helbig, che affermava invece la debolezza dell'ipotesi della realizzazione in loco di parte delle ceramiche figurate sostenuta dall'Accademico bolognese ${ }^{29}$. Ma soprattutto, definì etrusche le iscrizioni rinvenute nelle c.d. "palafitte" scavate da F.A. Bocchi, valorizzandole come importante testimonianza della fondazione della colonia da parte degli Etruschi come riportato dalle fonti antiche ${ }^{30}$. Quindi il Brizio, secondo il solido metodo scientifico che gli era proprio, utilizzò questo dato quale prova della veridicità del quadro storico fornito dalle fonti antiche.

In un lavoro edito nel medesimo 1879, V. Poggi affermò che la documentazione epigrafica dell'Italia settentrionale non fosse da riferire nella sua totalità alle iscrizioni c.d. "nord-etrusche", portando a dimostrazione le scoperte di Reggio, Mantova, Bologna e Adria in particolare, che invece rientravano secondo lo studioso nell'etrusco ${ }^{31}$. Il medesimo Poggi poneva comunque alcune iscrizioni adriesi in un gruppo gallico (-italico) ${ }^{32}$. Anche G.F. Gamurrini, nella redazione dell'Appendice al CII del Fabretti edita nel 1880, inserisce le iscrizioni rinvenute dal Bocchi fra le etrusche ${ }^{33}$. Pochi anni dopo, nel 1885 C. Pauli riconobbe l'etruscità dell' iscrizione CIE 21027, valorizzando la posizione sfumata del Mommsen nel 1853: «Diese Reserve Mommsens war wohlbegrundet, denn in der That ist hier alles rein etruskisch, Schrift wie Sprache ${ }^{34}$; inoltre non inserì le altre testimonianze epigrafiche adriesi allora note nel suo lavoro sulle iscrizioni venetiche del $1891^{35}$. Anche A. De Vit nel 1888 , in un lavoro considerato di importanza secondaria, affermò la presenza di una radice etrusca alla base dell'onomastica latina dell'Adria romana ${ }^{36}$. Questa linea interpretativa venne seguita nei seguenti decenni da Holder e quindi da Whatmough nel $1933^{37}$, ma non dal resto della critica.

Infatti, con l'inizio del XX secolo, in concomitanza con il rinvenimento delle prime testimonianze epigrafiche funerarie da parte di L. Conton, nel dibattitto scientifico emerse una posizione ben diversa da quella che si era affermata con gli ultimi decenni del secolo precedente. Già il Nissen nel 1902 affermò che «Solche kann auch füglich nicht in Zweifel gezogen werden, denn in Adria finden sich keine etruskischen, sondern die nämlichen venetischen Inschriften wie in Padua ${ }^{38}$, è nel 1905, durante il discorso di inaugurazione del Museo civico di Adria, che G. Ghirardini delineava con la sua autorevolezza quella che sarà la linea interpretativa delle iscrizioni adriesi fino agli ultimi decenni

27 Bocchi 1879, p. 445; Dallemulle 1993.

28 Cfr. Sassatelli $1984 a$, pp. 386-387.

29 BRIZIO 1879, in particolare p. 452. Sul violento dibattito scientifico, in particolare in merito al metodo, tra Brizio e Helbig, si veda SASSATELli 1984a, p. 389 e l'Appendice in coda al contributo.

30 BRIZIO 1879, pp. 455, 458.

31 Poggi 1879, p. 317.

32 Ibid., pp. 310, 314-315, nn. 53-57, con riferimento alle iscrizioni CIE 20006, 20020, 20051, 20776, 21021.

33 GamurRini 1880, p. 75, nn. 853-865.

34 PaUli 1885, pp. 43-44, 110.

35 Pauli 1891, pp. 230-231.

36 De Vit 1888.

37 Whatmough 1933, p. 178, con riferimenti precedenti.

38 Nissen 1902, II, p. 216. Tale posizione è contestata dal punto di vista del metodo da GHIRARDINi 1905, p. 127, nota 1 . Infatti quest'ultimo afferma che il Nissen non conosce le testimonianze epigrafiche adriesi (riferendosi con ciò al $C I I)$. 
del XX secolo ${ }^{39}$. Lo studioso infatti, pur esplicitando una certa difficoltà nell'interpretare la natura dell'alfabeto di queste iscrizioni, ne affermò l'attribuzione alla cultura epigrafica venetica «per quanto frammisti a qualche forma affine all'etrusco $\gg^{40}$. In ciò, criticava il Pauli che non teneva conto di Adria nella sua silloge sulle iscrizioni venetiche e anzi affermava il ruolo di questo sito come luogo di penetrazione dell'alfabeto nel mondo veneto. Solo pochi anni dopo, nel $1911 \mathrm{~F}$. Cordenons pubblicò un lavoro sulle iscrizioni venetiche, dedicando un capitolo alle iscrizioni di Adria ${ }^{41}$. Questi non solo non menziona in questa opera la letteratura anteriore a eccezione dei lavori di Bocchi, Schöne e Conton che pubblicarono di prima mano apografi e fac-simile di iscrizioni, ma propone una silloge di iscrizioni interpretate come venetiche ${ }^{42}$ ignorando le precedenti letture di alcune di queste come galliche da parte del Poggi (cfr. supra) e di altre come greche ${ }^{43}$.

Nel 1935, M. Buffa, nella sua Nuova Raccolta di Iscrizioni Etrusche, pose l'accento su alcune iscrizioni di ambito veneto che ritenne scritte in lingua etrusca, intese dunque come una probabile penetrazione di questa cultura in area veneta ${ }^{44}$. Il punto di riferimento del Buffa sono i lavori del Bocchi su Adria in Notizie degli Scavi di Antichità, come dimostra la selezione di iscrizioni che propone e la bibliografia di riferimento.

Con i lavori per l'inalveazione di un canale secondario del Canal Bianco iniziò nel 1938 l'indagine della più vasta area sepolcrale adriese (cfr. Introduzione). Le prime notizie relative a testimonianze epigrafiche da tale complesso necropolare risalgono al 1940, con la comunicazione preliminare della necropoli nella rivista Studi Etruschi da parte di G. Fogolari ${ }^{45}$. Tuttavia, una riflessione sistematica sulle iscrizioni fu affrontata solo successivamente da parte di G.B. Pellegrini a partire dal 1951, quando questi fu incaricato dello studio da parte della Soprintendenza alle Antichità di Padova ${ }^{46}$. Tale impegnò si concretizzò principalmente in due lavori editi negli anni 1958 e $1965^{47}$.

In questi lavori lo studioso ravvisa nell'epigrafia adriese quanto già colto in nuce dal Ghirardini cinquant'anni prima ${ }^{48}$, cioè una affinità paleografica con la cultura scrittoria venetica; a questo aggiunge l'osservazione di una concordanza sia nella scrittura che nell'onomastica con Spina ${ }^{49}$. Anche per Pellegrini dunque Adria (assieme a Spina) era centro di irradiazione della scrittura presso i Veneti. Nel 1967 scriveva che aveva intrapreso tale operazione perché «Si presentava urgente soprattutto una classificazione delle brevi epigrafi, oltre che una loro edizione, più corretta, riscontrabile su facsimili e foto $\gg^{50}$. Sebbene risulti importante rimarcare il nuovo approccio metodologico esplicitato

39 Sulla figura del Ghirardini, la sua impostazione metodologica e i dissensi rispetti ai modelli interpretativi del maestro Brizio, si veda SASSATELLI 1984b. Sul rilevante ruolo di Ghirardini nella tradizione di studi che ha sostenuto la veneticità di Adria da un punto di vista archeologico, si veda Fogolari, SCARFì 1970, in particolare p. 28, nota 20. In quest'ultimo lavoro, si ribadisce il carattere culturale veneto dominante del centro sulla base di argomentazioni di carattere archeologico e epigrafico (ibid., pp. 29-30, con riferimenti).

40 Ghirardini 1905, p. 148, nota 1.

41 Per il lavoro di F. CoRdenons (1911), si rimanda alle osservazioni in $L V$, II, pp. 5-6.

42 Cordenons 1912, pp. 223-226. Fra le iscrizioni che si riconoscono come etrusche, Cordenons menziona CIE 20006, 20020, 20028, 20030, 20214, 20236, 20683, 20736-20737.

43 SCHÖNE 1878, pp. 140-141, nn. 511-513, tav. XIX, 2-4, con riferimenti bibliografici. Per queste iscrizioni, si veda ora BALDASSARRA 2013, pp. 119-120, Adria IP 1, e pp. 122-124, Adria D 2-3. Per l'erronea lettura delle iscrizioni greche da parte di Cordenons, si veda anche Pellegrini 1956, p. 5.

44 NRIE, pp. 30-31.

45 Fogolari 1940.

46 Pellegrini, Fogolari 1958, p. 104.

47 Pellegrini, Fogolari 1958 e Pellegrini 1965. A questi va aggiunto il preliminare contributo Pellegrini 1957.

48 Le osservazioni di G. Ghirardini sono riportate dal Pellegrini nel primo intervento preliminare sulle iscrizioni di Adria (Pellegrini 1956, p. 6).

49 Pellegrini, Fogolari 1958, p. 104.

${ }^{50} L V$, I, p. 637. 
dallo studioso, va comunque osservato che la posizione scientifica del Pellegrini è sostanzialmente una mediazione di tutte quelle che dal secolo precedente si erano susseguite. Infatti, le iscrizioni nel contributo del $1958^{51}$ vengono suddivise dallo studioso in cinque sezioni, nominate con le prime lettere dell'alfabeto, sulla base della supposta origine etnica: etrusche (A), venetiche (B), etruscovenetiche $(C)$, incerte $(D)$, di transizione latina ma in onomastica gallica $(E)^{52}$. Le sezioni numericamente più rilevanti sono quella delle iscrizioni etrusche $(A)$, venetiche $(B)$ e incerte $(D)^{53}$; il criterio di raggruppamento, e in particolare la definizione della categoria delle iscrizioni etrusco-venetiche, è poi ribadito da Pellegrini nel contributo del $1965^{54}$. Secondo lo studioso, tutte le iscrizioni presentate si datano al periodo ellenistico e provengono da necropoli.

Nel 1966, M. Lejeune pubblicò un lavoro di analisi critica delle iscrizioni edite dal Pellegrini. Pur accettando l'impostazione generale del linguista veneto, non riconosce la sezione (E) come gallica, rinviandola piuttosto a una «série transitoire "vénéto-latine" », nella quale inserisce altre iscrizioni tra quelle edite da Pellegrini nel 1958 e nel 19655; inoltre propone una riformulazione delle iscrizioni ritenute venetiche o comunque transizionali (veneto-etrusche e veneto-latine), tanto che secondo Lejeune le iscrizioni adriesi manifesterebbero un imbastardimento ortografico rispetto a quelle dei centri veneti; ciò porta il linguista francese a definire una interessante distinzione tra gli alfabeti venetici, dei quali definisce con l'etichetta "euganeo" (secondo la definizione di tradizione erudita) quello proprio dei principali centri veneti (Este, Padova ecc.), e un altro come "di Adria" Infine, avanza due considerazioni importanti, che saranno alla base del prosieguo degli studi sull'epigrafia adriese: la paleografia delle iscrizioni etrusche rimanda alla norma scrittoria settentrionale; la puntuazione sillabica delle iscrizioni venetiche non può essere originaria di Adria, dove le iscrizioni vengono datate tutte al periodo ellenistico e riferite all'ambito settentrionale ${ }^{57}$. Tali argomentazioni, che per lo studioso sono fondative di una critica all' ipotesi di Pellegrini sull'origine adriese della scrittura venetica, colgono però una prospettiva storica legata alla cultura etrusca, innegabilmente presente ad Adria, che mancava ai lavori del Pellegrini ${ }^{58}$. Nel 1967, in occasione dell'edizione del corpus delle iscrizioni venetiche, Pellegrini ribadisce la sua ipotesi interpretativa pur accettando quanto osservato da Lejeune, in particolare il sostanziale "imbastardimento" della grafia venetica ad Adria (soprattutto in riferimento all' interpunzione sillabica) rispetto al problema di individuare il centro come originario del modello scrittorio venetico; più tecnicamente, accoglie la sezione sulle iscrizioni di transizione veneto-latine (3), accanto a quella delle iscrizioni etrusco-venetiche (1) e venetiche $(3)^{59}$. In coda al regesto adriese, Pellegrini aggiunge alcune iscrizioni etrusche da Spina e Adria; fra le adriesi, ricorda CIE 20822, che data dubitativamente al IV sec. a.C., 20680 e infine 20180, all'epoca inedita e attribuita a un contesto di necropoli ${ }^{60}$.

${ }^{51}$ Queste erano comunque una selezione rispetto alla totalità delle iscrizioni rinvenute dagli scavi del Canalbianco ma anche da quelli precedenti e dalle collezioni presenti nel Museo.

52 Pellegrini, Fogolari 1958, p. 106. In $L V$, I, p. 637, la sezione (E) è indicata come di transizione venetico-latina.

53 Interpretando come omicron il theta privo di punto centrale, il Pellegrini ritiene che la presenza di tale lettera ad Adria fosse dovuta all' influsso greco documentato dalle iscrizioni di questo ambito note già dal XIX secolo (PELLEGRINI, Fogolari 1958, pp. 105-106).

54 Pellegrini 1965, pp. 263-264, 272-273. Il medesimo Pellegrini sottolinea come anche J. UnTermann nel suo lavoro sull'onomastica venetica (1961) aveva recepito le letture da lui proposte.

55 LeJEUne 1966, pp. 14-15.

56 Ibid., pp. 16-23; la distinzione formulata da Lejeune è ribadita in FogolARI, SCARFì 1970, p. 39, nota 24.

57 LeJEUne 1966, pp. 24-25.

58 Questi, in $L V$, I, p. 639, su stimolo del lavoro di Lejeune, accenna cursoriamente al problema in termine di rapporto fra Adria e Spina e di provenienza dei coloni delle due città.

$59 L V$, I, pp. 637-638.

${ }^{60} \mathrm{Ibid}$., pp. 652-653, nn. 1-8. Le iscrizioni spinetiche sono aggiunte evidentemente sulla base delle considerazioni sul rapporto fra Adria e Spina e probabilmente anche in risposta al lavoro di Pfiffig, nel quale lo studioso austriaco vede in 
Ormai accessibile un nucleo consistente di iscrizioni con relativi apografi e fotografie, nel fondamentale lavoro del 1974, Ricerche sugli Etruschi e sugli Umbri a nord degli Appennini, G. Colonna afferma come le più antiche iscrizioni di Adria siano tutte in norma scrittoria etrusco-meridionale ${ }^{61}$. Quanto proposto da Colonna, che si pone nella linea delle problematiche tracciate circa dieci anni prima da Lejeune, se da un lato offre una soluzione al problema dell'origine dell'epigrafia venetica, dall'altro fornisce spessore storico all'epigrafia adriese, riconoscendone l'etruscità (meridionale) per la fase più antica.

Un punto di svolta significativo è da riconoscere nella critica al modello di G.B. Pellegrini offerta da L. Agostiniani nel 1982. Questi rifiuta il riconoscimento di un gruppo di iscrizioni etrusco-venetiche, proponendo per queste una lettura totalmente etrusca sulla base di nuove interpretazioni e di riflessioni sull'onomastica ${ }^{62}$, come già prima di lui C. de Simone aveva avanzato per l'unica iscrizione appartenente alla sezione (C) del Pellegrini (e A del Lejeune per quelle venetiche) ${ }^{63}$. L'inesistenza della categoria delle iscrizioni etrusco-venetiche è stata ribadita da M. Cristofani nel $1991^{64}$. Tale linea interpretativa è stata valorizzata anche da R. Mambella nel 1986, in occasione della pubblicazione del materiale già studiato per la tesi di Specializzazione a Bologna sotto la supervisione di G.A. Mansuelli. Analizzando nuovamente alcune delle iscrizioni di Canalbianco ${ }^{65}$, Mambella riafferma quando detto da Agostiniani e propone una nuova attribuzione all'ethnos etrusco per iscrizioni già lette come venetiche ${ }^{66}$. Mambella conclude asserendo l'esiguità delle attestazioni venetiche, e soprattutto una datazione di queste al II-I sec. a.C. con una evidente influenza latina ${ }^{67}$.

Radicatasi questa nuova linea interpretativa nella letteratura scientifica, A. Maggiani nel $2002^{68}$ afferma, sulla base delle nuove attribuzioni ad ambito etrusco di iscrizioni venetiche da parte di $\mathrm{H}$. Rix nel corpus da lui raccolto $(E T)$, la totale assenza di una documentazione epigrafica venetica ad Adria. Il contributo risulta di ampio respiro, indagando per la prima volta in maniera sistematica l'origine delle norme ortografiche e dei gentilizi attestati nelle epigrafi adriesi, per le quali conferma un' influenza orvietana tra fine VI e V sec. a.C., già riconosciuta da G. Colonna nel contributo del 1974 sopra menzionato. L'alfabetizzazione sarebbe stata dunque appannaggio unicamente della compagine etrusca della città, mentre tre soltanto sarebbero le attestazioni di onomastica venetica ma in iscrizioni etrusche per alfabeto e lingua, delle quali solo una da necropoli ${ }^{69}$; a queste lo studioso aggiunge un gruppo di iscrizioni che documenterebbero nomi attestati soltanto ad Adria, supposti di origine venetica benché privi di confronti onomastici, e un altro nutrito gruppo che per ampia diffusione nella penisola italica non consente puntuali attribuzioni culturali ed etniche ${ }^{70}$.

alcuni documenti spinetici ( $m a$ in misura minore anche da Bologna, Perugia e Chiusi) degli elementi onomastici venetici (Pfiffig 1961a, pp. 327-329, citato da G.B. Pellegrini in $L V$, I, p. 639).

${ }^{61}$ Colonna 1974b, pp. 23-24.

62 Agostiniani 1982, pp. 239-240; ma dubbi sulla completa etruscità esprime per le nn. 491-492 (adesso CIE 20461-20462).

63 DE Simone 1975, p. 136, n. 24 (adesso CIE 20461-20462).

${ }^{64}$ CRistofani 1991, pp. 120-121, nota 15.

65 Mambella 1986, dove non vengono riportati i numeri di inventario degli oggetti iscritti. In tale articolo Mambella propone una tabella, nella quale suddivide l'onomastica etrusca adriese in prenomi e gentilizi maschili e femminili, oltre a inserire l'onomastica greca attestata nella città.

66 Mambella 1986, coll. 268-270.

67 Ibid., col. 274, dove si ricollega all'assenza di una inclusione di Adria nell'area di influenza veneta nelle fonti antiche e non nega la presenza di Veneti nella città, secondo lo studioso comunque modesta alla luce delle attestazioni riportate.

68 Maggiani 2002a, in particolare p. 57.

69 Ibid., p. 57. Per queste iscrizioni, si rimanda alle letture proposte in CIE 20196 20273, 20614. In particolare, mentre la nuova lettura di CIE 20614 non la lega più all'onomastica venetica, per l'interpretazione delle altre due cfr. V.4.

70 Ibid., p. 60, con alcuni esempi, fra i quali per il primo gruppo Siliu (adesso CIE 20559-20560), connesso con l'idronimo venetico Silis, mentre per il secondo gruppo Tite (adesso CIE 20084), oppure Titi. Unioi (adesso CIE 20550). 


\section{I.2. La quantificazione delle iscrizioni}

La frammentarietà dei dati relativi alle iscrizioni, desumibili dal quadro critico sopra illustrato, è resa ancor più chiaramente evidente dall'ambiguità riscontrabile in merito al loro numero complessivo. G.B. Pellegrini nel 1959 in occasione del I Convegno di Studi Etruschi ${ }^{71}$ rende note circa cinquanta iscrizioni preromane. Queste provengono sia da necropoli che da abitato come si deduce dalle successive pubblicazioni del 1958 e 1965, benché lo studioso ne affermi la provenienza unicamente da contesti funerari. Tale cifra è successivamente riportata nel 1994 da L. Sanesi Mastrocinque ${ }^{72}$, mentre ancora nel 1970 G. Fogolari parla di una ventina di iscrizioni etrusche, riferendosi unicamente alla pubblicazione del Pellegrini del $1958^{73}$. Nel 1986 R. Mambella cita circa ottanta iscrizioni etrusche da lui esaminate provenienti da abitato e da necropoli ${ }^{74}$, mentre nel $1991 \mathrm{M}$. Cristofani fa riferimento a circa trenta iscrizioni etrusche ${ }^{75}$. Infine A. Maggiani per il IV-III sec. a.C. riferisce di circa settanta iscrizioni $^{76}$, a cui vengono aggiunte le tre di fine VI-V sec. a.C., già enucleate da G. Colonna nel 1974. Il medesimo Colonna, in un lavoro edito nel 2003, porta a sette le iscrizioni comprese tra il 520 e il 460 a.C. ${ }^{77}$. La cifra totale sale infine a 120 (comprensive di quelle greche) nel 2009: questo il numero di iscrizioni proposto da L. Haack, che però non ne esplicita la base documentaria ${ }^{78}$.

L'edizione del CIE IV, I, 1 ha portato a un notevole incremento della documentazione epigrafica etrusca (e non solo) di Adria e del suo territorio rispetto a quanto noto in letteratura. Più nello specifico, il lavoro di ricognizione preliminare ha consentito di acquisire nel corso degli anni un numero considerevole di iscrizioni inedite. Una prima comunicazione sui contesti funerari di epoca ellenistica,

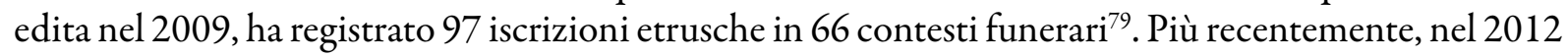
chi scrive ha proposto una analisi delle iscrizioni tardo-arcaiche, evidenziandone un aumento dalle tre registrate da G. Colonna nel $1974^{80}$, a 17 (già nel 2010 un lavoro preliminare ne aveva registrate $13^{81}$ ). Contestualmente si dava comunicazione di aver recuperato 180 iscrizioni comprese tra gli ultimi decenni del VI e il II sec. a.C. comprensive dei contesti abitativi e funerari, sia di Adria che del sito di Bottrighe ${ }^{82}$.

Il fascicolo adriese del CIE permette di aggiornare le quantità note in letteratura grazie a una registrazione sistematica di tutte le evidenze. Comprendendo tutti i documenti che presentano almeno due lettere (siano essi completi che lacunosi), si arriva a 212 iscrizioni localizzabili ad Adria e nel suo territorio tra la fine del VI e il II sec. a.C. Di queste, 183 appartengono alla città di Adria, con 32 iscrizioni databili approssimativamente tra la fine del VI e la fine del IV sec. a.C., 146 tra la fine del IV e il II, 5 di incerta datazione. Di quelle ellenistiche, 85 provengono da contesto funerario e 41 da contesto abitativo (le restanti 20 sono di incerta origine). Tra le più antiche, si datano tra la fine del VI e il V sec. a.C. 25 iscrizioni (CIE 20289 presenta due diverse iscrizioni sul medesimo supporto), 2 tra la fine del

${ }^{71}$ Pellegrini 1959, p. 190; tali iscrizioni sono quelle pubblicate in Pellegrini, Fogolari 1958 e Pellegrini 1965.

72 Sanesi Mastrocineue 1994, p. 127.

73 Fogolari, SCARFì 1970, pp. 37-40, p. 39 e nota 24.

74 Mambella 1986, p. 278. La cifra proposta da Mambella viene riportata anche in Bourdin 2006, p. 28, benché sia riferita, forse per una errata interpretazione, unicamente all'ambito funerario.

75 Cristofani 1991, p. 113, dove le iscrizioni da necropoli per il IV- III sec. a.C. risultano 31, e p. 120, dove risultano 32. In ibid., p. 113, le iscrizioni etrusche totali risultano 35.

76 Maggiani 2002a, p. 59; similmente G. Sassatelli riferisce di oltre 60 iscrizioni per il IV e III sec. a.C., nonché 4 o 5 iscrizioni databili al V sec. a.C. (SASSATELLI 2004, p. 25).

77 Colonna 2003, p. 166, nota 31. A questo elenco Colonna aggiunge come ottava l'iscrizione da Larda di Gavello, adesso letta come umbra e datata alla fine del II-I sec. a.C. (CIE 21031; REI LXXIX, pp. 348-350).

78 HAACK 2009, p. 46.

79 Gaucci, Pozzi 2009, p. 58, nota 27, e già incrementate di una unità nel 2012 (GAUCCi 2012a, p. 143, nota 1).

80 Colonna 1974b, pp. 23-24, nn. 1-3.

${ }^{81}$ GaUcCi 2010; ID. 2012a, in particolare p. 143.

82 Ibid., nota 1. 
VI e il IV, 2 tra il Ve il IV e sicuramente 3 al IV. Dal territorio provengono 29 iscrizioni: 20 da Bottrighe, di cui una di IV sec. a.C. e le restanti di III-II; da San Cassiano una iscrizione di V sec. a.C.; da Le Balone una di VI e una di V sec. a.C.; da San Basilio 5 iscrizioni di VI-V sec. a.C.; infine una iscrizione di III sec. a.C. genericamente rinvenute tra Adria ed Este (CIE 21027).

Nel complesso quindi si contano 33 iscrizioni di fine VI-V sec. a.C., 8 databili tra V e IV, 166 di III-II.

Alla luce di questi dati, le oscillazioni quantitative proposte nei precedenti lavori sopra menzionati sembrano dipendere da due fattori principali: l'incertezza di attribuzione all'alfabeto e alla lingua etrusca di molte iscrizioni, soprattutto fino agli anni Settanta del secolo scorso; l'assenza di una ricognizione sistematica. Meno influente pare invece l'incremento del corpus dovuto a nuovi scavi e scoperte, in quanto dal secondo dopoguerra, seppure l'attività di indagine ad Adria sia stata fervida e ricca di nuovi dati, non ha prodotto numeri tali in termini di iscrizioni da determinare le oscillazioni evidenziate. Infatti, solo 31 (e 3 non etrusche) sono state rinvenute con sicurezza dopo il 1970, in particolare durante l'indagine delle aree funerarie di via Spolverin (1990) e di Ca' Cima (1993-1995), e durante gli scavi di abitato di via San Francesco (1994) e via Ex Riformati (2000).

Uno sguardo al resto dell' Etruria padana permette di comprendere in maniera comparata l'effettiva entità di tali numeri, secondo un approccio che G. Sassatelli ha proposto per la documentazione epigrafica di Marzabotto nel $1994^{83}$. La seguente analisi è suddivisa in due periodi, quello compreso tra la fine del VI e il pieno IV sec. a.C. e quello dal pieno IV al II, seguendo così il range cronologico delle attestazioni adriesi. Lo spartiacque del pieno IV sec. a.C. è ovviamente concomitante con il periodo successivo all'invasione storica dei Celti e al venir meno della maggior parte dei siti etruscopadani.

\section{I.2.1. Adria e l'Etruria padana tra la fine del VI e gli inizi del IV sec. a.C.}

Un preliminare lavoro di confronto tra le attestazioni di Adria e del suo territorio rispetto al resto dell'Etruria padana per il periodo compreso tra la fine del VI e il V sec. a.C. è stato proposto nel $2012^{84}$. Il numero è ovviamente incrementato, mentre il quadro per il resto dell'Etruria padana non è sostanzialmente cambiato, se non per le novità di Marzabotto, cioè le iscrizioni dal tempio di Uni e la lamina di bronzo dal tempio di Tinia (in totale 6 iscrizioni) ${ }^{85}$, e inoltre le iscrizioni rinvenute a Mantova da Piazza Santa Barbara e quelle edite dall'abitato di Spina (si veda infra).

Si propone dunque un aggiornamento della quantificazione delle iscrizioni dell'Etruria padana note in letteratura. Queste sono state organizzate sulla base dei principali comprensori territoriali nei quali è per tradizione suddivisibile l'area interessata dal popolamento etrusco a nord degli Appennini. Si è infatti sentita l'esigenza di una impostazione che ponesse primariamente a confronto i centri principali e il loro territorio, anzi tutto perché questa equiparazione è possibile per la documentazione adriese (si veda supra). Inoltre, questa analisi pare ancora più rilevante da sviluppare in un'area, quella etrusco-padana, dove proprio con la seconda metà del VI sec. a.C. si assiste a una significativa riorganizzazione della rete insediativa, caratterizzata da una forte gerarchia dei $\operatorname{siti}^{86}$ che perdura fino all'invasione celtica.

83 Sassatelli 1994, pp. 194 e 199, dove si imposta una analisi comparativa tra le attestazioni di Marzabotto e del resto dell'Etruria padana.

${ }^{84}$ GaucCi 2012a, pp. 164-165.

85 Per queste iscrizioni si veda ora Govi 2014 (lamina dal tempio di Tinia); EAD., in REE LXXIX, pp. 306-312, nn. 63-64, in REE LXXX, pp. 236-241, nn. 2 e 4 e in REE LXXXII, pp. 221-225, n. 5 (per le iscrizioni dal tempio di Uni).

${ }^{86}$ Si rimanda alla recente analisi di QUIRINO 2017. Va inoltre puntualizzato, sebbene risulti secondario in questa sede, il ruolo rivestito dalle iscrizioni negli studi che affrontano i limiti dell'espansione etrusca in quest'area (cfr. SASSATELLI 2008a, passim e in particolare p. 86, nota 38). 
- Kainua-Marzabotto e la Valle del Reno. Il centro principale della valle, Kainua-Marzabotto, conta ben 61 iscrizioni comprese tra la seconda metà del VI e gli inizi del IV sec. a.C. ${ }^{87}$. A eccezione di quelle provienenti dai santuari di Tinia e di Uni (e di quella di destinazione sacra dalla c.d. "Grande Fornace" della Regio II, insula $1^{88}$ ), in numero di 10 , per il resto sono tutte da contesti abitativi. Non si registra invece nulla nel territorio a eccezione del caso, comunque eccezionale per molti versi, della stele di Monteacuto Ragazza del pieno V sec. a.C. da contesto sacro $\left(E T^{2}\right.$, Fe 3.3).

- Bologna. Il numero delle iscrizioni della principale città dell'Etruria padana non è particolarmente significativo rispetto alla sua importanza. Le attestazioni di Bologna sono prevalentemente di contesto funerario: si contano 19 iscrizioni su stele databili tra il V e gli inizi del IV sec. a.C.89, e 5 iscrizioni su supporti ceramici databili tra la fine del VI e il pieno IV sec. a.C. provenienti dai sepolcreti occidentali ${ }^{90}$. Solo 3 iscrizioni di V sec. a.C. provengono da contesto sacro (due frustuli di V sec. a.C. da Villa Cassarini) ${ }^{91}$ e abitativo (una iscrizione di medesimo periodo da via D’Azeglio) ${ }^{92}$. Nessuna iscrizione è documentata dal territorio attorno alla città.

- Emilia occidentale. Nel Modenese e nel Reggiano si ha la testimonianza di un significativo numero di attestazioni da siti diversi ( 14 da contesti abitativi ${ }^{93} \mathrm{e} 3$ da necropoli $\left.{ }^{94}\right)$; i centri principali dell'area presentano comunque quantità modeste (San Polo) se non nulle (Modena). Fra il materiale da necropoli, si ricorda l'iscrizione sulla stele di Tombarelle (e forse una simile dallo stesso luogo vista da Brizio

87 Si contano: 6 iscrizioni databili al 550-500 a.C. (SASSATELli 1994, nn. 261, 279; SASSATELli, GaUCCI 2010, nn. 434-436; REE LXXIX, n. 64); 13 databili alla fine VI-inizi V sec. a.C. (SAssatelLi 1994, nn. 1, 207-209, 277, 280; SASSATELli, GAUCCi 2010, nn. 437-440; REE LXXIX, n. 63; REE LXXX, n. 2; REE LXXXII, n. 5); 12 databili al 500-450 a.C. (SAsSATElli 1994, nn. 66, 293; SAsSATELli, Gaucci 2010, nn. 441-444; Govi 2014; REE LXXIX, nn. 59-62; REE LXXX, n. 4); 15 databili alla metà-seconda metà V sec. a.C. (SASSATELLI 1994, nn. 4, 8-9, 69-70, 90, 278, 292; SAssatelli, GAuCCi 2010, nn. 445-451); 15 databili al V-inizi IV sec. a.C. (SAssatelli 1994, nn. 2-3, 5-6, 10-11, 71-74, 91, 96-97, 210, 262).

88 Sassatelli 2011.

89 Un quadro aggiornato delle iscrizioni su stele è offerto in GaUcci, Govi, SAssatelli c.s. Rispetto a quanto precedentemente noto in letteratura, si sono aggiunti quattro frammenti iscritti inediti (stele Ducati 16, 18, 61, 83), le cui schede critiche sono edite in REE LXXXII, pp. 215-220, nn. 1-4.

90 Dal sepolcreto Arnoaldi: MaCellari 1994 (410-400 a.C.); si veda anche SAsSATElli 2008b, p. 336; ID. 2013 , p. 409 e ora REE LXXXII, pp. 281-283, n. 34. Dal sepolcreto della Certosa: PANdolfini, Prosdocimi 1990, pp. 58-59, n. III.9, tav. XXVII, dalla tomba Certosa 331-332, datata tra la fine del VI e gli inizi del V sec. a.C.; ET ${ }^{2}$, Fe 2.17 (nituna[?), dalla tomba Certosa 228 (ZANNONi 1876-1884, p. 80), già discussa in SASSATELli 1988, p. 243, nota 108 e letta [---] nituna, dove si riconosce un gentilizio; REE LXXXII, pp. 279-281, n. 33, dalla tomba Certosa 405 (per il contesto: Govi 1999, p. 34, nota 15; l'iscrizione è erroneamente datata al periodo recente e letta veltur in $E T^{2}$, Fe 2.20). A queste si aggiunge FABRETTI 1878, p. 77, n. 17, tav. I, iscrizione citata come problematica in SASSATELLI 1988, p. 243, nota 108, e sulla quale ci si ripropone di tornare in altra sede. Si espunge l'iscrizione dalla tomba Certosa 415 non più conservata e nota in letteratura come antis (FAbretti 1872, p. 12, n. 83, tav. IV; Govi 1999, p. 52), in quanto si ritiene da leggere come un insieme di trademarks greci.

91 Da ultimo Romagnoli 2014, pp. 204-205, nn. 80-81, fig. 161, con riferimenti (solo una delle due, la n. 81, recepita in $E T^{2}$, Fe 2.6).

92 Malnati 2010, p. 120, fig. 44.9. Più difficile proporre una cronologia certa per le altre due iscrizioni provenienti da questo scavo, per le quali ibid., p. 118, fig. 44.1, p. 119, fig. 44.19; per l'ultima citata, letta dubitativamente come teonimo, si rimanda anche alle osservazioni in SASSATELLi 2017, pp. 193-194.

93 San Prospero, loc. Campo del Castellazzo: MaCellari 2008, p. 116 (fine VI sec. a.C.); Monte Pèzzola: REE LXXIV, pp. 238-240, n. 5 (500-450 a.C.); Caciola di Scandiano: Losi 1989, p. 147, n. 11, tav. L (VI-V sec. a.C., contesto); Campo Pianelli presso la Pietra di Bismantova: Macellari 1981-1982, p. 279, n. 2, fig. 3,3 (V sec. a.C.); Montecchio, loc. Il Monte: $E T^{2}$, Pa 2.9 (V sec. a.C.); Coviolo, loc. S. Rigo: $E T^{2}$, Pa 2.8 (V sec. a.C.); Castellarano: Maggiani 1992a, pp. 211-212, n. 3 (V sec. a.C.); Buoite, Zamboni 2008, p. 126, n. 1031, fig. 32 (V sec. a.C.); Poviglio, loc. Case Carpi: Damiani, Pellegrini, Saltini 1990, pp. 266-267, n. 3, tav. LXXIX (450-400 a.C.); Poviglio, loc. Santa Rosa: REE LXXIV, pp. 237-238, n. 4, pp. 373-374, n. 126, pp. 374-376, n. 127 (tardo V sec. a.C.); Monte di Santa Maria: MACELLARI 2004, p. 151; Baggiovara, loc. Case Vandelli: Malnati 1989, p. 270, fig. 220,1 (?). Nell'elenco non è stato inserito il digramma $\chi u$ da Campo Pianella (MaCellari 1981-1982, pp. 278-279, n. 1, fig. 3, 1-2), in quanto probabile numerale.

${ }^{4}$ S. Polo d'Enza: ET², Pa 4.3, 9.1, 0.1 (V sec. a.C.); Crespellano, loc. Tombarelle: ET², Fe 1.12 (V sec. a.C.). 
riutilizzata all'interno di una casa di campagna) come appartenente a una pratica monumentale legata a contesti funerari di ambito felsineo. Le restanti 14 iscrizioni provenienti da contesti di tipo abitativo sono invece su supporto ceramico. In particolare, si ricorda che da San Polo provengono le due iscrizioni con le forme teonimiche vea $\left(E T^{2}, \mathrm{~Pa} 4.3\right)$ e rat $\left(E T^{2}, \mathrm{~Pa} 0.1\right)$, quest'ultima ora reinterpretata da G. Colonna come $\mathrm{ran}^{95}$.

- Mantovano. Nel Mantovano si contano almeno 19 iscrizioni, delle quali 16 dal sito del Forcello di Bagnolo San Vito ${ }^{96}$ e 3 da Mantova ${ }^{97}$, tutte da contesto abitativo. Gli scavi del Forcello mostrano una notevole ricchezza di documentazione per il periodo in esame, non confrontabile con quella di Mantova. È importante sottolineare che a fronte di una apparente scarsità di attestazioni a Mantova (ci si riferisce qui alle sole iscrizioni), la loro presenza attesta significativamente almeno una frequentazione dell'insediamento nel periodo cronologico in esame ${ }^{98}$, sebbene R.C. de Marinis ${ }^{99}$ vorrebbe Mantova fondata in coincidenza con l'abbandono del Forcello.

- Spina. Per Spina si contano 46 iscrizioni da necropoli e abitato edite fino al 1998 per le quali è stata proposta una cronologia comprensiva tra la fine del VI e gli inizi del IV sec. a.C. ${ }^{100}$. Per

95 Colonna 2015, p. 68. Su questo e su vea, si veda Sassatelli 2017, p. 196 e nota 31.

${ }^{9}$ REE LXXIV, p. 235, n. 2 (510-500 a.C.); DE MARINIs 2007², p. 65, nota 29, settore R 18, anno 1992, US 461 (inizi V sec. a.C.); ibid., p. 66, R 19 h-i 3-4, US 1383 (475-450 a.C.); ibid., p. 66, S 17 p-q 18-19, US 188 (500-450 a.C.);

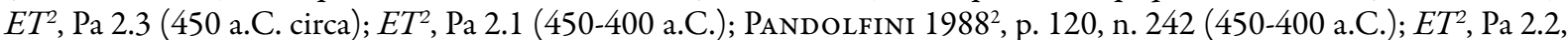
2.4-6 (450-400 a.C.); ET², Pa 2.7 e PANDolfini 1988², pp. 120-121, n. 244 (V-inizi IV sec. a.C.). Al Forcello sono state rinvenute 3 iscrizioni tutte provenienti dal medesimo contesto abitativo e datate alla prima metà del V sec. a.C., sulle quali si hanno notizie preliminari (LAMANna 2014, p. 57; Komp, QUiRINo, RaPi 2020, p. 231, fig. 14.3).

97 Rimane purtroppo difficile valutare nell'insieme le iscrizioni edite da D.F. Maras (in Menotti, Maras 2012) e recuperate dallo scavo di Piazza Santa Barbara a Mantova. L'editore propone ben 9 iscrizioni datate tra la fine del VI e il V sec. a.C. Tuttavia, sono presenti solo gli apografi e manca qualsiasi documentazione sui supporti, sia come caratteristiche (dimensioni, impasto, superficie) e riferimenti tipologici, sia come apparato grafico e fotografico, a eccezione delle iscrizioni nn. 1-2 e 8, per le quali sono forniti la fotografia del supporto della prima (un kantharos miniaturistico in ceramica grigia, definito impropriamente "bucchero grigio"), il disegno del supporto per la n. 2 (una olletta in ceramica grezza), e la fotografia del fondo interno della n. 8 (piede e fondo di una coppa in ceramica depurata). Il kantharos, altrimenti definito tazza biansata nel lessico tipologico dell'Atlante di C. MATTIOLI (2013), è documentato soltanto nell'Emilia occidentale e nella Romagna, in tutte le classi, seppure vada sottolineato che solo un esemplare in ceramica grigia è altrimenti noto da Faenza, loc. Persolino (Morpurgo 2013, p. 448, con riferimenti). L'esemplare di Mantova sembra appartenere al tipo I, 1, a. Come emerge dall'analisi offerta da G. Morpurgo, la forma miniaturistica necessita cautela, non solo per la rarità e la difficoltà di identificazione quando frammentaria, ma anche perché i confronti in Etruria propria rinviano al III-II sec. a.C. (cfr. MoRPURGo 2013, p. 445, con riferimenti). Pare quindi difficile accettare la datazione sulla base del supporto entro gli inizi del V sec. a.C. La paleografia sembra rimandare peraltro a esperienze di IV-III sec. a.C. proprio di ambito adriese (ma risulta affine anche alle caratteristiche paleografiche della n. 11, il cui supporto rimanda, dalla descrizione, plausibilmente grossomodo alla cronologia proposta di fine IV-inizi III sec. a.C.). L'olla in ceramica grezza appartiene invece al tipo X, 2, a dell'Atlante di C. MATTioli (2013, p. 343), che registra solo due attestazioni situate nel Reggiano, delle quali quella di Casale di Rivalta datata tra il pieno VI e il V sec. a.C. Non si capisce tuttavia la datazione puntuale del pezzo iscritto alla prima metà del V sec. a.C., considerando l'estrema difficoltà di lettura e quindi anche delle relative considerazioni di tipo paleografico, che non possono dunque meglio definire la cronologia ampia del supporto. Per il resto, alla luce delle caratteristiche paleografiche desumibili dagli apografi, sembra che possano appartenere con buona verosimiglianza a un periodo compreso tra la seconda metà del VI e il V sec. a.C., come proposto da Maras, almeno le iscrizioni nn. 3, 5, 7.

98 Per un quadro sintetico sulle ultime indagini di Mantova, si veda Menotti 2017, dove tuttavia non si comprende se la datazione al periodo tardo-arcaico di alcuni edifici (e un luogo di culto?) sia basata unicamente sulle iscrizioni (e sui vasi miniaturistici?).

99 Basti qui richiamare de Marinis, Casini, Rapi 2016, in particolare pp. 460-461. Per un quadro della documentazione di Mantova, si rimanda a MenOTTi 2017.

100 È stato pubblicato nel 1998 un lavoro di G. Uggeri sul suffisso - alu con alcune iscrizioni da Spina inedite (UGGERI 1998); dopo tale data, non sono state pubblicate novità epigrafiche fino al 2013. Un primo quadro comprensivo di 39 iscrizioni era stato proposto in GAUCCI 2012a, p. 164, nota 1. Si riporta qui l'elenco aggiornato: ET ${ }^{2}$, Sp 2.1; 0.6 (inizi V sec. a.C.); $E T^{2}$, Sp 2.110 (480 a.C.); $E T^{2}$, Sp 0.7; UGGERI 1978, p. 345, n. 5 a) (500-450 a.C.); UGGERI 1978, p. 353, 
l'abitato, al numero consistente di documenti degli scavi già edito da G. Uggeri e S. Patitucci ${ }^{101}$, si potrebbero oggi sommare i dati di abitato editi da L. Zamboni sugli scavi 1977-1981 e inoltre quelli degli scavi 2007-2009 della ex Soprintendenza per i Beni Archeologi dell'Emilia-Romagna; nessun documento epigrafico è noto attualmente per lo scavo dell'Università di Zurigo diretto da Ch. Reusser $^{102}$. Purtroppo l'edizione a cura di D.F. Maras dei documenti iscritti dagli scavi 2007-2009 (27 frammenti inediti con iscrizioni in alfabeto etrusco più o meno lacunose e digrammi a cui si aggiunge il frammento con iscrizione in greco e in etrusco ${ }^{103}$ ) non è di agevole utilizzo, mancando infatti dati sui supporti (solo intuitivamente desumibili dalle fotografie, che tuttavia sono perlopiù focalizzate sulle iscrizioni), proposte cronologiche (seppure di massima) dei documenti e infine rinvii agli specifici contesti di rinvenimento; non è dunque opportuno qui avventurarsi in quantificazioni di taglio diacronico che, sebbene prevalentemente orientate verso il periodo ellenistico, mancano di dati fondamentali per inquadrarle con la necessaria sicurezza. Il lavoro sugli scavi 1977-1981 ${ }^{104}$, pur presentando una edizione poco soddisfacente del materiale epigrafico ${ }^{105}$, permette di contare 29 documenti inediti su ceramica tra serie alfabetiche e altre tipologie di testi fino ai digrammi, da cui è peraltro lecito espungerne almeno due da ritenere greche ${ }^{106}$; la rassegna è completata dall' iscrizione mi taluś già nota alla critica (datata alla fine del V sec. a.C. da G. Uggeri ${ }^{107}$ ) e dal celebre ciottolo con iscrizione mi tular, per il quale si riporta la cronologia di prima metà del IV sec. a.C. accettata in letteratura ${ }^{108}$. Tutti i documenti inediti si dovrebbero collocare tra il periodo tardo-arcaico e gli inizi del IV sec. a.C., come dichiarato da Zamboni e sulla base della datazione proposta per le strutture analizzate ${ }^{109}$. Se si sommano queste iscrizioni alle 46 già conteggiate, si arriverebbe a 73 attestazioni edite da contesti funerari e abitativi (cifra che potrebbe variare con l'edizione del molto materiale ancora inedito e con uno studio accurato di quello già edito). A conclusione di questo quadro su Spina, si osserva una assenza di iscrizioni per il territorio circostante, per il quale si conosce con qualche sicurezza soltanto l'insediamento di $\operatorname{Voghenza}^{110}$, sebbene si sia tentato di ricostruirne l'areale

n. 13 a); UGGERI 1978, pp. 374-375, 5 e-g); $E T^{2}$, Sp 2.2 (475-450 a.C.); $E T^{2}$, Sp 2.4-8; REE LVII, p. 248 , n. 3 (450 a.C. circa); $E T^{2}$, Sp 2.3, 2.12, 7.1, 0.8 (450-400 a.C.); $E T^{2}$, Sp 2.11, 0.4 (450-425 a.C.); $E T^{2}$, Sp 2.13, 111, 0.9; UGGERI 1978, pp. 350-351, n. 10 a) (425-400 a.C.); $E T^{2}$, Sp 2.14-19, 121; Uggeri 1998, p. 495, n. 14; PANdolfini, Prosdocimi 1990, pp. 74-75 (fine V sec. a.C.); ET², Sp 2.9-10; velśu in Colonna 1993, p. 139 (V sec. a.C.); $E T^{2}$, Sp 2.20-21, 9.0, 9.5 (cronologia in Pandolfini, Prosdocimi 1990, pp. 75-76) (fine V-inizi IV sec. a.C.); ET ${ }^{2}$, Sp 2.22 (fine V-prima metà IV sec. a.C.); REE XLVIII, p. 356, n. 45 (G. Uggeri); ET², Sp 2.29-32 (inizi IV sec. a.C.). Va invece esclusa l'iscrizione letta a $\theta$ e pupuś in Colonna 1993, p. 140, nota 72, attribuita al V sec. a.C., e invece da leggere ahe pupuś e da attribuire al periodo ellenistico come proposto in GAUCCI 2016, p. 203, nota 125.

101 Sono 51 in totale le iscrizioni da abitato quantificate in Gaucci, Pozzi 2009, p. 52.

102 Sullo scavo diretto da C. Reusser, si vedano in particolare le comunicazioni in Reusser 2016; CAPpUCCINI, Mohr 2017.

103 Maras 2013a. Dal computo si è espunto il n. 19 del catalogo di Maras, di fatto composto da due aste lacunose come visibile dalla fig. 19 a p. 161.

104 Zamboni 2016.

105 Mancano infatti singole schede di approfondimento, articolate riflessioni su supporti e paleografia, gli apografi sono scarsamente leggibili essendo in scala 1:4 (fra le sequenze alfabetiche e le iscrizioni, manca l'apparato grafico per i documenti nn. 840,1307, 1320-1321) e vi è assenza di apparato fotografico (fotografie di molte iscrizioni si possono comunque visionare nella Tesi di Dottorato alla base del lavoro edito: ZAMBONI 2012).

106 Si tratta dei nn. 566 e 906. Il primo è da far rientrare nel novero dei marchi commerciali greci come sostenuto dal medesimo editore (che trascrive TE invece di $\Theta E$ ); gli altri segni individuati sono stati interpretati come etruschi (lettura $\theta l$ ), sebbene non si possa escludere che siano accidentali. Per il n. 906, si rimanda alle considerazioni nella scheda REE LXXXI, pp. 370-371, n. 59.

107 UGgeri 1998, p. 495, n. 14. Cfr. $E T^{2}$, Sp 2.121, con riferimenti.

108 Ma la paleografia potrebbe anche rialzare la cronologia proposta e diffusa in letteratura (cfr. SASSATELLI 2017, p. 185, nota 7).

109 Zamboni 2016, pp. 14 e 242.

110 Patitucci 1979. 
sulla base di puntuali attestazioni e notizie di rinvenimenti di imbarcazioni fluviali con carichi che da Spina potrebbero essere partiti ${ }^{111}$.

- Romagna. Si può aggiungere a questo quadro anche la Romagna, seppure vada sottolineato in misura prudenziale, come già fatto da G. Colonna, che per Imola ${ }^{112}$, Faenza (loc. Persolino) ${ }^{113}$ e San Martino in Gattara ( $E T^{2}$, Pa 9.3, V sec. a.C.) si registrano documenti che, sebbene manifestino una capacità scrittoria basata su apprendimento e uso dell'alfabeto etrusco, non testimoniano una specifica competenza linguista e pertanto potrebbero essere anche riferiti alla cultura umbra ${ }^{114}$. Se le iscrizioni di Imola e di San Martino ci documentano delle parziali sequenze alfabetiche di per sé indicative dell'alfabeto teorico, ma non necessariamente della lingua etrusca come affermato da G. Colonna, il documento di Faenza è ricondotto ora dal medesimo studioso a una onomastica schiettamente etrusca ${ }^{115}$. Si conferma per questa iscrizione quanto già sostenuto da G. Sassatelli, che vede in tutti i documenti romagnoli citati una testimonianza di cultura etrusca ${ }^{116}$. Eccezionale il quadro della valle del Marecchia, che restituisce 3 iscrizioni caratterizzate da una pratica scrittoria etrusco-meridionale ${ }^{117}$.

Il quadro delineato permette di cogliere come la documentazione di Spina, sebbene caratterizzata da importanti lacune nella completa edizione delle epigrafi rinvenute negli scavi, risulti comunque nettamente superiore a quella dei restanti centri, con l'unica eccezione di Marzabotto. In un'ottica meramente quantitativa, a questi due centri segue per quantità appunto Adria, che conta 25 iscrizioni databili tra la fine del VI e il V sec. a.C. e quindi Bologna con 17 iscrizioni e il Forcello di Bagnolo San Vito con 16. Ovviamente queste cifre vanno ritenute solo indicative, in quanto chiaramente influenzate da molti fattori, fra cui sicuramente la qualità, la durata e gli obiettivi delle ricerche effettuate nel tempo sono primariamente rilevanti. Ma si possono individuare altri fattori, definibili culturali. A Marzabotto, così come ad Adria e al Forcello, le iscrizioni sono tutte da contesti abitativi (e in misura minore da contesti sacri). Sebbene si possegga una conoscenza parziale di questo centro della valle del Reno, non essendo conservata la maggior parte del materiale rinvenuto negli scavi ottocenteschi delle necropoli, il dato può essere interpretato mettendolo a sistema con quanto noto per Bologna e per la medesima Spina. Se si guarda a Bologna, il numero molto ridotto di iscrizioni, 17 totali, dipende da diversi fattori. Il primo, sicuramente il più rilevante, è la scarsa documentazione di contesti abitativi di questa fase. In secondo luogo, colpisce come a fronte di circa un migliaio di sepolture datate tra la seconda metà del VI e il pieno IV sec. a.C. le attestazioni su supporto ceramico di ambito funerario siano piuttosto scarse. Una recente analisi ${ }^{118}$ ha portato peraltro a ipotizzare che alla base di tale scarsità ci debba necessariamente essere una prassi funeraria che non contemplava la manifestazione della scrittura a eccezione di casi speciali. Sempre a Bologna, la monumentalizzazione delle sepolture demandata alle stele documenta come la scrittura, comunque sempre in un numero limitato di casi, fosse destinata a questi speciali supporti assumendo dunque una forma pubblica, altrimenti non espressa in tutta l'Etruria padana a eccezione dei due più antichi cippi di Rubiera nel Reggiano (ET ${ }^{2}$, Pa 1.1-2), della stele di Tombarelle che comunque rientra nell'esperienza bolognese (si veda supra), e infine in Appennino nella stele di Monteacuto Ragazza del pieno V sec. a.C. appartenente a un ambito sacro (si veda supra). Le limitazioni della pratica scrittoria in necropoli sono confermate da Spina, dove l'importante raccolta sistematica di A. Pozzi permette di avere già da ora un quadro d'insieme (comprensivo dunque del materiale edito e di quello ancora inedito), con una maggior accuratezza per Valle Trebba dove

\footnotetext{
111 Su questo problema, si veda DesAntis 1995 e 1997.

112 Govi 1994 a (fine VI-prima metà V sec. a.C.).

113 SAsSatelli 1999, pp. 99-101, fig. 9 (fine VI-prima metà IV sec. a.C.).

114 Colonna 2008, pp. 48-50.

115 G. COLONNA, in REE LXXVIII, p. 222, n. 30, con riferimenti.

116 SASSATELLI 2008a, p. 87, con riferimenti.

$117 E T^{2}$, Um 2.1 (VI sec. a.C.); MACELLARI 1997 (450-425 a.C.); $E T^{2}$, Um 2.2 (V sec. a.C.).

118 Gaucci, Morpurgo, Pizzirani 2018, pp. 672-674.
} 
si posseggono tutti i dati dei contesti e cronologie più accurate grazie al progetto in corso di studio dell'intera necropoli ${ }^{119}$. Qui, su 1413 tombe (comprensive di quelle scavate negli anni 1922-1935 e quindi nel 1962), 106 presentano iscrizioni etrusche ${ }^{120}$. Se si restringe il campo alle sepolture comprese tra la fine del VI e il pieno IV sec. a.C., cioè circa il $40 \%$ del totale, poco meno di un decimo (cioè meno del $4 \%$ della cifra complessiva) presenta iscrizioni. Il gruppo funerario che ha il suo fulcro nella tomba 774 , recentemente analizzato ${ }^{121}$, contando il $20 \%$ circa del totale delle tombe con iscrizione dell'intera necropoli per il periodo in esame, esemplifica bene come tale pratica non sia diffusamente distribuita ma concentrata all'interno di pochi nuclei in nome di una consuetudine funeraria forse meno rigida (o comunque non episodica) rispetto a Bologna.

Più in generale, partendo dall'assunto che le iscrizioni in ambito funerario sono parte integrante del rituale e da questo dipendevano nelle loro manifestazioni, la registrazione di ricorrenze e anomalie, nelle varie accezioni dall'assenza alla diffusa presenza, può essere un utile strumento per comprendere gli aspetti che regolavano il costume funerario a livello dell'intera comunità o di singoli gruppi socia$\mathrm{li}^{122}$. Dunque proprio Spina suggerisce una tendenza che parrebbe applicabile anche agli altri centri padani più rilevanti, cioè una maggior diffusione della pratica scrittoria in ambito abitativo rispetto a quello funerario, caratterizzato da pratiche rituali che ne limitarono l'uso. Tale tendenza ben corrisponde a quanto noto per Bologna e Marzabotto, pur nella parzialità dei contesti a disposizione, ma anche per Adria e il suo territorio, dove la documentazione epigrafica è di ambito abitativo (e santuariale) mentre finora nulla (se non alcuni graffiti alfabetici e non alfabetici) proviene dalle sepolture.

A queste osservazioni, seppure sicuramente parziali e soggette a future revisioni delle cifre soprattutto per un sito come Spina, si aggiunge che il comprensorio adriese risulta attualmente l'unico padano dove la documentazione della città e quella del suo comprensorio forniscono un quadro articolato e complessivo. Ciò è sicuramente vincolato allo stato della ricerca, che qui ha avuto un fruttuoso impulso grazie ad attività di scavo e ricognizione. In ogni caso, l'eccezionalità della documentazione fa di questo comprensorio un importante osservatorio per comprendere le dinamiche sociali e politiche fra centro primario e insediamenti satelliti o complementari, in particolare San Basilio sulla costa, San Cassiano e Le Balone nell'interno.

\section{I.2.2. Adria e l'Etruria padana nel periodo ellenistico}

Come ben noto, la situazione muta con l'invasione storica dei Celti, che portò un profondo cambiamento non solo nell'assetto insediativo e organizzativo del territorio, ma anche da un punto di vista commerciale e sociale ${ }^{123}$. La documentazione adriese è molto avara per il pieno IV sec. a.C., restituendo soltanto tre iscrizioni attribuibili al periodo sulla base dei supporti (CIE 20008, 20256, 20899), e una più dubitativamente solo su base paleografica (CIE 20037); si datano più genericamente tra V e IV sec. a.C. le iscrizioni CIE 20184, 20823, 20832.

Durante il IV sec. a.C. anche nel resto dell'Etruria padana abbiamo un numero molto ridotto di iscrizioni, se si eccettua il caso di Spina. A Bologna l'unica iscrizione nota è quella in una tomba della prima metà del III sec. a.C. ${ }^{124}$. Lungo la Valle del Reno e nella città etrusca di Kainua-Marzabotto in questa fase non si hanno attestazioni ${ }^{125}$, mentre nella più orientale Valle dell' Idice il complesso inse-

119 Sul progetto di studio della necropoli di Valle Trebba, si rimanda a Govi 2017.

120 Pozzi 2011, pp. 395-399.

121 Gaucci 2015, p. 131.

122 Gaucci, Morpurgo, Pizzirani 2018, pp. 673-674.

123 Si veda il quadro bibliografico ragionato offerto in GaUCCi 2013, pp. 85-87.

124 Vitali 1992, p. 330, n. 1, p. 331 con precedente bibliografia, tavv. 45 e 71, dalla tomba 968 del sepolcreto Benacci datata entro la prima metà del III sec. a.C.; $E T^{2}$, Fe 2.18.

125 Sassatelli 1994, pp. 194 e 199; G. Sassatelli in Sassatelli, Gaucci 2010, p. 336. Si puntualizza che in $E T^{2}$, l'iscrizione Fe 3.4. viene erroneamente datata al IV-III sec. a.C., come puntualizzato da E. Govi, in REE LXXIX, pp. $297-$ 298, n. 59. 
diativo presso Monte Bibele registra la presenza di 7 iscrizioni dalle sepolture della necropoli di Monte Tamburino e 2 dall' insediamento della Pianella ${ }^{126}$, comprese tra la seconda metà del IV e il III sec. a.C. Nessun documento è registrato nell'Emilia occidentale. Diversamente, nel Mantovano sono note 3 iscrizioni della seconda metà del IV sec. a.C. dal Castellazzo della Garolda ${ }^{127}$ e una da Roncoferraro $\left(E T^{2}, \mathrm{~Pa} 2.14\right)$. A Mantova, oltre ai pochi (10) e perlopiù lacunosi documenti editi dagli scavi del XIX secolo (Piazza Sordello, 1872) e da quelli più recenti di Vicolo Pace (1986) datati tra IV e III sec. a.C. ${ }^{128}$, che ci attestano nomi etruschi (herini) assieme ad altri di origine celtica (eluveitie ${ }^{129}$ ) e venetica (fukis?, ripetuto ben quattro volte), dai più recenti scavi di Piazza Santa Barbara provengono sicuramente almeno altre quattro iscrizioni databili tra il IV e la prima metà del III sec. a.C. ${ }^{130}$. Ben più ricca la documentazione di Spina, che nel periodo compreso tra il pieno IV e il III sec. a.C. conta 52 iscrizioni dalla necropoli di Valle Trebba ${ }^{131}$, 12 edite dalla necropoli di Valle Pega ${ }^{132}$, e 30 da abitato fra quelle edite in $E T^{2}$ (questi numeri non fanno che rimarcare quanto ancora sia la documentazione "sommersa" e quindi la «non attendibilità sul piano statistico» dei dati di Spina, soprattutto per la fase recente, come già osservato da G. Colonna nel $1993^{133}$ ).

In particolare, tra la fine del IV e il III sec. a.C., accanto a insediamenti come Monte Bibele e Bologna che mostrano un controllo da parte della componente celtica, sebbene vi sia una persistenza di popolazione di cultura etrusca (puntualmente documentata appunto dalle iscrizioni), le città di Mantova, Spina e Adria continuano la loro vita. Nel complesso, sono Spina e Adria che impongono con i loro numeri un primato in ambito padano che gareggia con alcuni dei più importanti centri dell'Etruria tirrenica per quantità. Unica sostanziale differenza, è la pratica scrittoria, limitata a supporti vascolari sia in ambito abitativo che funerario, mentre in Etruria propria il grosso della documentazione è su monumenti funerari ${ }^{134}$.

A chiusura di questo capitolo va osservato che a differenza di tutti gli altri insediamenti, fra cui anche Mantova che ebbe continuità di vita in età romana, solo Adria testimonia una persistenza della lingua e della scrittura etrusca in Etruria padana fino al passaggio tra II e I sec. a.C.

126 Per le iscrizioni da necropoli, SASSATELli 2008b, pp. 339-341, nn. 1-7 (ET ${ }^{2}$, Fe 2.13-14, 30-34), e per quelle di abitato ibid., pp. 341-342, nn. 8-9 (ET ${ }^{2}$, Fe 2.15 e 3.5). In particolare si richiama la nuova proposta di lettura di G. Colonna in REE LXXIV, pp. 372-373, nn. 124-125 per le iscrizioni $E T^{2}$, Fe 2.32 e 3.5.

127 PANDOlFini $1988^{2}$, pp. 121-124, nn. 247-249.

128 DE MARINIS 2007², pp. 66-69, fig. 27 (dove sono elencate le iscrizioni più rilevanti); DE MARINIS, CASINI, RAPI 2016, p. 461, fig. 11 .

129 Su ciò, si vedano le considerazioni in Vitali, KaEnel 2000.

130 Menotti, Maras 2012, p. 881, nn. 10-13. Si veda quanto osservato precedentemente sull'edizione delle iscrizioni di questo scavo.

131 Per la quantificazione, basata sui dati in Pozzi 2011, si veda Gaucci, Morpurgo, Pizzirani 2018, p. 673, fig. 7.

132 Rispetto a Valle Trebba, è chiaro che le iscrizioni edite in $E T^{2}$ costituiscono una minima parte delle centinaia documentate dalla ricognizione di A. Pozzi (2011, p. 396 per il dato generale) e che attendono uno studio dei supporti e corredi di pertinenza per una datazione più puntuale.

133 Colonna 1998, p. 127.

134 Al riguardo, si rinvia alla sintesi sull'epigrafia di età recente offerta in BELLELLI, BENELLI 2018, pp. 188-209. 



\section{SUPPORTI, POSIZIONE, TECNICHE DI SCRITTURA}

\section{II.1. Supporti}

Tutte le iscrizioni sono su materiale ceramico. Nel periodo tardo-arcaico la prevalenza dei vasi iscritti appartiene alla ceramica depurata generalmente decorata con fasce dipinte in rosso (II.1.1), attribuibile a produzioni riconosciute come locali e definite etrusco-padane, e alla ceramica di importazione attica (II.1.2). Nel periodo ellenistico alla ceramica a v.n. di produzione locale (II.1.6), mentre classi ceramiche ben affermate come la ceramica alto-adriatica ${ }^{1}$ e quella depurata (caratterizzata dopo il III sec. a.C. da forme ben diverse da quelle etrusco-padane più antiche ${ }^{2}$ ) ospitano solo un numero molto ridotto di graffiti.

\section{II.1.1. Ceramica depurata etrusco-padana}

La classe è inquadrabile ad Adria tra la seconda metà del VI e il pieno III sec. a.C. ${ }^{3}$. Su 16 iscrizioni redatte su vasi di questa classe, la prevalenza (15) è su coppe (CIE 20037, 20125, 20126, 20159, 20160,

\footnotetext{
1 Si ha solo un caso di ceramica alto-adriatica graffita, cioè un segno a croce ripetuto sul pomello del coperchio e sul fondo esterno del bacile di una lekanis (CIE 20520).

2 Per l'orizzonte di III e II sec. a.C., gli scavi di abitato e anche le sepolture restituiscono ceramica depurata, che tuttavia presenta una selezione di forme molto diversa rispetto ai secoli precedenti, ormai fortemente influenzata da modelli greci di periodo ellenistico (tale classe è stata affrontata in studi parziali dedicati a specifici contesti sepolcrali: MANGANI 1982, p. 104, argilla tipo I; TAMASSIA 1993, p. 61); i quattro vasi con graffiti appartenenti a questa classe contano: un'anfora da tavola (CIE 20748), un askòs (CIE 20409), una lekanis (CIE 20972), un mortaio (CIE 20505).

${ }^{3}$ Il termine cronologico più basso per le attestazioni di questa classe ad Adria è indicato da alcune testimonianze di abitato relative allo scavo di via San Francesco (CAMERin, TAmAssia 1998-1999, in particolare pp. 217 e 231). Tale cronologia è sostanzialmente confermata dalle necropoli, dove non sembra più presente dopo gli inizi del III sec. a.C.: la coppa emisferica tipo Mattioli III,3,b della tomba 95 di Canalbianco databile tra fine III e inizi II sec. a.C. (CIE 20490) risulta un caso del tutto eccezionale. L'Atlante tipologico di C. MATTIOLI (2013) offre la possibilità di ordinare la prevalenza dei reperti che presentano iscrizioni e/o graffiti. La forma in assoluto più privilegiata per ospitare iscrizioni e graffiti è la coppa (75). Fra le restanti, si contano 5 piatti (solo CIE 20212 ha una iscrizione); un calice (CIE 20226) e un vaso situliforme che non rientra in tipologia (CIE 20220), entrambi supporto di graffiti; rimangono due frammenti di forma aperta (CIE 20268, 20871), due di forma chiusa (CIE 20088, 20154).
} 
20180, 20204, 20214, 20270, 20356, 20370, 20371, 20822, 20832, 20834). La posizione privilegiata è il fondo esterno, sistematicamente scelto a eccezione di un caso (CIE 20159, nella parete esterna). L'evidente predilezione della superficie del fondo esterno è da spiegare in relazione alla probabile pratica di riporle capovolte ${ }^{4} \mathrm{o}$ forse in alternativa quale cifra di un atto rituale compiuto in contesto sacro 5 . Nel primo caso, tale considerazione sembra applicabile alla parziale sequenza alfabetica CIE 20371, allusiva alla scrittura in quanto pratica socialmente elevata in un punto del vaso che doveva essere ben visibile una volta riposto ${ }^{6}$; per il secondo caso, si potrebbe portare come esempio l'iscrizione sul fondo esterno della coppa CIE 20214, proveniente dal Fondo Ornati, ritenuto contesto sacro (cfr. VI.1.2), per il quale si rimanda anche alle considerazioni sul calice in bucchero integro dal medesimo contesto (cfr. infra).

$\mathrm{Su} 5$ piatti con presenza di iscrizioni o graffiti ${ }^{7}$, quello a fondo piano CIE 20212 è l'unico con iscrizione e fa parte di un insieme di vasi con decorazioni a fasce o figurati definito da G. Colonna Gruppo di Adria e attribuito a una produzione locale adriese «opera di artigiani emigrati dal triangolo VulciOrvieto-Chiusi verso il 530-520 a.C.» ${ }^{8}$. E. Govi ha messo in dubbio il postulato della produzione locale di tali ceramiche, avanzando piuttosto l'idea alternativa di una possibile importazione dall'area tirrenica, in particolare vulcente 9 . Pur non escludendo l'ipotesi che il piatto iscritto possa essere una importazione dall'Etruria meridionale, in particolare da Vulci, l'affinità dell'argilla di questo con i vasi etrusco-padani in ceramica depurata ritenuti di produzione adriese ${ }^{10}$ non permette al momento di respingere l'ipotesi di una produzione locale su imitazione di modelli etrusco-meridionali. Riferito alla forma VI,4,a dell'Atlante tipologico, trova puntuale confronto con un esemplare dalla tomba 76 della Certosa di Bologna ${ }^{11}$ e ha come modello i piatti attici tipo broad rim datati tra 520 e 500 a.C. ${ }^{12}$. La ricorrenza in ambito padano di questi piatti, prodotti localmente o importati dall'Attica, sia figurati che verniciati di nero, in contesti funerari e sacri ha permesso di ipotizzarne privilegiate destinazioni in questi specifici ambiti, come suggeriscono anche i due fori per la sospensione. Tale destinazione è testimoniata nel nostro dall' iscrizione mi al apposta sul fondo esterno che ne esplicita il carattere votivo (cfr. V.2). Non si esclude che i fori passanti potessero essere presenti nella parte mancante dell'orlo, permettendo di appenderlo alla parete alla stregua di un $\operatorname{pinax}^{13}$. A tale proposito, vale la pena richiamare a confronto un simile piatto in ceramica acroma di probabile importazione attica dalla tomba $719 \mathrm{di}$ Valle Trebba a Spina: anch'esso con due fori, presenta nel fondo esterno l'iscrizione al che ne esplicita il carattere di dono funebre ${ }^{14}$. L'uso di questa forma nella produzione del Gruppo Spurinas ${ }^{15}$, e anche il ritrovamento di un piatto attico 'bilingue' della medesima forma dal santuario meridionale di Pyrgi della fine del VI sec. a.C. con una iscrizione greca di dedica graffita dopo la cottura in una posizione si-

${ }^{4}$ F. Cordano in Cordano, de Simone 1981, p. 134; Govi 1994b, p. 213; Ead. in Govi, Martelli, Sassatelli 2009, pp. 266, 274.

5 Per simili rinvenimenti, si rinvia in particolare al deposito di fondazione $\kappa$ e all'ipotesi di riti espiatori in relazione alla ristrutturazione dell'edificio $\gamma$ del Santuario meridionale di Pyrgi (CARLuCCI, Maneschi 2013, pp. 54-55, 62-64 con precedenti riferimenti). Pare rilevante porre in particolare l'attenzione sulla coppa monoansata e la kylix con iscrizioni onomastiche nel fondo esterno dal deposito $\kappa$ (Ps 28-29), interpretate come appartenenti a un atto rituale di carattere gentilizio (Maras 2013b, pp. 199-200).

6 Cfr. Gaucci 2012 b, p. 152.

7 Uno di forma ignota in quanto non recuperato (CIE 20224), due su basso piede (CIE 20209, 20344) e due su fondo piano (CIE 20212, 20391).

8 Colonna 2003, pp. $163-165$ e p. 166 , nota 31.

9 Govi 2012, in particolare p. 145.

10 Cfr. Donati, Parrini 1999, pp. 582-590.

11 Mattioli 2013, p. 227.

12 Agora XII, p. 144, nn. 1002-1005, tav. 36, fig. 9; cfr. anche Govi 1999, p. 127, n. 106, fig. 71, tavv. $15,29$.

13 Come lascia supporre per esempio l'esemplare figurato con due fori praticati nella tesa dal medesimo contesto (GovI 2012, pp. 109-113, n. 1 e in particolare p. 111, nota 30).

14 Gaucci et al. 2017, p. 129; G. Colonna, in REE LXXIX, p. 339, n. 93.

15 Bernardini 2002, Forma IV, pp. 18-19. 
mile a quella del nostro vaso, cioè nel fondo esterno (nella fattispecie esternamente ai cordoli a rilievo), aiutano a comprendere non solo la speciale funzione che questi piatti avevano, ma anche la scelta come supporto di iscrizioni di natura votiva.

\section{II.1.2. Ceramica attica}

Sebbene meno frequente come supporto di iscrizioni e graffiti rispetto alla ceramica etrusco-padana depurata, la ceramica attica rimane una scelta significativa tra il periodo tardo-arcaico e il IV sec. a.C. Si registrano vasi a figure nere $(6)^{16}$, a vernice nera $(19)^{17}$, oltre a frammenti di tecnica incerta $(17)^{18}$, mentre solo uno a figure rosse, che ospita un graffito non alfabetico (CIE 20868).

La ceramica a f.n. è documentata da tre vasi con iscrizioni databili attorno al 500 a.C. e nei decenni seguenti: un chous (CIE 20282), le cui valenze rituali legate alla celebrazione delle Antesterie sono note $^{19}$, e due kylikes (CIE 20287, 20305, a cui solo dubitativamente si potrebbbe aggiungere 20299 con un digramma di incerta interpretazione: etrusco $p i$ o greco $\lambda_{l}$ ). Tra gli ultimi decenni del VI e la prima metà del V sec. a.C. presentano iscrizioni: una kylix a v.n. (CIE 20143); una kylix (CIE 20289), una vicup (CIE 20290) e un cup-skyphos (CIE 20295) di tecnica indeterminata. Più genericamente tra $\mathrm{V}$ e IV sec. a.C. si datano due frammenti di parete entrambi con iscrizione lacunosa (CIE 20184, 20823), mentre si collocano nel IV sec. a.C. la ciotola del tipo outturned rim con digramma ra (CIE 20256) e il frammento di parete con decorazione a rotellature e iscrizione lacunosa ]na (CIE 20008). La notevole frammentarietà di tutti i reperti ceramici elencati e quindi l'incertezza nel riconoscimento della tecnica per molti, deriva dalla loro provenienza da scavi di abitato, mentre non sono attestati documenti epigrafici nelle ceramiche di importazione di necropoli.

Si nota nel complesso una netta prevalenza di forme potorie ${ }^{20}$, con una predilezione per la kylix, secondo una tendenza evidenziabile anche fra i supporti di graffiti: 2 su 3 nelle f.n.; 5 su 19 a v.n.; 9 su 15 fra i frammenti di tecnica indeterminata. Il fondo esterno del piede è la superficie scrittoria privilegiata; sono eccezioni il frammento di piede CIE 20289, dove sia la superficie inferiore sia quella superiore sono sfruttate per scrivere (cfr. infra), e il frammento di orlo di kylix CIE 20305, nel quale il digramma $k r$ è redatto nella parete esterna (cfr. III.4).

Come osservato dalla critica ${ }^{21}$, la scelta di apporre iscrizioni in vasi di importazione dall'Attica in ambito etrusco è una pratica diffusa nei contesti funerari e privilegiata rispetto ad altre classi ceramiche in quelli sacri, come ben esemplificato dall'area sacra di Gravisca e dal santuario meridionale di Pyrgi ${ }^{22}$. In area padana, la presenza di iscrizioni su ceramica attica in contesto sacro si riscontra a Bologna ${ }^{23}$, ma finora non a Marzabotto, dove lo scavo delle aree sacre urbane ha restituito un significativo numero di

16 Oltre ai 3 supporti con iscrizioni, si contano due kylikes (CIE 20299, 20300) e una c.d. figured bowl (CIE 20285; forma molto diffusa in ambito deltizio, soprattutto a Spina; cfr. GAUCCI et al. 2017, in particolare p. 129). Questi vasi ospitano singole lettere o digrammi che potrebbero essere attribuibili a un milieu culturale greco piuttosto che etrusco.

17 Nel complesso, si contano sei kylikes (CIE 20143, 20185, 20213, 20301, 20306, 20773), una olpe (CIE 20269), sette ciotole (CIE 20256, 20283, 20292, 20294, 20296, 20298, 20304), una c.d. saltcellar (CIE 20303) e quattro forme aperte non meglio determinabili (CIE 20008, 20184, 20195, 20823).

18 Si contano in totale dodici kylikes (CIE 20286, 20288, 20289, 20290, 20293, 20295, 20302, 20307, 20309, 20347, 20782, 20842), uno skyphos (CIE 20310), tre forme aperte (CIE 20184, 20291, 20823) e una chiusa (CIE 20311).

19 GaUCCi, VAllicelli 2011 per il vaso in questione; si richiama anche la recente analisi di Govi 2017, pp. 104-105 per i casi spinetici.

${ }^{20}$ In ZAмBOni 2016, p. 125, in merito all'analisi degli skyphoi attici dell'abitato di Spina si afferma che questi sono tra i supporti prediletti per iscrizioni e graffiti in alfabeto sia greco sia etrusco. Si osserva tuttavia che Zamboni registra solo un alfabetario (n. 587, tav. 11) all'interno del materiale iscritto proveniente dagli scavi 1977-1981, a cui aggiunge altre due serie alfabetiche già edite. L'affermazione pare quindi non comprovata a sufficienza, sebbene meritevole di approfondimento.

${ }^{21}$ STOPPoni 1990, p. 86; per i contesti votivi, si veda anche Maras 2009b, pp. 171-172.

22 Per Gravisca, cfr. PAndolfini 2000, in particolare p. 74. Per l'Area sud di Pyrgi, cfr. REE LVI, pp. 313-324; REE LXIV, pp. 369-422; REE LXIX, pp. 307-331; MARAs 2013b, p. 200.

23 Romagnoli 2014, p. 202. 
iscrizioni votive e di dedica tutte in bucchero (cfr. I.2.1). Ad Adria, la tendenza riscontrata dalla critica ben si accorderebbe con la ipotizzata (ma perlopiù non certa) provenienza di 6 dei 7 vasi attici iscritti (cioè tutti eccetto CIE 20143) dall'area della Tomba, probabile contesto santuariale (cfr. VI.1.4). È necessario però sottolineare che l'unico caso in cui si ha un testo, seppure frammentario, che per potenziale complessità è ragionevolmente attribuibile a una pratica votiva di santuario, è CIE 20289; questo infatti presenta due iscrizioni, ciascuna lungo il bordo di una delle due superfici opposte del piede, che parrebbero redatte da mani diverse, come proposto da S. Bruni ${ }^{24}$, una in probabile norma scrittoria meridionale nella superficie superiore del piede, l'altra in probabile norma settentrionale nel fondo esterno.

\section{II.1.3. Ceramica grezza}

Si contano 25 supporti di iscrizioni e graffiti, fra cui si riconoscono 9 olle e 14 coppecoperchio, un recipiente da fuoco ${ }^{25}$. Tre sono i vasi con iscrizioni tutte poste a crudo, due olle (CIE 20353, 20361) e una coppacoperchio (CIE 20265). Significativi contesti produttivi e sacri del mondo etrusco hanno permesso di mettere in evidenza come il ridotto numero di iscrizioni poste a crudo sulla ceramica grezza riguardi in prevalenza formule di possesso, le cui indicazioni di proprietà non sono indirizzate solo al contenitore ma anche al contenuto ${ }^{26}$. In questo quadro rientrerebbero le due iscrizioni su olla; queste sono state apposte in punti diversi, CIE 20353 nel fondo esterno, dove in effetti sono registrati quasi tutti i restanti graffiti su vasi di questa forma, e CIE 20361 sull'orlo, secondo una prassi altrimenti ignota ad Adria, ma che invece trova riscontro in altri siti ${ }^{27}$.

Il digramma della coppacoperchio CIE 20265 (forma III,1,a della tipologia Mattioli, età arcaica) è inciso a crudo sul fondo esterno del piede, dunque visibile quando il vaso era ribaltato e usato come coperchio (cfr. V.2). La tecnica di realizzazione e la posizione sono tendenzialmente coerenti con quelle dei graffiti alfabetici e non alfabetici redatti nei vasi della medesima forma. Fra questi ultimi infatti, solo tre casi si differenziano per posizione: un frammento con graffiti nella parete esterna e interna (CIE 20148), uno con una lettera nella parete esterna (CIE 20156) e uno con una lettera incisa nell' interno (CIE 20840).

Per il periodo ellenistico, l'evidenza di una unica iscrizione in ceramica grezza è coerente con l'estrema rarefazione di graffiti in questa classe nel medesimo orizzonte cronologico (su una olla e una coppacoperchio, rispettivamente CIE 20242 e CIE 20109, da contesti abitativi).

24 BRUni 2011, p. 126, nota 8. Per ulteriori commenti, si rimanda a GaUCCi 2012a, p. 144.

${ }^{25}$ Fra le olle, oltre ai due frammenti con iscrizione, si contano 6 frammenti di fondi piani (cioè forma J della tipologia dei piedi in MatTioli 2013, p. 379): CIE 20152, 20272 e 20354 (con graffiti incisi a crudo nel fondo esterno); 20165 e 20174 (con graffiti tracciati dopo la cottura nel fondo esterno); 20164 (con graffito inciso a crudo nella parete esterna). A questi si aggiunge CIE 20388 (con due graffiti incisi a crudo, uno nella parete e uno nel fondo esterno), che è l'unico caso integro (MatTioli 2013, forma IV,4,e) e da contesto funerario. Fra le coppecoperchio, oltre all'esemplare con digramma CIE 20265, si contano 11 frammenti di piede forma H, CIE 20035, 20091, 20161, 20186, 20351 con graffiti alfabetici e non alfabetici incisi a crudo nel fondo esterno, e CIE 20225, 20230, 20250, 20251, 20359, 20883 con graffiti alfabetici e non alfabetici graffiti dopo la cottura nel fondo esterno; un frammento di piede forma A, CIE 20205 e uno di forma F, CIE 20880, graffiti dopo la cottura nel fondo esterno. Non si registrano casi di supporti forati, come invece in almeno una coppacoperchio da San Cassiano (CIE 21034) e anche su vari documenti di questo tipo in ceramica depurata e bucchero di Adria (cfr. CIE 20134, 20344-20345, 20362, con riferimento a VI.1.1 e VI.1.4). Il recipiente da fuoco (CIE 20218: MaTtioli 2013, forma $I, 1, c)$ presenta un graffito non alfabetico redatto sul fondo esterno. Un frammento di forma aperta con graffito non alfabetico realizzato dopo cottura nel fondo esterno potrebbe invece appartenere a una coppacoperchio (CIE 20206).

26 Govi, Sassatelli, Martelli 2009, p. 265, con riferimento alla c.d. "Grande Fornace” della Regio II - insula 1 di Marzabotto e al Petriolo di Chiusi. Sul tema, anche Bellelli 2012, pp. 383-384, che richiama altri contesti come Cerveteri-Vigna Parrocchiale, Gravisca e Roma-Palatino.

27 Sassatelli, Gaucci 2010, p. 371, nota 26 con precedenti riferimenti; in particolare, per una ipotesi di unità metrologiche di capienza in relazione a graffiti non alfabetici interpretati come numerali, si veda NiJBOER 1998, pp. 318326. 


\section{II.1.4. Bucchero}

Il bucchero non è sicuramente supporto privilegiato per le iscrizioni, in quanto su 25 frammenti di coppe ${ }^{28}$ e un calice integro usati per scrivere, si documentano solo tre casi di lettere in legatura (CIE 20089, 20194, 20215) e nessuna iscrizione più lunga. Come per le altre classi, si rileva una sostanziale preferenza del fondo esterno. Caso speciale è il calice CIE 20215, attribuibile alla forma I,2,e, non attestata nell'Atlante tipologico. Tale vaso, oltre a essere unico per scelta del supporto, presenta un sistema di graffiti complesso che ne esplicita in maniera chiara la leggibilità solo in posizione capovolta (cfr. VI.1.2).

La scarsa presenza del bucchero e l'assenza del suo utilizzo per iscrizioni complesse nell'abitato di Adria risulta in controtendenza rispetto a una generale predilezione di questa classe come supporto di iscrizioni per i contesti etruschi di tipo abitativo/produttivo quali Marzabotto, il Petriolo di Chiusi, lo scarico e gli edifici di età arcaica dell'area della Vigna Parrocchiale a Caere, e sostanzialmente anche per quelli di tipo santuariale quali il Portonaccio di Veio, Gravisca e in ambito padano i santuari urbani di Marzabotto, per citare i casi più significativi ${ }^{29}$. Una scarsa rappresentatività di bucchero iscritto rispetto alla totalità di attestazioni di questa classe è registrata invece nel santuario di Campo della Fiera ${ }^{30}$. Ad Adria, le attestazioni epigrafiche nel bucchero sembrano forse perlopiù funzionali alla pratica di contrassegnare i vasi, come potrebbero suggerire i tre casi di lettere incise a crudo e in particolare i due sade a farfalla (cfr. III.2).

\section{II.1.5. Ceramica grigia}

Nel periodo tardo-arcaico fra le classi ceramiche scelte per le iscrizioni si distingue ad Adria anche la ceramica grigia, che presenta una propria autonomia tecnica e formale rispetto al bucchero e alla ceramica depurata ${ }^{31}$. La ceramica grigia è una classe ben documentata nel periodo ellenistico e soprattutto in abitato, sebbene per questa fase manchi ancora un inquadramento tipologico ${ }^{32}$.

In età tardo-arcaica, su 12 vasi lacunosi $(9$ coppe, due frammenti di forma aperta e due di forma chiusa), solamente due coppe ospitano iscrizioni graffite dopo la cottura ${ }^{33}$ : CIE 20189 al centro

${ }_{28}$ Si contano 15 casi di coppe graffite dopo cottura sul fondo esterno del piede (CIE 20089, 20090, 20094, 20146, $20147,20173,20211,20331,20358,20363,20574,20845,20872,20882$, a cui si aggiunge 20362 su una patera pertusa) e 3 incise a crudo (CIE 20092, 20157-20158); un caso nel fondo interno con graffitura dopo cottura (CIE 20252); uno sulla base del piede sempre dopo cottura (CIE 20355); uno sulla parete esterna graffito dopo cottura (CIE 20350); 3 casi che attestano più di un graffito presenti sul fondo esterno e in un'altra parte del vaso (CIE 20157, 20172, 20210), dei quali solo in uno sono associati un graffito dopo cottura e uno inciso a crudo (cioè CIE 20157). Si osserva un numero significativo di più graffiti sul medesimo supporto, posti coerentemente sempre nel fondo esterno oltre che in altri punti (parete esterna $o$ fondo interno). Si riconoscono 3 coppe emisferiche, 4 coppe carenate, 2 coppe troncoconiche, mentre i restanti frammenti sono parti di piedi con attacchi del fondo della vasca, dei quali 11 del tipo ad anello semplice, cioè forma A della tipologia dei piedi di Mattioli 2013 e 7 al tipo a disco, forma F. Per quanto è noto, non pare di rilevare una preferenza nella scelta tra coppe emisferiche, carenate e troncoconiche, se non forse per una lieve recenziorità cronologica di quelle emisferiche.

29 Sull'argomento, si veda SASSATELLI 1993, p. 202. Riguardo ai siti elencati, più in particolare: per la Casa 1, Regio IV - insula 2 di Marzabotto, cfr. Sassatelli, Gaucci 2010, pp. 338-339; per il Petriolo di Chiusi, cfr. Govi, Sassatelli, Martelli 2009, p. 265; per lo scarico arcaico della Vigna Parrocchiale di Cerveteri, cfr. Pandolfini 1989, pp. 69-70, mentre per gli edifici obliterati dal tempio di inizi V sec. a.C., cfr. da ultimo V. BELLELLI, in REE LXV-LXVIII, pp. 326327; per il santuario del Portonaccio di Veio, cfr. MARAs 2002, in particolare pp. 262-271; per Gravisca, cfr. PANDOLFINI 2000 , p. 74 , dove però le iscrizioni su bucchero sono seconde per quantità a quelle su importazione attica e presentano solo nomi propri e mai di divinità; per i santuari urbani di Marzabotto, cfr. I.2.1.

30 Capponi 2018, p. 81.

31 Su questo tema, si rimanda a Morpurgo 2013, p. 381.

32 Si veda Mangani 1982, p. 102; Tamassia 1993, pp. 60-61; Camerin, Tamassia 1998-1999, p. 231. L'orizzonte ellenistico non rientra nello studio tipologico di G. Morpurgo, che è circoscritto allo sviluppo cronologico della produzione etrusco-padana.

$33 \mathrm{Si}$ osserva che tutti i documenti sono graffiti dopo la cottura, a eccezione di un segno a croce a crudo nel fondo esterno di una delle due coppe con iscrizione (CIE 20368). Fra i frammenti con graffiti alfabetici e non alfabetici, se ne contano 
della vasca interna e CIE 20368 nella parete esterna. Sono tutti frammentari e provengono, quando noto, dagli scavi condotti nell'attuale sede del Museo archeologico, cioè da un'area ritenuta abitativoartigianale (cfr. IV.1). Il numero esiguo delle attestazioni non permette di stabilire linee di tendenza relativamente alla posizione.

Si datano tra la metà e la seconda metà del IV sec. a.C. 3 coppe con iscrizioni. Due coppe frammentarie provengono dalla sede del Museo come le altre più antiche; in una rimane l'ultima lettera di un testo ormai perduto, redatta nella vasca interna presso un segno a croce (CIE 20127) $)^{34}$, mentre nell'altra la legatura di più lettere ( $\widehat{a a}$ o meglio $\overparen{a i a}$ ) figura nel fondo esterno (CIE 20043). La terza invece appartiene al corredo della tomba 20 della necropoli di via Spolverin (CIE 20899) e conserva un antroponimo graffito nella vasca interna seguendo la circolarità della parete.

Tra la fine del IV e il II sec. a.C. si contano 173 vasi con iscrizioni e/o graffiti, in netta prevalenza coppe (150, a fronte di 9 piatti, 7 mortai, 2 skyphoi, una lekanis, una olla, tre frammenti di forma aperta). Solo 26 presentano iscrizioni, di cui 12 sicuramente databili tra la fine del IV e il III sec. a.C. ${ }^{35}$, mentre nessun vaso è databile con certezza tra la fine del III e il II sec. a.C. Lo stato di conservazione frammentario e la provenienza da contesti non chiusi permette di attribuire i restanti 14 solo genericamente al periodo ellenistico ${ }^{36}$. Colpisce il raro uso di questa classe come supporto di scrittura nei contesti funerari, due mortai e tre coppe (cfr. V.4 per la formula con il lessema helis che contraddistingue tutte le coppe), tutti non posteriori al III sec. a.C.

\section{II.1.6. Ceramica a v.n. di periodo ellenistico}

La ceramica a v.n. sostituisce in questa fase cronologica la ceramica depurata etrusco-padana del periodo tardo-arcaico quale classe privilegiata per la redazione di iscrizioni e graffiti. In generale, si contano 402 supporti con iscrizioni e/o graffiti attribuibili a una produzione locale e morfologicamente identificabili. Come per la ceramica depurata nella fase più antica, anche per questa classe le ciotole rappresentano quantitativamente la forma preferita con 264 unità. Molto più ridotto il numero di vasi di importazione (Fig. 3).

Sono 133 i vasi con iscrizioni etrusche, così suddivisi: uno degli ultimi decenni del IV sec. a.C.; 4 di fine IV-inizi III sec. a.C.; 50 di III sec. a.C.; 7 di fine III-inizi II sec. a.C.; 55 di II sec. a.C.; 3 di seconda metà II-prima metà I sec. a.C.; 16 più genericamente di periodo ellenistico. Senza entrare nel dettaglio delle specificità tipologiche, si potrebbe dividere i supporti con iscrizioni (comprensive dei digrammi) secondo lo schema alla Tab. 1.

Si nota anzi tutto l'assenza di forme chiuse. Tra le forme aperte destinate a ospitare iscrizioni, si conferma la preferenza della ciotola, almeno in contesto funerario, mentre in contesto abitativo sembra che non vi sia una netta preferenza tra ciotole e piatti (comprendendo anche la versione con cavetto centrale); seguono con un numero ridotto di attestazioni le kylikes. Rara la scelta di altre forme, tra cui spiccano solo una patera tipo Morel $2233 \mathrm{f} \mathrm{di} \mathrm{III} \mathrm{sec.} \mathrm{a.C.,} \mathrm{alcune} \mathrm{pissidi} \mathrm{e} \mathrm{una} \mathrm{tazzina}$ monoansata di II, che sono pertanto da ritenere scelte eccezionali, tutte effettuate in contesto funerario.

cinque tracciati nel fondo esterno (CIE 20145, 20167, 20170, 20357, 20372), due unicamente nella parete esterna (CIE 20166, 20352), uno nella parete interna (CIE 20369), uno in posizione non determinabile (CIE 20228). Casi eccezionali sono CIE 20002, con due lettere uguali, una nel fondo esterno e una in quello interno, e CIE 20163, con due graffiti non alfabetici, uno nella parete interna e uno nell'esterna.

${ }^{34}$ GAUCCI 2020.

35 Tra la fine del IV e gli inizi del III a.C. si collocano 2 coppe da abitato (CIE 20116, 20181) e una da necropoli (CIE 20595); nel III sec. a.C. un mortaio (CIE 20041) e 5 coppe emisferiche (CIE 20201, 20259, 20264, 20277, 20364) dall'abitato, 2 mortai (CIE 20457, 20895) e una coppa emisferica (CIE 20464) dalla necropoli.

${ }^{36}$ Si elencano 14 coppe, delle quali 11 dall'abitato (CIE 20044, 20051, 20079, 20084, 20120, 20121, 20133, 20190, 20236, 20263, e forse la forma aperta CIE 20258), una da necropoli (CIE 20601) e due da contesto incerto (CIE 20827, 20830). 
Tab 1. Tabella distributiva dei vasi con iscrizioni (comprensive dei diagrammi). Sono sottolineati i tituli appartenenti a vasi di produzione incerta, in corsivo quelli di importazione e in tondo quelli di produzione locale.

\begin{tabular}{|c|c|c|c|c|c|c|c|}
\hline Contesto & CrONOLOGIA & Ciotola & Piatto & KYLIX & $\begin{array}{l}\text { FORMA } \\
\text { APERTA }\end{array}$ & Altro & T. \\
\hline \multirow{4}{*}{ abitato } & $\begin{array}{l}\text { fine IV- } \\
\text { inizi III sec. }\end{array}$ & 20196 & & $\underline{20019}$ & & & 2 \\
\hline & III sec. & $\begin{array}{l}20006,20028,20030, \\
20182,20183,20188, \\
20191\end{array}$ & 20197 & 20029 & $\begin{array}{l}20107 \\
20253 \\
20273\end{array}$ & & 12 \\
\hline & II sec. & 20012 & $\begin{array}{l}\text { 20275, } \\
20279, \\
20318 \text { (?), } \\
20332(?), \\
20335 \text { (?) }\end{array}$ & & & & 6 \\
\hline & età ellenistica & 20020 & $\begin{array}{l}20007, \\
20333(?), \\
20336 \text { (?) }\end{array}$ & & 20257 & & 5 \\
\hline \multirow{6}{*}{ necropoli } & $\begin{array}{l}\text { fine IV- } \\
\text { inizi III sec. }\end{array}$ & & & & $\underline{20597}$ & & 1 \\
\hline & III sec. & $\begin{array}{l}20386,20387,20426, \\
20514,20523(?), \\
20547(?), 20563,20564, \\
20569,20570,20571, \\
20594,20603,20614, \\
20660,20662,20663, \\
20685(?), 21027\end{array}$ & $\begin{array}{l}20384, \\
20487, \\
20519, \\
20591 \text { (?), } \\
20627\end{array}$ & $\begin{array}{l}20385, \\
20477 \\
20511 \text { (?), }\end{array}$ & & 20904 (patera) & 28 \\
\hline & $\begin{array}{l}\text { fine III- } \\
\text { inizi II sec. }\end{array}$ & $\begin{array}{l}20436,20458,20489, \\
20709,20749,20992, \\
20993\end{array}$ & & & & & 7 \\
\hline & II sec. & $\begin{array}{l}20377,20404,20407, \\
20411,20420,20434, \\
20444,20461,20462, \\
20463,20472,20473, \\
20541,20553,20566, \\
20600(?), 20606,20607, \\
20621,20622,20640, \\
20697,20698,20699, \\
20762,20921,20923, \\
20924,20925,20926, \\
20932,20933,20997\end{array}$ & $\begin{array}{l}20375, \\
20390, \\
20422, \\
20474, \\
20475, \\
20610, \\
20654, \\
20694, \\
21002\end{array}$ & & & $\begin{array}{l}20376 \\
\text { (pisside), } \\
20534 \\
\text { (pisside), } \\
20628 \text { (tazzina } \\
\text { monoansata), } \\
20934 \\
\text { (pisside), } \\
20960 \\
\text { (pisside) }\end{array}$ & 47 \\
\hline & $\begin{array}{l}\text { seconda } \\
\text { metà II-prima } \\
\text { metà I sec. }\end{array}$ & 20559,20560 & & & & & 2 \\
\hline & età ellenistica & 20664 (?), 20678, 20679, & $\begin{array}{l}20677 \\
20680 \\
20683(?)\end{array}$ & & $\begin{array}{l}20419 \\
20736 \\
20737\end{array}$ & & 9 \\
\hline \multirow{5}{*}{$\begin{array}{c}\text { contesto } \\
\text { incerto }\end{array}$} & $\begin{array}{l}\text { ultimi decenni } \\
\text { del IV sec. }\end{array}$ & 20824 & & & & & 1 \\
\hline & III sec. & $\begin{array}{l}20323,20328,20330, \\
20772,20775,20776, \\
20788\end{array}$ & & $\begin{array}{l}20315 \\
20819\end{array}$ & 20316 & & 10 \\
\hline & II sec. & $\begin{array}{l}\text { 20777, 20794, 20817, } \\
20818,20820,20828, \\
20833 \text { (?), }\end{array}$ & 20785 & & & & 8 \\
\hline & $\begin{array}{l}\text { seconda } \\
\text { metà II-prima } \\
\text { metà I sec. }\end{array}$ & 20825 & & & & & 1 \\
\hline & età ellenistica & 20821,20826 & & & $\begin{array}{l}20320 \\
20829\end{array}$ & & 4 \\
\hline
\end{tabular}




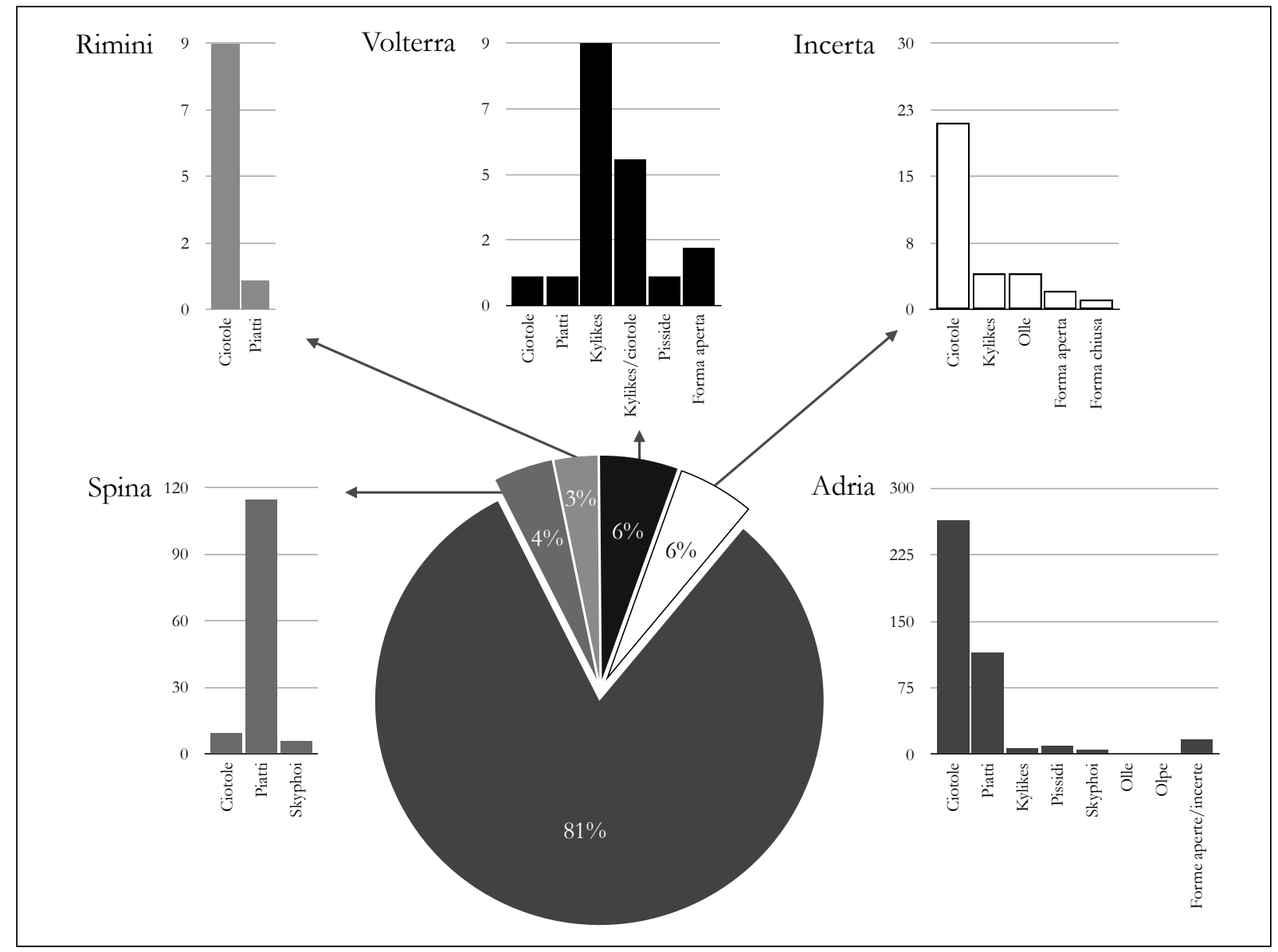

Fig. 3. Quantificazione del vasellame a v.n. di periodo ellenistico usato come supporto di iscrizioni.

Da un punto di vista diacronico, sembra rilevante osservare la notevole diminuzione delle occorrenze in abitato nel II sec. a.C. ( 1 caso su 15 databili), di contro a una quasi duplicazione in necropoli nello stesso secolo rispetto a quello precedente ( 47 casi contro 28 del III sec. a.C.). Sebbene questo potrebbe essere dovuto alla fortuità delle operazioni di scavo, tuttavia il quadro delineato suggerisce comunque come nel III sec. a.C. la scrittura su ceramica (assieme ai processi culturali che sottintende) possa essere un fenomeno diffuso in differenti tipi di contesti, mentre nel II sec. a.C. una manifestazione soprattutto funeraria ${ }^{37}$.

\section{II.1.7. Anfore greco-italiche}

Si contano 6 anfore greco-italiche con iscrizioni etrusche, tutte da contesto funerario (III sec. a.C.: CIE 20568; fine III-inizi II sec. a.C.: CIE 20951; II sec. a.C.: CIE 20405, 20408, 20704, 21013). Si ricorda che sono documentate nel complesso 24 anfore usate come supporto per iscrizioni e/o graffiti; tra queste, oltre alle 6 menzionate, se ne ricordano 6 dove figurano lettere o lessemi in alfabeto latino (CIE 20459, 20577, 20952, 20970, 21000, 21010), una con due iscrizioni attribuibili all'alfabeto venetico (CIE 21014), e inoltre 5 con numerali (CIE 20465, 20510, 20540, 20920, 21011) e 6 con graffiti non alfabetici (CIE 20470, 20585, 20613, 20898, 20936, 20955). La natura di contenitore da trasporto propria delle anfore rende nel complesso difficile valutare il luogo di realizzazione delle manifestazioni epigrafiche elencate, che in alcuni casi sono con tutta verosimiglianza da ritenere realizzate

37 Tra gli 8 vasi iscritti di contesto incerto databili al II sec. a.C., la metà di questi (CIE 20785, 20794, 20817, 20828) si presentano più o meno integri, suggerendo una origine da contesto funerario, a conferma dell'osservazione avanzata. 
fuori da Adria, come già ipotizzate a esempio per l'iscrizione latina sull'anfora CIE 20577 della prima metà del III sec. a.C. ${ }^{38}$. In ogni caso, e fino a prova contraria, almeno le iscrizioni etrusche non possono che essere attribuite al milieu adriese, anche per la tarda cronologia che perlopiù esclude Spina, dove una produzione di anfore greco-italiche è comunque ipotizzata ${ }^{39}$.

\section{II.2. Posizione e impaginazione}

La grande quantità di supporti con iscrizione di periodo ellenistico permette per questi una articolata analisi della posizione dei testi e come questi furono impaginati nello spazio scrittorio scelto. Per i periodi più antichi, si rinvia alle considerazioni sopra esposte nella trattazione delle singole classi ceramiche (cfr. II.1).

Nelle forme aperte, cioè la quasi totalità dei vasi con iscrizioni etrusche databili tra la fine del IV sec. a.C. e il passaggio tra il II e il I, le superfici privilegiate per la redazione dei testi sono l'interno del vaso (distinguendo il fondo piano e la parete inclinata, poiché richiedono posizioni diverse per praticare la scrittura e la lettura), la parete esterna e il fondo esterno. Grazie alla significativa quantità di documenti a disposizione, è possibile proporre una sistematizzazione delle occorrenze in una tabella distributiva (Tab. 2), dalla quale rimangono fuori tre iscrizioni su altrettante ciotole per le quali non è nota la posizione nel vaso (CIE 20600, 20736, 20737), 11 iscrizioni realizzate in piccoli vasi a v.n. (pissidi e una tazzina monoansata) e in anfore greco-italiche ${ }^{40}$.

Su un totale di 151 iscrizioni, la quasi totalità è redatta nell' interno del vaso (124), di cui 98 nel fondo interno e 26 nella parete interna. Il fondo interno del vaso è dunque la scelta nettamente privilegiata, sebbene si possano individuare diverse tipologie di impaginazione all'interno di questa categoria. Restano 17 iscrizioni redatte nella parete esterna e 9 nel fondo esterno. Solo un piatto presenta uno schema più complesso, con una iscrizione nell'interno e una nell'esterno (CIE 21002).

L'impaginazione più frequente asseconda la circolarità del vaso sia nel III che nel II sec. a.C., sebbene non manchino iscrizioni con andamento diritto, frequenti soprattutto nel II. Fra queste ultime, solo due (una da abitato di fine IV-inizi III sec. a.C. e una da necropoli di II sec. a.C.) sono posizionate al centro del fondo, e sebbene entrambe lacunose, verosimilmente dovevano risultare circoscritte in uno spazio delimitato dalla decorazione a rotellature (CIE 20019) o dall' impronta del disco di impilamento (CIE 20553). Casi speciali sono due testi redatti su più righe con a capo delle parole, quello di dedica ai Kulśnuter (CIE 21027), il cui andamento asseconda la circolarità del vaso, e la tarda iscrizione di possesso di Фaia Śliu (CIE 20560), dove invece il testo, breve e con lettere di grandi dimensioni, ha andamento diritto. Altro caso speciale è quello dell' iscrizione CIE 20563 di III sec. a.C., dove il lessema atakuś è graffito due volte sopra la decorazione a rotellature, in posizioni opposte rispetto al centro della vasca ma orientate nella medesima maniera.

Tra le iscrizioni ad andamento curvo su unica linea, la maggior parte dei testi è orientata verso l'esterno del vaso, mentre sono orientate verso il centro della vasca 7 iscrizioni su 23 totali di III sec. a.C., 7 su 18 totali di II sec. a.C., infine 10 sui 13 totali più genericamente databili al periodo ellenistico. Colpisce anzi tutto come i testi dei tre casi di triplice ripetizione della medesima formula (CIE 2056920571 di III sec. a.C., 20461-20463 di II, 20677-20679 più genericamente di età ellenistica) siano tutti i testi orientati verso il centro della vasca, suggerendo la possibilità che tale scelta possa rispondere

38 REI LXXIX, pp. 346-348.

39 Si vedano le considerazioni in GAUCCI 2016, pp. 196-197, con riferimenti precedenti.

${ }^{40}$ Le iscrizioni su pisside, tutte di II sec. a.C., sono perlopiù nella parete esterna (CIE 20376, 20534, 20934), e in un caso nel fondo esterno (CIE 20960); l'unica iscrizione su tazzina monoansata è stata tracciata nel fondo interno lungo il bordo (CIE 20628). Le sei iscrizioni su anfore greco-italiche sono sia sulla spalla (CIE 20408, 20568, 20704) sia sul collo (CIE 20405, 20951,21013), sempre orientate secondo la naturale posizione verticale del contenitore: tale puntualizzazione è necessaria perché invece le iscrizioni venetiche su anfora sono redatte con il vaso posizionato al contrario. Si tratta di una peculiarità che sicuramente dovrà essere indagata con maggiore attenzione. 
Tab 2. Tabella distributiva dei supporti con iscrizioni (comprensivi di diagrammi) secondo il tipo di impaginazione e su base cronologica.

\begin{tabular}{|c|c|c|c|c|c|c|c|}
\hline Posizione & $\begin{array}{c}\text { TIPO DI } \\
\text { IMPAGINAZIONE }\end{array}$ & $\begin{array}{l}300 \text { A.C. } \\
\text { CIRCA }\end{array}$ & III SEC. A.C. & $\begin{array}{l}200 \text { A.C. } \\
\text { CIRCA }\end{array}$ & II SEC. A.C. & $\begin{array}{l}\text { 150-50 A.C. } \\
\text { CIRCA }\end{array}$ & $\begin{array}{c}\text { Periodo } \\
\text { ELLENISTICo }\end{array}$ \\
\hline \multirow{6}{*}{$\begin{array}{l}\text { fondo } \\
\text { interno }\end{array}$} & $\begin{array}{l}\text { testo ad andamento } \\
\text { diritto, al centro }\end{array}$ & 20019 & & & 20553 & & \\
\hline & $\begin{array}{l}\text { testo ad andamento } \\
\text { diritto }\end{array}$ & & $\begin{array}{l}20183,20273, \\
20594,20660, \\
20819(?)\end{array}$ & 20458 & $\begin{array}{l}20012,20541, \\
20654,20694, \\
20699,20762, \\
20777,20794, \\
20817,20820, \\
20828,21002\end{array}$ & & $\begin{array}{l}20084,20257 \\
20258,20829\end{array}$ \\
\hline & $\begin{array}{l}\text { testo ad andamento } \\
\text { curvo }\end{array}$ & $\begin{array}{l}\text { 20181, } \\
20196(?)\end{array}$ & $\begin{array}{l}20030,20188, \\
20197,20201, \\
20253,20264, \\
20315,20330, \\
20384-20387, \\
20511,20514, \\
20519,20523, \\
20563,20569, \\
20570,20571(?), \\
20662,20685, \\
20776\end{array}$ & $\begin{array}{l}20489 \\
20992\end{array}$ & $\begin{array}{l}20407,20420, \\
20422,20434, \\
20461,20462, \\
20463,20472, \\
20473,20474, \\
20475,20566, \\
20610,20621, \\
20921,20926, \\
20933,20997\end{array}$ & 20559 & $\begin{array}{l}20020,20079, \\
20133,20190, \\
20236,20419, \\
20601,20677, \\
20678,20679, \\
20680,20821, \\
20826\end{array}$ \\
\hline & $\begin{array}{l}\text { testo ad andamento } \\
\text { curvo, su due righe }\end{array}$ & & 21027 & & & & \\
\hline & $\begin{array}{l}\text { testo ad andamento } \\
\text { diritto, su due righe }\end{array}$ & & & & & 20560 & \\
\hline & non determinato & & $\begin{array}{l}20029,20316, \\
20323,20328, \\
20426,20775\end{array}$ & $\begin{array}{l}20436 \\
20749\end{array}$ & $\begin{array}{l}20375,20411, \\
20833\end{array}$ & & 20121,20320 \\
\hline \multirow{5}{*}{$\begin{array}{l}\text { parete } \\
\text { interna }\end{array}$} & $\begin{array}{l}\text { testo assecondante } \\
\text { il bordo superiore }\end{array}$ & 20595 & $\begin{array}{l}20006,20028, \\
20464,20487, \\
20591,20663\end{array}$ & & 20622 & & \\
\hline & $\begin{array}{l}\text { testo ad andamento } \\
\text { diritto }\end{array}$ & & $\begin{array}{l}20457,20564 \\
20627,20895\end{array}$ & & $\begin{array}{l}20640,20698, \\
20923,20924 \\
20925,20932\end{array}$ & & \\
\hline & $\begin{array}{l}\text { testo ad andamento } \\
\text { curvo }\end{array}$ & & & & & & 20051 \\
\hline & $\begin{array}{l}\text { testo ad andamento } \\
\text { curvo opposto a } \\
\text { quello dell'orlo }\end{array}$ & & 20614 & 20709 & & & \\
\hline & non determinato & & 20191,20277 & & $\begin{array}{l}\text { 20001, 20390, } \\
20404\end{array}$ & & \\
\hline $\begin{array}{l}\text { interno } \\
\text { del vaso }\end{array}$ & non determinato & 20597 & & & & & \\
\hline \multirow{3}{*}{$\begin{array}{l}\text { parete } \\
\text { esterna }\end{array}$} & $\begin{array}{l}\text { testo presso l'orlo o } \\
\text { la tesa }\end{array}$ & & & & 20785 & & \\
\hline & $\begin{array}{l}\text { testo presso } \\
\text { l'attacco del piede }\end{array}$ & & $\begin{array}{l}20041,20107, \\
20477,20603, \\
20772,20904\end{array}$ & & $\begin{array}{l}20377,20444, \\
20606\end{array}$ & & 20120,20830 \\
\hline & $\begin{array}{l}\text { testo ad andamento } \\
\text { diritto }\end{array}$ & & 20182,20788 & & 21002 & 20825 (?) & $\begin{array}{l}20044(?) \\
20827\end{array}$ \\
\hline \multirow{2}{*}{$\begin{array}{l}\text { fondo } \\
\text { esterno }\end{array}$} & $\begin{array}{l}\text { testo ad andamento } \\
\text { diritto }\end{array}$ & & & & & & 20263 \\
\hline & $\begin{array}{l}\text { testo lungo } \\
\text { l'attacco della base } \\
\text { d'appoggio }\end{array}$ & 20116 & $\begin{array}{l}20259,20364, \\
20547\end{array}$ & 20993 & 20607,20697 & & 20664 \\
\hline
\end{tabular}


a una pratica canonizzata. Medesimo orientamento si manifesta anche nelle due iscrizioni associate a segni a croce posizionati al centro del fondo interno (CIE 20993 di II sec. a.C. e 20821 più genericamente di periodo ellenistico), evocando così un legame tra il testo scritto e la ripartizione dello spazio del vaso attraverso i quadranti del graffito centrale $e^{41}$.

Molto più diffusa la pratica di orientare l' iscrizione verso il centro del vaso tra quelle ad andamento diritto, 12 casi su 20 a cui si aggiunge l'iscrizione nel fondo interno del piatto CIE 21002. Tra queste, è peculiare l'iscrizione ana (CIE 20820): l'opposto orientamento dei due alpha permette infatti di leggere il nome palindromo in entrambi i versi.

Le iscrizioni redatte nella superficie obliqua della parete interna sono solo poco superiori per numero rispetto a quelle redatte nella superficie esterna. All'interno dei 26 documenti del primo gruppo, i testi redatti assecondando la curvatura dell'orlo si datano quasi tutti tra la fine del IV e il III sec. a.C. (7, mentre solo il digramma CIE 20622 appartiene al II sec. a.C.). Rimangono isolati i casi dell' iscrizione CIE 20051, redatta seguendo una curvatura difforme da quella del vaso, e le iscrizioni CIE 20614 e 20709 , che presentano una curvatura opposta a quella che ci si aspetterebbe. La pratica di redigere il testo diritto (10 casi), sia orientato verso l'esterno che verso l'interno del vaso, è attestata invece in entrambi i secoli.

Nella parete esterna sono redatte 17 iscrizioni a cui si aggiunge una delle due iscrizioni redatte nel piatto CIE 21002. Fra tutte, solo una di II sec. a.C. è tracciata seguendo il bordo della tesa e orientata verso l'interno, mentre la maggior parte segue l'attacco del piede con una prevalenza di orientamento verso l'esterno (solo CIE 20904 di III sec. a.C. e 20120 di periodo ellenistico sono orientate verso l'interno); pochi i casi di iscrizioni tracciate linearmente (incerte CIE 20044 e 20825).

Infine, è molto rara la casistica di iscrizioni nel fondo esterno. Dei 9 casi attestati, tutti eccetto uno (CIE 20263, un digramma in legatura) seguono il bordo dell'attacco del piede. Queste iscrizioni sono orientate sia verso l'interno che verso l'esterno; in particolare, sono orientati verso l'interno due digrammi su tre (CIE 20116, pu; 20607, nị; diversamente il digramma an di 20259 è verso l'esterno) e anche l'iscrizione circolare CIE 20697, kraiku.kaniu.

\section{II.3. Tecniche di scrittura, ductus, legature}

Le testimonianze epigrafiche mostrano una significativa difformità nel corso del tempo per tecnica di realizzazione (incisione a crudo/graffitura dopo la cottura). Ciò vale anche per la legatura delle lettere, mentre il ductus è coerentemente sinistrorso a eccezione di un piccolo nucleo di testi di periodo ellenistico.

Si possono datare al periodo compreso tra il VI, verosimilmente entro la seconda parte del secolo, e la prima metà del V sec. a.C. 29 iscrizioni comprensive dei digrammi e delle lettere in legatura. Sono perlopiù realizzate dopo la cottura ${ }^{42}$, a eccezione di 10 incise a crudo, fra le quali si contano 4 iscrizioni $(C I E 20265,20353,20361,20371)$ e i casi di due o più lettere in legatura, anch'essi a crudo: 2 di VI sec. a.C. (CIE 20089, 20194); 2 più puntualmente databili al periodo tardo-arcaico (CIE 20215, $20834)^{43}$; 2 di cronologia più incerta (CIE 20270, mentre la tecnica di realizzazione di CIE 20137 è dubitativa in quanto rimane solo la descrizione riportata dal Bocchi, oltre al suo apografo). Quan-

${ }^{41}$ Sui possibili sistemi di ripartizione della superficie del vaso attraverso graffiti, cfr. BAgNASCo GiANNi 2008, in particolare p. 279; si veda più recentemente l'analisi di A. GoBBI sul "Gruppo quadranti segnati all'interno" in BAGNASCO Gianni, Scoccimarro, Gobbi 2015. Per considerazioni su questo tema per Adria, si veda ora GaucCi 2020.

42 Agli ultimi decenni del VI sec. a.C. si data l'iscrizione CIE 20212, graffita dopo la cottura, e tra lo scorcio del secolo e gli inizi del successivo 8 iscrizioni, tutte graffite dopo la cottura: CIE 20143, 20180, 20189, 20287, 20289, 20305, 20368, 20370. Latamente al periodo arcaico si datano 7 iscrizioni, delle quali 3 graffite dopo la cottura (CIE 20194, 20356, 20834); alla prima metà del V sec. a.C. 8 iscrizioni (CIE 20159, 20160, 20204, 20214, 20282, 20290, 20295, 20822), a cui forse è da aggiungere per aspetto delle lettere la lacunosa CIE 20040, di cui rimane solo l'apografo del Bocchi.

43 La cronologia di questi ultimi documenti, redatti su vasi in bucchero, ceramica depurata e grezza in stato frammentario e non puntualmente databili, è prevalentemente basata sulla paleografia. 
do determinabile, il ductus delle iscrizioni è sempre sinistrorso. Rimane difficile la valutazione per il digramma CIE 20265, le cui due lettere (al) potrebbero essere tracciate secondo uno schema simmetrico (lambda sinistrorso e alpha destrorso con traversa discendente verso destra) oppure con ductus sinistrorso (nel caso, alpha con traversa ascendente verso sinistra). Il fenomeno della legatura sembra mostrare ad Adria una significativa incidenza nel periodo arcaico, in merito non solo alle occorrenze di coppie di lettere, ma anche a quelle che parrebbero più articolate (CIE 20137, 20215, 20371). I casi di due lettere si possono sciogliere con una relativa semplicità: $\widehat{a v}$ (CIE 20089), $\widehat{p v}$ o $\widehat{v p}$ (CIE 20194), $\widehat{k v}$ o $\widehat{v k}(C I E$ 20270), $\widehat{p e}$ (CIE 20834). In CIE 20089 e 20194 le lettere chiaramente speculari rendono incerta la direzione della lettura, mentre CIE 20834 mostra la scelta di orientare le lettere secondo un preciso ductus, come già osservato.

Il periodo compreso tra $\mathrm{V}$ e IV sec. a.C. è rappresentato da un numero di iscrizioni molto ridotto, tutte redatte dopo la cottura e con ductus sinistrorso. Unica iscrizione in legatura è CIE 20043 della seconda metà del IV sec. a.C., da leggere $\widehat{a a}$ o meglio $\overparen{\text { aia. }}$

Tutte le iscrizioni databili tra la fine del IV e il II sec. a.C., 165 comprensive dei digrammi, sono graffite dopo la cottura. La pratica di incidere a crudo non pare attestata in questa fase della cultura epigrafica adriese, se non raramente nella redazione di graffiti alfabetici e non alfabetici ${ }^{44}$ : si tratta nel complesso di pochi documenti da legare perlopiù a pratiche interne alla produzione ceramica, a cui però solo raramente si dovette ricorrere in questo periodo.

Quasi tutte le iscrizioni di periodo ellenistico mostrano un ductus sinistrorso. Soltanto 8 iscrizioni presentano ductus destrorso, con una prevalenza di casi (6) databili al II sec. a.C. ${ }^{45}$. Se durante il III sec. a.C., oltre a un inizio di serie alfabetica (cfr. V.1), l'unica con onomastica documenta il prenome Ata (cfr. IV.3.1.A) e l'uso di $n i$ al posto di $m i$ (unico caso adriese ${ }^{46}$ ), quelle di II sec. a.C. conservano invece nomi alloctoni di origine latina (i due Latiate) e venetica (Viza Muliu, Kani [u]). È possibile ipotizzare che il raro emergere di un ductus destrorso possa essere dovuto all'influenza di culture epigrafiche non etrusche, probabilmente espressione di individui detentori di una multi-competenza scrittoria. Infatti, la pratica scrittoria latina, generalmente destrorsa dal III sec. a.C. ${ }^{47}$, è attestata su ceramiche locali (CIE 20950) in questa città almeno dalla fine del III sec. a.C. e nel sepolcreto di via Spolverin si trovano documenti latini nei medesimi contesti di quelli etruschi ancora nel II sec. a.C. (es. tomba 106: CIE 21003). Simile osservazione può essere fatta per la prassi epigrafica venetica, dove il ductus può essere

${ }^{44} \mathrm{Si}$ contano poche testimonianze relative a graffiti non alfabetici incisi a crudo, sempre nel fondo esterno dei vasi: un segno a croce databile tra la metà del IV e la metà del III sec. a.C. da contesto funerario (CIE 20811), un segno a croce di una anfora da tavola da un contesto funerario di III sec. a.C. (CIE 20748), due serie di aste nella base di appoggio nel II sec. a.C. (CIE 20012, dove la serie è associata a una iscrizione nel fondo interno, da contesto abitativo; CIE 20912, da contesto funerario), un asterisco e un segno a croce da contesto abitativo (CIE 20055 e 20114) più latamente databili al periodo ellenistico. Il numerale $L$ inciso a crudo sul labbro di un'olla (CIE 20242) dal contesto abitativo/produttivo di via San Francesco, sembra invece interpretabile come unità di misura (cfr. SASSATELLI, GAUCCi 2010, p. 371, con riferimenti precedenti).

45 In particolare, tra la fine del IV e il III sec. a.C. si hanno solo due documenti: ataś ni da abitato (CIE 20364), iscrizione redatta con mano incerta sul fondo esterno di una coppa in ceramica grigia; il digramma ae, interpretabile come le prime due lettere della serie alfabetica, di incerto contesto (CIE 20775). Tra la fine del III sec. a.C. e gli inizi del II sec. a.C. si registra come destrorso il digramma la su un'anfora greco-italica (CIE 20951), graffito nel collo subito sopra la singola lettera tau, anch'essa destrorsa; al riguardo, vale peraltro la pena puntualizzare che nella stessa sepoltura, la tomba 56 di via Spolverin, si trova la più antica attestazione di iscrizione latina sicuramente redatta ad Adria (CIE 20950). Infine, nel II sec. a.C. sono redatte con ductus destrorso le due iscrizioni viza muliu su grandi ciotole a v.n. con due scanalature all'orlo (CIE 20923-20924) associate all' iscrizione forse da leggere kani $\times$ [ (CIE 20925, con alpha retrogrado) dalla medesima tomba 46 di via Spolverin (che però conta anche l'iscrizione CIE 20926 sinistrorsa), e l'iscrizione latiate dalla tomba 50 (il cui corredo conta anche iscrizioni sinistrorse: CIE 20933-20934) del medesimo sepolcreto; l'iscrizione latiate (CIE 20699) dalla tomba 75/1969 di Ca' Garzoni (che conta anche una iscrizione sinistrorsa: CIE 20698); infine un digramma da abitato ( $k a, C I E 20121)$, databile genericamente al periodo ellenistico.

46 Per la frequenza delle occorrenze, basti rimandare a $T h L E^{2}$, s.v.ni, 2. pronome.

${ }^{47}$ Cfr. REI LXXIX, p. 347, con riferimenti. 
sia sinistrorso che destrorso ${ }^{48}$; proprio il ductus destrorso è documentato nel medesimo sepolcreto di via Spolverin dalle uniche iscrizioni venetiche che si riconoscono ad Adria e nel suo territorio periferico (CIE 20911, sinistrorsa, dalla tomba 37; CIE 21014, con due lemmi destrorsi e in associazione all'iscrizione etrusca CIE 21013 nella tomba 112).

Tra III e II sec. a.C. sono rarissimi i casi di lettere in legatura a differenza del periodo compreso tra tardo VI e prima metà del V sec. a.C. I due unici documenti databili al III sec. a.C. sono associazioni di alpha e chi rovesciato (CIE 20041 e CIE 20426), forse allusivi della prima e dell'ultima lettera della sequenza alfabetica (cfr. V.1); in CIE 20041 le due lettere, se tali, presentano dei tratti sovrapposti piuttosto che una vera legatura, mentre nell'altro caso il tratto sinistro del chi va a costituire la traversa obliqua dell'alpha. Nel II sec. a.C. non si hanno invece attestazioni e anche il digramma a da una sepoltura di Ca' Garzoni (CIE 20377), si presenta diverso da quelli del secolo precedente, in quanto le lettere non sono legate e il chi non è rovesciato. Una ulteriore testimonianza di lettere in legatura, di difficile datazione sebbene comunque di periodo ellenistico, è graffita sul fondo esterno di una coppa in ceramica grigia dall'abitato (CIE 20263): le lettere alpha e kappa, che hanno in comune l'asta verticale, risultano fra loro speculari per ductus, rendendo difficile la lettura di questo digramma ( $\widehat{a k}$ oppure $\widehat{k a})^{49}$. Al contrario, la legatura di alpha e rho in una delle due iscrizioni alpi arnai dalla loc. Bettola (CIE 20737, alpi arnai) si ritiene una occorrenza anomala, non solo perché tale legatura sembra essere attestata in uno solo dei due casi identici per testo, ma anche perché è l'unica all'interno di tutti i testi con formule onomastiche. Considerando inoltre che l'unica testimonianza di questa iscrizione è l'apografo edito da L. Conton, sembra legittimo avanzare dei dubbi sulla attendibilità di tale legatura, forse piuttosto frutto di una erronea interpretazione dell'iscrizione da parte dell'editore.

48 MarinetTi 2002, p. 40.

49 Medesima legatura è attestata su ceramiche di produzione etrusca a Cerveteri e nel Midì francese in un periodo più antico; in questi casi è stata posta in connessione con i simili trademarks sulla ceramica attica e quindi interpretata come pratica legata al commercio (Benelli, Colivicchi, ZaCCAgnino 2017, p. 50, n. 22, con riferimenti, e fig. 4). Sembra tuttavia improbabile, soprattutto a fronte del diverso orizzonte cronologico, che la tarda sigla adriese possa riferirsi a questo complesso fenomeno epigrafico. 



\section{IL SISTEMA SCRITTORIO}

\section{III.1. Il quadro delle norme scrittorie tra VI e II sec. a.C.}

A partire dal fondamentale lavoro di G. Colonna del $1974^{1}$, si è sempre posta l'attenzione sulla meridionalità delle iscrizioni di Adria, fatto del tutto anomalo nell'Etruria padana, esclusa la zona della Valle del Marecchia (nella fattispecie l' iscrizione di Rimini) ${ }^{2}$. Questo assunto è stato posto in discussione in un preliminare lavoro del $2012^{3}$, che conviene richiamare nei suoi tratti essenziali in questa sede, integrandolo con alcune delle novità emerse con l'edizione del CIE IV, I, 1.

Per la fase tardo-arcaica, si contano dieci iscrizioni che offrono la possibilità di sviluppare alcune osservazioni sulla norma scrittoria.

Le iscrizioni CIE 20159 e CIE 20180 non pongono dubbi sulla norma scrittoria che le caratterizza, marcatamente meridionale. A queste, si aggiunge ora l'inizio di alfabetario CIE 20189, la cui sequenza comprensiva nella parte conservata di alpha gamma epsilon digamma, ne testimonia l'appartenenza a questa norma.

L'evidenza dell'uso della norma settentrionale, tipica dell' ambito padano, è data dall' iscrizione CIE 20368, che mostra la specificità del sade quale marca del genitivo I dell'antroponimo uiniaś. Se valida l'interpretazione proposta per le lettere fra loro legate e incise a crudo nel fondo di piede CIE 20371, cioè la parziale sequenza alfabetica composta dalle prime due lettere alpha ed epsilon e dall'ultima $c h i$, si avrebbe una possibile ulteriore testimonianza di tale norma. Va aggiunto che l'iscrizione lacunosa $C I E$ 20289 b) è stata recentemente riconosciuta da G. Colonna ${ }^{4}$ come terminazione del genitivo I espresso dal sade (a farfalla) e dunque sempre in norma settentrionale.

1 Colonna 1974b, pp. 23-24.

2 ET2, Um 2.1 da Rimini, mi titas in norma meridionale. Cfr. Sassatelli 2008a, p. 76, nota 14 con riferimenti; si conferma espunta dal novero quella del Marte di Ravenna (cfr. Cagianelli 2002, pp. 330-331, con bibliografia di riferimento), per la quale adesso si veda l'interpretazione in chiave sociale di TORELLI 2017.

3 Gaucci 2012a.

${ }^{4}$ G. Colonna, in REE LXXV, p. 260, n. 76. 
Già era stato rilevato come i casi delle iscrizioni CIE 20214, 20282, 20290, 20295, 20822 siano più complessi. G. Colonna ha ricondotto tutti questi documenti alla norma scrittoria meridionale ${ }^{5}$; successivamente chi scrive aveva rilevato alcune ambiguità nelle caratteristiche scrittorie ${ }^{6}$, che lascerebbero margini per una maggior articolazione interpretativa. Le considerazioni che seguono scaturiscono dalla resa della sibilante nel gentilizio di CIE 20822 e delle velari nelle iscrizioni CIE 20214, 20282, 20290, 20295.

La meridionalità dell'iscrizione CIE 20822 è riconoscibile nell'uscita in sigma del genitivo I del gentilizio Uselna. Proprio quest'ultimo nome, nella resa del grafema per la sibilante interna quale sigma e non sade come ci si aspetterebbe dal gentilizio orvietano, ha portato già G. Colonna a marcarne la specificità e chi scrive a ipotizzare una possibile settentrionalizzazione (su ciò, cfr. IV.1). In un orizzonte ormai di V sec. a.C., l'assenza di una esplicita opposizione grafemica tra le sibilanti sembra rimanere una problematica aperta nell' interpretazione del gentilizio. Come si vedrà di seguito, la possibilità che nel testo emergono elementi normativi non coerenti è comunque plausibile.

Altra particolarità delle iscrizioni adriesi è l'uso del kappa nelle iscrizioni CIE 20214, 20282, 20290, 20295. Come già accennato, nella prassi scrittoria meridionale il sistema a tre grafemi per la velare sor$\mathrm{da}(c i, c e, k a, q u)$ con l'ultimo quarto del VI sec. a.C. lascia progressivamente il passo all'uso indistinto di gamma ${ }^{7}$. Collocandosi nella prima metà del V sec. a.C., le quattro iscrizioni menzionate presentano una relativa recenziorità paleografica rispetto a questa fase di transizione ${ }^{8}$. In tutti e quattro i casi si ha la sola associazione di kappa con alpha, soluzione valida nel sistema arcaico a tre velari meridionale e in quello settentrionale. In CIE 20214, il prenome Venel con morfema del genitivo I è in norma meridionale. Ritenendo il fenomeno di attardamento di kappa come generalmente poco comune ${ }^{9}$, nonché l'evidenza dell'uso ad Adria anche della norma settentrionale, non si può escludere la possibilità che anche questa sia in norma ortografica mista come CIE 20822.

Nell'epigrafia etrusca, i casi di norma ortografica mista sono piuttosto pochi e i contesti di appartenenza sono generalmente un fattore determinante per la loro genesi. Si possono portare a esempio casi come quello della fascia di confine tra le due norme ortografiche presso il fiume Albegna (es. $E T^{2}$, AV 2.1, della prima metà del VII sec. a.C.); quello di città presso il confine fra le due norme che ebbe Orvieto, dove il quadro offerto da A. Maggiani sulla scrittura mette in risalto la presenza di alcuni documenti epigrafici in norma settentrionale o mista che manifestano l'influenza di Chiusi, con una continuità di contatti che perdurò fino al IV sec. a.C. ${ }^{10}$; il contesto coloniale di Aleria almeno fra IV e

\footnotetext{
5 Colonna 2003, pp. 166-167; per l'iscrizione CIE 20214, si rimanda a REE LXXIV, pp. 367-368, n. 111.

6 Gaucci 2012a, pp. 155-156.

7 Basti qui citare Cristofani 1972, pp. 480-481; Colonna 1976, pp. 16-17. In riferimento ad Adria, cfr. Colonna 2003, p. 167 e ID. REE LXXIV, pp. 367-368, n. 111.

8 Tale cronologia è confermata dai supporti nel caso di CIE 20282, 20290, 20295 (primi decenni del secolo, mentre si ricorda che CIE 20214 è perduta e la sua cronologia è su base paleografica).

9 Si aggiunge per completezza che a Orvieto, città alla quale sono rinviate le caratteristiche scrittorie e onomastiche di alcuni scriventi delle iscrizioni di Adria, ancora nel V sec. a.C. non mancano attestazioni di uso del kappa: in alcuni casi ciò è probabilmente dovuto alla persistenza del sistema a tre velari (e.g. $E T^{2}$, Vs 2.6, dove si ha l'associazione kappa+alpha, per la quale si veda DE SIMONE 1990, p. 78); in altri all'influenza della grafia settentrionale (cfr. infra).

10 Maggiani 2003. Si veda la parziale sequenza alfabetica in norma settentrionale la cui datazione è ipoteticamente collocata nella prima metà del V sec. a.C. (CIE 10658; PAndolfini, Prosdocimi 1990, pp. 64-65, n. III.17; MAgGiAnI 2003, pp. 380-381), e i gruppi di iscrizioni nelle due distinte norme della tomba Golini I degli inizi del IV sec. a.C. (RIX 1984, p. 458; MagGiani 1990, pp. 200-204, e più recentemente MaGgiani 2003, pp. 381-382); è possibile cogliere l'influenza chiusina nello sporadico uso del theta a croce e nelle due iscrizioni su oinochoai di bucchero (CIE 10592, $10593=$ $E T^{2}$, Vs 3.2-3). Queste ultime, databili al terzo quarto del VI sec. a.C., sono fra loro simili, ma una è totalmente in norma settentrionale e l'altra presenta l'uso di kappa + epsilon (indubitabilmente settentrionale) associato a quello di sigma per l'uscita del genitivo I: secondo A. Maggiani «questa ambiguità si può spiegare soltanto come il tentativo di adeguare un testo compilato secondo le regole ortografiche dell'Etruria settentrionale alle norme locali da parte di uno scrivente probabilmente chiusino» (MagGIAnI 2003, pp. 378-379).
} 
III sec. a.C. ${ }^{11}$. Infine, i casi del medesimo testo akius da Campiglia Marittima e da Marzabotto di fine V-inizi IV sec. a.C. ${ }^{12}$ attestano l'evidente mobilità individuale tra insediamenti etruschi dediti allo sfruttamento di risorse, alla produzione artigianale e al commercio. Caso forse diverso è rappresentato dalle testimonianze di VI-V sec. a.C. di Aleria e della Gallia meridionale (fra cui il piombo di Pech $M_{a h o}{ }^{13}$ ), per le quali G. van Heems ha sostenuto che «en lien avec le développement et l'utilisation de l'étrusque comme langue véhiculaire dans ces régions: les inscriptions [...] attesteraient ainsi un étrusque de "marchands", utilisé et diffusé aux $\mathrm{VI}^{\mathrm{e}}$ et $\mathrm{V}^{\mathrm{e}}$ siècles par les commerçants étrusques qui utilisaient les routes occidentales, celle d'Aléria, tout comme celle de la Gaule méridionale, et servant de langue de communication aver les communautés locales (celtes, ligures) ${ }^{14}$; si tratterebbe dunque dello sviluppo di una scrittura quale espressione di una lingua franca, finalizzata alla comunicazione con altre popolazioni all' intero di un florido bacino commerciale. La convergenza di individui provenienti da ambiti diversi del mondo etrusco e la proiezione commerciale verso i territori più settentrionali dominati da Veneti e Celti, fanno di Adria sicuramente un luogo privilegiato per lo sviluppo di manifestazioni scrittorie miste (per ulteriori considerazioni su questo aspetto, cfr. III.2).

Per il periodo compreso genericamente tra V e IV sec. a.C., le poche iscrizioni documentate mostrano l'uso della norma ortografica settentrionale (CIE 20037, 20184). Data la scarsità delle attestazioni e la prevalente impossibilità di datazioni puntuali per i supporti iscritti di questo periodo, rimane difficile poter circoscrivere in maniera dettagliata il passaggio di uso da diverse norme scrittorie anche con caratteri misti del periodo tardo-arcaico all'unicità della norma settentrionale, che oltre ai rari documenti di V-IV sec. a.C. si manifesta in maniera chiara e coerente dalla fine del IV sec. a.C. fino all'esaurirsi dell'esperienza epigrafica etrusca. A tale riguardo, va peraltro puntualizzato che l'ipotesi avanzata da F. Piva ${ }^{15}$ relativamente alla possibile presenza di almeno una iscrizione di pieno III sec. a.C. in norma meridionale (CIE 20487), si scontra con una diversa interpretazione di quest'ultima (cfr. V.4).

\section{III.2. La paleografia tra VI e II sec. a.C.}

L'analisi della paleografia adriese rimane a tutt'oggi l'unico tentativo aggiornato per l'Etruria padana che coinvolge un intero sito, con l'eccezione del pioneristico lavoro di G. Sassatelli su Marzabotto ${ }^{16}$, che alla luce delle più recenti acquisizioni richiederebbe una riflessione aggiornata ${ }^{17}$. Ma il quadro paleografico dei singoli centri, nella fattispecie quelli con più ricca documentazione (Marzabotto e Bologna nel settore gravitante sull'Appennino, il comprensorio mantovano, Spina e Adria nella fascia costiera: cfr. I.2), dovrebbe essere solo la prima tappa verso una analisi integrata di tutto l'areale padano, per comprenderne così nel dettaglio la fenomenologia della cultura epigrafica e individuarne le specificità interne, gli apporti esterni e la loro rielaborazione. Se quindi l'analisi di Adria può usufruire (anche e soprattutto metodologicamente) degli studi su Marzabotto (e prima ancora sulla Bologna orientalizzante ${ }^{18}$ ) impostati da G. Sassatelli, risulta attualmente più difficile orientarsi nella documentazione di Bologna di V-IV sec. a.C., che in particolare per le stele felsinee (ma non solo ${ }^{19}$ ) necessita di

\footnotetext{
11 VAN HeEms 2012, p. 213, con precedenti riferimenti (si veda anche Colonna 1990a, nota 12).

12 Maggiani 1992b, p. 184; Sassatelli 1994, p. 19, n. 13.

$13 E T^{2}, \mathrm{Na} 0.1$, datata al secondo quarto del V sec. a.C., per la quale G. Colonna evidenzia l'ambiente culturale periferico nel quale l'iscrizione è stata prodotta (Colonna 1990a).

14 VAN HeEMs 2012, pp. 452-453.

15 F. PIVA, in REE LXV-LXVIII, pp. 303-304, n. 3.

16 Sassatelli 1994.

${ }_{17} \mathrm{Si}$ fa qui riferimento alle iscrizioni di carattere sacro restituite dai templi urbani di Tinia e Uni, per le quali si veda Govi 2014; REE LXXIX, pp. 294-312. nn. 59-64; REE LXXX, pp. 236-241, nn. 2-4.

18 SASSATELli 1982-1983; ID. 1988, in particolare p. 235 ss.

19 Si richiamano qui le preliminari osservazioni sulle iscrizioni su ceramica offerte in GAUCCI, MorpUrgo, PIZzIRANI 2018, pp. 672-673.
} 
uno studio che parta dalla realizzazione di nuovi apografi, come ha dimostrato A. Maggiani con la stele Ducati n. 12 dei Giardini Margherita ${ }^{20}$. Inoltre, se per il Mantovano siamo ancora sostanzialmente fermi alle considerazioni di M. Pandolfini, assieme ad alcuni contributi di R.C. de Marinis (cfr. I.2), Spina rimane attualmente il grande convitato di pietra, in quanto non è possibile senza strumenti adeguati districarsi nella grande quantità di iscrizioni pubblicate (sia da necropoli che da abitato) secondo criteri editoriali diversi (non sempre funzionali a successive analisi), per non parlare dell'inedito: se la tesi di Dottorato di A. Pozzi dà contezza del sommerso per l'ambito funerario ${ }^{21}$, ciò è attualmente non quantificabile con esattezza per l'abitato (per alcune considerazioni, cfr. I.2).

Va peraltro aggiunto, come si vedrà, che è necessario superare una fase degli studi, forse ormai un po' sclerotizzata (e questo vale ancora di più per luoghi ritenuti di "frontiera"), dove la pratica epigrafica porta a distinguere ciò che è locale (sia per paleografia che per onomastica) da ciò che invece viene dall'esterno: da questo punto di vista i casi di Marzabotto con gli apporti chiusini e di Adria con gli scriventi orvietani sono esemplificativi. Infatti, i più recenti studi, soprattutto quelli che riguardano il ricchissimo e produttivo distretto tiberino, ci mostrano realtà molto complesse, dove la scrittura è strumento politico che si distingue nelle sue manifestazioni in base ai gruppi sociali che la praticano e ai contesti nei quali viene usata, come dimostra in maniera efficace il caso di Veio arcaica analizzato da D.F. Maras ${ }^{22}$. È pertanto evidente che va operato uno sforzo per superare l'equivalenza 'specifica manifestazione scrittoria = luogo di provenienza', per procedere verso modelli più complessi.

Adria permette di impostare una analisi che tenga conto di questi presupposti, non solo per la ricchezza della documentazione, ma anche per la possibilità di abbracciare un arco temporale di più di quattro secoli di scrittura etrusca, dalla seconda metà del VI sec. a.C. fino alle soglie della Romanizzazione nel I sec. a.C. Come già accennato (cfr. Introduzione), si tratta dunque di un laboratorio privilegiato, in particolare per l'Etruria padana dove sono rare le realtà urbane che superano il IV sec. a.C.

$\mathrm{Si}$ affronterà di seguito una trattazione analitica delle singole lettere, a eccezione dello iota e del lambda le cui forme non presentano sostanziali e significative variazioni nel corso del tempo. Per ogni lettera verrà analizzata l'evoluzione paleografica lungo tutto il corso dell'esperienza scrittoria etrusca ad Adria, per cogliere nella maniera più efficace possibile fenomeni di continuità e cambiamento.

Alpha (Fig. 4). Il grafema è attestato ininterrottamente e in quantità rilevante dal VI al II sec. a.C. ̇̀ così possibile tracciarne i cambiamenti formali nel corso di questo lungo periodo.

Nell'Arcaismo, l'alpha assume la forma angolata (CIE 20265 e 20371: in entrambe l'incertezza dell'orientamento del ductus non permette di comprendere se la traversa obliqua sia ascendente o discendente), e la forma con asta laterale spezzata (CIE 20353 e 20356). La lettera è attestata durante questa fase anche in iscrizioni dal territorio, rispettivamente a Le Balone (CIE 21041 ${ }^{23}$ ), dove è angolata con traversa discendente nel senso della scrittura, e a San Basilio (CIE 21065), che nell'insieme dei caratteri paleografici potrebbe trovare confronto, sebbene dubitativamente, nella pratica scrittoria veiente $^{24}$. In quest'ultima, la forma quadrangolare con traversa ascendente nel senso della scrittura e che va a congiungersi con l'angolo dell'asta laterale spezzata, si riscontra ad Adria soltanto nella iscrizione CIE 20370, poco più tarda.

$\mathrm{Al}$ periodo compreso tra gli ultimi decenni del VI e la prima metà del V sec. a.C. si datano 16 iscrizioni contenenti alpha. Nel complesso la lettera presenta una prevalenza di casi di aspetto con asta late-

\footnotetext{
20 Maggiani 1997 a.

21 Pozzi 2011.

22 Maras 2009a.

23 Per una disamina del documento, cfr. GaUCCi 2012a, pp. 162-163.

24 A. Gaucci, in REE LXXIX, pp. 292-293, n. 57.
} 
III. II sistema scrittorio

47

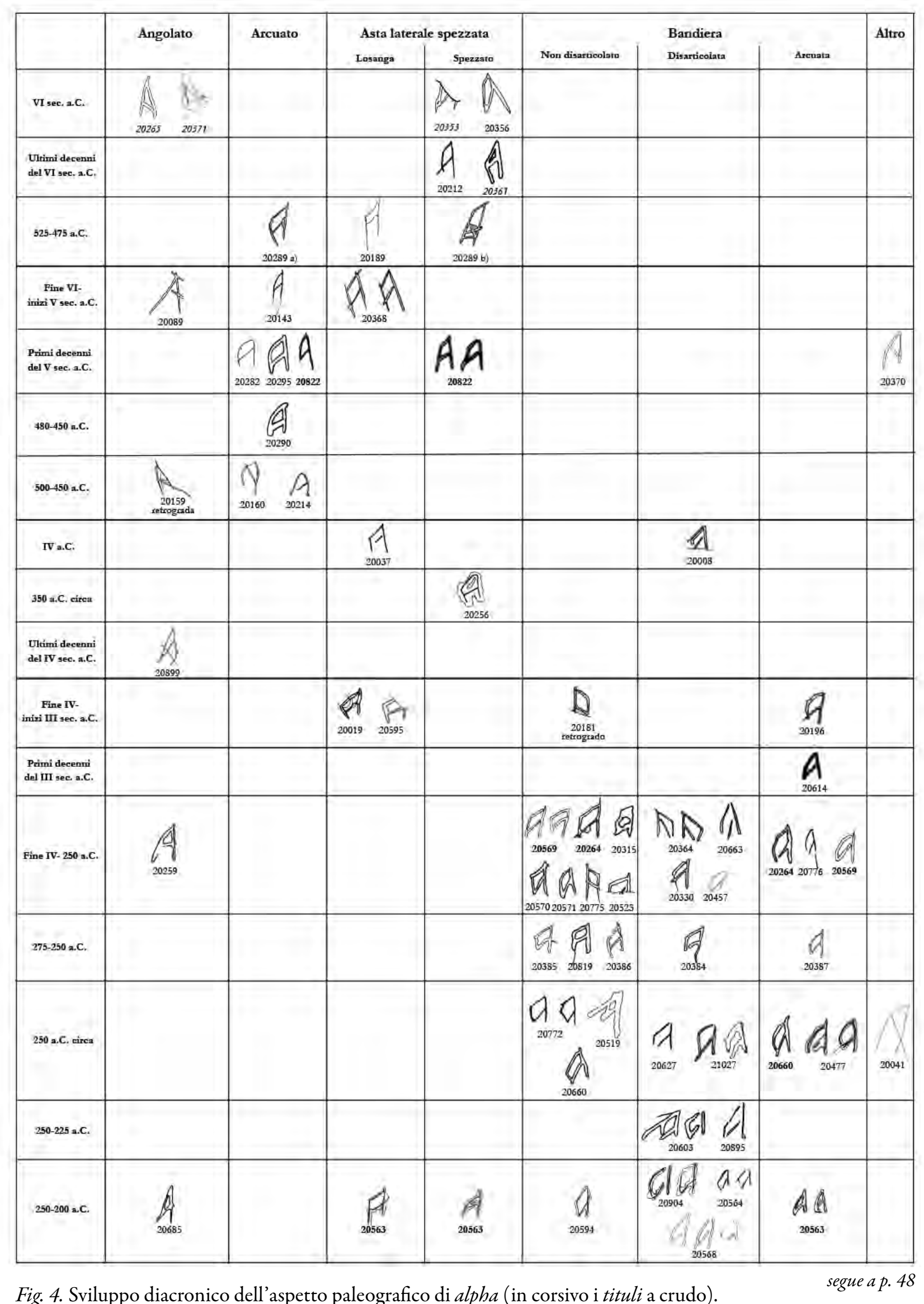


48

Iscrizioni della città etrusca di Adria

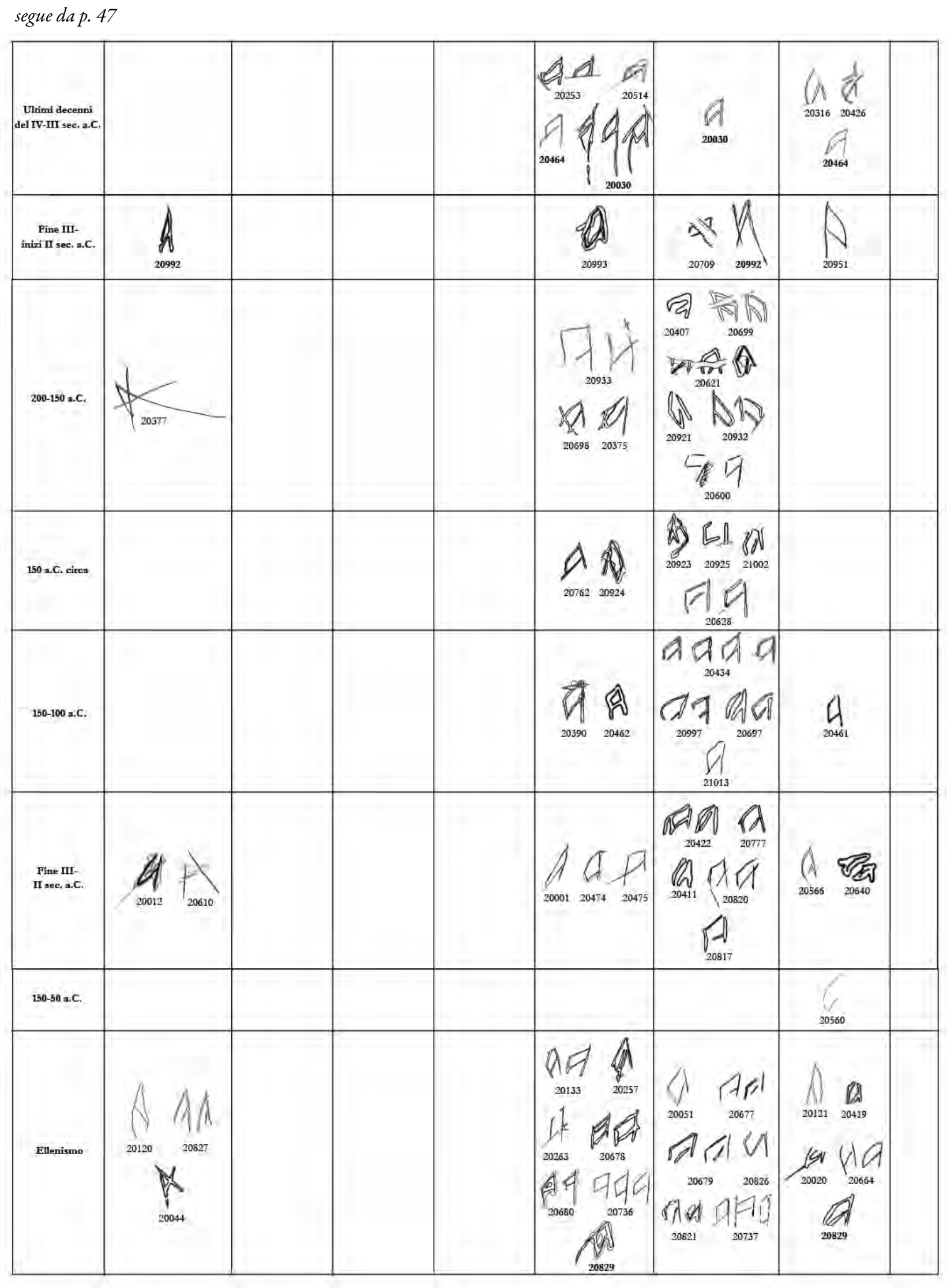


rale spezzata, con l'emergere in particolare di una variante con i quattro tratti a due a due paralleli, che si potrebbe definire squadrata o "a losanga" (come in CIE 20189); in particolare nell' iscrizione CIE 20368 la reiterazione conferma la coerenza grafica. L'aspetto di questa particolare variante richiama l'alfabetario di III fase di Roselle, la cui paleografia è avvicinata a quella radicata a Tarquinia e a Vulci del medesimo periodo, e anche uno simile da Gravisca, entrambi datati tra i decenni finali del VI e gli inizi del V sec. a.C. ${ }^{25}$. La forma squadrata sembra in effetti prevalente proprio in ambito tarquiniese e diffusa a Vulci e nel suo territorio ${ }^{26}$, oltre che attestata a Roselle ancora sulla fine del VI-inizi V sec. a.C. (CIE 11647), mentre in ambito padano, oltre ad Adria, questa variante risulta nel periodo in esame anche a Kainua-Marzabotto ${ }^{27}$, in maniera piuttosto coerente nell'abitato di Servirola e nel sito di Monte Pezzola in Emilia occidentale ${ }^{28}$, e almeno in un inizio di sequenza alfabetica da Spina ${ }^{29}$, tutte in norma settentrionale. Anche l'alpha di CIE 20289 b), sebbene abbia sicuramente presentato problemi redazionali ( $G$. Colonna ne ha supposto la correzione su un sottostante $l a m b d a^{30}$ ), suggerisce nell'aspetto con asta laterale spezzata e traversa prossima all'orizzontalità una affinità con alcuni casi di Vulci su ceramiche attiche degli ultimi decenni del VI sec. a.C. (CIE 10991, 10996). A questo orizzonte appartiene anche l'iscrizione di San Cassiano (CIE 21032), che si avvicina alla forma squadrata. Durante la prima metà del V sec. a.C., la prevalenza di occorrenze di alpha documenta l'aspetto con asta laterale arcuata con la traversa sempre discendente nel senso della scrittura; all'elenco è possibile aggiungere forse gli alpha delle iscrizioni CIE 20160 e $20214^{31}$. Dal quadro offerto si distinguono alcune occorrenze: CIE 20159; i tre alpha dell' iscrizione CIE 20822, fra loro piuttosto coerenti; CIE 20370, la cui forma con traversa ascendente nel senso della scrittura e asta laterale spezzata richiama più da vicino aspetti paleografici di ambito ceretano e veiente. Proprio in quest'ultima iscrizione, la forma con asta laterale spezzata della lettera potrebbe richiamare il modello alfabetico B in uso nel contesto urbano di Veio individuato nella sistematizzazione del repertorio paleografico di questo ambito da D.F. Maras ${ }^{32}$, e forse attestato anche nell'alpha dell' iscrizione arcaica di San Basilio CIE 21065, prima ricordata. Se ciò cogliesse nel vero, si avrebbe un utile indizio a supporto della via tracciata da A. Maggiani relativa al processo di riforma radicale della scrittura venetica durante l'Arcaismo, originato appunto dal comparto ceretano-veiente e arrivato a Padova forse attraverso un percorso adriatico costiero $^{33}$; percorso adriatico che potrebbe essere ancora stato attivo nel V sec. a.C., come testimonierebbe peraltro una iscrizione coeva di Spina ${ }^{34}$.

25 Pandolfini, Prosdocimi 1990, pp. 51-54, III.2 e 5.

26 STOpponi 1990, pp. 93-95. Per Vulci, si veda CIE 10982, su ceramica di impasto e datato alla fine del VI sec. a.C., e 10984, su ceramica attica di datazione non determinata, entrambi rinvenuti presso la Porta Ovest; 10991, su ceramica attica a f.r. datata al 520-500 a.C., 10992 del primo quarto del V sec. a.C. e 10997 del 470-460 a.C. rinvenuti presso Camposcala; 11078, rinvenuto in loc. Cavalupo; forse 11094. Dal territorio si richiama l'iscrizione su coppa in bucchero della seconda metà del VI sec. a.C. da Orbetello in norma scrittoria mista (CIE 11417), ma anche il più tardo piombo di Magliano di V sec. a.C. (CIE 5237; Massarelli 2014, pp. 241-244, figg. 1-2, tavv. I-II).

${ }_{27}$ Cfr. in particolare L. Malnati in Sassatelli 1994, p. 171, n. 277; cfr. anche ibid., p. 199. Più recentemente, E. Govi, in REE LXXX, pp. 239-241, n. 4, con un quadro generale sull'aspetto della lettera.

${ }_{28}$ Servirola: Maggiani 1992a, p. 211, nn. 1-2, con precedente bibliografia, e tav. IV, nn. 42 e 44 (per la n. 1, si veda ora G. Colonna, in REE LXXIX, n. 30, con una nuova proposta di lettura). Monte Pezzola: R. MACELLARI, in REE LXXIV, pp. 238-240, n. 5, il cui alpha è confrontato con quelli delle iscrizioni di Servirola.

29 Pandolfini, Prosdocimi 1990, pp. 59-60, n. III.10, dalla tomba 957 di Valle Trebba, datata sulla base della kylix attica a f.r. tra la fine del VI e gli inizi del V sec. a.C.

30 REE LXXV, p. 260, n. 76; tale interpretazione è stata accolta in GAUCCI 2012a, p. 144, n. 2.

31 Nel primo caso il ductus è solo dubitativamente destrorso (ma si tratta della parola al dove potrebbero entrare in gioco soluzioni grafiche di simmetria) e nel secondo ci rimane solo l'apografo del Bocchi, che non è possibile ritenere completamente affidabile.

32 Maras 2009a, p. 310.

33 Maggiani 2000a, pp. 92-95; ID. 2008, pp. 348-351.

34 Si tratta di una delle due iscrizioni della tomba 737 di Valle Trebba, della metà del V sec. a.C., che contiene un piattello in ceramica depurata con iscrizione letta usules (BENELLI 2004, p. 260). L'iscrizione, purtroppo ancora inedita, 
Nel IV sec. a.C., sebbene il numero di attestazioni sia scarso, si nota una notevole diversificazione dell'aspetto della lettera: con asta laterale spezzata, squadrato, angolato, con asta laterale e traversa convergenti sebbene disarticolate c.d. "a bandiera" 35 . Quest'ultima variante è attestata già precedentemente in Etruria settentrionale e padana ${ }^{36}$, ed è peraltro l'aspetto della lettera che si afferma nei documenti più antichi dell'epigrafia venetica riformata ${ }^{37}$.

Con la fine del IV sec. a.C. e gli inizi del successivo, la lettera si presenta prevalentemente quadrangolare, in due casi nella forma squadrata o "a losanga" (piuttosto schiacciato, con le aste verticali molto corte, come se rappresentasse una sorta di transizione verso il tipo "a bandiera") e negli altri due a bandiera (retrograda in CIE 20181, e con asta laterale arcuata in CIE 20196).

In effetti, nel III sec. a.C. si osserva la scomparsa definitiva dell'alpha squadrato. Le eventuali attestazioni (es. CIE 20563) possono essere spiegate come cursorie realizzazioni del tipo a bandiera, che si afferma da questo momento in maniera quasi assoluta, forse in continuità con la forma squadrata $\mathrm{o}$ "a losanga" che affonda le sue radici nel periodo tardo-arcaico. Durante il III sec. a.C., che vede ancora attestazioni epigrafiche etrusche diffuse in area padana, l'alpha a bandiera non trova corrispondenza nella documentazione di Monterenzio ${ }^{38}$; purtroppo manca un quadro completo per Spina, dove è comunque possibile affermare a grandi linee che l'alpha a bandiera è presente, sebbene sia contestuale all'uso di quello con asta laterale arcuata ${ }^{39}$.

Nella maggior parte dei casi le estremità inferiori dell' asta laterale e della traversa obliqua dell'alpha a bandiera sono convergenti e tangenti, ma si possono individuare anche casi di disarticolazione tra queste. Inoltre, la forma a bandiera può assumere un aspetto arcuato dell'asta laterale. Quest'ultima variante si riscontra dal III sec. a.C. fino al passaggio tra II e I. In ogni caso tale aspetto arcuato non è costante anche all'interno della medesima iscrizione. Più in generale, si coglie una certa variabilità di aspetto della lettera quando questa è ripetuta più volte in un testo ${ }^{40}$. Sebbene si possa far rientrare tutto ciò in una scarsa cura redazionale, questa spiegazione non sembra comunque del tutto soddisfacente quando l'alpha a bandiera con asta laterale arcuata (la cui resa è peraltro tendenzialmente più difficile nella graffitura della superficie ceramica) è associata a quella con asta laterale squadrata maggiormente attestata.

Tra le particolarità, colpisce anzi tutto una caratteristica che accomuna gli alpha delle iscrizioni CIE 20030, 20519 e 20776 di III sec. a.C., cioè il lungo tratto sottoavanzante, spesso disarticolato dall'asta verticale, che trova riscontro anche nelle lettere isolate della tomba 1 dell'Ospedale (CIE 20665-20667) e che potrebbe rappresentare la cifra grafica di una particolare moda scrittoria. Inoltre, ancora nel II sec. a.C. sono presenti casi di alpha angolato, che sembra però relegato a testi speciali quali i digrammi che evocano la serie alfabetica (CIE 20377).

Gamma e kappa (Fig. 5). Nel periodo tardo-arcaico, si contano solo due attestazioni di gamma (CIE 20180 della fine VI-inizi V sec. a.C.; CIE 20189 di cronologia più generica, entrambe riferibili alla

se non per le comunicazioni di G. Colonna (1993, p. 139) e di E. Benelli sopra riportata, presenta sicuramente il sigma a quattro tratti della sibilante interna. Si osserva che nella lettura autoptica data da A. Pozzi non pare essere presente il sigma finale del genitivo (Pozzi 2011, p. 196, n. 6).

35 Si veda a esempio Pellegrini 1959, p. 188, per l'uso di tale definizione nelle iscrizioni di Adria.

36 Si veda il quadro offerto in SASSATELLI 1994, p. 73, n. 91.

37 Maggiani 2000a, pp. 93-94.

38 Sassatelli 2008b, p. 342.

39 Pozzi 2011, p. 407.

40 Ancora nel II sec. a.C., tre casi (CIE 20628, 20697, 20997) mostrano l'associazione di più alpha fra loro diversi per aspetto: se in un caso (quello del prenome Ata di CIE 20628) la differenza riguarda semplicemente la disarticolazione dell'asta laterale, negli altri due riguarda invece l'andamento dell'asta laterale (squadrata o arcuata), evidenziando così come la diversa resa della lettera possa variare anche considerevolmente all' interno del medesimo testo, secondo quanto già riscontrato nel secolo precedente. 


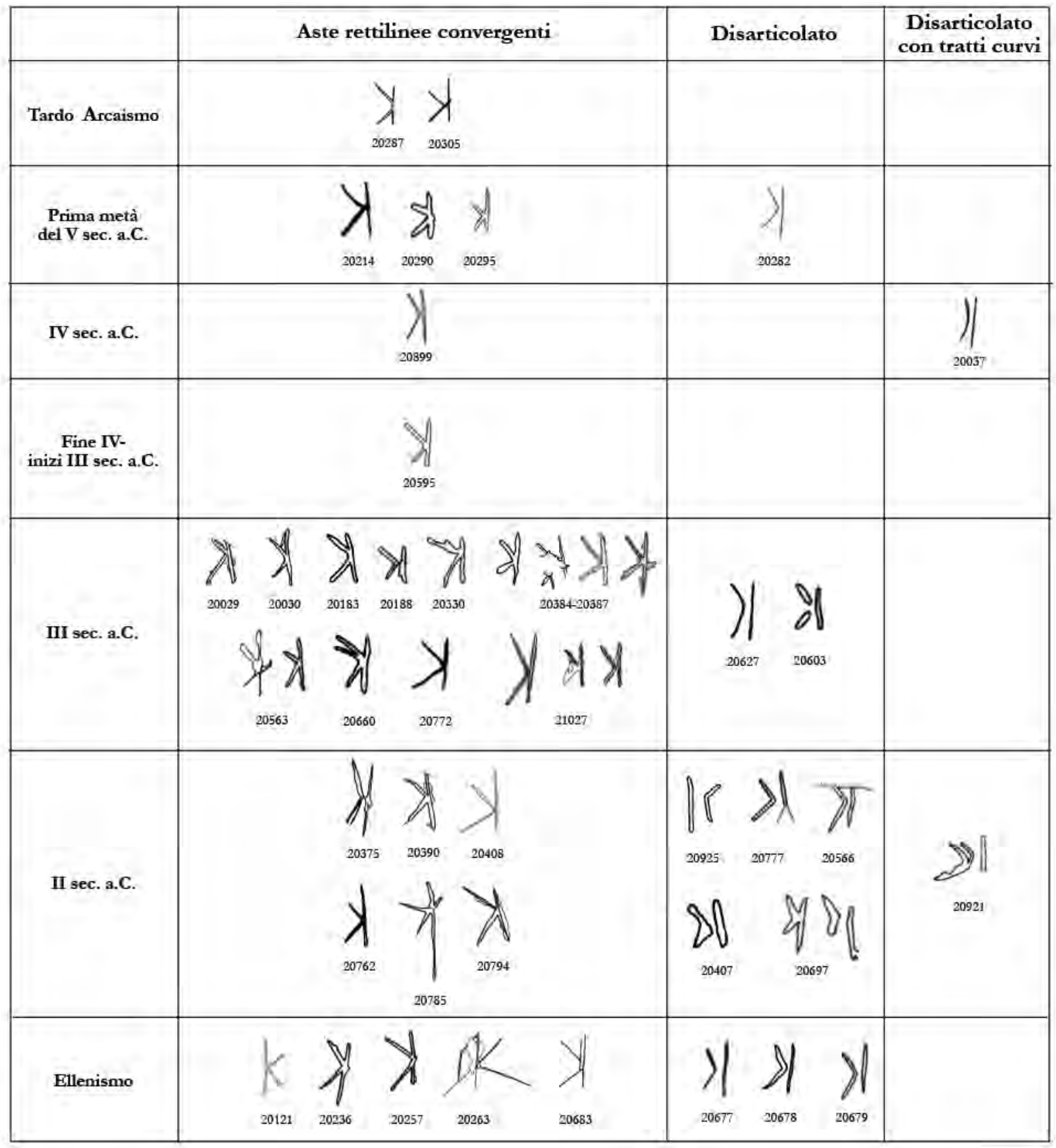

Fig. 5. Sviluppo diacronico dell'aspetto paleografico di kappa.

pratica scrittoria meridionale). Nel medesimo periodo, si collocano invece due attestazioni di kap$p a$ associato a rho in digrammi databili per il supporto attorno al 500 a.C. (CIE 20287 e 20305) e quattro attestazioni di kappa associato ad alpha in iscrizioni comprese entro la prima metà del V sec. a.C. (CIE 20214, 20282, 20290, 20295). Come già precedentemente evidenziato (cfr. III.1), l'associazione in CIE 20180 di gamma ed epsilon e in CIE 20214, 20282, 20290, 20295 di kappa e alpha non si discosta dal sistema a tre velari proprio della norma scrittoria etrusco meridionale arcaica, in uso fino agli ultimi decenni del VI sec. a.C. Va sottolineato che anche recentemente G. Colonna ha ribadito la meridionalità dell'iscrizione CIE $20214^{41}$, lasciando dunque presupporre che il kappa

${ }^{41}$ G. Colonna, in REE LXXIV, pp. 367-368, n. 111. 
associato ad alpha sia ancora retaggio della norma arcaica meridionale delle velari in una iscrizione il cui aspetto paleografico, seppure desunto dall'apografo di F.A. Bocchi, lascia pochi dubbi su una datazione al V sec. a.C.

Le uniche due occorrenze databili al IV sec. a.C. ci documentano l'uso del kappa all'interno della norma scrittoria settentrionale, che rimarrà in uso senza soluzioni di continuità fino a tutto il II sec. a.C., cioè al passaggio completo verso la cultura epigrafica latina. Unico caso che si discosta da questo quadro è l'iscrizione [---]× vercniś (CIE 20182) di III sec. a.C. Già A. Maggiani ha posto l'attenzione su questa iscrizione, ritenendola per il periodo ellenistico la più antica attestazione adriese del gam$m a^{42}$. La riforma del gamma nel passaggio tra IV e III sec. a.C. non ha però toccato la nostra città se non per l'unica occorrenza menzionata, che quindi è da ritenere episodica. Il mantenimento del kappa sembra caratterizzare anche il Mantovano ${ }^{43}$.

La lettera viene realizzata in due distinte maniere in periodo ellenistico: il maggior numero di attestazioni vede i tratti obliqui convergenti e tangenti l'asta verticale; alcuni casi mostrano invece i tratti obliqui convergenti o costituenti un unico tratto curvo, comunque non tangenti l'asta verticale. In particolare, le ultime due varianti si ritrovano a Spina ${ }^{44}$, e inoltre quella con due tratti convergenti ma non tangenti l'asta verticale nel Mantovano (Castellazzo della Garolda) nel IV sec. a.C.45, mentre quella con tratto unico curvo a Monterenzio ${ }^{46}$. Eccezionalmente entrambe queste due varianti sono presenti nella medesima iscrizione (CIE 20697), che potrebbe suggerirci come questa differenza dipenda più da un disordine redazionale che da una precisa scelta. Diversamente però, il kappa dell' iscrizione CIE 20603 mostra una disarticolazione dei tratti che caratterizza tutte le lettere del testo; in effetti, il numero delle occorrenze e soprattutto la coerenza nella resa del kappa nelle iscrizioni CIE 20677-20679, porta a ritenere plausibile l'idea che dietro la redazione della lettera con i tratti obliqui disarticolati dall'asta ci sia una pratica consapevole che si accentua nel II sec. a.C. Data la rarefazione di documentazione tra V e IV sec. a.C., rimane una questione aperta la possibilità che la coerenza di questa specificità epigrafica risalga indietro nel tempo fino al V sec. a.C., quando anche l'iscrizione CIE 20282 presenta la medesima caratteristica, che peraltro si ritrova anche a Marzabotto nella seconda metà del medesimo secolo ${ }^{47}$.

Epsilon (Fig. 6). Nelle iscrizioni generalmente databili al VI sec. a.C. questo risulta sempre in legatura con altre lettere rendendo incerta qualsiasi considerazione sull'aspetto. Nelle iscrizioni comprese tra

42 Maggiani 2002a, p. 61. Nel medesimo lavoro, lo studioso ha manifestato una sostanziale prudenza relativamente alla generale assenza del gamma ad Adria, affermando come non vi sia ragione di ritenere che anche qui l'uso si sia protratto a lungo dopo il 300 a.C., quando il kappa venne abbandonato pressocché ovunque, come anche nella vicina Spina (MAGGIANI 1998, p. 234); alla luce dello studio dei supporti e dei contesti con le relative datazioni riportate nel CIE IV, I, 1, adesso è possibile affermare che CIE 20182 rappresenta una eccezione rispetto a un uso altrimenti diffuso e sistematico del kappa quale grafema della velare sorda fino a tutto il II sec. a.C. Su ciò, si veda GaUCCI, Pozzi 2009, p. 58, sebbene qui alla nota 30 si elencassero tre attestazioni di gamma, nelle iscrizioni dalle tombe 55 e 257 di Canalbianco (CIE 20464 e 20581) e 45 di via Spolverin (CIE 20921), per le quali si esclude adesso la presenza di questa lettera.

43 Pandolfini $1988^{2}$, p. 117; anche nella più recente edizione delle iscrizioni da Mantova, piazza Santa Barbara, una iscrizione datata tra la fine del IV e gli inizi del III sec. a.C. presenta il kappa per rendere la velare sorda (MENOTTI, MARAS 2012, p. 881, n. 11, fig. 15).

${ }_{44}$ A titolo esemplificativo, la variante con tratti convergenti e disarticolati si riscontra nella necropoli di Valle Trebba alla fine del IV-metà del III sec. a.C. nell' iscrizione mi laris sekstaluś della tomba 98 (UGGERI 1998, n. 21, p. 496; ET², Sp. 2.71; per la cronologia, si veda BERTi 1983, p. 47; BENELLI 2004, p. 263); nell' iscrizione kulisia della tomba 417 del medesimo periodo (UGgeri 1978, n. 31; ET², Sp 2.87); nell' iscrizione kaisuś della tomba 1118 (UGGERI 1978, n. 26; ET ${ }^{2}$, Sp. 2.23). La variante con unico tratto curvo disarticolato è documentata nell' iscrizione letta perkño perki della tomba 106 di Valle Trebba (REE XLVI, p. 295, n. 8; UGgeri 1978, n. 44b, p. 380; ET², Sp 2.77; Benelli 2004, p. 264), anch'essa datata sulla base del corredo tra la fine del IV e la prima metà del III sec. a.C.

45 PANDOLFINI $1988^{2}$, p. 123, n. 249, fig. 49, dove non è espressa la cronologia che comunque può essere compresa plausibilmente entro il IV sec. a.C. (ibid., p. 116).

46 Sassatelli 2008b, pp. 341-342, n. 8, con bibliografia precedente.

47 Sassatelli 1994, p. 199. 


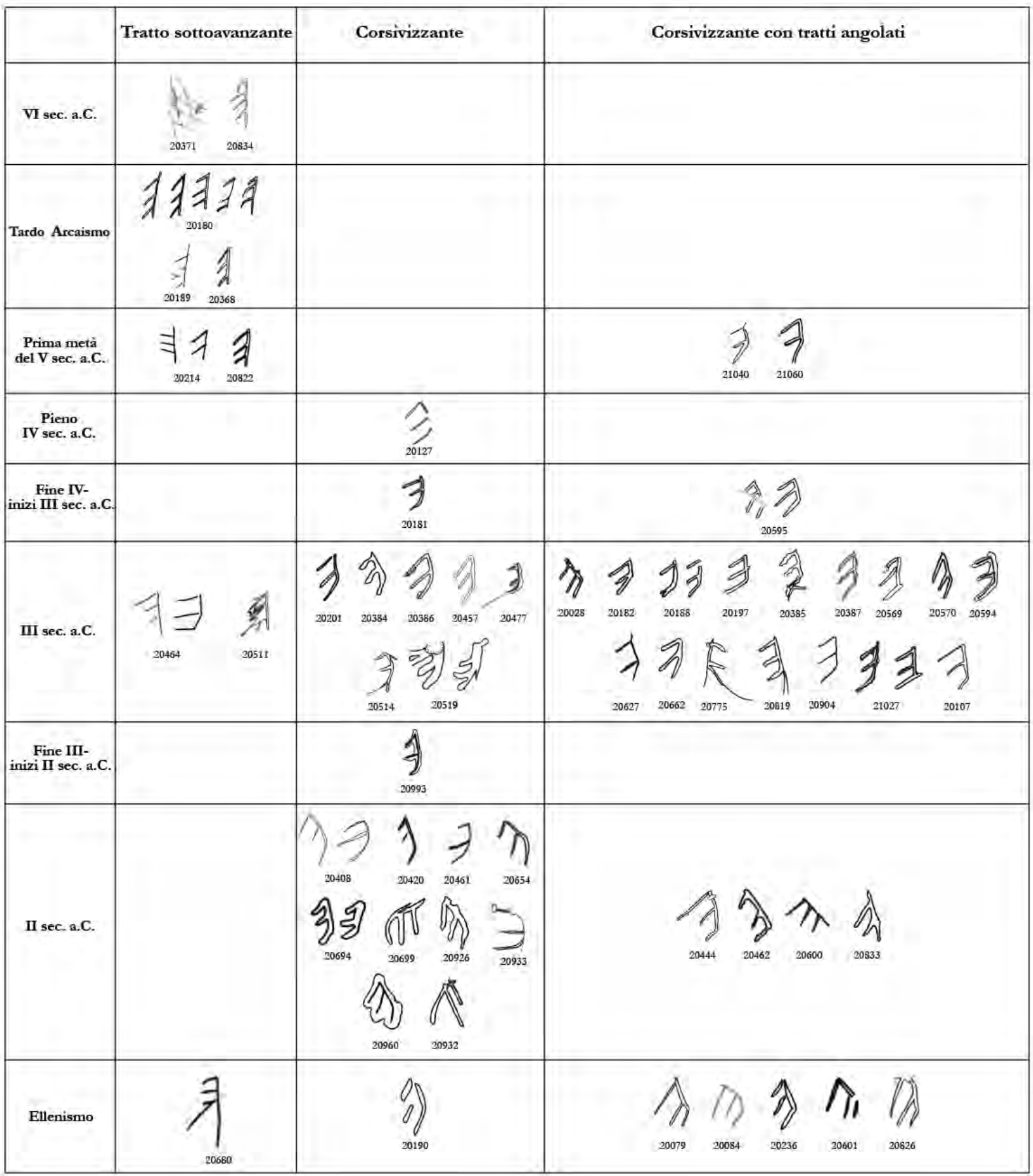

Fig. 6. Sviluppo diacronico dell'aspetto paleografico di epsilon (compresi CIE 21040 e CIE 21060 dal territorio).

gli ultimi decenni del secolo e la prima metà del successivo, la lettera presenta tratti obliqui regolarmente paralleli fra loro e asta verticale generalmente sottoavanzante secondo una tendenza comune alla paleografia etrusca del periodo. L'iscrizione CIE 20180 in particolare, che conta 5 occorrenze, mostra varietà di realizzazione e almeno un caso senza il tratto sottoavanzante. Nel medesimo intorno cronologico, l'iscrizione CIE 20214 evidenzia delle irregolarità che tuttavia non è possibile valutare quanto dipendano dall'apografo di F.A. Bocchi, data l'irreperibilità dell' iscrizione. Nel pieno V sec. a.C., l' iscrizione [m] i meslalus' di Le Balone (CIE 21040) e l'iscrizione lacunosa [---] upev [-- - di San Basilio (CIE 21060), dove l'epsilon è privo del tratto sottoavanzante e i due tratti obliqui inferiori convergono all'estremità dell'asta verticale, sembrano testimoniare l'acquisizione del tipo corsivizzante, 
formato appunto a Chiusi durante il V sec. a.C.48. A. Maggiani, in un importante lavoro del 1998, ha offerto un quadro della distribuzione delle più antiche manifestazioni del tipo corsivizzante in ambito padano, evidenziandone la comparsa su documenti di Bologna, di Marzabotto e del Mantovano allo scorcio del V sec. a.C., sebbene forse si possa alzare tale cronologia senza particolari difficoltà alla seconda metà del V sec. a.C. ${ }^{49}$. Le due testimonianze discusse provenienti dall'entroterra adriese non consentono di avanzare considerazioni sui possibili processi di diffusione di questo tipo grafico ad Adria, che solo con la metà del IV sec. a.C. restituisce una iscrizione di cui rimane unicamente la lettera epsilon, che è ormai pienamente quella caratteristica del tipo. In ogni caso, allo stato attuale della documentazione, sembra lecito ritenere che la sua ricezione in questo territorio sia comunque recenziore a quella spinetica ${ }^{50}$.

Il considerevole numero delle successive testimonianze di periodo ellenistico permette di osservare che in questa fase il tipo corsivizzante è sostanzialmente mantenuto, sebbene sia evidente la varietà nella resa formale di questa lettera. In particolare, l'ultimo tratto obliquo può essere in continuità con l'asta verticale producendo una curva oppure convergente all'estremità dell'asta medesima come già le due occorrenze di pieno $\mathrm{V}$ sec. a.C. Ma si osservano anche alcuni casi di tratto sottoavanzante distribuiti in tutto il periodo di III-II sec. a.C. (CIE 20464, 20511, 20680, e forse la perduta 20600). È probabile però che tale caratteristica rientri in una sostanziale assenza di uniformità nella resa delle lettere, come dimostra bene l'iscrizione CIE 20464, che documenta un epsilon con tratto sottoavanzante e uno quasi adagiato che ne è privo ${ }^{51}$.

Digamma (Fig. 7). Considerazioni simili a quelle esposte per epsilon si possono avanzare per il digamma, che sembra mantenere il tratto sottoavanzante fino alla prima metà del V sec. a.C. Nel periodo compreso tra V e IV sec. a.C. la lettera appare in una sola occorrenza (CIE 20037, forse databile su base paleografica proprio al IV sec. a.C.), ormai appartenente al tipo corsivizzante sebbene di incerta realizzazione. Anche per il periodo ellenistico, quanto osservato per l'epsilon è sostanzialmente valido per il digamma, che si presenta tendenzialmente nel tipo corsivizzante con alcune occorrenze di tratto sottoavanzante che sembrano dettate da una scarsa confidenza nella redazione del testo. In particolare si osserva come l'iscrizione CIE 20120 mostri la lettera con tratto sottoavanzante associata a un alpha angolato con tratto obliquo ascendente nel senso della scrittura, caratteri che parrebbero arcaicizzanti ma che figurano su una coppa in ceramica grigia di periodo ellenistico. Ciò potrebbe suscitare il dubbio che tale aspetto delle lettere possa essere il frutto di una diversificata competenza scrittoria del redattore; in particolare si ritrova il medesimo alpha nel digramma ma dell'anfora greco-italica CIE 21014, assieme al probabile kala sul medesimo supporto sicuramente riconoscibile come iscrizione venetica.

Zeta (Fig. 8). Nell'unica occorrenza di V sec. a.C., la lettera, se tale, si presenta senza tratto sottoavanzante, ma la resa disordinata, che ne rende incerta persino la lettura, non permette più puntuali

48 Maggiani 1990, pp. 182-183; ID. 1998, pp. 227-229; Benelli 2000, pp. 213-214. I due epsilon si avvicinano in particolare per realizzazione a quello dell' iscrizione dello stamnos di Petrignano datato al 460-450 a.C. (ET ${ }^{2}, \mathrm{Cl}_{2.24}$; cfr. per l'apografo CVA Mannheim, taf. 27, 1-4 e per ulteriori considerazioni BENELLI 2000, pp. 209-210), come peraltro confermerebbe in particolare il contesto di scavo dell'iscrizione di San Basilio, per il quale è stata proposta una datazione non oltre i decenni finali del V sec. a.C. (Salzani, Vitali 2002, p. 118).

${ }^{49}$ Maggiani 1998, p. 229; per Marzabotto, si veda più recentemente Govi 2014, p. 131, nota 127. Si sospende il giudizio sulla datazione alla prima metà del V sec. a.C. di una iscrizione lacunosa di Mantova (MenOTTI, Maras 2012, p. 881, n. 4, fig. 8), redatta secondo il tipo grafico corsivizzante e sulla quale si sono già espresse perplessità in merito alla lettura proposta (cfr. I.2).

50 Per Spina, si veda Maggiani 1998, pp. 229-230.

51 Con ciò si supera l'idea che questa caratteristica, comune ad altre lettere come appunto il digamma, possa indiziare aspetti della moda manierata (GAUCCI, Pozzi 2009, p. 60, nota 30). 


\begin{tabular}{|c|c|c|c|c|}
\hline & Tratto sottoavanzante & Corsivizzante & Corsivizzante con tratti angolati & Lacunoso \\
\hline VI sec. ac. & $\mathbb{N}_{20194} \underbrace{}_{20371} \mathbb{X}_{20353}$ & & & \\
\hline Tardo Arcaisme & $\left.\underset{20059}{A} \int_{20130}\right\}_{30199}$ & & & \\
\hline $\begin{array}{l}\text { Prima metá } \\
\text { del V sec. a.C. }\end{array}$ & $\underset{20214}{7}$ & & & \\
\hline IV sec, a.C. & & ) & & \\
\hline III sec, a.C. & 20464 & g & 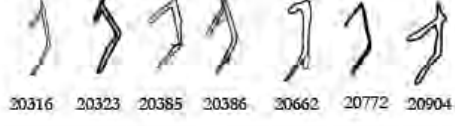 & $\lambda_{20162}$ \\
\hline II sec. a.C. & 2017 & $\prod_{20654} 9$ & $\int_{30420} \Gamma_{2022}$ & $\mathbb{R}_{2015}^{\alpha} \sqrt{20023}$ \\
\hline Ellenismo & 1 & 20133 20190 & & \\
\hline
\end{tabular}

Fig. 7. Sviluppo diacronico dell'aspetto paleografico di digamma.

considerazioni. Dal III sec. a.C., questa è realizzata prevalentemente senza tratto sottoavanzante, a eccezione di alcuni casi che invece si caratterizzano per un'asta più o meno accentuata verso il basso; fra questi ultimi, lo zeta dell' iscrizione CIE 20547 con i tratti ortogonali all'asta centrale pare una eccezione dovuta all'incertezza del redattore.

Chet (Fig. 9). La lettera è documentata a partire dalla fine del IV sec. a.C. e assume quasi sempre una forma romboidale con il tratto interno perlopiù posto al centro e ortogonale ai due verticali sui quali si impostano le sue estremità, forma che non può essere ritenuta variante del chet a cerchiello. Dunque, si può affermare con un certo grado di verosimiglianza che il chet a cerchiello, radicatosi nel tipo grafico corsivizzante sulla base di una riforma che pare avere il suo fulcro nella vicina Spina del tardo IV sec. a.C. ${ }^{52}$, non è stato mai acquisito in ambito adriese ${ }^{53}$. Rispetto a questo quadro, risultano eccezionali le attestazioni della lettera nelle iscrizioni CIE 20328 di III sec. a.C. e CIE 20133 più genericamente databile al periodo ellenistico, entrambe con due tratti interni paralleli; nel primo caso, questo ha una forma vagamente circolare, ma l'asta di destra è rettilinea e i tratti perlopiù

52 MagGiani 1998, pp. 232-233. Sugli aspetti storici di questa riforma, si vedano anche le considerazioni in GAUCCI 2016, p. 203.

53 Nello studio preliminare sulle iscrizioni funerarie edito nel 2009 si era osservato che il chet appare nella forma circolare nel III sec. a.C. (due casi riportati) e in quella a scaletta nel secolo seguente (GAUCCI, Pozzi 2009, p. 60, nota 30). Va tuttavia osservato che uno dei due casi riportati di chet a cerchiello è adesso letto in altra maniera (CIE 20591, $\chi u r \times \times$ rispetto al precedente huras proposto in $\left.E T^{2}, 2.24\right)$; per l'altro caso di III sec. a.C. (CIE 20594), si parlava di forma romboidale con tratto centrale. 


\begin{tabular}{|c|c|c|c|}
\hline & $\begin{array}{l}\text { Tratti obliqui } \\
\text { secanti }\end{array}$ & $\begin{array}{l}\text { Tratti obliqui non secanti } \\
\text { e asta sottoavanzante }\end{array}$ & $\begin{array}{l}\text { Tratti obliqui } \\
\text { non secanti }\end{array}$ \\
\hline $\begin{array}{l}\text { Prima metà } \\
\text { del V sec. a.C. }\end{array}$ & & & \\
\hline III sec. a.C. & & $\int_{20510}^{5} \underset{205+7}{5}$ & $\underset{20273.20514}{\mathbb{V}} \underset{20662}{ } \underset{20685}{N}$ \\
\hline $\begin{array}{l}\text { Fine III- } \\
\text { inizi II sec. a.C. }\end{array}$ & & & \\
\hline II sec. a.C. & & 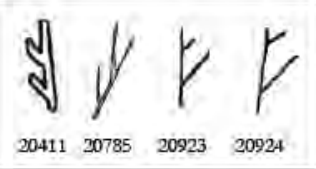 & $\mathbb{V}_{20621}^{i} \mathbb{N}$ \\
\hline
\end{tabular}

Fig. 8. Sviluppo diacronico dell'aspetto paleografico di zeta.

incerti. Se ci posso essere dubbi sull'interpretazione di questa particolare forma, questi sembrano fugati dal chet attestato nell' iscrizione CIE 20133, del tipo a scaletta con asta destra rettilinea sopravanzante e con due tratti obliqui.

Theta (Fig. 10). Questo è attestato nella forma romboidale senza punto in una iscrizione lacunosa su ceramica attica databile tra V e IV sec. a.C. e quindi tra III e II sec. a.C. in forma vagamente romboidale o più prossima alla circolarità senza punto centrale, secondo l'aspetto del più diffuso tipo corsivizzante ${ }^{54}$. Fanno eccezione i due casi di digramma $\theta i$ (cfr. V.4) compresi tra la fine del III e la prima metà del II sec. a.C., dove il theta presenta coerentemente il punto al centro. Tale aspetto è documentato in Etruria padana a partire dall'anforetta Melenzani di Bologna e del Cippo 1 di Rubiera con la fine del VII sec. a.C. ${ }^{55}$, e anche nel V sec. a.C. sempre a Bologna, a Marzabotto, nel Reggiano a Rubiera, nel Mantovano ${ }^{56}$, a cui è da aggiungere Spina, dove nel V sec. a.C. si ha la coesistenza del tipo a croce $^{57}$ e di quello puntato. Proprio a Spina, quest'ultimo si afferma come unico in uso e di forma romboidale dalla fine del V sec. a.C. (ET ${ }^{2}$, Sp 2. 20-21) fino alla più tarda iscrizione documentata con questa lettera, cioè $\theta$ aisi della tomba 417 di Valle Trebba della fine del IV-primi decenni del III sec. a.C. ${ }^{58}$. Colpisce in CIE 20606 il tratto verticale centrale, che si ritrova a Spina in una iscrizione di V sec. a.C. e in una di IV sec. a.C. $\left(E T^{2}, S p 2.6,42\right)$, oltre che in quella più tarda sopra citata. Non si può escludere dunque che l'aspetto della lettera possa dipendere da una speciale scelta, che pare coerentemente emergere con la scrittura della medesima parola in due vasi appartenenti a distinti contesti funerari.

A parte queste eccezioni, più in generale sembra di cogliere una distinzione fra l'aspetto grafico del theta a Spina e ad Adria. Mentre a Spina si osserva che la coesistenza dell'uso di due tipi di theta,

\footnotetext{
54 Maggiani 1990, p. 183; Benelli 2000, pp. 214-215.

55 Per la diffusione di tale grafema verso l'Etruria padana, si veda il quadro ancora valido in ColonNA, GAMBARI 1986, p. 145.

56 Per un quadro generale, si rinvia a BENELLI 2000, pp. 210-213; Govi 2014, p. 129, con relativi riferimenti.

$57 E T^{2}, S p 2.11,19,27$; diversamente, la lettura del theta a croce in Sp 2.12 è più dubbia (GAUCCI 2015, p. 131, nota 104, con riferimenti).

58 Per considerazioni generali, Benelli 2000, p. 212. Per la tarda attestazione della tomba 417 di Valle Trebba: UgGeri 1978, p. 360, n. 23; ET², Sp 2.62 (per la cronologia del corredo: UGgeri 1978, p. 367; S. PATITUCCI UGGERI, in REE LXVIII, p. 341, n. 16).
} 


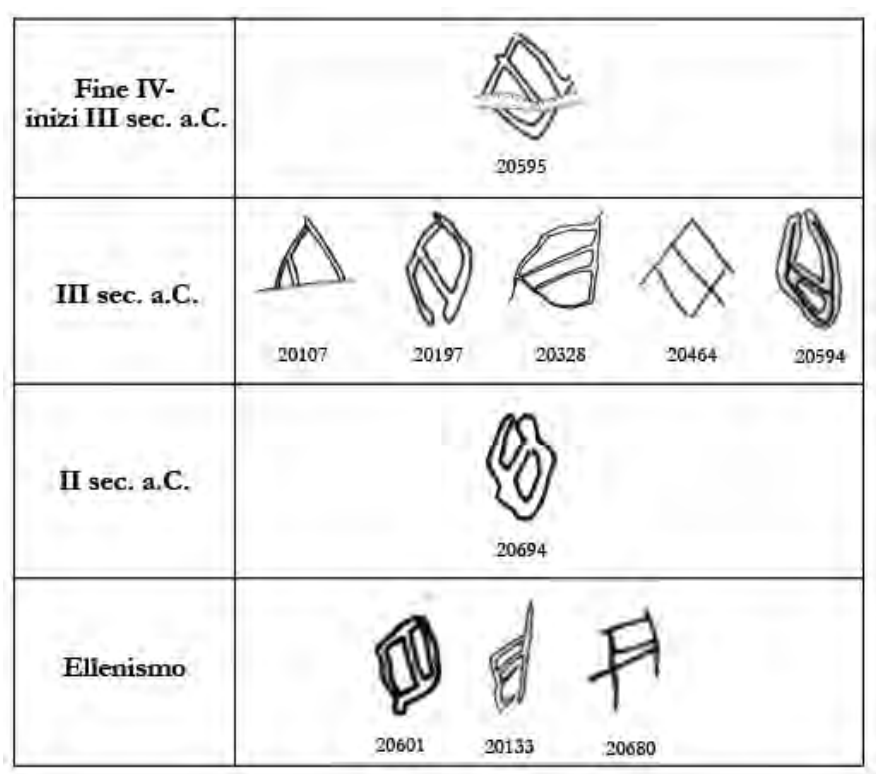

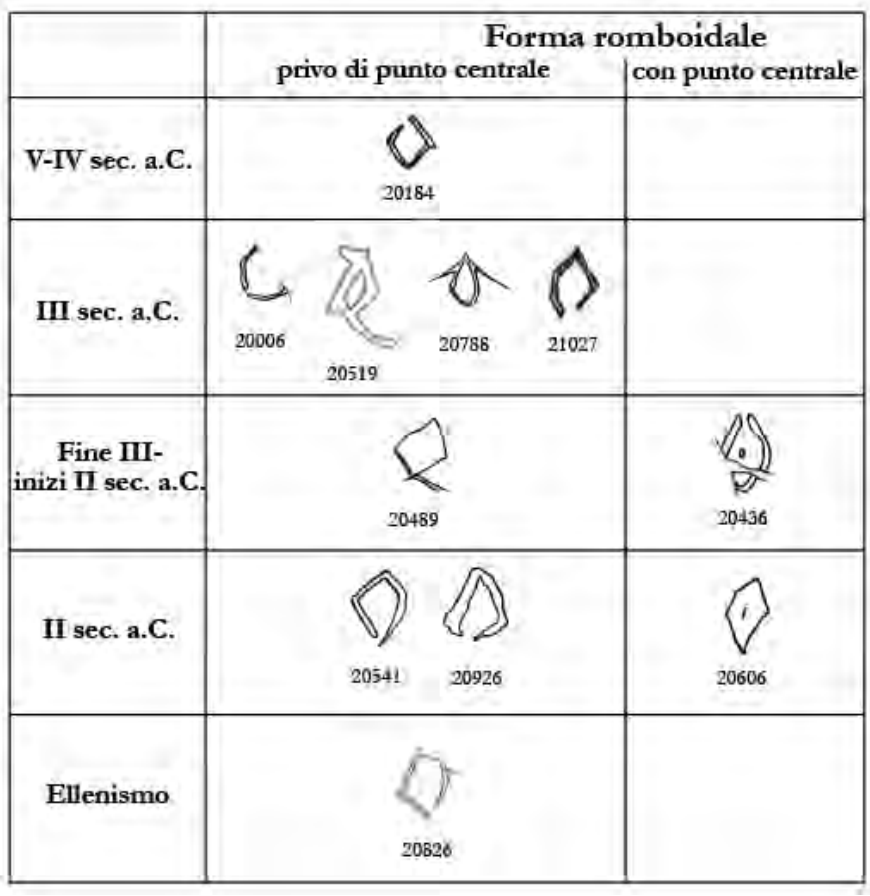

Fig. 9. Sviluppo diacronico dell'aspetto paleografico di chet.
Fig. 10. Sviluppo diacronico dell'aspetto paleografico di theta.

a croce e puntato, si risolve con la fine del V sec. a.C. per il secondo, ad Adria l'esiguità e l'incerta cronologia delle attestazioni note non permette di definire cronologicamente l'emergere della forma priva di punto sul lungo periodo. Anche le manifestazioni scrittorie di Monterenzio mostrano l'uso del theta privo di punto ${ }^{59}$ come ad Adria. Alla luce di queste considerazioni e nella dicotomia riscontrata tra i due principali centri deltizi, non si può escludere che i due casi di fine III-prima metà II sec. a.C. sopra discussi possano essere indizio di una puntuale manifestazione scrittoria originaria di Spina.

My e ny (Figg. 11-12). Queste due lettere entro il periodo tardo-arcaico presentano lunga asta verticale e brevi tratti obliqui, come si registra anche nell'iscrizione arcaica di Le Balone CIE 21041.

59 SASSATELli 2008b, p. 343, fig. 25. 


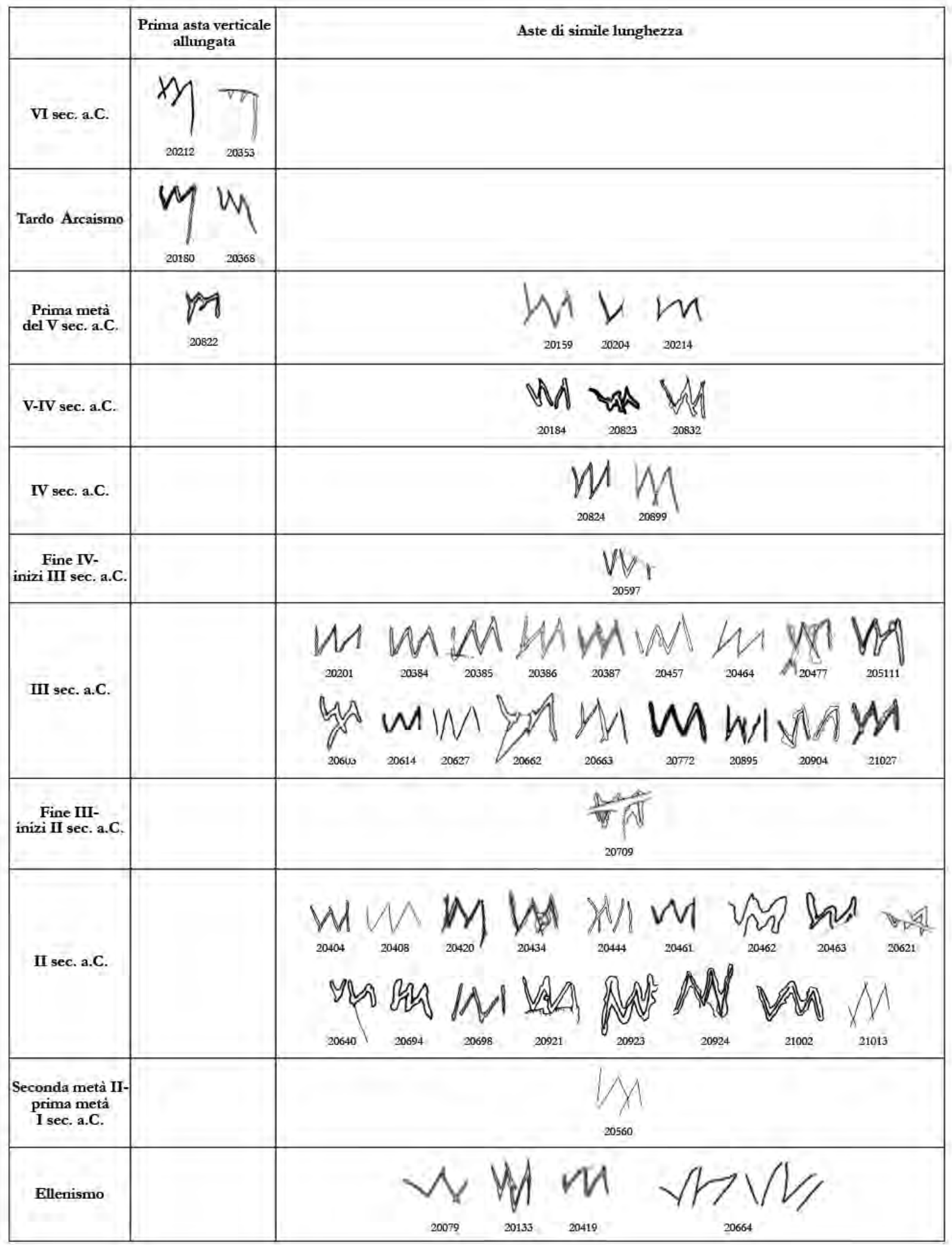

Fig. 11. Sviluppo diacronico dell'aspetto paleografico di $m y$.

Unica eccezione a questa tendenza arcaicizzante è apprezzabile in alcune iscrizioni, nelle quali il ny presenta tratti fra loro di uguale lunghezza, coerentemente con un aspetto recenziore anche delle restanti lettere del testo. 


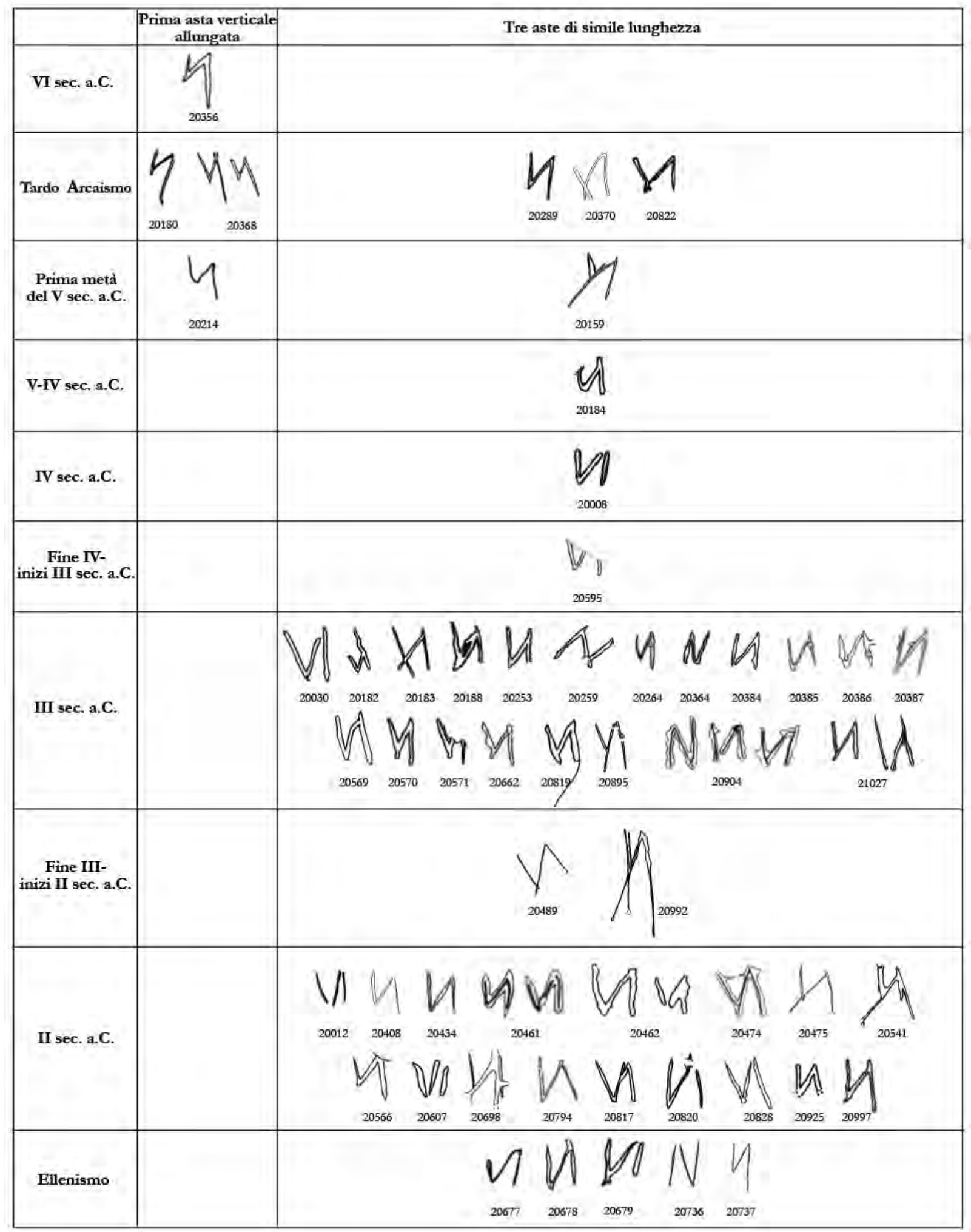

Fig. 12. Sviluppo diacronico dell'aspetto paleografico di $n y$.

A partire dagli inizi del V sec. a.C. e fino alla latinizzazione, non subirono cambiamenti sostanziali. In particolare, il $m y$, indicatore primario dei tipi grafici individuati da A. Maggiani per il periodo ellenistico, testimonia come il tipo corsivizzante si mantenga costante ad Adria, dove non sembra invece venga recepito il regolarizzato, che durante il corso del III sec. a.C. soppianta generalmente 
tutti gli altri diventando la grafia nazionale etrusca ${ }^{60}$. Rispetto a una maggior regolarità di realizzazione durante il III sec. a.C., nel II sec. a.C. queste lettere si presentano maggiormente disarticolate (soprattutto il my). Accade invece solo al $n y$ di essere scritto con ductus retrogrado ${ }^{61}$.

$P i$ (Fig. 13). Nel periodo arcaico è ravvisabile solo in due legature di lettere. Mentre in CIE 20194 il tratto obliquo assume un andamento lievemente curvo, in CIE 20834 questo si presenta chiaramente uncinato, come a Marzabotto e a Monteacuto Ragazza in iscrizioni della prima metà del V sec. a.C. ${ }^{62}$. Tale aspetto si ritrova anche nell'alfabetario di III fase di Roselle degli ultimi decenni del VI sec. a.C. ${ }^{63}$, che richiama l'epigrafia vulcente, dove in effetti si documenta il $p i$ con curvatura finale del tratto obliquo dal VII sec. a.C. ${ }^{64}$. Nel periodo arcaico, la lettera appare anche nell' iscrizione pisna da Le Balone (CIE 21041); sebbene l'asta presenti qualche incertezza nella linearità del tracciato (assegnabile a una resa cursoria), il tratto obliquo è in questo caso da ritenere rettilineo.

Dopo un vuoto di documentazione che copre tutto il IV sec. a.C., solo con la fine del medesimo secolo si registrano nuove occorrenze. Fino a tutto il II sec. a.C. il tratto obliquo è rettilineo sostanzialmente senza variazioni rilevanti, benché in alcuni casi l'incertezza della mano abbia portato a variazioni della forma quando la lettera è ripetuta più volte in una singola iscrizione (si veda il caso di papa in CIE 20933). Solo durante la prima metà del III sec. a.C. si potrebbero avere due casi di $p i$ con terminazione ad uncino (CIE 20819) o comunque con tratto terminale spezzato (CIE 20201). Tale aspetto della lettera si ritrova nel medesimo periodo anche nell' iscrizione petnei della tomba 14 (degli ultimi decenni del IV sec. a.C.) della necropoli di Monte Tamburino presso Monte Bibele nella valle dell' Idice ${ }^{65}$.

Sade (Fig. 14). Nel periodo tardo-arcaico, oltre alla più diffusa forma con aste laterali verticali e prolungate secondo l'aspetto canonico per il periodo, si registra anche un sade a farfalla. È noto come tale speciale forma sia usata quale sibilante in Etruria campana ${ }^{66}$, sporadicamente in Etruria meridionale nella seconda metà del VI sec. a.C.67, e ancora presente con i tratti sottoavanzanti nell' iscrizione del piombo di Magliano della metà del V sec. a.C. $\left(E T^{2}, \mathrm{AV} 4.1\right)$. In Etruria padana si registra in iscrizioni dal Forcello di Bagnolo San Vito ${ }^{68} \mathrm{e}$ in una da Spina ${ }^{69}$. Mentre il sade della nostra iscrizione lacunosa presenta lunghi tratti sottoavanzanti, sempre ad Adria altre attestazioni isolate della medesima forma a farfalla, graffite dopo cottura (CIE 20173) o incise a crudo (CIE 20092, 20157, 20845) su coppe in

60 Maggiani 1990, p. 191.

${ }^{61}$ Si veda il digramma CIE 20259 e uno dei tre ny dell'iscrizione CIE 20904 di III sec. a.C., nonché quello dell' iscrizione CIE 20736 genericamente databile al periodo ellenistico; si osserva in particolare come l'iscrizione CIE 20737 che presenta medesimo testo (e forse apparteneva al medesimo contesto) dell'ultima menzionata, abbia il ny solidale con il ductus generale.

62 Govi 2014, p. 128, nota 108, con riferimenti.

63 Pandolfini, Prosdocimi 1990, pp. 51-52, n. III.2.

${ }^{64}$ Cristofani 1973-1974, p. 158; Stopponi 1990, p. 95. Cfr. in particolare per il VII sec. a.C.: CIE 11058, 11146, 11157; per il periodo tardo-arcaico: CIE 10991, 10992, 11008, 11079, 11099, 11100, 11102, 11147 (caso distinto è CIE 11080); per il V sec. a.C., si veda in particolare il piombo di Magliano $\left(E T^{2}, A V 4.1\right)$. Si ritrova tale forma anche nelle due iscrizioni di dedica ad Apa su coppe in bucchero dal Belvedere di Orvieto (CIE 10525, 10535); si osserva in particolare come CIE 10535 presenti due alpha di aspetto vicino a quelli vulcenti.

65 Sassatelli 2008b, pp. 339-340, n. 2, figg. 17 e 25.

66 Pellegrino 2008, p. 430; cfr. anche per Calatia, F. Poli, in REE LXXI, pp. 224-225, n. 81.

67 Colonna, Gambari 1986, p. 140, nota 94; Sassatelli 1994, p. 33, n. 29.

68 PANDolfini $1988^{2}$, p. 120, nn. 242 e 244. Desta qualche dubbio l'attribuzione alla pratica scrittoria leponzia proprio in base alla presenza del segno a farfalla di una iscrizione lacunosa da Mantova, piazza Santa Barbara, per la quale è stata proposta la datazione al V sec. a.C. (D.F. Maras in MenotTi, Maras 2012, p. 881, n. 7, fig. 11, e p. 882, nota 8).

69 UGGeri 1978, pp. 354-355; cfr. Benelli 2004, p. 262, che pensa a una provenienza dall'ambiente mantovano. 


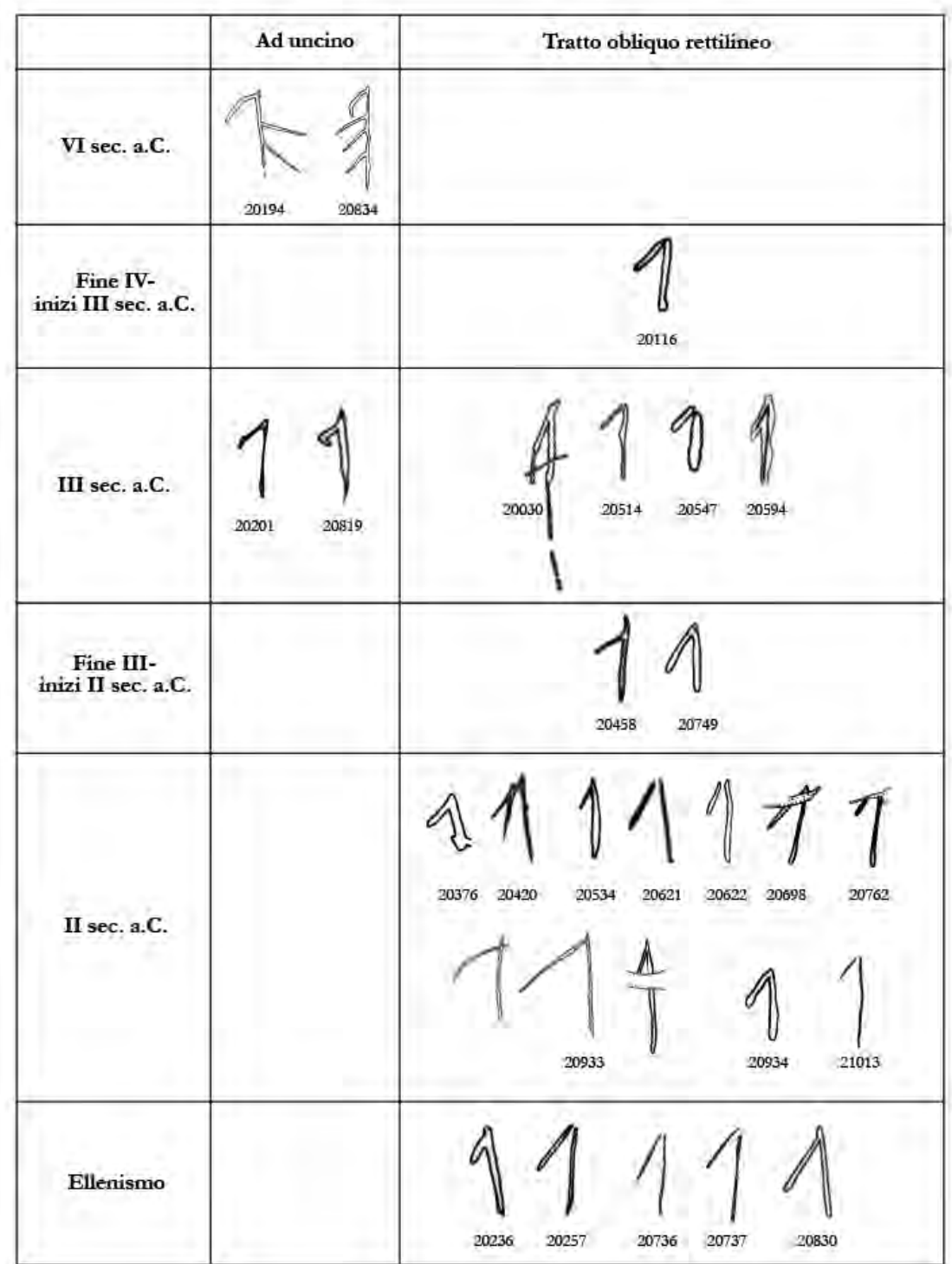

Fig. 13. Sviluppo diacronico dell'aspetto paleografico di $p i$.

bucchero della seconda metà-inizi del V sec. a.C., quasi tutte da contesto abitativo, non li presentano. Questi ultimi potrebbero essere piuttosto intesi come contrassegni, sebbene non si possa escludere che abbiano mantenuto un legame con un eventuale valore grafemico, come già ipotizzato per i medesimi segni isolati a Marzabotto ${ }^{70}$. L'utilizzo di tale sigla (definita anche "a clessidra") quale contrassegno è attestato anche in ambito etrusco-meridionale, dove si è avanzata invece l'idea che possa non essere necessariamente legato alla sua funzione di grafema ${ }^{71}$.

Le attestazioni della lettera tra V e IV sec. a.C. documentano la forma con aste laterali aperte, che rimarrà tale fino alle più tarde occorrenze comprese tra la seconda metà del II e la prima metà del I sec. a.C. A parte una scarsa coerenza formale, l'unico caso che spicca è quello del sade a farfalla dell' iscrizione CIE 20827, per la quale la forma a pettine degli alpha e il rho con pronunciato codolo richiamano, soprattutto per la coerenza complessiva di aspetto delle medesime lettere e per l'orizzonte cronologico, la più tarda espressione scrittoria in alfabeto leponzio ${ }^{72}$.

\footnotetext{
70 Govi 1994b, p. 222.

71 Si veda Benelli, Colivicchi, Zaccagnino 2017, p. 59, n. 55, fig. 7.

72 De Marinis, MotTa 1990-1991, in particolare p. 216.
} 


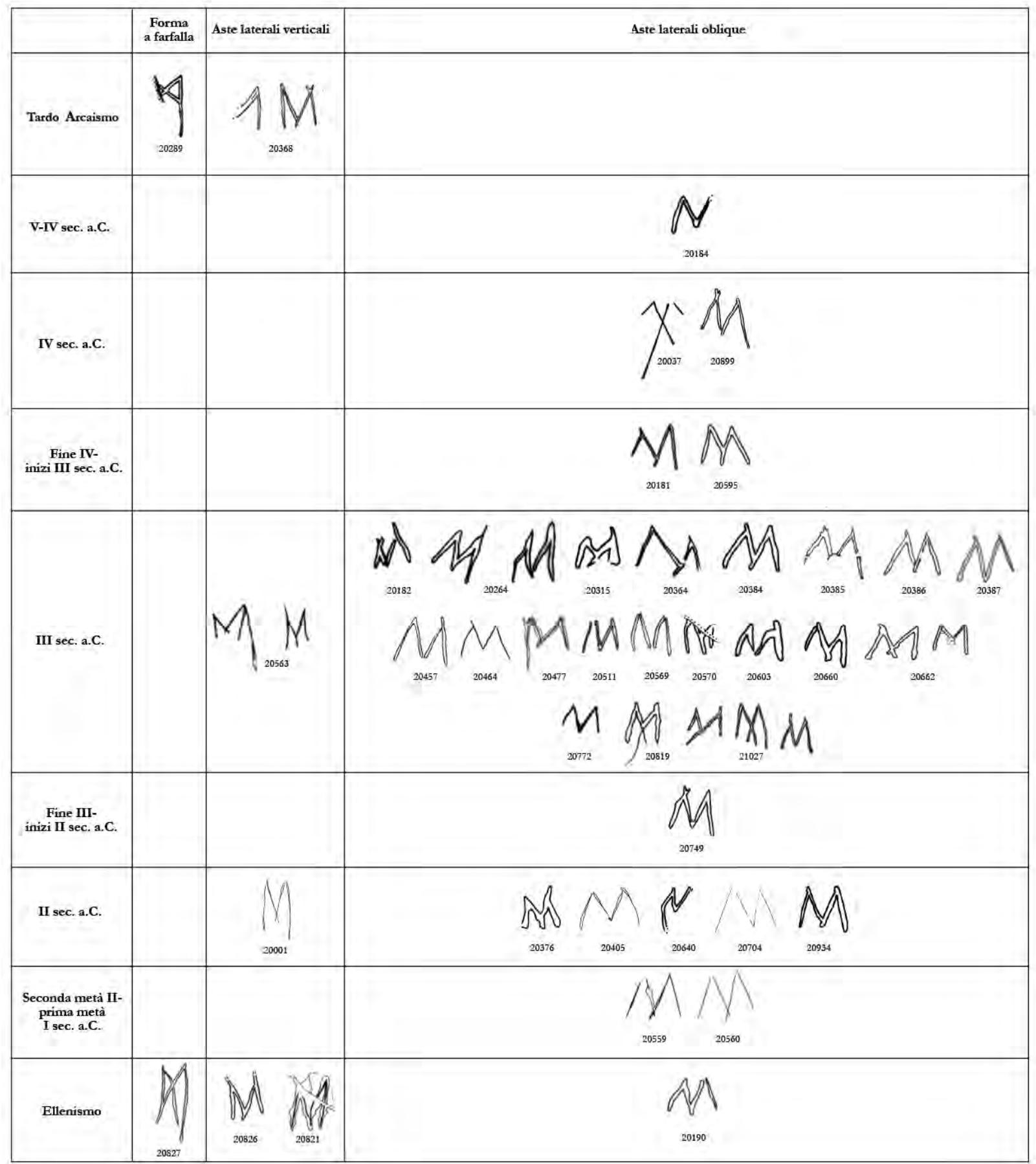

Fig. 14. Sviluppo diacronico dell'aspetto paleografico di sade.

Rho (Fig. 15). Nel periodo tardo-arcaico il rho è privo di codolo (l'accenno in CIE 20143 potrebbe dipendere da una corsiva realizzazione della lettera) e ha l'occhiello triangolare, sebbene in alcuni casi l'andamento di quest'ultimo possa risultare incerto (CIE 20287 e 20305) o anche lievemente arcuato (CIE 20361, ma ciò potrebbe dipendere dall'incisione nell'argilla cruda e dalla redazione sulla superficie curva dell'orlo). Nel complesso, la conformazione dell'occhiello triangolare richiama in maniera particolare la paleografia chiusina e vulcente di periodo tardo-arcaico ${ }^{73}$.

73 Cristofani 1977, p. 201, fig. 1; Benelli 2000, p. 213. 


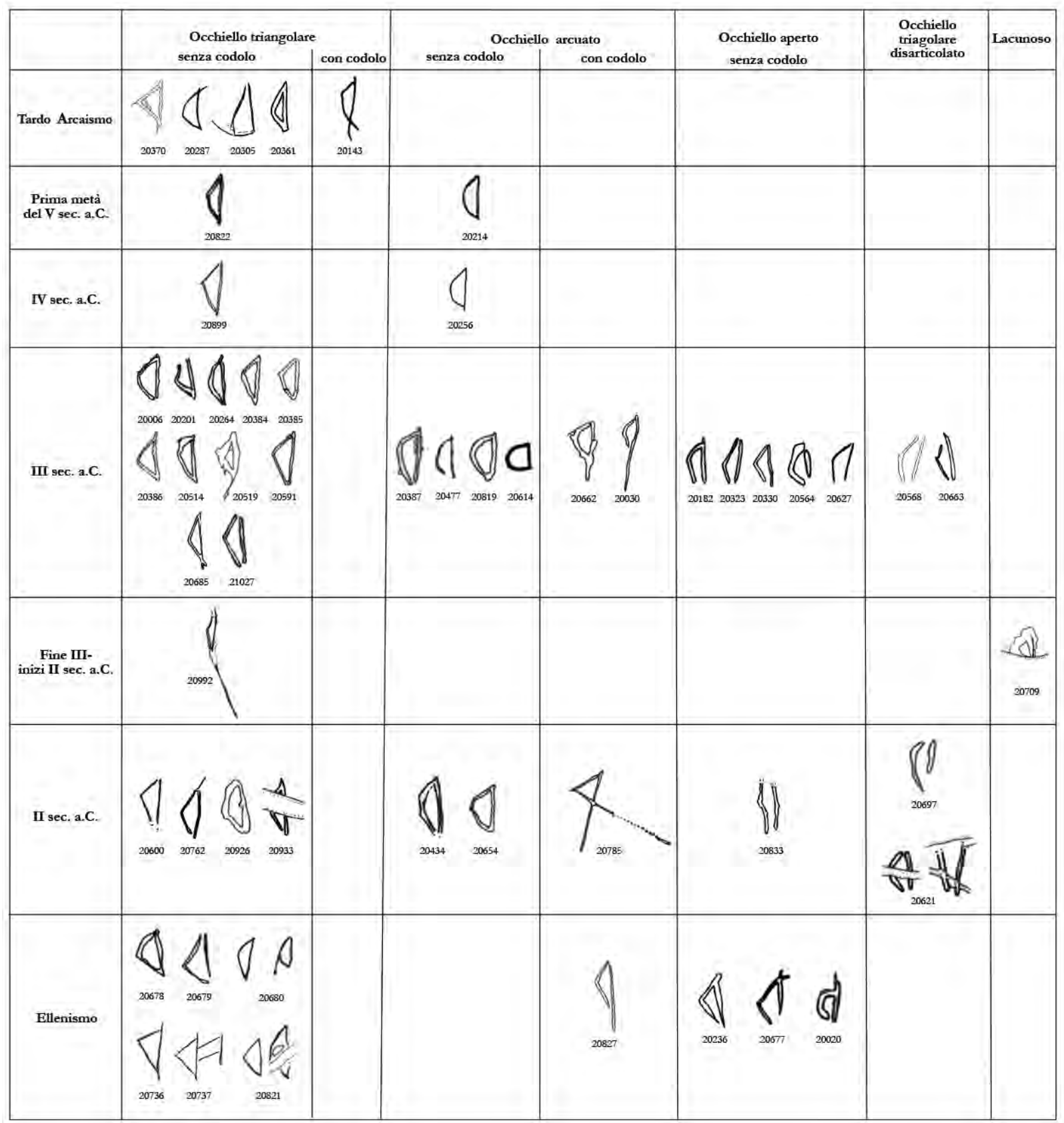

Fig. 15. Sviluppo diacronico dell'aspetto paleografico di $r h o$.

Con la prima metà del V sec. a.C. e fino a tutto il II sec. a.C. si continua perlopiù a realizzare la lettera senza codolo e con occhiello triangolare, sebbene non manchino casi con occhiello arcuato. Nel periodo ellenistico si documentano alcuni casi eccezionali di rho con codolo più o meno lungo (CIE 20030 e 20662 di III sec. a.C.; CIE 20785 di II sec. a.C.; CIE 20827 più latamente di periodo ellenistico), che non possono essere ritenuti indicativi dell'adesione a riforme grafiche esterne, data la episodicità delle attestazioni e l'incoerenza grafica interna alle iscrizioni medesime ${ }^{74}$. Nel III sec. a.C. pare significativo anche il numero di occorrenze (5) del rho con occhiello triangolare inferiormente aperto, che nel secolo seguente presenta invece una unica occorrenza (CIE 20833). Caso del tutto

${ }^{74}$ A eccezione di CIE 20662, si tratta comunque di iscrizioni speciali, dove anche le altre lettere presentano aste fortemente sottoavanzanti (CIE 20030, 20785) o comunque caratteristiche fuori dalla norma (CIE 20827). 


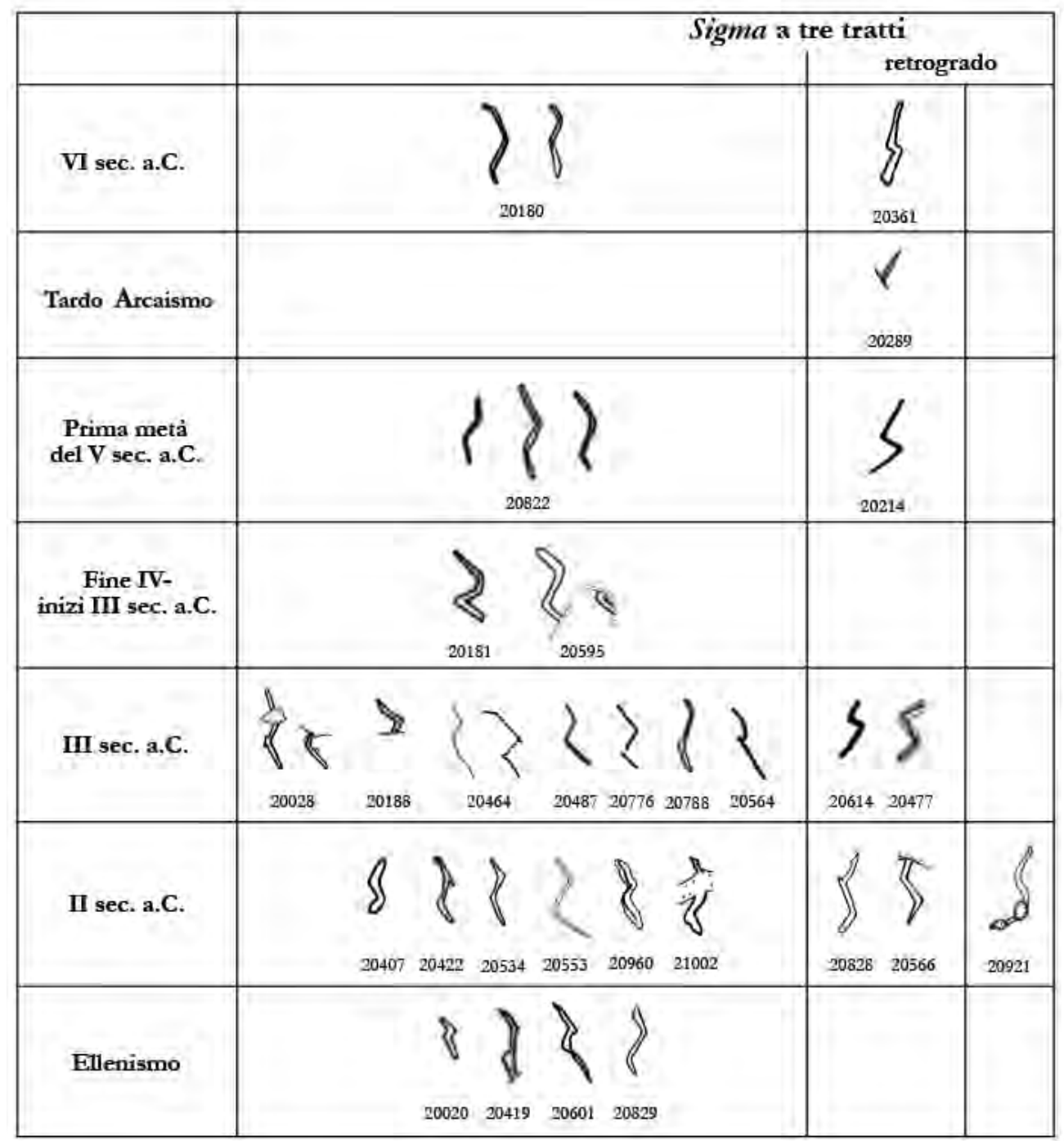

Fig. 16. Sviluppo diacronico dell'aspetto paleografico di sigma.

unico è quello del rho dell'iscrizione CIE 20020 (databile piuttosto genericamente al III-II sec. a.C.), aperto inferiormente e con occhiello circolare, ma soprattutto con tratto sopravanzante ${ }^{75}$.

Sigma (Fig. 16). Il sigma si presenta sempre realizzato con tre tratti. Tale grafema nel periodo compreso tra il VI e la prima metà del V sec. a.C. è generalmente documentato in iscrizioni dove esprime la sibilante post-dentale del genitivo I secondo la norma meridionale, mentre più dubitativo il caso di uselnas in CIE 20822, dove il sigma della sibilante interna potrebbbe esprimere una palatale se valida l'idea che il nome sia la settentrionalizzazione del meridionale Usélna (cfr. III.1 e IV.1). Caso sicuramente a parte è quello dell' iscrizione CIE 20361, dove la lettera è usata per la sibilante finale del prenome Laris, scritto sempre con il sigma indipendentemente dalla norma scrittoria vigente ${ }^{76}$. Rispetto al ductus sinistrorso uniformemente usato in questa fase, almeno in tre iscrizione il sigma è retrogrado ${ }^{77}$,

75 Rimane solo una suggestione il confronto con il grafema usato in ambito culturale umbro per esprimere la sibilante palatale (Agostiniani, Calderini, Massarelli 2011, p. 5).

76 Sul problema, si veda il sintetico quadro in Bellelli, Benelli 2018, p. 83.

77 Nell' iscrizione CIE 20214, dove è sicuramente riferibile all'uscita del genitivo I; nell' iscrizione lacunosa CIE 20289, dove la lettera segue il suffisso - na probabilmente pertinente a un gentilizio e quindi è forse da intendere come genitivo effettivo oppure come genitivo afunzionale; infine nel prenome Laris all'assolutivo di CIE 20361 (nell'iscrizione CIE 20822 il nome declinato al genitivo II presenta invece il sigma secondo il ductus del testo). 
come anche nell' iscrizione pisna di Le Balone di periodo arcaico (CIE 21041), dove ha verosimilmente valore di palatale. Tale caratteristica si riscontra significativamente nell'epigrafia orvietana e chiusina di periodo arcaico ${ }^{78}$, nonché in quella di Vulci e del suo territorio anche successivamente ${ }^{79}$.

A partire dalla fine del IV sec. a.C., il sigma esprime costantemente la sibilante palatale secondo la norma settentrionale. Nel III sec. a.C. gli unici due casi di sigma retrogrado pertengono alla redazione del prenome Laris, anche se queste evidenze non definiscono una regola in quanto l'iscrizione CIE 20564 presenta tale prenome scritto con il sigma sinistrorso e coerente con quello generale del testo. Nel II sec. a.C. non si hanno casi di questo prenome e i tre casi di sigma retrogrado sono pertinenti agli antroponimi snalu (CIE 20566), sinu (CIE 20828) e skamu (CIE 20921), dunque tutti a inizio di parola. Fra questi sigma, si osserva in particolare la resa curvilinea di quello dell' iscrizione CIE 20921, che potrebbe evocare una influenza della corsiva latina, in uso ad Adria già dalla fine del III sec. a.C. (CIE 20950).

Tau (Fig. 17). Già dal Tardo Arcaismo si registrano casi puntuali della forma costituita da un'asta verticale e un tratto obliquo ascendente nel senso della scrittura e non secante, che sarà uniformemente attestata tra III e II sec. a.C. ${ }^{80}$. Nella tradizione epigrafica etrusca tardo-arcaica è una variante piuttosto rara, ma comunque attestata in ambito padano nella prima metà del V sec. a.C. in una occorrenza a Marzabotto $^{81}$.

Rispetto a questo quadro molto uniforme, si distingue il caso, comunque dubbio (potrebbe essere un sade), dell' iscrizione CIE 20370, datata tra la fine del VI e i primi decenni del V sec. a.C. su base paleografica; il tau qui presenta il tratto obliquo ascendente nel senso della scrittura ma tangente l'estremità dell'asta verticale (e più volte ripassato). Se valida la suggestione di un orizzonte ceretano per l'alpha di questa iscrizione, il tau potrebbe essere coerentemente attribuibile a questo ambito scrittorio ${ }^{82}$.

Ypsilon (Fig. 18). La lettera si presenta quasi uniformemente priva di tratto sottoavanzante dal periodo tardo-arcaico fino alla seconda metà del II-prima metà del I sec. a.C.

Nel periodo tardo-arcaico fa eccezione l'iscrizione CIE 20368, dove l'ypsilon si caratterizza per un tratto marcatamente sottoavanzante dell' asta ascendente nel senso della scrittura, mentre in CIE 20370 si osservano brevi tratti sottoavanzanti per entrambe le aste oblique (più prolungato quello ascendente nel senso della scrittura) non qualificabili con certezza come elementi arcaicizzanti. Rare occorrenze di tale caratteristica si ritrovano anche tra III e II sec. a.C., sebbene in questi casi si osservi una generale tendenza all'allungamento dei tratti di tutte le lettere dei testi (cfr. CIE 20519, 20680, 20785). Diverso il caso dell'ypsilon di CIE 20273 di fine IV-III sec. a.C., con marcato tratto

78 Per Orvieto, Maggiani 2003, p. 373, dove si osserva come il sigma retrogrado venga sostituito da quello progressivo a partire dal pieno Arcaismo fino all'età tardo-arcaica, quando quest'ultimo è l'unico attestato; per Chiusi, CRISTOFANI 1977 , p. 269 , fig. 1.

79 Per lo scorcio del VII sec. a.C.: CIE 11006-11007, 11054,11146 (interno di antroponimo), 11158, 11160-11161, 11234, 11274: per la seconda metà del VI sec. a.C.: CIE 10988, 11168, 11224, e PANdolfini, Prosdocimi 1990, pp. 48-51; per il V sec. a.C.: CIE 5303, 11073, 11226; e ancora nel IV sec. a.C.: CIE 10953.

80 Sebbene si osservi una generale scarsa attenzione nella cura redazionale della lettera nel periodo ellenistico, si individua un caso che sembra distinguersi dai restanti, quello dell' iscrizione CIE $20191 \mathrm{di}$ III sec. a.C. Qui il tau presenta il tratto obliquo secante l'asta verticale nella sua parte superiore. Tale variante non è tipica dell'Etruria padana né più in generale del tipo grafico IA corsivizzante; risulta tuttavia azzardato, a fronte di quest'unico caso, proporre il rinvio ad altri tipi grafici come ad esempio il tipo II regolarizzato, che si diffonde nell'Etruria settentrionale nel III sec. a.C. (MAGGIANI 1990, p. 190). Attualmente non si riscontrano elementi che possano permettere di confermare l'uso di questo tipo grafico ad Adria, come invece era stato precedentemente affermato (GAUCCI, Pozzi 2009, p. 60).

81 SASSATELLi 1994, p. 54, n. 66. Si richiama qui anche il tau dell' iscrizione della stele di via Saffi a Bologna, con tratto ascendente nel senso della scrittura ma secante l'asta verticale (MARAs 2014).

82 Cfr. Stopponi 1990, p. 92, e più recentemente Maras 2016. 


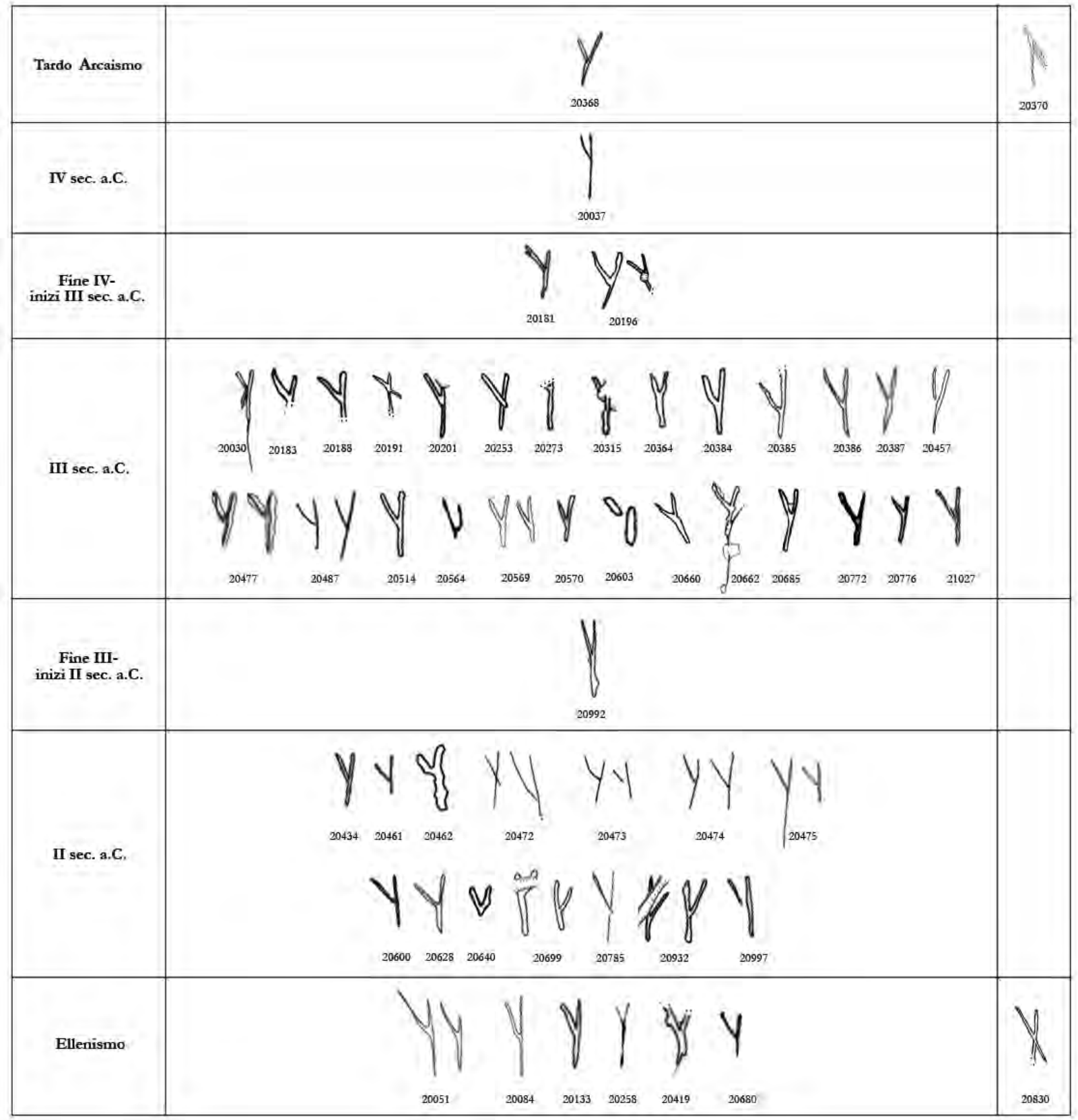

Fig. 17. Sviluppo diacronico dell'aspetto paleografico di tau.

sottoavanzante dell'asta discendente nel senso della scrittura che non pare associabile a una generale tendenza riscontrabile nelle altre lettere del testo come dimostra lo zeta, che ne è privo (cfr. supra).

Degno di nota è l'ypsilon di CIE 20591, rovesciato all'interno di una iscrizione lacunosa e dall' impaginazione piuttosto incerta.

Phi, chi e segno ad otto. L'aspetto dell'unico phi attestato (CIE 20560, seconda metà del II-prima metà del I sec. a.C.) si allinea con quello del chet e del theta di periodo ellenistico (cfr. supra), anch'essi romboidali.

Circoscritte al periodo ellenistico e caratterizzate da una notevole varietà formale sono le attestazioni di $\operatorname{chi}$ (Fig. 19). Colpisce in particolare la coerenza di due casi di III sec. a.C., dove la lettera si presenta rovesciata e sempre in legatura con alpha (cfr. V.1). 


\begin{tabular}{|c|c|c|c|c|}
\hline & 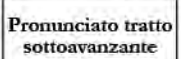 & Privo di pronunciato tratto sottoavanzante & Lacunosi & Altri casi \\
\hline Tardo Arcaismo & $Y_{20365}$ & $\bigvee_{20180}$ & $\underset{20289}{Y}$ & \\
\hline $\begin{array}{l}\text { Prima metà } \\
\text { del V sec. a.C. }\end{array}$ & & $\underset{\substack{20214 \\
20822}}{V_{202}}$ & & \\
\hline V-IV sec. a.C. & & $\underset{20032}{V} \vee \underset{20037}{V} \underset{20099}{Y}$ & & \\
\hline $\begin{array}{l}\text { Fine IV- } \\
\text { inizi III sec. a.C. }\end{array}$ & & $20019 \quad 20016$ & $\begin{array}{ll}11 \\
20181 \quad 20196\end{array}$ & \\
\hline III sec. a.C. & $\int_{2051}^{20273}$ & 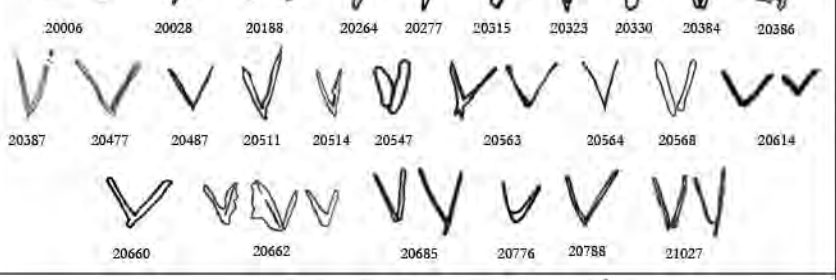 & 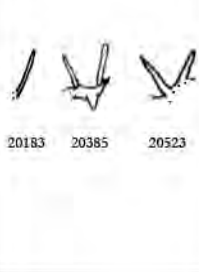 & $\bigwedge_{20591}$ \\
\hline $\begin{array}{c}\text { Fine III- } \\
\text { inizi II sec, a.C. }\end{array}$ & & $\underset{20458}{V} \underset{20489}{\underset{2009}{L}} \underset{20092}{\longrightarrow}$ & & \\
\hline II sec. a.c. & $\Upsilon_{20755}$ & 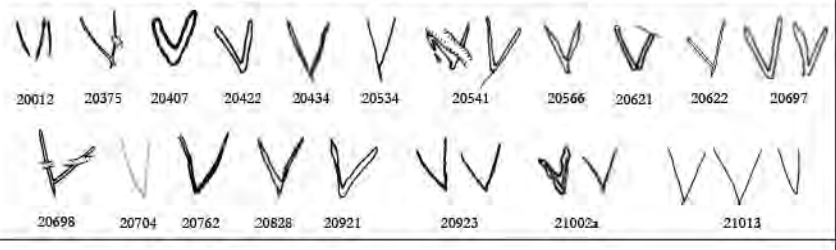 & 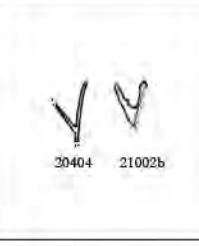 & $\underset{20024}{20026}$ \\
\hline $\begin{array}{c}\text { Seconda metá II- } \\
\text { prina meta. } \\
\text { I sec. a.C. }\end{array}$ & & $\underset{20559}{V}$ & (1) & \\
\hline Ellenismo & Y & 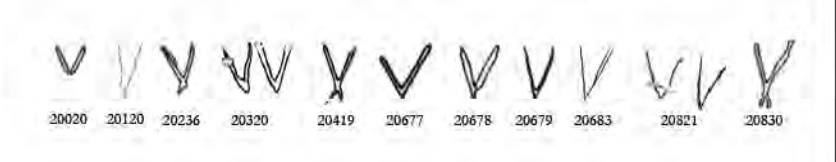 & $\underset{20029}{\mathbb{M}}$ & $\underset{20190}{\mathbb{N}}$ \\
\hline
\end{tabular}

Fig. 18. Sviluppo diacronico dell'aspetto paleografico di ypsilon.

Il segno a otto, che esprime il fonema /f/, è documentato da 3 occorrenze di periodo ellenistico (Fig. 20). La tecnica di redazione sembra per tutti la medesima, cioè tracciando due linee spezzate fra loro speculari: i due rombi cosi formati possono essere fra loro tangenti oppure costituiti da due linee spezzate che non si toccano al centro, come risulta anche negli alfabetari spinetici e mantovani di IV sec. a.C. ${ }^{83}$.

Come ricordato precedentemente, nella fase più antica, compresa tra il VI e la prima metà del V sec. a.C. ${ }^{84}$, sono documentate entrambe le norme, meridionale e settentrionale, oltre che possibili casi di

83 Pandolfini, Prosdocimi 1990, pp. 76-77, III.28, pp. 79-80, III.31-32 (?).

${ }^{84} \mathrm{Il}$ numero complessivo di iscrizioni (24, comprensive dei digrammi) permette un approfondimento sicuramente più puntuale rispetto a GAUCCI 2012a, in particolare pp. 154-156. 


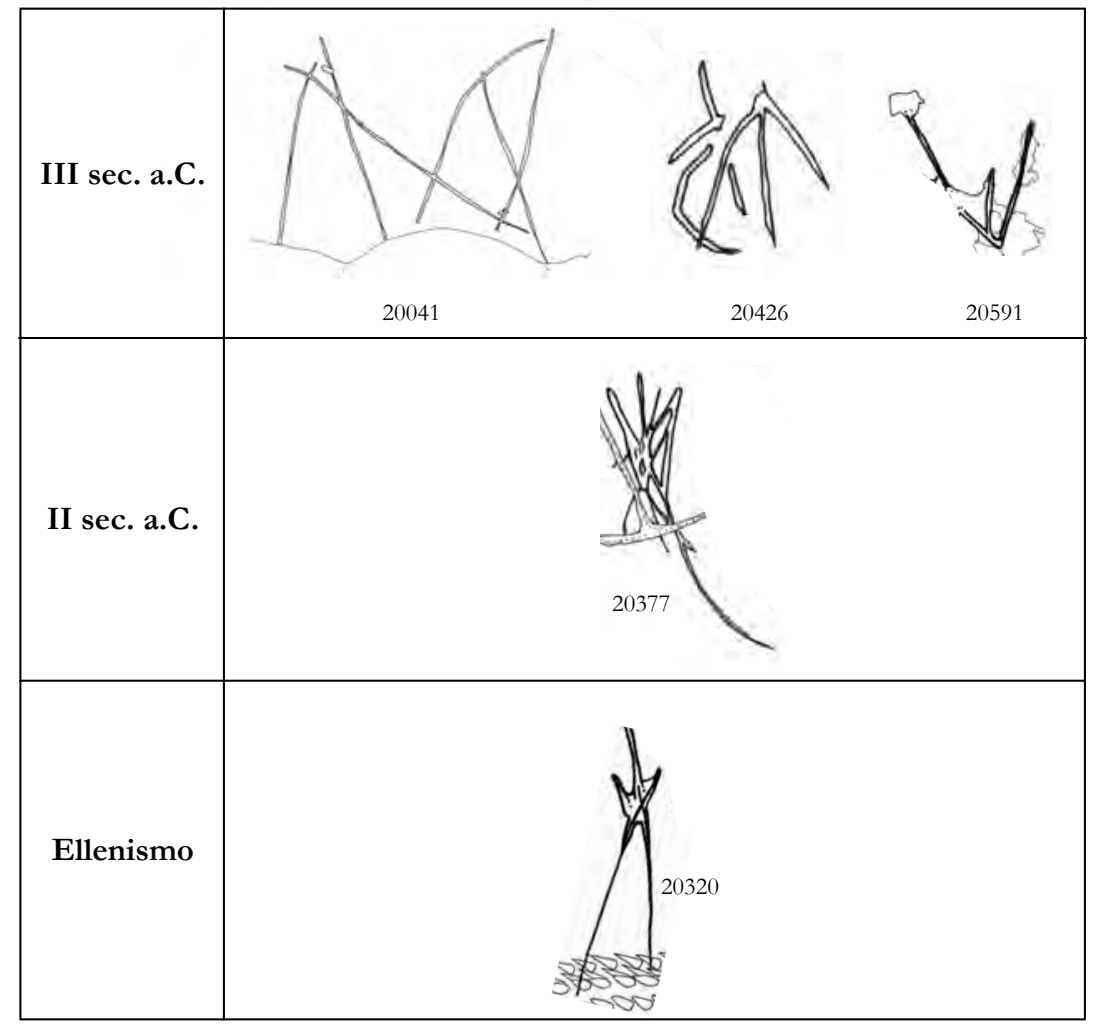

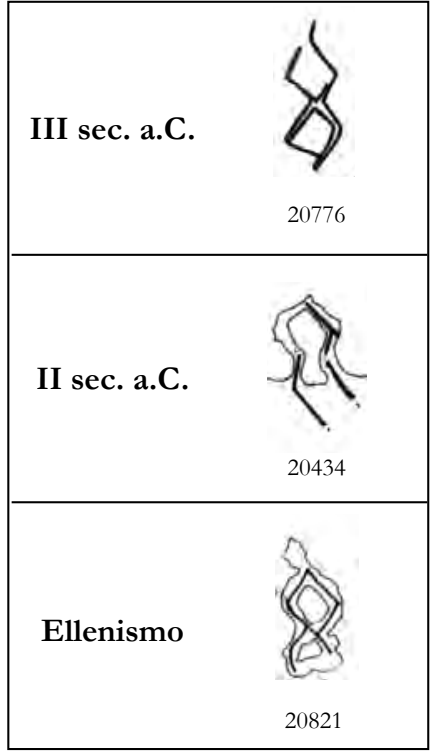

Fig. 20. Aspetto paleografico del segno a otto.

Fig. 19. Aspetto paleografico di chi.

norma mista. Questa mescolanza sembra essere una caratteristica di centri e luoghi di frontiera, sia geografica che culturale, e che ben si accorda anche con la natura di Adria, porto adriatico proiettato verso l'Europa continentale posto nell'estrema periferia settentrionale del mondo etrusco. Sulla base delle principali caratteristiche della paleografia adriese di questo periodo (Fig. 21), il centro tirrenico più affine ad Adria sembra essere Vulci. Qui infatti si assommano in maniera significativa caratteri che si ritrovano ad Adria: l'alpha di forma squadrata o "a losanga", accanto a quello con asta laterale spezzata e traversa orizzontale e a quello con asta arcuata; l'uso del $p i$ con tratto obliquo curvato nella sua estremità e del rho con occhiello triangolare senza codolo; la frequenza del sigma retrogrado; la presenza del sade a farfalla (sebbene documentato nel V sec. a.C.). Inoltre, anche questa città dell'Etruria meridionale mostra caratteristiche epigrafiche di un centro di frontiera (come già sostenuto nel 1976 da G. Colonna ${ }^{85}$ ). Infatti, accanto alla più diffusa norma meridionale, si documentano iscrizioni in norma settentrionale e iscrizioni in norma mista ${ }^{86}$. Pare peraltro importante sottolineare che proprio l'affinità

85 Colonna 1976, pp. 15-16.

86 Anzi tutto va ricordato l'alfabetario dalla necropoli dell'Osteria in norma settentrionale graffito su fusaiola e datato agli inizi del VI sec. a.C. (CIE 11061): secondo G. Colonna questo doveva appartenere a una donna di origine settentrionale ed essere così indizio di mobilità individuale (per il documento, si rimanda a PANDolfini, Prosdocimi 1990, p. 38; per l'interpretazione, cfr. Colonna 1976, p. 16, nota 30; M. Bonamici, in REE LV, pp. 317-319, n. 87; BENELLI 2000, p. 207, nota 10). Più significative le due sequenze alfabetiche in norma settentrionale redatte sulla parete di una tomba a Magliano in loc. Le Mollaie e di cui la più antica è stata datata da M. Martelli nell'inoltrato VI sec. a.C. (M. Martelli, in REE LiII, pp. 217-218, n. 32; Pandolfini, Prosdocimi 1990, pp. 48-51; ET², AV 9.2-3): se per la studiosa queste iscrizioni sono indicatori della loro localizzazione nella zona del distretto scrittorio settentrionale (risultando così il più tardo e famoso Piombo in norma meridionale una importazione), per M. Pandolfini non ci sono elementi per riconoscere come primaria una delle due norme soprattutto considerando che il sito è su un'area di confine (Pandolfini, Prosdocimi 1990, p. 49). A Vulci è riferita l'iscrizione min larekés datata alla seconda metà del VI sec. a.C (CIE 11164), redatta su una coppa di "impasto buccheroide" e ritenuta da G. Colonna la più "settentrionale" (per aspetto) delle iscrizioni vulcenti (G. ColonnA, in REE XXXIX, pp. 336-337, n. 7; ET², Vc 2.12: mi\{n\} larekeśi). Nel 


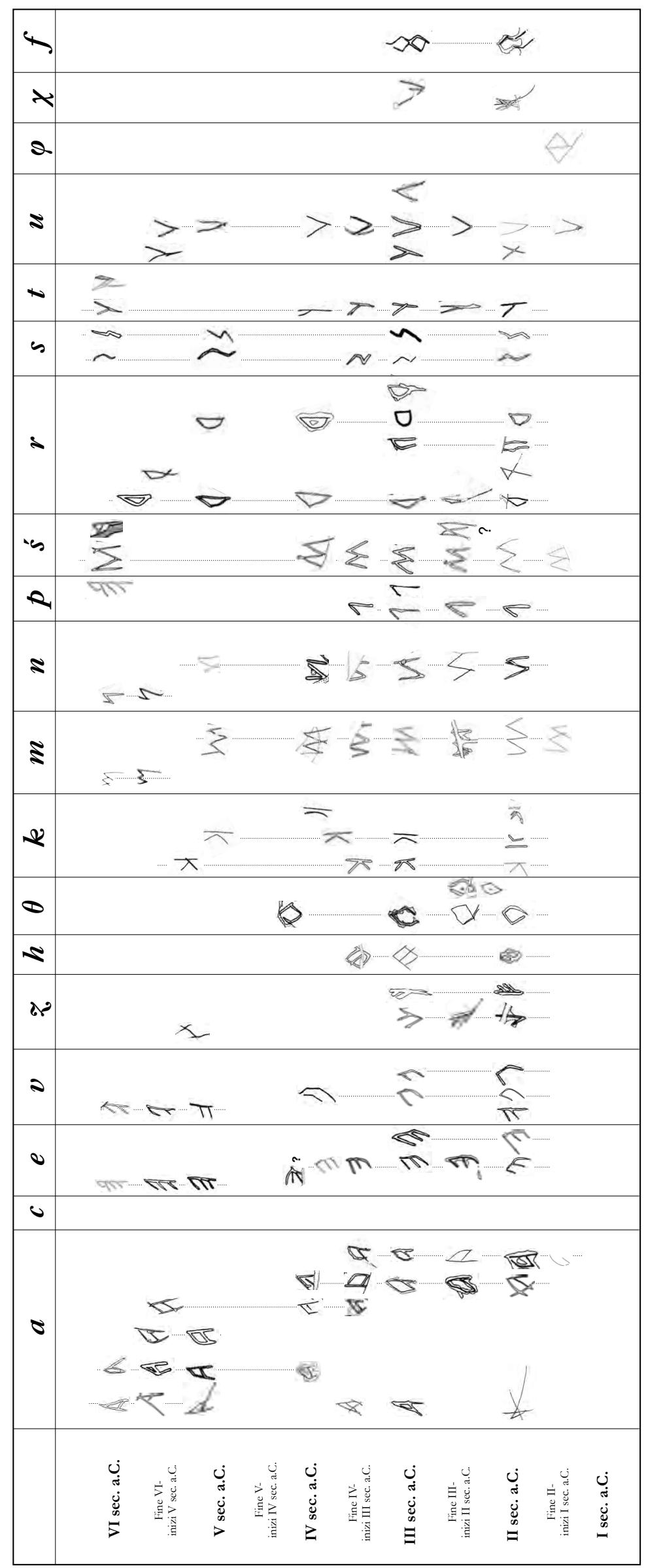


tra queste iscrizioni e quelle di Roselle ${ }^{87}$ (già richiamate come confronto di quelle adriesi per i caratteri paleografici) suggerisce forme di affinità scrittoria e modelli di uso che valicano la semplice distinzione geografica fra le norme. A Roselle le norme ortografiche non dovevano essere troppo rigide $^{88}$ : nel periodo tardo-arcaico, l'emergere della pratica scrittoria meridionale all' interno di un quadro di generale coerenza della norma settentrionale dalle più antiche del pieno VII sec. a.C. fino a quelle ellenistiche, è stato interpretato come dipendente dall'accoglienza in città di individui provenienti dai distretti più meridionali ${ }^{89}$. Una nuova iscrizione di recentissimo rinvenimento ha consentito a E. Benelli di tornare sul problema, evidenziando come il cambio di norma a Roselle si mostri in realtà come sistematico (settentrionale fino al pieno VI sec. a.C.; meridionale nel Tardo Arcaismo; ancora settentrionale nella fase recente), presupponendo scelte consapevoli e coerenti da parte della comunità locale e non frutto di dinamiche di mobilità contingenti ${ }^{90}$.

Proprio il richiamo alla tradizione epigrafica vulcente può stimolare qualche ulteriore osservazione sull'uso dei grafemi per le velari in ambito adriese (cfr. III.2). Infatti, nel Vulcente si riscontra la persistenza del kappa associato ad alpha fino al V sec. a.C. Da questo punto di vista pare significativa l'iscrizione [---] kapes sli del primo quarto del V sec. a.C. (CIE 10992): oltre alla presenza del grafema citato per la velare, anche l'aspetto di alpha e pi trova riscontro ad Adria. Ma a Vulci, situazioni di fluidità scrittoria si trovano già nel VI sec. a.C., quando l'uso settentrionale del kappa è associato all'uscita del genitivo I in sigma, o al contrario l'uso del gamma al sade quale grafema di marca del caso. Sebbene Adria non documenti casi così espliciti di questa fluidità nell'uso dei grafemi di velari e sibilanti, è possibile che anche qui la scelta grafemica non sia strettamente legata all'applicazione rigida di una norma scrittoria come nel territorio di Vulci.

È stato già rimarcato come l'emergenza di sporadiche manifestazioni dubitativamente da attribuire a una pratica scrittoria veiente dal pieno VI sec. a.C. possano orientare verso la conferma di un percorso adriatico verso il Delta padano battuto già durante l'Arcaismo da individui e gruppi di provenienza etrusco-meridionale ${ }^{91}$, e che avrebbe veicolato tale cultura epigrafica verso il comparto veneto grazie al ruolo di tramite del comprensorio adriese. Queste evidenze sembrano inserirsi in un quadro culturalmente più complesso, che con la fine del VI sec. a.C. potrebbe manifestare esperienze epigrafiche molto vicine a quelle di Vulci e del suo territorio. Il sistema scrittorio di quest'ultimo centro si mostra capace di adattarsi e assorbire senza urti diverse istanze culturali, in quanto da sempre in un'area di confine in dialogo con la più settentrionale Vetulonia ${ }^{92}$. Nel Tardo Arcaismo ad Adria la tradizione epigrafica vulcente forse rappresentò la risposta più efficace ai molteplici stimoli culturali di questo centro di frontiera, dove peraltro la componente proveniente dalla città tirrenica non doveva essere esigua. Infatti, a parte le importazioni di bronzi ${ }^{93}$, indizi quali le influenze artigianali sulla produzione locale (?) figurata ${ }^{94}$ e la concentrazione di kyathoi attici ${ }^{95}$, trovano spiegazione solo nell' ipotesi di una attiva presenza di individui originari da questo importante centro dell'Etruria meridionale. Ovviamente

territorio, comunque a sud di quella linea che corre lungo l'Albegna suggerita dalla Martelli come possibile confine dei distretti scrittori, a Sovana abbiamo l'iscrizione mi lakenas di VI sec. a.C. (CIE 11360) e a Orbetello l'iscrizione mi lareceś súpelniieśs $\theta$ afna della seconda metà del secolo (CIE 11417): entrambe sono testimonianza di una norma scrittoria mista.

87 Cfr. M. MARTELli, in REE LIII, p. 218.

${ }^{88}$ H. RIX, in REE XLIX, p. 251 con riferimento a $E T^{2}, \mathrm{Ru} 2.2-3$.

89 S. ZAMBELli, in CIE III, 4, p. 5.

90 E. BENELLI, in REE LXXXI, pp. 329-330, n. 16.

${ }^{91}$ Cfr. REE LXXIX, pp. 292-293, n. 57, con riferimenti.

92 Non è un caso che la nuova analisi intorno alla nascita della scrittura nell'Etruria settentrionale proposta da A. Maggiani veda in Vulci il luogo privilegiato di impulso di questa importante elaborazione avvenuta a Vetulonia (MAGGIANI 2017).

93 BonOmi 2004, pp. 65-69.

${ }_{94}$ Colonna 2003, pp. 164-165; Govi 2012, in particolare pp. 143-148, dove si esprimono dubbi sulla natura locale della produzione figurata etrusca.

95 Tonglet 2018, in particolare p. 259. 
queste considerazioni non possono ignorare il ruolo di Orvieto, in letteratura dai lavori di Colonna in poi visto come centro primario di origine di alcuni di quegli individui che ad Adria scrivevano. Tuttavia di Orvieto si conosce bene la cultura epigrafia arcaica e prettamente monumentale ${ }^{96}$, mentre quasi per nulla quella su ceramica e di periodo tardo-arcaico che più direttamente sarebbe confrontabile con Adria ${ }^{97}$. È possibile comunque osservare che i testi dei lapicidi orvietani sono solo limitatamente confrontabile con le iscrizioni adriesi.

L'insieme di queste osservazioni sembra indebolire il processo di uguaglianza tra luogo di origine di un individuo e pratica scrittoria. Se l'iscrizione mi venelus leceniies (CIE 20180) può in effetti essere pienamente identificata come concepita in norma meridionale e forse redatta da un individuo portatore di una cultura epigrafica che si potrebbe continuare a ritenere direttamente orvietana, ben diverso è il caso dell'iscrizione CIE 20822. Ammessa infatti l'origine orvietana anche per Laris Uselna(s), il fatto che il gentilizio sia stato eventualmente "settentrionalizzato" mostrerebbe che in un contesto culturale e identitario è nuovo, ben distinto da quello originario di questo individuo o comunque del suo gruppo familiare.

Nel complesso e indipendentemente dalle specificità del modello proposto, è lecito ritenere che per Adria vada superato il paradigma "sito = singola norma scrittoria vigente". Vale la pena qui richiamare il concetto di sistema scrittorio così come espresso da D.F. Maras, cioè un insieme di usi grafici, che a volte assumono la forza di vere e proprie norme che regolano l'applicazione della scrittura da parte di un gruppo umano per la rappresentazione dei suoni della propria lingua ${ }^{98}$. Ci si può dunque domandare se ad Adria sia presente un sistema scrittorio coerente e codificato, che segue nell'uso dell'alfabeto precise regole (e relativi insegnamenti) ${ }^{99}$. Ammesso sia esistito, il modello attraverso il quale comprendere la cultura epigrafica dell'Adria tardo-arcaica va cercato in un sistema non rigidamente monolitico, la cui origine sembra essere individuabile in quello vulcente, che risponde bene a quella fluidità culturale caratteristica di un luogo dove confluivano individui da tutti i comparti territoriali del mondo etrusco, da quello tiberino fino all'area padana. Il sistema scrittorio vulcente, se valido quanto osservato, arrivò ad Adria forse attraverso la via tiberina e quindi adriatica che passava per Orvieto, veicolato da quei gruppi che evidentemente nella città padana si stabilirono. Sulla base di queste premesse, una iscrizione come mi venelus kar (CIE 20214) rientra dunque pienamente in questo sistema scrittorio di matrice meridionale, ma che fin dalle origini presenta caratteristiche che, stando alla recente lettura di A. Maggiani, sono alla base dell'elaborazione della norma scrittoria settentrionale. Così anche i tre kai (CIE 20282, 20290, 20295) vi potrebbero rientrare. Ovviamente, il modello avanzato prevede una situazione dove tale sistema scrittorio, che per natura tollerava pratiche caratterizzate da specificità distinte ma necessariamente alternative (es. i grafemi delle sibilanti), rappresenta forse la soluzione primaria recepita da uno o più gruppi sociali dominanti (quale il ruolo dell'ambito sacro che pure doveva essere ben presente?) all'interno di una comunità socialmente e culturalmente complessa.

Non è purtroppo possibile stabilire con certezza cosa succeda nel periodo compreso tra il pieno $\mathrm{V}$ e la prima metà del IV sec. a.C. In questo lasso di tempo si attua sicuramente il consolidamento della norma scrittoria settentrionale quale unica in uso nella comunità, sebbene la scarsità di dati a disposizione non permetta di formulare ipotesi solide riguardo i processi di affermazione. Inoltre, avviene l'acquisizione del tipo grafico corsivizzante, usato in maniera matura ad Adria nel IV sec. a.C. e di cui troviamo documentazione nel territorio a San Basilio e a Le Balone nel V sec. a.C.

La recente riflessione sulla documentazione epigrafica delle stele funerarie di Bologna ${ }^{100}$ ha permesso di collocare subito dopo la metà del V sec. a.C. la fase di acquisizione di questa importante riforma

\footnotetext{
96 Si rinvia ai lavori di A. MAggiani (2003 e 2013a).

97 Ibid., pp. 379-380; si veda anche MAgGiani 2013a, p. 173.

98 Maras 2012, p. 332.

99 A.L. Prosdocimi, in Pandolfini, Prosdocimi 1990, p. 159.

100 Gaucci, Govi, Sassatelli c.s.
} 
grafica nella metropoli, rialzando dunque di qualche decennio la cronologia proposta da A. Maggiani alla fine del secolo medesimo per la maggior parte dell'Etruria padana ${ }^{101}$. La datazione è peraltro in sostanziale accordo con quanto già osservava Maggiani per il Mantovano ${ }^{102}$, e più recentemente documentato a Marzabotto ${ }^{103}$; diversamente, per Spina non vi sono novità rispetto a quanto già osservato ${ }^{104}$, sebbene manchi una riflessione d'insieme sulla paleografia e il molto materiale inedito potrebbe portare nuovi dati.

In particolare, l'iscrizione della stele Ducati 42 dal sepolcreto Arnoaldi di Bologna ${ }^{105}$ sembra testimoniare il momento di ricezione ed elaborazione da parte di una scuola scrittoria locale della riforma grafica corsivizzante messa a punto a Chiusi nella parte iniziale del secolo ${ }^{106} \mathrm{e}$ arrivata a Bologna con ogni probabilità attraverso il Fiesolano e la Valle del Reno. Infatti, l'acquisizione della riforma nel territorio fiesolano e volterrano entro la prima metà del V sec. a.C. sarebbe documentata dall' iscrizione vipia veteśsu una stele di Artimino, per la quale Maggiani propone una cronologia entro la prima metà del V sec. a.C. o meglio il primo quarto ${ }^{107}$, e da due frammenti iscritti dal santuario in località Ortaglia presso Peccioli, editi da S. Bruni ${ }^{108}$. Il settore del medio e basso Valdarno dunque recepì precocemente la riforma chiusina e forse non è da escludere che da questo comparto venne veicolata in Etruria padana, dove fu fatta propria dalla scuola scrittoria bolognese dopo la metà del secolo e forse da qui diffusa verso gli altri centri fra cui Adria ${ }^{109}$. Non va escluso che la ricezione di tale tipo grafico in un orizzonte ancora formativo possa aver portato al mantenimento ad Adria di proprie specificità scrittorie, cioè alla formazione di una tradizione epigrafica epicoria. Ciò si coglierebbe nell'aspetto squadrato dell'alpha che già nel IV sec. a.C. assume la classica forma "a bandiera" tipica del periodo ellenistico; in quello prevalentemente angolato del rho; nelle forme spigolose di epsilon e digamma; nell'uso non diffuso ma piuttosto coerente del sigma retrogrado.

Durante il III e II sec. a.C. Adria mostra una tenace tradizione scrittoria che si potrebbe definire senza esitazione e in questo caso giustamente "monolitica" (Fig. 21). La ricca documentazione raccolta, rispetto a quanto si poteva ritenere anche solo un ventennio fa, testimonia infatti come la cultura epigrafica di questa città fosse refrattaria alle riforme grafiche provenienti dall'Etruria propria, ma anche a quella del chet a cerchiello che parrebbe essere stata maturata nella vicina Spina ${ }^{110}$.

101 Maggiani 1998, p. 228.

102 Maggiani 1998, p. 228, nota 17. Sul Mantova, si veda anche Menotti, Maras 2012.

103 Maggiani 1998, p. 229; per Marzabotto, si veda più recentemente Govi 2014, p. 131, nota 127 e GaUCCI c.s. Si sospende il giudizio sulla datazione alla prima metà del V sec. a.C. di una iscrizione lacunosa di Mantova (MENOTTI, Maras 2012, p. 881, n. 4, fig. 8), redatta secondo il tipo grafico corsivizzante e sulla quale si sono già espresse anche perplessità in merito alla lettura proposta.

104 Per Spina, si veda Maggiani 1998, pp. 229-230.

105 Gaucci, Govi, Sassatelli c.s. Per l'iscrizione della stele, si rimanda a Sassatelli 1988, p. 237, n. 6, nota 99 con bibliografia di riferimento e $E T^{2}$, Fe 1.7+7.2.

106 A. Maggiani, che per primo ha definito questo tipo grafico, ha individuato in Chiusi il centro di elaborazione, suggerendo peraltro che il modello ispiratore fosse greco, nella fattispecie cumano. Se durante la fase ancora formativa di $\mathrm{V}$ sec. a.C. questo tipo si riscontra in un gruppo di iscrizioni di Chiusi (o comunque qui prodotte) legate soprattutto a epigrafi di impegno (monumentale e sacrale; MAGgiani 1998, p. 229), dove ancora le innovazioni si associano a persistenze di retaggio arcaicizzante, appaiono anche documenti sporadici a Vulci (CIE 11097; per l'iscrizione, cfr. M. CRISTOFANI, in REE LV, pp. 344-345, n. 125; per interpretazione e lettura, si veda da ultimo Colonna 2004a, p. 85, nota 107), Populonia e nel territorio lungo la media e bassa valle dell'Arno.

107 M.C. Bettini (in Perazzi, Poggesi, Sarti 2016, p. 91, n. 4), non esclude una posteriorità del testo rispetto alla realizzazione del monumento.

108 REE LXXIII, pp. 375-377, nn. 98-99.

109 Nel complesso, pare da rivedere la linea di sviluppo tracciata ormai circa venti anni fa da E. Benelli (BENELLI 2000, pp. 215-216), che vedeva l'arrivo in Etruria padana del tipo grafico con lo scorcio del V sec. a.C. sulla base di quanto precedentemente osservato da A. Maggiani (Maggiani 1998, in particolare p. 228) e una maggior affinità con le elaborazioni messe a punto nel territorio chiusino (tra la Val d'Orcia e Bettolle) piuttosto che nel centro urbano.

110 Ibid., pp. 232-233. 
Proprio il theta, ad Adria generalmente privo di punto, è esemplificativo di esperienze episodiche, come la variante con punto centrale tipica di Spina in due occorrenze comprese tra la fine del III e la prima metà del II sec. a.C. e in associazione con il medesimo digramma (o meglio, il medesimo lessema $\theta i$ : cfr. V.4). Questa particolarità potrebbe essere imputata, in assenza di dati più cogenti, all'emergere di una tradizione scrittoria spinetica in un periodo nel quale il più meridionale centro deltizio doveva essere ormai collassato ${ }^{111}$. Altro caso riguarda epsilon, digamma, zeta, rho, ypsilon, che possono presentare tratti sottoavanzanti ${ }^{112}$, forse frutto di una attenzione redazionale piuttosto cursoria, come traspare anche nella frequenza di tratti disarticolati e nel differente aspetto di quelle lettere ripetute più volte all'interno degli stessi testi. Non va tuttavia escluso che in alcuni casi questi tratti sottoavanzanti possano essere indizio di multi-competenze scrittorie latenti, come nel caso dell'iscrizione CIE 20827, dove il rho con codolo si associa a un sade a farfalla e un alpha a pettine che sembrano tradire la conoscenza dell'alfabeto leponzio se non l'uso diretto di tale alfabeto per esprimere un nome etrusco quale Larza (ma nella variante Larśa, forse con una confusione tra fonemi e grafemi). Tra queste puntuali emergenze di periodo ellenistico c'è anche l'unico caso di gamma: mentre questo sostituisce il kappa pressocché ovunque in ambito etrusco attorno al 300 a.C., come anche nella vicina Spina, ad Adria si continua a utilizzare quest'ultimo in maniera pressoché costante.

\section{III.3. Puntuazione}

Solo tre iscrizioni, tutte di periodo ellenistico, mostrano l'uso della puntuazione, due di II sec. a.C. da contesti funerari fra loro prossimi (CIE 20694, 20697), e una da abitato databile più genericamente al III-II sec. a.C. (CIE 20020).

Le due iscrizioni funerarie, he.me (CIE 20694) e kraiku.kaniu. (CIE 20697), appartengono rispettivamente alle tombe di adulto 38/1966 e 43/1966 di Ca' Garzoni, entrambe della seconda metà del II sec. a.C. e fra loro prossime all' interno di un'area caratterizzata da una prevalenza di tombe infantili (cfr. VI.2.1 $)^{113}$. Nel primo caso la puntuazione divide due digrammi. Risulta complesso, date le difficoltà di interpretazione, comprendere qui la funzione della punteggiatura. Supposto che la punteggiatura sia funzionale alla distinzione del primo digramma dal secondo (sia esso abbreviazione o no), rimane difficile comprenderne significato e associazione. Il secondo caso rappresenta l'unica iscrizione ad andamento circolare sul fondo esterno di una ciotola (cfr. II.2). Qui la puntuazione è chiaramente interverbale, forse per evitare che la circolarità dell'impaginazione inducesse errori di lettura.

La terza iscrizione menzionata, $a$ : riusiu se valida la lettura proposta (CIE 20020), proviene dall'area abitativa sotto l'attuale sede del Museo Archeologico, e si caratterizza per un singolo alpha distinto mediante due punti dal seguente elemento onomastico risiu. Si tratta di un fenomeno di abbreviazione puntuata, che nel comparto territoriale adriese trova come unico confronto certo la tarda iscrizione umbra da Larda di Gavello (CIE 21031) ${ }^{114}$.

I testi illustrati documentano nel complesso come la puntuazione sia una pratica molto rara e priva di una coerenza d'uso, forse usata con lo scopo primario di disambiguare i testi.

\section{III.4. Abbreviazioni e lettere in legatura}

All'Arcaismo è da ascrivere un numero molto limitato di digrammi, tre su ceramica attica (CIE 20143, 20287, 20305) e uno su ceramica depurata (CIE 20356). La possibilità che ar di CIE 20143 sia l'abbreviazione di un prenome (es. $\operatorname{Arn} \theta$ ) appare più verosimile che l'alternativa interpretazione votiva,

111 GaUcci 2016, p. 203.

112 Sulla base di alcune evidenze, si era precedentemente supposto la possibile influenza della moda manierata che tuttavia non si sarebbe mai affermata diffusamente (GAUCCI, Pozzi 2009, p. 60).

113 Mangani 1982, in particolare p. 100.

114 Per una analisi del documento, cfr. REI LXXIX, pp. 348-350. 
cioè ar come base di un verbum faciendi ${ }^{115}$ (per il contesto, cfr. VI.1). Il digramma $k r$ in due kylikes attiche a f.n. (CIE 20287, 20305) potrebbero invece essere interpretabili come abbreviazioni consonantiche del termine kara già riconosciuto nell' iscrizione CIE 20214. In tal maniera, $k r$ alluderebbe al nome del vaso (cfr. IV.1). Tale osservazione parrebbe peraltro rafforzata proprio dalla redazione di uno dei due casi nella parete esterna, quindi in un punto ben visibile del vaso medesimo. Rimane più difficile proporre una interpretazione del digramma an (CIE 20356), verosimilmente abbreviazione, ma senza significativi confronti che possano orientare la discussione ${ }^{116}$.

Colpisce invece il numero di legature (5), per le quali l'operazione di individuazione delle singole lettere che compongono i monogrammi non è sempre facile ${ }^{117}$. Si osserva infatti che solo in tre casi, cioè CIE 20089, 20194 e 20834, le lettere sono di agevole lettura. Tuttavia, mentre per CIE 20089 e 20834 si possono avanzare delle considerazioni sull'interpretazione, è invece molto più complesso circoscrivere un numero ragionevole di ipotesi per CIE 20194 ( $\widehat{p v}$ o $\widehat{v p})$.

Il digramma $\widehat{a v}$ di $C I E 20089$ è verosimilmente da intendere come abbreviazione di antroponimo quale Avele/Avile; come già anticipato (cfr. II.1.4), la scelta della coppa in bucchero quale supporto risulta un evento speciale nel panorama adriese, dove sono privilegiate le ceramiche depurate locali e quelle di importazione dall'Attica.

Più complesso il caso di CIE 20834, $\widehat{p e}$. Questo digramma trova confronto in una coppa di bucchero della seconda metà del VI sec. a.C. di una tomba di Fratte ${ }^{118}$, sempre nel VI sec. a.C. a Veio (CIE 6510), nel V sec. a.C. a Cerveteri, Pyrgi, Tarquinia, Sovana, e a Chiusi attorno al 450 a.C. nel fondo esterno di uno skyphos attico a f.r. ${ }^{119}$. Nel periodo ellenistico, pe è interpretato come abbreviazione di nome familiare nelle iscrizioni onomastiche delle urne della tomba dei letiu, secondo una pratica diffusa in ambito perugino ${ }^{120}$, e sempre come probabile abbreviazione di nome familiare, ma in ciotole di corredo, a Volterra ${ }^{121}$ e a Monterenzio ${ }^{122}$; ancora in ciotole appartenenti a corredi funebri, si trova pe nel Perugino a Marsciano (loc. Villanova), dove è peraltro associato ad altre ciotole con graffita la singola lettera $p i^{123}$, e a Spina ${ }^{124}$. Caso del tutto particolare è il medesimo digramma inciso a crudo su un peso da telaio di età ellenistica da Campo della Fiera ${ }^{125}$. S. Stopponi ha ipotizzato quest'ultima occorrenza come abbreviazione del gentilizio Pe $\theta$ na oppure del teonimo Pe $\theta n^{126}$; secondo G. Colon$\mathrm{na}^{127}$ è da preferire la seconda alternativa, essendo rinvenuto in un' area sacra, nella fattispecie una zona marginale del santuario dove sarebbero state dunque venerate $V e i$ e $\operatorname{Pe} \theta(a) n$, quest'ultima quale dea infera, come dimostra l'epiteto Caluśnal (gen.) a questa attribuito nella dedica dell'avanzato III sec. a.C. rinvenuta probabilmente a Corciano ${ }^{128}$. Secondo lo studioso, Vei e Pe $\theta$ an sono da identificare con

115 Cfr. GaucCi 2012a, p. 147, con riferimenti.

116 Si ricordano unicamente il simile digramma CIE 10627 di Orvieto (pe an secondo la lettura di GAMURRINI 1880, n. 640, e già CII 2053, ma in CIE è data come incerta).

117 Fra le legature si ricordano anche i due alpha contigui e fra loro tangenti di CIE 20372 da abitato.

118 CIE 8822. In COLONNA 1990b, pp. 301-309, non si spiega la presenza del digramma, forse implicitamente ritenuto una ripetizione dell'antroponimo femminile iniziale peiOrasia? (ibid., pp. 303-304, n. 2).

119 Si veda $T h L E^{2}$, s.v. pe; in particolare per Chiusi, CVA Chiusi 2, tav. 34, 1-3.

120 A. Maggiani, in REE LI, p. 277, nn. 22-23, p. 278, n. 27, e p. 279.

121 È attestato nella tomba 8 della necropoli delle Ripaie a Volterra (L. RosselLI, in REE LXXV, pp. 201-202, n. 14), graffito in una ciotola a v.n. serie Morel 2538 e in associazione con un' altra ciotola simile iscritta con il gentilizio maschile petini (ibid., pp. 200-201, n. 13).

122 Anche a Monte Bibele si documentano nella stessa tomba le iscrizioni petnei e pe (D. VITALI, in REE LI, p. 261, n. 2).

123 Gens antiquissima 1990, p. 287, n. 4.60, da una tomba da Marsciano, località Villanova, datata agli ultimi decenni del IV sec. a.C. o posteriormente (coppetta in ceramica grigia, assieme ad altre coppette con ripetuto il singolo $p i$ ).

124 REE XLVII, p. 297, n. 2; REE L, p. 261, n. 2; PAOLI, PARrini 1988, p. 67, n. 134.

125 G. COLONNA, in REE LXXIV, p. 380, n. 135, con riferimenti precedenti.

126 Stopponi 2007, pp. 501-502. Su Pe日n si veda da ultimo Rigobianco 2017, pp. 194-195, con riferimenti.

127 REE LXXIV, pp. 382-383.

${ }^{128} E T^{2}$, Pe 3.2; Maras 2009b, pp. 328-329, Pe do.4. 
la coppia greca di Demetra e Kore, osservando che evidentemente nel III sec. a.C. Pe $\theta n$ avrebbe preso il posto di $\operatorname{Cav}(a) \theta \mathrm{a}$, venerata nella stessa Orvieto come $\operatorname{Cavu} \theta a$ nel santuario del Belvedere ${ }^{129}$. Sempre a Campo della Fiera, è documentata anche una coppa in bucchero della seconda metà del VI sec. a.C. rinvenuta sopra la preparazione pavimentale del tempio $\mathrm{C}^{130}$, con iscrizione atial redatta sul bordo dell'orlo e i digrammi pe e $h u^{131}$ nella vasca interna. Nel caso di CIE 20834, non potendo escludere che $\widehat{p e}$ sia l'abbreviazione di un nome familiare, è suggestivo il richiamo a una possibile abbreviazione teonimica quale $\operatorname{Pe} \theta(a) n$, sebbene questo nome sia generalmente riconosciuto come in uso nel periodo ellenistico. Purtroppo risulta del tutto sconosciuto il contesto di rinvenimento, che potrebbe supportare o al contrario inficiare questa suggestione. Non è peraltro fuoriluogo ritenere che la legatura delle due lettere iniziali possa rappresentare una forma di occultamento, come nel caso (sicuramente più strutturato dato il numero di attestazioni) discusso da G. Bagnasco Gianni in merito alla c.d. "Cetamura ligature", anche questa di periodo ellenistico, per la quale è stato proposto lo scioglimento alp ${ }^{132}$.

I restanti tre monogrammi, CIE 20137, 20215, 20270, presentano un intreccio di lettere più complesso da districare:

- CIE 20137: nel monogramma, documentato solo dall'apografo e dalla descrizione del Bocchi, che lo descrive come situato nella parete esterna di una coppa forse in bucchero (o ceramica grigia) e redatto a crudo, è riconoscibile un alpha con asta laterale spezzata a sinistra dell'asta verticale centrale e un epsilon a destra. I due triangoli speculari ai lati dell'asta centrale sono forse da intendere come phi (meno probabile vedervi almeno a destra un $r h o$, in quanto non si spiegherebbe il tratto obliquo che attraversa l'occhiello dell'alpha), tale da leggere da sinistra verso destra il lessema a $e$ e. Se valido il confronto con l'alpha tracciato nell'interno del vaso, caratterizzato da un'asta laterale spezzata dal ductus sinistrorso, forse potremmo avere quindi una disposizione speculare di alpha ed epsilon rispetto al phi centrale. A e è un nome divino finora unicamente attestato nella $T C^{133}$. Va comunque ribadito che tale lettura si basa su una operazione di individuazione delle lettere nel monogramma caratterizzata da un forte grado di incertezza, a cui si aggiunge l'irreperibilità del documento e quindi non si hanno garanzie che l'apografo tracciato da F.A. Bocchi sia fedele.

- CIE 20215: oltre al monogramma sul fondo esterno, in questo eccezionale documento abbiamo anche una raffigurazione graffita sempre dopo la cottura nella parete esterna, complessa per organizzazione nella superficie usata e apprezzabile tenendo il vaso con la vasca verso il basso (cfr. VI.1.2). Proprio questa posizione permette di vedere anche il monogramma nel fondo esterno, che appunto è interpretabile come la legatura di più di due lettere ${ }^{134}$.

129 G. Colonna, in REE LVI, p. 318 (in riferimento al n. 30).

130 S. STOPPONI, in REE LXXV, pp. 241-242, n. 58.

131 Quest'ultimo interpretato da S. Stopponi come abbreviazione di husiur (il digramma è peraltro ripetuto anche nel fondo esterno).

132 Bagnasco GiAnni 2014. Una diversa forma di occultamento è ipotizzata da D.F. Maras per l'iscrizione sulla base in nenfro dal tempio di San Leonardo ad Orvieto: qui la posizione del nome della dea Pe $\theta n$, realizzato in disparte in un punto di difficile lettura, è ritenuta una scelta legata alla consacrazione del monumento medesimo (cfr. MARAS 2009b, Vs do. 3, pp. 439-440, e anche il candelabro Fa co.2, p. 289 con medesima considerazione).

133 CRistofani 1995, p. 68.

134 Difficile stabilire con un certo grado di verosimiglianza il possibile scioglimento. Solo come tentativo, fra molti possibili, nel groviglio di tratti si potrebbe discernere la parola zare, come già proposto in CIE IV, I, 1. La radice zar- è individuata da $\mathrm{K}$. Wylin nel plurale locativo zarve attestato nel $L L$, in $z$ aru caratterizzato dal morfema $-u$ in funzione aggettivale nella $T C$ e nella forma plurale o aggettivale zarua legata a un ambito cleromantico nella sors di Punta della Vipera (WYLIN 2000, pp. 225-226, nota 545). Secondo lo studioso la radice sarebbe afferente alla sfera dell'espiazione e secondo D.F. Maras espliciterebbe il risultato di una azione rituale (cioè vicino al significato di "perfetto" che aveva già proposto $\mathrm{H}$. Rix istituendo un parallelo con i testi religiosi umbri; MARAs 2009b, p. 87, nota 1). Ma rimarrebbe la difficoltà del morfema -e, forse indizio di una forma verbale di tipo ingiuntivo o modo indicativo (WyLIN 2000, pp. 97-107, con riassunte le diverse posizioni) difficilmente accettabile. Se nel monogramma si isola anche un digamma, sostanzialmente ricavabile dai due tratti obliqui superiori dell'epsilon, si avrebbe zarve, identificabile come locativo di 
- CIE 20270: le lettere in legatura, incise a crudo, sono state individuate dubitativamente in digamma e kappa, mentre è da escludere un pi (che in questo orizzonte, per quanto noto, è piuttosto del tipo con tratto obliquo ricurvo); si sospende il giudizio sulla possibile individuazione di un rho con occhiello triangolare e codolo molto lungo. Altre lettere non sembrano presenti. La lettura $\widehat{k v}$ non dà stimolo a soluzioni convincenti in questo orizzonte cronologico; infatti il lessema cver, che potrebbe essere la soluzione ottimale, è tendenzialmente attivo in età ellenistica ${ }^{135}$, sebbene appaia (dubitativamente) nel piombo di Santa Marinella e sia stato ipotizzato nella forma abbreviata $c v$ incisa a crudo in un peso da telaio rinvenuto a cinque miglia di distanza da Vulci e in due bacini fittili a Roselle in contesto sacro $(C I E 12006,12020)^{136}$. In alternativa, $k v$ potrebbe essere inteso come $k u$ e dunque richiamare principalmente le iniziali di un teonimo tra quelli legati alla parola "porta" (culs: Culśanś, Culśsu) ${ }^{137}$. Se letto $v k$, questo digramma potrebbe essere una variante per $u k$, dove la scrittura del digamma per $y p s i$ lon è documentato nel distretto etrusco-meridionale cerite-veiente tra VII e VI sec. a.C. ${ }^{138}$ e a Lattes e St. Blaise nel Midì francese nel VI sec. a.C. ${ }^{139}$; il digramma $u k$ appare isolato a Spina e a Casale di Rivalta in legatura ${ }^{140}$, e potrebbe essere sciolto nella base onomastica riferita all'antroponimo maschile $u k e /$ $u k u$, ritenuto di derivazione celtica da G. Colonna e piuttosto attivo dal VII sec. a.C., nonché presente a Bologna nella forma $u k v a$ di fine VII sec. a.C. nell'anforetta Melenzani ${ }^{141}$.

Tra i numerosi digrammi documentati nella fase ellenistica, molti non si prestano a possibilità di verosimili proposte di interpretazione o scioglimento (es. la, $m e, m i, n i, p u, s u, r e, s e / s a, s e$ ). Inoltre, rimane di incerta lettura il digramma CIE 20044, graffito nella parete esterna di una coppa in ceramica grigia dall'abitato (se letto al potrebbe indicare un 'dono', ma anche la lettura au è possibile).

A parte il caso del lessema $\theta i$, piuttosto limpido (cfr. V.4), grazie ai contesti di rinvenimento, si possono avanzare alcune ipotesi per i seguenti digrammi:

- ka: documentato in una occorrenza da abitato (in ductus destrorso) databile genericamente al periodo ellenistico (CIE 20121) e in una da necropoli databile al II sec. a.C. (CIE 20390). In altre più antiche occorrenze questo è stato interpretato come deittico isolato ${ }^{142}$ oppure dubitativamente come abbreviazione di teonimo (Marzabotto: KavaAa) ${ }^{143}$; nel caso del digramma da corredo funebre, quest'ultima ipotesi potrebbe essere suggestiva, soprattutto se si vede in Kalu il possibile riferimento.

- ku: documentato su un piatto appartenente a un contesto funerario purtroppo perduto (CIE 20683), potrebbe essere ipoteticamente l'abbreviazione dell'appellativo kul'́nuteraś (CIE 21027; cfr. V.3).

- hi: documentato sulla vasca interna di una ciotola a v.n. locale integra, solo ipoteticamente da contesto funerario (CIE 20328), potrebbe trovare un confronto in un simile digramma dal Forcello di Bagnolo San Vito, da un contesto abitativo datato al 510-500 a.C.; per quest'ultimo, G. Colonna ha

zaru (RIx $2001^{3}$, p. 212), già documentato nel $L L$ ma che dovrebbe avere la medesima forma in periodo più antico, come ci documenta la $T C$ (cfr. CRISTOFAni 1995, p. 62). Nel $L L$ zarve è identificato con un tipo di vittima del rituale (BELFIORE 2010, p. 94).

135 Cfr. Maras 2009b, pp. 84-85.

136 Cfr. G. ColONNA, in REE LXXIV, pp. 396-397, n. 155, in particolare per il peso da telaio.

137 Cfr. MARAs 2009b, p. 441, con riferimento alla più tarda iscrizione sull'altare di nenfro da Bagnoregio $\left(E T^{2}\right.$, Vs 3.6)

138 G. Colonna, in REE LXV-LXVIII, p. 461, n. 139.

139 Colonna 2006, p. 665.

140 Rispettivamente NRIE, n. 143, e MaCellari, SQUAdRINI, Bentini 1990, p. 183, tav. LIV, n. 4, dove è ritenuto abbreviazione di nome (V sec. a.C.).

${ }^{141}$ Per questa base onomastica, cfr. Colonna 2006, p. 665.

142 Colonna 2006, pp. 664-665.

143 E. Govi, in REE LXXX, p. 241 (in riferimento al n. 4). 
inteso hi come riferibile al contenuto del vaso, possibile abbreviazione delle voci lessicali hia/bil/hinu/ meno probabilmente $\operatorname{hinth}(i) u$, oppure al nome del suo possessore ${ }^{144}$.

- pl/pu: digramma documentato su una ciotola (CIE 20622) appartenente al medesimo corredo (tomba 334 di Canalbianco) dell'iscrizione mura larza pili (CIE 20621): si può dunque con buona ragionevolezza ipotizzare che possa essere un relitto consonantico $p l$ del cognomen pili.

- sp: documentato tre volte, in tre vasi a v.n. locale da corredi funerari di II sec. a.C. (due pissidi: CIE 20376, 20934; una ciotola: CIE 20749); è invece poco probabile che il digramma sull'anfora greco-italica della tomba 109 di via Spolverin (CIE 21010) sia da leggere śp in ductus destroso, quanto piuttosto $M P$ in alfabeto latino. L'uso del sade in associazione all'occlusiva (ci si aspetterebbe sigma in area settentrionale per un fenomeno di palatalizzazione: si veda spiu, CIE 20534) ${ }^{145}$ fa ipotizzare che il digramma possa essere allusivo della parola *spura ${ }^{146}$, dove il carattere istituzionale del termine quale imprestito dall'Etruria meridionale ne ha preservato la grafia originaria nel passaggio verso le aree a sistema scrittorio settentrionale. Se di qualche valore questa ipotesi, rimane tuttavia difficile comprendere quale pratica (comunque molto rara da un punto di vista epigrafico) possa sottintendere l'allusione a questo termine istituzionale.

${ }_{144}$ G. ColOnNa, in REE LXIV, p. 235, n. 2.

145 Rix $2001^{3}$, p. 209.

146 Per un quadro aggiornato del termine e le sue occorrenze in ambito padano, cfr. Govi 2014, pp. 119-120, con riferimenti. 



\section{ONOMASTICA}

La principale difficoltà nello studio dell'onomastica in ambito padano è insita nella fenomenologia dei testi. In Etruria propria, le migliaia di iscrizioni funerarie su urne o di tipo più monumentale esplicitano in maniera più o meno articolata le formule onomastiche degli individui sepolti, consentendo così analisi complesse delle società, soprattutto per alcuni centri quali in età arcaica Orvieto, e nel periodo ellenistico Chiusi e Perugia, di cui si possono ricostruire intere prosopografie familiari (e.g. la famosa tomba dei Cai Cutu di Perugia ${ }^{1}$ ). Proprio questa messe di dati è alla base del più importante lavoro relativo alla Namengebung, l'Etruskische Cognomen di H. Rix del $1963^{2}$, ancora oggi un punto di riferimento fondamentale per gli studi sull'onomastica etrusca non solo ellenistica. In Etruria padana, a parte alcune casistiche di monumenti in pietra - comunque non posteriori al IV sec. a.C. ${ }^{3}$ - che ospitano testi dove si esplicita l'identità del defunto proiettandola pubblicamente, la scrittura del nome in ambiente funerario è destinata a formule graffite sui vasi dei corredi che accompagnavano i defunti, rispondendo così a logiche diverse. A causa di questa diversa destinazione sociale della propria identità, non si riscontra il dispiegamento di formule onomastiche più o meno complete e ciò già nel passato ha portato a una errata interpretazione del quadro sociale della vicina Spina, ritenendolo costituito in prevalenza da individui liberi ma privi del gentilizió.

Di seguito si affronterà l'analisi delle formule onomastiche documentate nell'epigrafia adriese nell'arco di oltre 4 secoli. Come si vedrà, il periodo tardo-arcaico restituisce qualche documento significativo (IV.1) e non è del tutto priva di dati anche la fase di V-IV sec. a.C. (IV.2). Ma è il ricco repertorio di formule onomastiche datate tra la fine del IV e il II sec. a.C. (IV.3.1) che consentirà per quest'ultima fase di impostare una classificazione delle iscrizioni (IV.3.2) e di formulare alcune considerazioni in particolare sui nomi familiari (IV.3.3).

\footnotetext{
1 Feruglio 2010-2013.
}

2 Rix 1963.

${ }^{3}$ Le uniche eccezioni documentabili rispetto a questa considerazione sono i cippi di Rubiera di fine VII-inizi VI sec. a.C. e le stele bolognesi di V e IV sec. a.C. (cfr. I.2.1).

${ }^{4}$ Una sintesi della questione con riferimenti bibliografici in GaUCCI 2015, p. 136. 


\section{IV.1. Il periodo tardo-arcaico}

Si contano quattro formule onomastiche all'interno di iscrizioni caratterizzate dall'uso del pronome personale mi e del genitivo dell'antroponimo (CIE 20180, 20214, 20368, 20822), mentre due sono i casi di antroponimi isolati, uno all'assolutivo databile entro il VI sec. a.C. (CIE 20361) e uno al genitivo I della prima metà del V sec. a.C. (CIE 20040). Come si vedrà, anche il trigramma kai (CIE 20282, 20290, 20295) è forse da intendere come nome completo all'assolutivo piuttosto che abbreviato. Infine, esprime con buona verosimiglianza un gentilizio con morfema del genitivo I l'iscrizione lacunosa sulla superficie superiore (a) del piede CIE 20289 ([-- - ] ynas [-- ]; ovviamente la lacuna non permette di avere la certezza che l'uscita del genitivo non sia afunzionale; per ulteriori considerazioni sul documento, cfr. VI.1).

Il pronome $m i$ in quattro iscrizioni identifica il vaso come soggetto parlante ${ }^{5}$. La varietà di uso di questo pronome è sottolineata anche da CIE 20212, dove si esprime l'identità tra il vaso e il sostantivo al che indica il dono (cfr. V.2). Solo dubitativamente il pronome potrebbe essere riconosciuto in un trigramma realizzato nel fondo di un'olla in ceramica grezza (CIE 20353) e anche nell' iscrizione lacunosa CIE 20204. In particolare, la possibile associazione del pronome $m<i>$ e dell'abbreviazione $a v$ in CIE 20353 espliciterebbe il possesso del vaso (interpretazione più difficile se si leggesse $m a v^{6}$ ). L'incisione a crudo su un contenitore in ceramica grezza, classe nella quale le iscrizioni di proprietà sono generalmente frequenti (cfr. II.1.3), potrebbe sostenere questa ipotesi.

Nei testi con pronome $m i$, due casi contengono formule onomastiche bimembri caratterizzate da prenome e gentilizio maschile, entrambi espressi al genitivo: mi venelus leceniies (CIE 20180), mi larisal uselnas (CIE 20822). A eccezione del prenome Laris di CIE 20822 declinato al genitivo II, gli altri elementi onomastici sono al genitivo I e seguono la norma meridionale, sebbene l'iscrizione CIE 20822 possa forse essere considerata di norma mista (cfr. infra). Per il gentilizio leceniies di CIE 20180, G. Colonna ha sottolineato la corrispondenza con l'area latina $(\text { Licinius })^{7}$ e successivamente A. Maggiani ha riconosciuto nel nome una base *lece, documentata da tardi confronti ${ }^{8}$, unita ai suffissi -na e $-i e$, quest'ultimo canonico in Etruria con la fine del VII sec. a.C. ${ }^{9}$. Per il gentilizio uselnas, è già stato rilevato da G. Colonna ${ }^{10}$ come questo sembri presentare l'anomalia della resa della sibilante interna rispetto alle altre attestazioni note. Infatti ad Orvieto, dove indubbiamente si registrano i confronti più stringenti per il periodo arcaico, il prenome da cui deriva $(u s ́ e l e)^{11}$ e il gentilizio stesso sono resi

5 Sul tema, si rimanda al fondamentale Agostiniani 1982.

${ }^{6}$ Cfr. Gaucci 2012a, p. 147.

7 Colonna 1974b, p. 23, nota 1.

8 Come puntualizza A. Maggiani, la base onomastica *lece presenta solo tardi confronti, cioè lecu da Volterra $\left(E T^{2}, \mathrm{Vt} 1\right.$. 128-129), e soprattutto in ambito volsiniese sveitu lecnies (ET ${ }^{2}$, Vs 1.301) e vel lecnies da Castel Giorgio di Orvieto (ET ${ }^{2}$, Vs 2.48; Morandi TaRabella 2004, p. 277, n. CCCVII, 1-2, che richiama anche la forma lecne/lecni nel territorio senese: $E T^{2}$, AS 1.318-333, 489), tarde occorrenze del nostro gentilizio.

9 A. Maggiani, in Akeo 2002, p. 182, nota 15; per il suffisso -ie, Marchesini 2007, pp. 96-103. Per il fenomeno della geminazione, nella fattispecie come riduzione della vocale /a/ del suffisso derivativo -na, BELFIORE 2011, in particolare p. 43.

10 Colonna 2003, p. 167, nota 34; Gaucci 2012a, p. 155.

11 Per una sintesi delle più recenti posizioni in merito alla formazione del prenome, si rinvia a DE SIMONE 2009a, in particolare pp. 114-123 (diminutivo con suffisso -le dal prenome *use/*uśu collegato ad uśil, 'sole'); G. ColonNA, in REE LXIV, p. 282 (dal teonimo *uśel, dall'originario sab. *ausel- / umbro *ozel, più conservativo a Orvieto, mentre invece sarebbe stato già precocemente adeguato a Cerveteri nella forma usil come testimonia il prenome usile, per il quale si veda anche RiX 1998, pp. 220-221, 227). Più recentemente, E. BENELLI, in REE LXXIX, p. 334, in riferimento alle iscrizioni nn. 87-88, conferma l'ipotesi di C. de Simone e afferma l'impossibilità di una derivazione dal teonimo, a differenza della forma usile documentata a Cerveteri tra VII e VI sec. a.C. (ET2 , Cr 3.1 della metà del VII sec. a.C., dove ha funzione di prenome e 2.64 di VI sec. a.C., dove invece è Individualnamengentile) e per la quale tale derivazione rimarrebbe una possibilità. Se si riconosce dunque l'estraneità della forma usé(le) dal teonimo usil, cade la distinzione territoriale nella resa delle sibilanti per i derivati di questo già espressa da MAGGIANI (1990, p. 202, nota 108: sigma a Cerveteri, Vulci, Adria, nel $L L$ e in altre iscrizioni meridionali; sade ad Orvieto). Si nota che a Spina è documentato su un piattello di produzione locale l'isolato 
con il sade $e^{12}$, marcando così la sibilante palatale secondo il sistema scrittorio della città (cfr. III.1). Se dunque validi questi presupposti, Uselna potrebbe essere inteso come un gentilizio già naturalizzato in un ambiente a norma settentrionale.

Nell' iscrizione mi uiniaś anteș́[ (CIE 20368), l'antroponimo non lacunoso uiniaś segue invece palesemente la norma scrittoria settentrionale. Come già rilevato ${ }^{13}$, sebbene questo trovi puntuale confronto ad Aleria nel IV sec. a.C. (ET ${ }^{2}$, Cs 2.8 e 2.9), dove è stato interpretato da J. Heurgon quale etruschizzazione di un nome greco ${ }^{14}$, tuttavia almeno quello adriese potrebbe derivare ragionevolmente dall'onomastica celtica ${ }^{15}$. Nel corpus delle iscrizioni 'galliche d'Italia' si trovano antroponimi (alkouinos, uini ${ }^{16}$ ) riferibili alla radice uind- (> uinn- > uin-) secondo un processo di assimilazione che M. Lejeune ritiene proprio del 'celtico d'Italia' e influenzato direttamente dal c.d. leponzio (intendendo con ciò il celtico parlato nei territori della Cultura di Golasecca) ${ }^{17}$. In alternativa uiniaś potrebbe teoricamente trovare confronto in una serie di nomi derivanti dalla radice oino-, cioè il numerale $1^{18}$, e A. Morandi ha suggerito di estendere il campo di confronto anche all'ambiente camuno, che avrebbe condiviso il milieu onomastico celtico ${ }^{19}$. Indipendentemente dalle problematiche di riconoscimento della radice, per uinia basti dire che pare verosimile, accanto all'ipotesi di derivazione greca, l'etruschizzazione di un nome celtico verosimilmente già caratterizzato con il suffisso derivativo femminile $-i a$, funzionale anche in etrusco ${ }^{20}$. Anche il secondo elemento della formula, il lacunoso anteș́, non trovando riscontro in etrusco, va similmente ritenuto derivato da un altro bacino onomastico. In questo caso non offrendo il mondo greco un convincente confronto, si possono elencare varie corrispondenze legate sempre al mondo celtico. Anzi tutto, si ricorda la base andeto- di alcune iscrizioni venetiche su ciottoloni patavini di V-IV sec. a.C., riferita a un nucleo familiare di origine celtica e per la quale l'ipotesi più probabile è la derivazione da un toponimo (Andes?) ${ }^{21}$. La base ande-/ando- è in generale molto attiva nell'onomastica celtica ${ }^{22}$. La lacuna non permette purtroppo una analisi più approfondita

usule con sigma a quattro tratti che punta a un sistema scrittorio cerite-veiente (BENELLI 2004, p. 260 con bibliografia di riferimento; DE Simone 2009a, p. 114 nota 36, pp. 120-122).

${ }^{12}$ In tutte le attestazioni del gentilizio è evidente la caduta della seconda epsilon, che H. Rix spiega non dovuta a sincope (troppo antiche le attestazioni), ma a indebolimento della vocale in contatto con la nasale del suffisso già avvenuta in età arcaica: Usele $>$ Usele $+n a>U s e l(e) n a\left(\operatorname{Rix} 2001^{3}\right.$, p. 206). Rimane incerta la lettura per la possibile attestazione del gentilizio a Volterra, che - se tale - sarebbe caratterizzata dalla sincope della prima epsilon (ET ${ }^{2}$, Vt 1.162, mi arn $\theta i a l$ ú́lnieś, cioè $T L E^{2} 383$, mi arntial uslnieśs).

13 REE LXXIX, pp. 288-291, n. 56.

${ }_{14}$ J. Heurgon ha avanzato l'ipotesi di una etruschizzazione del nome greco maschile Oinias (HeURGON 1973, pp. 560562, n. 50, ma anche p. 549, n. 5; su quest'ultima, si veda ora D. BriQuel, G. van HeEms, REE LXXXI, pp. 392-393, n. 71, dove viene confermata la natura greca del testo, ritenuta etrusca da $\mathrm{H}$. Rix, ET ${ }^{2}$, Cs 2.2).

15 Tale lettura in chiave celtica viene accolta da A. MorAndi, in REE LXXX, pp. 280-281, n. 25.

16 Alkouinos da San Pietro di Stabio presso Mendrisio, di fine II-inizi I sec. a.C.: Lejeune 1971, pp. 55-56; P. Solinas, in REI LX, p. 328, n. 21, tav. LCXIII c). Uini da Arsago Seprio, attribuibile al La Tène D1/2: Morandi 2004, p. 602, n. 119.

17 LEJEUNE 1971, p. 24.

18 Cfr. Holder 1904, c. 840; Delamarre 2003, p. 240.

19 A. Morandi, in REE LXXX, pp. 280-281, n. 25.

20 Per il celtico, cfr. Мотта 2009, pp. 313-315; per l'etrusco, cfr. da ultimo Rigobianco 2013, p. 127 con riferimenti interni ed esterni.

21 Prosdocimi 1988, pp. 290-291; Marinetti, Solinas 2014, p. 79.

22 Ellis Evans 1967, pp. 137-141; Delamarre 2003, pp. 45-46. Va puntualizzato che M. Lejeune ha avanzato l'ipotesi dell'assimilazione del gruppo consonantico -nd->-nn->-n-nel 'celtico d'Italia' e anteriormente nel 'leponzio' (cfr. anche uind-, supra), ipotesi ancora dibattuta (cfr. Solinas 1993-1994, p. 898; EAD. 1998, p. 114, nota 7; UHLICH 2007, pp. 373-405). Tenendo presente queste problematiche, il nostro anteś[ potrebbe essere messo a confronto anche con il tardo anteśilu di Solduno, per il quale è stata ipotizzata la formazione *ande-tessi-, attestata anche altrove: MoTTA 2000, p. 215, n. 26, con precedenti riferimenti (LeJEune 1971, pp. 25 e 54; cfr. DelamarRe 2003, p. 294); in questo caso, la presenza del tau è risolta da Lejeune grazie alla natura composita del nome (LEJEUNE 1971, p. 54, n. 136; cfr. MotTA 2000 , p. 215 , n. 26, con riferimenti). 
di questo elemento onomastico, seppure sia plausibile alla luce di una possibile derivazione celtica e dei confronti proposti, una continuazione del nome dopo il sade. Non è così possibile cogliere appieno la struttura di questa formula di possesso. L'associazione di uinia con un elemento onomastico di probabile origine celtica ha portato chi scrive più convincentemente a ritenere che anche questo potesse avere la medesima origine (e non necessariamente collegabile alla trafila che ha originato uinia di Aleria, peraltro di tutt'altra cronologia). Ciò sembra la lezione più economica, anche alla luce dei fenomeni di mobilità individuale che dovevano interessare l'Italia settentrionale ${ }^{23}$.

Da queste attestazioni di formule di possesso si distingue CIE 20214, che mostra, secondo la più recente interpretazione di G. Colonna, l'identità tra il pronome mi e kar, quest'ultimo specificato dal prenome Venel al genitivo I secondo la norma scrittoria meridionale ${ }^{24}$. Il termine $k$ ar secondo Colonna sarebbe da integrare $k a r<a>$, cioè con l'omissione dell'alpha finale per ragioni di spazio. Il lessema kara

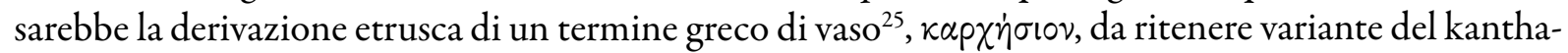
ros e da intendere in ambito etrusco quale denominazione di vasi potori di forma diversa anche con la finalità di nobilitarli. A tale riguardo, ci si domanda se invece il lessema, forse sempre da intendere come nome del vaso, possa piuttosto avere una base puramente etrusca con un suffisso derivativo -ra. La base potrebbe essere $k a$ o meglio ${ }^{*} k a r$, radice di un verbum faciendi $i^{26}$ (kar+ra con aplologia $>$ kara).

Due sono gli antroponimi isolati, uno databile entro il VI sec. a.C. (CIE 20361) e uno alla prima metà del V sec. a.C. (CIE 20040). L'iscrizione CIE 20361, che esplicita il prenome Laris, è l'unico documento dell'orizzonte cronologico in esame nel quale si ha una sicura formula onomastica all'assolutivo (secondo un noto procedimento di identità come appartenenza dell'oggetto iscritto ${ }^{27}$ ). L'iscrizione CIE 20040 esplicita invece un gentilizio al genitivo I (amnz), probabile forma sincopata $\mathrm{di}^{*}$ amnas $\mathrm{o}$ *amnis, con confronti nelle formule onomastiche a Volterra e Chiusi in epoca recente ${ }^{28} \mathrm{e}$ presumibilmente già documentato ad Orvieto nei gentilizi amanas (anche Veio) e amuna della fine del VI sec. a.C. ${ }^{29}$, che secondo E. Benelli potrebbero essere varianti con diverse oscillazioni vocaliche del medesimo antroponimo ${ }^{30}$. Tuttavia, si potrebbe rinviare anche all'arcaico amina [ di Pontecagnano, che G. Colonna riconosce come gentilizio formato dal nome *ame; più recentemente E. Benelli ne individua la base nel prenome femminile amia di una iscrizione tardo-arcaica di origine incerta ma di concezione ceretana $^{31}$. Si aggiunge che la probabile uscita in /z/ di amnz, come proposto da A. Maggiani, conforterebbe il supposto legame con Orvieto, tenendo comunque conto che in quest'ultimo contesto la sostituzione di sigma con zeta è frequente soprattutto dal IV sec. a.C. ${ }^{32}$.

Tirando le fila del discorso, nei due testi in norma meridionale (CIE 20180 e 20822) è palesata una struttura onomastica appartenente a individui ingenui portatori di gentilizio. Si riscontrano i medesimi prenomi Laris e Venel in altre due iscrizioni dove però non è esplicitato il gentilizio, sebbene

${ }^{23}$ Da ultimo, cfr. SASSATELLi 2008b, pp. 397-427.

${ }^{24}$ REE LXXIV, pp. 367-368, n. 111. Si ricorda che la precedente lettura proposta da G. Colonna era mi venelus kaîs (Colonna 1974b, p. 23, n. 3, fig. 5), dove già allora si poneva l'accento sulla meridionalità della norma ortografica (ibid., p. 24).

${ }^{25}$ G. Colonna trova confronto per il lessema con l' iscrizione di dono della kylix in bucchero dell'ultimo quarto del VII sec. a.C. da Narce (ET ${ }^{2}$, Fa 3.3; adesso G. ColonnA, in REE LXXIV, pp. 411-413, n. 167).

${ }^{26}$ Cfr. Belfiore 2014a, pp. 36-37. Dell'avviso che alla base di kara ci possa essere *kar-, sembra anche la stessa V. BeLFiORE (2014b, p. 84, nota 11).

27 Colonna 1983, p. 49. Su questo, si veda ora Poccetti 2019.

28 Maggiani 2002a, p. 57.

29 Morandi Tarabella 2004, nn. XXV e XXVI p. 57. Si aggiunge che confronti si trovano anche nell'onomastica italica (es. lat. Amnius, Amunius; cfr. Hirata 1967, p. 34). Se valido il passaggio amuna/amana > amna/amni si avrebbe nell'amnz di Adria una doppia sincope.

30 E. BENELLI, in REE LXXIX, p. 334, in merito alle iscrizioni nn. 87-88.

31 Bailo Modesti 1984; $E T^{2}$, Cm 2.20; Colonna 1995, pp. 330-331, che ricostruisce *ame in base all'alternanza morfologica -a/-e da *ama da cui amana di Orvieto. Per il prenome amia, si veda REE LXXIX, pp. 333-334, nn. 87-88.

32 VAN HEEMs 2003. 
sia verosimile che lo possedessero. Le iscrizioni amnz e ] unaș conservano gli altri due gentilizi noti, anch'essi come i precedenti declinati al genitivo secondo la norma meridionale. L'unica iscrizione in norma settentrionale mostra un nome femminile di origine celtica, Uinia, associato a un altro antroponimo probabilmente del medesimo ambito culturale, Anteś[, per il quale tuttavia non è possibile comprenderne la funzione sociale (nome individuale? gentilizio con base onomastica celtica?). L'ipotesi più plausibile a questo livello cronologico è che Uinia sia una donna ingenua, la cui onomastica potrebbe per ipotesi essere simile a quella della più tarda iscrizione rei $\theta v i$. keisnaś della stele di Tomba-

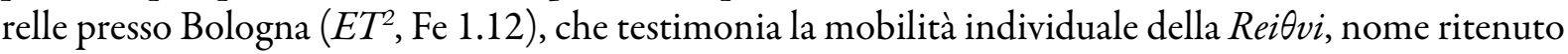
derivante dall'etnonimo dei Reti ${ }^{33}$.

Rimane da affrontare l' isolato kai delle tre iscrizioni CIE 20282, 20290, 20295, sul quale merita di richiamare l'intero percorso ermeneutico.

Il kai adriese è stato generalmente interpretato come antroponimo maschile di origine italica all'assolutivo, con confronti in particolare a Vulci ${ }^{34}$. Infatti, a un contesto funerario vulcente M. Martelli ${ }^{35}$ riferisce le tre iscrizioni caial redatte sul fondo esterno di altrettante anfore attiche ${ }^{36}$, che la studiosa attribuisce a una medesima mano e interpreta come antroponimo femminile declinato al genitivo. Tale interpretazione è basata sulla lettura di CIE 20214 offerta da G. Colonna nel 1974, che restituisce l'antroponimo kaîs, risolvendo così quello che nel fac-simile tipografico del Bocchi edito nel 1879 (unica documentazione dell' iscrizione all'epoca) sembra un $r h o^{37}$. Richiamando dunque il documento adriese, M. Martelli ha tentato di spiegare caial come esito femminile di cai $(e)+i a$, evocando una precoce monottongazione del suffisso $-i e$ in $-i^{38}$. Tuttavia, il medesimo Colonna ha più recentemente proposto di leggere kar e non kais ${ }^{39}$, venendo così meno il più forte elemento che poneva in parallelo le iscrizioni di Vulci con quelle di Adria (ma anche il più forte elemento dell' interpretazione della Martelli). Ovviamente l'iscrizione adriese non è l'unica che documenterebbe l'antroponimo, ma era l'unica nel quale questo si inseriva in una formula onomastica più articolata.

Espunta dunque l' iscrizione CIE 20214, il lessema kai appare isolato in tre attestazioni, tutte redatte su vasi attici fra loro coevi: un chous a figure nere datato al 490-480 a.C. (CIE 20282), una vicup di tecnica indeterminata datata al 480-450 a.C. (CIE 20290) e un cup-skyphos probabilmente figurato datato al 500-470 a.C. (CIE 20295). Le singole lettere risultano coerenti per aspetto fra le tre iscrizioni.

Anzi tutto, va osservato che la graffitura nel fondo esterno di vasi attici evoca simili sigle greche ${ }^{40}$. Già M. Martelli in merito a caial aveva posto l'attenzione sui trademarks elencati da A.W. Johnston ${ }^{41}$, e in particolare sulle due oinochoai da Karlsruhe databili entro la prima metà del V sec. a.C. ${ }^{42}$ che presentano iscrizioni simili a quelle in esame ${ }^{43}$.

33 Per questa iscrizione, si veda da ultimo Gaucci, Govi, Sassatelli c.s.

34 Colonna 2003, p. 167, seguito in Gaucci 2010, p. 36. Si ricorda che in Antonetti 2005, p. 122, nota 32, i casi in esame sono ritenuti di dubbia interpretazione.

${ }^{35}$ REE LVIII, pp. 295-298, nn. 26-28.

36 CIE 11169: anfora a f.r., Euphronios o sua maniera (A/ Eracle con arco; B/ hydra), 510-500 a.C.; CIE 1170: anfora a f.r., maniera di Euphronios (A/ Eracle che incede verso destra; B/ amazzone Barkida con arco), 510-500 a.C.; CIE 10993: anfora a f.r., maniera di Euphronios (A/ Eracle che corre verso destra con arco e clava; B/ arciere con berretto frigio), 500 a.C. circa.

37 Colonna 1974b, p. 23, n. 3, nota 113.

38 M. Martelli, in REE LVIII, p. 296.

39 G. Colonna, in REE LXXIV, pp. 367-368, n. 111.

40 Johnston 2006, p. 153, tipo 11F, iv).

${ }^{41}$ M. MARTELLI, in REE LVIII, p. 298, nn. 26-28.

42 Johnston 2006, p. 153, nn. 24-25. Per i vasi, cfr. CVA Karlsrube I, tav. 31, 4, oinochoe configurata a testa femminile del Sabouroff Group, datata attorno al 470 a.C. (iscrizione sinistrorsa); ibid., tav. 35, 4: oinochoe forma 1 a v.n., datata alla metà del V sec. a.C. (iscrizione destrorsa).

43 Johnston afferma, come poi ribadito da M. Martelli, che l'iscrizione sinistrorsa sull'oinochoe configurata a testa femminile sia più affine a quelle adriesi (Johnston 1979, p. 228; M. MARTELLI, in REE LVIII, p. 298). 
Si ricorda che cai in età recente a Perugia è gentilizio maschile (femminile caia), formato con il suffisso $-i$ tipico di questa città e ritenuto di probabile origine italica ${ }^{44}$. Si tratta di una forma locale rispetto a cae, il più diffuso dei gentilizi chiusini ${ }^{45}$.

In questa più tarda fase, si contano altre occorrenze di cai:

- a Volterra, nel santuario dell'acropoli, dove appare isolato sul fondo di un piattello di ceramica presigillata della prima metà del II sec. a.C., interpretato come gentilizio epiceno ${ }^{46}$;

- a Roselle, dove appare più volte, isolato (CIE 11636, di fine IV sec. a.C.; 11816, di fine III sec. a.C.), associato a numerali (CIE 11658, della prima metà del III sec. a.C.), nell' iscrizione mi avleś cai (CIE 11703, su un'anfora di III-II sec. a.C., dove si ritiene omesso il morfema del genitivo in cai $)^{47}$;

- a Orvieto, dalla favissa Malvolti, dove appare isolato in un anello distanziatore (inciso a crudo?; CIE 10564);

- forse a Vulci, nel fondo interno presso la rosetta stampigliata di una ciotola a v.n. di periodo ellenistico dalla collezione del Principe Vittorio Massimo conservata a Scorano ${ }^{48}$.

Dunque, nell'orizzonte tardo-arcaico la base $c /$ kai è documentata unicamente ad Adria e a Vulci. Per le occorrenze vulcenti, M. Martelli aveva ipotizzato l'assolutivo caia (noto in questa forma in età recente), rispetto al quale kai sarebbe il corrispettivo maschile (inteso come gentilizio sulla base della lettura di CIE 20214, non più valida). G. Colonna ${ }^{49}$ ritiene kai modellato sull'umbro cais attestato nel IV sec. a.C. (ST, Um 22), ma che troverebbe un parallelo in falisco arcaico nella forma kaios, ipotizzato appunto da H. Rix un umbrismo di fronte alla forma normale cauio ${ }^{50}$. L'etruschizzazione del nome sarebbe avvenuta in Etruria meridionale oppure nella medesima Adria secondo lo studioso. Seguendo questa ipotesi, sembra lecito distinguere il nome dalla base kav-, anch'essa di origine italica ma con altro percorso formativo (cfr. kavi di III sec. a.C.: IV.3.1.B, categoria a).

A tutto ciò, va aggiunto che L. Rigobianco ha messo in evidenza che il genitivo II di caial lascia aperta la possibilità che l'assolutivo sia ${ }^{*} c a i(e)+i$ e non $c^{c}{ }^{51}{ }^{51}$. Questo in effetti non stupirebbe, se si mette a sistema questa considerazione con una di carattere archeologico che riguarda l'oinochoe configurata a testa femminile di origine incerta. Tale vaso appartiene infatti a una forma vascolare generalmente associata a culti inferi di Persefone/CavaAa in ambito sacro ma anche funerario, dove i defunti sono riconosciuti come di genere femminile ${ }^{52}$. Non è quindi azzardato supporre che anche i kai di Adria potrebbero essere interpretati come assolutivo del medesimo nome femminile.

La reiterazione del testo ha portato chi scrive ad avanzare la possibilità di interpretare il trigramma come sigla con probabile funzione commerciale ${ }^{53}$. A tale riguardo, si era osservato che la graffitura nel fondo esterno di vasi attici e inoltre la somiglianza di kai con simili sigle greche raccolte da A.W. Johnston, porterebbe a interpretare kai come sigla di un proprietario o intermediario commer-

44 Benelli 2002a, p. 522.

45 Benelli 2014, p. 61.

46 M. BonAmici, in REE LXXVII, pp. 310-311, n. 16.

47 A questo elenco si potrebbe ipoteticamente aggiungere l'iscrizione lacunosa çaiz[ (CIE 11644, fine V-inizi IV sec. a.C.), soprattutto se si propone la divisione çai . $z$ [, come intuibile dalla fotografia in REE XLIV, tav. XLIII, n. 18, e in parallelo con CIE 11658, anch'esso con puntuazione interverbale. Sempre a Roselle merita ricordare anche l'iscrizione lacunosa caina [---] su un frammento della seconda metà del IV sec. a.C. (CIE 11648 e REE XLII, tav. XXX, n. 104 per la fotografia), ma che forse si potrebbe leggere in alternativa cai na [-- ], data la diversa dimensione dello iota rispetto al $n y$ e alla differente inclinazione fra le due lettere. La maggior parte delle attestazioni citate viene da strati sottostanti al foro (CIE 11636, 11644, 11648, 11658).

48 M.T. Falconi Amorelli, in REE XXXIX, p. 360, n. 48; ET², OA 2.59.

49 Colonna 2003, p. 167. Si puntualizza che tali osservazioni sono anteriori alla nuova lettura proposta dal medesimo Colonna per CIE 20214.

50 Rix 1998, p. 227.

51 Rigobianco 2013, p. 106.

52 G. Morpurgo, in Gaucci, Morpurgo, Pizzirani 2018, pp. 657-663, con riferimenti.

53 Gaucci, Vallicelli 2011; Gaucci 2012a, pp. 143-144. 
ciale $^{54}$, e quindi di potervi leggere un antroponimo completo (appunto kai) oppure eventualmente l'abbreviazione di un nome etrusco (es. kaile ${ }^{55}$ ) o greco in grafia etrusca (es. Kaĩke1os ${ }^{56}$ ). In tal modo, benché non strettamente legato al contesto sacro, l'interpretazione del reiterato trigramma su ceramica attica ben si allineerebbe alle osservazioni sull'importante lotto di trademarks di Adria ${ }^{57}$. Tale ipotesi è forse alternativa a quella, comunque più convincente, di vedervi un triplice testo votivo composto unicamente dal nome del dedicante ${ }^{58}$, forse una donna portatrice di un nome (gentilizio?) che trova il più tringente parallelo a Vulci. Ma ciò presuppone, come probabile, che le tre iscrizioni provengano da un unico contesto di natura sacra (una favissa?). In effetti, proprio la mancanza di dati di contesto non può che limitare e rendere opinabile qualsiasi linea interpretativa ${ }^{59}$.

\section{IV.2. V e IV sec. a.C.}

Il nucleo di documenti epigrafici databili a questa fase è molto ridotto, limitandosi a brevi testi di carattere onomastico, molti dei quali lacunosi in quanto provenienti perlopiù da contesti abitativi.

È databile genericamente al V-IV sec. a.C. l'iscrizione lacunosa ?] $\operatorname{smin} \theta[$ (CIE 20184), graffita dopo la cottura nella parete interna di una forma aperta attica a v.n. Il lessema è da interpretare come antroponimo con evidente richiamo al più tardo śmin $\theta i$ dell'iscrizione CIE 21027 (cfr. IV.3.1.B, categoria c).

Entro il IV sec. a.C. si datano più puntualmente cinque iscrizioni. Tra queste, quattro si collocano in questo secolo sulla base del supporto o del contesto: le lacunose an [ (CIE 20008), ]e (CIE 20127), e inoltre kramuriś della tomba 20 di via Spolverin (CIE 20899), che risulta difficilmente spiegabile attualmente sia all' interno del bacino onomastico etrusco, che in quello venetico e celtico (all'ambito lateniano rimanderebbero le decorazioni a stampiglie nei due skyphoi in ceramica grigia $\left.^{60}\right)$; a queste si aggiunge l'iscrizione CIE 20043 della seconda metà del IV sec. a.C., da leggere come $\widehat{a a}$ o meglio $\overparen{a i a}$, in entrambi i casi in legatura. Se valida la lettura aia, allora potrebbe essere

${ }^{54}$ La possibilità di intermediari etruschi che siglano più vasi attraverso l'abbreviazione del proprio nome è esplicitata in Johnston 1985, pp. 250-251, nota 15, fig. 5.

$55 E T^{2}$, Cs 2.3 (450-425 a.C.); Vc 7.24 (IV sec. a.C.); Vs S.4 (IV-III sec.a.C.).

56 Osborne, Byrne 1994, Atene, 510 a.C.

57 BALDASSARRA 2013. Ė plausibile ritenere che proprio queste pratiche epigrafiche greche, ascritte ad Adria in prevalenza all'alfabeto eginetico e interpretate come sigle di proprietà o commerciali (JoHNSTON 1979, p. 13), abbiano potuto stimolare la medesima pratica anche tra gli intermediari commerciali etruschi (soprattutto nei casi di reiterazione della sigla), come ha riconosciuto A. Maggiani per la piazza commerciale di Vulci, alla quale lo studioso ascrive il TM Johnston 8 E, che riconosce come legatura di due lettere etrusche (alpha e zeta), verosimilmente le iniziali di un antroponimo (MAGGIANI 2013b). Si rimanda al riguardo anche alle considerazioni di A.W. Johnston sul riconoscimento e i relativi problemi di interpretazione di trademarks etruschi su ceramica attica figurata (JoHNSTON 1985). Seguendo questa ipotesi, kai, graffito su più vasi attici e con stringenti paralleli in un tipo di trademark greco ben attestato (tipo 11F, iv), potrebbe essere l'esito di una pratica etrusca mutuata da quella greca che è adesso riconosciuta anche a Cerveteri in ceramiche di produzione locale, che presentano trademarks simili a quelli rinvenuti su prodotti attici (BENELLI, ColiviCCHI, ZACCAGNINO 2017, p. 44). Tale tema, sul quale vi è una incipiente attenzione da parte della critica, risulta sicuramente difficile da indagare data l'esiguità dei testi e spesso l'ambiguità della competenza scrittoria che questi esplicitano.

58 Su tale tipologia di formulario devozionale, si veda Maras 2009b, p. 451.

59 A tale riguardo, merita aggiungere, per completezza, che si ritiene più difficile che il lessema possa essere interpretato come locativo di $(i) k a$. La forma dell'assolutivo privo (così precocemente) dello iota iniziale è sicuramente attestata nel piombo di Pech Maho (CRistofani 1993, p. 834) e ipotizzata anche per il digramma $k a$ su un frammento di olla da un contesto abitativo di Lattes in Francia meridionale datato all'ultimo quarto del VI sec. a.C. (da intendere per G. Colonna come indicazione della destinazione di un determinato coperchio: "questa" è l'olla; Colonna 2006, pp. 664-665, fig. 4). Tuttavia, la forma cei (cfr. Rix $2001^{3}$, p. 217) si ritrova già nella $T C$ allo stesso livello cronologico dei nostri (CRISTOFANI 1995, pp. 83-85 e nota 61 per precedenti interpretazioni; cfr. anche FACCHETTI 2002a, p. 27, nota 57): quest'ultimo è stato ipotizzato locativo di tipo strumentale dal Cristofani e così è confermato più recentemente da HADAS-LEBel (2016, p. 119).

60 A. Ruta Serafini, in Gambacurta, Ruta Serafini 2018, p. 68, fig. 49.a. 
interpretabile come antroponimo femminile che ha in aie di probabile origine umbra il suo corrispettivo maschile ${ }^{61}$.

È invece databile al IV sec. a.C. su base paleografica kutvaluś [ (CIE 20037), antroponimo con suffisso - alu tipico dei gentilizi padani (cfr. IV.3.3.B) declinato al genitivo I. Forse da intendere come nome di famiglia, potrebbe avere come base onomastica kutu diffuso in ambito perugino, così come già riconosciuto G.F. Gamurrini e quindi confermato più recentemente da G. Sassatelli e C. de Simone ${ }^{62}$; ciò induce a ipotizzare la formazione kutu $+a l u>k u t v a l u$, oppure $k u t u+v a+a l u$, quindi su una base $k u t u+v a^{63}$ (per il suffisso -va, cfr. IV.3.1.B, categoria d).

\section{IV.3. Fine IV-II sec. a.C.}

\section{IV.3.1. Le formule onomastiche}

La significativa varietà qualitativa e quantitativa delle formule onomastiche di questo periodo, porta ad affrontarne l'analisi sulla base della loro articolazione. Si sono dunque distinte quelle costituite da più elementi onomastici (IV.3.1.A) dagli antroponimi isolati (IV.3.1.B). Si ritengono di interpretazione incerta alcuni nomi che presentano l'associazione di una lettera o un digramma: pe zurta (CIE 20514) di fine IV-III sec. a.C., ksnalu (CIE 20566) e kzurtiu (CIE 20785) di II sec. a.C., a:risiu (CIE 20020) più genericamente di III-II sec. a.C. Sull'interpretazione di questi testi, si veda oltre (IV.3.2.A). Qui si può anticipare che il primo è probabilmente da intendere come nome individuale (cfr. IV.3.1.A), mentre gli altri come gentilizi ${ }^{64}$.

Rimangono escluse dalla seguente trattazione anche alcune iscrizioni lacunose, per le quali le parti conservate sono riferibili alla sfera dell'onomastica. Queste sono:

- III sec. a.C.: ]× vercniś (CIE 20182); mi petr[ (CIE 20201), <e >mi karku[ (CIE 20627),

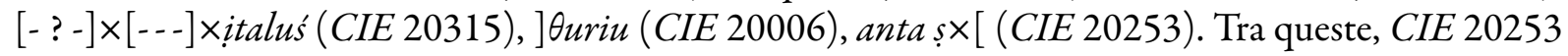
potrebbe suggerire una formula all'assolutivo introdotta dal prenome anta forse seguito dal gentilizio. ] xịtaluś invece potrebbe segnalare un gentilizio con suffisso - alu all' interno di una formula complessa, purtroppo fortemente lacunosa. Anche ] x vercniś manifesta nella parte conservata un probabile gentilizio, la cui base onomastica (che si preferisce alla lettura percniś proposta da A. Maggiani ${ }^{65}$ ) è diffusa tra Perugia ed Orvieto nella forma vercna. Più difficile identificare con sicurezza la funzione di $m i$ petr [, che presenta un diretto confronto con la base onomastica petr-, molto attiva in ambito chiusino $\mathrm{e}$ presente ad Adria anche nel secolo seguente nell'altrettanto lacunoso petra a [ (CIE 20600). Purtroppo entrambe queste ultime due formule non permettono di comprendere appieno la funzione di questi elementi onomastici nei rispettivi testi, ma petra potrebbe comunque essere composto con il suffisso di mozione $-a$, formante nomi femminili ${ }^{66}$.

- II sec. a.C.: ] euna (CIE 20012), kanix [ (CIE 20925), petra a[ (CIE 20600). La prima potrebbe trovare completamento nel gentilizio volterrano cnevna/cneun ${ }^{67}$ attestato anche a Tarquinia e Vulci

${ }^{61}$ Cfr. Colonna 2004b, p. 304, n. 10.

62 Gamurrini 1880, p. 75, n. 861; Sassatelli 1991, p. 710, n. 21; De Simone 2011, p. 156, nota 166.

${ }^{63}$ Cfr. Colonna 2009, p. 219; Belfiore 2014b, pp. 91-92.

${ }^{64}$ Per zurtiu, si rinvia a IV.3.1.A. Per snalu si potrebbe ipotizzare una base *sne/sna a cui è aggiunto il suffisso -alu; tale base potrebbe avere un legame con Snenat/Snenat del corteggio di Turan (rispettivamente ET ${ }^{2}$, Um S.2, S.4, OI S.34; Vc S.27), a sua volta formante il lessema snenaziulas della TC (rr. 16-17), riconosciuto da M. Cristofani come una ministra del culto (CRISTOFANi 1995, p. 83; tale lettura è ripresa più recentemente in Belfiore 2014b, p. 165): Snena $\theta$ sarebbe dunque composto su una base * sne con l'aggiunta del suffisso derivativo -na e del suffisso agentivo $-\theta$. Rimane senza confronti significativi risiu.

65 Maggiani 2002a, p. 60, fig. 10.

66 Rigobianco 2013, p. 105, nota 586; lo studioso ritiene che tale suffisso sia attivo solo in opposizione al maschile -e.

${ }^{67}$ Per Volterra, cfr. in particolare $E T^{2}$, Vt 1.45, 103, 104, 105, 106, 113, 4.6; per le iscrizioni sui coperchi riferiti alla probabile tomba della gens in località Marmini datate entro il I sec. a.C., cfr. Maggiani 1976, in particolare p. 7. 
per il comparto etrusco-meridionale ${ }^{68}$ e a Chiusi per quello interno $\left(E T^{2}, \mathrm{Cl} 1.1999\right)$, oppure nel gentilizio di ampia diffusione perugina leuna, attestato nel femminile leunei/leunal. Il lessema kani-trova invece confronto nella formula kraiku.kaniu. (cfr. IV.3.1.A).

Si datano più genericamente al periodo ellenistico quattro occorrenze: lei[ (CIE 20079), ]×luva (CIE 20120), ] e aiteś (CIE 20826), [- ? - f]astiu m[i] (CIE 20419). lei[ potrebbe essere correlato al leiu(ś) di III sec. a.C., per il quale non è certo un valore onomastico (cfr. IV.3.2); ]luva invece richiama la forma lessicale cilva del $L L$, in quanto è l'unica che potrebbe prevedere una formazione $-l+\mathrm{V}+v a^{69}$. Nell'ultima iscrizione, aiteś potrebbe avere un valore onomastico, ma l'assenza di sicuri confronti e il carattere lacunoso del testo inducono a sospendere il giudizio. Per CIE 20419, se valida l'integrazione fastiu, si veda IV.3.1.B, categoria d).

\section{IV.3.1.A. Formule onomastiche tri- e bimembri}

Si datano al III sec. a.C. 11 distinte formule bimembri complete, tutte appartenenti a uomini: CIE 20188, venu kleuste [; CIE 20264: larza śiluniś; 20477: laris tetialuś mi; CIE 20519: venza velOuriu; 20564: laris ațiu; 20569-20570, e forse 20571: ata śetina; 20614: mi laris fuliu; CIE 20662: venuś zurtiuśmi; 20904: venza malni. Solo un caso fra quelli elencati presenta la formula al genitivo, mentre in tutti gli altri è all'assolutivo, con due chiare attestazioni di genitivo afunzionale, cioè larza śiluniś e laris tetialuś (la lacuna impedisce di conoscere la terminazione del secondo elemento onomastico di CIE 20188). Si distinguono due iscrizioni composte da un nome maschile associato a un nome femminile al genitivo II (CIE 20030: ata krapnal; 20568: arza lausial).

Tra la fine del III e la prima metà del I sec. a.C., a differenza della fase cronologica precedente, le occorrenze, ancora tutte maschili, presentano sistematicamente entrambi gli elementi onomastici all'assolutivo e non vi sono casi di genitivo afunzionale, come è da aspettarsi. Si contano 2 formule trimembri (CIE 20434: anta afr mura; CIE 20621: mura larza pili) e 5 formule bimembri (CIE 20992: larza lintialu; CIE 20697: kraiku.kaniu.; CIE 20923-20924: viza muliu; CIE 21013: apu muliu; CIE 20560: qạia mi śiliu).

Non mancano alcune occorrenze prive di una datazione puntuale. Tra queste è sicuramente significativa l'unica formula femminile documentata ad Adria (CIE 20736-20737: alpi arnai).

Nel III sec. a.C. i primi elementi delle formule sono da riconoscere come prenomi e presentano una varietà molto limitata: Arza, Ata, Laris, Larza, Venza, Venu. Eccetto Ata, questi sono tutti ben documentati in Etruria padana: Venu dall'Orientalizzante recente a Bologna $\left(E T^{2}, \mathrm{Fe} 2.1\right)$; Laris tra fine VI e primi decenni del V sec. a.C. a Kainua-Marzabotto ${ }^{70}$ e nella medesima Adria (CIE 20822); Arza, Larza e Venza in area deltizia a Spina tra IV e III sec. a.C. Per quanto concerne in particolare i prenomi con suffisso diminutivo quali Arza e Larza, fuori dall'area padana questi sono registrati in periodo ellenistico soprattutto in territorio chiusino, ma anche a Perugia e sulla tavola di Cortona $(T C o)^{71}$. Il calcolo delle occorrenze proposto da L. Agostiniani nello studio sulla $T C o$, ha portato lo studioso a ritenere che questi sono di bassa frequenza (con rapporto rispettivamente di 1:19 e 2:23) rispetto agli altri documentati fra i quali si ricorda Laris (6:94 $)^{72}$. Più in generale, Arza, Larza e Venza sarebbero composti con il suffisso diminutivo - $z a$ dai comuni Arth, Lar $\theta$ e Venel. Secondo G. van Heems, questi costituirebbero una "sotto-categoria funzionale", cioè avrebbero potuto avere uno specifico valore

${ }_{68}$ Morandi Tarabella 2004, p. 138, nn. 1-2 (rispettivamente $E T^{2}$, Ta 1.236 e Vc 1.88 ).

69 Belfiore 2014b, pp. 163, nota 6, p. 171.

70 Govi 2014, in particolare pp. 124-125.

71 Si rinvia agli elenchi in VAN HeEms 2008, pp. 103-104; in particolare per Cortona, Agostiniani, Nicosia 2000 , pp. 66-67.

${ }^{72}$ Ibid., p. 67 e nota 92. Il calcolo è stato effettuato sulla base di Agostiniani, HJORDT-VeTLESEN 1988. 
sociale oppure la funzione di discriminare le classi di età (anche relative) ${ }^{73}$. In ogni caso, i prenomi adriesi sembrerebbero a tutti gli effetti forme sclerotizzate e sistematicamente adottate all'interno della comunità degli aventi diritto ${ }^{74}$.

Ata, già riconosciuto come prenome da A. Maggiani ${ }^{75}$, è attestato unicamente ad Adria; sembra tuttavia di ritrovarlo composto con il suffisso femminile -ia nella formula mi caviaś ataiaś di Monte Bibele $\left(E T^{2}, \mathrm{Fe} 2.15\right)^{76}$. Ata potrebbe essere inteso come alternativo di Ate; quest'ultimo non è attestato come prenome nelle coeve iscrizioni dell' Etruria settentrionale ${ }^{77}$, ma risulta attivo in ambito chiusino come gentilizio $^{78}$ ed è ritenuto alla base delle forme derivative atainei/ataini. Secondo H. Rix, il medesimo Ate deriverebbe dall'italico Attus $^{79}$. A tale riguardo, sembra opportuno qui richiamare l'analisi effettuata da A. Marinetti e successivamente ripresa da A.L. Prosdocimi sul latino atta e il sabino ${ }^{*} a p p a^{80}$. Atta è antroponimo ricordato da Svetonio per l'antenato della gens Claudia (Tib. 1). Questo sarebbe un "nome parlante", di forma sabina secondo Svetonio e invece latina seguendo l'ipotesi di A. Marinetti (in ogni caso comunque di ambito latino/sabellico), nonché un termine appartenente alla sfera della parentela, come indicato da Festo (11 e $13 \mathrm{~L})$. Dunque, in quanto termine paraonomastico, secondo Prosdocimi atta può identificarsi con un prenome all'interno della formula binomia assimilandosi così all'onomastica. Viene pertanto da chiedersi se proprio in questa forma si possa trovare l'origine del prenome etrusco Ata documentato ad Adria almeno dal III sec. a.C. ${ }^{81}$. Anche C. de Simone ha avvicinato il nostro prenome al latino atta, sebbene inteso come Lallname dai confini semantici sfuggenti e di larga diffusione interlinguistica ${ }^{82}$. Queste osservazioni aprono ovviamente il problema della trafila che ha portato all'acquisizione del nostro Ata, a cui è attualmente difficile rispondere in maniera circostanziata e senza espandere il ragionamento a un areale ben più ampio di quello adriese.

Tutti i prenomi esaminati sono associati ad antroponimi caratterizzati da terminazioni generalmente riconosciute come suffissi formativi di nomi derivati. Nelle occorrenze con nomi entrambi maschili, la terminazione più frequente è - $\mathrm{C}+i u$ con 4 casi (atiu, velOuriu, zurtiu, fuliu); seguono -ni con 2 casi (malni, śluniś), e quindi -alu (tetialuś), -na (śetina), -te (kleuste[ ) con un caso ciascuno. Di seguito si riportano alcune considerazioni puntuali per i nomi maschili caratterizzati da tali suffissi:

- ațiu: altrove non attestato. Potrebbe derivare dal termine ati, "madre", con l'aggiunta di un suffisso - $u$ oppure di un suffisso - iu. Risulta invece meno probabile riconoscere la base nel prenome Ata, in quanto l'ataiaś di Monte Bibele sopra ricordata mostra il mantenimento dell'alpha finale in associazione alla semivocale del suffisso; ma nel nostro non si può escludere la caduta di tale alpha (cfr. infra, zurtiuś). Si richiama quanto osservato da G. van Heems, il quale individuerebbe nell'atiu di una iscrizione chiusina $\left(E T^{2}, \mathrm{Cl} 1.2232\right.$; avvicinato anche alla forma ativu di Vulci: $\left.E T^{2}, \mathrm{Vc} 1.17\right)$, un valore di appellativo relativo al defunto, da attribuire secondo lo studioso alla sfera semantica della madre (forse "madre adottiva") 83 . Questo ovviamente non può essere il significato primario nella formula laris ațiu; tuttavia non pare da escludere che l'eventuale appellativo atiu possa aver svolto il ruolo secondario di nome familiare.

73 Ibid., pp. 90-94.

74 van HeEms 2008, pp. 77-78, 90.

75 Maggiani 2002a, p. 60.

76 Questa forma è già attestata nell'iscrizione di periodo orientalizzante mi ataias (ET ${ }^{2}$, Cr 2.41), nella quale l'antroponimo è riconosciuto come prenome in MARCHESINI 1997, p. 36, n. 41, e p. 105.

77 Sulla scarsa frequenza dei prenomi usati come gentilizi, cfr. Rix 1963, p. 349 (con riferimento specifico ad Ate), e quindi BENELLI 1998, p. 250.

78 Sul problema, BENELLI 2002a, in particolare p. 520; ID. 2011.

79 Rix 1963, p. 316.

80 MarinetTi 1982, pp. 169-181; Prosdocimi 2009, pp. 105-126.

81 Secondo un processo di formazione onomastica già indagato da A.J. Pfiffig (1971, pp. 35-39).

82 DE Simone 2001-2002, p. 101.

83 VAN HeEMs 2008, p. 84. 
- kleuste [: attestato come gentilizio con quattro occorrenze a Bolsena, una a Chiusi e nella lamina di Monti Pitti $\left(E T^{2} \text {, Po 4.4, del II sec. a.C. }\right)^{84}$. Il nome è un derivato del poleonimo di Chiusi, manifestando così in maniera chiara il suo processo formativo ${ }^{85}$.

- velOuriu: attestato nel III sec. a.C. come prenome con suffisso diminutivo -iu a Norchia in questa medesima forma ${ }^{86}$. Sebbene così sia stato interpretato da G. van Heems ${ }^{87}$, l'associazione con il sicuro prenome venza nell'occorrenza adriese porta a escludere che tale sia la funzione. V. Belfiore ha invece riconosciuto il nostro quale gentilizio e, come per le altre occorrenze adriesi che terminano in -riu, ipotizzato la possibilità che si abbia una trafila $-r a+i e+u /-r a+i u>-r i u^{88}$. Seguendo questa linea interpretativa, la base velOura è documentata ad Orvieto in periodo arcaico $\left(E T^{2}, 1.15,1.127\right)$, in Campania $\left(E T^{2}, \mathrm{Cm} 2.33\right)$, a Cerveteri $\left(E T^{2}, \mathrm{Cr} 2.16\right)$; durante l'età ellenistica la forma velouri ${ }^{89}$, modulata con il suffisso $-i e>-i^{90}$, è attestata come gentilizio maschile in ambito cortonese $\left(E T^{2}\right.$, Co 3.2) e femminile in ambito perugino $\left(E T^{2} \text {, Pe } 1.83\right)^{91}$, mentre il femminile velOuria è diffuso soprattutto in ambito chiusino $^{92}$. Ritenute le forme velOura/i derivanti dal prenome velOur ${ }^{93}$, l'ipotesi di una formazione dal gentilizio di area perugina potrebbe essere la soluzione più plausibile.

- zurtiuś: attestato in un'altra iscrizione adriese di II sec. a.C. (CIE 20785: $k$ zurtiu; cfr. supra). La base onomastica è nota anche in zurta e zurtalu, entrambi di III sec. a.C. A. Maggiani ha recentemente richiamato l'attenzione su questo gruppo onomastico ${ }^{94}$, che potrebbe trovare confronto con surte, attivo come cognome a Perugia ${ }^{95}$ (ma già noto nell'arcaico śurtenas a Montaperti) e riconosciuto da G. Colonna come probabile teoforico ${ }^{96}$; Rix ne aveva ipotizzato precedentemente la derivazione da una base umbra *sordo-, latino sordus (cioè sporco) ${ }^{97}$, e il medesimo Maggiani non ha escluso una derivazione da un ambito venetico (e.g. lat. Surtius di Aquileia). Se si ipotizzasse una base etrusca, bisognerebbe anzi tutto postulare un affricamento della sibilante palatale iniziale, che appunto in area perugina è possibile riscontrare nelle forme alternate zerturi $\left(E T^{2}\right.$, Pe 1.1053) in luogo del più diffuso serturi ${ }^{98}$ e zatna $\left(E T^{2}\right.$, Pe 1.232) per satna. Come già anticipato, la base zurt- è documentata ad Adria anche nella forma zurta (CIE 20514, dove è associato alla probabile abbreviazione pe: IV.3.2.A) che secondo G. Colonna è composto da un prenome teoforico sure con l'aggiunta del suffisso $-\theta a /$-ta (ritenuto dallo studioso non necessariamente femminile) ${ }^{99}$. Tale prenome teoforico è attestato nel territorio chiusino; in una occorrenza aretina ha invece la funzione di gentilizio ${ }^{100}$. Zurta potrebbe dunque essere interpretato come nome individuale, forse servile nel nostro caso (?), e alla base delle forme derivative zurtiu e zurtalu. Se valida l'idea di una generazione

${ }^{84}$ Si veda anche Morandi Tarabella 2004, p. 137.

85 Maggiani 2002a, p. 60.

${ }^{86} E T^{2}$, AT 1.167; Moranti Tarabella 2004, p. 468.

87 VAN HeEMS 2008, p. 83.

88 Belfiore 2014b, pp. 121, 145-146. Questa ipotesi tuttavia potrebbe risolvere, in via ipotetica, solo un caso delle molte terminazioni $-i u$, che si ritiene vanno analizzate nella loro totalità.

89 Cfr. Rix 1963, pp. 261 e 264.

90 Belfiore 2014b, p. 122.

91 La terminazione - $i$ per maschili e femminili è registrata come una prassi tipicamente perugina (cfr. BENELLI 2002a, p. 522).

92 Per un quadro generale delle attestazioni, cfr. Belfiore 2014b, p. 122.

93 Ibid., p. 122, nota 13 con riferimenti.

94 Maggiani 2002a, p. 60.

95 Rix 1963, p. 205.

96 Colonna 2009, p. 109.

97 Rix 1963, p. 227.

98 Benelli 2014, p. 65.

99 G. Colonna, in REE LXXIV, p. 310, n. 65.

100 Per le attestazioni: $E T^{2}$, AS 1.204, 1.284; Cl 1.421, 1.1047-1048, 1.2429; Ar 1.24. Su questo nome, si rimanda alle osservazioni di H. Rix e D. STEINBAUER, in REE L, pp. 263-264, n. 6. 
diretta di questi antroponimi da $z u r t a$, allora pare del tutto plausibile che il nome zurtiu sia formato direttamente sul suffisso $-i u(<z u r t a+i u)$.

- malni: attestato solo ad Adria (anche isolato: CIE 20895; cfr. IV.3.1.B, categoria b), derivante dalla radice mal(e) che produce la parola malena (cioè specchio ${ }^{101}$ ) e che è documentata nella $T C o \mathrm{e}$ nel lessema malstria del $L L^{102}$. Tale base non è tuttavia altrimenti nota come formativa di gentilizio in ambito etrusco. Nel caso di malni, è verosimilmente ritenere che alla base sia stato aggiunto il suffisso $-n i<-n i e$, attivo in ambito perugino ${ }^{103}$.

- setina: attestato in più occorrenze dalla medesima tomba (CIE 20569-20571). Per il gentilizio, Maggiani accoglie la proposta di G.B. Pellegrini di una derivazione venetica di ambito atestino ${ }^{104}$. Tuttavia, è forse più economico avvicinarlo al gentilizio perugino zetna (ET ${ }^{2}$, Pe 1.768-769), con un passaggio da fricativa a sibilante (ed eventuale anaptissi dello iota).

- siluniś: altrove non attestato. Maggiani propone una base venetica attiva anche nell'idronimo Silis, e già secondo G.B. Pellegrini legato al termine preromano sila (canale) ${ }^{105}$. Sebbene Maggiani ritenga tale base altrimenti ignota in Etruria, richiama comunque il perugino silai $\theta$, che parrebbe un etnico su base ${ }^{*}$ silai- o ${ }^{*}$ silaie- ${ }^{106}$. È comunque possibile ravvisare una spiegazione interna all'onomastica etrusca, se si richiama zilniś di Populonia $\left(E T^{2}\right.$, Po 2.10) del tardo IV sec. a.C., già avvicinato da Maggiani alla forma gentilizia zilini/zilni di Chiusi $\left(E T^{2}, \mathrm{Cl} .1 .259-260\right)^{107}$, nonché silu, attestato sempre a Chiusi come cognome $\left(E T^{2}, \mathrm{Cl}\right.$. 1.1139).

- tetialus: altrove non attestato. Si potrebbe ritenere plausibile una formazione da tetie, documentato come nome individuale nella Tomba delle Iscrizioni di Tarquinia di periodo tardo-arcaico (tetiie: $\left.E T^{2}, \mathrm{Ta} 7.16\right)^{108}$ e quindi attivo in età ellenistica in area chiusina come gentilizio $\left(E T^{2}, \mathrm{Cl} .1 .442\right)$, oltre che omografo del nome etruschizzato della greca Teti $\left(E T^{2}, \mathrm{Um}\right.$ S.1).

- fuliu: la forma con terminazione -iu non è altrove attestata. Fulu (bulu in area chiusina ${ }^{109}$ ) ha una ampia diffusione: documentato come Individualnamengentile nell'Arcaismo a Cerveteri ${ }^{110}$, è noto come gentilizio ma anche cognome in età ellenistica e il gentilizio fulni (/hulni) da questo trae la sua origine ${ }^{111}$. Sembra lecito ritenere che una derivazione dalla base fulu (peraltro attestata anche a Monterenzio: $E T^{2}$, Fe 2.30) sia possibile solo se si ipotizza che il suffisso -iu abbia sostituito la vocale terminale $-u$ della base (forse secondo un meccanismo di tipo analogico con altri gentilizi terminanti in -iu). Risulta peraltro da escludere che la forma fuliu derivi da un meccanismo di sincope, che in questo caso avrebbe eventualmente mantenuto la vocale finale della base onomastica ${ }^{112}$ (si ricorda a tal proposito il femminile fului con attestazioni tutte di età recente ${ }^{113}$ ) e piuttosto assorbito il suffisso.

Per i due nomi derivati al genitivo II e riconosciuti come femminili, si avanzano le considerazioni che seguono:

101 VAN DER MEER 1985, p. 95.

102 Agostiniani, Nicosia 2000, p. 106; Belfiore 2010, p. 141.

103 BeNELli $2002 \mathrm{a}, \mathrm{p} .522$.

104 Maggiani 2002a, p. 60, nota 38.

105 Ibid., p. 60, nota 37.

106 Ibid., nota 36.

107 Maggiani 1992b, p. 180, nota 15, p. 192, n. 50, fig. 15.

108 Morandi Tarabella 2004, pp. 534-535.

109 Cfr. Belfiore 2012, pp. 431-432, con riferimenti.

110 Marchesini 2007, p. 53, n. 15.

111 BENELLi 2014, p. 67 per un quadro di sintesi.

112 Rix 2000, p. 205.

113 Benelli 2014, p. 67. Attestazioni di fului: $E T^{2}$, Ru 2.12, Pe 1.1274, Vt 1.94, 2.21, 2.22. Si sottolinea che un passaggio da ypsilon a digamma prima dello iota avrebbe potuto portare confusione con la base onomastica *fulve, di imprestito latino; a tale proposito, si vedano le simili considerazioni in MARCHESINI 2007, pp. 59-60 per il bvuluves di Veio (CIE $6405,6445)$. 
- krapnal: questo gentilizio è di probabile area chiusina, dove è attestata la forma craupania $\left(E T^{2}\right.$, $\mathrm{Cl} 1.1390)$, dalla quale, come già riconosciuto da A. Maggiani, si avrebbe una riduzione del dittongo $-a u->-a_{-1}{ }^{114}$. Tale passaggio si attesta a Perugia e anche nella medesima Chiusi ${ }^{115}$ e dunque non è forse necessario pensare a una mediazione umbra come supposto da Maggiani.

- lausial: il nome è attestato isolato in altre due occorrenze ad Adria (CIE 20422, 20829; cfr. IV.3.1.B, categoria c). Nella tavola di Cortona è attestato lausa, che L. Agostiniani ritiene non altrimenti noto nell'onomastica etrusca ${ }^{116}$, e lausisa. Il secondo è interpretato come cognomen in -sa, la cui base onomastica è riconosciuta nella stessa di lausini attestata a Spina $\left(E T^{2}, S p\right.$ 2.72), a Cetamura in Chianti $\left(E T^{2}, \mathrm{Vt} 2.19\right)$ e a Chiusi $\left(E T^{2}, \mathrm{Cl} 1.1317\right)^{117}$, e che secondo H. Rix è formato sul nome individuale lausie ( $>$ recente *lausi), attestato a Fiesole in età arcaica $\left(E T^{2}\right.$, Fs 1.8: lavsieś), e composto con il suffisso $-n i^{118}$ (generalmente maschile; f. -nei). Più difficile secondo Agostiniani l'interpretazione di lausa. Escluso adesso il confronto con il graffito di Adria prima letto laus ta e ora appunto lausia (CIE 20422), per la base onomastica rimane anche in questo caso la trafila che ha a capo il nome individuale fiesolano di età arcaica. Se così, Agostiniani ritiene non chiara la finale $-a$, non escludendo un caso di aplografia $(\text { lau }<s i>s a)^{119}$. Diversamente, secondo C. de Simone, lausisa sarebbe una filiazione, dove il prenome lausi viene riconosciuto di derivazione dubitativamente umbra $\left({ }^{*}\right.$ Loukyo-s $>{ }^{*}$ Lousyo-s $>$ ${ }^{*}$ Lousis $){ }^{120}$. Nel nostro caso, la base onomastica, etrusca di area settentrionale o di derivazione umbra, sembra essere composta con il suffisso femminile -ia.

Nel complesso, i nomi di famiglia analizzati mostrano tutti basi onomastiche che con buona verosimiglianza possono essere ritenute etrusche. Alcuni sono da ritenere per formazione e suffisso sicuramente dei gentilizi (m. kleustẹ [, malni, śetina, siluniś; f. krapnal, lausial), mentre quelli con suffissi -iu (probabile in tutti i casi) e -alu sembrano perlopiù formati sulla base di nomi familiari.

Meritano un approfondimento le formule bimembri che presentano il gentilizio al genitivo I c.d. afunzionale (śluniś, tetialuśs ${ }^{121}$ e quelle con il gentilizio declinato al genitivo II, chiaramente femminile (krapnal, lausial) e che potrebbe esplicitare un legame familiare (metronimico) o una manifestazione di subalternità sociale ${ }^{122}$.

Per quanto concerne il genitivo afunzionale, vista la rarità del fenomeno ad Adria, ci si può domandare quale sia la diffusione in ambito padano. Una rassegna delle occorrenze (beninteso, quelle che non lasciano spazio a dubbi ${ }^{123}$ ), permette di documentare cinque casi, di cui il più antico è $A r n \theta$ Kapruśs della lamina di Marzabotto dei primi decenni del V sec. a.C. ${ }^{124}$; dopo un ampio vuoto documentale tra il V e il IV sec. a.C. ${ }^{125}$, seguono nel periodo ellenistico tre occorrenze di Spina: Larza

114 Maggiani 2002a, p. 59.

115 Colonna 2004b, p. 302, n. 3.

116 Agostiniani, Nicosia 2000, pp. 75-76.

117 Ibid., p. 76.

118 H. RIX, in REE LII, pp. 276-277, n. 1.

119 Agostiniani, Nicosia 2000, pp. 75-76.

120 De Simone 2009b, p. 538, A, 12.

121 Maggiani 2002b. Si richiama anche la precedente interpretazione di de Simone, che vi vedeva una Gruppenflexion (DE Simone 1970, p. 223). Per completezza, si rimanda anche all'iscrizione arza viufraluś (CIE 20821), di cronologia incerta, anch'essa caratterizzata da un gentilizio con genitivo afunzionale (cfr. infra).

122 Benelli 2013, p. 452.

123 Fra i dubbi, $E T^{2}$, Sp 2.4 .

124 Govi 2014, p. 121.

125 L'iscrizione della stele bolognese Ducati n. 17 degli inizi del IV sec. a.C. (ET'2, Fe 1.1: skaiva karinaś ex Maggiani), è adesso stata corretta in skapvas <a> karinaś (GAUCCI, Govi, SASSATElli c.s.). Secondo questa nuova proposta di lettura, ancora da porre al vaglio della critica, la defunta (la stele sembra con tutta evidenza femminile) è stata definita unicamente dal nome del marito a cui è stato successivamente aggiunto un secondo nome maschile con enclitico -sa (quello del padre? più improbabile ritenerlo il nome del padrone secondo lo schema arcaico dei nomi servili), secondo un principio di preminenza delle figure maschili che trova una chiara corrispondenza nella stele Ducati n. 14 del medesimo periodo. 
Farakanaś (come anche in $E T^{2}, \mathrm{Sp} 2.58$, o meglio Larz Afarakanas ${ }^{126}$ : i problemi di lettura non influenzano comunque il dato) della tomba 3 di Valle Trebba, datata al 320-280 a.C. ${ }^{127}$, e redatta sull'esterno di una ciotola a v.n. (sicuramente restaurata successivamente alla redazione del testo); Larza Atruś della tomba 156 di Valle Trebba ${ }^{128}$, databile entro la prima metà del III sec. a.C. e Laris Vipleś della tomba 1171 di Valle Trebba, della fine IV-inizi III sec. a.C. ${ }^{129}$, entrambe redatte su ciotole a v.n. Nell' iscrizione di Marzabotto, data la sicura destinazione monumentale, il nome appartiene sicuramente a un individuo ingenuo e di rango. Le attestazioni spinetiche risultano coeve a quelle adriesi, e anche queste non appartengono a iscrizioni di carattere "pubblico" (da intendere cioè visibili da tutti) o comunque di destinazione monumentale, ma furono tracciate su vasi destinati al corredo del defunto. Sempre a Spina, nel medesimo periodo l'iscrizione seslasa dalla tomba 1089 di Valle Trebba evidenzia l'uso del dimostrativo enclitico -sa per indicare verosimilmente un individuo di condizione servile tramite il nome del padrone, secondo una pratica dal sapore arcaico ${ }^{130}$. La probabile persistenza di questa modalità di identificazione per gli schiavi non dovrebbe stupire per un luogo come Spina, nel quale l'onomastica locale non sembrerebbe in effetti aver raggiunto il livello di standardizzazione (con le relative implicazioni sociali) dell'Etruria propria e quindi poteva eventualmente risultare difficile distinguere un ingenuo da uno schiavo sulla base del genitivo del gentilizio. Le tre formule spinetiche lasciano in ogni caso pochi dubbi, essendo i gentilizi associati a prenomi generalmente di ingenui quali Laris e Larza. Nel complesso, in Etruria padana il gentilizio con genitivo afunzionale sembra essere in uso almeno dal V sec. a.C. (l'Individualnamengentile Kapruś) e manifestarsi a Spina in età ellenistica, dove probabilmente risultava ben distinto dalle formule che esplicitavano una condizione servile. Ad Adria entrambe le occorrenze che lo registrano presentano suffissi derivativi nei gentilizi e anche qui i prenomi Laris e Larza.

Nelle due formule con secondo elemento al genitivo II, i prenomi maschili Arza e Ata sembrano associati a donne identificate dal solo nome familiare. L'uso di prenomi del pool onomastico già ritenuto pertinente agli individui ingenui e la possibilità che la condizione servile potesse essere indicata similmente che a Spina (e comunque non altrimenti documentata ad Adria), portano a ritenere che sia più probabile che i due nomi femminili esplicitino il metronimico. La preferenza del nome materno rispetto alla filiazione potrebbe dipendere dal più efficace valore di disambiguazione che questo poteva avere, attingendo a uno stock ben più nutrito e variegato di quello dei prenomi maschili generativi della filiazione ${ }^{131}$, che anche ad Adria almeno per il III sec. a.C. sembra piuttosto limitato. La specificità dei contesti, abitativo in un caso e da corredo funerario nell'altro, spiega il carattere incompleto di queste formule onomastiche. Sembra invece meno plausibile, ma non da escludere a priori, che l'uso del gentilizio femminile possa esplicitare il legame fra liberto e patrona. A tale riguardo, i bolli con iscrizione vel numnal ${ }^{132}$ mostrano una struttura formulare affine su cui vale la pena soffermarsi. Vel è ritenuto da G. Colonna servo della Numnei, evidentemente proprietaria dell'officina alla quale appartengono anche altri individuo di sicura condizione servile e nome non etrusco ( $\Theta a n s e: E T^{2}$, Vs 6.24). Certo, il nome ingenuo $\mathrm{Vel}$ per un servo rimane fatto ambiguo, tanto che il medesimo Colonna per spiegare l'anomalia ha avanzato l'ipotesi che questi possa essere stato un lautni che attorno al 100 a.C.

126 G. Colonna, in REE LXXIV, pp. 368-369, n. 113; A. MAGgiani, REE LXXXII, p. 292 (n. 38).

127 Muggia 2004, pp. 53-54.

$128 E T^{2}$, Sp 2.76; si veda anche BENELLi 2004, p. 262.

$129 E T^{2}$, Sp 2.59; per il contesto, si veda UGGERI 1978, p. 342, n. 2.

$130 E T^{2}$, Sp 2.83. Per il suo riconoscimento, cfr. Colonna 1993, p. 139. Nello stesso orizzonte temporale (fine IVprima metà III sec. a.C.), si riscontra una simile testimonianza anche a Populonia, dove un individuo è designato come umrke pelkasa, dove il primo elemento è da intendere l' Umbro", mentre il nome pelkaś( $+s a)$ non è altrimenti documentato (ET², Po 2.44; per l'iscrizione, cfr. Maggiani 1992b, pp. 184, 192, n. 48, fig. 15).

131 Cfr. Benelli 2002b, p. 97.

132 Si riporta qui l'elenco delle attestazioni desunto da $E T^{2}$ : $\mathrm{Cm}$ 6.2, La 6.1, Ta 6.13, AT 6.1, Vs 6.23, Vc 6.21, AV 6.7, OA 6.3, OI 6.2, 6.3, 6.6 . 
cambiò il suo nome servile con uno ingenuo nel momento di passaggio dalla formula onomastica di tipo etrusco a quella di tipo latino ${ }^{133}$. In ogni caso, lo speciale valore di questi bolli non consente di ritenerli efficacemente paragonabili alle due iscrizioni adriesi, per le quali dunque rimane più plausibile l'ipotesi che i gentilizi femminili siano dei metronimici.

Tra la fine del III e la prima metà del I sec. a.C. diminuisce sensibilmente l'uso dei prenomi attinti dal ristretto pool onomastico del secolo precedente, ora associati a gentilizi già ben noti come Mura (Anta, Larza) o di "aspetto" padano (Larza Lintialu). In particolare, Lintialu trova probabilmente la sua base onomastica nel greco Lindios ("il Lindio"; > etr. *lintie), mentre è meno probabile una derivazione celtica (se la base è lindon?) ${ }^{134}$. In effetti, a parte le occorrenze menzionate (anta afr mura, CIE 20434; mura larza pili, CIE 20621; larza lintialu, CIE 20992), le formule bimembri presentano entrambi gli elementi di origine alloctona, tutte concentrate dopo la metà del II sec. a.C. (kraiku.kaniu., CIE 20697; viza muliu ripetuta due volte, CIE 20923-20924; apu muliu, CIE 21013; фạia mi śiliu, CIE 20560). In queste sembra piuttosto evidente che il secondo elemento onomastico funga da gentilizio, come dimostra la diffusione del nome Muliu in varie sepolture della necropoli di via Spolverin a Bottrighe (nella fattispecie le tombe $46,106,112$ ) all' interno di quello che con tutta evidenza si può interpretare come un recinto funerario familiare ${ }^{135}$. Al riguardo, merita qui riprendere le fila delle riflessioni onomastiche su muliu e sui nomi viza e apu a questo associato, già avviate in precedenti lavori.

In occasione della prima edizione delle tombe 46 e $106^{136}$, dove muliu appare associato a viza nella prima (CIE 20923-20924) e isolato nella seconda (CIE21002), si era offerto un primo inquadramento degli antroponimi. La più recente identificazione dell' iscrizione apu muliu su un'anfora greco-italica della tomba 112 (CIE 21013) conferma quanto già supposto precedentemente, cioè il probabile valore di gentilizio per il nome muliu. Inoltre, quest'ultima iscrizione si aggiunge a quelle di viza muliu, offrendo dunque la possibilità di portare ulteriori riflessioni su questo gruppo familiare.

Per prima cosa conviene affrontare il nome di famiglia, Muliu. Già nella prima edizione delle tombe

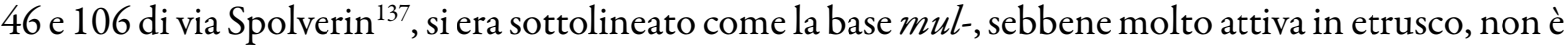
però produttiva nell'onomastica. Si è dunque avanzata l'ipotesi che tale radice possa trovare piuttosto origine nell'onomastica venetica, soprattutto nel nome Molon attestato a Padova nel V-IV sec. a.C. ( $L V, \mathrm{~Pa} 13)$ e Molo a Este e a Montebelluna nell'area plavense ( $L V$, Es 72 e Tr 1). A.L. Prosdocimi riconosce in questo nome la medesima radice del verbo latino molo (macinare) ${ }^{138}$, secondo una antroponimia, quella veneta, spesso costruita su basi con valore lessicale. Inoltre, riconosce in Mollo, Mollonia, Mollio la resa latina del nome venetico ${ }^{139}$. Va comunque ricordato che tale base è attiva anche nell'onomastica latina e in quella greca, entrambe presenti ad Adria nel corso del II sec. a.C.: Mul-/ Mull- latino è molto diffuso in Italia soprattutto centro-meridionale ${ }^{140}$, mentre per il greco si richiama fra altri il nome Mìnos, piuttosto diffuso tra il VI sec. a.C. e il periodo ellenistico, oppure altre forme

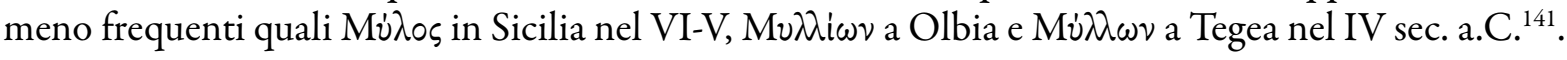

133 G. Colonna, in REE XXXV, pp. 560-561. Per una sintesi del problema con i relativi riferimenti bibliografici, MoRANDi TARABELla 2004, p. 336.

134 Holder 1904, 2, coll. 227-229; Delamarre 2003, p. 203.

135 Gaucci, Morpurgo, Pizzirani 2018, pp. 674-675, con riferimenti precedenti, fig. 16.

136 GaUCCI 2008.

137 In particolare, GAUCCI 2008, p. 88.

138 Per tale base si veda $L V$, vol. I, pp. 143-144. Qui, la base mol- è equiparata a molt-, sebbene quest'ultima adesso sia riconosciuta attiva in moltevebos della lamina di Este, come termine indicante un animale (cfr. MARINETTI 1998), forse l'ariete (MAGNIN 2014).

139 LV, II, p. 144; Prosdocimi 1988, p. 355.

140 ZGLE, pp. 424-428; PANCIERA et al. 1991, p. 384. Si ricorda anche l'iscrizione Appi Muli Philini dipinta su un'anfora (fine del I sec. a.C. - prima metà del I sec. d.C.) dal sito di La Nautique nella regione di Narbonne (Liou 1993, p. 140).

${ }^{141}$ Per questi nomi, cfr. $L G P N$ online. 
Anche per Viza e Apu non convince un inquadramento all'interno dell'onomastica etrusca. Viza non è altrimenti documentato nel corpus etrusco ${ }^{142}$, sebbene alcune forme derivate di Chiusi (vezra quale probabile cognomen: $E T^{2}, \mathrm{Cl} 1.1538,2743^{143}$; vizurisa quale gamonimico: $\left.E T^{2}, \mathrm{Cl} 1.2347\right)$ permettano di ricostruire una possibile base ${ }^{*}$ veze, sulla quale recentemente V. Belfiore ha fornito un quadro ${ }^{144}$. apu- ̀̀ solo dubitativamente isolato come formante gentilizi (apuna, apunies) $)^{145}$, ma non si può escludere che sia una variante fonetica rispetto al radicale di gentilizi quali apena e api$n a^{146}$. Scartata l'onomastica di base etrusca, che più plausibilmente ci si aspetterebbe, e premesso che molte potrebbero essere le possibili proposte di origine per questi due idionimi, è sicuramente più produttivo guardare all'esterno e il primo ambito da richiamare è quello del venetico.

Il mondo veneto non offre in realtà particolari sponde per viza, se non l'occorrenza viseio bo [ios di Este ${ }^{147}$, che comunque sembra suggerire per il legame con boios e per la sua unicità una base celtica (uesu-/uid-? $\left.{ }^{148}\right)$. Ammessa una frequenza significativa dell'onomastica di base celtica nel mondo veneto, dovuta a mobilità di individui oppure a fenomeni di moda ${ }^{149}$, rimane comunque difficile giustificare la terminazione - $a$ del nostro, se non come maschile (da originario tema maschile in $-/ \bar{a} /$ oppure eventualmente come analogia dei prenomi del tipo Larza). Il nome $A p u$ non trova invece alcun riscontro. Sicuramente si potrebbe richiamare l'appioi in dativo della tarda iscrizione in alfabeto latino e lingua venetica di Belluno ${ }^{150}$, ma questo non soddisfa pienamente la terminazione etrusca $-u$ del nostro. Una base celtica $a b$-/ap-attiva nell'onomastica in nomi monotematici e composti ${ }^{151}$ e il tema -on potrebbero spiegare la resa etrusca apu. Va aggiunto che tale base potrebbe essere riconosciuta nel toponimo/teonimo latino Aponus, che secondo Prosdocimi avrebbe forse alla base il possibile venetico *apon- (cioè "cosa che ha a che fare con l'acqua") ${ }^{152}$. Se quanto proposto può avere qualche sostanza, è possibile che $V i z a$ e $A p u$ siano due nomi di base plausibilmente celtica in uso all'interno di un milieu familiare di cultura originariamente veneta. Ciò non stupirebbe in linea generale e nella fattispecie nella necropoli di via Spolverin, dove due testimonianze epigrafiche di tradizione venetica possono confortare l'interpretazione. Infatti la tomba 112, oltre all'iscrizione apu muliu, conserva un digramma e un lessema in alfabeto venetico (CIE 21014); sia il testo etrusco che i due venetici sono su anfore greco-italiche (Fig. 22). In particolare, la lettura kala per il lessema venetico, più probabile che $k a p a$, orienterebbe ancora una volta verso una onomastica di base celtica, come rilevato da A.L. Prosdocimi per il kala[ $n]$ ioi dell' iscrizione su tripode bronzeo ${ }^{*}$ Es $121^{153}$. Anche la tomba 37 di II sec. a.C., prossima alla 46, sebbene divisa da questa da un corso d'acqua (Fig. 22), conserva l'iscrizione venetica .o.ne (CIE 20911), che va intesa come nome monotematico di base celtica ${ }^{154}$. Nel complesso, tutte queste testimonianze supportano l'idea di un nucleo di

${ }_{142} \mathrm{Si}$ ritroverebbe in ambito etrusco nel nome individuale vize, così riconosciuto da G. Colonna in una iscrizione da Vulci (G. Colonna, in REE XLVI, pp. 278-279, n. 132), sebbene tale lettura non sia accolta in $E T^{2}$, Vc 2.45, e in una iscrizione da Spina $\left(E T^{2}\right.$, Sp 2. 102: pitamn [---]vize), purtroppo fortemente lacunosa e dove il lessema individuato potrebbe non essere completo (si veda al riguardo l'apografo in UGGERI 1978, p. 369, fig. 2 ).

${ }_{143}$ REE LXXIII, p. 309, n. 40, dove l'editore intende il binomio ane vezra come nomen.

144 Belfiore 2014b, p. 121.

145 Morandi Tarabella 2004, p. 81.

146 BELFIORE 2014b, p. 42.

147 LeJEUNe 1974, n. 63, già $L V$, Es 8.

148 Delamarre 2003, p. 318.

149 Marinetti, Solinas 2016, p. 44.

150 Cfr. $L V$, Bl 1 e vol. II, p. 54, s.v. appioi.

151 Delamarre 2003, pp. 29-30.

152 Prosdocimi 1988, pp. 390-391. A completamente del quadro, si ricorda che una iscrizione lacunosa di V-IV sec. a.C. dal santuario lacustre di Montegrotto celerebbe forse il nome della divinità che poi in epoca romana sarà Aponus (Prosdocimi 1988, pp. 292-293; A. Marinetti, F. Veronese in Gamba et al. 2013, p. 336, 8.42).

153 Prosdocimi 1988, p. 255.

154 Delamarre 2003, p. 242. 


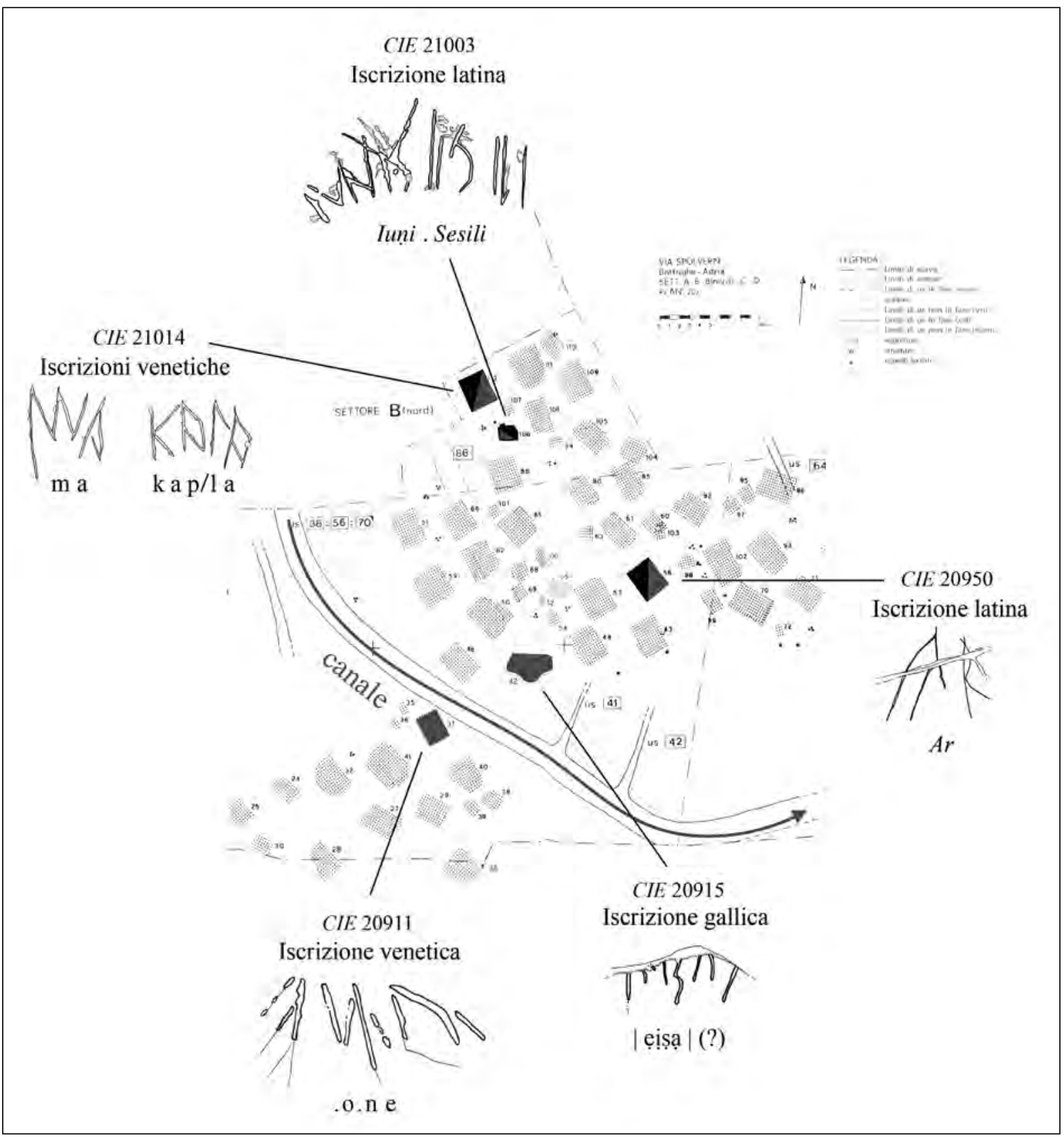

Fig. 22. Dettaglio della planimetria della necropoli di via Spolverin in loc. Bottrighe (da Bonomi, Peretto, TAMAssia 1993; cfr. Fig. 44). Sono indicate le sepolture che conservano le iscrizioni non etrusche più significative (apografi non in scala).

individui portatori di nomi monotematici di base celtica ma partecipi della tradizione epigrafica e più latamente culturale del mondo veneto, che si inseriscono nella comunità etrusca di Adria e dove almeno un gruppo familiare assume il gentilizio Muliu.

Altra formula bimembre è Kraiku Kaniu. Il primo elemento deriva chiaramente dall'etnonimo che identifica il "Greco". Questo potrebbe avere una derivazione diretta dal gr. Гpaıkós, oppure si può forse pensare a un percorso indiretto attraverso il venetico Graikoi, documentato in una iscrizione funeraria della necropoli di Casa di Ricovero di Este del medesimo periodo ${ }^{155}$. In entrambe le eventualità, ci

$155 L V$, Es 76 e vol. II, pp. 62 e 101. 
si aspetterebbe l'etr. creice, mentre kraiku presuppone il mantenimento del dittongo /ai/, comunque attestato in altri casi ${ }^{156}$, e il passaggio della terminazione $-o s>-u$. Rispetto a quanto osservato in altre occasioni ${ }^{157}$, l'ipotesi più economica è che l'antroponimo derivi direttamente dal greco. Se così, il secondo elemento Kaniu trova riscontro nell'onomastica greca di periodo ellenistico senza particolari

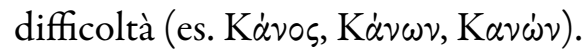

Sempre fra le formule bimembri va inserito il tardo Фaia Śiliu, che sembra tradire un'origine greca: il primo elemento può essere riconosciuto come l'etruschizzazione del maschile $\Phi \alpha i \tilde{\xi} \xi^{158}$, mentre il secondo può derivare da una base onomastica $\Sigma \hat{i} \lambda$ - ben documentata (forse il diffuso $\Sigma i \lambda \omega \nu$ ?) con la probabile aggiunta del suffisso -iu. Questa proposta permette di spiegare l'origine di entrambi i nomi. Diversamente, Maggiani ha visto in Śiliu una radice di origine venetica ${ }^{159}$, mentre non fornisce spiegazione per Фaia. Se valida l'interpretazione offerta, l'affinità con il Siluniś di III sec. a.C. (CIE 20264) potrebbe risultare pertanto solo formale. A queste osservazioni, si può aggiungere che due iscrizioni latine su stele funeraria con terminazione a disco da Canalbianco e Retratto, di fine I sec. a.C. - inizi I sec. d.C., testimoniano l'uso del femminile Silia, nel caso di Retratto come liberta, mentre in quello di Canalbianco associato al cognome Advena, che ne indica l'origine servile ${ }^{160}$. Nell'Adria tardo-repubblicana e primo-imperiale è pertanto presente una gens Silia, che potrebbe essere in continuità con il nostro Śiliu di circa un secolo prima.

Questi uomini di origine veneta e greca non dovevano essere servi affrancati. Infatti, tale condizione presupporrebbe l'adozione di prenomi etruschi fra i pochi già circoscritti nel III e che paiono ricorrere isolati anche nel II sec. a.C. (cfr. infra). Si ha piuttosto l'impressione di trovarci di fronte a individui liberi che ottengono i diritti sanciti dall'adozione del gentilizio, se tale il valore del secondo elemento della formula. Sembra pertanto plausibile ritenere che in questi casi i nomi familiari possano essere rimodulati su patronimici, sulla base di un meccanismo di analogia del suffisso -ios con l'etrusco -iu. D'altra parte, è ipotizzabile che il processo di sempre più accentuato avvicinamento alla cultura romana, che nel pieno del II sec. a.C. investe fra le altre cose l'urbanistica della città ${ }^{161}$, abbia favorito un indebolimento dell'onomastica etrusca quale strumento sociale, favorendo così nuove e più fluide dinamiche di integrazione prima del definitivo passaggio nell'orbita romana. Non sembra fortuito che proprio in questi contesti funerari più tardi come il recinto dei Muliu, si trovino testimonianze epigrafiche che rimandano al mondo celtico, veneto e anche latino (cfr. Fig. 22 e VI.4).

Esclusa l'iscrizione larza lintialu della fine III-inizi II sec. a.C., per il resto le uniche formule non monomie in onomastica di tradizione etrusca durante il secolo sono attribuibili solo alla gens Mura e manifestano l'uso del cognomen (Afr attestato nel territorio senese nella forma cognominale afur ${ }^{162} \mathrm{e}$

156 Si vedano le iscrizioni CIE 20330 e 20826, dove il dittongo è in sillaba interna; CIE 21002, dove il dittongo è in prima sillaba; si tenga conto anche delle uscite in -nai dei gentilizi femminili: arnai (CIE 20736-20737), lentnai (CIE 20461-20462, e forse 20463), titinai (CIE 20474-20475). Sebbene queste evidenze non si possano meccanicamente mettere a sistema, il mancato passaggio /ai/ > / ei/ tipico dell'etrusco recente sembra emergere come cifra caratteristica di Adria.

157 Gaucci 2008, p. 88; Gaucci, Pozzi 2009, p. 62, nota 38.

158 Per $\Phi \alpha i \alpha \xi$, attestato tra V e III sec. a.C. in Attica e nelle isole dell'Egeo, cfr. Osborne, Byrne 1994, p. 438, e Fraser, Matthews 1987, p. 452; per $\Phi \alpha i \alpha \xi$, attestato in Acarnania, a Corcira, Leucade e Agrigento tra V sec. a.C. e I sec. d.C., cfr. Fraser-Matthews $2001^{2}$, p. 441.

159 Maggiani 2002a, p. 60.

160 Forlati Tamaro 1956, p. 64, n. 27, e quindi Solin 1998, p. 95.

161 Bonomi, Robino 2007.

162 Una breve sintesi del lessema afrs e delle forme onomastiche affini è offerta in MASSARELLI 2014, pp. 83-84 e quindi pp. 112-113. Il lessema, già inteso come "antenati, parenti" dal Trombetti, successivamente seguito anche da Pallottino (cfr. ibid., p. 83, nota 5 con riferimenti), è ritenuto nome divino da Facchetti e Wylin (FACCHETTi 2000b, p. 262; WYLIN 2004, p. 215). Tale ultima interpretazione è richiamata anche da Massarelli, che ne riconosce il valore di genitivo e l'indipendenza grammaticale (MASSARELLi 2014, p. 113). Le forme onomastiche riconosciute dallo studioso come affini sono 
Pili privo di confronti). Nel caso dell' iscrizione mura larza pili, l'inversione fra prenome e gentilizio trova riscontro a Vulci e Tarquinia ${ }^{163}$, marcando così una pratica spiccatamente etrusco-meridionale che ben si accorda con le attestazioni note per il gentilizio di origine vulcente (mura: $E T^{2}$, Vc 1.27; f. murai: $\left.E T^{2}, 1.32,47,55,57\right)$.

Rimangono fuori dal quadro delle formule bimembri due maschili e l'unica femminile, più difficili da collocare cronologicamente:

- arza viufraluś (CIE 20821): mentre sul prenome Arza si è già detto, il genitivo afunzionale è associato al suffisso -alu del gentilizio come nel tetialuś di III sec. a.C. Una eventuale base onomastica *viufre non trova confronti in etrusco ${ }^{164}$. Alla luce delle considerazioni proposte per le attestazioni delle due fasi cronologiche precedentemente esposte, non pare fuori luogo ritenere che l'iscrizione CIE 20821 (il frammento di ciotola su cui è graffita non è facilmente tipologizzabile) sia pertinente al III sec. a.C.

- arza heturiu (CIE 20680): il gentilizio heturiu è stato recentemente oggetto di attenzione da parte di V. Belfiore in relazione all'analisi della morfologia dei suffissi derivativi. Nella fattispecie, la studiosa ipotizza per heturiu una formazione ${ }^{*}$ he $\theta u r /{ }^{*}$ vhetur ${ }^{165}+i u$ oppure con $+r a+u^{166}$, sebbene ritenga più probabile, data la tendenza alla caduta di /a/ finale davanti a semivocale, che a essere rimorfologizzata sia una forma già suffissata, es. $-i(e)+u^{167}$. Va anzi tutto puntualizzato però che, nel formulare questa considerazione, V. Belfiore seleziona alcune terminazioni in -riu (si intende ovviamente parlare di quelle del comparto adriese), senza affrontare tuttavia l'intera problematica dei nomi con terminazione -iu, di cui quelli in -riu sono un sottoinsieme (cfr. infra). Quanto alla base onomastica, sebbene le proposte della Belfiore manchino di altre occorrenze che possano sostanziarle, si ritiene comunque verosimile che il radicale abbia una origine interna all'onomastica etrusca $^{168}$.

- alpi arnai (CIE 20736-20737): due iscrizioni ripetono la medesima formula, eccezionale in quanto unica bimembre di genere femminile attestata ad Adria. Mentre in alpi si riconosce la base lessicale alp- (attiva nell'onomastica e nella sfera del sacro ${ }^{169}$ ) con l'aggiunta del suffisso di mozione femminile $-i$, arnai ha la sua radice in arne, documentato nella necropoli di Tolle in periodo tardo-arcaico ${ }^{170}$. Per quanto attiene il prenome, a fronte di una base riconoscibile come etrusca, questo è attualmente un hapax.

il nostro afr, e inoltre afrce, afur (ibid., p. 84, nota 2 per l'elenco delle attestazioni; si ricorda che tale affinità tra l'afr adriese e le altre basi è già evidenziata in MAGgiani 2002a, p. 60). Vale qui la pena richiamare anche una precedente lettura di Rix, che vede afur di origine italica, non escludendo una uguaglianza con il lat. Afer, supponendo che la presenza di ypsilon sia dovuta a una anaptissi (RIx 1963, p. 276). Di un legame con il lat. Afer, da intendere quindi con il significato di originario dell'Africa, è dell'avviso anche G. CoLONNA, in REE LXXIV, p. 369.

163 Morandi Tarabella 2004, pp. 90, 289, 384.

${ }^{164}$ Come già ricordato da A. Maggiani (in Akeo 2002, p. 183, n. 16), che tuttavia all'etrusco sembra comunque ricondurla.

165 Per l'ipotesi del passaggio grafo-fonetico da *vheturiu a heturiu, cfr. BELFIORE 2012, p. 433.

166 Belfiore 2014b, p. 130. La studiosa ipotizza invece una base *hetura ${ }^{*}$ heture a p. 145.

167 Belfiore 2014b, p. 145.

168 La stessa Belfiore, per la simile base heӨaria (comunque posta in alternanza a le $\theta a r i a$ su base archeologica e prosopografica; su questo, cfr. anche BENELLI 2014, p. 64), avanza l'idea di una possibile derivazione, ritenuta prudenzialmente speculativa, da una base *he $\theta$ - (BELFIORE 2014b, p. 130, nota 5), riconosciuta come verbale nel $L L$ (BELFIORE 2010, p. 180).

169 Cfr. Maras 2009b, pp. 82-83.

170 Benelli 2015, pp. 410-411; G. Paolucci, E. Benelli, in REE LXXIX, pp. 319-320, n. 73. Per la forma femminile Arni, si veda Morandi Tarabella 2004, p. 87. 


\section{IV.3.1.B. Antroponimi isolati}

Gli antroponimi isolati possono essere suddivisi in 4 categorie (Tab. 3).

Tab 3. Categorie degli antroponimi isolati.

\begin{tabular}{|l|c|c|c|}
\hline & FINE IV-III SEC. A.C. & FINE III-II SEC. A.C. & III-II SEC. A.C. \\
\hline a) prenomi maschili & 2 & 4 & 2 \\
\hline b) nomi familiari maschili & 6 & 9 & 4 \\
\hline $\begin{array}{l}\text { c) nomi femminili } \\
\text { (prenomi e gentilizi) }\end{array}$ & 1 & 8 & 2 \\
\hline $\begin{array}{l}\text { d) nomi individuali o di incerta } \\
\text { interpretazione }\end{array}$ & 8 & 7 & 1 \\
\hline
\end{tabular}

a) Tra III e II sec. a.C. due prenomi maschili ricorrono isolati: Ata (CIE 20364, III sec. a.C.; CIE 20628, II sec. a.C.; CIE 20258, III-II sec. a.C.) attestato anche nelle formule binomie (a cui si rimanda), e Kavi (CIE 20603, 20772, III sec. a.C.; CIE 20777, II sec. a.C.), non documentato in associazione a gentilizi. Quest'ultimo trova puntuale confronto in una coeva iscrizione di Spina, kaviśtitaluś, dove figura come prenome ${ }^{171}$. Risalendo indietro nel tempo, cavi è documentato isolato in una iscrizione arcaica da Poggio Colla nel Mugello, redatta secondo A. Maggiani da un individuo probabilmente proveniente dall'Etruria meridionale (Tarquinia, Vulci o meglio Orvieto) ${ }^{172}$; nell'iscrizione in norma meridionale di uno specchio della prima metà del V sec. a.C., forse da intendere come prenome maschile, esprimente filiazione seguendo l'interpretazione di V. Belfiore ${ }^{173}$; in un testo votivo dal santuario di Sant'Antonio di Cerveteri datato al pieno V sec. a.C. ${ }^{174}$, nel quale D.F. Maras ritiene cavi nome individuale di un artigiano preposto al restauro dell'oggetto iscritto piuttosto che un Individualnamengentile o un prenome. Proprio in merito a quest'ultima occorrenza, la terminazione $-i$ viene risolta da Maras ipotizzando questioni di pronuncia o un passaggio cavie $>$ cavi, plausibile per il parallelo con altri antroponimi in ambiente ceretano ${ }^{175}$. Più recentemente, V. Belfiore, in merito a kaviieśi di una iscrizione ceretana di VII sec. a.C. ipotizza che questo sia costruito su un originario *kave, trasformato in gentilizio con il suffisso -ie; la base onomastica sarebbe secondo la studiosa di derivazione latina ${ }^{176}$. Va tuttavia evidenziato che la forma in questione si localizza nelle attestazioni di questo nome nel sud-piceno, nell'osco e nel falisco rispetto a quella priva della labiovelare diffusa invece in area umbra e latina (cioè Gavius vs. Gaius) ${ }^{177}$, come già evidenziato per i kai della prima metà del V sec. a.C., di probabile origine umbra (cfr. IV.1). Proprio l'ambito falisco conserva nel III sec. a.C. il valore prenominale di kavie in una iscrizione dal santuario capenate di Lucus Feroniae ${ }^{178}$. Tale funzione sembra già essere documentata in età arcaica, come dimostra la formula etrusca con prenome+gentilizio kavie pẹsente del cippo in pietra fetida di una sepoltura di

$171 E T^{2}$, Sp 2.125; G. ColonNa, in REE LXXIV p. 372, n. 123.

172 Maggiani 2015, p. 152 e tav. XXIIII a.

${ }^{173} E T^{2}$, OA 2.95. Per l'interpretazione, si veda BELfiore 2011, p. 53; tale interpretazione è confermata da D. Briquel e R. Hirata, che sulla base di nuova documentazione grafica e fotografica, rilevano la geminazione dello iota anche in spuriies (D. BriQuel, R. Hirata, in REE LXXX, pp. 308-309, n. 46).

${ }^{174} E T^{2}$, Cr 3.44 e Maras 2009b, pp. 271-274, Cr do 2-3, con ampia bibliografia.

175 Maras 2009b, p. 274.

176 Belfiore 2014b, p. 54 e nota 8 con riferimento a Solin, SAlomies 1994, p. 51. Si veda al riguardo anche ZGLE, p. 76.

177 Agostiniani, Calderini, Massarelli 2011, p. 33.

$178 E T^{2}$, Fa 2.25; G. Colonna, in REE XLIV, p. 251, n. 64. 
Tolle ${ }^{179}$. Dunque, rispetto a una forma cavie attestata almeno dal VII sec. a.C., tra VI e V sec. a.C. è documentata in Etruria meridionale una forma cavi, con probabile valore di prenome maschile almeno nel V sec. a.C. ${ }^{180}$. Se, come sottolineato da Maggiani, l'occorrenza di Poggio Colla traccia un percorso verso l'area padana, allora è forse tramite questa direttrice che il prenome si è diffuso nelle comunità etrusche del comparto transappenninico, dove emerge in periodo ellenistico a Spina $\mathrm{e}$ ad Adria in questa forma e sempre come prenome. Ciò può trovare conferma anche nel femminile cavia dall'abitato della Pianella di Monte Tamburino, anch'esso di III sec. a.C. e interpretato come prenome ${ }^{181}$. Che peraltro kavi non sia un caso episodico è confermato dalla sua iterazione ad Adria sia nel III che nel II sec. a.C. L'incidenza di forme kavi (m.) / cavia (f.) in ambito padano di III-II sec. a.C., è così forse la tarda emergenza di un prenome in uso da epoca più antica.

Nel II sec. a.C. si contano anche possibili forme abbreviate o per meglio dire relitte (laz<larza?, CIE 20411; vna < venza?, CIE 20817). Rimane infine di cronologia più incerta l'iscrizione larśa (CIE 20827), che testimonia il prenome etrusco Larza reso con un sade a farfalla al posto dello zeta. In effetti, a ben vedere, l'associazione di questo grafema e dell'aspetto a pettine dell'alpha sembra indirizzare verso forme grafemiche proprie dell'ambito epigrafico celtico (come già detto, cfr. III. 2). Lo stesso uso del sade in concorrenza al molto raro zeta in quest'ultimo ambito sembra confermare questa osservazione ${ }^{182}$. Dunque, dietro l'emergere di queste peculiarità distanti dalla pratica epigrafica etrusca di Adria, ci potrebbe essere un individuo detentore di multi-competenze linguistiche e scrittorie non pienamente dominate, forse cresciuto e istruito in un milieu culturale celtico. Stanti queste considerazioni, basate sulle evidenze epigrafiche, solo come suggestione e se valida l'identificazione fra nome e individuo scrivente (cfr. IV.3.2), viene da chiedersi se questo Larza/Larśa non possa essere un liberto, che dunque ha assunto un prenome etrusco dal pool onomastico degli ingenui una volta affrancato dal padrone. Purtroppo, l'assenza di formule onomastiche più esplicite relative a questa categoria sociale ad Adria, come nel resto dell'Etruria padana, non permette di avere la certezza che i nomi acquisiti con l'affrancamento seguano le stesse dinamiche di quelle note per l'Etruria propria.

b) Nel III sec. a.C., nomi familiari con suffissi derivati si riconoscono con sicurezza negli antroponimi isolati malni (CIE 20895) e mura (CIE 20663). Il primo è noto come nome familiare nell'iscrizione venza malni, mentre il gentilizio di origine vulcente Mura è stato già menzionato per le formule trimembri di II sec. a.C. (cfr. per entrambi IV.3.1.A). Sempre durante questo secolo, si registrano alcuni nomi isolati aventi suffisso derivato e non altrimenti attestati: preznaś; tre attesta-

179 G. Paolucci, E. Benelli, in REE LXXIX, pp. 319-320, n. 73. Nei restanti casi, l'antroponimo risulta isolato o in testi di discussa interpretazione. Per l'iscrizione rupestre arcaica dalla Banditaccia di Cerveteri (CIE 5993), il nome nella formula mine zineke kavie è interpretato da Colonna come Individualnamengentile (G. ColonNA, in REE LXXVIII, p. $247, \mathrm{n} .62)$. Per l'iscrizione mi cavias su un piattello di origine incerta, probabilmente di V-IV sec. a.C. e ritenuto da A. Maggiani di area volsiniese: REE XLVII, pp. 326-327, n. 33. Per altre occorrenze: Morandi Tarabella 2004, p. 260. Cavies isolato è noto anche nell' iscrizione di IV sec. a.C. cavies huze cena: G. ColonNA, in REE XLVI, p. 349 , n. 102 e $E T^{2}$, Cr. 2.74. Per l'iscrizione arcaica con prenome maschile avente funzione patronimica da Orvieto: $E T^{2}$, Vs 1.99; Moranti Tarabella 2004, p. 242.

${ }_{180}$ Briquel e Hirata avanzano la possibilità che il Cavi Spuriie dello specchio di Tokyo, sopra menzionato, possa essere di diretta derivazione italica (indiziata dalla differenziazione dei due nomi e con richiamo all'osco), come suggerirebbe la terminazione - $i$ del prenome e -iie del gentilizio (D. BRIQUEL, R. HiRATA, in REE LXXX, p. 309). Sempre in età arcaica si registra anche il femminile cavia (per l'iscrizione arcaica femminile del Museo di Coutrai mi kavias kalaisias: ET ${ }^{2}$, OA 2.11; De Simone 1975, p. 137, n. 26; MARCHESINI 2007, p. 25, che ipotizza un imprestito dall'italico con integrazione del morfema -ia; Rigobianco 2013, pp. 114-115, che ritiene il nome diretta derivazione dall'onomastica italica oppure femminile in - $a(-)$ dal maschile cavie) .

${ }^{181} E T^{2}$, Fe 2.15; SASSATELLi 2008b, p. 341, n. 8, fig. 23. Peraltro, il gentilizio ataiaś potrebbe confermare un legame con il comparto adriese (cfr. supra).

182 Cfr. Motta 2000, p. 184. 
zioni con suffisso -alu (estaluś, zurtalu e anche ]ialu, che sebbene lacunoso deve essere con buona verosimiglianza isolato); cinque attestazioni con terminazione -iu (kraiu, leiuś, sesiu, siӨiu, fastiu).

Preznaś (CIE 20819) è da riconoscere come gentilizio e trova un confronto puntuale a Cortona, dove è attestato come metronimico (preznal: $E T^{2}$, Co 1.26$)^{183}$ e nella forma con suffisso -te nel territorio chiusino (presnte, f. presnti). Per quanto riguarda gli antroponimi con suffisso -alu, oltre al lacunoso ]ialu (CIE 20019) e a zurtalu (CIE 20685, la cui base onomastica è senza alcun dubbio zurta: cfr. IV.3.1.A), si ha estaluś (CIE 20181). Per quest'ultimo C. De Simone ha richiamato il retico ${ }^{*}$ estua (la cui base è piuttosto attiva in questo corpus onomastico ${ }^{184}$ ) e proposto l'interpretazione come gentilizio con suffisso - $a l u^{185}$. Più in generale, il medesimo de Simone ha osservato che nelle basi onomastiche composte con tale suffisso, la vocale finale è elisa prima dell'alpha di -alu ${ }^{186}$.

Cinque lessemi (kraiu, leiuś, sesiu, siliu, fastiu) presentano la terminazione -iu, attiva nella designazione di gentilizi (cfr. IV.3.1.A). Tuttavia, non si reputa di poter riconoscere come gentilizio sesiu (CIE 20028) e sitiu (CIE 20788), non essendovi ragioni per ritenere che tali basi siano attive nell'onomastica (la terminazione -iu non risolve di per sé il problema; per questi lessemi, cfr. V.4). Leiu(ś) lascia pochi spiragli a una lettura in chiave diversa da quella onomastica, con una base riconosciuta da Maggiani attiva in ambito volsiniese, come attesta la gens Leinies (lei-na-ie-s) della Tomba Golini I di Porano $^{187}$. Va tuttavia ricordato che proprio il gentilizio leinies è stato interpretato da G. Colonna come teoforico da $\operatorname{Lein} \theta$ (riconosciuta con Persefone) e generato secondo una trafila ${ }^{*}$ Lein $\theta i e>{ }^{*}$ Leinnie $>$ Leinie $^{188}$. Il teonimo alla base del nome è inoltre accostato al predicato leine, circoscritto alla cultura epigrafica volterrana; se il predicato significa "morire" o "essere morto" per K. Wylin ${ }^{189}$, per Colonna la sfera semantica è più ampia, cioè quella del "passare" (da uno stato all'altro), e quindi morire oppure nascere. Si potrebbe riconoscere la base lei-anche nel cognome lei $\chi u$, noto solo in ambito chiusino ${ }^{190} \mathrm{e}$ che potrebbe essere un Verbalnomen con funzione simile a $z i \chi u^{191}$. Se valida l'idea che il radicale possa essere $l e i(n)-{ }^{192}$, allora per il nostro leiu, forse dalla trafila $l e i(n)+u / i u$, è forse da vedere non tanto una base onomastica (comunque possibile), quanto piuttosto un lessema pertinente alla sfera della morte intesa come passaggio. In questo caso, la formula di possesso mi leiuś indicherebbe la pertinenza del vaso al leiu, che potrebbe dunque essere un appellativo (il defunto?) piuttosto che un nome proprio.

Rimangono kraiu (CIE 20330) e fastiu (CIE 20776). Per kraiu una base onomastica sembra essere l'unica soluzione. Questa potrebbe trovare un possibile confronto con i Kreinie di Pieve a Socana, che G. Colonna ha proposto di derivare dal greco Graios ${ }^{193}$, sebbene nel nostro non si abbia il passaggio del dittongo /ai/ verso la monottongazione, similmente ad altre occorrenze adriesi (su ciò, cfr. IV.3.1.A, $k r a i k u)$. Tale derivazione presuppone il passaggio gr. -os $>$ etr. $-u$, non altrimenti attestato in un orizzonte di III sec. a.C., ma supposto per quello seguente nel nome Kraiku ${ }^{194}$. Non si può escludere che

183 Cfr. già Maggiani 2002a, p. 59, dove si richiama anche la base onomastica prezu, attestata a Orvieto agli inizi del IV sec. a.C. (ET $T^{2}$ Vs 3.4).

184 De Simone 1970, p. 223, nota 49. Per tale base, cfr. Morandi 1999, pp. 79-80 e A. Marinetti, in Akeo 2002, p. 191, nn. 22.7-8, con ulteriore bibliografia.

185 Così anche Sassatelli 1991, p. 710, n. 18.

186 DE Simone 1970, p. 223.

187 Maggiani 2002a, p. 59. Per il gentilizio, cfr. Morandi Tarabella 2004, pp. 281-285.

188 G. Colonna, in REE LXXIII, pp. 305-306, con riferimento alla scheda di N.T. DE GRUmmOnd, in ibid., pp. 303-304, n. 37, relativa a un frammento di fondo di forma aperta a v.n. con rotellature e iscrizione lacunosa, dove si legge mi lein [---] o mi leim [---].

189 WyLIN 2000, p. 105.

190 Benelli 2014, p. 66.

191 Rix 1963, p. 189.

192 Su questo, ibid., p. 104.

193 Colonna 1985, 9.3.C.

${ }_{194}$ Per kraiku nel II sec. a.C., cfr. IV.3.1.A; per altre terminazioni in -e e - $u$ da basi onomastiche di origine allogena, si veda infra, d); per considerazioni sul suffisso etr. -iu e il suo rapporto con gr. -os, cfr. IV.3.3). 
kraiu sia piuttosto da intendere come derivato in -iu, cioè come nome di famiglia. Fastiu ha la sua base onomastica nel nome femminile fasti/fastia ${ }^{195}$; proprio sulla base di fastiu, G. van Heems ritiene che il suffisso -iu sia epiceno ${ }^{196}$, benché questo sarebbe valido se avessimo la certezza che il nostro fosse un diminutivo di fasti e non un antroponimo derivato.

Nel II sec. a.C., alcuni gentilizi registrati in formule trimembri e bimembri ricorrono anche isolati (cfr. IV.3.1.A) nelle seguenti iscrizioni: mura (CIE 20709), muliu (CIE 21002), e il più tardo siliu (CIE 20559). Fra i nomi isolati non altrimenti attestati, si riconoscono come gentilizi at [vacat] na (CIE 20997), spiu (CIE 20534) e unitiu (CIE 20489, 20541). Atna e Spiu trovano puntuale corrispondenza in Etruria settentrionale, rispettivamente a Perugia ${ }^{197} \mathrm{e}$ in loc. Guistrigona presso Castelnuovo Berardenga ${ }^{198}$. In particolare, le iscrizioni su coperchi di urna della tomba a camera nel Senese hanno permesso a A. Maggiani di ricostruire una prosopografia familiare di tre generazioni (due accertate direttamente dalle iscrizioni) compresa tra gli ultimi decenni del III e la metà del II sec. a.C. ${ }^{199}$; tale cronologia è in fase con quella della tomba 175 di Canalbianco nella quale è conservata la nostra iscrizione, documentando quindi con buona probabilità come nel medesimo periodo un gruppo di questa gens fosse stanziato ad Adria. Rimane più complessa da interpretare la base onomastica di uni si hanno due occorrenze in tombe diverse (CIE 20489 di fine III-inizi II sec. a.C.; 20541 di II sec. a.C.). F. Piva ha proposto il confronto con uneiӨaś dall'anforetta Melenzani di Bologna della fine del VII sec. a.C. $\left(E T^{2}, \mathrm{Fe} 2.1\right)$ e da una iscrizione chiusina di periodo arcaico $\left(E T^{2}, \mathrm{Cl} 2.5\right)$; tale nome è formato da un suffisso di mozione $-\theta a^{200}$ e G. Colonna lo interpreta adesso come un antroponimo teoforico maschile formato sulla base unei/uni ${ }^{201}$, mentre il nostro ne sarebbe una forma diminutiva. Diversamente,

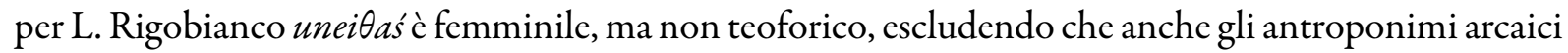
prima menzionati e le nostre più tarde occorrenze adriesi lo siano ${ }^{202}$. Si può nel complesso ritenere che unitiu derivi dall'arcaico unei $\theta a s$, attestato significativamente anche in ambito padano. Come suggeriscono le osservazioni di Colonna ${ }^{203}$, si potrebbe qui avere un parallelo con $z$ urta $>$ zurtiu.

Fra i nomi familiari isolati privi di suffisso derivativo, si reputa di poter inserire sinu (CIE 20828) di II sec. a.C., benché questo non trovi puntuale confronto. È ben attestata la forma con sibilante iniziale dentale śinu quale cognomen (ma usato anche come gentilizio) in ambito chiusino (Monteroni d'Arbia: $E T^{2}$, AS 1.7, 1.8; Cl 1.610, 1.957, 1.2261); tale base onomastica è nota quale nome individuale anche a Marzabotto alla fine del V sec. a.C. $\left(E T^{2}\right.$, Fe 2.21). Si osserva in particolare che nel Chiusino la forma femminile analogica śinunia $\left(E T^{2}, \mathrm{Cl} 1.42,100,101,2599,2262\right)^{204}$ è attestata anche con sigma iniziale: $\operatorname{sinunia}^{205}$. Sebbene non si possa escludere che il nostro sinu sia un nome individuale, alla luce delle considerazioni riguardo le attestazioni di nomi familiari isolati redatti sul vasellame adriese, non pare inappropriato darne quest'ultima interpretazione (cognomen?). Per l'antroponimo di Marzabot-

195 Rigobianco 2013, pp. 126-127.

196 VAN HeEms 2008, p. 84.

197 Gli Atna sono anche titolari di un ipogeo a Rosavecchia presso Tarquinia, da cui si sarebbero spostati verso Perugia nel IV sec. a.C. (Morandi TARABELla 2004, p. 634).

198 Cfr. già F. PIVA, in REE LXV-LXVIII, pp. 306-307, n. 7 su questo gentilizio ad Adria. Per le occorrenze senesi, cfr. A. MAGgiAni, in REE LIV, pp. 233-235, nn. 26-29 (ET ${ }^{2}$, AS 1.42, 43, 44, 45), da una tomba a camera.

199 Ibid., p. 235.

200 Rigobianco 2013, pp. 93-94, nota 483.

201 G. Colonna, in REE LXXIV, p. 310 (n. 65).

202 Rigobianco 2013, p. 111, n. 18, p. 141, nota 899.

203 G. Colonna, in REE LXXIV, p. 310 (n. 65).

204 BENELLi 2014, p. 67.

${ }^{205}$ Questo vale almeno per $E T^{2}, \mathrm{Cl} 1.143$. Invece $E T^{2}, \mathrm{Cl} 1.142$ presenta l'iniziale di sinunia ma anche il metronimico con sigma e non sade (come invece $E T^{2}, \mathrm{Cl} 1.143$ ), e in $E T^{2}, \mathrm{Cl} 1.2598$ sinunias presenta entrambe le sibilanti dell'iniziale e del genitivo I quali sigma; si tratterebbe quindi di redazioni che seguono la norma meridionale, al pari del più ovvio caso di Bolsena (sinun $[i a], E T^{2}$, Vs 1.239; MoRANTI TARABELLA 2004, p. 467), dove il sigma è dovuto alla pratica scrittoria meridionale. 
to, la più antica delle attestazioni, Colonna ha proposto una origine greca da $\sum i \nu \omega \nu^{206}$. Tuttavia tale base onomastica potrebbe trovare corrispondenza in un possibile radicale */si/-ipotizzato da V. Belfiore per alcune occorrenze del $L L$, ed in particolare sin, inteso come forma verbale legata forse alla sfera semantica dell'accoglienza ${ }^{207}$ e attestato anche a Spina sul fondo di una coppa in depurata datata al più tardi alla fine del V sec. a.C., già avvicinata all'antroponimo di Marzabotto da G. Sassatelli ${ }^{208}$. Solo una suggestione, priva purtroppo di ulteriori dati che la possano supportare, è il richiamo a una iscrizione latina su stele funeraria di Adria del I sec. d.C., che conserva il testo ${ }^{1}$ Amarylidi ${ }^{2} C$ Senti ${ }^{3}$ an $X X I^{209}$, dove il padrone della schiava Amaryllis è un Sentius. Ciò evoca l'iscrizione bilingue di Chianciano dove compare il cognomen śinu nella parte etrusca e appunto il gentilizio Sentius in quella latina ${ }^{210}$.

Altri nomi isolati con suffissi derivativi con probabile valore gentilizio possono essere datati solo genericamente al periodo ellenistico: ankariu (CIE 20677-20679) e kepriu (CIE 20236) con terminazione -iu; titalu (CIE 20051) con terminazione -alu; haltva (CIE 20133) con probabile terminazione -va.

Ankariu è formato su una base onomastica ancar, attiva in diverse forme derivate con valore di gentilizio in area chiusina (ancari, ancaru, ancarni), ma anche in Etruria meridionale (anxarie e ancare) ${ }^{211}$. Due indizi concorrono per vedere in Ankariu un nome familiare: la terminazione -iu e l'attestazione dell'iscrizione latina ${ }^{1} C$ Ancharius ${ }^{2} C l$ Philastus su una stele funeraria con terminazione a disco del I sec. a.C. recuperata a Canalbianco il 15 febbraio $1938^{212}$. La base onomastica di kepriu ${ }^{213}$ potrebbe avvicinarsi all'etrusco cepen ${ }^{214}$ (a cui forse sono da affiancare cepta e cepar? ${ }^{215}$ ). Si potrebbe in alternativa, ipotizzare un Palatal-Umlaut ${ }^{216}$, documentato in etrusco, tale da avere kapr-/kepr-. In tale senso, kepriu potrebbe essere una attestazione tarda di un Individualnamengentile già noto in Etruria padana poco dopo il 500 a.C. a Marzabotto (Kapruś) $)^{217}$, e quindi in età recente in territorio chiusino (m. Capru; f. ${ }^{*}$ Caprunei $)^{218}$. Se questa ipotesi può avere qualche fondamento, allora nel nostro kepriu la /u/ finale potrebbe essere caduta quando la base è stata rimorfologizzata con il suffisso -iu.

Titalu potrebbe derivare da un originario tite con l'aggiunta del suffisso -alu. Il confronto con lo spinetico kaviś titaluś ( $E T^{2}$, Sp 2.125; già citato per kavi della categoria a) ne chiarifica il valore di nome familiare.

Si segnalano inoltre zuхu (CIE 20320) e tite (CIE 20084) di più generica datazione al periodo ellenistico. Fra questi, tite è sicuramente attivo e molto diffuso come nome familiare ${ }^{219}$. Zuれu è attivo come

206 G. Colonna in Guida 1980, p. 114. Per una trattazione completa dell' iscrizione di Marzabotto, si veda la scheda di G. Sassatelli, in SASSATElli 1994, p. 18, n. 2 (dove si ricorda una iscrizione bilingue di Chianciano, per la quale si veda oltre).

207 Belfiore 2010, pp. 122-123, in particolare nota 5 con riferimento a Cortsen e Pfiffig per tale interpretazione.

208 Sassatelli 1994, p. 18; per l'iscrizione di Spina, cfr. G. Uggeri, in REE XLVIII, p. 356, n. 46 (ET², Sp 2.17).

209 Forlati Tamaro 1956, p. 69.

${ }^{210}$ BeNELLi 1994, p. 23, n. 13, p. 57. In questa iscrizione all'etrusco śinu corrisponderebbe il latino Sentius, sebbene nel primo caso śinu sia il cognomen con gentilizio sottinteso e nel secondo Sentius il gentilizio.

211 Belfiore 2014b, pp. 132-133, con riferimenti. Per l'area etrusco-meridionale, cfr. Morandi Tarabella 2004, p. 70.

${ }^{212}$ CIL V, 2317; Forlati TAmaro 1956, p. 57, n. 15. Alla stessa gens potrebbe appartenere anche un bollo su laterizi (si veda Cipriano, Mazzocchin 2007, passim).

213 Non considerato nell'analisi delle terminazioni onomastiche in -riu di BELFIORE 2014b.

214 Su cui da ultimo Adiego 2006, che ne analizza le occorrenze per scioglierlo dal significato di "sacerdote" finora prevalso in letteratura (analisi accettata anche nel quadro di sintesi offerto in Agostiniani 2013, p. 470).

215 Per cepar, Belfiore 2010, p. 144, nota 5.

216 Rix 1987-1988, p. 188.

217 Govi 2014, p. 121.

218 BeNELLi 2014, p. 65.

$219 T h L E^{2}$, pp. 391-392, con le relative voci riferibili a questo nome. Per l'ipotesi di una derivazione dall'arcaico titaie, da intendere come Echte Gentile e non Vornamengentile, si veda BENELLi 2011, p. 197. 
cognomen in ambito chiusino $\left(E T^{2}, \mathrm{Cl} 1.1619,1769,1770,1771,2173\right)$ e già attestato in età arcaica ad Orvieto, dove è interpretato come Individualnamengentile, e sulla fine del IV sec. a.C. a Corchiano ${ }^{220}$.

c) Nel III sec. a.C. un solo antroponimo isolato è forse identificabile come femminile, cioè śmin $\theta i$ della dedica ai Kulśnuter (CIE 21027), nome già attestato in abitato tra V e IV sec. a.C. in una iscrizione lacunosa (cfr. IV.2). Derivante dall'epiclesi greca di Apollo $\Sigma \mu \nu \theta \varepsilon \dot{v} \varsigma^{221}$, è noto ad Acquapendente nel prenome maschile śminte della seconda metà del III sec. a.C. (ET ${ }^{2}$, Vs 1.307; SECI, n.

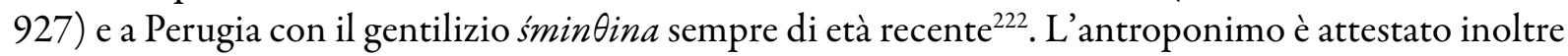
nell'osco smintís di IV sec. a.C. a Capua (ST, Cp 4.5). P. Poccetti ha offerto un articolato contributo su questo nome e la sua acquisizione nell'onomastica dell' Italia preromana ${ }^{223}$. Secondo lo studioso, l'impiego dell'epiclesi divina (e anche del lessema $\sigma \mu i v \theta 0 \varsigma$ = topo, che ha un legame etimologico e cultuale con Apollo $\Sigma \mu \nu v \theta \varepsilon \dot{v} \varsigma$ ) quale antroponimo è uno sviluppo interno alle culture dell' Italia preromana e nella fattispecie attecchisce nel mondo etrusco, mentre quello osco capuano ne sarebbe un riflesso. Poccetti ricostruisce sulla base di questi indizi il processo di ricezione e diffusione del nome: dagli ambienti etruschi della Campania (probabilmente tramite Cuma), questo si diffuse nel comparto tiberino e nel Polesine. Il nome si sarebbe propagato però non tanto per il suo legame diretto con il culto di Apollo $\Sigma \mu \nu v \varepsilon \dot{v} \varsigma$, quanto piuttosto per le pratiche mantiche che a questo erano legate. Riguardo all'occorrenza adriese, Poccetti ipotizza un femminile con suffisso di mozione dal prenome $\operatorname{Smin} \theta e(+i>S \min \theta i)$ o in alternativa un gentilizio teoforico maschile con terminazione $-i$ di area perugina. Verso un prenome femminile è orientato D.F. Maras, mentre precedentemente H. Rix lo aveva interpretato come Individualnamengentile ${ }^{224}$. Lo scarso numero di nomi femminili adriesi nell'ampio orizzonte di V/IV-III sec. a.C. e la duplice occorrenza della base onomastica isolata (nel caso più antico lacunosa) in un arco cronologico di almeno un secolo non consentono purtroppo ulteriori considerazioni.

Nel II sec. a.C. il numero dei nomi femminili isolati aumenta in maniera significativa: kusia (CIE 20407), lausia (CIE 20422), lentnai (CIE 20461-20462, e forse 20463), puinia (CIE 20698), titi (CIE 20472-20473), titinai (CIE 20474-20475) ${ }^{225}$; forse lai (CIE 20610; cfr. infra) e pulevi (CIE 20420). Lausia e forse Pulevi si ritrovano sempre isolati anche in iscrizioni più genericamente databili al periodo ellenistico (lausia, CIE 20829; ]uleviś, CIE 20190).

Oltre che in un frammento di ceramica a vernice nera locale di incerta cronologia (CIE 20829), Lausia è già noto dal III sec. a.C. come probabile metronimico in una bimembre maschile (cfr. IV.3.1.A), mostrando così la continuità di questo gentilizio, attestato finora solo nella versione femminile.

Letnai, puinia, titi e titinai trovano riscontro in nomi familiari dell'Etruria propria, rispettivamente ad Orvieto il primo, a Volterra come cognomen il secondo (m.puine; f. puinei) ${ }^{226}$, mentre titi è molto diffuso

220 Per Orvieto, si veda $E T^{2}$, Vs 1.136 (cfr. MARCHesini 2007, pp. 92-93); per Corchiano, $E T^{2}$, Fa 2.15. In particolare, per le occorrenze meridionali, cfr. MORANDi TARABELLA 2004, p. 496. Forme con suffisso derivativo sono documentate

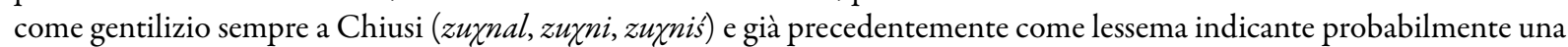

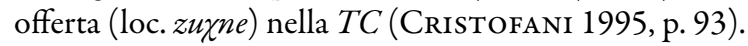

221 Colonna 1984-1985, p. 81.

222 Sul lessema ismintians di uno specchio da Bolsena (CIE 10840), si vedano le considerazioni in PocCETTI 2019.

223 Poccetti 2019.

${ }^{224}$ Rispettivamente Maras 2009b, p. 218, dove si propone il genere femminile, e RIX 1986, pp. 29-30.

${ }^{225}$ Per completezza del quadro, va ricordata l'ipotesi di una origine etrusca (e servile) del M'Titenus (Titnnell'iscrizione di quella che viene identificata come la moglie) ritenuto a capo della stirpe dei Titini della tomba Benvenuti 125 di Este, datata tra la fine del II e la seconda metà del I sec. a.C. (CApuis, Chieco Bianchi 2006, pp. 301-319, e in particolare pp. 317-319 per le considerazioni epigrafiche; per la diversa ipotesi di una diretta origine dalla gens dei Titini di Minturno, cfr. Di Filippo Balestrazzi 2013, p. 165). Se l'ipotesi di una origine etrusca cogliesse nel segno, sarebbe sicuramente da valutare il rapporto con l'attestazione del gentilizio nella forma femminile ad Adria.

${ }^{226}$ Si veda la tabella defixionis di San Girolamo $\left(E T^{2}\right.$, Vt 1.137, 4.1), ma anche il bollo da Rocchette Pannocchiesche $\left(E T^{2}\right.$, AS 6.2), che manifesta un legame con la gens volterrana (A. CIACCI, in REE LXV-LXVIII, pp. 446-448, n. 129). 
nel Chiusino e titinai a Cortona (dove è documentato un lart cusu titinal) ${ }^{227}$. In particolare, colpisce la formazione, sistematica ad Adria, di gentilizi femminili in -nai dal suffisso maschile -na. Si ricorda che in età recente tale terminazione rimane prevalentemente inalterata in Etruria meridionale, mentre in quella settentrionale la resa è -nei così come nell'area emiliana (Spina non offre testimonianze al riguardo): esempi ne sono la Satlnei della stele 12 dei Giardini Margherita di Bologna di fine V-inizi IV sec. a.C. e la Petnei della necropoli di Monte Tamburino presso Monte Bibele di fine IV sec. a.C. Non è da escludere che nell'etrusco di Adria ciò sia dipeso dal mancato avvio del processo di monottongazione (/ai/ > /ei/) in epoca più antica, come altre attestazioni potrebbero far supporre (cfr. IV.3.1.A).

Kusia sembra avere come base onomastica cusu, documentato a Arezzo e Cortona: L. Agostiniani vede in cusu un gentilizio tipicamente cortonese, dato che qui sono documentate quasi tutte le occorrenze note in letteratura ( $E T^{2}, T C o$; Co 1.5; f. kusui, 1.20; 3.5), a esclusione dell'attestazione da San Quirico d'Orcia $\left(E T^{2}\right.$, AS 1.279 ${ }^{228}$. Nella TCo è noto anche il femminile cusual, cioè cusui all'assolutivo ${ }^{229}$. Altre attestazioni della base si riscontrano nell' isolato cusine di Chiusi $\left(E T^{2}, 1.574,575\right)$, e già nel tardo-arcaico gentilizio kusiunaś da Bettolle $\left(E T^{2}, \mathrm{Ar} 1.53\right)$, mentre più incerto il legame con il falisco $c u s u l$ di età arcaica ${ }^{230}$. Pare pertanto plausibile riconoscere nel nostro kusia un gentilizio femminile costituito su una base onomastica di origine probabilmente cortonese, a cui è aggiunto il suffisso femminile -ia.

Rimane più incerto lai, attestato isolato a partire dal periodo tardo-arcaico ${ }^{231}$ e documento nel II sec. a.C. su un fondo di piattello a v.n. da Bolsena (loc. Poggio Moscini) ${ }^{232}$. Le occorrenze tardo-arcaiche sono interpretate da M. Martelli come abbreviazione di laive ${ }^{233}$, mentre G. Colonna ha precedentemente ritenuto lai una variante di lae, a sua volta forma recente di laive e documentato in un'olletta di III-II sec. a.C. di provenienza incerta ${ }^{234}$. L'occorrenza su un peso da telaio di Roselle $\left(E T^{2}, \mathrm{Ru} 0.14\right)$ e quella di Bolsena, dove lai è associato al prenome femminile lar $\theta i$, lasciano pensare che questo possa indicare, almeno in età recente, un nome femminile (lae+i $>$ lai ?), forse nel caso di Bolsena da intendere come gentilizio ${ }^{235}$.

d) Si fanno rientrare in questa categoria tutti i nomi isolati che non sono riconoscibili con certezza come prenomi o gentilizi e che potrebbe essere interpretati perlopiù come individuali.

Tra questi figurano anzi tutto quegli antroponimi interpretabili come diretta etruschizzazione di basi onomastiche allogene, da intendere con buona verosimiglianza come nomi individuali. Per il III sec. a.C. si possono elencare i maschili Ataku, Verkantu, Ipu. Ataku (CIE 20563, 20660) è documentato in due diverse tombe di Canalbianco, sempre isolato. Questo potrebbe essere letto come composto

227 Agostiniani, Nicosia 2000, pp. 65, 75. L. Agostiniani osserva il parallelo della medesima forma femminile tra Adria e Cortona; inoltre vede nel titie di Tarquinia di III-II sec. a.C. (ET ${ }^{2}$ Ta 1.247$)$ la base su cui si è formato il derivato in -na.

228 Agostiniani, Nicosia 2000, p. 70. Si veda inoltre la nota 99 per la lettura dell'iscrizione $E T^{2}$, Co 3.5, che presenta la parziale abbreviazione di entrambi i nomi familiari della formula $a(r n t)$.vels $(i) . c u s(u)$.

229 Ibid., p. 74.

${ }^{230} E T^{2}$, Fa 1.6. L. Agostiniani invece ritiene che cusul sia di «struttura morfologica non immediatamente trasparente», espungendolo prudenzialmente dal quadro della base onomastica Cusu (Agostiniani, Nicosia 2000, p. 70, nota 100). Un affondo su questo nome è offerto anche in DE SimONE 2009b, pp. 547-549, dove si afferma la netta distinzione tra la base settentrionale in sibilante palatale Kus- e quella meridionale in sibilante post-dentale Cus-, oltre che una derivazione da prenomi maschili originari (nord: ${ }^{*}$ Kuse $/{ }^{*}$ Kusie; sud: ${ }^{*}$ Cuse $/{ }^{*}$ Cusie). Su ciò, si veda anche Morandi Tarabella 2004, p. 389, s.v. Puiuna, con bibliografia precedente; Rigobianco 2013, pp. 116-117, n. 34.

231 REE LIX, pp. 272-273, n. 46.

232 CIE 10813; M. Pandolfini, in REE LVI, p. 342, n. 58 b.

${ }^{233}$ REE LIX, pp. 272-273, n. 46. Non spiega però l'occorrenza più tarda di Bolsena; così anche in PANDOLFINI 2000, p. 100.

${ }^{234}$ REE LVI, pp. 331-332, n. 44; ET², OA 1.4.

235 In Ambrosini 2000, p. 155, h), lai è inteso invece come gentilizio abbreviato. 
del prenome Ata con il suffisso - $k u$, formante diminutivi; tuttavia tale suffisso, analizzato da G. van Heems e attivo nei prenomi a partire dal III sec. a.C., appare solo nei femminili ${ }^{236}$ e si lega sempre a - $i$-, tanto che lo studioso si chiede se sia questa vocale o piuttosto direttamente il suffisso -cu a costituire la marca del genere ${ }^{237}$. Allo stato attuale degli studi, è quindi più plausibile che Ataku vada inteso come nome di origine celtica (da atacos, 'molto rapido' ${ }^{238}$ ). Su Verkantu (CIE 20384-20387), K. Tamassia ha ipotizzato a ragione una derivazione celtica. Secondo la studiosa, questo sarebbe nome teoforico, da Vercana ${ }^{239}$, dea delle fonti, con l'aggiunta del suffisso $-t u^{240}$. È però più probabile che il nome sia composto dal prefisso ver-, molto attivo nell'onomastica celtica con il valore di "sopra" (es. il famoso Vercingetorige) ${ }^{241}$, e -canto- molto frequente in ambito onomastico ${ }^{242}$. Infine, Ipu (attestato nella forma ipuz, da intendere come genitivo I; CIE 20547) è probabilmente da spiegare come l'etruschizzazione del greco $\mathrm{I} \pi \tau \omega \nu$, nome di ampia diffusione e cronologia ${ }^{243}$.

Tra la fine del III e il II sec. a.C. si contano i maschili Aku, Latiate, Pulu, Pruka, Skamu, nonché

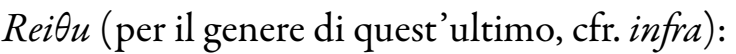

- dal latino: Latiate ${ }^{244}$, attestato nel medesimo periodo ad Adria e a Bottrighe (CIE 20699 e 20932) e già individuato come etnico formato con -(a)te da Latium > etrusco Lati(e);

- dal celtico: Aku (CIE 20375), da acus/aco ("rapido") ${ }^{245}$; Pruka (CIE 20762), forse maschile in analogia a Viza (cfr. IV.3.1.A) e derivante dalla base onomastica celtica brog-, attestata in forma maschile e affine anche nell'onomastica venetica dove è sempre di derivazione celtica ${ }^{246}$;

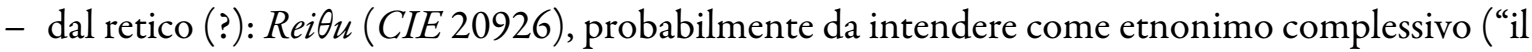
retico/a") che diventa idionimo (femminile? $)^{247}$;

- dal greco: Skamu (CIE 20921), di probabile origine greca secondo G. Colonna ${ }^{248}$, come anche

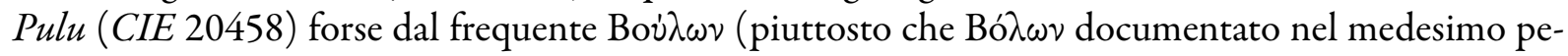
riodo ad Alicarnasso in Asia Minore $)^{249}$.

L'uso di nomi di origine non etrusca quali prenomi in associazione a nomi familiari (cfr. IV.3.1.A) almeno dal pieno II sec. a.C. induce a ritenere ambigui gli antroponimi isolati, che potrebbero essere portati da individui privi di diritti oppure in alternativa appartenenti a gentes di recente formazione.

Alcuni nomi suffissati isolati, perlopiù di III sec. a.C., per alcune caratteristiche che li contraddistinguono non possono essere interpretati con sicurezza come gentilizi, ma piuttosto come nomi individuali composti con suffissi derivativi (-ke, -va; incerta l'individuazione di - vi, come si dirà) e diminutivi $(-l e)$. La base onomastica può essere sia etrusca che allogena.

Tra la fine del IV e gli inizi del III sec. a.C. si data l'iscrizione $m i\langle x \times\rangle$ helis nalskeś (CIE 20595), dove l'antroponimo nalskeś sembra espresso al genitivo I. Il radicale nal-potrebbe trovare confronto

236 van Heems 2008, pp. 105-106, nn. 141-154. Su tale suffisso, cfr. anche Rigobianco 2013, p. 93, nota 483.

${ }^{237}$ Ibid., pp. 85-86.

${ }^{238}$ Delamarre 2003, p. 144.

239 Cfr. Heichelheim 1955, p. 979.

240 K. Tamassia, in Akeo 2002, p. 176, n. 14.1.

${ }^{241}$ Delamarre 2003, p. 314.

${ }^{242}$ Ellis Evans 1967, p. 67; Delamarre 2003, pp. 103-104. Si richiama il simile Vercatus (Ellis Evans 1967, p. 167). Più difficile spiegare questo antroponimo partendo da una base verk- (cfr. Delamarre 2003, p. 315; Prosdocimi 2009, p. 88).

${ }^{243}$ Cfr. LGPN online.

${ }^{244}$ GAUCCI 2016, p. 208, nota 151, con riferimenti.

245 Delamarre 2003, p. 144.

${ }^{246}$ Ellis Evans 1967, pp. 158-159, Delamarre 2003, p. 91.

${ }^{247}$ Per una disamina, cfr. GaUCCI 2008, p. 90. Si rinvia in particolare a Colonna 2004a, p. 76, nota 48, dove si ritiene che l'aspirazione della dentale possa essere in analogia ai nomi femminili in - $\theta u$ (cfr. anche Rigobianco 2013, p. 124).

${ }^{248}$ G. Colonna, in REE LXXIV, p. 368, n. 112.

${ }^{249}$ Si considerano le occorrenze in Fraser, Matthews 1987, ID. 2000, Osborne, Byrne 1994, s.v., che ci documentano il nome a partire dal IV sec. a.C. in Attica, a Delfi e a Delos. 
in nali di Genova ${ }^{250}$, con riferimenti all'onomastica chiusina e perugina, ma anche a Volterra nel femminile nalui $\left(E T^{2}, \mathrm{Vt} 1.131\right)$ e forse in nelaiuś da Aleria $\left(E T^{2}\right.$, Cs 2.12$)$, se valida la possibile allofonia iniziale $e / a^{251}$. Il segmento sillabico finale potrebbe apparire come -ske, suffisso raro in ambito etrusco e ipotizzato di derivazione italica quale generativo di etnonimi secondo P. Poccetti ${ }^{252}$. Ma è possibile formulare anche un percorso alternativo e forse più convincente: se valido il confronto con la base nal-, questa è stata collegata da G. Colonna al gentilizio chiusino navlis $\left(E T^{2}, \mathrm{Cl} 1.2011-2012,2736,2170\right)$ che avrebbe subito una riduzione naul- > nal-, secondo un passaggio interno all'area chiusina ${ }^{253}$. In questo caso, la base nalis sarebbe stata composta con il suffisso $-k e^{254}$.

Nalske è attestato in un testo che ha caratteristiche strutturali identiche a CIE 20464: mi helis uvasaveś. La radice dell'antroponimo di quest'ultima iscrizione potrebbe rinviare all'onomastica celtica, trovando apparentemente parallelo nella base di Prestino in alfabeto leponzio e lingua celtica di V sec. a.C. (uvamokosis) ${ }^{255} \mathrm{e}$ in una stele antropomorfa della Lunigiana di periodo arcaico $\left(E T^{2}, \mathrm{Li} 1.5\right)$, mentre perde la più tarda attestazione etrusca dal sepolcreto Arnoaldi ora da leggere $m i t e i^{256}$. D.F. Maras ha più recentemente edito una iscrizione etrusca piuttosto lacunosa da Mantova, Piazza Santa Barbara, letta uva $\varphi$ el [---] e realizzata su una coppa in ceramica depurata per la quale è stata proposta una datazione alla prima metà del V sec. a.C. ${ }^{257}$. Tenuto conto che si potrebbero avanzare alcuni dubbi sulla lettura proposta (in particolare riguardo ypsilon e la divisione delle parole) e sulla relativa cronologia in rapporto all'aspetto paleografico delle lettere ${ }^{258}$, Maras suggerisce il rimando alla iscrizione arcaica dalla Lunigiana già menzionata e al nome uvi di Aleria ( $E T^{2}$, Cs 2.15), confrontato con l'italico Ovius $^{259}$. Nel nostro caso sembra da escludere una derivazione dall'onomastica italica, mentre per l'ambito celtico la difficoltà è insita nella problematica della base $u v-\left(<{ }^{*} u p-\right)$, ancora attiva nel V sec. a.C. nell'iscrizione di Prestino di area golasecchiana e che tenderebbe a trasformarsi successivamente in consonante; tuttavia, non si può escludere che nel passaggio all'etrusco tale base si mantenesse viva, almeno in nomi entrati nell'onomasticon prima di questo passaggio. Se questa osservazione può avere qualche validità, nell'antroponimo adriese si potrebbe riconoscere la base onomastica celtica uass(os)("servo") ${ }^{260}$. Sembra invece meno convincente riconoscere nella seconda parte il lessema celtico auos, cioè "discendente" ${ }^{261}$, che porterebbe ad avere un nome composto, altrimenti non documentato nell'epigrafia celtica o di derivazione celtica: ${ }^{*}$ uassauos > etr. uvasave, cioè un nome parlante che espliciterebbe l'origine servile del possessore. Si potrebbe invece pensare che il nome sia composto con il suffisso etrusco - va, tale da avere uvasa $+v a$ : in tale caso uvasaveś sarebbe dunque ablativo di origine: "io (sono) l'helis da Uvasava" (sul lessema helis, cfr. V.4). Se tale interpretazione avesse qualche fondamento, allora è probabile che anche nalskeś per analogia di formula sia ablativo di Nalske.

Il suffisso -va è attivo nell'onomastica padana, come documenta l'adriese haltva di IV-III sec. a.C. (CIE 20133). Questo nome è stato recentemente discusso da G. Colonna, che vi ha riconosciuto

250 Colonna 2004b, p. 302, n. 3 (ET 2 , Li 0.3).

251 Poccetti 1997 , pp. 286-287.

${ }^{252}$ Ibid., pp. 284-285. Lo studioso richiama il feluske (o Oeluske: cfr. Agostiniani 2011) della stele figurata di età orientalizzante di Vetulonia (ET ${ }^{2}, \mathrm{Vn}$ 1.1). Sull'antroponimo e l'individuazione del suffisso -ce, si veda invece BELFIORE 2014b, p. 84 .

253 Colonna 2004b, p. 302, n. 3.

254 Su questo si veda il quadro critico offerto in BELFIORE 2014b, pp. 82-84.

255 Per il documento si rimanda a MotTa 2000, pp. 197-198, n. 2.

256 Su questa iscrizione, finora letta mi uva (cfr. $E T^{2}$, Fe 2.25), una anticipazione in GAUCCI, Morpurgo, Pizzirani 2018, p. 672, mentre per la nuova lettura, si rimanda alla REE LXXXII, pp. 281-283, n. 34.

257 Menotti, Maras 2012, p. 881, n. 4, fig. 8.

258 A parte il tipo corsivizzante del digamma e l'alpha quadrangolato, ci si può domandare se il phi non possa piuttosto essere letto come chet circolare. Per considerazioni paleografiche più generali, cfr. III.

259 Menotti, Maras 2012, p. 883.

260 Delamarre 2003, pp. 307-308.

261 Ibid., pp. 60-61. 
un suffisso - va attivo nella morfologia derivativa dei gentilizi, mettendo in parallelo l'attestazione adriese a quella spinetica mi kuripeś haltuva $\left(E T^{2}, 2.93\right)$ e a quella mantovana (haltuv $\left[{ }^{262}\right)$, con i gentilizi haltu/haltunei a Chiusi e haltnas a Orvieto ${ }^{263}$. Recentemente il medesimo Colonna ha inoltre affermato che il testo spinetico è composto da due distinti enunciati, nel secondo dei quali vi sarebbe l'ellissi del verbo di dono: haltuva ( $\mathrm{mi}$ ha donato) ${ }^{264}$, così rifiutando la precedente ipotesi da lui stesso formulata che haltuva potesse essere l'appellativo dell'oggetto iscritto ${ }^{265}$. Sebbene sia possibile riconosce a - va una funzione derivativa ${ }^{266}$, non è certo che haltva avesse funzione di gentilizio. In effetti le reiterate occorrenze padane non mostrano associazione con prenomi (anche in quella lacunosa il nostro è il primo lessema del testo), e questo basta a far sorgere dubbi ${ }^{267}$.

Nell'antroponimo pulevi di II sec. a.C. (CIE 20420; si veda anche CIE 20190, lacunoso ma di facile integrazione), dove si riconosce la base onomastica pule $e^{268}$, la terminazione - $v i$ è isolabile con buona approssimazione. Se valido ciò, questa si potrebbe plausibilmente intendere come variante di - va (forse femminile, se ha qualche valore il confronto della struttura testuale con altre iscrizioni femminili: cfr. IV.3.2).

Per il suffisso - va e anche per la terminazione - vi, se coglie nel segno la sua identificazione come variante del primo, è lecito ritenere che lo scarso numero di occorrenze possa dipendere dalla difficoltà di isolarli con certezza. Ciò è ben dimostrabile in due occorrenze di V-IV sec. a.C., cioè kutvaluś di Adria e ]uśvaluś di Bologna (ET $\left.{ }^{2}, \mathrm{Fe} 1.5\right)$, dove -va potrebbe essere agglutinato al più attivo -alu, sebbene altre soluzioni siano possibili (cfr. supra, IV.2).

Un altro caso adriese di nome isolato può portare qualche ulteriore considerazione riguardo la difficoltà di individuazione del suffisso: Urvi, sempre attribuibile all'orizzonte di fine IV-III sec. a.C. (CIE 20323). Questo potrebbe mostrare in apparenza la terminazione -vi quale risultato della rimodulazione del radicale verbale $u r^{269}$. Tuttavia questa soluzione desta perplessità, perché i casi sopra discussi lasciano intendere che la rimodulazione avvenga su una base onomastica che qui non si avrebbe ${ }^{270}$. Sebbene si possa escludere la formazione dal perfettivo $u r u$, merita comunque sottolineare che più in generale casi con terminazione - $v i$ intesi come rimodulazioni dal morfema femminile $-\mathrm{C} u+i>-\mathrm{C} v i$ potrebbero celarlo ${ }^{271}$. Ma altre soluzioni sono comunque possibili per Urvi: si potrebbe avvicinare a una base latina (orbus, orfano) e forse italica ${ }^{272}$, o piuttosto celtica (e come imprestito

262 Pandolfini $1988^{2}$, p. 123, n. 249, fig. 49.

263 Colonna 2007, p. 219; più recentemente sulla questione, Belfiore 2014b, p. 92. Per haltnas, cfr. anche Morandi TARABELla 2004, p. 226.

264 ColonNa 2007, p. 219, nota 38. Su questa iscrizione, cfr. Rix 1981, p. 114. Sul valore di gentilizio per halt(u)va, si è pronunciata anche V. BELFIORE (2014b, p. 92).

${ }^{265}$ Colonna 1993, p. 138. Si ricorda che L. Agostiani (1997, p. 9) ha richiamato proprio haltva di Adria assieme ad haltuva di Spina quali lessemi con un formante aggettivale -va (smentendone contestualmente una possibile forma plurale).

266 BELFIORE 2014b, pp. 171-172.

267 Altri nomi padani sembrano solo apparentemente composti con il suffisso -va, come ad esempio $u k v a\left(E T^{2}, \mathrm{Fe} 2.1\right)$, per il quale cfr. Rigobianco 2013, p. 126, n. 58. Si ricorda invece che per l'iscrizione skaiva karinaś (ET ${ }^{2}, \mathrm{Fe} 1.1$ ), è adesso proposta una nuova lettura (skapvas< < > karinaś, in GAUCCI, Govi, SAsSATELLI c.s.).

268 Tale base potrebbe essere già attiva in ambito padano nel puleisnai del II cippo di Rubiera: $E T^{2}$, Pa 1.2; BELFIORE 2014b, p. 33, con riferimenti. Tuttavia recentemente A. Maggiani ha proposto di leggere hulesnai (A. MAGGIANI, in REE LXXVI, pp. 277-278, n. 34).

269 Belfiore 2014a, pp. 38-39.

270 Il radicale $u r$ è comunque attivo nell'onomastica con altre forme, fra le quali si ricorda ure della vicina $S$ pina $\left(E T^{2}\right.$, Sp 2.55; cfr. G. Colonna, in REE LXXIV, pp. 399-401, n. 158, e adesso E. Govi, in REE LXXXII, pp. 221-225, n. 5, e pp. 284-285, n. 35.

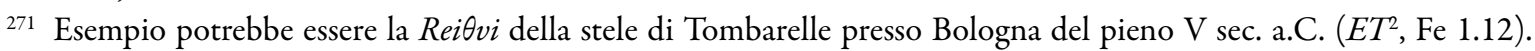
Qui, sebbene la formazione $r e i \theta u+i$ rimanga la lectio facilior, non si può escludere rei $\theta u+v i$, che darebbe conto di un valore derivativo del nome come riconosciuto per il suffisso -va.

272 A. CALDERINI, in REI LXXII, p. 278. 
anche in ambito venetico ad Altino) ${ }^{273}$, sulla quale sarebbe stato generato il nome (in questo caso individuale), poi rimodulato con il morfema femminile $-i$.

Fra le forme suffissate rientra anche Hapile della seconda metà del III sec. a.C. (CIE 20594), unico caso di nome (individuale?) composto con suffisso diminutivo. Alla base hapi è aggiunto -( $i)$ $l e$, attestato in età recente ma forse più antico e attivo nei prenomi e anche nei gentilizi ${ }^{274}$. L'area di diffusione del suffisso secondo G. van Heems è Chiusi, Bomarzo e Todi. Come già riconosciuto da A. Maggiani, la forma hapile si documenta come formante gentilizio nel sincopato haplnal di Chiusi $\left(E T^{2}, \mathrm{Cl} 1.232\right)^{275}$.

Alcuni antroponimi isolati risultano di più problematica interpretazione, sebbene sia probabile una loro funzione come nomi individuali. A quelli che di seguito si illustrano, è forse possibile aggiungere zurta, già discusso in merito ai nomi di famiglia zurtalu e zurtiu (cfr. IV.3.1.A). Fra questi, si richiamano Mei (CIE 20457) per il III sec. a.C., Ana (CIE 20820) e Veri (CIE 20654) per il II sec. a.C. Rimane di più incerta cronologia l'iscrizione mama (CIE 20664; il vaso iscritto non è reperibile). Mei si confronta con il gentilizio chiusino Meie, forma riconosciuta da $\mathrm{H}$. Rix come di derivazione italica, da Mai(us), Mais, che a Chiusi è attestata con il suffisso $-i e^{276}$. Nel nostro caso si avrebbe un nome individuale $\mathrm{Me} i$ oppure una forma con suffisso - $i(m e i+i)$ attiva a Chiusi e formante nomi familiari (cfr. supra, categoria b). Considerando che la formula pertinente all'antroponimo (meiśta) ricalca quella con il prenome Kavi (cfr. supra, categoria a), nel complesso sembra più plausibile che il nostro sia un nome individuale di origine italica. Il nome Ana ha una documentazione circoscritta al periodo orientalizzante e arcaico, isolato o in testi dove ha funzione ambigua ${ }^{277}$, come nell'anforetta Melenzani di Bologna, dove è associato a ×remiru, inteso da G. Colonna come cognome o appellativo atto a qualificare l'artefice dell'opera. Dunque, stupisce che questo nome riemerga dopo secoli e in ogni caso è improbabile che sia mai entrato a far parte dei prenomi in uso. L'antroponimo isolato Veri presenta una base documentata nella $T C o$; qui la forma vêrề è riconosciuta da L. Agostiniani come un predicato. Questa base verbale avrebbe formato anche il cognomen/gentilizio Veru attestato perlopiù a Chiusi e Perugia (ma anche in singole occorrenze a Vulci e Gravisca) ${ }^{278}$. Nel nostro si riconosce pertanto una base onomastica di derivazione verbale con suffisso $-i$, ben noto in ambito perugino come formativo di gentilizi, sebbene non si possa escludere del tutto un valore sostantivale o verbale ${ }^{279}$. Infine, Mama è riconosciuto da Colonna come nome individuale maschile (Lallname) attestato in falisco già nel VII sec. a.C. e diffuso in ambito latino e italico, oltre che etrusco dal periodo tardo-arcaico nella Tomba delle Iscrizioni graffite e quindi nell'Etruria interna in periodo ellenistico (dove secondo il medesimo studioso sarebbe attivo come gentilizio nella forma dissimilata Mana $)^{280}$.

273 MarinetTi 2009, p. 90, n. 20.

274 VAN HEEMS 2008, pp. 86-87.

275 Maggiani 2002a, p. 59.

276 Rix 1963, p. 345.

277 Per il periodo orientalizzante: $E T^{2}$, Fa 6.1 e Fe 6.1 (cfr. da ultimo Colonna 2014, pp. 51-52 con riferimenti). Nei casi accertati di periodo arcaico, ana è isolato oppure in testi dove non è limpida la sua funzione $\left(E T^{2}, \mathrm{Ta} 7.13\right.$, dalla Tomba delle Iscrizioni di Tarquinia, e Fs 1.5, il cippo di Capalle della prima metà del VI sec. a.C. per il quale cfr. A. Maggiani, in REE LI, pp. 248-249, n. 58 e MAggiani 2016, p. 74). Tutti i casi certi sono circoscrivibili tra VII e VI sec. a.C. e collocati perlopiù in Etruria meridionale, a eccezione di quello bolognese e del cippo fiesolano, per il quale tuttavia Maggiani evidenzia una connessione con gli alfabeti meridionali nell'aspetto delle lettere (ibid.). Va ricordato anche che ana è riconosciuto come base di derivativi: anaśnies di Volterra $\left(E T^{2}, \mathrm{Vt} 1.58\right)$, ricostruito da V. Belfiore come *ana+/s/ (BELFIORE 2014b, p. 28).

278 Agostiniani, Nicosia 2000, p. 104.

279 WYLIN 2000, pp. 122-124, 199-200.

280 G. Colonna, in REE LXXI, p. 180, n. 29, e p. 221, n. 75. Sul problema dei c.d.Lallnamen, cfr. anche Prosdocimi 2009, pp. 104-105. 


\section{IV.3.2. Classificazione delle iscrizioni con formule onomastiche}

La seguente classificazione (Tab. 4) ha come obiettivo la sistematizzazione dei testi con le formule onomastiche non lacunose precedentemente discusse (C: cognome; G: gentilizio; NI: nome individuale; NND: nome non determinato; P: prenome; ${ }_{\mathrm{f}}$ femminile; ${ }_{\mathrm{m}}$ : maschile; ${ }_{\mathrm{i}}$ : genere incerto; $\mathrm{a}$ : assolutivo/nominativo; g: genitivo; loc: locativo; ?: non determinabile). Nella strutturazione delle classi viene privilegiata l'assenza / presenza del pronome $m i$, e inoltre l'uso dell'assolutivo o del genitivo.

L'iscrizione CIE 21027, unica formula con verbo espresso, non è stata inserita nella classificazione proprio per il suo carattere di eccezionalità. Il testo prevede il destinatario al caso genitivo (kulśnuteraś) secondo la norma nelle iscrizioni di dono votivo ${ }^{281}$, un antroponimo probabilmente femminile all'assolutivo quale soggetto dell'azione (śmin $\theta i$, IV.3.1.B, categoria c) e quindi in posizione finale il verbo akśke, preterito sincopato nel quale pare plausibile riconoscere il significato di "donare"282. Sempre nella struttura classificatoria non sono stati inseriti alcuni testi che presentano l'associazione di una lettera o un digramma con un nome all'assolutivo, in quanto rappresentano un gruppo non chiaramente definibile e coerente, che si tratterà a parte (cfr. IV.3.2.A $)^{283}$.

La maggior parte delle iscrizioni è composta solo da formule onomastiche all'assolutivo, cioè in nominativus pendens (I). Sono invece poco frequenti quelle all'assolutivo associate al pronome mi (II) e quelle al genitivo con / senza il pronome mi (classi III-IV). Del tutto eccezionali le due iscrizioni dove il vaso si identifica con il lessema helis (classe $\mathrm{V}$ ): in queste il caso espresso dall'antroponimo rimane in sospeso (genitivo I, che le assimilerebbe alla classe IV, o ablativo?).

Nella classe I, le trimembri (I.a) e le bimembri (I.b-e) provengono quasi tutte da contesto funerario. I nomi isolati (I. f-i) si contano sia da necropoli che da abitato, evidenziando così una pratica trasversale, ma sicuramente concentrata in prevalenza in ambito funerario durante il II sec. a.C. e che riguarda soprattutto gentilizi maschili e femminili, nomi individuali.

Per quanto concerne la classe II, è noto che la formula all'assolutivo associata al pronome $m i$ esprime una diretta corrispondenza tra il vaso e la persona, secondo un processo di identità come appartenenza dell'oggetto iscritto su cui ha posto l'attenzione G. Colonna ${ }^{284}$. Processo sul quale adesso si è soffermato P. Poccetti, puntualizzando che deve essere invertito, cioè non è l'identità che definisce l'appartenenza, ma piuttosto il contrario ${ }^{285}$. Le tre bimembri (II.a) appartengono a uomini, mentre le monomembri prevalentemente a donne (II.b). Colpisce la varietà di posizione del pronome, perlopiù posposto al nome quasi a voler marcare l'accento sull'individuo. Si è già detto che l'iscrizione pulevi $m$ [i] da necropoli e l'unica da abitato mi haltva (II.c) non sembrano chiaramente identificabili come gentilizi (cfr. IV.3.1.B): mentre la prima sembra essere assimilabile per struttura alla sottoclasse II.b, cioè quella dei nomi femminili, la seconda, pur esprimendo un nome maschile, non può essere messa in parallelo con la sottoclasse II.a sia per struttura sia per contesto.

Sempre riguardo la classe II, sembra rilevante il confronto con la vicina Spina, dove tra la fine del IV e il III sec. a.C. si ritrova un numero molto ridotto di iscrizioni con simile struttura ${ }^{286}$ : ure $m i$

281 Maras 2009b, pp. 20-29.

282 Belfiore 2014a, p. 30.

283 Altri testi si presentano invece lacunosi e pertanto non classificabili. Presentano nomi all'assolutivo in formule

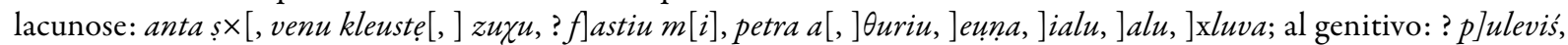
]× vercniś, ] ×italuś; non sono determinabili: mi petr[, <e >mi karku[, ] mi, kani $\times$ [. L'iscrizione ]ṣ́ta mi di II sec. a.C. (CIE 20640), sebbene lacunosa, sembra rientrare nella classe IV, vicina alla sottoclasse c).

284 Colonna 1983.

285 Poccetti 2019.

286 Non si fa rientrare nell'elenco l'iscrizione della tomba 80 di Valle Trebba, per la quale non è condivisibile la lettura larke laus mi fornita da G. Uggeri, mentre si ritiene corretta la proposta di H. Rix larke lausini $\left(E T^{2}, \mathrm{Sp} 2.72\right.$, con riferimenti precedenti). 
Tab 4. Classificazione delle iscrizioni con formule onomastiche.

\begin{tabular}{|c|c|c|c|c|c|c|c|}
\hline & & \multicolumn{3}{|c|}{ Aвitato } & \multicolumn{2}{|c|}{ NeCropoli } & \multirow{2}{*}{$\begin{array}{c}\text { CONTESTO } \\
\text { NON DET. }\end{array}$} \\
\hline & & $\begin{array}{l}\text { FINE IV-III } \\
\text { SEC. }\end{array}$ & $\begin{array}{l}\text { FINE III-II } \\
\text { SEC. }\end{array}$ & INCERTO & $\begin{array}{l}\text { FINE IV-III } \\
\text { SEC. }\end{array}$ & FINE III-II SEC. & \\
\hline \multirow{9}{*}{$\begin{array}{l}\text { I. Formule } \\
\text { onomastiche } \\
\text { all'assolutivo }\end{array}$} & $\begin{array}{l}\text { a) } \mathrm{P}_{\mathrm{m}} \mathrm{a}+\mathrm{G}_{\mathrm{m}} \mathrm{a}+\mathrm{C}_{\mathrm{m}} \mathrm{a} \\
\text { (ordine variabile) }\end{array}$ & & & & & $\begin{array}{l}\text { anta afr mura, } \\
\text { mura larza pili }\end{array}$ & \\
\hline & b) $\mathrm{P}_{\mathrm{m}} \mathrm{a}+\mathrm{G}_{\mathrm{m}} \mathrm{a}$ & & & & $\begin{array}{l}\text { ata śetina }(\mathrm{x} 3) \\
\text { laris atiu, venza } \\
\text { velOuriu, venza } \\
\text { malni }\end{array}$ & $\begin{array}{l}\text { apu muliu, arza } \\
\text { heturiu, viza } \\
\text { muliu (x2), } \\
\text { kraiku.kaniu., } \\
\text { larza lintialu }\end{array}$ & \\
\hline & c) $P_{m} a+G_{m} g$ & larza śiluniś & & & & & $\begin{array}{l}\text { larza viufralus' } \\
\text { (III sec.) }\end{array}$ \\
\hline & d) $\mathrm{P}_{\mathrm{m}} \mathrm{a}+\mathrm{Gg}_{\mathrm{f}}$ & ata krapnal & & & arza lausial & & \\
\hline & e) $\mathrm{P}_{\mathrm{f}}+\mathrm{G}_{\mathrm{f}} \mathrm{a}$ & & & & \multicolumn{2}{|c|}{ alpiarnai (x2) } & \\
\hline & f) $\mathrm{P}_{\mathrm{m}} \mathrm{a}$ & & & & & laz & kavi (II sec.) \\
\hline & g) $G_{m} a$ & kraiu & & $\begin{array}{l}\text { kepriu, } \\
\text { titalu, tite }\end{array}$ & $\begin{array}{l}\text { zurtalu, malni, } \\
\text { mura }\end{array}$ & $\begin{array}{l}\text { ankariu (x3), } \\
\text { atna, muliu (x2), } \\
\text { mura, śiliu, spiu, } \\
\text { unitiu (x2) }\end{array}$ & $\begin{array}{l}\text { fastiu (III sec. } \\
\text { a.C.)?, sinu (II } \\
\text { sec.) }\end{array}$ \\
\hline & h) $G_{f} \mathrm{a}$ & & & & & $\begin{array}{l}\text { kusia, lai, lausia, } \\
\text { titi (x2), titnai } \\
(\mathrm{x} 2)\end{array}$ & lausia \\
\hline & i) $\mathrm{NI}_{\mathrm{m} / \mathrm{f}} \mathrm{a}$ & urvi (?) & & & & 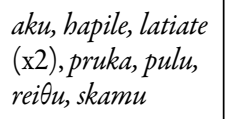 & ana (II sec.) \\
\hline \multirow{3}{*}{$\begin{array}{l}\text { II. Formule } \\
\text { onomastiche } \\
\text { all'assolutivo } \\
\text { con pronome }\end{array}$} & $\begin{array}{l}\text { a) } m i+\mathrm{P}_{\mathrm{m}} \mathrm{a}+\mathrm{G}_{\mathrm{m}} \mathrm{a} \\
\text { (ordine variabile) }\end{array}$ & & & & $\begin{array}{l}\text { mi laris fuliu, } \\
\text { laris tetialus'mi }\end{array}$ & Qaia mi siliu & \\
\hline & b) $\mathrm{G}_{\mathrm{f}} \mathrm{a}+m i$ & & & & & $\begin{array}{l}\text { lentnaimi } \\
\text { (x2, più } \\
\text { plausibilmente } \\
\text { x 3),puinia mi }\end{array}$ & \\
\hline & c) $m i+\mathrm{NND}_{\mathrm{m} / \mathrm{f}} \mathrm{a}$ & & & mi haltva (?) & & pulevi $m[i]$ & \\
\hline \multirow{3}{*}{$\begin{array}{l}\text { III. Formule } \\
\text { onomastiche } \\
\text { al genitivo }\end{array}$} & a) $G_{m} g$ & estalus' & & & & & $\begin{array}{l}\text { preznaśs } \\
\text { (III sec. a.C.) }\end{array}$ \\
\hline & b) $\mathrm{NI}_{\mathrm{m}} \mathrm{g}$ & & & & $\begin{array}{l}\text { atakuś, atakuś } \\
(\mathrm{x} 2), i p u z\end{array}$ & & \\
\hline & c) $\mathrm{NI}_{\mathrm{m}} \mathrm{g}+t a$ & & & & meiśta & & \\
\hline \multirow{4}{*}{$\begin{array}{l}\text { IV. Formule } \\
\text { onomastiche } \\
\text { al genitivo } \\
\text { con pronome }\end{array}$} & a) $P_{m} g+G_{m} g+m i$ & & & & venuśzurtiuśmi & & \\
\hline & b) $\mathrm{P}_{\mathrm{m}} \mathrm{g}+m i$ & ataśni & & & & & \\
\hline & c) $\mathrm{P}_{\mathrm{m}} \mathrm{g}+\mathrm{ta}+m i$ & & & & kaviśta mi (x2) & & \\
\hline & d) $\mathrm{NI}_{\mathrm{m}} \mathrm{g}+m i$ & & & & $\begin{array}{l}\text { mi verkantuś } \\
(\mathrm{x} 4)\end{array}$ & & \\
\hline $\begin{array}{l}\text { V. Formule } \\
\text { onomastiche } \\
\text { (all'ablativo?) } \\
\text { con pronome } \\
\text { e lessema helis }\end{array}$ & a) $m i+h e l i s+\mathrm{NND}_{\mathrm{m}} \mathrm{g}$ & & & & $\begin{array}{l}\text { mi } \times \times \text { belis } \\
\text { nalskes, mi helis } \\
\text { uvasaves' }\end{array}$ & & \\
\hline
\end{tabular}


$\left(E T^{2}\right.$, Sp $\left.2.55^{287}\right)$; i due testi fra loro identici $m i$ : ziltu dalla tomba 269A di Valle Pega ${ }^{288}$; mi fasi dalla tomba 656A di Valle Pega ${ }^{289}$; infine la formula di consacrazione a Van $\theta$ (mi vant: $E T^{2}$, Sp 2.114). Più complessa appare la formula mi sureś: letiu dalla tomba $41 \mathrm{~B}$ di Valle Pega ${ }^{290}$, forse da ritenere affine a mi kuripeś haltuva $\left(E T^{2}, \mathrm{Sp} 2.93\right)$, che recentemente G. Colonna ha interpretato come composta da due enunciati, di cui il secondo con antroponimo e verbo di dono sottinteso (cfr. IV.3.1.B, categoria d). Entrambe le città deltizie mostrano dunque una ridotta frequenza di uso di questo tipo di formula (si puntualizza che il confronto è circoscritto alla fine IV-III sec. a.C., in quanto nel secolo successivo Spina non è più vitale), ma sembra ravvisabile una differenza d'uso che sottende pratiche epigrafiche diverse. Infatti, nei rari casi spinetici (rimane incerto $E T^{2}$, Sp 2.4) il pronome mi è associato ad antroponimi isolati maschili (almeno per ure e ziltu) e a un nome divino (vant) in una formula di consacrazione, distinguendosi così dalle occorrenze adriesi per il medesimo periodo che mostrano nomi maschili composti da prenome e gentilizio.

Entrambe le classi I e II sono interessate dalla ripetizione del medesimo testo su tre vasi distinti, secondo una formula rituale nota nel mondo etrusco ${ }^{291}$. Si tratta di un fenomeno che ricorre in formule bimembre e monomembre all'assolutivo (I.b: ata śetina; I.g: ankariu) e monomembre con pronome mi (II.b: lentnai mi). Più in generale le classi I e II (assolutivo senza / con pronome) sembrano prevalentemente destinate all'ambito funerario e mentre nel III sec. a.C. interessano formule bimembri o gentilizi isolati maschili, in quello successivo perlopiù donne ingenue identificate dal solo gentilizio.

L'unica iscrizione femminile che esprime il prenome, cioè alpi arnai, appartiene alla classe I. L'omissione del prenome nei nomi femminili trova una significativa incidenza (circa 20\%) nelle iscrizioni di Chiusi e Perugia come evidenziato da E. Benelli, che su base cronologica suggerisce di svincolare il fenomeno da una diretta influenza dell'onomastica romana ${ }^{292}$. In ambito padano, tale fenomeno epigrafico si distingue nettamente da quanto noto in un altro corpus di monumenti, quello delle stele bolognesi di V-IV sec. a.C., dove le donne sono identificate da prenome/nome individuale e gamoni-

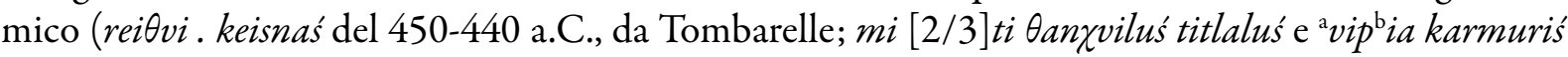
di fine V-inizi IV sec. a.C., rispettivamente stele Ducati nn. 105 e 106), anche qui con un'unica eccezione: Rakvi Satlnei della stele Ducati $12^{293}$. Le pratiche epigrafiche della Bologna di V-inizi IV sec. a.C. e dell'Adria di II sec. a.C. relative alle iscrizioni femminili evidenziano fenomeni distinti e tendenzialmente coerenti al loro interno, che sottendono gli aspetti sociali dell'identità femminile nelle rispettive società. Nella stele la proiezione della donna all'esterno è soprattutto vincolata al suo ruolo di moglie, mentre nella pratica adriese di scrittura sui vasi destinati al defunto la donna è identificata dal solo gentilizio paterno, dunque senza marcare la propria individualità (prenome) o l'eventuale famiglia del marito (gamonimico).

Entro le classi III-IV, eccetto le iscrizioni estaluś e ataś ni da abitato, tutte le restanti sono da necropoli. Inoltre, si collocano tutte nel III sec. a.C., con l'eccezione della formula con pronome e deittico di II sec. a.C. (CIE 20640: ]ś ța mi), non inserita perché lacunosa del nome (indiziato solo dal morfema

${ }^{287} \mathrm{Su}$ ure come antroponimo, cfr. G. ColonNA, in REE LXXIV, p. 399, n. 158, dove è ritenuto derivato onomastico dalla base verbale ur. Non è condivisibile la lettura proposta in UGGERI 1978, p. 396, n. 64 (urv mi).

288 Pozzi 2011, p. 317, inedite.

289 Ibid., p. 335, inedito.

290 Ibid., p. 339, dove è letta erroneamente mi streś: letiu. Sure è prenome raro ma attivo in area chiusina (ET ${ }^{2}$, s.v. sure) ed è attestato al confine con il territorio aretino a Lucignano come probabile gentilizio ( $\left.E T^{2}, \operatorname{Ar} 1.24\right)$. Si osserva l'uso della puntuazione per disambiguare il caso.

291 Si veda Pfiffig 1975, p. 363.

292 Benelli 2015, p. 408. Sulla problematica, si veda anche Belelli, Benelli 2018, pp. 130-131.

${ }^{293}$ Per un quadro aggiornato su queste iscrizioni, si veda GAUCCI, Govi, SAsSATELli c.s. In questo contributo, la nuova proposta di lettura della stele B del Polisportivo skapvas<a> karinaś, se effettivamente appartenente a una stele femminile, potrebbe indicare l'omissione completa del nome della defunta, identificata in questo caso solo dalla filiazione e dal patronimico. 
del genitivo I) ma che potrebbe rientrare nella classe IV. Ci si può domandare pertanto se le formule delle classi III-IV, che sembrano scomparire quasi del tutto nel II sec. a.C., identifichino pratiche da distinguere nettamente da quelle delle formule all'assolutivo, con forse l'eccezione dei gentilizi isolati (III.a) se si riconoscesse in questi la presenza del genitivo afunzionale (non accertabile, ma cfr. IV.2.1.A per preznaś). Il caso delle quattro iscrizioni con l'antroponimo Verkantu (IV.d) porta a ritenere che il set di vasi su cui il testo è ripetuto sia verosimilmente proprietà o destinato al defunto, unico in tutte le tombe adriesi note ad avere un' arma nel corredo e dunque caratterizzarsi come guerriero in deroga alla prassi funeraria etrusco-padana (cfr. VI.2.2). Ciò potrebbe indurre a formulare l'ipotesi che l'intera classe possa marcare la proprietà del vaso o la destinazione del dono, tendenzialmente al defunto data la prevalenza di occorrenze dell'ambito funerario.

La distinzione tra la classe I e la classe IV potrebbe trovare conforto nell' interpretazione proposta da G. Colonna per l'iscrizione di Spina mi kuripeś haltuva (ET $\left.{ }^{2}, \mathrm{Sp} 2.93\right)$, già discussa (cfr. supra). Riconoscendo dei nomi nei due lessemi, lo studioso ha visto in questa due enunciati distinti: il primo, composto dal pronome mi e da kuripeś al genitivo I, è la formula dell'iscrizione parlante, cioè esplicita la proprietà o la destinazione; il secondo enunciato sarebbe costituito dal solo lessema haltuva all'assolutivo, privo secondo Colonna del verbo che lo identificherebbe quale donatore ${ }^{294}$. Donatore/dedicante all'assolutivo e destinatario al genitivo ritornano in una tipologia di testi votivi nella quale rientra anche l'iscrizione di dedica ai Kulśnuter. All'interno della classificazione di questi tipi di testi elaborata da D.F. Maras, tale iscrizione appartiene a un modulo piuttosto diffuso in età recente identificato dalle tre componenti: dedicante all'assolutivo + destinatario divino al genitivo + verbo, secondo una sequenza mai uguale ma che comunque non prevede il verbo in testa ${ }^{295}$, come ci si aspetta dall'ordine SOV che caratterizza la lingua etrusca ${ }^{296}$.

Alla luce di queste considerazioni, non sembra azzardato ritenere che all'interno della tradizione epigrafica adriese di periodo ellenistico la classe I, cioè la formula onomastica all'assolutivo senza pronome (nominativus pendens) possa rappresentare il donatore dell'offerta, almeno quando pertinente alla sfera funeraria (cioè la maggior parte delle occorrenze).

\section{IV.3.2. A. Singole lettere e digrammi associati a nomi}

Rimangono esclusi dal quadro sopra tratteggiato alcuni testi che presentano l'associazione di una lettera o un digramma a un nome: pe zurta (CIE 20514) di fine IV-III sec. a.C., $k$ snalu (CIE 20566) e $k$ zurtiu (CIE 20785) di II sec. a.C., a:risiu (CIE 20020) più genericamente di III-II sec. a.C. Si potrebbero far rientrare in questa casistica anche due iscrizioni di II sec. a.C. da necropoli, nelle quali un prenome sembra essere associato a un numerale: vna $V$ (CIE 20817) e $L$ a ata (CIE 20628; ma per questa è possibile una diversa interpretazione, per la quale si rinvia a V.1).

L'unica iscrizione da abitato di questo tipo, a:risiu, è redatta nell'interno di una ciotola a v.n. locale di III-II sec. a.C. Le altre, analoghe per scelta di supporto e posizione, sono da contesto funerario (per $k$ zurtiu il contesto è solo supposto data l'integrità del supporto). Supposto che snalu, zurtiu e risiu abbiano il valore di gentilizio (cfr. IV.3.1 per una riflessione sulle basi onomastiche), come lascerebbe pensare l'uscita in -iu e-alu, si potrebbe allora ritenere in via ipotetica che l'abbreviazione riguardi i prenomi (kavi?, anta/arza/ata?). Il sistema delle abbreviazioni onomastiche, sebbene strutturato in Etruria tirrenica per le iscrizioni di ambito ellenistico e basato su un pool onomastico poco variato, è stato proposto per altri documenti anche di epoche più antiche ${ }^{297}$, come anche per alcune iscrizioni tardo-arcaiche di Adria (cfr. IV.1) e dunque potrebbe essere qui ipotizzato, anche se in forma molto dubitativa ${ }^{298}$.

${ }^{294}$ Colonna 2007, p. 219, nota 38. Cfr. anche IV.3.1.B, categoria b).

295 Maras 2009b, p. 457, modulo 28.

296 Agostiniani 1993.

297 PANDOLFINi 2000, pp. 95-96, per un quadro del problema nel periodo arcaico.

298 È già stata proposta in passato la possibilità che alcune delle formule di periodo ellenistico ad Adria possano manifestare una pratica di abbreviazione del primo o di entrambi gli elementi di una formula onomastica più complessa (GAUCCI 
Diverso appare invece il caso del più antico pe zurta, dove quest'ultimo nome è da ritenere individuale e il digramma pe non è confrontabile con i prenomi di ambito adriese. Questo digramma è noto isolato dal periodo arcaico e quale abbreviazione perlopiù di nome familiare in varie iscrizioni funerarie ellenistiche ${ }^{299}$. In particolare, tra le attestazioni del digramma isolato graffito su vasi, sono di qualche interesse i casi documentati a Volterra dalla necropoli delle Ripaie ${ }^{300}$, e a Monterenzio dalla necropoli di Monte Tamburino ${ }^{301}$, entrambi già discussi per le lettere in legatura pe di periodo tardo-arcaico (CIE 20834; cfr. III.4). In queste attestazioni pe è ritenuto una abbreviazione di gentilizi attestati in altre iscrizioni dei medesimi contesti (petini a Volterra, petnei a Monterenzio). Purtroppo, nel nostro caso il corredo del vaso iscritto non è noto (data l'erronea attribuzione), ed è forse in ogni caso da escludere che pe sia l'abbreviazione di un gentilizio, dato che introduce un nome da ritenere individuale. Altre attestazioni del digramma provengono dal contesto sacro di Campo della Fiera (cfr. III.4), dove è interpretato come abbreviazione del teonimo Pe $\theta n$ (sebbene questo non sia altrimenti attestato nel santuario in forma sciolta). Per provare a districare il nodo interpretativo, pare di qualche utilità richiamare, anche in questo caso per la sua limpidità, la struttura dell'iscrizione ai Kulśnuter. In questa formula di dedica, il destinatario ha il primo posto ed è declinato al genitivo mentre il donatore al secondo e declinato all'assolutivo, chiude il verbo. Se si prova per ipotesi ad applicare tale struttura all' iscrizione pe zurta, potremmo supporre che il destinatario sia abbreviato e il verbo sottinteso. Sulla base di tale interpretazione, pe potrebbe evocare il defunto oppure la divinità femminile $P e \theta n$, appartenente alla cerchia di $C_{a l u^{302}}$. In particolare, l'abbreviazione permetterebbe di alludere alla divinità senza esplicitarne il nome, di fatto secondo un meccanismo che riguarda anche la medesima parola Kulśnuter, che, se valida l' interpretazione di Colonna, evoca demoni dell'Oltretomba attraverso una epiclesi, definendoli "Guardiani della Porta”. Il nome all'assolutivo può rientrare nella classe I, già analizzata precedentemente come indicazione del donatore. Viene da chiedersi se anche nelle iscrizioni $k$ snalu e $k$ zurtiu, il kappa non sia da intendere come abbreviazione di un prenome, ma allude piuttosto all'epiclesi discussa oppure a una divinità quale l'infero Kalu, documentato altrove in ambito padano (cfr. V.3) e per il quale potrebbe essere riconosciuta una attestazione adriese nell' iscrizione lacunosa della tomba femminile 160/A di Canalbianco della prima metà del III sec. a.C. (CIE 20523: ] kalu; cfr. V.3). Seguendo questo ragionamento, ci si può domandare se anche la lacunosa e davvero poco perspicua iscrizione $p k a[$ dall'area di abitato (forse sacra; cfr. VI.1.3) in località Bettola (CIE 20257) possa alludere a questa coppia divina (quindi da intendere: $p k a$ [ ?), piuttosto che essere un relitto onomastico.

In alcuni casi, anche le singole lettere possono essere interpretate come abbreviazioni. Esclusi alpha, ypsilon e $c h i$, ma anche theta e phi, che, sebbene di bassissima attestazione, possono comunque avere valore di numerale o essere funzionali a operazioni di conteggio ${ }^{303}$, le restanti provengono per la quasi totalità da necropoli:

- epsilon: 2 occorrenze (CIE 20192, 20688), di cui una da necropoli;

- digamma: una occorrenza incerta da necropoli (CIE 20889);

- zeta: 3 occorrenze probabilmente tutte da necropoli (CIE 20329, 20635, 21016);

2008, in particolare p. 102), purtroppo sulla base di letture ormai superate (cfr. CIE 21003).

$299 T h L E^{2}$, s.v.pe, 2. varie, dove sono elencati i casi di abbreviazioni, tutti di nomi familiari a eccezione di $E T^{2}$, As 1.200, che è ritenuto già dal Buffa abbreviazione di prenome, e dell' incerto CIE 10627; 3. lettere isolate.

300 L. RosSELLI, in REE LXXV, pp. 201-202, n. 14.

301 D. Vitali, in REE L, p. 261, n. 2.

302 Cfr. Maras 2009b, pp. 328-329, Pe do.4 (ET2, Pe 3.2), pp. 439-440, Vs do.3 (ET², Vs 4.4). Pare più complesso il caso dei digrammi $k a$, prevalentemente da contesto abitativo (CIE 20032, 20121, 20263?) e con una sola occorrenza da necropoli (CIE 20390), per il quale non si può escludere l'interpretazione come particella deittica isolata.

303 Qui basta rinviare a Govi $1994 \mathrm{~b}$. 
- kappa: 10 occorrenze da necropoli (CIE 20394, 20395, 20566, 20590, 20656, 20785, 20800, 20843, 20900, 20994) e una da abitato (CIE 20083)

- chet: 3 occorrenze (CIE 20522, 20657, 20841), di cui due da necropoli;

- lambda/pi: due occorrenze da necropoli (CIE 20924, 20935);

- my: due occorrenze da necropoli (CIE 20928, 20929);

- sade: due occorrenze (CIE 20036, 20326), di cui una forse da necropoli;

- sigma: una occorrenza da necropoli (CIE 20498);

- tau: una occorrenza certa da necropoli (CIE 20951).

Tra queste, i due my della tomba 46 di via Spolverin di pieno II sec. a.C. (CIE 20928-20929), uniche attestazioni isolate di questa lettera, sembrano con tutta verosimiglianza da interpretare quali iniziali del gentilizio Muliu ${ }^{304}$, che ricorre in due vasi (viza muliu, CIE 20923-20924) nel medesimo corredo. Anche il kappa in una coppa in ceramica grigia della tomba 20 di via Spolverin degli ultimi decenni del IV sec. a.C. (CIE 20900) può forse richiamare il nome individuale kramuriś graffito su un'altra ciotola del corredo (CIE 20899).

Nella ciotola a v.n. di produzione locale CIE 20657, il chet è situato in prossimità del foro pervio al centro della vasca che identifica il vaso come una patera pertusa, dunque forse da ritenere legato a pratiche di natura rituale. Tale associazione fra lettera e foro potrebbe qui essere indizio di una allusione a un lessico di natura sacra ${ }^{305}$. A tale proposito, si ricorda come la recente analisi di V. Bellelli su alcuni documenti ceretani abbia rimarcato il ruolo di parole scritte e simboli grafici graffiti nelle c.d.paterae pertusae ${ }^{306}$.

Più in generale, il valore allusivo di queste lettere, sia riferibile a un lessico sacro sia a un ambito onomastico, non offre mai la possibilità di scioglierne il senso con sicurezza, sebbene in qualche caso il contesto, come già evidenziato, possa aiutare in questa operazione.

Rimangono infine da discutere i due casi di prenome associato a numerale. In CIE 20817, il probabile prenome, vna > venza, è seguito da un ypsilon rovesciato, cioè il numerale $V^{307}$. La struttura sembra piuttosto diversa dall'altra iscrizione, CIE 20628, che, se seguiamo il ductus sinistroso suggerito dal prenome, presenta il testo introdotto da un $c h i$ rovesciato, cioè il numerale $L$, seguito da un alpha e da ata. L'alpha isolato può essere inteso come falsa partenza del prenome oppure si potrebbe ipotizzare che la lettera e il $c h i$ rovesciato evochino la sequenza alfabetica (cfr. V.1). Se quest'ultima lettura cogliesse nel segno, l'iscrizione vna $V$ sarebbe un caso del tutto isolato sul quale risulta difficile avanzare ipotesi concrete.

\section{IV.3.3. I nomi familiari}

L'analisi dell'onomastica di III-II sec. a.C. (cfr. IV.3.1) ha permesso di riconoscere, seppure in alcuni casi in maniera ipotetica, almeno 44 nomi familiari, che si possono suddividere secondo diverse terminazioni (Tab. 5).

304 Così già in GAUCCI 2008, p. 85, nn. 12-13.

${ }^{305}$ Un interessante parallelo potrebbe essere individuato in un frammento di kylix attica dal santuario di Gravisca. Questo è caratterizzato da un chet presso il bordo del foro pervio al centro della vasca (CIE 10338; PANDOLFINI 2000, p. 81, n. 433). La frattura subito dopo la lettera impedisce di comprendere se questa fosse stata isolata oppure parte di un testo più lungo.

306 Da ultimo, Bellelli 2014, pp. 47-49, con riferimenti precedenti.

307 Per le precedenti letture, si veda la scheda nel fascicolo CIE IV, I, 1 e più in particolare per la proposta di leggervi vnat con un poco verosimile tau, G. Colonna, in REE LXXIII, p. 369, n. 93. Un caso del tutto simile a quello di CIE 20817 proviene dall'abitato di Spina, dove vna è associato a un segno a croce (G. UGGERI, in REE XLVIII, p. 342, n. 18, fig. 1), probabilmente da intendere come numerale 10; in questo caso il supporto è datato alla metà circa del V sec. a.C. 
Tab. 5. Nomi familiari suddivisi sulla base delle terminazioni.

\begin{tabular}{|c|c|c|c|}
\hline & FINE IV-III SEC. A.C. & FINE III-PRIMA METÀ I SEC. A.C. & III-II SEC. A.C. \\
\hline$-\mathrm{C}+i u$ & $\begin{array}{l}\text { atiu, velouriu, zurtiu, kraiu (?), } \\
\text { fastiu, fuliu }\end{array}$ & kaniu, muliu, siliu, spiu, uniӨiu & $\begin{array}{l}\text { ankariu, kepriu, heturiu, } \\
\text { risiu, fastiu }\end{array}$ \\
\hline$-\mathrm{V} / \mathrm{C}+a l u$ & $\begin{array}{l}\text { estalu(s's?), zurtalu, tetialus', } \\
\text { ]×italus' }\end{array}$ & lintialu, snalu & viufralus', titalu \\
\hline$-\mathrm{V} / \mathrm{C}+n a / n a i$ & krapnai (?),prezna(s'?), setina, & atna, lentnai, titinai & arnai \\
\hline$-\mathrm{V}+r a$ & mura & $\begin{array}{l}\text { mura afr, } \\
\text { murapili }\end{array}$ & \\
\hline$-\mathrm{C}+i a$ & lausia & kusia, lausia, puinia & \\
\hline$-\mathrm{V} / \mathrm{C}+n i$ & vercni(s's), malni, siluniś & & \\
\hline$-\mathrm{V} / \mathrm{C}+i$ & & veri (?), lai, titi & \\
\hline$-\mathrm{C}+u$ & & $\sin u$ & $z u \chi u$ \\
\hline$-\mathrm{C}+t e$ & kleuste & & \\
\hline
\end{tabular}

A questi può essere aggiunto anche l'isolato tite, la cui cronologia resta incerta. Questo si presenta come un Vornamengentile, e recentemente E. Benelli ha ipotizzato che sia un Echte Gentile (si veda IV.3.1, categoria b).

Nel II sec. a.C. sono attivi due cognomina, Afr e Pili. Anche Zuðu e Sinu, di più incerta cronologia, potrebbero dubitativamente avere medesima funzione. Sebbene ci si possa domandare quanto l'uso del cognomen fosse attivo ad Adria (e se, ad esempio, prevedesse dinamiche di sostituzione al gentilizio come in ambito perugino), il tipo di documentazione a disposizione non consente purtroppo affondi di questo tipo, che restano quindi sospesi alle due occorrenze di più limpida interpretazione, entrambe associate al gentilizio Mura.

I nomi con suffisso derivativo -na, -ni, -ra appartengono a gentilizi di diretta origine cisappenninica, con una prevalenza dell'Etruria settentrionale interna (Chiusi, Perugia, Cortona), ma anche meridionale (Vulci, Orvieto). All'Etruria propria possono rimandare anche Kleuste e Tite, sempre di ambito

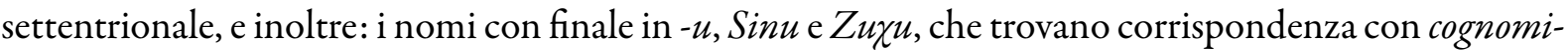
na chiusini, ma che sono già attivi prima come prenomi e nomi individuali anche nel settore padano; quelli con finale -i, di cui Veri e Titi attestati a Cortona e Chiusi, e Lai, forse femminile, attestato a Bolsena e Roselle.

Nel complesso, a partire almeno dal III sec. a.C. e ancora nel secolo a seguire, colpisce il legame molto forte con l'Etruria settentrionale interna, in particolare Chiusi e Perugia, ma anche Cortona e Volterra $^{308}$. Da questi luoghi sembra che provengano individui appartenenti a gentes di lunga tradizione, ma forse anche altre di più recente formazione e forse di origine servile. Più debole la presenza di nomi familiari di acclarata origine padana, riconoscibili nel suffisso -alu, al quale si dedica un approfondimento (IV.3.3.B), mentre rimane sub iudice l'attività di -va (e -vi?) nella formazione di gentilizi (cfr. IV.3.1.B, categoria d).

Sicuramente i nomi con terminazione -iu sono i più diffusi e dimostrano l'attività di questo suffisso con basi onomastiche etrusche (se effettivamente sono valide le considerazioni per il suo riconoscimento; cfr. IV.3.1). Solo in una fase prossima alla romanizzazione del centro tale suffisso sembra usato per la formazione di nomi familiari di origine alloctona, in un quadro che pare tuttavia ormai completamente destrutturato rispetto al secolo precedente, come qui di seguito si prova a illustrare.

308 I confronti dipendono dalla quantità delle occorrenze nei diversi ambiti e quindi questo rende più o meno rilevante il dato. Per Cortona, L. Agostiniani ritiene significativa l'occasionalità dei rapporti con Volterra e l'area settentrionale transappenninica (Agostiniani, Nicosia 2000, p. 80). 


\section{IV.3.3. A. I nomi familiari con terminazione -iu}

I nomi terminanti in $-\mathrm{V} / \mathrm{C}+i u$ rappresentano sicuramente la casistica più attestata tra i nomi familiari, con 15 distinte occorrenze. Un quadro di sintesi su questa terminazione è stato di recente offerto da $\mathrm{E}$. Govi $^{309}$. Sembra utile qui riprendere in maniera sintetica il problema per inserirvi la documentazione adriese.

Il suffisso -iu non lascia dubbi sulla sua esistenza, peraltro con funzioni diverse, in quanto attivo come diminutivo di prenomi, formativo di appellativi e anche come derivativo di nomi familiari ${ }^{310}$. Tale isovalenza è già documentata entro il VII-VI sec. a.C. ${ }^{311}$. Tuttavia vi è una sostanziale difficoltà insita nel suo riconoscimento, in quanto formazioni suffissali diverse posso avere come risultato la terminazione -iu.

Il suffisso in questione non è però problematico solo nella sua individuazione, ma lo è anche nella sua ermeneutica. Infatti, se è pacifico che questo sia attivo nell'onomastica etrusca, dove H. Rix per l'ambito tirrenico e la fase recente ne ha riconosciuto il valore di cognomen ${ }^{312}$, M. Cristofani ne ha proposto per l'ambito padano la derivazione dal morfema -io degli appositivi venetici ${ }^{313}$, mentre A. Maggiani il valore di morfema di filiazione ${ }^{314}$; suffisso derivativo di tipo patronimico è anche per $\mathrm{G}$. van Heems, almeno per il settore padano, dove riconosce meccanismi di formazione onomastica differenti rispetto all'Etruria tirrenica e una possibile isofunzionalità tra i suffissi -iu e-alu $u^{315}$. Anche secondo E. Govi, che da ultima si è occupata del problema, sussiste tale isofunzionalità, documentando così il forte particolarismo dell'onomastica padana. G. van Heems inoltre ha rimarcato la similarità del nostro suffisso con il greco -10V, soprattutto perché neutro in rapporto al carattere epiceno che acquisirebbero i composti con il suffisso etrusco ${ }^{316}$. Sempre secondo lo studioso, se si dovesse pensare a una acquisizione, non sarebbe comunque il greco da tenere come riferimento, ma piuttosto il latino o l'umbro.

Recentemente l'ambito padano si è arricchito di due nuove attestazioni di V sec. a.C., una da Marzabotto, dove nella lamina di bronzo dal tempio di Tinia è attestato Lar $\theta$ Lentiu $^{317}$, e una da Bologna, dove in una stele di metà V sec. a.C. è attestato il gentilizio Atiniu (possibili lacune iniziali della base onomastica non inficiano il valore dell'attestazione $)^{318}$. In particolare, D.F. Maras ritiene quest'ultimo formato dall'agglutinazione dei suffissi -na+iu, dove -iu è posto in parallelo con -ie di origine italica e ricondotto all' ipotesi di A. Maggiani di intendere questa rideterminazione quale affermazione dell'appartenenza ad antiche origini nobiliari da parte dei gruppi gentilizi che la adottarono ${ }^{319}$. A completamento del dossier anteriore al periodo ellenistico, si ricorda sempre a Marzabotto il śakiu [-- - d di fine VI sec. a.C. (altrimenti letto da Maggiani śakn[---] con differente interpretazione ${ }^{320}$ ), e akius di fine V sec. a.C., nome individuale probabilmente da mettere in connessione diretta con il distretto minerario dell'Etruria settentrionale costiera $^{321}$. E. Govi aggiunge a questo quadro, seppure in maniera dubitativa data la lacunosità del testo in quel punto, anche venes [---]iu dell' anforetta Melenzani di fine VII-inizi VI sec. a.C. ${ }^{322}$. A eccezione di akius, tutti i casi sono interpretati come nomi familiari ${ }^{323}$.

309 Govi 2014, pp. 134-136.

310 VAN HEEMS 2008, pp. 82-85.

311 Govi 2014, p. 135, nota 159.

312 Rix 1963, pp. 153-161.

313 Cristofani 1991, p. 62.

314 Maggiani 2002a, p. 61.

315 VAN HeEms 2008, p. 85, nota 1.

316 Ibid., p. 84.

317 Govi 2014, pp. 134-135.

318 Maras 2014, pp. 337-338.

319 Maggiani 2000b, p. 267.

320 Maggiani 2009, p. 227.

321 Maggiani 1992b, p. 184; SASSATElli 1994, p. 19, n. 13.

322 Morigi Govi, Colonna 1981, pp. 88-89.

323 Anche nel ridotto dossier di attestazioni note nell'Etruria tirrenica, sintetizzato da E. Govi (2014, p. 135, nota 159), a fronte di alcuni casi interpretati come prenomi, ve ne sono altri dove il suffisso -iu è ritenuto formante gentilizi. 
Passando al periodo ellenistico, si ricorda anzi tutto il caso di laiu plaiskna [ di Mantova, dove la terminazione è documentata nel prenome, che D.F. Maras riconosce come di provenienza italica (lai$v e$ ), pur non escludendo una terminazione di origine celtica ${ }^{324}$. In questa fase cronologica più tarda si situa anche la documentazione di Spina, molto limitata: per Valle Trebba si hanno tre occorrenze, due in formula binomia (venuś puliuś e larzl perciuś, rispettivamente $E T^{2}, S \mathrm{p} 2.56$ e 2.75 ) e due monomie (karsius: $E T^{2}, 2.81$; vipiu ${ }^{325}$ ); per Valle Pega le occorrenze enumerabili sono tutte inedite, 3 sicuramente in formula binomia, 3 in formula monomia ${ }^{326}$.

Ad Adria, rispetto al quadro già fornito in passato ${ }^{327}$, una analisi complessiva porta qualche elemento di novità. Anzi tutto è necessario premettere che non tutte le occorrenze adriesi terminanti in -iu sono univocamente interpretabili come composte con il relativo suffisso. La maggior parte dei casi considerati in effetti può presentare in tal senso delle ambiguità (come è già stato rilevato ${ }^{328}$ ), perché più di uno possono essere i processi di rimorfologizzazione di basi onomastiche che determinano i nomi in -iu (e comunque tale ambiguità non è limitata alla potenziale eterogenità dei processi, ma anche alla loro possibile natura non onomastica come è stato ipotizzato per leiu: IV.3.1.B, categoria b). Tuttavia almeno due nomi familiari, Zurtiu e Fuliu (cfr. IV.3.1.A, categoria a; fine IV-III sec. a.C.) sono formati con buona verosimiglianza con il suffisso -iu, sostituito alla vocale terminale della base onomastica ${ }^{329}$. Durante il III sec. a.C. tale processo sarebbe generato da basi gentilizie (Fuliu), ma anche da nomi individuali (es. $z$ urta+iu > zurtiu).

Un processo che determina nomi in -iu è stato messo a fuoco da A. Maggiani e riguarda un gruppo di iscrizioni su urne in travertino in collezioni private lucchesi e verosimilmente originariamente concentrate in un unico complesso funerario di ambito perugino ${ }^{330}$. Le iscrizioni documentano un gruppo caratterizzato da due nomi familiari, Petru e LeӨiu, che Maggiani riconosce nello schema Vornamengentilicium-Patronusgentilicium già elaborato da H. Rix ${ }^{331}$. Petru non presenta suffissi derivativi (cioè non fa parte degli Echte Gentilicia) ed è pertanto possibile ritenerlo a tutti gli effetti uno dei rari casi di Vornamengentile, non venendo così colpito dalla recente rilettura di questa ultima categoria di nomi da parte di E. Benelli ${ }^{332}$. Letiu, secondo Maggiani e sulla base di una delle iscrizioni ([-- ] petru.le日eu.au: $E T^{2}$, Pe 1.879), sarebbe invece costruito sul gentilizio del patronus le $\theta e(s)^{333}$ con l'aggiunta del suffisso $-u$, da considerare come forma aggettivale derivativa, e da cui si avrebbe infine la forma le $\theta i u$ (tenendo conto però, come puntualizzato da Maggiani, che l'iscrizione in questione non è facilmente collocabile nella prosopografia familiare). Nella trafila prosopografica, il Vornamengentile sarebbe dunque abbreviato in favore del nome derivato dal patrono, assunto come nome familiare identificativo. È possibile che anche nei casi adriesi si abbiano formazioni in $-u$ da gentilizi di patroni, secondo un meccanismo già concluso al momento dell'arrivo di questi gruppi ad Adria?

324 Menotti, Maras 2012, p. 884.

325 Tale iscrizione, edita da G. UGGERI, in REE LVII, p. 269, n. 40 (ma non presente in $E T^{2}$ ), è stata erroneamente riferita alla tomba 128, come rilevato da A. Pozzi, che più correttamente la attribuisce alla tomba 262 (Pozzi 2011, p. 89).

326 Pozzi 2011: le binomie dalle tombe 843B, 294C, 339A; le monomie dalle tombe 683A, 448B, 66C. Si è deciso di non inserire prudenzialmente in questo conteggio l'iscrizione dalla tomba $41 \mathrm{~B}$, che comunque non influenza in maniera significativa il dato.

327 Cfr. Gaucci, Pozzi 2009, pp. 60 e 62; Gaucci 2016, p. 200, nota 114.

328 BeNELli 2014, p. 70.

329 Così anche van HeEms 2008, p. 82.

330 REE L, pp. 275-280, nn. 20-27.

331 Rix 1963, pp. 376-378.

332 Benelli 2011.

333 Il problema di questo come Vornamengentile, espresso da Maggiani, che ne fa, richiamando Rix 1963, p. 378, un caso di precoce concessione di diritto di cittadinanza (REE L, p. 279), viene ora di fatto risolto da E. Benelli, che vede in questo nome un Echte Gentile sulla base della trafila le $\theta a i e>l e \theta a e>l e \theta e$ (BENELLI 2014, p. 197). 
Se quest'ultima domanda ha qualche fondamento, è plausibile ritenere in via teorica che la terminazione - $i u$ sia il frutto di meccanismi onomastici differenziati, prodotti direttamente ad Adria oppure già compiuti nel momento in cui i nomi vennero fissati tramite la scrittura. Inoltre, anche ammessa l'esistenza di percorsi formativi differenziati, è possibile che almeno ad Adria nel tempo si sclerotizzi l'uso del suffisso -iu, tanto da risultare nel tardo II sec. a.C. la terminazione usata in gentilizi acquisiti da gruppi familiari di probabile origine alloctona. In questi è forse possibile (ma non necessario) che la somiglianza della terminazione in esame con il morfema venetico e greco -io $(s)$ abbia in qualche maniera influito nella scelta di usare forme rideterminate quali gentilizi formalmente identici a quelli con terminazione -iu.

Sulla base delle considerazioni qui sviluppate e di quelle sui singoli nomi offerte precedentemente (cfr. IV.3.1), potremmo azzardare il seguente quadro:

- Gentilizio direttamente originario dell'Etruria settentrionale: Spiu (II sec. a.C.);

- Gentilizi etruschi rimorfologizzati con il suffisso -iu: Fuliu (III sec. a.C.), e forse Kepriu;

- Gentilizi etruschi (prevalente l'Etruria interna) rimorfologizzati con - $u$ oppure -iu: Ankariu e Heturiu, VelOuriu (III sec. a.C.);

- Nomi individuali rimorfologizzati con il suffisso -iu: Zurtiu (III sec. a.C.), e forse UniӨiu (fine III-II sec. a.C.);

- Basi onomastiche di origine alloctona rimorfologizzate con il suffisso -iu: Kaniu, Muliu, Śiliu (tutti tra il pieno II e la prima metà del I sec. a.C.);

- Altre basi onomastiche etrusche rimorfologizzate con - $u$ oppure -iu: Atiu, Risiu, Fastiu.

Ammesso che anche Kraiu sia un nome familiare, non è facilmente inseribile in questo schema data l'incertezza dell'origine della radice (cfr. IV.3.1.B, categoria b).

Si osserva che le basi onomastiche almeno fino circa alla metà del II sec. a.C., come anche quelle più antiche prima menzionate, sono sostanzialmente etrusche. Si tratta cioè di un fenomeno che non sembra riguardare nomi allogeni etruschizzati, dunque apporti diretti dall'esterno, ma piuttosto interno all'onomastica etrusca. Va peraltro considerato che solo dopo la metà del II sec. a.C. alcuni nomi da intendere come gentilizi (Kaniu, Muliu, Siliu) sembrano derivare direttamente dall'onomastica greca e venetica in un momento in cui, come già osservato, anche il sistema dei prenomi pare allentare le sue maglie verso un'apertura maggiore a forme di derivazione alloctona (Apu, Viza, Kraiku, Фaia). Ma, come già detto, questi potrebbero comunque essere stati adattati per analogia al bacino onomastico etrusco conformandosi ai nomi familiari in uso nel periodo.

Per quanto riguarda i derivati su base gentilizia, ci si può chiedere quale sia la differenza di questi nomi rimorfologizzati rispetto ad altri familiari sempre di origine cisappenninica che non hanno subito tale rimorfologizzazione (es. il piuttosto diffuso Mura). In effetti, i processi differenziati individuati concorrono a ritenere che a monte della formazione dei gentilizi in - $i u$ adriesi ci possano essere perlopiù categorie sociali subalterne (quali servi e stranieri) caratterizzate da molteplici vicende prosopografiche, che possono essere approdate ad Adria già in possesso di un gentilizio (es. liberti), oppure averlo assunto localmente. Se nel primo caso può essere probabile una formazione con $-u$, nel secondo si può ritenere plausibile che il suffisso sia -iu attivo in area padana almeno dal V sec. a.C.

A chiusura delle riflessioni su tale terminazione, non sembra inappropriato affrontare il caso dei nomi femminili terminanti in -ia. Si documentano le occorrenze di Lausia, attestato in ben tre iscrizioni distinte tra III e II sec. a.C., inoltre Kusia e Puinia nel II sec. a.C. Si tratta di gentilizi sempre del comparto etrusco-settentrionale interno. Mentre ci si aspetterebbe almeno per due occorrenze le forme femminili Kusui e Puinei, in quanto altrove documentate, si coglie qui una rimorfologizzazione diversa. Si è osservato che la scelta delle formule onomastiche nei testi sembra dipendere da precise scelte sociali, che possono cambiare nel tempo. Ciò non permette di comprendere se i nomi con terminazione -iu possano avere anche valore femminile. Allo stato attuale della documentazione, solo come ipotesi di lavoro, si può avanzare l'idea che -ia terminale sia la forma femminile di -iu, che dunque non avrebbe valore epiceno. 


\section{IV.3.3.B. I nomi familiari con terminazione -alu}

Per quanto riguarda le occorrenze con terminazione -alu, a tutti gli effetti un suffisso, queste sono pari numericamente a quelle del suffisso -na e si concentrano soprattutto nel III sec. a.C., confermando il dato statistico già osservato in passato ${ }^{334}$. Diversamente dai nomi con terminazione -iu, quelli con suffisso - alu sembrano formarsi da basi onomastiche di origine alloctona (Estalu o Estaluś, Lintialu; incerto il caso di Viufraluś) o da probabili nomi individuali (Zurtalu, Tetialuś; cfr. IV.3.1).

$\mathrm{Al}$ fine di avere un quadro il più possibile esaustivo, è opportuno ripercorrere a grandi linee le tappe della critica sulla funzione dei nomi con tale suffisso. Un primo tentativo di discussione si deve a B.A. Terracini nel 1931 (che identificava un gruppo-ale/-alu con valore diminutivo) soprattutto in merito al rapporto con -alos attivo dell'onomastica leponzia (allora ritenuta ligure, ora celtica) come già precedentemente discusso dal Pedersen e dal Kretschmer ${ }^{335}$. La questione è stata poi ripresa da A.J. Pfiffig, che, sospendendo il giudizio sul dibattito tra i precedenti studiosi, ha ritenuto il suffisso come "risolutamente" aggettivale, portando a conferma il confronto con altre iscrizioni di area nord-orientale (Sanzeno, Magré, Lagole), e lo ha interpretato come formante patronimici piuttosto che gentilizi ${ }^{336}$. È stato poi ritenuto formante gentilizi ma di origine patronimica da $\mathrm{H}$. $\mathrm{Rix}^{337}$, e tale funzione è stata accettata anche da C. de Simone nelle occorrenze tirreniche, mentre nei casi padani quest'ultimo si è dichiarato incerto sulla funzione gentilizia o patronimica, soprattutto a causa della scarsa affidabilità statistica delle poche occorrenze disponibili ${ }^{338}$; la contiguità geografica ha portato inoltre de Simone a ritenere più probabile una derivazione dall'onomastica venetica e istriana, dove tale suffisso era attivo, piuttosto che leponzia ${ }^{339}$. Nel $1971 \mathrm{M}$. Lejeune ha avanzato l'ipotesi che -alo- fosse la derivazione del genitivo II etrusco (e retico) tematizzato ${ }^{340}$. Sull'argomento ${ }^{341}$ è quindi tornato G. Colonna nell'importante contributo sull' iscrizione di Castelletto Ticino ${ }^{342}$. Lo studioso è partito dall' iscrizione di dono di due kotylai in bucchero da Castelnuovo Berardenga datate al 625 a.C. circa $^{343}$ : qui l'antroponimo del donatore keivale è ritenuto l'etruschizzazione di un nome con suffisso - alo- di ambito leponzio e ligure, mentre la base troverebbe confronto in ambito ligure. Sempre dall'ambito ligure verrebbe enistale da Ameglia ${ }^{344}$. Colonna ha puntualizzato che tali occorrenze si distinguono da quelle etrusche con terminazione -alu, per le quali ha riconosciuto la mediazione dell'Etruria padana.

Si deve a G. Sassatelli il primo quadro esaustivo sull'argomento ${ }^{345}$ : «Esiste in area padana un consistente gruppo di gentilizi caratterizzati dal formante -alu che si ricollega al noto suffisso patronimico - alo di ambito leponzio e ligure. Anche se spesso viene riaperto il problema della loro effettiva funzione sul piano onomastico, è certo comunque che questi nomi in -alu da un probabile originario valore patronimico si sono evoluti in gentilizi, se non altro per la necessità di adeguarsi al sistema onomastico etrusco di cui fanno parte ${ }^{346}$. Sassatelli, riconoscendo una derivazione «da nomi in-

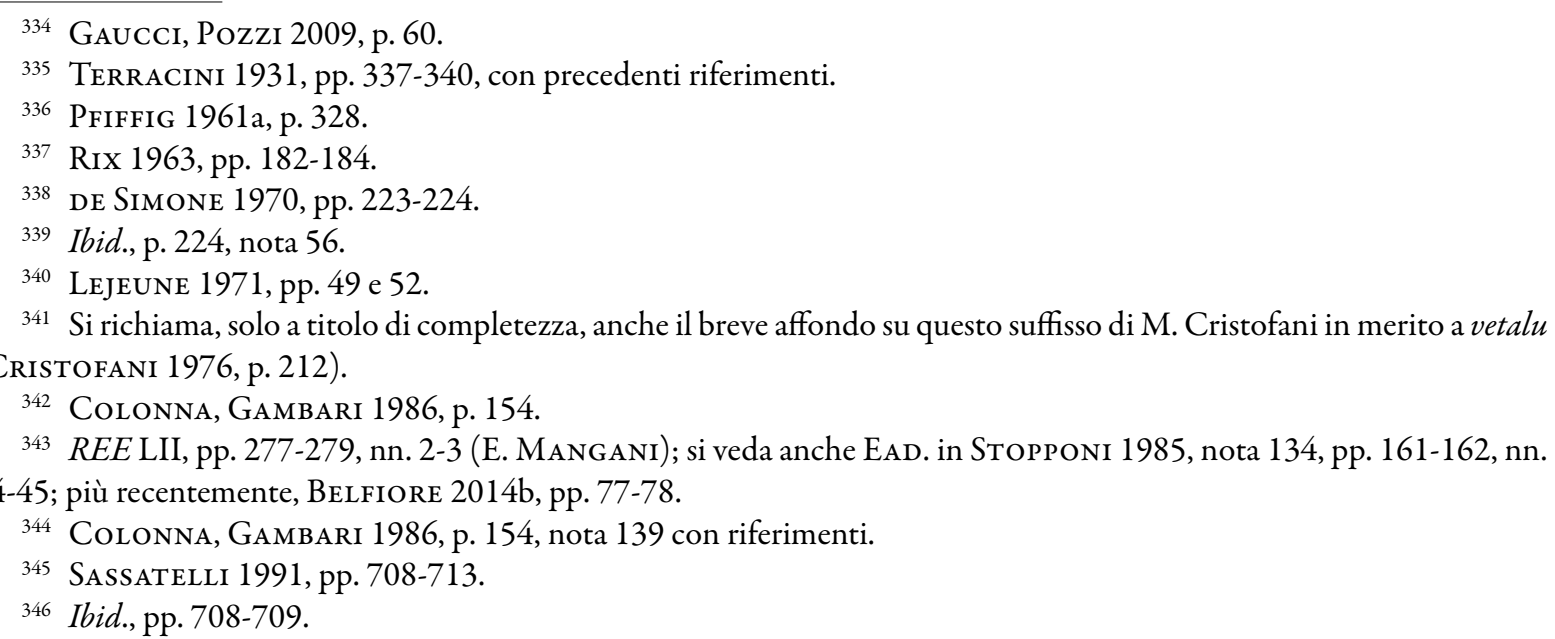


dividuali, o più precisamente da prenomi», offre un quadro aggiornato delle occorrenze di ambito padano e tirrenico, che risulta allo stato attuale delle ricerche incrementato di poco ${ }^{347}$. Inoltre, lo studioso ha sottolineato come la documentazione tirrenica sia concentrata nel periodo ellenistico, di contro a quella padana che rimonta almeno all'anforetta Melenzani della fine del VII-inizi VI sec. a.C. con remeśalu, di cui è riconosciuta la radice formativa etrusca ${ }^{*}$ remeśs ${ }^{348}$. Più recentemente, A. Maggiani, nel lavoro del 2002 sulle iscrizioni di Adria, è tornato a proporre la possibilità di una conservazione dell'accezione patronimica almeno per i nomi in -alu (diverso per lo studioso il caso di quelli con genitivo afunzionale in formule binomie, da intendere come gentilizi) ${ }^{349}$.

L'elenco aggiornato di ambito padano vede nel periodo tardo-arcaico i casi di Marzabotto e di Spina databili alla fine del VI-primi decenni del V sec. a.C., formati su basi onomastiche da ritenere etrusche o in un caso di probabile derivazione greca ${ }^{350}$. Nel V sec. a.C. si registrano a Bologna pluxśaluś, titlaluś, ] nalu[, ]uśvaluś, a Marzabotto axalu (x2), kraikaluśs śualuś e nel territorio di Adria meslaluś (CIE 21040, da Le Balone), di cui l'unico di derivazione plausibilmente celtica è quello adriese; tra la fine del V e il IV sec. a.C. a Spina mitalus', mutaluś, tulaluś, tumalus's51 e ad Adria kutvalus'; infine tra la fine del IV e il III sec. a.C., oltre alle occorrenze adriesi, si registrano a Spina akalu[, veialus', plaiskalu, platunalus', prasalu, prataluś, rautialu, sekstalus's, suntalu, taluś, titaluś, tutalu [, e a Monte Bibele laӨialus ${ }^{352}$, mentre nulla è attestato più a ovest. Espungendo il ni kaluś e il ] titelu del Mantovano (Castellazzo della Garolda) ${ }^{353}$, colpisce come il suffisso non sia documentato (e il corpus noto non è irrilevante) nei settori a più diretto contatto con il mondo di cultura celtica, ma sembra piuttosto caratterizzare il settore orientale dell' area padana ${ }^{354}$, concentrandosi soprattutto nella direttrice tra il Delta e la valle del Reno.

347 Cfr. Uggeri 1998 e G. Colonna, in REE LXXIV, pp. 369-372, nn. 114-122. Va sottolineato che la recente edizione dell'Additamentum a CIE, II, 1, ha portato C. de Simone a ipotizzare che l'iscrizione apalus datata al V sec. a.C. da Narce (CIE 8899) sia una formazione in -alu dell'appellativo apa (DE Simone 2007, p. 236), sebbene gli editori del fascicolo lo ritengano piuttosto un teonimico (Apulus $>$ aplu).

348 Morigi Govi, Colonna 1981, p. 87.

349 Maggiani 2002a, p. 59.

350 Per Marzabotto: Sassatelli, Gaucci 2010, pp. 315-319, nn. 434-435 (rakaluś e śataluś), fine VI-inizi V sec. a.C.; ibid., pp. 328-329, n. 443 (vetaluś), prima metà del V sec. a.C.; SASSATELLi 1994, p. 54, n. 66 (niritalu, lettura non recepita in $E T^{2}$, Fe 3.2, dove si riporta quella precedente di G. Colonna), prima metà del V sec. a.C. Si osserva che tutte le basi onomastiche sono etrusche, a eccezione di niritalu, per la quale S. Marchesini ha supposto una derivazione dall'aggettivo gr. vท́pı ${ }^{*}$ Nerite+alu > niritalu (MARCHESINI 1994; cfr. anche MARAS 2009b, pp. 322-323, Pa do.2); pare convincente la spiegazione dal greco (si ricordi anche il poco più tardo kraikaluś, per il quale cfr. SASSATELLi 1994, p. 59, n. 69), a fronte di una spiegazione fragile dal celtico nerto- offerta in UGGERI 1998, p. 498, n. 35 (dove si rimanda a Ellis Evans 1967, p. 267, fraintendendo peraltro la spiegazione della radice: “forte”, non “povero" come dice l'Uggeri). Una sola è l'occorrenza da Spina in questo orizzonte cronologico: f. sakśalua: ET ${ }^{2}$, Sp 2.1; ma diversa lettura propone G. ColonNA, in REE XLVI, p. 379, n. 139, dove si preferisce (ma non univocamente, data la circolarità dell'impaginazione) aksaluaś, vedendovi un cumulo di suffissi, -alu+va (tale ultima lettura è ripresa in UGGERI 1998, p. 499, n. 40, sebbene il medesimo Colonna avesse già precedentemente accolto quella di Rix: Colonna 1993, p. 139; in merito alla lettura di Rix, vale la pena richiamare la radice verbale ${ }^{*}$ sac- della $T C$ in CRISTOFANi 1995, in particolare p. 73).

351 Cfr. UgGeri 1998, pp. 495, 497, nn. 14, 15, 24, 26; per tumaluś anche G. Colonna, in REE LXXIV, p. 370, n. 117.

352 Per Spina e Monterenzio, si rinvia al catalogo sostanzialmente ancora valido di UGGERI 1998, recepito in $E T^{2}$.

353 Il primo è interpretato come antroponimo in UGGERI 1998, p. 492, n. 1, sebbene già dal primo editore, R. de Marinis e fino al repertorio del Rix (ora $E T^{2}, \mathrm{~Pa} 2.10$ ), sia ritenuto un teonimo (così anche nello studio sul dono votivo di MARAS 2009b: pp. 320-321, Pa co.3). Il secondo invece (lacunoso) è stato introdotto nel dossier sulla base dell'interpretazione come variante di ${ }^{*}$ titalu da parte di SASSATELLI (1991, p. 710, n. 26), poi accettata da UGGERI (1998, p. 492, n. 1).

354 Questa osservazione differisce da quanto già osservato in UGGERI 1998, p. 501, proprio per la prudenziale omissione delle testimonianze del Mantovano. 
Confermata allo stato attuale degli studi la più alta cronologia delle occorrenze padane, come già sostenuto da $\mathrm{H}$. Rix nel 1963, che ne aveva appunto riconosciuto il ruolo di gentilizi ${ }^{355}$ (poi ribadito da G. Sassatelli ${ }^{356}$ ), il centro originario di questo suffisso quale produttore di nomi familiari nell'onomastica etrusca sembra essere Bologna e il suo territorio tra la fine del VII e il VI sec. a.C. ${ }^{357}$.

A chiosa di queste osservazioni, sembra utile aggiungere un altro elemento di riflessione. Negli stessi anni e poco dopo il lavoro di Sassatelli, gli studi di A.L. Prosdocimi hanno portato a scardinare l'idea che i nomi leponzi in -alo- per pertinenza primaria fossero patronimici (che rimane comunque il mezzo generativo più immediato per il secondo elemento della formula), ma piuttosto appositivi sul modello dei nomi familiari etruschi (senza averne però la funzione sociale e civica) ${ }^{358}$; in sostanza l'appositivo avrebbe avuto funzione culturale ma non strutturale/istituzionale e per ciò si poteva sovrapporre al patronimico (che ha una funzione istituzionale propria) ma non collimare con questo.

Nel caso specifico di Adria, oltre all'isolato kutvaluśs di V o più probabilmente IV sec. a.C., i gentilizi certi e quelli isolati, per i quali tuttavia una funzione di nome familiare è comunque probabile, sembrano perlopiù derivare da nomi individuali e prenomi, come già sostenuto da Sassatelli per il quadro più ampio padano. Questo peraltro in parallelo con quanto si può osservare per Spina per il medesimo periodo. Viene allora da domandarsi se tale suffisso, indipendentemente dalla sua formazione, nella tarda etruscità padana ( $\mathrm{ma}$ forse anche prima?) fosse generativo di nomi familiari derivanti da individui cooptati nella comunità degli aventi diritti, non necessariamente di origine non etrusca.

355 Rix 1963, p. 182: «Der Bildungstyp hat offenbar seinen Ursprung bei den Etruskern der Poebene, wo er auffallend reich vertreten ist. Seine Analyse, die mit verschiedenen ethnisch-linguistischen Komponenten zu rechnen hat, ist viel diskutiert; eine ursprünglich patronymische Bildung ist das Wahrscheinlichste. Jedenfalls besteht kein Anlaß zu der Annahme, daß die Namen auf - alu jemals etwas anderes gewesen sind als Familiennamen bzw. in terminologischem Sinn Gentilizia».

356 Sassatelli 1991, p. 711.

357 Con ciò, non si concorda con le osservazioni avanzate in UGGERI 1998, p. 501, che, pur riconoscendo un primato cronologico padano, mette sullo stesso piano i porti del Delta e la metropoli dell'entroterra, collocando l'acquisizione del suffisso - alu, ritenuto un imprestito celtico (va sottolineato come a fronte di un ricco catalogo, non si mettano a sistema i dati ricavati dalle singole interpretazioni) in un più generico «vasto fenomeno di osmosi culturale e sociale che interessa la pianura padana tra il V e il III sec. a.C.».

358 Cfr. Prosdocimi 2009, pp. 82-83, con riferimenti. 



\section{ALFABETARI, TEONIMI, LESSICO}

In questo capitolo si illustrano tutte quelle categorie di contenuto che esulano dall'onomastica. Nella fattispecie, si trattano le sequenze alfabetiche (V.1), le attestazioni del lessema al (V.2), i teonimi e il lessico del sacro (V.3) e infine altre voci di lessico di periodo ellenistico (V.4) a cui non si riconosce un valore onomastico e che non rientrano nelle precedenti categorie.

\section{V.1. Sequenze alfabetiche}

La categoria degli alfabetari è presente ad Adria con alcune occorrenze che si concentrano, come d'altra parte la maggior parte della documentazione epigrafica, nel periodo tardo-arcaico e in quello ellenistico.

Nel periodo tardo-arcaico, si registra una sequenza con lettere in legatura in probabile norma settentrionale (CIE 20371) e una lacunosa in norma meridionale (CIE 20189), entrambe da abitato. In quest'ultima, la posizione delle prime quattro lettere dell'alfabeto (quelle conservate) al centro della vasca suggerisce che la sequenza doveva prevedere un numero limitato di lettere. Dunque, in entrambi i casi la graffitura delle prime lettere della serie alfabetica veicola il valore allusivo all'intero alfabeto, come manifestazione della conoscenza della scrittura e con probabili connessioni con la sfera magicoreligiosa ${ }^{1}$. In particolare, la legatura delle lettere in CIE 20371, con probabile intento decorativo, e la loro incisione a crudo nel fondo esterno sono ulteriore prova di questa volontà di esibizione, come già rimarcato da G. Sassatelli per simili documenti rinvenuti a Kainua-Marzabotto ${ }^{2}$.

Risulta più difficile proporre una cronologia puntuale per il frammento di piede di coppa in ceramica depurata etrusco-padana con le prime due lettere della sequenza settentrionale graffite nella vasca in vicinanza di una stella a cinque punte (CIE 20125). Come la sequenza meridionale, anche questo frammento iscritto proviene dalla sede dell'attuale Museo archeologico.

Durante il periodo ellenistico, si contano due occorrenze delle prime due lettere della sequenza settentrionale, entrambe di III sec. a.C. e da contesto funerario (certa la provenienza per CIE 20993, solo ipotetica data l'integrità del supporto per CIE 20775). Le due lettere sono state redatte su ciotole a v.n., in un caso nel fondo esterno (CIE 20993) e nell'altro nella vasca interna (CIE 20775).

\footnotetext{
1 M. Pandolfini, in Pandolfini, Prosdocimi 1990, pp. 9-10.

2 Sassatelli, Gaucci 2010, p. 339.
} 
Nel complesso, si osserva una netta distinzione di destinazione fra le occorrenze più antiche, tutte $\mathrm{da}$ abitato e quelle invece più recenti da contesti funerari. Tale tendenza non stupisce, ma anzi si allinea con quanto già osservato in un precedente lavoro che mirava alla messa a fuoco dei processi che stimolarono la redazione degli alfabetari latini nell'Italia preromana ${ }^{3}$ : le sequenze alfabetiche da abitato sono circa un terzo del totale e concentrate soprattutto tra VI e V sec. a.C. Non deve inoltre sfuggire che sempre questa analisi aveva mostrato come la maggior parte delle sequenze alfabetiche da abitato si concentri in area padana e appartenga perlopiù a contesti coerenti e complessi, come lascerebbe intuire il rinvenimento di almeno due occorrenze su tre (CIE 20125 e 20189) dagli scavi presso la sede del Museo archeologico (ma anche per CIE 20371 è stata ipotizzata la medesima provenienza ${ }^{4}$ ). Alla luce di queste considerazioni, conviene qui richiamare la recente analisi offerta da M.-L. Haack sugli alfabetari di Spina. Secondo le conclusioni della studiosa, le sequenze alfabetiche parziali rinvenute nell'abitato di Spina e concentrate in settori di scavo fra loro prossimi, presenterebbero un carattere anomalo e se si associa tali speciali documenti ai relativi contesti, anch'essi ritenuti anomali, allora ciò inviterebbe gli studiosi «au moins à penser que tous les temples et sanctuaires n'étaient peut-être pas rejetés en périphérie et que, comme ailleurs en Étrurie, un ou plusieurs abécédaires ont pu être déposés à l'intérieur d'un bâtiment à fonction religieuse $\gg^{5}$. Ma cosa spinge la Haack a questa suggestiva conclusione? Ebbene, tra le considerazioni della studiosa, si richiama primariamente il numero di alfabetari da abitato, alto in Etruria padana e molto basso in Etruria propria. Se ciò può essere valido di per sé, va tuttavia osservato che a Kainua gli alfabetari si concentrano nella Casa 1, Regio IV-Insula 2, un contesto abitativo-produttivo ${ }^{6}$. La seconda considerazione riguarda i contesti: sulla base di pochi oggetti particolari come a esempio una olla biconica in impasto non tornito, i settori 11 e 12 presenterebbero «caractères inhabituels». Ovviamente, semplici questioni di metodo mettono in guardia dall' interpretare interi contesti sulla base di oggetti speciali, ignorando il resto e la loro messa a sistema nel contesto. Peraltro, la recente edizione di questi settori da parte di L. Zamboni chiarifica il loro carattere di edifici abitativi ${ }^{7}$. Anche la redazione di testi speciali nel fondo esterno dei vasi non pare un argomento significativo. Al riguardo, merita richiamare le parole di G. Sassatelli: «attraverso di essi si voleva quindi alludere alla pratica della scrittura non in quanto tale, ma come attività socialmente elevata, praticata all' interno della casa dove evidentemente questi vasi [...] erano ben visibili, forse appesi alle pareti, costituendo di fatto un elemento di prestigio per la casa stessa e per i suoi abitanti» ${ }^{8}$. Suggestivo infine il richiamo della studiosa ai pochi alfabetari greci costituiti da sequenze interrotte, i più antichi dei quali si daterebbero al VI sec. a.C. e si concentrerebbero nel santuario di Zeus Sémios sul Monte Imetto, quali votivi al dio dei segni celesti e di quelli scritti. Ma anche qui, oltre alle differenze culturali e cronologiche, va rimarcato da un lato come il contesto sopracitato di Kainua-Marzabotto metta in guardia da facili schematismi, e dall'altro che all'interno del settore 8 degli scavi di Spina, evocato appunto dalla studiosa per la presenza di un alfabetario parziale, fosse stato recuperato anche un fondo di skyphos con una doppia sequenza alfabetica completa?.

Sempre nel lavoro sugli alfabetari latini, si era offerto un quadro dei simili testi etruschi da necropoli ${ }^{10}$. Se nel complesso il numero di questi rispetto alla totalità sia significativo (circa il $40 \%{ }^{11}$ ), la cifra

${ }^{3}$ GaUCCI 2012b, pp. 73-74.

${ }^{4}$ GAUCCI 2012a, p. 161.

${ }^{5}$ HAACK 2016, pp. 197-201.

${ }^{6}$ Si rimanda alle statistiche e alle osservazioni sviluppate in GAUCCi 2012b, p. 74, con relativa bibliografia.

ZAMBONi 2016, in particolare pp. 16-31.

8 Sassatelli, Gaucci 2010, p. 339.

9 Si rimanda a GaUCCI 2012b, p. 74, nota 56 e fig. 6 per un quadro generale delle attestazioni.

${ }^{10}$ GAUCCI 2012b, pp. 70-71.

${ }^{11}$ Rispetto al lavoro del 2012, il quadro potrà essere cambiato di qualche unità, come dimostrano a esempio le attestazioni di Adria, allora inedite. In ogni caso, la percentuale non dovrebbe discostarsi di molto e il suo valore si misura in rapporto a quelli degli alfabetari dalle altre tipologie di contesto, cioè abitativo (circa il 30\%) e sacro (circa 20\%). 
diminuisce sensibilmente limitandosi al periodo compreso tra IV e II sec. a.C. Esclusi quelli di Adria, gli alfabetari che si datano a questo periodo noti in letteratura sono 6: uno da Pontecagnano e uno da Tarquinia di IV sec. a.C. (ThLE $E^{2}$, nn. 11 e 31), uno da Bomarzo e uno da Aleria di III sec. a.C. (ThLE $E^{2}$, nn. 44 e 92), uno da Vetulonia e uno rinvenuto vicino Acquapendente (Trevinano) di II sec. a.C. ${ }^{12}$. Si tratta di sequenze in norma meridionale, a eccezione di quella di Aleria ${ }^{13}$ e dei due documenti più tardi rinvenuti in ambito etrusco-settentrionale (comunque prossimi al confine con quello meridionale). Per questi ultimi è tuttavia importante sottolineare che la sequenza delle lettere presenta la sostituzione del kappa in favore del gamma, sulla base dell' influenza del tipo grafico regolarizzato ${ }^{14}$. Colpisce nel complesso l'assenza di documenti nella gran parte dell'Etruria settentrionale e anche nella città padana di Spina, ancora vitale almeno fino al III sec. a.C. Le due occorrenze adriesi in norma settentrionale sembrano dunque essere la tarda testimonianza di una pratica molto rara e che nel medesimo periodo si registra soltanto a Vetulonia in una lastra di pietra e in località Castellazzi presso Trevinano alla sinistra del fiume Paglia in una tegola destinata a una tomba del tipo chiusino a nicchiotto, dunque su supporti diversi. Non è facile inquadrare culturalmente il fenomeno delle nostre due sequenze su ciotole a v.n., che sembrano piuttosto richiamare la pratica delle attestazioni di IV e III sec. a.C. La presenza nell'Adria di II sec. a.C. di una gens di area meridionale quale quella dei Mura, che sembra replicare pratiche epigrafiche proprie del territorio vulcente e tarquiniese, suggerisce di non escludere che anche dietro le due sequenze alfabetiche vi possa essere l'influenza dell'ambito etrusco-meridionale.

All'interno del quadro offerto deve essere compresa un' altra tipologia di documento, cioè il digramma $a \chi$, che la critica più aggiornata interpreta come richiamo compendiario all'intero alfabeto tramite la prima e l'ultima lettera della sequenza. Infatti, in tutti gli alfabeti parziali noti se presente la lettera di chiusura della sequenza, questa è il chi, cioè quella finale prima della regolarizzazione del segno a otto $^{15}$. Purtroppo manca ancora oggi un quadro aggiornato e complessivo delle occorrenze della coppia alpha-chi, sebbene recentemente l'argomento sia stato trattato in maniera articolata da N. Scoccimarro all'interno di uno studio sui c.d. "segni eloquenti" coordinato da G. Bagnasco Gianni ${ }^{16}$. Scoccimarro vede tre schemi ricorrenti: lettere unite da un cerchio; lettere in punti diversi dello stesso vaso; due chi ai lati dell'alpha. Senza avere la pretesa di fornire un quadro esaustivo del fenomeno, si ricorda come l'Etruria padana offra molte occorrenze a partire dagli inizi del VII sec. a.C. a Bologna, e nei secoli seguenti a Marzabotto e a Spina ${ }^{17}$. Ad Adria è documentato due volte nel III sec. a.C., una da abitato (CIE 20041, più dubitativa nella lettura) e una da sepoltura (CIE 20426): in entrambe il chi è rovesciato e legato all'alpha (cfr. III.2); una volta nel II sec. a.C., sempre da sepoltura (CIE 20377), dove alpha e chi non sono legati e hanno medesimo orientamento secondo un ductus che sembrerebbe sinistrorso. Il vincolo fisico esplicitato nei due documenti di III sec. a.C. sembra richiamare in particolare il gruppo di lettere unite da un cerchio dello schema di Scoccimarro. Al riguardo, vale la pena ricordare in particolare un frammento di coppa rinvenuta nell'area delle insulae 3-4 della Regio V di Kainua-Marzabotto, per la quale G. Sassatelli osserva che il cerchio a unione delle lettere ne sottolineerebbe «l'inscindibilità e l'appartenenza a un corpo unico, chiaramente la serie alfabetica, pur essendo segni singoli e autonomi ${ }^{18}$. Non sembra azzardato ritenere che la legatura fra le lettere delle due occorrenze adriesi

$12 T h L E^{2}$, n. 57 e REE LXXIII, pp. 389-391, n. 118 (A. Maggiani).

13 Rimane di incerta cronologia l'inizio di sequenza ac rinvenuta a Fiesole $\left(T h L E^{2}\right.$, n. 57$)$ e attribuito alla quarta fase degli alfabetari etruschi di M. Pandolfini (Pandolfini, Prosdocimi 1990, IV.5).

14 Maggiani 1990, p. 190.

15 Govi 1994a, p. 74. Per un quadro delle occorrenze di periodo tardo, si veda Colonna 2004b, p. 307, nn. 29-30.

16 Bagnasco Gianni, Scoccimarro, Gobbi 2015, pp. 260-261, figg. 7-9 (si veda anche le tabelle in coda al testo). Nel lavoro si propone una direttrice di diffusione di tale coppia di lettere che dall'Etruria meridionale attraverso un asse tiberino arriverebbe in Etruria padana. Questo sembra però in netto contrasto con la cronologia delle attestazioni, fra le quali quelle di Bologna sembrano essere fra le più antiche.

17 Sassatelli 1985 , p. 109 e fig. 3.

18 Sassatelli 1994, p. 211 (e scheda n. 254 a p. 155). 
possa avere la medesima funzione. Colpisce inoltre il $c h i$ rovesciato. Attestato in altri casi ${ }^{19}$, ci si può domandare se non sia un ulteriore espediente per sottolineare l'opposizione delle due lettere, estremi della sequenza alfabetica. Vale qui la pena richiamare anche il caso dell' iscrizione sulla tazzina monoansata a v.n. di produzione locale della tomba 348 di Canalbianco di fine III-inizi II sec. a.C. (CIE 20628; cfr. IV.3.2.A), che potrebbe presentare una sequenza da sinistra a destra di $a l p h a^{20}$ (ma rimane dubitativa la lettura di quest'ultimo) e chi rovesciato. Se tale interpretazione cogliesse nel vero, si avrebbe qui un prenome (Ata) con ductus sinistrorso associato alle due lettere che evocano la sequenza alfabetica redatte con ductus destrorso; non si può tuttavia escludere che il chi sia un numerale, come almeno in un'altra occorrenza (CIE 20817; cfr. IV.3.2.A), mentre l'alpha isolato potrebbe essere interpretato come una falsa partenza, similmente all' iscrizione $<t>$ titinai di II sec. a.C. (CIE 20474).

Nel complesso, sembra di cogliere tre distinte pratiche legate alla redazione di sequenze di lettere che richiamano più o meno esplicitamente gli alfabetari. Nell'Adria tardo-arcaica e classica, le sequenze provengono da abitato (probabilmente concentrate nella medesima area) e sono redatte in norma meridionale e settentrionale, marcando una pratica allusiva della capacità scrittoria, peraltro condivisa da individui formati a differenti tradizioni epigrafiche, ed evocatrice di poteri magicoreligiosi che la sequenza doveva avere. Nel periodo ellenistico sembra di poter distinguere invece due distinte pratiche, quella dell'esplicitazione delle prime due lettere della sequenza (ae), destinata ai vasi dei corredi funerari e solo ipoteticamente portata da individui di origine etrusco-meridionale, l'altra relativa alla prima e all'ultima lettera della sequenza, che si ritrova sia in abitato sia in necropoli e che sembra piuttosto radicata nelle tradizioni di area padana. In particolare, fra queste ultime due pratiche le distinzioni formali, redazionali e contestuali lasciano supporre anche diversità di funzione, che tuttavia non è possibile dettagliare maggiormente. Infatti, solo uno studio specifico e analitico di tutte le attestazioni note nel mondo etrusco e dei loro contesti potrebbe forse portare qualche elemento per approfondire il problema.

\section{V.2. $A l$, il "dono"}

Tra VI e V sec. a.C. si documentano tre occorrenze del lessema al, forma sostantivata della radice di un verbo di dono. Questo è isolato in due casi (CIE 20160, 20265), mentre in CIE 20212 è associato al pronome personale mi. Proprio quest'ultima iscrizione esplicita l'identità tra il dono e l'oggetto donato, che si potrebbe estendere anche alle due attestazioni isolate del sostantivo, come già osservato da G. Colonna per altri casi dove compare sempre isolato ${ }^{21}$. Tuttavia, la posizione della parola sulla coppacoperchio in CIE 20265, oltre all'umile natura del supporto, lascia piuttosto supporre che fosse il contenuto di un'olla che il nostro supporto proteggeva a essere il "dono" piuttosto che il vaso medesimo. Se valida questa osservazione, le occorrenze della parola al celerebbero almeno due distinte pratiche di dono: l'iscrizione mi al sul piatto chiarifica che esso stesso è il dono, mentre la parola sulla coppacoperchio rimanda a offerte di natura deperibile che il vaso contiene o protegge.

Inoltre, si coglie il carattere assoluto delle offerte: in nessun caso è menzionato un antroponimo o una formula onomastica. Tale carattere si ritrova anche nelle altre occorrenze attestate in Etruria

19 Si richiama a titolo esemplificativo l'occorrenza su coppa in bucchero dalla collezione del Museo di Stato della Repubblica di San Marino (RAVAsio 1995, pp. 159-160, figg. 6-7) e quella sul fondo esterno di una forma chiusa di ceramica grezza dalla plateia A di fronte alla Casa 1 della Regio V-insula 5 di Kainua-Marzabotto (L. Malnati in SAssatelli 1994, p. 174, n. 279), entrambe datate alla seconda metà del VI sec. a.C. Se l'integrità della prima suggerisce la provenienza (comunque ignota) da un contesto funerario, la seconda proviene dagli strati di preparazione del piano stradale e non si può escludere uno speciale valore simbolico della lettera legato a una pratica rituale, forse non distante da quella documentata dai frammenti iscritti di anfora da tavola in bucchero recuperati nei muri di fondazione del tempio di Uni della Regio I (E. Govi, in REE LXXIX, p. 309; su queste forme rituali che implicano la pratica scrittoria, si veda ora GAUCCI c.s.).

${ }^{20}$ Per l'aspetto della lettera, si veda in particolare l'alpha del digramma ca dall'abitato di Spina, graffito su un frammento di anfora datato al IV sec. a.C. (G. UGgerI, in REE XLVIII, p. 350, n. 32, fig. 2).

21 G. Colonna, in REE LXV-LXVIII, p. 401 con riferimento al n. 93. 
padana, cioè quella nel piatto attico della tomba 719 di Valle Trebba a Spina ${ }^{22}$ e le tre documentate a Kainua-Marzabotto, di cui la più significativa è sicuramente quella del disco fittile dalla c.d. "Grande Fornace" della Regio II - insula $1^{23}$. Ma estendendo lo sguardo, anche le occorrenze del lessema al inteso come "dono" in Etruria tirrenica, tutte circoscritte tra VI e V sec. a.C., sono isolate ${ }^{24}$. La forma diminutiva alza è invece attestata nell' iscrizione mi alza ceriies in una kylix a v.n. di fine V-inizi IV sec. a.C. da una tomba di Pontecagnano ${ }^{25}$ : il dono è dedicato alla dea etrusca $V e i$, a cui ci si riferisce con una forma etruschizzata del nome latino-italico Cerere. L'uso del pronome personale ricalca il nostro CIE 20212, ma l'iscrizione di Pontecagnano si configura come l'unica nella quale il lessema al $(z a)$ non è semplicemente identificato con l'oggetto, ma ha la specifica della divinità a cui l'oggetto medesimo è dedicato. In effetti, ci si potrebbe domandare se l'uso (e la scelta dunque) del lessema senza altra specifica del donatore o del destinatario non celi una formula più complessa omessa ma sottintesa (cfr. VI.1.2-3, per la contestualizzazione delle iscrizioni).

\section{V.3. Teonimi e lessico del sacro}

Alcuni lessemi delle iscrizioni adriesi sembrano appartenere alla sfera del sacro. Anzi tutto, Adria restituisce alcune iscrizioni nelle quali in maniera più o meno dubitativa è possibile riconoscere dei teonimi. Il primo lessema dell' iscrizione kulśnuteraśśsmin $\theta i$ akśke di pieno III sec. a.C. (CIE 21027) è sicuramente il caso più significativo: $\mathrm{H}$. Rix ha proposto di vedere in akśke il preterito di un verbum faciendi in forma sincopata, mentre ha inteso kulśnuteraś come "Custodi della Porta"26, intepretazione poi seguita da Cristofani e Belfiore ${ }^{27}$. G. Colonna, sebbene abbia accettato l'interpretazione dell'autorevole linguista tedesco, ritiene che questi custodi si riferiscano alla porta degli Inferi, cioè siano Xaru et $\operatorname{Van} \theta$, e pertanto che la formula sia votiva. Tale lettura è stata poi accolta da K. Wylin, A. Maggiani e D.F. Maras ${ }^{28}$. In particolare, Maggiani ha scritto che questi "custodi" sono i Ianitoribus terrestribus descritti da Marziano Capella (De nuptiis Mercurii et Philologiae I, 60, Regio XVI). Questa interpretazione, del tutto condivisibile, assieme all' integrità della ciotola, permette di ritenere che la ciotola, per la quale rimane solo la notizia che fu rinvenuta fra Adria ed Este, fosse stata deposta in una tomba.

Altre iscrizioni, di più incerta lettura in quanto lacunose, potrebbero restituire teonimi o epiclesi divine:

- CIE 20370, ?] tanur (fine del VI-primi decenni del V sec. a.C. circa);

- CIE 20523, ]kalu (prima metà del III sec. a.C.);

- CIE 20933, papa (prima metà del II sec. a.C.).

Si affronta anzi tutto CIE 20370, per la quale pare più probabile la lettura ?]tanur rispetto a ?] śanur. Se valida la lettura tanur, comunque incerta, si avrebbe un teonimo all'assolutivo, che rinvierebbe al nome della dea $\Theta a n r$, di cui è possibile ricostruire una forma presincopata ${ }^{*} \Theta$ anur sulla base del termine $\theta$ anurari della $T C$ del primo quarto del V sec. a.C., ricollegato al teonimo ${ }^{29}$. Come già

22 Gaucci et al. 2017, pp. 128-129, note 20-21, taf. 65.b; G. Colonna, in REE LXXIX, p. 339, n. 93.

23 SASSATELli 1994, pp. 63-64, n. 74, tav. IXa-b (sul valore del disco in rapporto al significato del lessema, cfr. anche Sassatelli 2011, p. 157, nota 32). Sempre da Marzabotto, è documentato il medesimo lessema dai vecchi scavi (ibid., pp. 24-25, n. 10, tav. IIa-b), e dai livelli di fondazione dell'edificio di V sec. a.C. della Regio V - insula 5 (L. Malnati, in ibid., pp. 174-175, n. 280, tav. XXXVa, dove però è letto au; Sassatelli invece propone per il medesimo la lettura al: ibid., p. 205).

24 ThLE', p. 18, s.v. al.

25 M. Mancusi, G. Colonna, in REE LXV-LXVIII, pp. 400-402, n. 93.

${ }^{26}$ Rix 1986, pp. 29-30; sul verbo, cfr. più recentemente BELFIORE 2014a, pp. 30, 41. Sul nome śmin $\theta i$, si rinvia a IV.3.1.B, categoria b).

27 Cristofani 1991, p. 121, nota 16; Belfiore 2014a, pp. 30, 41.

28 Wylin 2000, p. 165, nota 410; Maggiani 2002a, pp. 59, 62, note 54-55; Maras 2009b, pp. 217-218, Ad do.I.

29 Cristofani 1995, p. 70; Maras 1998, p. 180; si veda da ultimo sul teonimo e la sua radice, G.M. FacChETTI, in REE LXXXI, pp. 385-386, n. 67. 
affermato in altra sede, il supposto passaggio * $\Theta$ anur $>$ Tanur può essere postulato, benché la causa del medesimo potrebbe essere imputata a fattori fra loro diversi (scambio; influenza italica; errore $\left.{ }^{30}\right)$. La dea è attestata anche nella vicina Spina verso la metà del V sec. a.C. (ET ${ }^{2}$, Sp 0.4; cfr. infra). Si aggiunge, solo come suggestione, che l'aspetto paleografico di alcune lettere, nella nostra affine alla tradizione scrittoria cerite-veiente, potrebbe ben accordarsi con una influenza latina o di lingua affine (es. falisco) nel passaggio $\theta>t$. Nonostante la lacunosità iniziale dell'iscrizione, una integrazione del pronome di prima persona, cioè $m i+$ teonimo all'assolutivo, pare poco convincente, in quanto si scontra con una frequenza piuttosto rara in una formula di questo tipo, mentre è più plausibile ritenere che il testo sia sostanzialmente completo, cioè una forma di consacrazione del vaso o del suo contenuto attraverso la graffitura del teonimo al quale è consacrato ${ }^{31}$, secondo un processo di identità come appartenenza di sapore arcaico $^{32}$.

Si potrebbe avanzare l'ipotesi che anche l'iscrizione lacunosa ]kalu (CIE 20523) non appartenga a un nome con suffisso - alu, ma piuttosto identifichi il teonimo Calu ed essere così una formula di consacrazione ${ }^{33}$. Questo teonimo è già attestato in ambito padano (cfr. infra) e nella fattispecie potrebbe indicare, come la dedica ai Kulśnuter sopra citata, una forma di culto funerario. Ciò sarebbe suggerito dal contesto, una sepoltura della prima metà del III sec. a.C. Tale interpretazione porta a ritenere questa uno dei due casi di consacrazione funeraria a divinità (l'altro è CIE 21027 sopra discussa). Merita comunque ricordare che a Spina, su un numero maggiore di tombe ed iscrizioni ${ }^{34}$, si documenta un unico caso, mi vant su una ciotola a v.n. $\left(E T^{2}, S p 2.114\right)$, configurando dunque il fenomeno come eccezionale (ma comunque documentato).

Tra le possibili consacrazioni si ricorda anche il ciotolone con due scanalature all'orlo della tomba 50 di via Spolverin della prima metà del II sec. a.C., probabilmente appartenente al recinto dei Muliu (cfr. VI.3), con i testi papa e pre fra loro opposti e attorno a un segno a croce (CIE 20933) ${ }^{35}$. Papa, oltre a significare notoriamente "nonno", è ora riconosciuto quale appellativo di divinità maschili e forme di consacrazione funeraria con questo appellativo sono altrove documentate ${ }^{36}$. Si sospende invece il giudizio sul testo opposto rispetto al segno a croce, cioè $p r e^{37}$.

Nel complesso, questi teonimi ed epiclesi di Adria, se effettivamente tali, arricchiscono il quadro dell'Etruria padana, sul quale G. Sassatelli ha offerto una recente analisi ${ }^{38}$ :

- Tinia (/ Tina; fine del VI-prima metà del V sec. a.C.) e Uni (verso la fine del VI sec. a.C.) a Kainua-Marzabotto ${ }^{39}$;

30 Si veda GaUcci 2012a, p. 149, per un quadro delle possibilità e più recentemente Govi 2014, pp. 116-119 in merito al lessema muntie della lamina di bronzo dal tempio di Tinia di Kainua-Marzabotto.

31 Maras 2009b, p. 452, modulo n. 8, e pp. 449-450, modulo n. 2.

32 Colonna 1983, p. 49 (si veda anche Poccetti 2019).

33 Maras 2009b, pp. 449-450, A.2 (teonimo all'assolutivo), più frequente, o p. 452, B.8 (pronome mi + teonimo all'assolutivo) più sporadico e nel quale c'è anche il mi vant di Spina $\left(E T^{2}, \mathrm{Sp} 2.114\right)$.

34 Quantificazione preliminare offerta in GaUCCI, Pozzi 2009, p. 53 (aggiornata al 2008); GaUCCI, Morpurgo, Pizzirani 2018, p. 674, fig. 15, per la necropoli di Valle Trebba.

35 Sul possibile legame tra il segno a croce e i due lessemi, si rimanda a GAUCCI 2020.

36 Cfr. G. Colonna, in REE LXXVIII, pp. 248-249, n. 63, per l'incidenza in tomba.

37 Rimane solo una suggestione il richiamo alla forma verbale presente nella lamina di bronzo conservata al British

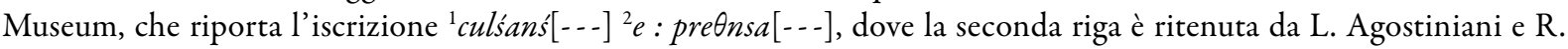
Massarelli una forma negativa di una prescrizione (AgOSTINIANI, MASSARELLI 2012, p. 120), già proposta come tale

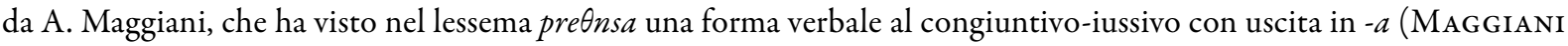
1994, p. 74).

38 Sassatelli 2017.

39 I documenti epigrafici che attestano il teonimo Tinia sono tre, dei quali due vengono dal Tempio urbano della Regio I - Ins. 5 (SAsSATElli, Govi 2005, pp. 38-47; SASSATElli 2009, p. 333; E. Govi, in REE LXXIX, pp. 301-306, nn. 60-62) e uno dalla c.d. "Grande Fornace” della Regio II - insula 1 (SASSATELli 2011, pp. 156-157, figg. 10-11); per il documento relativo al teonimo Uni, cfr. E. Govi, in REE LXXIX, pp. 310-312, n. 64. 
- Vei sempre a Kainua (fine del VI-inizi del V sec. a.C.), a S. Polo (prima metà del V sec. a.C.), a Mantova (fine IV-prima metà del III sec. a.C.), e forse Bologna ${ }^{40}$;

- $\Theta a n r$ (metà del V sec. a.C.), $\operatorname{Van} \theta$ e $\Theta e s a n$ (III sec. a.C.) a Spina ${ }^{41}$;

- dubitativamente Rat (?) a S. Polo (metà del V sec. a.C.) ${ }^{42}$;

- dubitativamente Kalu in località Castellazzo della Garolda (seconda metà del V-prima metà del IV sec. a.C. $)^{43}$.

- sipinaial (sulle problematiche relative alla cronologia del supporto, cfr. I.2.1), forma femminile da D.F. Maras messa in rapporto al teonimo Zipna, divinità della cerchia di Turan, e da G. Colonna ritenuta piuttosto variante troncata di Versipinai, cioè la Persefone etrusca ${ }^{44}$.

Sempre alla sfera del sacro sembra appartenere il lessema kemne (CIE 20408), e forse anche il lacunoso aisi $\times \times$ u (CIE 21002, b).

Kemne, graffito sulla spalla di un'anfora di II sec. a.C., trova confronto nella voce cemna- del $L L$, ritenuta da V. Belfiore un epiteto in -na di divinità, e che potrebbe avere più puntualmente il senso di "celeste/superiore"; la base cem- è comunque attiva anche in formazioni onomastiche (cemnil, cemu, cemunia $)^{45}$. La possibile forma locativa del nostro potrebbe più plausibilmente richiamare la sfera divina di cemna- piuttosto che un antroponimo.

Anche il lacunoso aisi $\times \times u(C I E 21002$, b) della tomba 106 di via Spolverin, graffito nella parete esterna di un piatto a v.n. di pieno II sec. a.C., potrebbe appartenere alla sfera sacra. Il medesimo piatto conserva nella vasca interna il gentilizio Muliu isolato, che suggerisce la pertinenza della tomba al relativo recinto (cfr. VI.3). Nell'edizione del contesto, ais $i \times \times u$ era stato interpretato come antroponimo ${ }^{46}$. Nella prima parte meglio conservata si può riconoscere senza difficoltà ais (nel nostro senza traccia del processo di monottongazione $a i->e i-/ e$-, secondo una tendenza comune alle iscrizioni adriesi ), cioè "dio", che si trova documentato altrove, (eventualmente al plurale), e in forme derivate del lessico ${ }^{47} \mathrm{e}$ dell'onomastica (es. il gentilizio femminile aisinal, comunque dubbio, ipotizzato formato dal nome individuale maschile aisiu ${ }^{48}$ ). Nella parte lacunosa, la quinta lettera potrebbe essere alpha (un po' più stretto del primo, ma sempre disarticolato) oppure $r h o$. Il $r h o$ porterebbe a leggere aisir $\times u$, che, escludendo un plurale (sempre nella forma aiser/eiser), sarebbe piuttosto da spiegare come derivativo in -ra. Una simile dedica votiva è stata riconosciuta da Colonna nell' iscrizione esle su una ciotola a v.n. degli inizi del III sec. a.C., ritenuta la flessione al pertinentivo II di ais $\left(\text { ai- }>e_{-}^{-}\right)^{49}$ : per Colonna tale dio non può che essere infero. Il nostro lessema tuttavia sembra avvicinarsi maggiormente ad *aisia, recentemente oggetto di nuove riflessioni da parte di V. Belfiore, che indaga formazione e funzione delle due

40 Kainua-Marzabotto: E. Govi, in REE LXXX, pp. 236-238, n. 2; Mantova: Menotti, Maras 2012, pp. 880-881, n. 10 e n. 13; S. Polo: $E T^{2}$, Pa 4.3; Bologna: Malnati 2010, p. 120, fig. 44.19, dove si esplicita che la frattura del vaso dopo iota non permette una serena lettura dell' iscrizione (cfr. anche SASSATELli 2017, p. 194, nota 25). Si aggiunge che per Kainua, E. Govi ha ipotizzato che il digramma ka nel contesto sacro del santuario di Uni e in associazione con l'iscrizione Vei possa essere una forma abbreviata per KavaAa (REE LXXX, p. 241, in riferimento al n. 4).

41 Ganr: S. Patitucci Uggeri e M. Cristofani, in REE XLVI, pp. 289-291, n. 1, tav. LII; Maras 1998, p. 181; Maras 2009b, Sp do.1, p. 372; ET², Sp 0.4. Vant: Maras 2009b, p. 371, Sp co.1. Eesan: Maras 2009b, p. 373, Sp do.2, con precedenti riferimenti; $E T^{2}, \mathrm{Sp} 3.1$.

$42 E T^{2}$, Pa 0.1; Maras 2009b, p. 320, Pa co.2. Ma sull'iscrizione, cfr. adesso G. Colonna che preferisce la lettura ran (REE LXXVIII, p. 222, n. 30).

43 REE LI, n. 13; Maras 2009b, pp. 320-321, Pa co.3; ET², Pa 2.10.

44 Si rimanda rispettivamente a MenotTi, Maras 2012, p. 881, n. 1, figg. 4-5, p. 885, e G. Colonna, REE LXXIX, pp. 337-338, n. 91, con precedente riferimento.

45 Belfiore 2010, p. 118.

46 Gaucci 2008, p. 96, n. 1.

47 Cfr. Maras 2009b, pp. 71-72.

48 Morandi Tarabella 2004, p. 396.

49 G. Colonna, in REE LXXVIII, p. 249, n. 65. 
attestazioni note: nella forma aisias in una tomba di Crocefisso del Tufo ad Orvieto, dove la parola, apposta sopra il nome del defunto non completamente abraso, è stata ritenuta una forma di damnatio memoriae, e in uno specchio da Palestrina di V sec. a.C., dove esia è associato ad Arianna trascinata verso l'Ade da Artemide ed è stato interpretato da G. Colonna come "consacrato (agli dei)" "50. Alla luce di queste osservazioni, vi sarebbero più concrete ragioni per riconoscere un alpha nella quinta lettera. Ciò consentirebbe inoltre una più facile integrazione della sesta: aisia $[l] u$ o aisia $[t] u$. Se la prima soluzione rinvia all'onomastica, per aisiạ $[t] u$ si può pensare a una derivazione da aisia con aggiunta del suffisso $-t u(/ \theta u)$, intendendo il lessema come appellativo da riferire forse a un individuo preposto ai sacra, o più probabilmente con aggiunta del suffisso agentivo $+u$, intendendo il lessema come forma di consacrazione del vaso. L'idea di una forma derivativa non onomastica (aisir $\times u$ o meglio aisia $[t] u$ ) è sostenibile anche in base alla presenza del gentilizio Muliu (cfr. IV.3.1.A) nella vasca del medesimo vaso: se il nostro lessema fosse un antroponimo, questo sarebbe l'unico caso documentato di associazione di due formule onomastiche in parti distinte di un vaso. Sempre a sfavore di una lettura onomastica, almeno per la possibile integrazione aisia $[l] u$, sarebbe la collocazione al pieno II sec. a.C. di un probabile gentilizio con suffisso -alu, che non sembra più attivo in questa cronologia (cfr. IV.3.3.B).

\section{V.4. Altre voci di lessico (belis, $\theta i$, sesiu, tutis, usti/uzti)}

$\mathrm{Si}$ analizzano di seguito alcuni lessemi riconosciuti come voci lessicali, che non sembrano avere un valore onomastico o di esplicito ambito sacro. Queste sono helis (CIE 20464, 20595, 20601), $\theta i$ (CIE 20436, 20606), sesiu (CIE 20028), tutis (CIE 20487), usti/uzti (CIE 20196, 20273).

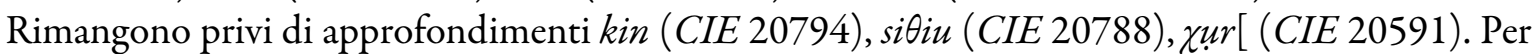
questi non è possibile avanzare concrete proposte interpretative, sebbene si porti qui qualche considerazione:

- kin, graffito su un vaso datato al II sec. a.C., richiama unicamente l'hapax cina del cippo c.d. dei Marmini di Volterra, nel quale A. Morandi ha visto il termine "giaciglio", inteso in senso funerario, come derivato in -na della base $c i$ - di origine latamente indoeuropea ${ }^{51}$. Se questa la lettura, ovviamente pare improbabile che il nostro possa avere un legame se non puramente formale con il cina volterrano. In alternativa, più recentemente $\mathrm{K}$. Wylin ha proposto di vedere in cina un gentilizio ${ }^{52}$.

- sitiu, graffito su un vaso di II sec. a.C., trova corrispondenza nelle iscrizioni ellenistiche $E T^{2}, \mathrm{Cl}$

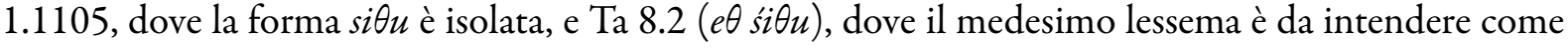
una forma verbale ${ }^{53}$.

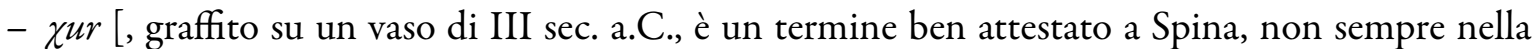
sequenza canonica di lettere e in alcuni casi associato a graffiti non alfabetici con verosimile valore numerale $^{54}$. Questo trigramma non ha ancora avuto uno studio complessivo di tutte le attestazioni.

50 Si rinvia alle osservazioni della studiosa in BELFIORE 2017.

51 Morandi 1990, p. 143.

52 WYLIN 2000, p. 265.

53 Agostiniani, Nicosia 2000, p. 96; Belfiore 2017, p. 174.

${ }^{54}$ Per le attestazioni, cfr. Gaucci 2016, p. 180, note 51-52. Si ricorda che per $\chi u r$ sono state elaborate due ipotesi, la prima per la quale l'associazione del termine con numerali e la relazione con il $\chi u r v a r$ delle lamine di Pyrgi, porta a ritenerlo una voce di lessico associabile ai numerali stessi (S. Patitucci), mentre l'altra ipotesi vede nel termine ripetuto della tomba

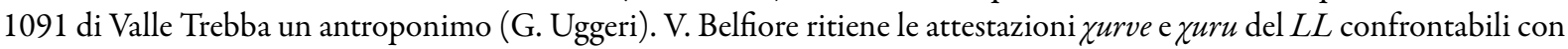

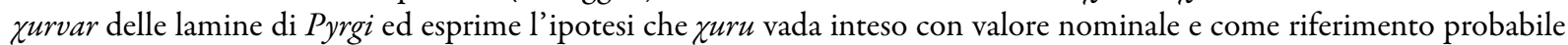
a un tipo di sacrificio o cerimonia (BELFIORE 2010, pp. 164-165). A tale riguardo, risulta di qualche interesse il contesto di rinvenimento dell' iscrizione $\chi u r$ a crudo su olla di Donoratico edita da M. Pistolesi, parte di un nucleo di documenti epigrafici associati a materiale votivo rinvenuti in un luogo connesso con l'acqua e la sua conservazione (M. PISTOLESI in REE LXXII, p. 159, n. 12, tav. XXXIV). Si aggiunge che D.F. Maras ha proposto, per alcune iscrizioni da recenti scavi di abitato dove figurerebbe questo termine (nella combinazione rqu), la lettura rat (MARAs 2013a, pp. 156-158, 160, nn. 9-11, tutte e tre nella parete esterna di ceramiche a v.n., in almeno due casi, nn. 10-11, riconoscibili come kylikes con anse non ripiegate ad orecchia forma Morel 82), interpretando così l' iscrizione come la consacrazione in caso zero al dio $R a \theta$. 


\section{belis}

Il lessema helis è ripetuto su due iscrizioni con medesimo formulario (CIE 20464, 20595; cfr. IV.3.2, classe V) e attestato anche isolato (CIE 20601, periodo ellenistico). Nelle prime due, l'associazione con il pronome $m i$ e con un nome individuale declinato al genitivo I o all'ablativo, lascia ipotizzare che il lessema sia all'assolutivo e non sia un nome, sebbene la base hel- sia attiva nell'onomastica dei nomi familiari (es. heli, helia, helusni, helusnei).

Il nostro è verosimilmente un composto della base hel-con il suffisso - $i$ s, attivo secondo la proposta di E. Vetter come nomen agentis, in lessemi con funzione di appellativi. H. Rix in particolare ne tratta per gli antroponimi uscenti in -is, documentati come appellativi e quindi attivi nell'onomastica a partire da questi ${ }^{55}$. Helis potrebbe dunque essere rimorfologizzato dal lessema hel, che si documenta in particolare nella formula hels atrś attestata in alcune iscrizioni funerarie di Vulci.

I lessemi aturś/atrś e hels ricorrono isolati oppure in associazione ${ }^{56}$. L'interpretazione di M. Pallottino di atrś e della formula hels atrś, cioè"'familiari" e "propri familiari" ${ }^{7}$, è stata ripresa da Pfiffig, Olzscha, Cristofani e più recentemente $\mathrm{da} \mathrm{G}$. van Heems ${ }^{58}$, che traducono la locuzione hels atrś come "a sé (hels) e ai parenti (atrś)". K. Wylin invece propone "costruttore dello spazio tombale" 59 . Più recentemente, V. Belfiore ha fornito un nuovo quadro contestualizzato delle occorrenze. La studiosa rileva che dove hels compare isolato (e dunque interpretabile in forma compendiara con atrśs sottinteso), l'iscrizione è redatta su un oggetto mobile (in $E T^{2}$, Vc 1.32 su un cippo; nel testo latino $E T^{2}$, Vc 1.65 su un puteale (?) in nenfro dalla tomba dei Sarcofagi; in $E T^{2}$, Vc 1.16 su un cippo a dado); quando il lessema è associato ad atrś, l'iscrizione si trova su architravi e pareti. Postulato che atr'śsi riferisca allo spazio tombale, per la Belfiore hel-s avrebbe quindi a che fare con una qualifica del defunto, come documenterebbe l'associazione con la formula latina in L. Obili L. F. Hels $\left(E T^{2}\right.$, Vc 1.65 ${ }^{60}$. Nel Cippo di Perugia compare invece helu quale predicato al preterito e la medesima Belfiore lo individua come "stabilire, definire, decretare" oppure "costruire", richiamando anche il parallelo con l'iscrizione lemnia di Efestia dove il preterito heloke avrebbe la medesima funzione, come proposto da C. de Simone ${ }^{61}$. La Belfiore conclude che la forma hel delle iscrizioni vulcenti possa indicare la costruzione tombale, sebbene non escluda comunque che possa riferirsi ad altre sfere semantiche come "volere, richiedere" o alla sfera del "fare" materiale ${ }^{62}$, con parallelo con la forma eluku di alcune iscrizioni di dono retiche ${ }^{63}$.

Quale che siano gli sviluppi dell'analisi di dettaglio relativi a hels, nel caso dei nostri helis la sfera funeraria è decisamente pertinente e non sembra fuori luogo pensare che questo funga da appellativo del vaso medesimo.

A quanto osservato, si può aggiungere che helis appare solo su tre vasi, gli unici su ceramica grigia tra tutte le iscrizioni funerarie e probabilmente concentrati nel III sec. a.C. (almeno due sicuramente, mentre CIE 20601 è di incerta cronologia sebbene plausibilmente in fase con le altre). Si aggiunge

55 Rix 1963, pp. 267-270, 274 (anche per la proposta di Vetter). V. Belfiore (2014, p. 82) ipotizza per il suffisso una marca di animatezza.

56 Un quadro delle attestazioni in BeLFiore 2015, pp. 110-111.

57 Sull'interpretazione, cfr. M. Pallottino, in REE XXXII, p. 198. Cfr. anche Cristofani 1995, p. 93.

58 Belfiore 2015, pp. 110-111, con riferimenti. MASSARELLI 2014 (p. 161), notando che la terminazione -cu è associata a temi verbali, participi passati forse, spesso sostantivati, per il termine helucu richiama A.J. Pfiffig, che ha proposto il significato di “eigen”, proprio (PFIFfig 1961b, p. 143, già da una idea di Torp; ID. 1968, pp. 74-76); questa ipotesi è stata recuperata da FACCHETTI (2002b), che avanza l'idea (priva però di indizi concreti) che possa fungere anche da radice verbale.

59 WyLin 2000, pp. 243-246.

60 Belfiore 2015, p. 111, nota 48.

61 Belfiore 2017, pp. 189-190, con riferimenti; cfr. anche van Der MeEr 2017, p. 209. Per la forma verbale del lemnio heloke, si veda DE Simone 2009c, che traduce il termine Aaura belu come "la tomba eretta".

${ }^{62}$ Belfiore 2017, p. 190, nota 145.

63 MLR 14, 154, 194. 
che nelle due formule con elementi onomastici, questi sono entrambi nomi individuali (cfr. IV.3.1.B, categoria d). Appare evidente che vi sia una pratica codificata dietro la scelta d'uso di questo specifico lessema e forse con ciò si voglia rimarcare una speciale destinazione per queste coppe in ceramica grigia che forse non fa parte della normale pratica rituale.

Sebbene esplicitato solo su questa classe ceramica e verosimilmente nel III sec. a.C., ci si può domandare se si possa riconoscere il termine helis nel digramma puntato dell' iscrizione he.me da una tomba di Ca' Garzoni databile alla seconda metà del II sec. a.C. (CIE 20694). Il secondo digramma, $m e$, raramente attestato ${ }^{64}$, potrebbe verosimilmente essere inteso come allografo di mi. Sembra essere invece piuttosto improbabile riconoscere questo lessema in due iscrizioni lacunose su ceramica a v.n. dall'abitato, cioè su una forma aperta di fine IV-III sec. a.C. (CIE 20107: he [ ) e su un piatto da pesce di III sec. a.C. (CIE 20197: hex[ ).

\section{$\theta i$}

Il digramma $\theta i$, isolato in due occorrenze della fine del III-prima metà del II sec. a.C. (CIE 20436, 20606), è con buona verosimiglianza da intendere come "acqua" secondo una interpretazione ormai comunemente accettata ${ }^{65}$. Il lessema è documentato in due vasi da contesto funerario, forse graffito con la finalità di indicarne il contenuto e la probabile destinazione rituale (cfr. infra, usti/uzti). Va osservato che in entrambi i casi, la parola è scritta con il theta puntato, aspetto paleografico caratteristico della vicina Spina dalla fine del V sec. a.C. in poi ma altrimenti non documentato ad Adria (cfr. III.2). La peculiare scelta grafica è dunque un carattere significativo nella redazione di questa parola, sebbene non si possa facilmente cogliere se ciò dipenda da una influenza della tradizione epigrafica spinetica oppure dalla volontà di marcare lo speciale valore (rituale?) della parola scritta, distinguendola così dalla più consueta pratica scrittoria adriese.

\section{sesiu}

Il lessema sesiu, attestato isolato dall'abitato nel III sec. a.C. (CIE 20028), non sembra da inserire tra gli antroponimi, come già anticipato (cfr. IV.3.1.B, categoria b). Il radicale trova confronto con il séś della Tomba delle iscrizioni graffite, dove è associato al verbum faciendi arce $e^{66}$, poi in età ellenistica a Populonia nella forma sesa con due occorrenze forse dal medesimo contesto ${ }^{67}$ e nel locativo sese del $L L$; questo lessema è ormai riconosciuto come indicante una cosa atta a essere offerta (così G. Colonna), o con una sostanza alimentare o il contenitore (così M. Bonamici) ${ }^{68}$. Se dunque può essere valido quanto richiamato, questa base lessicale non sembra essere attiva nell'onomastica (come già affermato $\mathrm{da} \mathrm{M}$. Bonamici in merito a sesa). Ciò porta a ritenere che anche il nostro sesiu possa essere un lessema indicante pertinenza piuttosto che un antroponimo. In tale senso si potrebbe ipotizzare una trafila sesa $+i e+u>$ sesae $+u>$ seseu $>$ sesiu.

\section{tutis}

Tutis, attestato isolato su un piatto a v.n. nel pieno III sec. a.C. (CIE 20487), è stato inteso da F. Piva come antroponimo con terminazione in sigma all'assolutivo ${ }^{69}$; se così, sarebbe l'unico caso ad Adria e nel suo territorio in periodo ellenistico, espunto m.atis di Gavello (CIE 21031), per il quale è stata

${ }^{64} T h L E^{2}$, s.v. $m e$, isolato solo a Chiusi; sempre dal Chiusino viene l'urna etrusca ellenistica con l'iscrizione la. cale. me, dove quest'ultimo è inteso come abbreviazione (REE XLV, pp. 308-309, n. 43).

${ }_{65}$ Cfr. Rix 1991, p. 680. Sulla parola anche Facchetti 2000, p. 18, nota 65.

66 G. Colonna, in REE LXXI, pp. 177-179, n. 27.

${ }^{67}$ Maggiani 1992a, p. 192, n. 47 con riferimenti, fig. 15 (ET², Po 2.18) e M. Bonamici, in REE LXX, pp. 281-282, n. 8 (ET $T^{2}$, Po 2.53).

68 Per una sintesi, cfr. Belfiore 2014b, p. 114.

69 F. PIVA, in REE LXV-LXVIII, pp. 303-304, n. 3. 
proposta una lettura in chiave $u_{m b r a}{ }^{70}$. Il richiamo alla base $t u \theta-/ t u \theta i$ potrebbe suggerire una soluzione alternativa. Tale base è stata infatti recentemente discussa da $\mathrm{R}$. Massarelli, problematizzando il suo riconoscimento nella sfera semantica dei nomi di città e comunità; questa è identificata in forma deaspirata nel $L L$ e attiva in derivativi e basi onomastiche ${ }^{71}$. Se Massarelli non si esprime sul significato di questa base lessicale ${ }^{72}$, per la Belfiore sembra appartenere alla sfera del sacro (nella fattispecie del "consacrato"). Nel nostro caso una possibile formazione dalla base deaspirata con il suffisso -is attivo per gli aggettivi (cfr. supra, helis), potrebbe indicare l'oggetto come appartenente alla sfera della base $t u \theta-/ t u \theta i$, forse dunque quale vaso "consacrato".

\section{usti/uzti}

Si conoscono due attestazioni di usti, entrambe dall'abitato:

- CIE 20273, uzti [, fine del IV-III sec. a.C.: questa documenta l'affricamento della sibilante palatale prima dell'occlusiva.

- CIE 20196, ] usti ta, fine del IV-inizi del III sec. a.C.: questa è l'unica attestazione di concordanza di caso con il deittico $t a$; infatti, nelle altre, la particella regge sempre il genitivo I (cfr. IV.3.2, classi III.c e IV.c).

A introduzione del problema interpretativo del lessema usti, merita anzi tutto soffermarsi sul deittico ta. La rassegna delle attestazioni di formule con tale deittico, già avviata da M. Cristofani per l'analisi del testo della $T C$, mostra chiaramente che la concordanza di caso avviene tra questo e il pronome personale $m i$ o tra questo e il nome del vaso, come a esempio $\theta a p n a\left(E T^{2} \text {, Po } 2.21\right)^{73}$, ma non con antroponimo. Nelle restanti attestazioni adriesi, tale identità si instaura appunto tra $m i$ (cioè il vaso che si esprime in prima persona) e $t a$. Viene dunque preliminarmente da chiedersi se usti possa non essere un antroponimo (di origine venetica, come genericamente riconosciuto dalla $\mathrm{critica}^{74}$ ), ma piuttosto un appellativo o un nome da riferire al vaso o al suo contenuto.

Seguendo questa ipotesi di lavoro, occorre volgere lo sguardo verso Spina, altro sito dove il nostro lessema ricorre. E. Benelli ha fornito un breve quadro delle attestazioni, perlopiù da ritenere sostanzialmente ancora inedite ${ }^{75}$ : sei le testimonianze isolate; una in cui funzionerebbe da gentilizio; cinque dove invece sarebbe prenome. Se integriamo le iscrizioni edite con quelle inedite documentate dal lavoro di A. Pozzi sulle necropoli, riusciamo a ricostruire un quadro un po' più articolato di quello delineato sinteticamente da Benelli. Usti appare:

- isolato, dieci volte all'assolutivo ( 9 da necropoli ${ }^{76}$; una da abitato: $E T^{2}, S p$ 2.67) e una volta al genitivo I (da abitato: $\left.E T^{2}, \mathrm{Sp} 2.66\right)$;

- una volta associato al pronome $m i$ ripetuto prima e dopo il lessema ( $m i$ usti $m i$, da abitato: $E T^{2}$, Sp 2.92);

- una volta al genitivo I e associato a un nome (gentilizio?) all'assolutivo (ustiz tite, ET ${ }^{2}, S p 2.101$ ). Sembra essere qui condivisibile la suggestione di G. Uggeri riguardo la redazione delle due parole in due momenti distinti, date le loro diverse dimensioni (quindi per certi versi anche in questo caso ustiz poteva essere almeno inizialmente isolato $)^{77}$.

70 REI LXXIX, pp. 348-350.

71 Massarelli 2008; ID. 2014, pp. 43-48; Belfiore 2014b, p. 48.

72 Cfr. più recentemente, Massarelli 2014, pp. 88-89.

73 Cristofani 1995, p. 84.

${ }_{74}$ Basti qui richiamare MAGgIANI 2002a, p. 57.

75 Benelli 2004, pp. 265-266.

76 Per le 9 occorrenze da Valle Pega: Pozzi 2011, p. 321, IG 14678 e 14683 dalla tomba 367A; p. 322, IG 14796, dalla tomba 380A; p. 329, senza IG, dalla tomba 514 A; p. 334, IG 36360 dalla tomba 656A; p. 359, IG 43413 dalla tomba 916B; p. 382, IG 32738 dalla tomba 582C; pp. 386-387, IG 38769 dalla tomba 670C; p. 390, IG 34400 dalla tomba 127D).

77 G. UGgerI, in REE XLVII, p. 303, n. 15. 
- tre volte inserito nella medesima formula, ustiscnetatis/usticnetatis. Si tratta di iscrizioni sinistrorse e quasi identiche su tre vasi a v.n. citate da Benelli (che ritiene qui usti prenome) ma sostanzialmente inedite, provenienti da distinte tombe di Valle Pega (tombe 410A, 98B, 474C) ${ }^{78}$.

- una occorrenza lacunosa da abitato $\left(E T^{2}, S p 2.52\right)$.

Manca traccia bibliografica di due ulteriori iscrizioni citate da Benelli che ne documenterebbero il valore di prenome, una dall'abitato e una erratica dal dosso B di Valle Pega non rilevata da A. Pozzi.

Fuori da Spina e Adria, tale base sembra documentata solo nell' iscrizione recente tita ustiuś dal Chiusino (CIE 4871), che già W. Schulze aveva messo in parallelo con il ben più tardo $P$. Ustius Erotis di Roma (CIL VI, 29607; cfr. ZGLE, p. 306).

Tenendo da parte quest'ultima particolare occorrenza, del tutto isolata, conviene focalizzare l'attenzione sui dati di Spina e Adria. Colpisce anzi tutto il numero di occorrenze del lessema isolato (11 su 17), e la concentrazione della documentazione spinetica dalla necropoli a Valle Pega (nessuna testimonianza viene invece dalla più settentrionale Valle Trebba). Si osserva inoltre che il lessema è sempre all'assolutivo a eccezione di due casi al genitivo I da abitato. Si è già visto che entrambi possono considerarsi isolati: il nome tite in uno dei due è forse aggiunto dopo, suggerendo così una iscrizione redatta in due tempi diversi.

Senza approfondire ulteriormente la questione, su cui ci si ripromette di tornare in altra sede con particolare attenzione alle tre formule usti(s)cnetatis, qui conviene solo sottolineare come l'insieme degli indizi rilevati portino a ritenere che ust $i$ non sia da interpretare come antroponimo, ma piuttosto si riferisca al vaso o al suo contenuto.

78 Pozzi 2011, p. 325, IG 42297 dalla tomba 410A; p. 341, IG 7958 dalla tomba 98B; p. 379, IG 31516 dalla tomba 474C. Le tre iscrizioni sono cursoriamente citate da E. Benelli senza esplicitarne i testi, affiancandole a una iscrizione erratica dal dosso B di Valle Pega e a una da abitato, quali testimonianze dell'uso di usti come prenome (BENELLI 2004, p. 265, dove si cita la tomba 297C al posto della 474C). 


\section{EPIGRAFIA IN CONTESTO}

Il presente capitolo si pone come l'ultima tappa dello studio dell'epigrafia adriese, avendo come obiettivo l'analisi in contesto della documentazione precedentemente discussa. Sebbene il lavoro sia focalizzato sulle iscrizioni, seguendo un corretto approccio metodologico l'analisi tiene nella debita considerazione anche tutti i graffiti alfabetici e non alfabetici presenti nei contesti in associazione alle iscrizioni medesime. Solo in questa maniera si può infatti approdare a un quadro che possa ritenersi il più possibile completo ed esaustivo.

La trattazione viene affrontata distinguendo i contesti di abitato (VI.1) da quelli di necropoli (VI.2), toccando anche i siti ritenuti periferici alla città di Adria (VI.3), cioè gli insediamenti testimonianti dalla necropoli di via Spolverin e da quella in tenuta Campelli, oltre ai singoli documenti provenienti da quella parte di territorio più prossimo al centro principale.

Molte iscrizioni (CIE 20772-20883), comunque sicuramente rinvenute ad Adria, rimangono prive di contesto accertato. Tra queste, CIE 20774-20816 appartengono in prevalenza alle collezioni del Museo Domestico e del Museo Civico.

\section{VI.1. Abitato}

I reperti di provenienza locale derivanti in prevalenza dall'attività di scavo dei componenti della famiglia Bocchi durante il XVIII e XIX secolo oppure da acquisiti da altri concittadini anteriormente al 1870, sono confluiti nella collezione del Museo privato c.d. "Domestico" della famiglia'. Gli scavi condotti nel XVIII secolo e di cui rimane memoria, sono quelli nei terreni della famiglia Lupati nella parte meridionale di Adria (località Bettola, Chiusa e Confortin), dei quali non si sa quanto materiale confluì nelle successive raccolte ${ }^{2}$. Dagli inizi del XIX secolo condussero in vari punti della città fra cui i più importanti nella località della La Tomba, dove è ormai accettata la presenza di un santuario ${ }^{3}$; fuori

\footnotetext{
1 Wiel-Marin 2005, p. 41; Baldassarra 2013, p. 9.

2 BALDASSARra 2013, pp. 7-9.

3 GaUcci 2012a, pp. 158-159, con riferimenti.
} 


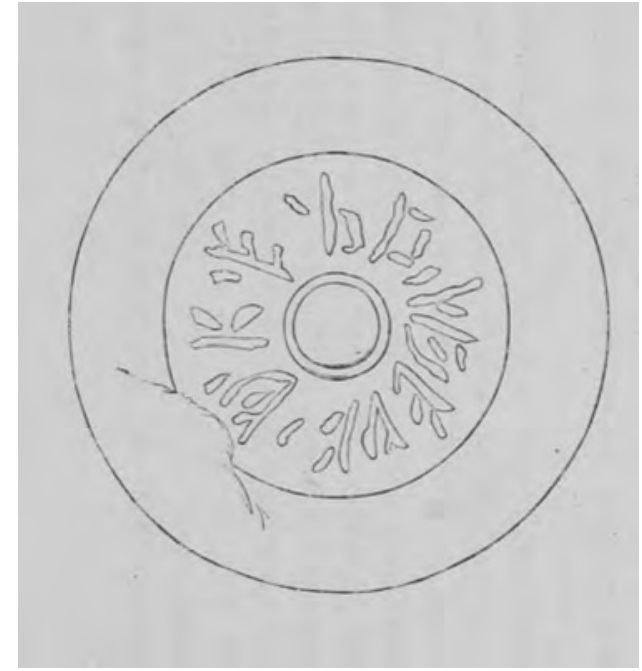

Fig. 23. Apografo edito da R. SCHöNE (1878) dell'iscrizione rinvenuta nel 1740 .

Adria nei pressi di Gavello ${ }^{4}$; inoltre, nella località Cantarane, a ovest dell'abitato moderno ${ }^{5}$. Sebbene siano esistiti inventari dettagliati degli oggetti recuperati dall'attività di scavo ${ }^{6}$, questi non sono giunti a noi (se non per quelli relativi al primo Museo Civico di Adria per gli anni 1815-1819 e 1820), e unico riferimento disponibile è l'inventario manoscritto del Museo Domestico redatto da F.A. Bocchi tra il 1867 e il $1887^{7}$ in maniera similare a quello del Museo Civico ${ }^{8}$.

I Bocchi non furono però l'unica famiglia adriese dedita a scavi per il recupero di documenti che celebrassero l' identità della propria comunità. Fra queste famiglie, oltre ai Silvestri, della cui collezione conosciamo almeno due documenti iscritti (CIE 20603, 20772), vanno ricordati anche i Grotto. In particolare R. Schöne ci dà memoria di una iscrizione su un fondo di vaso di «color cenericcio» (così riporta l'editore) conservata in casa di Alvise Grotto e di cui pubblica l'apografo' (Fig. 23). Questa proveniva dal terreno detto nel "Ritratto di Dragonzo", di proprietà di Biagio Crepaldo soprannominato Caratino, e fu rinvenuta il 6 aprile 1740. Nelle Memorie per servire all'istoria letteraria ${ }^{10}$, P. Valvasense richiama il rinvenimento di alcuni reperti sempre nei terreni dei fratelli Biagio e Antonio Crepaldi (detti Caratini) nell'ottobre del medesimo anno. Si specifica che il terreno, in vicinanza della loro casa, era situato nella "Pressa di Dragonzo" in località "Ritratto", dietro il Convento dei Padri Riformati, dirimpetto al coro della loro chiesa: rispetto alla topografia antica, oggi siamo in grado di collocarlo al confine con la necropoli, nella parte sud-occidentale della città preromana. Dall'apografo edito, si potrebbe ipotizzare che l'iscrizione possa essere stata redatta sulla superficie esterna di un piede a disco con rilievo centrale o più probabilmente di un piede su stelo (in entrambi i casi forse in ceramica attica piuttosto che bucchero o ceramica grigia, viste le linee concentriche del disegno?). L'apografo pare disturbato dalla presenza di brevi tratti, probabilmente segni accidentali. Si potrebbe leggere con molta incertezza paiteś[?]ak/te. Se questa lettura può avere qualche fondamento, paite trova riscontro in un nome individuale documentato a Gravisca nel secondo quarto del V sec. a.C. ${ }^{11}$; la

${ }^{4}$ Baldassarra 2013, pp. 10-11.

5 Wiel-MARIN 2005, pp. 37-38, con riferimenti.

${ }^{6}$ Ibid., pp. 44-45, con riferimenti; BALDASSARRA 2013, p. 22, con riferimenti.

7 In CIE, IV, I, 1 si è seguita la redazione nota come I stesura, compilata dal 1867 fino al 1887, mentre la II stesura pare una copia della I stesura limitata alla versione del 1868. Purtroppo, in entrambe le stesure del Catalogo non sono riportate per buona parte dei reperti editi indicazioni relative al contesto di provenienza.

8 WIEL-MARIN 2005, pp. 45-49, con riferimenti.

9 SChÖNE 1878 , p. 146, n. 617.

10 VAlvasense 1758, p. 177.

${ }^{11} E T^{2}$, Ta 0.17, da Gravisca, datato al 470-460 a.C. Per questa iscrizione, cfr. in particolare Pandolfini 2000, p. 71, n. 387a), p. 77, e Maras 2009b, pp. 378-379, Ta co.12. 


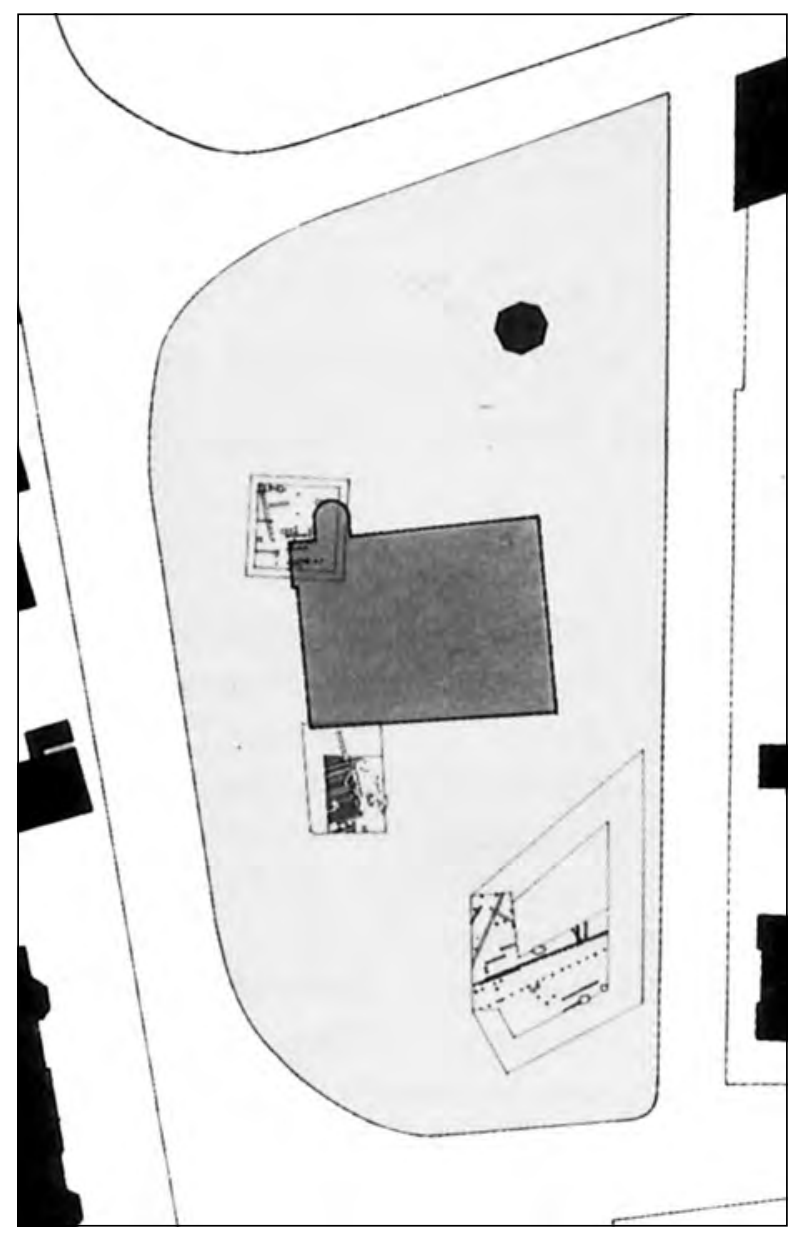

Fig. 24. Sede del Museo Archeologico Nazionale: le aree indagate nel 1878-1879 e nel 1936-1939 (da BoNOMI 1993).

forma (se realmente tale) di alcune lettere, come alpha, epsilon, pi, potrebbe rimandare alla paleografia adriese di questo medesimo orizzonte cronologico. Rimangono ovviamente forti dubbi su questa interpretazione desunta unicamente dalle informazioni che a noi sono giunte.

Si può illustrare un quadro topografico dei rinvenimenti proprio partendo dalla zona a nord del convento (attuale Ospedale), in prossimità dei limiti meridionali dell'abitato preromano, dove si conta un buon numero di siti indagati tra il XIX e i primi decenni del XXI secolo. Tra i più importanti, si ricorda l'attuale sede del Museo Archeologico Nazionale, spazio che fu parco prima di essere destinato al Museo Civico, costruito a partire dal 1957 e inaugurato nel 1961 (diventato poi Nazionale nel 1972). Il parco aveva forma ellittica ed era diviso in quattro settori (c.d. "triangoli") da due viali ortogonali. Nel tempo venne ricordato come Pubblico Giardino (XIX sec.) e poi Prato dell'Ospedale (nel XX sec. fino alla costruzione del Museo) trovandosi subito a nord dell' ingresso principale dell'Ospedale civico di Adria. Il sito è stato scavato a più riprese tra il XIX e il XX sec. (Fig. 24), portando in luce strutture insediative in materiale deperibile riferite probabilmente a un contesto abitativo-produttivo costeggiato da un canale irregimentato con materiale databile dal pieno VI sec. a.C. fino all'età ellenistica ${ }^{12}$. I primi interventi si devono a F.A. Bocchi, che qui effettuò tra il 1878 e il 1879 due ampi scavi nei settori Nord-Ovest e Sud-Est del giardino, tuttora molto importanti per l'acribia con la quale furono condotti. Nel settore $S E^{13}$, il Bocchi scavò strutture attribuite alla fase romana, fra le quali riconosce

12 Per una recente analisi del contesto, cfr. GaUcci 2012a, pp. 160-161, con riferimenti. Per più recenti scavi relativi alla fase di II sec. a.C., cfr. BonOMI, RoBIno 2007, p. 88.

13 BocсHi 1879, pp. 90-94; SCARANi 1971, pp. 23-25. Nel settore la realizzazione di una prima trincea di scavo, profonda $5 \mathrm{~m}$ rispetto al piano di campagna (BосCHI 1888, tavola sinottica finale) permise il recupero di CIE 20001-20002, 
un possibile impianto viario e complessi produttivi, e approfondendosi rinvenne resti di materiale organico, piccoli pali a costipamento del terreno. Tale palificazione, definita dal Bocchi «palancolata» o «palafitta», si ritiene adesso la struttura di consolidamento di un canale navigabile ${ }^{14}$. Lo scavo di un quadrato di $15 \mathrm{~m}$ di lato nel settore $\mathrm{NO}^{15}$ arrivò invece a mettere in luce un edificio ligneo oltre $\mathrm{i}$ 4 metri di profondità e successivamente raggiunse in un saggio di scavo ristretto la profondità di $8 \mathrm{~m}$ dal piano di campagna ${ }^{16}$. Una riflessione sulle evidenze archeologiche rinvenute dal Bocchi nello scavo di questo settore è proposta da $\mathrm{S}$. Bonomi ${ }^{17}$, e quindi ripresa da L. Donati ${ }^{18}$. S. Bonomi ha ricostruito una sequenza stratigrafica che prevede un primo strato di riporto di epoca post-romana spesso un paio di metri, quindi sotto questo uno strato di origine alluvionale di spessore variabile che sigilla i livelli arcaici scavati tra i 3,5 e i $4 \mathrm{~m}$ di profondità ${ }^{19}$. L. Donati ha proposto di vedere nella sequenza dei livelli arcaici descritta dal Bocchi due fasi edilizie, una più antica, definita Adria I e sigillata da un evento alluvionale datato entro il terzo quarto del VI sec. a.C. e una più recente di età tardo-arcaica, definita Adria II, della quale rimangono maggiori evidenze strutturali ${ }^{20}$. Un consistente numero di documenti epigrafici, appartenenti alla collezione del Museo Domestico, è indicato come proveniente dalle palafitte, solo ipoteticamente individuabili in quelle indagate nello scavo del Pubblico Giardino.

Durante il XX sec. furono indagati i settori di Sud-Ovest e di Nord-Est. Lo scavo nel primo fu supervisionato da G.B. Scarpari sotto la direzione della Soprintendenza alle Antichità del Veneto. Dapprima attraverso due trincee nel 1936 e poi più estensivamente nel 1938-1939 ${ }^{21}$, l'indagine consentì di portare alla luce a 3,6 $\mathrm{m}$ dal piano di campagna un vasto tavolato ligneo e altre file di pali infissi nel terreno, riferibili a un orizzonte di VI-V sec. a.C. Tale opera è stata interpretata da S. Bonomi come area portuale o di lavorazione all'aperto ${ }^{22}$. Nel settore di NE, lo scavo aperto nell'inverno tra il 1954 e il 1955, sotto la direzione scientifica di G. Fogolari, consentì invece di intercettare, sotto le strutture romane e a partire da una profondità di $2 \mathrm{~m}$ circa dal piano di campagna, uno strato ricco di materiale ceramico, che G. Fogolari riteneva di riporto e trasportato dall'acqua, suggerendo così la presenza di un canale ${ }^{23}$.

Si deve a S. Bonomi una sistematizzazione delle informazioni relative a questi scavi ${ }^{24}$, mentre $\mathrm{L}$. Donati e A. Parrini hanno più recentemente analizzato le fasi più antiche dello scavo del settore di $\mathrm{NO}^{25}$. Nel complesso, dalle indagini dell'area del Museo si desume nel periodo etrusco che un canale doveva attraversarla nella sua parte orientale, con un andamento circa nord-sud. La porzione occi-

per i quali non è possibile stabilire con maggior precisione la profondità di rinvenimento. Allo "strato romano" del Bocchi sono attribuiti CIE 20003-20011, tutti databili all'età ellenistica.

14 Bonomi 1993, p. 78; Donati, Parrini 1999, p. 576.

15 Indicazioni dello scavo del Bocchi si trovano in SCARANI, 1971, pp. 25-29; Giangiulio 1984, pp. 41-42. Le pareti dello scavo erano a scarpata, portando per ragioni di sicurezza a un restringimento sensibile dell'area di scavo mano a mano che questa veniva approfondita.

16 Bоссні 1879, tab. VI. Vale la pena ricordare che dalla metà di maggio fino alla metà di giugno del 1879 il Bocchi raccolse materiale soprattutto dalle sezioni, mentre prima della chiusura impiegò una settimana a raccogliere materiale dal terreno smosso (ibid., p. 217). Per le quote si veda Bocchi 1888, tavola sinottica.

17 BonOMi 1993, pp. 76-77.

18 Donati, PARrini 1999 , pp. 567-581

19 BONOMI 1993, pp. 76-77.

20 Donati, Parrini 1999, p. 578.

21 Battaglia 1958-1959, pp. 341-342, fig. 129.

22 Bonomi 1993, p. 78. Lo scavo di via Ex Riformati porta a rivedere questa ipotesi (comunicazione personale di S. Bonomi).

${ }^{23}$ Fogolari, 1955, p. 196, n. 2479; EAD. 1956, p. 541; cfr. Scarani 1971, p. 29, 9-IV. Si ricorda che abbassandosi ulteriormente, a 3,6 m di profondità affiorò un unico trave orizzontale associato a bucchero e quindi a 3,8 m di profondità altri due pali. L'area di scavo non è riportata nella ricostruzione qui alla Fig. 24. Infatti, sebbene esista nella documentazione di scavo uno schizzo con la sua collocazione, questa non è riportabile in pianta senza grossi margini di errore.

24 BONOMI 1993.

25 Donati, PARrini 1999. 
dentale dell'area era invece occupata da strutture lignee, edifici e una vasta banchina. Più difficile, allo stato attuale degli studi, distinguere fasi cronologiche coerenti con queste strutture. Il rapporto tra i documenti epigrafici e i relativi contesti di questo importante sito è oggetto di uno specifico approfondimento (VI.1.1).

Lì presso si situa lo scavo in località Retratto, avvenuto nel 1970 e che portò in luce strutture di I sec. a.C. ${ }^{26}$, e quello più significativo del contesto abitativo-produttivo compresivo di più fasi in via Ex Riformati. Grazie a successivi interventi di scavo $(2001,2004)$ a lato di questa via è stata indagata una stratigrafia che ha mostrato il susseguirsi di strutture arcaiche ed ellenistiche con medesimo orientamento e costeggiate da canali ${ }^{27}$, successivamente bonificati per costruirvi una via con cloaca nella prima metà del II sec. a.C. ${ }^{28}$. Alla fase tardo-arcaica ${ }^{29}$ appartengono i documenti CIE 20203-20211. Fra questi si ricorda il lacerto di iscrizione su ceramica depurata (CIE 20204, dall'US 9), che assieme ai due frammenti di ceramica grezza con graffiti non alfabetici realizzati dopo la cottura (CIE 20205-20206, dalle UUSS 9 e 9A) proviene da strati di detriti e scarti delle attività dell'edificio, rinvenuti nello spazio tra questo e il canale e datati ai primi decenni del V sec. a.C. ${ }^{30}$. Dalla fase di età ellenistica dello scavo di via Ex Riformati (nello specifico, US 1080, datata alla prima metà del III sec. a.C.) proviene l'iscrizione lacunosa CIE 20201 (probabilmente di possesso: mi petr [ ), graffita nel fondo interno di una coppa in ceramica grigia ${ }^{31}$. Alla fase ellenistica appartengono i quattro frammenti di vasi a v.n. graffiti dallo scavo in loc. Retratto (CIE 20197-20200). Fra questi, si datano tra III e II sec. a.C. un lacerto di iscrizione graffita dopo cottura nella vasca di un piatto da pesce a v.n. locale (CIE 20197, III sec. a.C.) forse attribuibile a un nome piuttosto che al lessema helis (cfr. V.4), un singolo alpha (CIE 20199) e un segno a croce (CIE 20200), questi ultimi due graffiti nel fondo interno di ciotole locali a v.n. Tra la seconda metà del II e gli inizi del I sec. a.C. si data invece una iscrizione gallica per alfabeto (c.d. leponzio) e onomastica (iara) graffita nella parete esterna di una ciotola a v.n. locale (CIE 20198), l'unica da contesto abitativo attribuibile a questa cultura epigrafica.

Verso est, non molto distante da questa zona e subito a nord dell'attuale via Angeli, lo scavo di F.A. Bocchi nel cortile di Antonio Ornati nel 1878 ha messo in luce strutture lignee e materiali databili tra il periodo tardo-arcaico e quello ellenistico, il cui contesto è riconosciuto ipoteticamente come votivo $^{32}$. Data la sua importanza, viene offerto un approfondimento su questo contesto (VI.1.2). Non

26 Wiel-Marin 2005, p. 84.

27 In particolare, in quest'ultimo una prima campagna di scavo (2001) ha portato in luce un complesso edilizio di III sec. a.C. indiziato da un focolare e da un piano pavimentale, forse interrotto da un incendio, cui è seguita una seconda fase testimoniata da tre vani separati da due passaggi (BONOMI, RoBıno 2007, p. 88). Un successivo intervento di scavo (2004) ha permesso di condurre l'indagine della stratigrafia fino allo sterile, recuperando così una sequenza di strutture lignee con destinazione abitativo/produttiva succedutesi nel tempo fra il pieno VI e il V sec. a.C. A partire dalla seconda fase costruttiva queste furono delimitate a nord e ad est da due canali ortogonali fra loro, dei quali quello orientale era orientato NNO-SSE e aveva la sponda occidentale attrezzata con un camminamento costituito da una piattaforma di travi di legno con riporti di terra e fascine per tenere asciutto il passaggio. Il più recente scavo del 2015-2016 non è ovviamente compreso in questo studio. Per quest'ultimo, si rinvia a Gennuso, Marchesini, Vallicelli 2018 e a Bonomi, Vallicelli, BALISTA 2020.

28 Robino 2008, pp. 10-11.

29 Bonomi, GambacurTa 2017, pp. 69-74.

30 Sempre da uno scarico, ma più antico, cioè l'US 72 datata agli ultimi decenni del VI sec. a.C. e legata ad attività artigianali, provengono i frammenti di coppe in bucchero con graffiti non alfabetici CIE 20210-20211. Tra i documenti epigrafici di questo contesto, si ricordano anche i due frammenti di coppe in ceramica depurata con singole lettere (CIE 20207-20208) e l'anfora da trasporto di produzione greco-occidentale del 550-490 a.C. con graffito non alfabetico nel puntale (CIE 20203).

${ }^{31}$ Questa e un graffito non alfabetico su un frammento di ceramica locale a v.n. di II sec. a.C (CIE 20202) rinvenuto nel riempimento di una sepoltura di periodo alto-medievale, sono già discussi da M.T.A. Robino (2004, pp. 361-364).

${ }^{32}$ Nel 1878 F.A. Bocchi aprì un saggio di scavo, intercettando, a 5,5 m di profondità dal piano di campagna, un tavolato ligneo (identificato come rovere), ricco di materiale ceramico fra cui molte iscrizioni e graffiti (BocCHI 1879, pp. 95-97). La maggior parte dei documenti epigrafici è riferita al mese di ottobre del 1878, quando il Bocchi si approfondì 
molto distante, sempre nel XIX secolo fu scavato il pozzo presso la via che oggi è dedicata a Vittorio Emanuele III, dalle cui profondità furono recuperati alcuni frammenti con graffiti databili al VI sec. a.C. $(\text { CIE 20250-20252) })^{33}$.

A sud del cortile Ornati, in via San Francesco, nel 1994 è stato effettuato uno scavo archeologico indagando la stratigrafia dallo spoglio di strutture di età romana fino alla fase di V sec. a.C. ${ }^{34}$. Nella porzione settentrionale dello scavo (settore A), a 3,9 $\mathrm{m}$ di profondità dal piano di campagna fu individuata una fascinata di andamento parallelo al canale che doveva rappresentare parte delle piattaforme di fondazione di una struttura edilizia riferita al V sec. a.C., di cui però non fu rinvenuta alcuna traccia ${ }^{35}$. Tra la metà del III e il II sec. a.C. fu costruito un edificio a uso abitativo-artigianale lungo la sponda del canale attrezzata con assi di legno (fase II), colmato tra la fine del II e gli inizi del I sec. a.C. (fase III) per la costruzione di una strada con cloaca sottostante e di un complesso monumentale porticato (fase IV). Da questo scavo non provengono iscrizioni, ma solo 4 lettere isolate, di cui una identificabile come numerale (unico graffito inciso a crudo), 8 graffiti non alfabetici (dei quali tre nel medesimo supporto) e due non determinabili (CIE 20238-20249). Nel complesso, si nota come nessuno dei frammenti graffiti sia in contesto significativo, ma piuttosto siano tutti in strati di obliterazione o riporto e quindi non direttamente da riferire all'impianto abitativoartigianale. La prevalenza è ceramica grigia di periodo ellenistico, ma anche frammenti di ceramica a v.n. fra cui due attribuibili a una produzione volterrana (CIE 20248-20249). L'unico frammento di olla in ceramica grezza (CIE 20242) testimonia, eccezionalmente per il periodo ellenistico, una lettera incisa a crudo (cfr. II.1.3), riconoscibile con tutta evidenza come un numerale con probabile valore di capacità. I graffiti sono per lo più non alfabetici e di difficile definizione spesso a causa dello stato lacunoso (CIE 20238, 20239, 20241, 20243a-b, 20246, 20247). Oltre al numerale suddetto, si riconoscono tre lettere singole, delle quali due databili al periodo ellenistico (alpha, CIE 20249; ypsilon, CIE 20240) e il pi di forma uncinata graffito sul fondo esterno di una coppa etrusco-padana in ceramica depurata (CIE 20244), che richiama aspetti paleografici documentati nel VI sec. a.C. (cfr. III.2) e che potrebbe essere molto più antico del contesto di età ellenistica (un focolaio inutilizzato) nel quale è stato rinvenuto. Si conferma così l'impressione del raro uso della scrittura e di altri tipi di segni nell'ambito produttivo di periodo ellenistico.

Una serie di rinvenimenti sembrano concentrarsi lungo una fascia presso quello che doveva essere il limite orientale della città etrusca e a diretto contatto con il settore funerario, cioè nella zona della Tomba e della Bettola. Il sito sicuramente più rilevante è presso la chiesa di Santa Maria della Tomba, dove è stato riconosciuto un santuario dedicato ad Apollo e forse Iride, frequentato da Egineti nel periodo tardo-arcaico ${ }^{36}$. Da questo santuario è probabile che vengano almeno tre, se non quattro iscrizioni su ceramica attica $(C I E 20282,20289,20290,20295)^{37}$, nonché forse molto del materiale epi-

oltre i 2,5 $\mathrm{m}$ e fino ai $6 \mathrm{~m}$ dal piano di campagna. Il contesto è stato recentemente interpretato come sacro e connesso con il vicino scavo della località La Tomba (GAUCCi 2012a, pp. 159-160; Govi 2012, p. 142). Rispetto al quadro noto, il rinvenimento della planimetria originale inviata dal Bocchi a Roma per la pubblicazione in Notizie degli Scavi, mostra chiaramente che la posizione del contesto è differente dalla tavola poi edita (FACCHI, VALLICELLI, WiEL-Marin c.s.; si ringrazia la Direttrice del Museo Archeologico Nazionale di Adria, Dott.ssa Alberta Facchi, per l'anticipazione della preziosa notizia); il contesto va collocato infatti a ovest dell'attuale corso Vittorio Emanuele II e non a est, come invece si è sempre ritenuto, trovandosi così circa $200 \mathrm{~m}$ dalla località La Tomba (cfr. Figg. 1-2 rispetto alle medesime figure in CIE IV, I, 1, pp. 3 e 5$)$.

33 BocCHI 1879, p. 92. Tre frammenti, due di coppecoperchio in ceramica grezza (CIE 20250-20251) e un fondo di coppa in bucchero (CIE 20252), furono rinvenuti nel giugno 1878 a 6-7 $\mathrm{m}$ di profondità dal piano di campagna scavando il pozzo.

34 Camerin, Tamassia 1998-1999, pp. 212-235; Bonomi, Camerin, Tamassia 2002b, pp. 201-213.

35 Tale assenza è stata interpretata da S. Bonomi come frutto del degrado dell'area dopo la fine del V sec. a.C., colpita da un evento alluvionale collocato dopo i primi decenni o la metà del IV sec. a.C. (fase I).

36 Colonna 1974a, p. 8, nota 23.

37 GaUcci 2012a, pp. 158-159. 
grafico conservato nel Museo Domestico della famiglia Bocchi e purtroppo privo di certa provenienza, al quale viene dedicato uno specifico approfondimento (VI.1.4).

In località Bettola, in quello che doveva essere il margine sud-orientale dell'abitato antico, sono state rinvenute tra il 1874 e il 2000 varie testimonianze epigrafiche con cronologie che vanno dalla fase arcaica fino al II sec. a.C., tra cui sicuramente le più significative sono quelle del fondo Lodo (di difficile localizzazione) e del fondo Calisti, ai quali viene dedicato uno specifico approfondimento (VI.1.3) ${ }^{38}$. Sempre in questa località, ma in uno scavo del 2000 lungo il lato est di via Bettola, è stato portato in luce un ambiente interno e una probabile zona cortilizia di un edificio di III-II sec. a.C. ${ }^{39}$; in un piano di occupazione è stata rinvenuta una coppa in ceramica grigia lacunosa di gran parte dell'orlo e della vasca (CIE 20277), databile tra la fine del IV e la prima metà del III sec. a.C. e che presenta nella vasca interna, presso la decorazione impressa, una iscrizione fortemente lacunosa (]$\times u)$.

Rispetto a questo quadro, alcuni documenti epigrafici compresi tra VI e V sec. a.C. di incerta provenienza rinvenuti nel XX sec. (CIE 20368-20374), sono da ritenere provenienti da contesti abitativi, ma non meglio specificati. Tra questi, due frammenti di coppe in ceramica grigia di periodo tardo arcaico (CIE 20368-20369) ${ }^{40}$, furono forse rinvenuti durante lavori di scavo in profondità (es. la realizzazione di un pozzo). La provenienza da contesti arcaici è confermata per almeno tre documenti (CIE 20370-20372), mentre per altri due è comunque plausibile (CIE 20373-20374). Stante questo proesupposto, per questi ultimi cinque dobbiamo ritenere che, non essendo registrati nei Cataloghi del Bocchi, siano ritrovamenti successivi ${ }^{41}$. Gli scavi a cui quindi possono appartenere sono quello del 1904-1905 nel fondo Osellieri, quello del 1910 dietro l'abside della Chiesa della Tomba e quelli del 1936-1939 nell'area dell'attuale Museo Archeologico. Altri scavi documentati e anteriori a quello edito di via San Francesco si presume che non abbiano intercettato livelli tardoarcaici da cui questi documenti iscritti possano giungere. Sulla base della documentazione nota, vi è pertanto più probabilità che questi appartengano agli scavi 1936-1939 nell'area dell'attuale Museo Archeologico, i cui materiali sono andati in prevalenza dispersi o confusi con quelli del Museo Civico. A parte il fondo di coppa in ceramica depurata con lettere in legatura che paiono evocare una serie alfabetica settentrionale (CIE 20371), si richiama l'attenzione sulla possibile formula di consacrazione a $\Theta a n r$ databile tra la fine del VI e i primi decenni del V sec. a.C. sul fondo esterno di una coppa in ceramica depurata (CIE 20370; cfr. V.3); se valida l'idea che possa provenire dagli scavi del Museo Archeologico del 1936-1939, allora potrebbe rientrare assieme ad alcune c.d. paterae pertusae (CIE 20134, 20362), ma anche all' iscrizione al (CIE 20160; cfr. V.2) all'interno di un sistema di pratiche cultuali di natura non chiaramente perspicua in un complesso strutturale che sembra piuttosto destinato in prevalenza a funzioni di tipo lavorativo.

\footnotetext{
38 Altri rinvenimenti epigrafici riferibili al periodo compreso tra il tardo Arcaismo e l'età ellenistica, di natura più puntuale, sempre in località Bettola sono quelli recuperati negli scavi del 1904-1905 nel fondo Osellieri (cfr. CONTON 1908, p. 76), e nel recente scavo del 2000 di un probabile edificio (poche sono le evidenze nello scavo che rimandano con certezza alla presenza di una struttura) di III-II sec. a.C. lungo il lato est di via Bettola. A questi si aggiungono i frammenti di ceramica databili tra III e II sec. a.C. con graffiti alfabetici e non alfabetici rinvenuti da F.A. Bocchi lungo il canale della Tomba poco a nord del ponte di Santo Stefano (CIE 20278-20281). Sempre nella parte orientale della città, livelli tardo-arcaici intercettati tramite i carotaggi effettuati nel 1964 e 1967 in località Bettola presso il Canalbianco (SCARFì 1964 p. 396), non hanno restituito testimonianze epigrafiche.

39 Robino 2004, pp. 175, 182-184.

${ }^{40}$ I frammenti sono stati recuperati all'interno di una cassa di materiale frammentario con la sola indicazione esterna "Sig. Ceragioli - Wanda Marin". Non è stato possibile risalire al sig. Ceragioli menzionato, mentre è noto che W. Marin è stata una rilevante personalità filantropica della società adriese durante il XX sec. Si potrebbe congetturare che la Marin abbia agito da intermediario per il Ceragioli nella consegna del materiale archeologico (?). Si rimanda ora alla riflessione su questa iscrizione edita in REE LXXIX, pp. 288-290, n. 56.

41 Si richiama qui una ipotesi già formula in GAUCCI 2012a, p. 147.
} 


\section{VI.1.1. Gli scavi presso l'attuale Museo Archeologico Nazionale}

Da quest'area sono stati recuperati nel corso degli scavi 53 documenti tra iscrizioni e digrammi, tutti dopo cottura a eccezione delle iscrizioni CIE 20353 e 20361, incise a crudo. Si riporta qui di seguito un quadro delle occorrenze strutturato su base cronologica (Tab. 6).

Tab. 6. Iscrizioni (compresive di diagrammi e lettere in legatura) dagli scavi effettuati presso il Museo Archeologico, organizzate su base cronologica.

\begin{tabular}{|c|c|c|c|c|}
\hline$C I E$ & Testo & Posizione & Classe & FORMA \\
\hline \multicolumn{5}{|c|}{ Ultimi decenni del VI - inizi del V sec. a.C. } \\
\hline 20089 & $\widehat{a v}$ & fondo esterno & bucchero & coppa \\
\hline 20143 & ar & fondo esterno & v.n. attica & kylix \\
\hline 20180 & mi venelus leceniies & fondo esterno & ceramica depurata & coppa \\
\hline 20189 & sequenza alfabetica & fondo interno & ceramica grigia & coppa \\
\hline 20194 & $\widehat{p v} \circ \widehat{v p}$ & fondo esterno & bucchero & forma aperta \\
\hline 20353 & mav & fondo esterno & ceramica grezza & olla \\
\hline 20356 & an & fondo esterno & ceramica depurata & coppa \\
\hline 20361 & laris & orlo & ceramica grezza & olla \\
\hline \multicolumn{5}{|c|}{ Prima metà del V sec. a.C. } \\
\hline 20159 & $a m n z$ & parete esterna & ceramica depurata & coppa \\
\hline 20160 & al & fondo esterno & ceramica depurata & coppa \\
\hline \multicolumn{5}{|c|}{ IV sec. a.C. } \\
\hline 20008 & an $[---]$ & fondo interno & v.n. attica & forma aperta \\
\hline 20037 & kutvaluś [- ? - ] & fondo esterno & ceramica depurata & coppa \\
\hline 20043 & $\widehat{a a}$ о $\overparen{a i a}$ & fondo esterno & ceramica grigia & coppa \\
\hline 20127 & {$[---] e$} & fondo interno & ceramica grigia & coppa \\
\hline \multicolumn{5}{|c|}{ V-IV sec. a.C. } \\
\hline 20125 & sequenza alfabetica & fondo interno & ceramica depurata & coppa \\
\hline 20184 & {$[-?-] \operatorname{sinin} \theta[---]$} & parete & v.n. attica & forma aperta \\
\hline \multicolumn{5}{|c|}{ Incerta datazione, comunque non posteriore al IV sec. a.C. } \\
\hline 20040 & {$[---] n ̣ a$} & esterno & non det. & non det. \\
\hline 20126 & {$[-?-a] r n z a$} & fondo interno & ceramica depurata & coppa \\
\hline 20135 & non det. & esterno & ceramica depurata & coppa \\
\hline \multicolumn{5}{|c|}{ IV-III sec. a.C. } \\
\hline 20133 & mi haltva & fondo interno & ceramica grigia & coppa \\
\hline \multicolumn{5}{|c|}{ Fine del IV - prima metà del III sec. a.C. } \\
\hline 20019 & [---]ialu & fondo interno & v.n. volterrana & kylix \\
\hline 20029 & $k a[-?-]$ & parete interna presso l'orlo & v.n. volterrana & kylix \\
\hline 20116 & $p u$ & fondo esterno & ceramica grigia & coppa \\
\hline 20181 & estaluś & fondo interno & ceramica grigia & coppa \\
\hline 20196 & [?] usti ta & fondo interno & v.n. locale & ciotola \\
\hline 20364 & ataśni & fondo esterno & ceramica grigia & coppa \\
\hline \multicolumn{5}{|c|}{ Ultimi decenni del IV - III sec. a.C. } \\
\hline 20006 & {$[---]$ ouriu } & parete interna presso l'orlo & v.n. locale & ciotola \\
\hline 20028 & sesiu & parete interna presso l'orlo & v.n. locale & ciotola \\
\hline 20030 & ata krapnal [-? -] & parete interna presso l'orlo & v.n. locale & ciotola \\
\hline 20107 & ḅe[---] & parete esterna presso il piede & v.n. volterrana & ciotola/kylix \\
\hline
\end{tabular}




\begin{tabular}{|c|c|c|c|c|}
\hline CIE & Testo & Posizione & Clansse & Forma \\
\hline \multicolumn{5}{|c|}{ III sec. a.C. } \\
\hline 20041 & alpha e chi in legatura? & parete esterna & ceramica grigia & mortaio \\
\hline 20182 & {$[---] \times$ vercniś } & parete esterna & v.n. locale & ciotola \\
\hline 20183 & [---]tnakiu o [---]knakiu & parete interna & v.n. locale & forma aperta \\
\hline 20188 & venu kleustẹ[-? -] & fondo interno & v.n. locale & ciotola \\
\hline 20191 & $t a[---]$ & parete interna presso l'orlo & v.n. locale & ciotola \\
\hline \multicolumn{5}{|c|}{ III-II sec. a.C. } \\
\hline 20001 & {$[---] \dot{s} a$} & parete esterna & ceramica grezza & coppacoperchio \\
\hline 20020 & a:risiu & fondo interno & v.n. locale & ciotola \\
\hline \multicolumn{5}{|c|}{ II sec. a.C. } \\
\hline 20012 & [---]euna & fondo interno & v.n. locale & ciotola \\
\hline \multicolumn{5}{|c|}{ Età ellenistica } \\
\hline 20010 & $i u$ o $V I$ & fondo interno & v.n. locale & forma aperta \\
\hline 20032 & $k a$ & parete & v.n. non det. & forma aperta \\
\hline 20044 & alo au & parete esterna & ceramica grigia & coppa \\
\hline 20051 & titalu & parete interna presso l'orlo & ceramica grigia & coppa \\
\hline 20079 & mi leị $[---]$ & fondo interno & ceramica grigia & coppa \\
\hline 20084 & tite & parete interna presso l'orlo & ceramica grigia & coppa \\
\hline 20100 & testo non leggibile & interno del vaso & ceramica grigia & coppa \\
\hline 20110 & {$[-?-] \operatorname{aru} u[---]$} & interno del vaso & ceramica grigia & coppa \\
\hline 20120 & {$[---] \times l u v a$} & parete esterna & ceramica grigia & coppa \\
\hline 20121 & $k a$ & fondo interno & ceramica grigia & coppa \\
\hline 20124 & $i u$ o $V I$ & parete esterna presso il piede & ceramica grigia & coppa \\
\hline 20139 & $v \times r \times[---]$ & fondo interno & ceramica grigia & coppa \\
\hline 20190 & {$[? p]$ uleviś } & fondo interno & ceramica grigia & coppa \\
\hline \multicolumn{5}{|c|}{ Età incerta } \\
\hline 20013 & $\operatorname{arnt}[---]$ o arnz $[---]$ & interno & non det. & forma aperta \\
\hline 20111 & [-- - ]tuśvalu[ [- ?-] & parete esterna presso l'orlo & ceramica acroma & piatto \\
\hline
\end{tabular}

Si riportano di seguito alcune considerazioni sul rapporto fra i contesti dei quattro settori indagati e le iscrizioni recuperate, richiamando, dove necessario, anche i graffiti alfabetici e non alfabetici. Per la descrizione dei contesti, si rimanda a quanto sopra esposto.

Settore SE. Da quest'area provengono cinque iscrizioni etrusche, purtroppo tutte in stato molto lacunoso (CIE 20001, 20006, 20008, 20012, 20013). Si tratta di testi graffiti dopo cottura prevalentemente su forme aperte a v.n. e in un caso su una coppacoperchio in ceramica grezza. I frammenti sono databili tra III e II sec. a.C. a eccezione di CIE 20008 che risulta una delle rarissime attestazioni di IV sec. a.C. A queste è possibile aggiungere CIE 20010, un frammento di parete presso l'attacco del piede di una forma aperta a v.n. di produzione locale attribuibile all'età ellenistica, nel quale è graffito dopo cottura il digramma $i u$ oppure più verosimilmente un numerale $(V I)$. 
Nel complesso, si osserva come, tutti i frammenti iscritti e graffiti ${ }^{42}$ appartengano latamente al periodo ellenistico, a eccezione di CIE 20002. Non è da sottovalutare che quest'ultimo, assieme a CIE 20001, possa provenire da quello che pare il riempimento di un canale, probabilmente in uso fino al suo tombamento, forse da collocare al pieno II sec. a.C. se estendiamo a queste evidenze le considerazioni di S. Bonomi e M. Robino ${ }^{43}$. Diversamente, i restanti documenti provengono da uno strato definito dal Bocchi "romano" e al quale attribuisce infrastrutture che potrebbero con buona verosimiglianza appartenere alla ristrutturazione dell'abitato avvenuta appunto nel pieno II sec. a.C. Non è possibile approfondire ulteriormente il problema del contesto, se non notando l'uniformità di frammenti compresi tra IV e II sec. a.C., la presenza di onomastica (seppure molto lacunosa) in almeno tre casi su quattro e la selezione di vasi di forma aperta.

Settore NE. È verosimile che l'inizio di alfabetario CIE 20189 riferibile al tardo Arcaismo, le iscrizioni lacunose CIE 20188 (una formula onomastica forse all'assolutivo nella parte conservata) e 20191 (che ci testimonia nella parte conservata forse il deittico ta) databili al pieno III sec. a.C., 20190 (un probabile nome femminile), la lettera isolata CIE 20192 e il graffito non alfabetico CIE 20193 di periodo ellenistico, appartengano tutti a un contesto rimescolato. Sembra diverso il caso di CIE 20194-20195, il primo costituito da due lettere in legatura di difficile scioglimento e il secondo una lettera singola, rinvenuti il 5 febbraio 1955 scavando a $3,8 \mathrm{~m}$ di profondità nei pressi di due pali infissi nel terreno ${ }^{44}$. Il primo è attribuibile al VI sec. a.C. e il secondo è verosimilmente un frammento di ceramica attica, dunque non posteriore al IV. Pare significativo il loro rinvenimento in profondità e in rapporto a strutture lignee suggerendo in questo caso la loro coerente pertinenza al periodo tardo-arcaico.

L' iscrizione CIE 20196, databile tra la fine del IV e gli inizi del III sec. a.C. e forse allusiva al vaso o al suo contenuto (cfr. V.4), risulta senza indicazioni puntuali di rinvenimento.

Settore NO. Molte sono le iscrizioni rinvenute in questo settore di scavo. Sebbene la complessa stratigrafia descritta dal Bocchi non possa essere analizzata secondo i più moderni metodi della ricerca archeologica, vale tuttavia la pena provare ad avviare una riflessione sui contesti descritti dallo scavatore, per comprendere se questi possano risultare coerenti rispetto alla documentazione epigrafica recuperata.

Dai contesti stratigrafici più superficiali il Bocchi recupera due iscrizioni, CIE 20019 ([-- - ] ialu) e 20020 (a:risiu), oltre a una singola lettera chi (CIE 20021), tutte graffite dopo cottura su ceramica a v.n. e rinvenute nel terreno fortemente rimaneggiato entro il primo metro dal piano di campagna, fra molte "rovine".

Provengono da un contesto inferiore caratterizzato da terreno povero di materiale e percorso da due fossati, le iscrizioni CIE 20028, 20029, 20030, 20032, tutte su ceramica locale a v.n. e datate perlopiù tra gli ultimi decenni del IV e il III sec. a.C., e inoltre alcuni frammenti con singole let-

${ }^{42}$ Si contano due frammenti di piedi di coppe in ceramica grigia con singole alpha graffite la dopo cottura (CIE 20002, 20017). In entrambi i casi il vaso presenta graffiti sia nel fondo interno che in quello esterno (reiterazione della lettera in CIE 20002, associazione con asterisco in CIE 20017); i due frammenti appartengono a periodi diversi, essendo il primo attribuibile tra gli ultimi decenni del VI e la prima metà del V sec. a.C., mentre il secondo all'età ellenistica. Tra le lettere vanno inoltre contati due casi di $c h i$, uno sicuramente graffito dopo la cottura nel fondo interno di un frammento di piede e fondo di coppa in ceramica grigia (CIE 20015), mentre l'altro (purtroppo perduto), forse graffito dopo la cottura nel fondo (non si sa se interno o esterno) di una forma aperta a v.n. con circoli incisi nella vasca. In entrambi i casi i documenti sono verosimilmente databili al periodo ellenistico. Si contano poi 9 graffiti non alfabetici tutti redatti dopo la cottura in ceramica grigia o a v.n. (tra questi, tre asterischi: CIE 20017 già menzionato, 20018 e 20016; quattro segni a croce: CIE 20007, 20009, 20011, 20014).

43 Bonomi, Robino 2007.

44 Ibid., p. 44. 
tere ${ }^{45}$ e diversi graffiti non alfabeti ${ }^{46}$. Forse questi documenti, che si attestano in prevalenza entro il III sec. a.C. (solo alcuni scendono fino al II), provengono proprio dai fossati. Mentre le ceramiche registrate come C.e. (cioè quelle a v.n.) nel Catalogo del Museo Civico sono associate a date (più o meno puntuali) di rinvenimento, in quelle registrate come F.i. (cioè CIE 20040-20041, 20043-20044, $20051,20079,20084^{47}$ ), prevalentemente in ceramica grigia e piuttosto coerentemente collocabili tra la seconda metà del IV e il III sec. a.C. (quando databili più puntualmente), si ha invece solo un generico riferimento al novembre 1878. La coerenza tra cronologia e periodo di rinvenimento induce a ipotizzare che tutte queste iscrizioni possano appartenere a un medesimo contesto, nella fattispecie i due fossati ricchi di materiale. Colpisce che queste, quando sono riconoscibili degli antroponimi, documentino la formula all'assolutivo priva di pronome personale (CIE 20030, 20043?, 20051, 20084), per la quale è stata ipotizzata una speciale pratica che in ambito funerario si potrebbe interpretare come dono al defunto (cfr. IV.3.3); a queste si associano lessemi che potrebbero evocare una sfera rituale (cfr. V.4, e in particolare sesiu, CIE 20028; forse mi leị [ , CIE 20079). In definitiva, ci si può domandare se questi documenti epigrafici, tutti databili entro il III sec. a.C., originariamente possano essere stati deposti in aree sacre come doni o con altre funzioni rituali e poi confluiti nel riempimento dei fossati.

L' iscrizione CIE 20037, una coppa etrusco-padana in ceramica depurata databile su base paleografica al IV sec. a.C., dovrebbe essere stata rinvenuta a una profondità maggiore nella porzione orientale dell'area, sulla quale il Bocchi si concentrò dopo aver raggiunto $2 \mathrm{~m}$ di profondità, ormai prossima alle strutture lignee più antiche. Questa iscrizione sembra coerente con una sequenza archeologica lineare e potrebbe dunque essere la testimonianza di una fase di IV sec. a.C. che ad Adria si fatica sempre a individuare.

Approfondendosi nella porzione occidentale (dal 20 marzo 1879), a partire da $2 \mathrm{~m}$ di profondità e fino a circa 2,4-2,6 $\mathrm{m}$ il Bocchi rinvenne una grande quantità di materiale fra cui molte iscrizioni. Furono recuperate tra il 20 e il 28 marzo le iscrizioni CIE 20100, 20110-20111, 20116, 20120-20121, 20124, tutte coppe in ceramica grigia di periodo ellenistico (forse anche la perduta CIE 20111), e CIE 20107 che è realizzata invece su un frammento di vaso volterrano a v.n. Non è chiaro purtroppo il contesto di rinvenimento e quali di queste siano state recuperate nell'ampliamento di nord-ovest, per il quale Bocchi descrive una stratigrafia complessa dove strati ricchi di materiale e carbone si alternavano a strati di terreno alluvionale (un canale?) che seguì fino alla profondità di 2,6 m; rimane tuttavia significativa, anche in questo caso, la sostanziale coerenza cronologica e tipologica dei supporti, con una netta predilezione per le coppe in ceramica grigia. Approfondendosi ulteriormente (dal 29 marzo), Bocchi rinvenne le iscrizioni CIE 20125-20127, 20133, 20135, 20137, 20139. Si tratta di un nucleo interessante, caratterizzato dalla presenza di coppe in ceramica grigia e in ceramica depurata databili sommariamente tra $\mathrm{V}$ sec. a.C. e periodo ellenistico (gli esemplari in ceramica grigia databili con maggior accuratezza non scendono oltre il IV-III sec. a.C.); unica eccezione potrebbe essere il perduto CIE 20137, il cui schema delle lettere in legatura richiama altri documenti di periodo tardo-arcaico (cfr. III.4). Il documento recuperato qui più in profondità è l'iscrizione lacunosa CIE 20139 (di lettura disperata), attribuibile verosimilmente al periodo ellenistico. Nel complesso, nella porzione occidentale del settore, si osserva una sostanziale coerenza nella scelta del supporto per realizzare le iscrizioni, prevalentemente coppe in ceramica grigia, altrimenti raramente selezionate. Queste si trovano nei con-

\footnotetext{
45 Sade (CIE 20036), ypsilon (CIE 20022) e chi (CIE 20024): il primo su ceramica grigia e gli altri su vasi a v.n. di produzione locale.

${ }^{46}$ Due stelle a cinque punte (CIE 20023 e 20026), entrambe su frammenti a v.n. di IV-III sec. a.C., un ramo secco (CIE 20025) su v.n. di produzione locale databile al II sec. a.C., tre segni a croce (CIE 20027, 20034, 20035) su supporti vari, di cui eccezionalmente uno inciso a crudo su ceramica grezza databile tra la seconda metà del VI e il V sec. a.C. (CIE 20035), e infine due graffiti non determinabili (CIE 20031, 20033) su frammenti a v.n. e in ceramica grigia compresi tra III e II sec. a.C.

47 Si sottolinea che CIE 20040 è perduta: l'aspetto paleografico desumibile dall'apografo del Bocchi suggerirebbe una datazione più antica, ma su supposizioni di questo tipo occorre comunque cautela.
} 
testi più superficiali e nei riempimenti dei canali, ma anche a una maggior profondità in associazione con coppe in ceramica depurata. È molto rara invece la presenza di ceramica a v.n.

A partire dal 10 marzo 1879 Bocchi avviò l'indagine delle strutture lignee già rinvenute a 4,4 m di profondità nel dicembre 1878, partendo dalla porzione orientale dello scavo. Riguardo l'attività di dicembre, quando mise in luce il tavolato di quercia con grande quantità di materiale, lo scavatore non registra documenti epigrafici. Diversamente, nello scavo di marzo, a 4,5 $\mathrm{m}$ di profondità, proprio tra le travi di legno (il 12 marzo per esattezza) recuperò il digramma in legatura $\widehat{a v}$ (CIE 20089), redatto su un frammento di coppa di bucchero della fine del VI sec. a.C., assieme a un altro frammento di bucchero e due di ceramica grezza con lettere singole (CIE 20088, 20090-20091); alla profondità di 5 $\mathrm{m}$, verosimilmente sotto le strutture lignee, il frammento di una coppa troncoconica in bucchero della seconda metà del VI sec. a.C. con un sade a farfalla inciso a crudo (CIE 20092), probabile marchio di fabbrica (cfr. III.2). Dopo aver smontato la struttura, alla profondità di 5,5 m rinvenne un graffito complesso su bucchero non più conservato (CIE 20093) e scendendo oltre una stella a cinque punte graffita sul fondo esterno di una coppa sempre di bucchero (CIE 20094). Non è infine chiara la profondità di rinvenimento dell'epsilon arcaicizzante ancora una volta redatto su bucchero (CIE 20095, non più conservato), indicato come trovato «in mezzo terreno carbonoso e legnoso», e forse appartenente alla stratigrafia sottostante le strutture lignee ${ }^{48}$. Con ciò si conferma che la rigida distinzione operata da L. Donati e A. Parrini tra due fasi cronologiche e strutturali Adria I e Adria II, benché sia un importante stimolo per la riflessione sul tema, non è appropriata; infatti non si vede uno scarto cronologico netto fra i documenti, tutti trovati in mezzo e sotto ai travi, che possa giustificare la distinzione delle fasi.

Ai primi di aprile 1879 si data l'intervento nella porzione meridionale dell'area occidentale, dove a partire dai $3,75 \mathrm{~m}$ di profondità il Bocchi rinvenne le travi trasversali in continuazione della porzione orientale già indagata. Presso "la palafitta", dunque lungo il limite settentrionale di quest'area, a 3,5 m circa recuperò alcuni frammenti ceramici con graffiti alfabetici (CIE 20168, in ceramica depurata) e non alfabetici (CIE 20166-20167, in ceramica grigia; CIE 20165, in ceramica grezza). Dopo Pasqua, scendendo a $4 \mathrm{~m}$ di profondità sempre in questa porzione, mise in luce parte di un edificio ligneo di cui si conservavano anche le pareti verticali. Subito dopo la festività e ormai in aprile avanzato, è registrato il rinvenimento di CIE 20143, il digramma ar su kylix attica tipo C a v.n. databile al 500 a.C. circa, assieme a un graffito complesso su coppacoperchio in ceramica grezza (CIE 20148), un doppio chi redatto su una coppa di bucchero (CIE 20147) e un segno a croce su una coppa emisferica su alto piede in ceramica depurata (CIE 20149, databile tra la fine del VI e la prima metà del V sec. a.C. $)^{49}$. Negli stessi giorni, sicuramente a $4 \mathrm{~m}$ di profondità, è documentato il rinvenimento dei frammenti iscritti CIE 20150-20160. Tra questi, si contano due iscrizioni su coppe in ceramica depurata, cioè amnz (CIE 20159) collocabile entro la prima metà del V sec. a.C., e al (CIE 20160), a cui si aggiungono frammenti con lettere singole e graffiti non alfabetici ${ }^{50}$, di cui diversi incisi a crudo fra cui si contano in particolare due casi piuttosto significativi di singole lettere su fondo di coppe in bucchero (CIE 20157-20158, sade a farfalla e ypsilon). Subito sotto, a $4,15 \mathrm{~m} \mathrm{di}$

48 Infatti a questo frammento, indicato con l'inventario F.i. 256 nel Catalogo del Museo Civico, seguono quelli rinvenuti il 20 marzo nell'area occidentale dello scavo. A questo nucleo di materiale, va ascritto forse anche un $c h i$, ora non più reperibile e già discusso in BALDASSARRA 2013, pp. 155-156, Adria TM $p 5$.

49 A questi va forse aggiunto anche il segno a croce inciso a crudo sulla parete di un'olla in ceramica grezza (CIE 20164).

50 Tra le lettere, si conta un significativo numero di alpha, uno ancora arcaicizzante su una coppa in ceramica depurata databile tra la fine del VI e la metà del V sec. a.C. (CIE 20155) e altri di forma più recente su ceramiche di varie classi (CIE 20153, 20156, 20157, 20158); tra i graffiti non alfabetici, un segno a croce su una parete di forma chiusa in ceramica depurata (CIE 20154) e un graffito non alfabetico sul fondo di un'olla in ceramica grezza (CIE 20152) entrambi incisi a crudo, e inoltre un segno a croce e un ramo secco graffiti dopo la cottura in coppe in ceramica depurata (CIE 2015020151). Sono invece più generiche le informazioni sul rinvenimento del frammento di ceramica depurata con $y p$ silon e segno a croce (CIE 20162) e dell'orlo di coppa in ceramica grigia con alpha e ramo secco (CIE 20162); il Bocchi infatti scrive di averli trovati tra 3 e $4 \mathrm{~m}$ di profondità «in vari strati ma sempre preromani». 
profondità, Bocchi rinvenne il frammento di coppacoperchio con segno a croce e aste incisi a crudo (CIE 20161). Tale concentrazione di graffiti redatti prima della cottura si ritrova nella parte orientale dello scavo, oltre i 4,5 m di profondità, come già descritto sopra. Ciò denota una sostanziale coerenza di fondo nella scelta del supporto, la coppa in bucchero, e l'uso in un numero significativo di casi dell'incisione prima della cottura, che suggerisce l'utilizzo della scrittura, e nella fattispecie di lettere, nella fase di produzione dei vasi. Tale preferenza risulta ancora più marcata se si considera la sostanziale rarità del bucchero nella fase tardo-arcaica di Adria (cfr. II.1.4) e la scarsa incidenza dell'incisione a crudo, come dimostrato anche dall'analisi dei segni a croce ${ }^{51}$.

Oltre i $4 \mathrm{~m}$ di profondità in questa porzione di scavo (maggio 1879) non sono registrate iscrizioni, ma solo frammenti di coppe in ceramica depurata, grigia e in bucchero con graffiti non alfabetici databili al periodo tardo-arcaico (CIE 20169-20172), e più sotto ancora un frammento di coppa in bucchero della seconda metà del VI sec. a.C. con sade a farfalla graffito in questo caso dopo la cottura (CIE 20173). Infine un frammento di olla in ceramica grezza con asterisco (CIE 20174) fu rinvenuto «presso il fascinaio, tra questo e la seconda travata orizzontale», quindi con buona verosimiglianza presso le strutture di bonifica e fondazione dell'edificio. Sono riferibili alla fase tardo-arcaica dello scavo anche i quattro frammenti rinvenuti nel 1880 «sulle palafitte o lì presso»: CIE 20176-20179, tra cui si contano graffiti non alfabetici e un alpha collocabile nella prima metà del V sec. a.C. (CIE 20176), tutti su ceramica depurata.

A conclusione del settore NO, è necessario ricordare i venti frammenti di vasi iscritti appartenenti al Museo Domestico dei Bocchi (CIE 20347-20367), recuperati nel 1882 (solo CIE 20347 nel 1887) dalle "palafitte" (così l'indicazione nel Catalogo), verosimilmente identificabili con le strutture lignee scavate tra il 1878 e il 1879 ed evidentemente rimaste a vista per lungo tempo ${ }^{52}$.

Si datano al periodo arcaico 5 frammenti di ceramica grezza (tre olle e due coppecoperchio), dei quali uno solo graffito dopo la cottura (CIE 20359); 12 frammenti di coppe ( 5 in bucchero, 5 in ceramica depurata, 2 in ceramica grigia), in tutti i casi graffite dopo la cottura. Alcuni di questi si annoverano fra i documenti iscritti più antichi di tutta la città: si tratta dei singoli chi redatti nelle pareti esterne di due coppe, una carenata in bucchero della seconda metà del VI sec. a.C. (CIE 20350) e una in ceramica grigia con due solchi nell'orlo della metà del VI sec. a.C. (CIE 20352).

Due frammenti di olle conservano due iscrizioni tardo-arcaiche, entrambe incise a crudo, delle quali la prima è mav su un fondo esterno (CIE 20353), e la seconda il prenome laris (CIE 20364) sulla superficie esterna di un orlo. Il terzo frammento mostra un segno a croce legato a un digamma (o meno probabilmente zeta?) (CIE 20354), anch'esso inciso a crudo. In entrambi i frammenti di coppacoperchio, si ha un unico segno, un chi inciso prima della cottura (CIE 20351) e un segno a croce dopo la cottura (CIE 20359), sempre sul fondo esterno.

Fra le coppe si documenta solo in un caso un digramma, an (CIE 20356), graffito dopo la cottura sul fondo esterno in ceramica depurata. L'aspetto delle lettere situa questo documento entro gli inizi del V sec. a.C..$^{53}$. All'interno della significativa concentrazione di frammenti di coppe in bucchero, è sicuramente da segnalare il caso con foro pervio al centro del fondo e una serie di aste disposte a raggiera nella superficie esterna (CIE 20362), probabilmente rientrante nella categoria dei "segni visivamente eloquenti” di G. Bagnasco Gianni, richiamata da V. Bellelli per simili occorrenze ceretane valorizzando il rapporto tra il graffito e il foro chiaramente legato a usi rituali ${ }^{54}$. A tale riguardo,

51 Gaucci 2020.

52 Cfr. Gaucci 2012a, p. 160, nota 3.

53 Si ricorda che un simile digramma è presente su una coppa in ceramica grigia di periodo ellenistico dalla località Bettola (CIE 20259).

${ }^{54}$ Bellelli 2014, pp. 48-49. Per V. Bellelli (ibid., p. 51), in riferimento a un simile caso ceretano, «se, come è evidente, la resa grafica ha lo scopo di articolare lo spazio concavo del bacino della scodella in settori convergenti in un foro funzionale a far fuoriuscire una offerta alimentare indirizzata a divinità ctonie, la funzione rituale dell'oggetto non 
è opportuno richiamare simili documenti di medesimo orizzonte cronologico: un frammento di coppa in ceramica depurata con aste disposte a raggiera attorno al foro nel fondo esterno e stella a cinque punte nel fondo interno (il foro è al centro del graffito), anch'esso rinvenuto nel settore NO del Pubblico Giardino (CIE 20134, marzo del 1879); una coppacoperchio da San Cassiano (CIE 21034), che presenta un foro pervio e una serie di aste disposte a raggiera e unite fra loro da una linea curva, tutte graffite dopo la cottura, nel fondo esterno, mentre nel fondo interno sempre vicino al foro è tracciato un segno non alfabetico di difficile interpretazione. Indipendentemente dalla natura dei contesti di rinvenimento ${ }^{55}$, le occorrenze elencate testimoniano con tutta evidenza attività rituali.

Fra i restanti documenti in ceramica depurata e grigia, è di qualche interesse il graffito non alfabetico interpretabile come àncora, graffito sul fondo esterno di una coppa in ceramica grigia di periodo tardo-arcaico (CIE 20357), che richiama un trademark documentato nella ceramica greca $^{56}$. A tale riguardo, si ricorda che recentemente E. Benelli, in merito a un contributo sul contesto della Vigna Marini di Cerveteri, ha posto l'attenzione su graffiti redatti su vasi di produzione etrusca che richiamano i trademarks sul vasellame greco catalogati da A.W. Johnston ${ }^{57}$. Ciò suggerisce che gli Etruschi non si limitarono ad adottare tali pratiche commerciali per le importazioni, come già riconosciuto da A. Maggiani per il mercato di Vulci ${ }^{58}$.

Solo tre sono i documenti riferibili al periodo ellenistico: l'iscrizione ataśni (CIE 20364) ${ }^{59}$, l'unica proveniente da abitato del ristretto numero di iscrizioni con formula di possesso al genitivo con pronome di III sec. a.C. (cfr. IV.3.2, classe IV.b), e due graffiti non alfabetici (CIE 20360 e 20366) da riferire al periodo ellenistico, tutti i restanti documenti sono di età arcaica. Questi documenti sembrano suggerire che il materiale recuperato dal Bocchi nel 1882 fosse sì a livello delle palafitte, ma probabilmente in un terreno in qualche maniera contaminato (profonde buche? smottamenti dei livelli più superficiali delle pareti dello scavo?).

Settore SO. Nella documentazione di scavo redatta dagli assistenti A. Nicolussi e G. Longo, conservata nell'archivio del Museo Archeologico, non vi è notizia del rinvenimento delle iscrizioni che da questo scavo provengono (CIE 20180-20184), a eccezione di una, definita «un coccio con inciso una riga di lettere paleovenete », registrata il 20 agosto 1936 nello strato III della trincea I, tra 1,4 e 1,65 m di profondità rispetto al piano di campagna. Seppure manchino informazioni al riguardo, tale frammento potrebbe essere l'iscrizione di fine IV-inizi III sec. a.C. CIE 20181 o quella di III sec. a.C. CIE 20182, attribuite dall'etichetta che le accompagna genericamente agli scavi del 1936-1939 (medesima etichet-

può che essere connessa a questa funzionalità pratica. Fra gli usi possibili, si può immaginare allora quello di una qualche forma di lecanomanzia legata alla osservazione della distribuzione spaziale dei residui di sacrifici incruenti, all'interno della superficie concava del recipiente, che veniva gradualmente a svuotarsi durante il rito sacrificale attraverso il foro centrale».

55 Più volte è stata ribadita la natura artigianale-abitativa per l'area scavata dal Bocchi nella sede dell'attuale Museo, mentre una destinazione cultuale è stata attribuita a una specifica area del complesso residenziale di San Cassiano indagato da M. Harari (da ultimo PALtineri, Robino 2016 in particolare pp. 281-282).

56 Johnston 1979, pp. 192-193, 36A.

57 Benelli, Colivicchi, ZacCagnino 2017, p. 44 e per il graffito a forma di àncora, in particolare pp. 44 e 46, n. 1, fig. 3 .

58 Si rimanda alla bibliografia e alle considerazioni di IV.1.

59 Si ricorda che questa iscrizione è stata catalogata da chi scrive in precedenti lavori (GAUCCI 2010, p. 40, n. 6; ID. 2012a, p. 145, n. 6, fig. 2) sulla base della documentazione fornita da F.A. Bocchi, sebbene non fosse a suo tempo stato riconosciuto il documento. L'interpretazione sulla base del contesto di provenienza (le "palafitte", cioè il livello più profondo che si suppone il Bocchi abbia raggiunto nello scavo del Pubblico Giardino) e la descrizione del supporto, avevano portato a ritenere questa iscrizione attribuibile al periodo tardo-arcaico. Un'ultima ricognizione finalizzata alla redazione del fascicolo adriese del CIE ha fortunosamente portato al riconoscimento del pezzo, che è da attribuire più correttamente al periodo ellenistico. 
ta generica è presente sul frammento CIE 20180). È da riferire sempre all'indagine del 1936 anche l'iscrizione di III sec. a.C. CIE 20183, rinvenuta, come indicato nell'etichetta, nello strato V a 2,2 m di profondità. Nel 1938 durante lo scavo delle strutture lignee, fu rinvenuta l'iscrizione su ceramica attica CIE 20184, secondo quanto segnato sempre nell'etichetta del reperto. Al 25 settembre 1939 a $4 \mathrm{~m}$ di profondità durante il lavoro di messa in luce delle strutture sono riferite le singole lettere CIE 20185-20187, come indicato nella scatola che conteneva questi frammenti iscritti ${ }^{60}$.

Le informazioni sul contesto delle iscrizioni sono nel complesso molto poche e non sono significative, dato il sostanziale silenzio relativo a queste nella documentazione di scavo. I testi presentano tutti elementi onomastici, dalla tardo-arcaica CIE 20180, alle restanti di periodo ellenistico. Degna di nota per supporto e contesto, [- ? - ] $\operatorname{sinin} \theta[---]$ (CIE 20184), con nome lacunoso (cfr. IV.2), fu rinvenuta in corrispondenza delle strutture lignee e appartiene significativamente a un orizzonte di V-IV sec. a.C.

\section{VI.1.2. Il cortile di Antonio Ornati}

Da questo importante scavo, per il quale purtroppo si posseggono solo poche informazioni, provengono 4 iscrizioni (fra queste, due di periodo tardo-arcaico e una ellenistica), il calice in bucchero con lettere in legatura e una raffigurazione realizzata a graffito (CIE 20215), a cui si aggiungono 10 lettere isolate (di cui una dubitativamente greca, CIE 20213) su altrettanti supporti, 11 graffiti non alfabetici su 9 supporti, due graffiti andati perduti (CIE 20222 e metà di una coppa in ceramica depurata con due tratti lacunosi convergenti graffiti dopo cottura nel fondo esterno, vista nel 2009 e ora dispersa, già n. inv. F.i. 157 del Catalogo del Museo Civico).

Le attestazioni attribuite da Bocchi alla categoria C.e. del Catalogo del Museo Civico (dunque le ceramiche con superficie verniciata di nero) sono dette provenienti dallo "strato etrusco" (CIE 20212) e dallo "strato preromano" (CIE 20213); i frammenti si inquadrano tra gli ultimi decenni del VI e gli inizi del V sec. a.C. Nella categoria F.i. (ceramiche senza vernice), si contano cinque documenti (CIE 20214-20218) detti rinvenuti nello "strato preromano" e inquadrabili nel periodo tardo-arcaico, mentre i restanti (CIE 20219-20230) sono provenienti dalla "stazione preromana", termine con il quale allora si indicava l'insediamento antico e che può riconoscersi nel tavolato ligneo sopra descritto; sono anch'esse riferibili al periodo tardo-arcaico (CIE 20221-20224, 20228 non reperibili). Dunque, dallo "strato etrusco/preromano" (definizioni di fatto identiche per il Bocchi), sono stati recuperati coerentemente frammenti databili tra gli ultimi decenni del VI e i primi decenni del V sec. a.C. Tre dei documenti più significativi, cioè il piatto con l'iscrizione mi al graffita dopo la cottura sul fondo esterno (CIE 20212), la coppa con iscrizione mi venelus kar sempre sul fondo esterno (CIE 20214), il calice con la scena complessa graffita nella parete esterna e le lettere in legatura (CIE 20215), furono recuperati tutti integri o quasi, facendo sospettare che il contesto intercettato dallo scavatore fosse coerente e i vasi deposti volontariamente forse come parte di una o più azioni rituali. Anche il contenuto epigrafico orienta verso questa ipotesi. Ciò si evince dalla dedica di dono ( $m i$ al) e dalla formula parlante del kar(a) di venel (CIE 20214), dove in kar (a) si potrebbe vedere la denominazione del vaso medesimo. Ma anche il calice in bucchero è veicolo di un messaggio pregnante che ben si accorda a un contesto sacro. Questo rappresenta l'unico vaso in bucchero graffito dell'intero contesto, che altrimenti documenta (per quanto registrato dal Bocchi) l'uso di ceramica depurata e ceramica grezza. Oltre al monogramma di difficile scioglimento sul fondo esterno, nella parete esterna si sviluppa una complessa raffigurazione graffita sempre dopo la cottura: verosimilmente una nave in viaggio verso destra, fortemente abbozzata ma la cui identità dovrebbe

\footnotetext{
${ }^{60}$ Mentre le lettere singole CIE 20185-20186 appartengono all'età arcaica e quindi potrebbero essere pertinenti alle strutture lignee più antiche, CIE 20187, per la cronologia di fine IV-inizi III sec. a.C. del supporto, difficilmente può essere pertinente al contesto messo in luce a tale profondità e quindi è verosimile ritenere quanto meno problematica la sua localizzazione.
} 
essere suggerita dalla disposizione del timone, di due remi, dell'albero e forse dei tiranti che danno la forma triangolare all'immagine (le altre linee interne a destra del presunto albero potrebbero essere una goffa resa della vela quadrangolare? sicuramente nessun uomo è ritratto a bordo), e un mostro marino diretto verso sinistra (dal corpo anguiforme e pinna caudale verticale ${ }^{61}$ ) sono disegnati in posizioni diametralmente opposte rispetto all'asse del vaso, la prima sopra e il secondo sotto una linea di incerto andamento graffita orizzontale che percorre tutta la circonferenza della parete e che rappresenta il mare; nave e mostro si vengono così incontro, evocando il pericolo (ben sottolineato dalle fauci spalancate della creatura) imminente durante il viaggio marittimo, immagine che dall' $\mathrm{O}$ rientalizzante in poi venne usato nel mondo etrusco anche come metafora di quello oltremondano del defunto ${ }^{62}$. La raffigurazione si può apprezzare ribaltando il calice. Proprio questa posizione permette di vedere anche il monogramma nel fondo esterno. Non è chiaro se la scena rappresentata possa essere una metafora oppure davvero alludere ai pericoli della navigazione, ma in ogni caso sembra legittimo chiedersi se il vaso capovolto non rappresenti l'offerta compiuta a seguito di un evento dal buon esito e che dunque il monogramma possa veicolare il messaggio, occultato dall'intreccio delle lettere, relativo all'atto compiuto una volta lasciato il vaso capovolto nel luogo sacro ${ }^{63}$.

Se ha qualche peso l'ipotesi di una natura sacra del contesto, allora pare interessante porre l'attenzione da un lato sull'assenza di vasi iscritti che esplicitino nomi divini, dall'altro sull'associazione tra una formula nella quale compare solo un prenome sul fondo esterno di una coppa e il calice in bucchero dalla probabile funzione rituale concepito per essere deposto rovesciato. Questi indizi, sebbene labili, richiamano alcune caratteristiche del deposito $\kappa$ del Santuario Meridionale di Pyrgi, e in particolare il gruppo di offerte meridionali dove sono documentati vasi rovesciati e testi costituiti dai soli nomi dei dedicanti, che marcano secondo D.F. Maras un atto rituale di carattere gentilizio ${ }^{64}$. Questo materiale, sebbene possa essere stato recuperato attingendo a oggetti già precedentemente votati nel medesimo santuario pyrgense, è stato interpretato dagli scavatori come parte di un atto propiziatorio legato all'ampliamento dell'area sacra, cioè un rito di fondazione per l'impianto dell'altare monumentale $\lambda$, e strettamente legato sulla base di altre iscrizioni alla coppia ctonia Suri-Cavata, nonché alla sfera dionisiaca della divinità maschile $(\text { fuflunusra })^{65}$. In definitiva, si può ribadire che i nostri vasi potrebbero appartenere ad azioni votive e rituali, di cui purtroppo non sarà possibile ricostruire con precisione il contesto archeologico, sebbene qualche maggior dettaglio potrebbe venire da uno studio complessivo di tutto il materiale catalogato da Bocchi.

Diversamente, non è purtroppo attualmente perspicua la natura di questo contesto in periodo ellenistico. In effetti, la maggior parte dei documenti rinvenuti in questo scavo appartiene a questa fase cronologica, ma il Bocchi nel Catalogo non fornisce alcun dettaglio sul contesto (CIE 20233-20237). Tra questi si ricorda in particolare l'iscrizione kepriu di IV-III sec. a.C. su coppa in ceramica grigia (CIE 20236) e verosimilmente anche una iscrizione lacunosa (rimane solo l'epsilon finale leggibile) e perduta (CIE 20237) sempre su ceramica grigia.

${ }^{61}$ Seppure molto schematico e di realizzazione poco attenta, evoca il parallelo in particolare con le creature che popolano il mare della Stele Ducati n. 10 dai Giardini Margherita di Bologna, in particolare per le fauci molto allungate (SASSATElli 2009, p. 838, fig. 5), che nel nostro sono spalancate.

62 Basti citare Cerchiai 2002; Pizzirani 2005, p. 256. Diversa posizione è espressa in Martelli 2018, passim.

${ }^{63}$ Quest'ultimo aspetto richiama in qualche maniera quanto osservato da A. Maggiani in merito a uno specchio con una sorta di anagramma dell'iscrizione di proprietà: secondo lo studioso, il redattore dell'iscrizione intendeva così dichiarare occultamente la proprietà, salvaguardando l'oggetto da un potenziale aggressore che attraverso la lettura del nome avrebbe potuto far proprio non solo lo specchio, ma forse magicamente la stessa personalità del legittimo proprietario (MAGGIANI 2002b, pp. 7-8). Sulla possibilità di espedienti per camuffamenti grafici di nomi di divinità infere, si veda MARAs 2009b, p. 477 (dove si cita l'iscrizione CIE 20817, per la quale Maras segue l'ipotesi di G. Colonna, cioè che sia il nome di $\operatorname{Van} \theta \operatorname{con}$ metatesi delle prime due lettere; su questa, cfr. IV.3.2.A).

${ }^{64}$ Maras 2013b, pp. 199-200.

65 Carlucci, Maneschi 2013, pp. 47, 56. 


\section{VI.1.3. I fondi Lodo e Calisti nella zona sud-orientale della città}

Nel fondo di Giuseppe Lodo in località Bettola, nel 1878 F.A. Bocchi aprì un saggio di scavo, intercettando a circa 3,2 m di profondità dal piano di campagna un tavolato ligneo ${ }^{66}$. Lo scavo fu interrotto per ragioni logistiche poco sotto il tavolato. Lo scavatore ritrovò nel terreno sopra la struttura, presumibilmente ricco di materiale organico, una notevole quantità di bolli, sigle e iscrizioni graffite ${ }^{67}$. Indipendentemente da quella che poteva essere la reale stratigrafia, i pochi documenti noti (CIE 2025320267) mostrano la mancanza di una coerenza cronologica ${ }^{68}$.

Nove frammenti di vasi (CIE 20268-20276) provengono dal fondo Calisti in località Bettola (non facilmente collocabile topograficamente allo stato attuale della documentazione), scoperti nel $1879 \mathrm{e}$ acquistati da F.A. Bocchi per il Museo Civico nel maggio 1880. Un probabile digramma in legatura ( $\widehat{k v}$ oppure $\widehat{v k}, C I E 20270$ ) e alcuni graffiti alfabetici e non alfabetici (CIE 20271-20272), sono verosimilmente riferibili a una fase di VI-IV sec. a.C., mentre sono quattro i frammenti iscritti in ceramica a v.n., sicuramente di periodo ellenistico (CIE 20273-20276), tra i quali si conta solo una iscrizione lacunosa (uzti [ , CIE 20273). Dal medesimo fondo provengono anche significative iscrizioni e sigle commerciali greche $^{69}$, fra cui forse da conteggiare sono anche i $p h i$ isolati su ceramica attica CIE 20268 e 20269, oltre a una iscrizione sempre greca in anfora ${ }^{70}$.

Tenuto conto delle scarse informazioni relative al fondo Lodo, per quanto riguarda il digramma arcaico al (CIE 20265) è già stato osservato in altra sede la possibile pertinenza a un contesto di natura votiva. Riguardo a questo documento, vale la pena richiamare la recente analisi di V. Bellelli sui vasi in ceramica grezza (olle e coppecoperchio) come supporto di documenti epigrafici in contesti sacri, che ha suggerito allo studioso l'evidenza di una pratica legata a culti ctonii, e in particolare per le coppecoperchio al culto di divinità patriarcali maschili corrispondenti a un Ade dalla forte connotazione dionisiaca ${ }^{71}$. Sempre dal contesto in esame, provengono due iscrizioni greche con antroponimi lacunosi datate al V sec. a.C., di cui almeno una interpretata da D. Baldassarra come votiva ${ }^{72}$. Passando al periodo ellenistico, nell'analisi epigrafica, l'iscrizione ellenistica CIE 20264 è una delle rare occorrenze

${ }^{66}$ Bocchi 1879, pp. 94-97.

67 Ibid., p. 95.

68 CIE 20253-20255 furono trovati dal Bocchi nel settembre e riferiti allo "strato romano"; il chi graffito su ceramica a v.n. CIE 20254 non è reperibile, mentre l' iscrizione anta ș× [-- - CIE 20253 e il graffito non alfabetico CIE 20255 sono entrambi su forme aperte locali a v.n. della fine del IV-metà del III sec. a.C., forse parte di uno strato di rimescolamento superiore. Cinque iscrizioni (CIE 20256-20260), delle quali due non reperibili (CIE 20258 e 20260), e vari graffiti, dei quali solo tre reperibili (CIE 20261-20263), provengono dal c.d. "strato etrusco" scavato da Bocchi sempre in settembre. Mentre il digramma ra graffito sul fondo esterno di una ciotola attica a v.n. del tipo incurving rim (CIE 20256) è tra le rare iscrizioni riferibili al pieno IV sec. a.C., le brevi iscrizioni pka[-- ] (CIE 20257), an (CIE 20259), due segni a croce CIE 20261-20262 e il digramma in legatura da leggere $\widehat{a k}$ o $\widehat{k a}$, graffito nel fondo esterno e associato a un ramo secco nella vasca interna (CIE 20263: in CIE IV, I, 1 il supporto è classificato come bucchero, ma è preferibile la definizione ceramica grigia data la cronologia al periodo ellenistico), sono tutti realizzati dopo la cottura su coppe o comunque forme aperte in ceramica grigia e databili al periodo ellenistico. Anche le due iscrizioni non reperibili ata [- ? -] (CIE 20258, associata a un segno a croce) e [---]alu (CIE 20260) sono da ritenersi su ceramica grigia. Infine, due iscrizioni (CIE 20264-20265) e molti graffiti, dei quali solo due reperiti (CIE 20266-20267), sono attributi da Bocchi al c.d. "strato preromano", del quale non si hanno ulteriori informazioni; mentre l'iscrizione larza siluniś (CIE 20264) graffita su una coppa in ceramica grigia è riferibile alla fine del IV - prima metà del III sec. a.C., l'iscrizione a crudo al sul fondo esterno di una coppacoperchio in ceramica grezza (CIE 20265) al periodo arcaico; anche i due graffiti non alfabetici CIE 20266-20267 sono incisi prima della cottura su fondo esterno di olle in ceramica grezza.

${ }^{69}$ Baldassarra 2013, p. 126, Adria D*6, p. 127, Adria D p 1, pp. 205-206, Adria TM 72, p. 218, Adria TM 89, inoltre la croce non più reperibile ibid., p. 200, Adria TM $p 17$.

${ }^{70}$ N. inv. Ad 24001, già n. inv. F.i. 455 del Catalogo del Museo Civico.

71 Bellelli 2014, p. 53.

${ }^{72} \mathrm{P} \imath$ [--- ], sul fondo esterno di un'olpe miniaturistica (BALDASSARra 2013, p. 126, Adria D* 6) e ${ }^{1} \mathrm{Ti} \tau^{2} \mathrm{o} v[---]$, perduta, forse su ceramica a v.n. (BALDASSARRA 2013, p. 127, Adria D $p$ 1, che sottolinea la rarità del nome, con una documentazione circoscritta ad Atene). 
della formula all'assolutivo priva di pronome proveniente dall'abitato, possibile indizio di una pratica relativa alla sfera del dono (cfr. IV. 3.2, classe I): in questo caso eventuali 'coefficienti di sacralità' (secondo la definizione data da D.F. Maras ${ }^{73}$ ) potrebbero essere la struttura della formula e il contesto. Infine, non è da escludere che l'iscrizione lacunosa CIE 20257 possa essere letta come abbreviazione di teonimi inferi secondo una pratica di allusione (cfr. V.3), che potrebbe valere anche per il digramma in legatura CIE 20263.

Nel fondo Calisti, fra i frammenti di periodo più antico, si registra in particolare il possibile digramma in legatura $\widehat{k y}$ oppure $\widehat{v k}$ (CIE 20270) e anche un alpha sul fondo di olla (CIE 20272), entrambi incisi a crudo. Le lettere in legatura CIE 20270 potrebbero dubitativamente alludere a un lessico sacro o a forme teonimiche (cfr. III.4). Per il periodo ellenistico, si osserva che tutti i frammenti iscritti sono su ceramica a v.n., diversamente da quanto si registra nel vicino fondo Lodo. In quest'ultimo contesto, spicca l' iscrizione $u z t i[-?$ - ], graffita dopo la cottura nella vasca interna di una forma aperta di importazione databile tra la fine del IV e il III sec. a.C. e associata a un ypilon nel fondo esterno (CIE 20273). Si ricorda che per usti/uzti è stata avanzata la proposta di interpretarlo come lessema piuttosto che come antroponimo (cfr. V.4).

Nel complesso, i documenti dei fondi Lodo e Calisti, comunque di incerta interpretazione, potrebbero a orientare verso l'idea di contesti sacri, forse da mettere a sistema fra loro e con una continuità di vita tra il periodo arcaico e quello ellenistico. Sebbene rimanga dubitativa la possibilità che alcune delle iscrizioni possano alludere effettivamente a divinità infere, non va comunque dimenticato che la zona in questione era marginale dell'abitato e soprattutto in prossimità di un settore funerario.

\section{VI.1.4. I reperti del Museo Domestico della Famiglia Bocchi}

Anche se per molti dei 65 documenti epigrafici (CIE 20282-20346) del Museo Domestico acquisiti anteriormente al 1870 è ipotizzabile una origine dal pluriennale scavo in località Tomba, perlopiù questi non presentano certa provenienza. La loro prevalente frammentarietà induce a escludere che siano stati rinvenuti in contesti funerari. Inoltre, si osserva percentualmente una prevalenza di ceramiche a v.n. e figurate rispetto a quelle di altre classi di materiali per il periodo compreso grossomodo tra il tardo Arcaismo e l'inizio dell'Ellenismo. Si contano infatti 30 frammenti attribuibili a una produzione attica, di cui 14 con iscrizioni e singole lettere etrusche, 7 con lettere di incerta natura (piuttosto greche che etrusche), 9 con graffiti non alfabetici; 5 frammenti in ceramica depurata e uno in bucchero con singole lettere (in un caso un numerale); 3 in ceramica depurata con graffiti non alfabetici. Tale netta sproporzione dipende almeno in parte da una selezione del materiale conservato. Infatti, se si analizza la sezione F.i. (cioè le ceramiche prive di vernice) del Catalogo del Museo Domestico e la medesima sezione del Catalogo del Museo Civico, c’è una netta differenza quantitativa difficilmente riconducibile unicamente alla aleatorietà dei rinvenimenti: nella prima si contano 59 numeri, dei quali solo i primi 16 acquisiti anteriormente al 1874; di contro F.A. Bocchi cataloga 460 frammenti frutto dei suoi scavi.

Tra i frammenti iscritti, si conosce la data di rinvenimento solo del chous a f.n. con iscrizione kai (CIE 20282), recuperato nell'autunno del 1808, forse proprio dagli scavi di Santa Maria della Tomba ${ }^{74}$. Dal medesimo luogo, seppure in anni diversi, provengono anche alcune iscrizioni greche (indicate con la sigla del Catalogo di D. Baldassarra): Adria IP 1, IP 2, D 1, D 2, D 3, I $1^{75}$. Tra queste si segnalano almeno tre sicure iscrizioni di dedica (D 1-3), due di possesso (IP 1-2) e un possibile elenco di vasellame (I 1), che Baldassarra ipotizza relativo a possedimenti di santuario piuttosto che di destinazione commerciale. È stato già sottolineato come si ritenga fortemente probabile che anche gli altri due frammenti con iscrizione kai (CIE 20290 e 20295) possano avere medesima origine, così come il frammento di

\footnotetext{
73 Maras 2009b, p. 451.

74 Gaucci 2012a, p. 158, con riferimenti.

75 Cfr. BAldassarra 2013, pp. 299-300.
} 
piede di kylix con iscrizione su ambedue i lati (CIE 20289). In particolare, quest'ultimo frammento presenta alcune peculiarità degne di nota. Come già sottolineato da G. Colonna, l'iscrizione della parte superiore conserva il lacerto di un possibile gentilizio [---] unas con sigma retrogrado, il quale, indipendentemente dalla sua funzione (genitivo afunzionale?), evoca la pratica scrittoria meridionale. Ben diverso per aspetto delle lettere (forse una diversa mano, come già suggerito da S. Bruni ${ }^{76}$ ), è il lacerto [- - - ] aś [---], che invece richiama la pratica scrittoria settentrionale e l'uso di un grafema, il sade a farfalla, molto circoscritto ai territori più settentrionali compresi tra Spina e il Forcello, ma documentato anche a Vulci. Si tratta pertanto di due diversi testi, tracciati verosimilmente in momenti distinti. Casi simili sono ipotizzati per le iscrizioni votive, dove il nome della divinità è verosimilmente già graffito prima della dedica, parte di una dinamica commerciale interna al santuario medesimo. Per quanto attiene le offerte che coinvolgono il vasellame attico, tale pratica, manifestata da due iscrizioni in diverse parti del vaso, si ritrova in una kylix a figure rosse da Gravisca, datata al secondo quarto del V sec. a.C.: in questa alla consacrazione alla divinità in genitivo (vea) nella parete esterna, si associa il nome del dedicante (paite) con altra grafia nella vasca interna ${ }^{77}$. Va sottolineato che questa risulta l'unico caso di tal genere tra le iscrizioni votive su ceramica attica ${ }^{78}$, supporto privilegiato per i testi etruschi in molti contesti santuariali ${ }^{79}$. Se questo modello di preparazione dell'offerta potesse essere ipoteticamente valido anche per il nostro caso, si avrebbe una consacrazione con il nome della divinità al genitivo I secondo la norma settentrionale (fra le più probabili: Menerva, Tinia, CavaAa, e l'epiteto $A p a$ ) e sopra il piede il nome del dedicante, di cui si conserva il relitto di una sua parte (il gentilizio?) in norma meridionale.

Anche altre brevi iscrizioni potrebbero manifestare la predilezione del vasellame attico per una destinazione sacra. Anzi tutto va richiamato il lessema kai, presente su tre differenti supporti, un chous attico a f.n. datato al 490-480 a.C. (CIE 20282), una vicup attica di tecnica indeterminata datata al 480-450 a.C. (CIE 20290) e un cup-skyphos attico probabilmente figurato datato al 500-470 a.C. (CIE 20295). Molteplici potrebbero essere le interpretazioni di questo lessema, benché sia forse da preferire un nome femminile all'assolutivo, che dunque dichiarerebbe solo il nome del dedicante (cfr. IV.1). Malgrado queste incertezze, risulta acclarato, almeno nel caso del chous, il rinvenimento dalla località Tomba, che ne certifica una possibile destinazione sacra. Sempre a un contesto sacro punta l'interpretazione del digramma $k r$ delle kylikes attiche a f.n. CIE 20287 e 20305, datate attorno al 500 a.C., quale possibile relitto consonantico del termine kara; se tale fosse lo scioglimento, il lessema alluderebbero al vaso, forse con una precisa destinazione sacrale (cfr. III.4). Infine, una notevole frequenza della scelta della ceramica attica come supporto si documenta anche per le lettere singole il cui l'aspetto ne disambigua l'appartenenza all'alfabeto etrusco ${ }^{80}$.

A un contesto legato a pratiche rituali potrebbero essere riferibili anche CIE 20344-20345, che sono coppe forate (paterae pertusae) in ceramica di produzione locale ${ }^{81}$, indizio di forme di ritualità volte al mondo ctonio e tipiche della cultura etrusca, già ricordate per l'area dell'attuale Museo (cfr. VI.1.1).

\footnotetext{
76 BRUNi 2011, p. 126, nota 8.

77 Maras 2009b, pp. 378-379, Ta co.12. Per la realizzazione in momenti diversi delle iscrizioni, si veda anche Maggiani 1997 b, p. 38.

78 Ibid., p. 22, A 3.

79 Colonna 1989-1990, in particolare p. 877.

80 Alpha: CIE 20303, sul fondo esterno di una c.d. saltcellar (500-475 a.C.); CIE 20306, sul piano d'appoggio di un piede di vicup a v.n. (475-450 a.C.). Epsilon (o digamma): CIE 20288, sul fondo esterno di una vicup (470-460 a.C.). Digamma: CIE 20311, nel fondo esterno presso l'attacco del piede di un vaso non meglio determinato (500-450 a.C.). $N y$ (con segno a croce opposto): CIE 20293, nel fondo esterno di un piede di acrocup di tecnica indeterminata (500-475 a.C.). Ypsilon: CIE 20310, nel fondo esterno di uno skyphos di tecnica indeterminata (475 a.C. circa). Chi: CIE 20300, nel fondo esterno di un cup-skyphos a f.n. (fine VI sec. a.C.).

81 In particolare, CIE 20344 documenta un chi nel fondo esterno di un piatto in ceramica depurata non più recente della metà del V sec. a.C.; 20345 una serie di aste disposte a raggiera attorno al foro centrale realizzato nella vasca di una coppa emisferica, non più recente del IV sec. a.C.
} 
Nel complesso, dunque, si può ritenere plausibile che una buona parte dei vasi iscritti compresi tra il tardo Arcaismo e l'inizio dell'Ellenismo provenga da un contesto votivo, forse proprio quello della Tomba.

Diversa la casistica delle testimonianze di periodo ellenistico, tra le quali si documentano almeno tre iscrizioni su vasi a v.n. locali integri databili tra gli ultimi decenni del IV e il III sec. a.C. (CIE 20323: urvi; 20328: ḥi; 20330: kraiu), a cui va aggiunto il caso di una kylix volterrana databile tra la fine del IV sec. e il 275 a.C. con due alpha graffite dopo la cottura, una nel fondo esterno e una nel centro della vasca (CIE 20308). L'assenza di entrambe le anse in quest'ultimo vaso, per il resto sostanzialmente integro, suggerisce la possibilità di un rito di defunzionalizzazione. Proprio lo stato di conservazione di questi vasi, come anche del piatto a v.n. di II sec. a.C. con iscrizione latina Spu (CIE 20335), lascia supporre che almeno una parte di questi possa provenire da contesti verosimilmente funerari.

\section{VI.2. Sepolcreti urbani}

Le più antiche sepolture di Adria preromana testimoniano l'occupazione dei terreni a est della città (presso Ca' Cima) già dal pieno VI sec. a.C. e di quelli a sud (presso Canalbianco) almeno dal periodo tardo-arcaico ${ }^{82}$. Dopo il quasi totale vuoto documentale del V e IV sec. a.C., che attende ancora delle risposte in merito al destino dell' insediamento, tra III e II sec. a.C. le centinaia di tombe, di cui si ha notizia sistematica a partire dagli inizi del XX secolo, sembrano delineare una vasta cintura cimiteriale che doveva estendersi da Ca' Garzoni a sud-ovest fino a Ca' Cima a nord-est, lambendo dunque le propaggini sud-orientali dell'abitato antico ed estendendosi per molte centinaia di metri verso ovest e verso nord ${ }^{83}$. Il vasto paesaggio funerario, che doveva svilupparsi in alti morfologici lambiti da canali, sembra dunque essere problematicamente sproporzionato rispetto al nucleo insediativo di pochi ettari che si conosce.

Sebbene non si distinguano significative soluzioni di continuità nella cintura sepolcrale cittadina, per ragioni di opportunità ai fine della trattazione, questa viene divisa di seguito in due settori, meridionale (VI.2.1, da Ca' Garzoni a ovest fino a Piantamelon a est) e orientale (VI.2.2, dalla Bettola a sud fino a Ca' Cima a nord).

\section{VI.2.1 Il settore meridionale}

Sicuramente l'area funeraria più estesamente indagata è quella nota come Canalbianco, scavata tra il 1938 ed il 1940 per la realizzazione dell'omonimo canale, chiamato all'epoca "Mussolini” e che lambisce ancora oggi a sud l'attuale centro cittadino. Qui sono state portate in luce 396 tombe, delle quale 2 comprese tra il periodo tardo-arcaico e il V sec. a.C. (tombe 333 e 347 ) 84,245 di fine IV-II sec. a.C. (prevalentemente ad inumazione; in alcuni casi queste "tombe" sono piuttosto interpretabili come contesti votivi) e 133 di periodo romano ${ }^{85}$. Pesa allo stato attuale degli studi l'assenza di una analisi sistematica dei corredi e di una pianta generale dell'area, di cui è edita solo una versione muta e di scarsa utilità ${ }^{86}$. Un corso d'acqua, che si immetteva nell'abitato da sud-ovest, dovette pro-

${ }^{82}$ Gaucci 2015, pp. 115-116, con riferimenti.

83 Un quadro di sintesi dei rinvenimenti funerari è offerto in Tamassia 1993, p. 8 e nota 7 con riferimenti. Per le scoperte successive, presso Ca' Cima a nord-est e nella zona di Retratto presso l'Ospedale civile a sud, si veda infra.

${ }^{84}$ Gaucci 2015, pp. 115-166.

85 Fogolari 1940,pp. 431-442.

86 Scarpari 1977, p. 46, fig. 8; cfr. le osservazioni in Gaucci, Pozzi 2009, p. 61, nota 39; Robino 2016, pp. 95-97. La planimetria potrebbe essere in prevalenza ricostruibile sulla base delle misure di triangolazione per ciascuna tomba (quando queste sono state registrate) presenti nei giornali di scavo, come dimostrato in RoBINO 2016, pp. 95-97. I dati di archivio della necropoli riguardano i giornali di scavo dell'ispettore onorario arch. G.B. Scarpari e degli assistenti A. Nicolussi (fino alla tomba 252) e G. Longo (dalla 259 alla 354) (ibid., p. 92); l'elenco tipologico dei materiali elaborato dal Nicolussi (fino alla tomba 214) (ibid., pp. 91-92); le fotografie di scavo per un numero limitato di sepolture (ibid., p. 92). Le note vicende della sede del Museo Archeologico di Adria e i numerosi spostamenti del 
babilmente costituire una delle principali direttrici extra-urbane attorno alla quale si coagularono le sepolture ${ }^{87}$.

In Canalbianco si contano 100 contesti con documenti epigrafici ${ }^{88}$, tutti di periodo ellenistico: 92 sepolture ( 72 quelle i cui vasi con epigrafi sono di certa attribuzione: Tab. 7; mentre 20 quelle dove tale attribuzione è incerta: Tab. 8.A;) e 8 invece interpretabili come di natura votiva (si distinguono per essere introdotti da "n.”: Tab. 8.B). A questo quadro, vanno aggiunti 59 vasi con iscrizioni e/o graffiti per i quali il contesto indicato nell' Inventario Generale è sicuramente errato oppure non è noto (Tab. 8.C-D). Una riflessione sul rapporto tra contesti con testimonianze epigrafiche e la totalità di quelli di periodo ellenistico non potrà pertanto tenere conto di questi ultimi 59 vasi, in quanto è impossibile desumerne il numero effettivo di contesti. Se ci limitiamo ai 100 documentati, rispetto al totale di 245 il rapporto è con buona approssimazione 2:5. Si tratta di un valore non solo significativo, ma anche potenzialmente più alto a causa dei molti documenti privi di un contesto di pertinenza. Se si restringe il campo ai contesti con iscrizione, si conta un totale di 33 tombe e 3 contesti votivi, dunque il $15 \%$ circa del totale ${ }^{89}$. Tra questi, conservano iscrizioni non appartenenti alla tradizione epigrafica e alla lingua etrusca, 3 sepolture (tombe 250, 251,257) e un contesto votivo (n. 296), per le quali si veda oltre. Rimangono fuori da questo quadro le 12 iscrizioni di erronea attribuzione o prive di contesto, che alzerebbero sensibilmente il rapporto.

Tab. 7. Tombe di Canalbianco con documenti epigrafici di certa attribuzione (tutti realizzati dopo la cottura).

\begin{tabular}{|l|c|l|l|l|}
\hline \multicolumn{1}{|c|}{ ConTESTO } & \multicolumn{1}{|c|}{ CIE } & \multicolumn{1}{c|}{ TIPO DI EPIGRAFE } & \multicolumn{1}{c|}{ PosIzIONE } & \multicolumn{1}{c|}{ SUPPORTO } \\
\hline $\begin{array}{l}\text { T. 368, inumazione (forse femminile), } \\
\text { ultimi decenni del IV sec. a.C. }\end{array}$ & 20653 & $p h i$ & fondo esterno & ciotola, v.n. volterrana \\
\hline $\begin{array}{l}\text { T. 309, inumazione, } \\
\text { inizi del III sec. a.C. }\end{array}$ & 20595 & $m i<\times \times>$ helis nalskeś & fondo interno & coppa, ceramica grigia \\
\cline { 2 - 5 } & 20596 & segno a croce & fondo esterno & coppa, ceramica grigia \\
\hline $\begin{array}{l}\text { T. 327, inumazione, } \\
\text { primi decenni del III sec. a.C. }\end{array}$ & 20614 & mi laris fuliu & fondo interno & ciotola, v.n. locale \\
\cline { 2 - 5 } $\begin{array}{l}\text { T. 8, forse inumazione, } \\
\text { prima metà del III sec. a.C. }\end{array}$ & 20615 & segno a croce & fondo interno & ciotola, v.n. locale \\
\hline
\end{tabular}

materiale della necropoli (e delle altre collezioni del Museo) portarono nel tempo a una notevole confusione nella composizione dei singoli corredi. Tale confusione rimase quando si iniziò a redigere l'Inventario Generale negli anni Settanta del XX sec. e pertanto ancora oggi permangono molti problemi nella ricostruzione dei corredi di Canalbianco. Se in particolare l'elenco tipologico di A. Nicolussi e le rare fotografie di scavo permettono una maggior certezza nelle attribuzioni dei vasi ai corredi nelle prime 214 tombe (cfr. ibid., pp. 92-93), per quelle successive rimane quasi sempre l'incertezza dell'attribuzione.

87 Peretto, Vallicelli, Wiel-Marin 2002, pp. 92-93, fig. 5. Su questo caso, si veda adesso Bonomi, Vallicelli, BALISTA 2020, p. 202.

${ }^{88}$ Come già ricordato per Ca' Cima, le cronologie proposte risultano più puntuali per i corredi editi (si rimanda ai tituli del CIE IV, I, 1 per riferimenti bibliografici puntuali) o per quelli che ospitano iscrizioni in quanto studiati nel dettaglio nei lavori di tesi di chi scrive (GAUCCI 2007; ID. 2009).

${ }_{89}$ Tra questi, si conta circa il $40 \%$ di tombe con solo iscrizioni, il $40 \%$ di tombe con iscrizioni e un graffito e il restante $20 \%$ di tombe con iscrizioni e graffiti in numero maggiore di uno. Tale tendenza appare per certi versi simile a quella delle tombe che registrano graffiti ma non iscrizioni, dove il $75 \%$ presenta un solo graffito e il $25 \%$ più di uno. Se si osserva la tendenza in una prospettiva diacronica, le tombe con iscrizioni si distribuiscono diffusamente lungo tutto l'arco cronologico del III e del II sec. a.C., così come le tombe con iscrizioni e un graffito, mentre più discontinua appare la distribuzione delle tombe che associano iscrizioni e più graffiti. Tale fenomeno è evidente anche tra le tombe prive di iscrizioni, dove quelle con più graffiti si incontrano solo tra la seconda metà del III e la prima metà del II sec. a.C. Nel complesso, si potrebbe avanzare l'idea che i due fenomeni, cioè la presenza di iscrizioni e quella di graffiti, non siano necessariamente vincolati fra loro, ma appartengano a logiche differenti che possono intersecarsi. 


\begin{tabular}{|c|c|c|c|c|}
\hline Contesto & $C I E$ & TIPO DI EPIGRAFE & Posizione & SUPPORTO \\
\hline \multirow{3}{*}{$\begin{array}{l}\text { T. } 160 / \mathrm{A} \text {, inumazione in fossa, } \\
\text { prima metà del III sec. a.C. }\end{array}$} & 20523 & {$[---] k a l u$} & parete interna & $\begin{array}{l}\text { ciotola, v.n. di prod. } \\
\text { incerta }\end{array}$ \\
\hline & 20524 & $c h i$ & parete interna & $\begin{array}{l}\text { ciotola, v.n. di prod. } \\
\text { incerta }\end{array}$ \\
\hline & 20525 & asterisco & parete interna & ciotola, v.n. locale \\
\hline \multirow{3}{*}{$\begin{array}{l}\text { T. } 243 \text {, inumazione, } \\
\text { prima metà del III sec. a.C. }\end{array}$} & 20569 & ata śetina & parete interna & ciotola, v.n. locale \\
\hline & 20570 & {$[---] \times a$ śetina } & fondo interno & ciotola, v.n. locale \\
\hline & 20571 & {$[--] n a$} & parete interna & ciotola, v.n. locale \\
\hline \multirow{4}{*}{$\begin{array}{l}\text { T. } 67 \text {, inumazione in fossa, } \\
\text { metà del III sec. a.C. }\end{array}$} & 20477 & laris tetialuśmi & parete esterna & kylix, v.n. locale \\
\hline & 20478 & segno a croce & fondo interno & $\begin{array}{l}\text { piccola ciotola, v.n. } \\
\text { locale }\end{array}$ \\
\hline & 20479 & segno a croce & fondo esterno & ciotola, v.n. locale \\
\hline & 20480 & segno a croce & fondo esterno & piatto, v.n. locale \\
\hline $\begin{array}{l}\text { T. } 85 \text {, inumazione in fossa, } \\
\text { metà del III sec. a.C. }\end{array}$ & 20487 & tutis & fondo interno & piatto, v.n. locale \\
\hline $\begin{array}{l}\text { T. 92, inumazione in fossa, } \\
\text { metà del III sec. a.C. }\end{array}$ & 20488 & segno a croce & fondo esterno & coppa, ceramica grigia \\
\hline \multirow{3}{*}{$\begin{array}{l}\text { T. } 132 \text {, inumazione in fossa, } \\
\text { metà del III sec. a.C. }\end{array}$} & 20511 & mi leiuśs & fondo interno & $\begin{array}{l}\text { kylix, v.n. di prod. } \\
\text { incerta }\end{array}$ \\
\hline & 20512 & $c h i$ & parete esterna & coppa, ceramica grigia \\
\hline & 20513 & aste disposte a raggiera & fondo esterno & $\begin{array}{l}\text { piatto da pesce, } \\
\text { ceramica grigia }\end{array}$ \\
\hline $\begin{array}{l}\text { T. } 294 \text {, inumazione in fossa (forse } \\
\text { bambino), } \\
\text { pieno III sec. a.C. }\end{array}$ & 20589 & $c h i$ & parete interna & $\begin{array}{l}\text { piatto da pesce, v.n. } \\
\text { locale }\end{array}$ \\
\hline \multirow{2}{*}{$\begin{array}{l}\text { T. } 311 \text {, inumazione, } \\
\text { metà-seconda metà del III sec. a.C. }\end{array}$} & 20598 & phi & fondo esterno & ciotola, v.n. locale \\
\hline & 20599 & segno a croce & fondo interno & ciotola, v.n. locale \\
\hline $\begin{array}{l}\text { T. } 66 \text {, inumazione in fossa (forse } \\
\text { femminile?), } \\
\text { seconda metà del III sec. a.C. }\end{array}$ & 20476 & segno a croce & fondo esterno & $\begin{array}{l}\text { ciotola, v.n. forse } \\
\text { riminese }\end{array}$ \\
\hline $\begin{array}{l}\text { T. 201, inumazione in fossa, } \\
\text { seconda metà del III sec. a.C. }\end{array}$ & 20551 & segno a croce & fondo interno & ciotola, v.n. locale \\
\hline $\begin{array}{l}\text { T. } 204 \text {, inumazione in fossa (forse } \\
\text { femminile), } \\
\text { seconda metà del III sec. a.C. }\end{array}$ & 20552 & stella a cinque punte & fondo interno & ciotola, v.n. locale \\
\hline $\begin{array}{l}\text { T. } 157 \text {, inumazione (forse } \\
\text { femminile?), } \\
250-225 \text { a.C. }\end{array}$ & 20520 & $\begin{array}{l}\text { segno a croce }(a) \\
\text { segno a croce }(b)\end{array}$ & $\begin{array}{l}\text { pomello (a), } \\
\text { fondo esterno } \\
\text { (b) }\end{array}$ & $\begin{array}{l}\text { pisside con coperchio, } \\
\text { ceramica alto-adriatica }\end{array}$ \\
\hline $\begin{array}{l}\text { T. } 56 \text {, inumazione in fossa, } \\
\text { seconda metà del III } \\
\text { (-inizi del II) sec. a.C. }\end{array}$ & 20467 & $\begin{array}{l}\text { tre aste }(a) \\
\text { segno a croce }(b)\end{array}$ & $\begin{array}{l}\text { parete interna } \\
\text { (a), segno a } \\
\text { croce }(\mathrm{b})\end{array}$ & coppa, ceramica grigia \\
\hline \multirow{3}{*}{$\begin{array}{l}\text { T. } 164 \text {, inumazione in fossa, } \\
\text { seconda metà del III } \\
\text { (-inizi del II) sec. a.C. }\end{array}$} & 20527 & ypsilon & fondo esterno & ciotola, v.n. locale \\
\hline & 20528 & chi & parete esterna & ciotola, v.n. locale \\
\hline & 20529 & segno a croce & parete esterna & ciotola, v.n. locale \\
\hline \multirow{2}{*}{$\begin{array}{l}\text { T. } 187 \text {, inumazione in fossa, } \\
\text { seconda metà del III } \\
\text { (-inizi del II) sec. a.C. }\end{array}$} & 20543 & ypsilon & fondo interno & ciotola, v.n. locale \\
\hline & 20544 & segno a croce & fondo interno & ciotola, v.n. locale \\
\hline
\end{tabular}




\begin{tabular}{|c|c|c|c|c|}
\hline Contesto & $C I E$ & TIPO DI EPIGRAFE & Posizione & SUPPORTO \\
\hline $\begin{array}{l}\text { T. } 158 \text {, inumazione, } \\
\text { seconda metà del III sec. a.C. }\end{array}$ & 20521 & ypsilon & parete interna & ciotola, v.n. locale \\
\hline \multirow{3}{*}{$\begin{array}{l}\text { T. 236, inumazione infantile, } \\
\text { seconda metà del III sec. a.C. }\end{array}$} & 20563 & atakus 'b atakuś & fondo interno & ciotola, v.n. locale \\
\hline & 20564 & laris ațiu & parete interna & ciotola, v.n. locale \\
\hline & 20565 & $c h i$ & fondo interno & ciotola, v.n. locale \\
\hline $\begin{array}{l}\text { T. 291, inumazione, } \\
\text { seconda metà del III sec. a.C. }\end{array}$ & 20587 & segno a croce & fondo interno & ciotola, v.n. locale \\
\hline $\begin{array}{l}\text { T. } 301 \text {, inumazione, } \\
\text { seconda metà del III sec. a.C. }\end{array}$ & 20594 & hapile, segno a croce & fondo interno & ciotola, v.n. locale \\
\hline \multirow{5}{*}{$\begin{array}{l}\text { T. } 349 \text {, inumazione (di adolescente } \\
\text { secondo gli scavatori), } \\
\text { seconda metà del III } \\
\text { (-inizi del II sec. a.C.) }\end{array}$} & 20629 & alpha & fondo interno & ciotola, v.n. locale \\
\hline & 20630 & alpha & fondo interno & ciotola, v.n. locale \\
\hline & 20631 & segno a croce & fondo interno & $\begin{array}{l}\text { ciotola, v.n. forse } \\
\text { riminese }\end{array}$ \\
\hline & 20632 & segno a croce & fondo interno & $\begin{array}{l}\text { ciotola, v.n. forse } \\
\text { riminese }\end{array}$ \\
\hline & 20633 & graffito non det. & parete esterna & $\begin{array}{l}\text { ciotola, v.n. forse } \\
\text { riminese }\end{array}$ \\
\hline $\begin{array}{l}\text { T. 131, cremazione, } \\
\text { fine del III sec. a.C. }\end{array}$ & 20510 & $X X X$ & spalla & anfora greco-italica \\
\hline $\begin{array}{l}\text { T. 177, cremazione, } \\
\text { (fine del IV-) III sec. a.C. }\end{array}$ & 20538 & tre aste & parete interna & ciotola, v.n. locale \\
\hline $\begin{array}{l}\text { T. } 3 \text {, rito incerto, } \\
\text { III sec. a.C. }\end{array}$ & 20425 & graffito non det. & parete esterna & coppa, ceramica grigia \\
\hline \multirow{2}{*}{$\begin{array}{l}\text { T. } 6 \text {, rito incerto, } \\
\text { III sec. a.C. }\end{array}$} & 20426 & $\widehat{a \chi}$ & fondo interno & ciotola, v.n. locale \\
\hline & 20427 & $c h i$ & interno & ciotola, v.n. locale \\
\hline \multirow{2}{*}{$\begin{array}{l}\text { T. } 9 \text {, inumazione in fossa, } \\
\text { III sec. a.C. }\end{array}$} & 20430 & ramo secco & fondo interno & ciotola, v.n. locale \\
\hline & 20431 & ramo secco & fondo interno & ciotola, v.n. locale \\
\hline $\begin{array}{l}\text { T. 59, inumazione (forse femminile?), } \\
\text { III sec. a.C. }\end{array}$ & 20469 & asterisco & fondo interno & ciotola, v.n. locale \\
\hline $\begin{array}{l}\text { T. } 115 \text {, inumazione, } \\
\text { III sec. a.C. }\end{array}$ & 20506 & $c h i$ & parete interna & $\begin{array}{l}\text { piatto da pesce, v.n. } \\
\text { locale }\end{array}$ \\
\hline \multirow{2}{*}{$\begin{array}{l}\text { T. } 134 \text {, inumazione in fossa, } \\
\text { III sec. a.C. }\end{array}$} & 20515 & $c h i$ & fondo esterno & ciotola, v.n. locale \\
\hline & 20516 & ypsilon & fondo esterno & ciotola, v.n. locale \\
\hline $\begin{array}{l}\text { T. } 154 \text {, inumazione femminile, } \\
\text { (III sec. a.C.) }\end{array}$ & 20519 & venza velOuriu & fondo interno & piatto, v.n. locale \\
\hline $\begin{array}{l}\text { T. } 160 \mathrm{~B} \text {, inumazione, } \\
\text { (III sec. a.C.) }\end{array}$ & 20526 & $\begin{array}{l}\text { ypsilon }(\mathrm{a}) \\
\operatorname{chi}(\mathrm{b})\end{array}$ & $\begin{array}{l}\text { fondo interno } \\
\text { (a), parete } \\
\text { esterna (b) }\end{array}$ & ciotola, v.n. locale \\
\hline \multirow{2}{*}{$\begin{array}{l}\text { T. 95, inumazione in fossa, } \\
\text { fine del III-inizi del II sec. a.C. }\end{array}$} & 20489 & 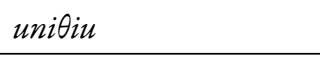 & fondo interno & ciotola, v.n. locale \\
\hline & 20490 & segno a croce & parete esterna & $\begin{array}{l}\text { piccola coppa, } \\
\text { ceramica depurata }\end{array}$ \\
\hline \multirow{3}{*}{$\begin{array}{l}\text { T. } 97 \text {, inumazione in fossa, } \\
\text { fine del III-inizi del II sec. a.C. }\end{array}$} & 20491 & quattro $y p$ silon & fondo interno & ciotola, v.n. locale \\
\hline & 20492 & segno a croce & fondo esterno & ciotola, v.n. locale \\
\hline & 20493 & $\begin{array}{l}\text { segno a croce }(a), \\
\text { segno a croce }(b)\end{array}$ & fondo esterno & ciotola, v.n. locale \\
\hline
\end{tabular}




\begin{tabular}{|c|c|c|c|c|}
\hline Contesto & $C I E$ & TIPO DI EPIGRAFE & Posizione & SUPPORTO \\
\hline \multirow{4}{*}{$\begin{array}{l}\text { T. } 21 \text {, inumazione in fossa forse } \\
\text { femminile, } \\
\text { fine del III-inizi del II sec. a.C. }\end{array}$} & 20436 & $\theta i$ & parete interna & $\begin{array}{l}\text { piccola ciotola, } \\
\text { v.n. locale }\end{array}$ \\
\hline & 20437 & segno a croce & fondo interno & coppa, ceramica grigia \\
\hline & 20438 & segno a croce & fondo esterno & pisside, v.n. locale \\
\hline & 20439 & asterisco & fondo esterno & coppa, ceramica grigia \\
\hline \multirow{2}{*}{$\begin{array}{l}\text { T. 197, inumazione in fossa, } \\
\text { fine del III-inizi del II sec. a.C. }\end{array}$} & 20549 & segno a croce & parete esterna & piatto, v.n. locale \\
\hline & 20550 & alpha & fondo interno & ciotola, v.n. locale \\
\hline $\begin{array}{l}\text { T. 348, inumazione, } \\
\text { fine del III-inizi del II sec. a.C. }\end{array}$ & 20628 & 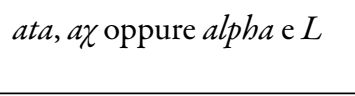 & fondo interno & $\begin{array}{l}\text { tazzina monoansata, } \\
\text { v.n. locale }\end{array}$ \\
\hline \multirow{5}{*}{$\begin{array}{l}\text { T. } 40 \text {, inumazione bisoma in fossa } \\
\text { (un individuo forse femminile), } \\
\text { seconda metà del III-prima metà } \\
\text { del II sec. a.C. }\end{array}$} & 20450 & ui o $I V$ & fondo interno & ciotola, v.n. locale \\
\hline & 20451 & $u i \mathrm{o} I V$ & fondo interno & ciotola, v.n. locale \\
\hline & 20452 & segno a croce & fondo interno & ciotola, v.n. locale \\
\hline & 20453 & segno a croce & fondo interno & piatto, v.n. locale \\
\hline & 20454 & segno a croce & fondo interno & piatto, v.n. locale \\
\hline \multirow{2}{*}{$\begin{array}{l}\text { T. } 166 \text {, inumazione in fossa, } \\
\text { seconda metà del III-prima metà del } \\
\text { II sec. a.C. }\end{array}$} & 20530 & ypsilon & parete interna & piatto, v.n. locale \\
\hline & 20531 & ypsilon & parete interna & piatto, v.n. locale \\
\hline $\begin{array}{l}\text { T. } 221 \text {, inumazione, } \\
\text { III-II sec. a.C. (ma dubitativamente) }\end{array}$ & 20557 & segno a croce & fondo interno & ciotola, v.n. locale \\
\hline $\begin{array}{l}\text { T. 103, inumazione in fossa, } \\
\text { fine del III-prima metà del II sec. a.C. }\end{array}$ & 20498 & sigma & fondo interno & piatto, v.n. locale \\
\hline \multirow{12}{*}{$\begin{array}{l}\text { T. } 363 \text {, inumazione in fossa, } \\
\text { fine del III-prima metà del II sec. a.C. }\end{array}$} & 20641 & $c h i$ & fondo interno & $\begin{array}{l}\text { grande ciotola con due } \\
\text { scanalature all'orlo, } \\
\text { v.n. locale }\end{array}$ \\
\hline & 20642 & $c h i$ & fondo interno & ciotola, v.n. locale \\
\hline & 20643 & $c h i$ & fondo interno & ciotola, v.n. locale \\
\hline & 20644 & $c h i$ & fondo interno & ciotola, v.n. locale \\
\hline & 20645 & $c h i$ & fondo interno & ciotola, v.n. locale \\
\hline & 20646 & $c h i$ & fondo interno & ciotola, v.n. locale \\
\hline & 20647 & $c h i$ & fondo interno & ciotola, v.n. locale \\
\hline & 20648 & $c h i$ & fondo interno & piatto, v.n. locale \\
\hline & 20649 & $c h i$ & fondo interno & piatto, v.n. locale \\
\hline & 20650 & $c h i$ & fondo interno & piatto, v.n. locale \\
\hline & 20651 & $c h i$ & fondo interno & ciotola, v.n. locale \\
\hline & 20652 & $c h i$ & fondo interno & ciotola, v.n. locale \\
\hline \multirow{2}{*}{$\begin{array}{l}\text { T. } 47 \text {, cremazione, } \\
\text { inizi del II sec. a.C. }\end{array}$} & 20458 & pulu & fondo interno & ciotola, v.n. locale \\
\hline & 20459 & $F$ e segno a croce & spalla & anfora greco-italica \\
\hline \multirow{2}{*}{$\begin{array}{l}\text { T. } 13 \text {, inumazione in fossa, } \\
\text { prima metà del II sec. a.C. }\end{array}$} & 20432 & ypsilon & fondo interno & $\begin{array}{l}\text { ciotola, v.n. forse } \\
\text { riminese }\end{array}$ \\
\hline & 20433 & segno a croce & parete esterna & ciotola, v.n. locale \\
\hline \multirow{2}{*}{$\begin{array}{l}\text { T. } 113 \text {, inumazione in fossa, } \\
\text { prima metà del II sec. a.C. }\end{array}$} & 20503 & ypsilon e graffito non det. & fondo interno & ciotola, v.n. locale \\
\hline & 20504 & $i u$ o $V I$ & fondo esterno & ciotola, v.n. locale \\
\hline
\end{tabular}




\begin{tabular}{|c|c|c|c|c|}
\hline Contesto & $C I E$ & TIPO DI EPIGRAFE & Posizione & SUPPORTO \\
\hline \multirow{4}{*}{$\begin{array}{l}\text { T. } 175 \text {, inumazione in fossa, } \\
\text { prima metà del II sec. a.C. }\end{array}$} & 20534 & $\begin{array}{l}\operatorname{spiu}(\mathrm{a}) \\
X I \text { o } I X(\mathrm{~b})\end{array}$ & $\begin{array}{l}\text { parete esterna } \\
\text { (a), fondo } \\
\text { interno (b) }\end{array}$ & pisside, v.n. locale \\
\hline & 20535 & $c h i$ & fondo esterno & piattello, v.n. locale \\
\hline & 20536 & segno a croce & parete esterna & ciotola, v.n. locale \\
\hline & 20537 & due segni a croce $(X X$ ? $)$ & parete esterna & ciotola, v.n. locale \\
\hline $\begin{array}{l}\text { T. } 183 \text {, cremazione, } \\
\text { prima metà del II sec. a.C. }\end{array}$ & 20539 & ypsilon & parete esterna & piatto, v.n. locale \\
\hline $\begin{array}{l}\text { T. } 313 \text {, inumazione, } \\
\text { prima metà del II sec. a.C. }\end{array}$ & 20600 & petra a $[---]$ & non det. & non det. \\
\hline \multirow{3}{*}{$\begin{array}{l}\text { T. } 324 \text {, inumazione, } \\
\text { prima metà del II sec. a.C. }\end{array}$} & 20606 & $\theta i$ & parete esterna & ciotola, v.n. locale \\
\hline & 20607 & $\begin{array}{l}\text { graffito non det. (a), } \\
n i(\mathrm{~b})\end{array}$ & $\begin{array}{l}\text { fondo interno } \\
\text { (a), fondo } \\
\text { esterno (b) }\end{array}$ & fr. di ciotola, v.n. locale \\
\hline & 20608 & segno a croce & fondo interno & ciotola, v.n. locale \\
\hline \multirow{5}{*}{$\begin{array}{l}\text { T. } 334 \text {, inumazione, } \\
\text { prima metà del II sec. a.C. }\end{array}$} & 20621 & mura larza pili & fondo interno & ciotola, v.n. locale \\
\hline & 20622 & pu o meglio $p l$ & parete interna & ciotola, v.n. locale \\
\hline & 20623 & segno a croce & fondo interno & ciotola, v.n. locale \\
\hline & 20624 & segno a croce & fondo interno & ciotola, v.n. locale \\
\hline & 20625 & segno a croce & fondo interno & ciotola, v.n. locale \\
\hline \multirow{2}{*}{$\begin{array}{l}\text { T. } 370 \text {, inumazione (forse infantile), } \\
\text { prima metà del II sec. a.C. }\end{array}$} & 20654 & veri & fondo interno & ciotola, v.n. locale \\
\hline & 20655 & segno a croce & fondo esterno & ciotola, v.n. locale \\
\hline \multirow{2}{*}{$\begin{array}{l}\text { T. 18, cremazione, } \\
\text { seconda metà del II sec. a.C. }\end{array}$} & 20434 & anta afr mura & fondo interno & ciotola, v.n. locale \\
\hline & 20435 & chi & parete esterna & piatto, v.n. locale \\
\hline \multirow{2}{*}{$\begin{array}{l}\text { T. 32, inumazione in fossa, } \\
\text { seconda metà del II sec. a.C. }\end{array}$} & 20444 & me & fondo esterno & ciotola, v.n. locale \\
\hline & 20445 & alpha & fondo esterno & ciotola, v.n. locale \\
\hline \multirow{3}{*}{$\begin{array}{l}\text { T. 52, inumazione, } \\
\text { seconda metà del II sec. a.C. }\end{array}$} & 20461 & lentnaimi & fondo interno & ciotola, v.n. locale \\
\hline & 20462 & $\begin{array}{l}\text { lentnaimi }(\mathrm{a}) \\
\text { ypsilon }(\mathrm{b})\end{array}$ & $\begin{array}{l}\text { fondo interno } \\
\text { (a), parete } \\
\text { esterna (b) }\end{array}$ & ciotola, v.n. locale \\
\hline & 20463 & {$[---] m i$} & fondo interno & ciotola, v.n. locale \\
\hline \multirow{2}{*}{$\begin{array}{l}\text { T. 186, inumazione in fossa, } \\
\text { seconda metà del II sec. a.C. }\end{array}$} & 20541 & unitiu & fondo interno & ciotola, v.n. locale \\
\hline & 20542 & graffito non det. lacunoso & cavetto & $\begin{array}{l}\text { piatto da pesce, v.n. } \\
\text { locale }\end{array}$ \\
\hline $\begin{array}{l}\text { T. } 46 \text {, cremazione, } \\
\text { fine del III-II sec. a.C. }\end{array}$ & 20456 & $\begin{array}{l}\text { asterisco legato a chi }(\mathrm{a}) \text {, } \\
\text { segno a croce }(\mathrm{b})\end{array}$ & $\begin{array}{l}\text { fondo interno } \\
\text { (a), fondo } \\
\text { esterno (b) }\end{array}$ & coppa, ceramica grigia \\
\hline \multirow{2}{*}{$\begin{array}{l}\text { T. } 36 \text {, inumazione in fossa, } \\
\text { II sec. a.C. }\end{array}$} & 20447 & segno a croce & parete esterna & ciotola, v.n. locale \\
\hline & 20448 & segno a croce & parete esterna & piatto, v.n. locale \\
\hline $\begin{array}{l}\text { T. 42, inumazione in fossa, } \\
\text { II sec. a.C. }\end{array}$ & 20455 & stella a cinque punte? & fondo interno & ciotola, v.n. locale \\
\hline $\begin{array}{l}\text { T. } 49 \text {, inumazione in fossa, } \\
\text { II sec. a.C. }\end{array}$ & 20460 & segno a croce & fondo interno & ciotola, v.n. locale \\
\hline $\begin{array}{l}\text { T. } 60 \text {, inumazione in cassa di legno, } \\
\text { II sec. a.C. }\end{array}$ & 20471 & segno a croce & fondo interno & ciotola, v.n. locale \\
\hline $\begin{array}{l}\text { T. } 76 \text {, cremazione, } \\
\text { II sec. a.C. }\end{array}$ & 20482 & alpha & fondo interno & ciotola, v.n. locale \\
\hline $\begin{array}{l}\text { T. 100, inumazione in fossa, } \\
\text { II sec. a.C. }\end{array}$ & 20495 & segno a croce & fondo interno & piatto, v.n. locale \\
\hline
\end{tabular}




\begin{tabular}{|c|c|c|c|c|}
\hline Contesto & $C I E$ & TIPO DI EPIGRAFE & Posizione & SUPPORTO \\
\hline $\begin{array}{l}\text { T. 102, inumazione in fossa, } \\
\text { II sec. a.C. }\end{array}$ & 20497 & segno a croce & fondo interno & ciotola, v.n. locale \\
\hline $\begin{array}{l}\text { T. } 159 \text {, inumazione in fossa, } \\
\text { II sec. a.C. }\end{array}$ & 20522 & chet & parete esterna & ciotola, v.n. locale \\
\hline $\begin{array}{l}\text { T. 171, inumazione in fossa, } \\
\text { II sec. a.C. }\end{array}$ & 20533 & chi e graffito non det. & parete esterna & ciotola, v.n. locale \\
\hline $\begin{array}{l}\text { T. } 211 \text {, } \\
\text { dubitativamente II sec. a.C. }\end{array}$ & 20555 & $\begin{array}{l}\text { triangolo con asta } \\
\text { al centro }\end{array}$ & fondo interno & ciotola, v.n. locale \\
\hline $\begin{array}{l}\text { T. } 362 \text {, inumazione in fossa, } \\
\text { II sec. a.C. }\end{array}$ & 20640 & [-- ] śta mi & fondo interno & $\begin{array}{l}\text { grande ciotola con due } \\
\text { scanalature all'orlo, } \\
\text { v.n. locale }\end{array}$ \\
\hline \multirow{3}{*}{$\begin{array}{l}\text { T. } 228 \text {, cremazione, } \\
\text { seconda metà del II-prima metà } \\
\text { del I sec. a.C. }\end{array}$} & 20559 & $\operatorname{siliu}(\mathrm{a})$, tre aste $(\mathrm{b})$ & $\begin{array}{l}\text { fondo interno } \\
\text { (a), base del } \\
\text { piede (b) }\end{array}$ & ciotola, v.n. locale \\
\hline & 20560 & Qạ̣a mi siliu & fondo interno & ciotola, v.n. locale \\
\hline & 20561 & segno a croce & fondo interno & $\begin{array}{l}\text { piccola ciotola, v.n. } \\
\text { locale (IV-III sec. a.C.) }\end{array}$ \\
\hline T. 125 , cremazione, età ellenistica & 20508 & $c h i$ & parete esterna & coppa, ceramica grigia \\
\hline $\begin{array}{l}\text { T. } 104 \text {, cremazione, } \\
\text { prima metà del I sec. a.C. }\end{array}$ & 20500 & Titiunio & parete esterna & $\begin{array}{l}\text { ciotola, v.n. di prod. } \\
\text { incerta }\end{array}$ \\
\hline
\end{tabular}

Tab. 8. Documenti epigrafici (tutti realizzati dopo la cottura) da Canalbianco da corredi di attribuzione incerta (A) e contesti votivi (B), di erronea attribuzione (C), erratici o privi di informazioni (D).

\section{A. SEPOLTURE CON ELEMENTI Di CORREDO Di ATTRIBUZIONE INCERTA}

T. 310, inumazione, inizi del III sec. a.C.: 20597, [---] mi, interno, forma aperta di produzione incerta. T. 250, inumazione: 20577 , C. Titio e segno a croce in spalla, anfora greco-italica, fine del IV-prima metà del III sec. a.C.

T. 285, inumazione: 20585 , graffito non det. (due triangoli?), nel collo di anfora greco-italica, fine del IV-prima metà del III sec. a.C.

T. 325, inumazione: 20609 , chi e stella a cinque punte in legatura, fondo interno, ciotola a v.n. di produzione incerta, fine del IV-prima metà del III sec. a.C.

III sec. a.C. T. 258, inumazione: 20583, due aste, fondo interno, ciotola a v.n. locale, metà del III sec. a.C.

T. 378, inumazione: 20660 , atakuś, parete interna, ciotola a v.n. locale, metà del III sec. a.C.

T. 240, inumazione: 20567, chi, fondo interno, piatto a v.n. locale, seconda metà del III-inizi del II sec. a.C. T. 332, inumazione, fine del III sec. a.C.: 20618, segno a croce, fondo interno, ciotola a v.n. locale; 20619, segno a croce, fondo interno, ciotola a v.n. locale; 20620, segno a croce, fondo interno, ciotola a v.n. locale.

T. 245, inumazione: 20572, numerale $(L I)$, fondo interno, piatto da pesce a v.n. locale, III sec. a.C.

T. 248, inumazione: 20573 , segno a croce, fondo interno, kylix a v.n. locale, III sec. a.C.

T. 359, rito incerto: 20636, ypsilon, parete esterna, piatto a v.n. locale, III sec. a.C.

T. 326, inumazione, prima metà del II sec. a.C.: 20610, lại, fondo interno, piatto a v.n. locale; 20611, ypsilon, fondo interno, piatto a v.n. locale; 20612, asterisco, fondo esterno, ciotola a v.n. locale; 20613, segno a croce, collo, anfora greco-italica.

T. 320, inumazione: 20604, segno a croce, fondo interno, ciotola a v.n. locale, metà-seconda metà del II sec. a.C.

II sec. a.C.

T. 65, inumazione, femminile: 20474: $<t>$ titinai, fondo interno, piatto a v.n. locale, II sec. a.C.; 20475 : titinai, fondo interno, piatto a v.n. locale, II sec. a.C.

T. 251, inumazione: 20578 , susas (iscrizione in alfabeto leponzio), interno del vaso, ciotola a v.n. locale, II sec. a.C.

T. 288, rito incerto: 20586 , due ypsilon e segno a croce, fondo interno, ciotola a v.n. locale, II sec. a.C.

T. 342, rito incerto: 20626, chi, parete esterna, piatto a v.n. locale, II sec. a.C.

T. 352, inumazione: 20634 , segno a croce, fondo interno, piatto a v.n. locale, II sec. a.C. 


\begin{tabular}{|c|c|}
\hline III-II sec. a.C. & $\begin{array}{l}\text { TT. 54-55, III-II sec. a.C.: 20464, mi helis uvasaves, fondo interno, coppa in ceramica grigia; } 20465 \text {, } \\
\text { numerale (XXXII), collo, anfora greco-italica; } 20466 \text {, graffito non det., parete esterna, ciotola a v.n. } \\
\text { locale. } \\
\text { T. } 257 \text {, inumazione: } 20580 \text {, susas (iscrizione in alfabeto leponzio) nel fondo interno e segno a croce nel } \\
\text { fondo esterno, ciotola a v.n. locale, II sec. a.C.; } 20581 \text {, | eisa | (iscrizione in alfabeto leponzio), fondo } \\
\text { interno, ciotola a v.n. locale, II sec. a.C.; 20582, graffito non det. (triangolo), fondo interno, ciotola a } \\
\text { v.n. locale, III sec. a.C. }\end{array}$ \\
\hline \multicolumn{2}{|r|}{ B. Contesti votivi } \\
\hline III sec. a.C. & $\begin{array}{l}\text { n. } 194 \text {, prima metà del III sec. a.C.: } 20547 \text {, ipuz, fondo esterno, piccola ciotola a v.n. di produzione } \\
\text { incerta. } \\
\text { n. } 296 \text {, pieno III sec. a.C.: } 20590 \text {, keus (iscrizione in alfabeto leponzio), chi e kappa, fondo interno, } \\
\text { grande ciotola a v.n. locale o spinetico; } 20591, \chi u r \times \times \text {, fondo interno, piatto da pesce a v.n. locale o } \\
\text { spinetico; } 20592 \text {, graffito non det., interno, ciotola a v.n. locale; } 20593 \text {, segno a croce, parete esterna, } \\
\text { coppa in ceramica grigia. } \\
\text { n. } 57 \text {, fine del IV-III sec. a.C.: } 20468 \text {, graffito non det. (due segni a croce in legatura?), parete esterna, } \\
\text { mortaio in ceramica grigia. } \\
\text { n. } 24 \text {, seconda metà del III-inizi del II sec. a.C.: } 20442 \text {, graffito non det., parete esterna, ciotola a v.n. } \\
\text { locale. } \\
\text { n. } 129 \text {, III sec. a.C.: } 20509 \text {, cinque } y \text { psilon disposti a raggiera, fondo interno, piatto a v.n. locale. } \\
\text { n. } 229 \text {, III sec. a.C.(?): } 20562 \text {, graffito non det., fondo esterno, mortaio in ceramica grigia. }\end{array}$ \\
\hline II sec. a.C. & $\begin{array}{l}\text { n. } 31 \text {, II sec. a.C.: } 20443 \text {, graffito non det., fondo esterno, grande ciotola con due scanalature all'orlo } \\
\text { a v.n. locale. } \\
\text { n. } 237: 20566, k \text { snalu, fondo interno, ciotola a v.n. locale, II sec. a.C. }\end{array}$ \\
\hline \multicolumn{2}{|r|}{ C. VASI DI ERRONEA ATTRIBUZIONE AL CORREDO } \\
\hline Età arcaica & 20574: segno a croce, fondo esterno, coppa in bucchero. \\
\hline IV sec. a.C. & $\begin{array}{l}\text { 20605: chi, fondo esterno, ciotola a v.n. volterrana, seconda metà del IV sec. a.C. } \\
\text { 20481: ypsilon, fondo interno, ciotola a v.n. di produzione incerta, fine del IV sec. a.C. }\end{array}$ \\
\hline III sec. a.C. & $\begin{array}{l}\text { 20483: due chi contrapposti, fondo interno, kylix a v.n. locale, fine del IV-prima metà del III sec. a.C. } \\
\text { 20499: graffito non det., fondo interno, kylix a v.n. volterrana, fine del IV-prima metà del III sec. a.C. } \\
\text { 20584: graffito non det. (forse un segno a croce o due chi contrapposti ?), fondo esterno, coppa in cera- } \\
\text { mica grigia, fine del IV-prima metà del III sec. a.C. } \\
\text { 20588: chi, interno, piattello a v.n. locale, fine del IV-metà del III sec. a.C. } \\
\text { 20658: chi, parete esterna, ciotola a v.n. locale, prima metà del III sec. a.C. } \\
\text { 20457: meiś ta, parete interna, mortaio in ceramica grigia, prima metà-metà del III sec. a.C. } \\
\text { 20659: asterisco, fondo interno, ciotola a v.n. locale, metà del III sec. a.C. } \\
\text { 20656: kappa, fondo esterno, ciotola a v.n. locale, metà del III sec. a.C. } \\
\text { 20602: alpha, parete esterna, ciotola a v.n. locale, metà del III sec. a.C. } \\
\text { 20627: <e >mi karku[---], fondo interno, piatto da pesce a v.n. locale, pieno III sec. a.C. } \\
\text { 20603: kaviś ta mi e chi, parete esterna, ciotola a v.n. locale, metà-terzo quarto del III sec. a.C. } \\
\text { 20657: chet, fondo interno presso il foro pervio centrale, ciotola a v.n. locale, 250-225 a.C. circa. } \\
\text { 20494: graffito non det. (chi e due aste?), parete esterna, ciotola a v.n. locale, seconda metà del III-inizi } \\
\text { del II sec. a.C. } \\
\text { 20501: ypsilon, fondo interno, ciotola a v.n. locale. } \\
\text { 20502: chi, parete esterna, ciotola a v.n. locale. } \\
\text { 20505: graffito non det., parete esterna, mortaio con beccuccio in ceramica depurata. } \\
\text { 20507: chi, fondo interno, ciotola a v.n. riminese. } \\
\text { 20514: pe zurta, fondo interno, ciotola a v.n. volterrana. } \\
\text { 20518: ypsilon, fondo interno, piatto da pesce a v.n. locale. } \\
\text { 20532; ypsilon, parete esterna, piatto da pesce a v.n. locale. } \\
\text { 20554: segno a croce, fondo interno, ciotola a v.n. locale. } \\
\text { 20558: graffito non det., fondo interno, kylix a v.n. volterrana. } \\
\text { 20576: stella a cinque punte nel fondo interno e graffito non det. lacunoso nel fondo esterno, coppa in } \\
\text { ceramica grigia. } \\
\text { 20579: chi, parete esterna, kylix a v.n. volterrana. }\end{array}$ \\
\hline
\end{tabular}




\begin{tabular}{|c|c|}
\hline II sec. a.C. & $\begin{array}{l}\text { 20540: numerale }(X X X X V I) \text { nel collo e graffito non det. nella spalla, anfora greco-italica, seconda } \\
\text { metà del III-primo quarto del II sec. a.C. } \\
\text { 20568: arza lausial, collo, anfora greco-italica, seconda metà del III-inizi del II sec. a.C. } \\
\text { 20470: graffito non det., collo, anfora greco-italica, prima metà del II sec. a.C. } \\
\text { 20517: iu o VI, fondo esterno, piatto a v.n. locale, prima metà del II sec. a.C. } \\
\text { 20635: zeta, parete esterna, olla a v.n. di produzione incerta, prima metà del II sec. a.C. } \\
\text { 20428: chi, fondo interno, ciotola a v.n. locale. } \\
\text { 20446: alpha (?), parete esterna, ciotola a v.n. di produzione incerta. } \\
\text { 20449: graffito non det., parete esterna, ciotola a v.n. locale. } \\
\text { 20472: titi, fondo interno, ciotola a v.n. locale. } \\
\text { 20473: titi, fondo interno, ciotola a v.n. locale. } \\
\text { 20484: segno a croce, fondo interno, ciotola a v.n. locale. } \\
\text { 20546: } y \text { psilon, fondo esterno, pisside a v.n. locale. } \\
\text { 20548: due aste, parete esterna, ciotola a v.n. locale. } \\
\text { 20553: se o meglio sa, fondo interno, ciotola a v.n. locale. } \\
\text { 20556: chi, fondo interno, ciotola a v.n. locale. } \\
\text { 20616: graffito non det. lacunoso, parete esterna, ciotola a v.n. locale. } \\
\text { 20617: asterisco, fondo interno, ciotola a v.n. locale. } \\
\text { 20637: chi, fondo esterno, skyphos a v.n. locale. } \\
\text { 20638: } \text { (lat.), parete esterna, skyphos a v.n. locale. } \\
\text { 20661: chi nel fondo interno e } c h i \text { nella parete esterna, piatto a v.n. locale. }\end{array}$ \\
\hline III-II sec. a.C. & $\begin{array}{l}\text { 20440: segno a croce, fondo interno, coppa in ceramica grigia. } \\
\text { 20441: asterisco (?), fondo interno, ciotola lacunosa a v.n. locale. } \\
\text { 20485: asterisco, fondo interno, coppa in ceramica grigia. } \\
\text { 20486: asterisco, fondo interno, coppa in ceramica grigia. } \\
\text { 20496: ramo secco, fondo interno, coppa in ceramica grigia, età ellenistica. } \\
\text { 20545: ypsilon, fondo esterno, coppa in ceramica grigia. } \\
\text { 20575: due graffiti non det. nel fondo interno e nel fondo esterno, coppa in ceramica grigia. } \\
\text { 20601: helis nella vasca interna e tre aste nella parete esterna, coppa in ceramica grigia. } \\
\text { 20639: graffito non det., fondo esterno, coppa in ceramica grigia. }\end{array}$ \\
\hline \multicolumn{2}{|r|}{ D. VASI ERRATICI O PRIVI DI INFORMAZIONI } \\
\hline III sec. a.C. & $\begin{array}{l}\text { 20662: venuś zurtiuś mi, fondo interno, ciotola a v.n. locale, metà del III sec. a.C. } \\
\text { 20663: mura, parete interna, ciotola a v.n. locale, prima metà del III sec. a.C. }\end{array}$ \\
\hline Non det. & 20664: mama, fondo esterno, ciotola a v.n. di produzione e cronologia incerta. \\
\hline
\end{tabular}

All'interno delle 92 sepolture (Tabb. 7 e 8.A), colpisce anzi tutto il dato relativo al rito funerario. Ben consci della diffusa pratica inumatoria che caratterizza le sepolture adriesi di III e II sec. a.C., si osserva una interessante tendenza che purtroppo non è possibile estendere all'intera necropoli, mancando uno studio d'insieme di tutte le sepolture: il rito crematorio è documentato da 9 contesti, cioè circa un decimo del totale. Questi, a parte un caso di III sec. a.C. (tomba 177) e uno genericamente databile al periodo ellenistico (tomba 125), si concentrano dopo gli inizi del II e tre conservano una iscrizione etrusca (tombe 18, 47, 228). Pertanto, se si considera che tra IV e III sec. a.C. su 45 sepolture una sola è a cremazione, mentre nel secolo successivo su 41 se ne contano 7, pur con la necessaria cautela dovuta alla parzialità e alla selezione del dato, si coglie l'emergere di un biritualismo che solo uno studio sistematico dell' intera area funeraria permetterà di comprendere nella sua pienezza. A conferma della rilevanza di questa situazione, si anticipa che nelle altre aree funerarie non si registra il rito crematorio fra le tombe con documentazione epigrafica.

Tra le 72 sepolture con corredo di certa attribuzione (Tab. 7), a fronte di un solo caso ancora databile entro gli ultimi decenni del IV sec. a.C. (con una singola lettera graffita) e di 4 più genericamente attribuibile al III-II sec. a.C., si osserva una concentrazione relativamente maggiore durante il III sec. a.C. (Fig. 25). Tale tendenza è confermata anche nelle 20 sepolture con corredo di attri- 


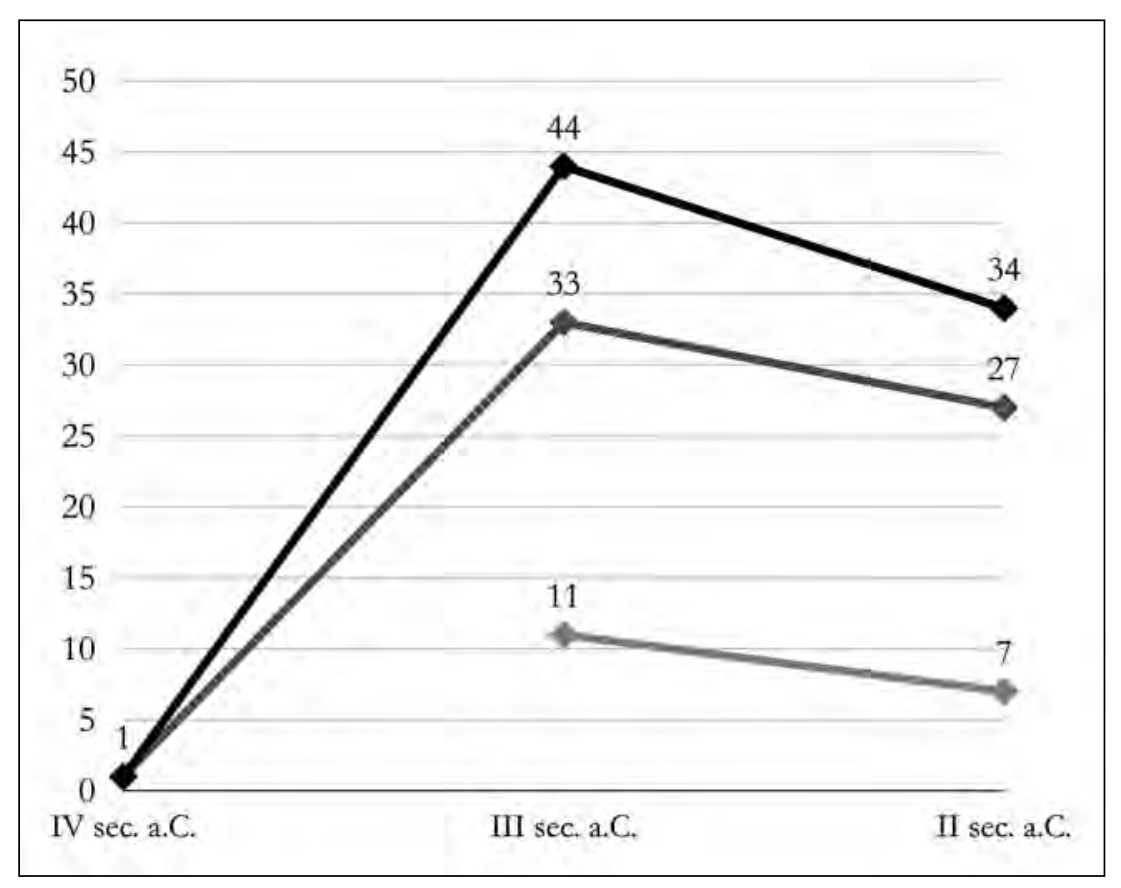

Fig. 25. Quantificazione dei contesti funerari di Canalbianco con documenti epigrafici. In nero la loro totalità, in grigio scuro le tombe di certa attribuzione e in grigio chiaro quelle di incerta attribuzione. Non sono state considerate le tombe datate a cavallo di due secoli e quelle di generica datazione.

buzione incerta e negli 8 contesti probabilmente votivi ${ }^{90}$, dei quali 6 di III e 2 di II sec. a.C. Anche i 56 vasi di erronea attribuzione al corredo (1 di età arcaica, 2 di IV sec. a.C., 24 di III sec. a.C., 20 di II sec. a.C., 9 più latamente di III-II sec. a.C.) e le 3 iscrizioni su vasi definiti erratici o privi di informazioni sui contesti ( 2 di III sec. a.C. e uno di cronologia non determinabile), suggeriscono la medesima tendenza.

Il quadro diacronico risulterebbe però lacunoso senza preliminari considerazioni sulla distribuzione spaziale. Purtroppo, come già detto, la necropoli di Canalbianco a oggi soffre dell'assenza di una planimetria di dettaglio (Fig. 26) ${ }^{91}$. Sono note solo due planimetrie parziali, una edita da G. Fogolari nel $1940^{92}$ e una recentemente ricostruita da M.T.A. Robino sulla base di uno studio accurato della documentazione di $\operatorname{archivio}^{93}$. Queste due planimetrie si riferiscono a due settori rispettivamente a est (Fig. 27) e a ovest del ponte ferroviario (Fig. 28). Sebbene vi siano problematiche relative all'assenza di sepolture in pianta o a misure che non trovano corrispondenza in quelle indicate nel Ms. Giornale di scavo di Scarpari ${ }^{94}$, rimane tuttavia un utile esercizio provare ad approcciare una analisi spaziale della distribuzione delle iscrizioni. A scanso di equivoci, va comunque puntualizzato che il traguardo di una pianta completa della necropoli non potrà comunque essere integrata con i dati di tutte le sepolture con documenti epigrafici, che si sa essere di attribuzione incerta o errata per un numero significativo di casi. A ovest del ponte si nota una bassa concentrazione di sepolture con documentazione epigrafica: la tomba 362 (II sec. a.C.) e la 378 (III sec. a.C.) fra quelle con iscrizioni, le tombe 349 (non posizionata da M.T.A. Robino, ma sicuramente vicina alla 348 secondo le misure riportate da Scarpari: Fig. 29), 359 (III sec. a.C.), 348 (fine III-inizi II sec. a.C.) e 342, 363 (II sec. a.C.) fra quelle con graffiti, perlopiù alfabetici. A est del ponte, nel settore

90 Per l'identificazione di questi particolari contesti nella necropoli, cfr. RoBINo 2016, p. 95, nota 22.

91 È nota solo una pianta muta e non comprensiva di tutte le tombe elencate nel giornale di scavo dell'ing. G.B. (SCARPARi 1977, p. 46, fig. 8). Si attende l'edizione della planimetria recentemente recuperata da M.C. Vallicelli nell'archivio della SABAP di Padova. Si ringrazia la suddetta per l'anticipazione.

92 Fogolari 1940, p. 432, fig. 1.

93 Robino 2016, p. 101, fig. 7.

${ }_{94}$ Per la spiegazione di queste informazioni, si rimanda a RoBINo 2016. 


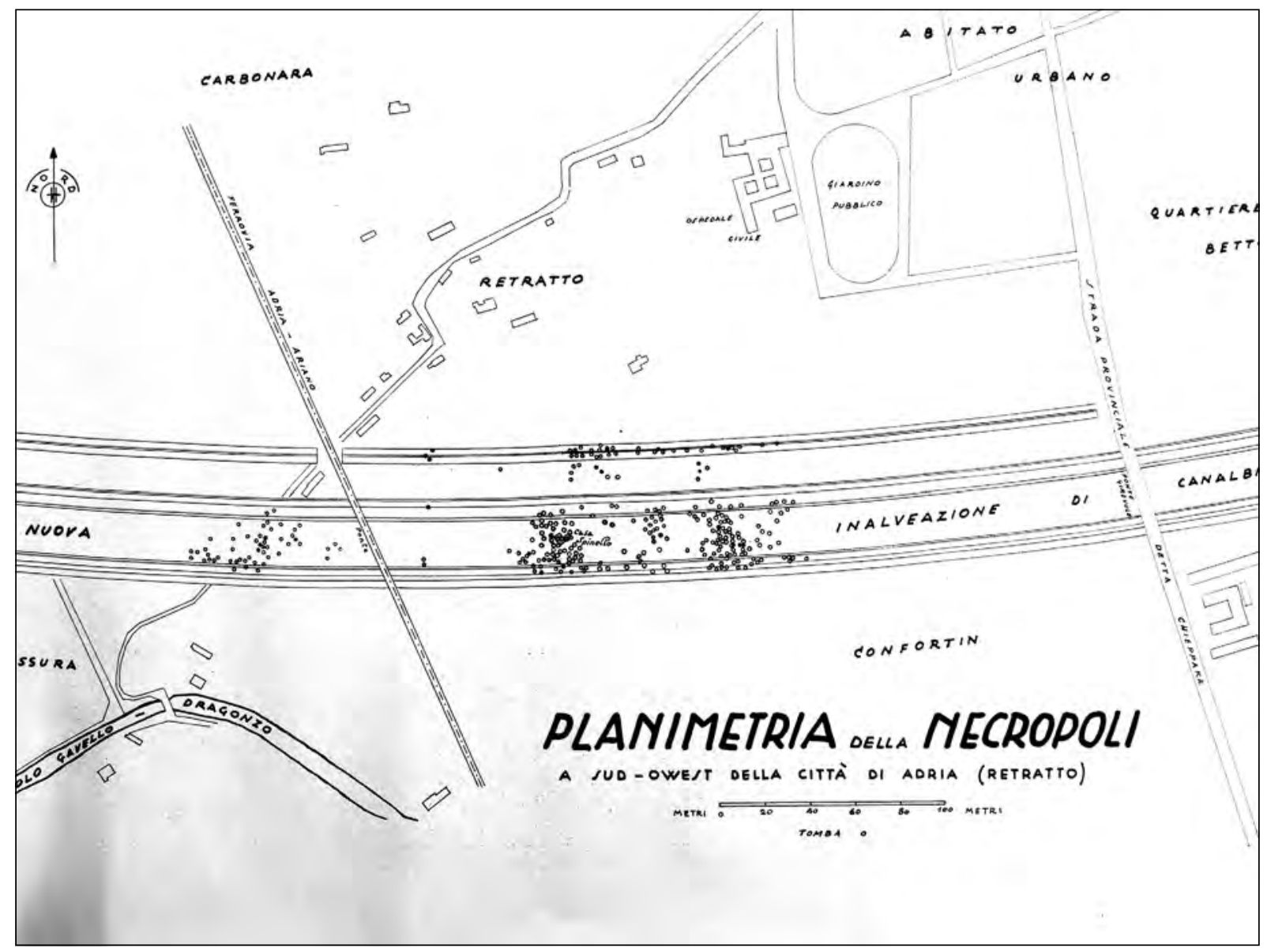

Fig. 26. Planimetria muta della necropoli di Canalbianco (dall'Archivio del Museo Archeologico Nazionale di Adria).

edito da G. Fogolari, purtroppo non facilmente collocabile nella planimetria generale, si osserva una concentrazione maggiore, tuttavia senza una particolare associazione di antroponimi o lessemi fra loro simili in tombe vicine. Questa potrebbe essere una riprova che la maggior parte di queste iscrizioni pertengano a pratiche di dono funerario trasversali ai singoli gruppi familiare, che pure dovevano essere organizzati in recinti come dimostra l'eccezionale caso dei Muliu di via Spolverin a Bottrighe (cfr. VI.3). In quest'ultimo contesto però la reiterazione del gentilizio potrebbe essere connessa a una comunità più circoscritta e periferica rispetto alla città.

Subito a nord di Canalbianco, la zona di Retratto (chiamata Aretratto nella sua parte più orientale) è stata indagata nel corso del tempo in maniera purtroppo molto puntuale, non permettendo così che la conoscenza di un numero ridotto di contesti funerari. La rarefazione delle testimonianze non permette dunque osservazioni d'insieme come per Canalbianco. Qui insiste l'area occupata attualmente dall'Ospedale civile, dove i più recenti scavi degli anni 2007, 2010-2011 hanno dimostrato che questo era il confine tra abitato e necropoli ${ }^{95}$. Data la lunga tradizione di scavi e l'attenzione dimostrata a partire dal Settecento dalla comunità cittadina verso le antichità, non mancano in questa zona rinvenimenti ottocenteschi.

Praticamente nulla si può dire per il vasellame a v.n. perlopiù di produzione locale con iscrizioni e/o graffiti rinvenuto nell'Aretratto il 2 ottobre 1877 (tre ciotole, CIE 20685-20687) e il 2 novem-

95 Bonomi 2011, pp. 62-66; Gambacurta, Bacci, Marcassa 2012, pp. 44-50. Questi scavi non sono stati oggetto di ricognizione finalizzata alla redazione del fascicolo adriese del CIE. 


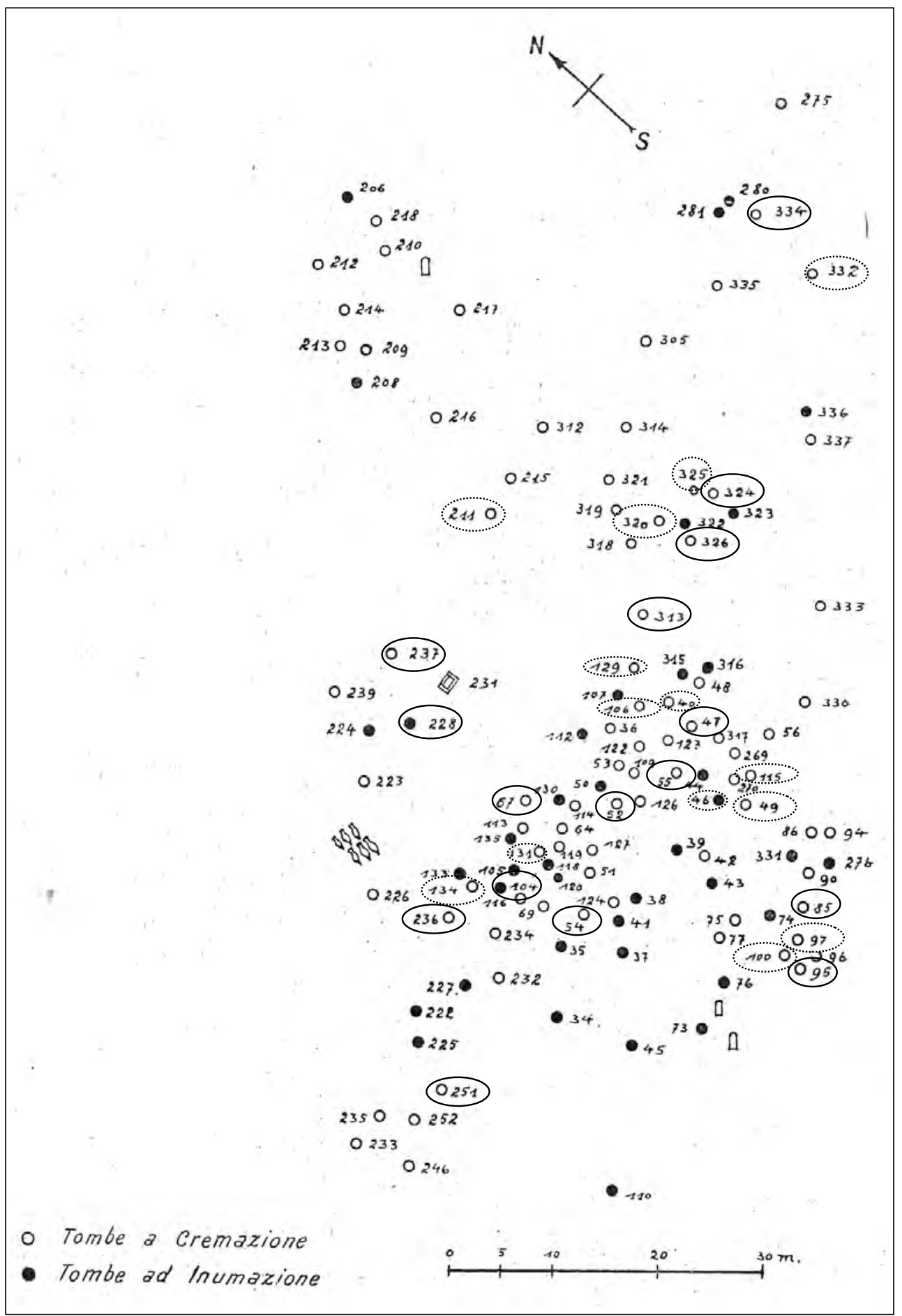

Fig. 27. Pianta parziale del settore della necropoli di Canalbianco ad est del ponte ferroviario, edita in Fogolari 1940. Cerchiate in nero le tombe con iscrizioni, in tratteggio quelle con graffiti alfabetici e non alfabetici.

bre dello stesso anno dal contadino Ruzza (due ciotole, CIE 20688, 20690; una kylix, CIE 20689; un piatto, CIE 20691). Tutti questi vasi, poi confluiti nel Museo Domestico della famiglia Bocchi, per la loro integrità potrebbero appartenere a sepolture. Il più significativo è sicuramente la ciotola di produzione incerta della seconda metà del III sec. a.C. con l'iscrizione zurtalu e una stella a cinque punte realizzati in posizione opposte rispetto alla stampiglia impressa al centro della vasca interna (CIE 20685). Sempre in zona Retratto, con gli inizi del Novecento, si deve a L. Conton e il gruppo degli Archeofili lo scavo di alcune sepolture ellenistiche nel fondo del sig. Trombini e dunque più estensivamente in quello di Luigi Ferro, dove nell'inverno 1904-1905 scavarono cinquanta tombe 


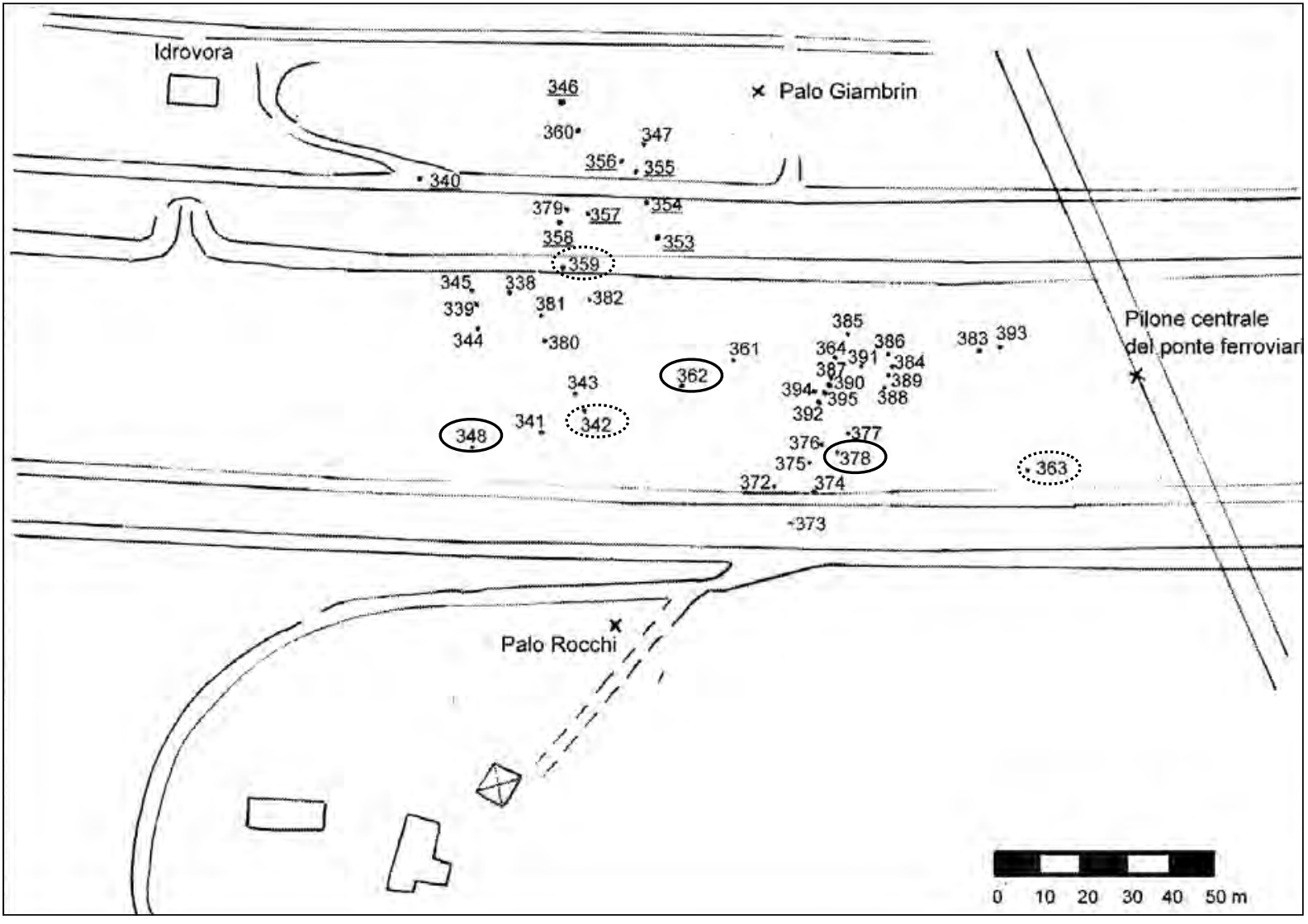

Fig. 28. Settore della necropoli di Canalbianco ad ovest del ponte ferroviario, elaborata da M.T.A. Robino (2016). Cerchiate in nero le tombe con iscrizioni, in tratteggio quelle con graffiti alfabetici e non alfabetici.

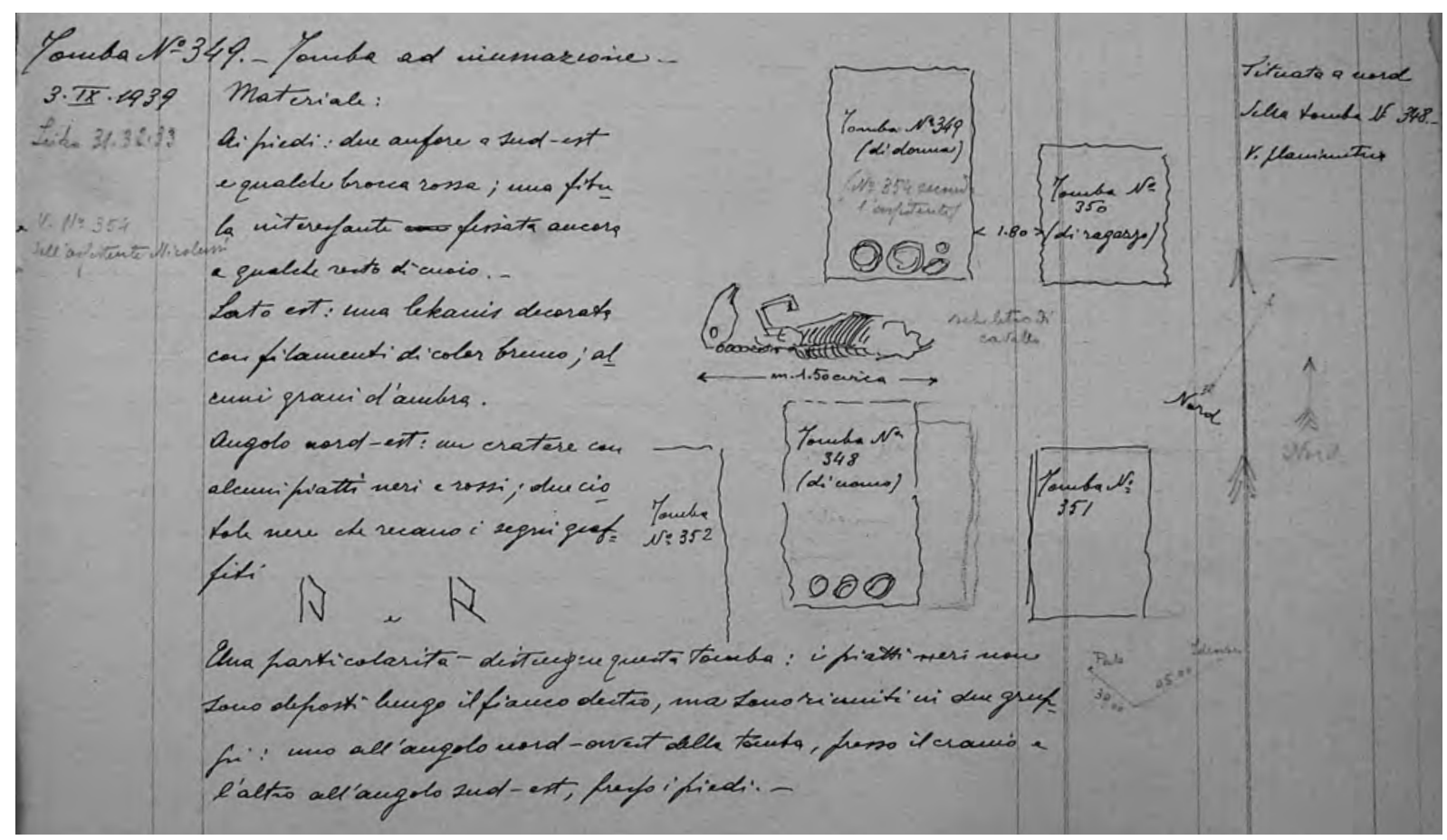

Fig. 29. Dettaglio del Giornale di Scavo di G.B. Scarpari relativo alla tomba 349, con schizzo della posizione relativa delle tombe circostanti (dall'Archivio del Museo Archeologico Nazionale di Adria). 
comprese tra il periodo ellenistico e romano ${ }^{96}$, il cui materiale è andato perlopiù disperso. Non è dato sapere quanti documenti epigrafici furono rinvenuti se non per la tomba 17 , probabilmente infantile, per la quale la descrizione del corredo (che non è stato possibile rintracciare) rimanda al periodo ellenistico e conserva un digramma $(k u)$ e alcuni graffiti ${ }^{97}$.

Successivamente alla seconda guerra mondiale, nuove indagini hanno interessato l'area di Retratto (Tab.9). Nel 1957 presso il fondo Donà, terreno attualmente appartenente all'Ospedale, fu effettuata una campagna di scavo che portò al rinvenimento di 15 sepolture, datate tra il III e il II sec. a.C. ${ }^{98} \mathrm{e}$ tutte a inumazione a eccezione della tomba 5 , per la quale l'assenza di evidenze può cautamente orientare verso l'interpretazione di un contesto votivo ${ }^{99}$. Purtroppo, le vicende successive alla messa in luce hanno causato lo smembramento dei nuclei originari, spesso inficiandone la coerenza filologica, non sempre ricostruibile ${ }^{100}$, come è documentato da K. Tamassia anche per le uniche due sepolture (tombe 11 e 15) che registrano documenti epigrafici ${ }^{101}$. Sempre nell'area dell'Ospedale Civile di Adria, nel 1993 durante la costruzione di nuovi edifici si rinvenne la tomba 1 di inumato adulto probabilmente entro cassone ligneo, datata alla prima metà del III sec. a.C. e dalle interessanti affinità con i contesti spinetici del medesimo periodo ${ }^{102}$, e due contesti votivi collocabili entro il III sec. a.C. ${ }^{103}$. La sepoltura e uno dei due depositi votivi (anch'esso indicato con il numero 1) datato alla metà del III sec. a.C. ${ }^{104}$, conservano graffiti alfabetici e non alfabetici.

Tab. 9. Tombe da Retratto (scavi 1957 e 1993) eon documenti epigrafici (tutti realizzati dopo la cottura).

\begin{tabular}{|c|c|c|c|c|}
\hline Contesto & $C I E$ & TIPO DI EPIGRAFE & Posizione & SUPPORTO \\
\hline \multirow{7}{*}{$\begin{array}{l}\text { Fondo Donà, T. } 11 \text {, inumazione in fossa, } \\
\text { II sec. a.C., probabilmente seconda metà }\end{array}$} & 20670 & alpha & parete esterna & piatto, v.n. locale \\
\hline & 20671 & alpha & fondo interno & ciotola, v.n. locale \\
\hline & 20672 & $\begin{array}{l}\text { alpha }(\mathrm{a}) \\
\text { segno a croce }(\mathrm{b})\end{array}$ & $\begin{array}{l}\text { fondo interno } \\
\text { (a), parete } \\
\text { esterno (b) }\end{array}$ & ciotola, v.n. locale \\
\hline & 20673 & ypsilon & parete esterna & ciotola, v.n. locale \\
\hline & 20674 & segno a croce & fondo interno & ciotola, v.n. locale \\
\hline & 20675 & segno a croce & fondo interno & ciotola, v.n. locale \\
\hline & 20676 & $\begin{array}{l}\text { due segni a croce } \\
\text { (numerale?) }\end{array}$ & parete esterna & ciotola, v.n. locale \\
\hline
\end{tabular}

96 Conton 1908 (in particolare, per le poche informazioni sullo scavo nel terreno del sig. Trombini, si veda p. 8).

97 Ibid., pp. 25-26. Da questa sepoltura provengono un digramma $(k u)$ su un piatto a v.n. detto di grandi dimensioni (CIE 20683) e un segno a croce nel fondo esterno di una ciotola a v.n. (CIE 20684), editi dallo scavatore ma non più conservati. Si documenta anche un secondo segno a croce descritto come realizzato nel fondo interno di un vaso a v.n., di cui purtroppo non fu edito l'apografo (ibid., p. 26).

98 TAmassia 1993. Le quindici sepolture del fondo Donà si distribuiscono tra III e II sec. a.C., con solo una di III sec. (tomba 9) e una compresa tra III e prima metà del II sec. a.C. (tomba 15), mentre le restanti si collocano nel II sec. a.C.

$99 \mathrm{Ibid}$., in particolare p. 56 e nota 27 per la tomba 5 , oltre a p. 17, nota 21 , dove si sottolinea l'assenza di informazioni sui resti del defunto e molto prudenzialmente si equipara alle c.d. "strutture" scavate nella necropoli di via Spolverin a Bottrighe.

${ }^{100}$ Ibid., pp. 10-11. Si ricorda che in CIE, IV, I, 1 sono stati considerati segni accidentali quelli del piatto n. inv. Ad 8634, appartenente alla tomba 8 (ibid., p. 29, n. 135).

101 Tamassia 1993, pp. 34 e 48.

102 Bellintani et al. 1995, pp. 43-45.

103 Ibid., pp. 44-47, 52-57.

${ }^{104}$ Ibid., pp. 53-55. 


\begin{tabular}{|c|c|c|c|c|}
\hline Contesto & $C I E$ & TIPO DI EPIGRAFE & Posizione & SUPPORTO \\
\hline \multirow{6}{*}{$\begin{array}{l}\text { Fondo Donà, T. 15, inumazione, } \\
\text { III-prima metà II sec. a.C. }\end{array}$} & 20677 & ankariu & fondo interno & $\begin{array}{l}\text { piattello su alto } \\
\text { piede, v.n. locale }\end{array}$ \\
\hline & 20678 & ankariu & fondo interno & ciotola, v.n. locale \\
\hline & 20679 & ankariu & fondo interno & ciotola, v.n. locale \\
\hline & 20680 & arza beturiu & fondo interno & $\begin{array}{l}\text { piatto da pesce, } \\
\text { v.n. locale }\end{array}$ \\
\hline & 20681 & chi & fondo interno & ciotola, v.n. riminese \\
\hline & 20682 & chi & fondo interno & ciotola, v.n. locale \\
\hline \multirow{4}{*}{$\begin{array}{l}\text { Ospedale civile } 1993, \text { T. } 1 \text {, } \\
\text { inumazione in fossa, } \\
\text { prima metà del III sec. a.C. }\end{array}$} & 20665 & alpha & fondo interno & $\begin{array}{l}\text { piattello su alto } \\
\text { piede, v.n. spinetica }\end{array}$ \\
\hline & 20666 & alpha & fondo interno & $\begin{array}{l}\text { piatto, v.n. } \\
\text { probabilmente } \\
\text { spinetica }\end{array}$ \\
\hline & 20667 & alpha & fondo interno & $\begin{array}{l}\text { kylix, v.n. } \\
\text { probabilmente locale }\end{array}$ \\
\hline & 20668 & graffito non det. & fondo esterno & $\begin{array}{l}\text { ciotola, v.n. locale } \\
\text { o spinetica }\end{array}$ \\
\hline
\end{tabular}

Verso sud-ovest, in località Ca' Garzoni doveva estendersi parte dell'estrema propaggine occidentale della necropoli. Qui, lungo il canale Dragonzo, furono effettuate tre campagne di scavo nel 1966, 1969 e 1972, che permisero il recupero di 211 tombe, databili genericamente tra il III sec. a.C. e la fine del I sec. d.C. ${ }^{105}$. Si contano 18 sepolture con documenti epigrafici (Tab. 10), distribuite diacronicamente secondo una tendenza inversa a quella di Canalbianco ( 5 di III sec. a.C., 2 databili tra la seconda metà del III e la prima metà del II sec. a.C., 10 di II sec. a.C. e 1 più genericamente databile tra III e II sec. a.C.).

Tab. 10. Tombe da Ca' Garzoni con documenti epigrafici (tutti realizzati dopo la cottura).

\begin{tabular}{|c|c|c|c|c|}
\hline Contesto & $C I E$ & TIPO DI EPIGRAFE & Posizione & SUPPORTO \\
\hline \multirow{3}{*}{$\begin{array}{l}\text { T. 107/1969, inumazione, } \\
\text { seconda metà del III sec. a.C. }\end{array}$} & 20705 & ypsilon & parete esterna & $\begin{array}{l}\text { ciotola, v.n. di prod. } \\
\text { incerta }\end{array}$ \\
\hline & 20706 & $\begin{array}{l}\text { graffito non det. } \\
\text { (àncora?) }\end{array}$ & fondo esterno & ciotola, v.n. riminese \\
\hline & 20707 & $I X \circ X I$ & fondo esterno & $\begin{array}{l}\text { ciotola v.n. di prod. } \\
\text { incerta }\end{array}$ \\
\hline $\begin{array}{l}\text { T. } 155 / 1972 \text {, inumazione in fossa, } \\
\text { seconda metà del III sec. a.C. }\end{array}$ & 20727 & ypsilon & fondo esterno & $\begin{array}{l}\text { coppa, ceramica } \\
\text { grigia }\end{array}$ \\
\hline \multirow{2}{*}{$\begin{array}{l}\text { T. } 116 / 1969 \text {, inumazione in fossa } \\
\text { (forse femminile), } \\
\text { fine III sec. a.C. }\end{array}$} & 20724 & ypsilon & fondo interno & ciotola, v.n. locale \\
\hline & 20725 & graffito non det. e $X X I$ & fondo interno & ciotola, v.n. riminese \\
\hline $\begin{array}{l}\text { T. } 157 / 1972 \text {, forse inumazione, } \\
\text { III sec. a.C. }\end{array}$ & 20729 & $\begin{array}{l}\text { due } A \text { (lat.) } \\
\text { contrapposto }\end{array}$ & parete esterna & ciotola, v.n. locale \\
\hline
\end{tabular}

${ }_{105}$ Atria 1989, pp. 458-460, n. 398. Soltanto le 55 tombe scavate nel 1966 sono state pubblicate (MANGANi 1982, pp. 5-107), mentre le restanti sono ancora inedite (per una notizia preliminare dello scavo del 1969, cfr. FogolARI, SCARFì 1970 , p. 24, nota 22). 


\begin{tabular}{|c|c|c|c|c|}
\hline Contesto & $C I E$ & TIPO DI EPIGRAFE & Posizione & SUPPORTO \\
\hline $\begin{array}{l}\text { T. } 198 / 1972 \text {, inumazione in fossa } \\
\text { forse femminile, } \\
\text { III sec. a.C. }\end{array}$ & 20733 & segno a croce & fondo interno & ciotola, v.n. locale \\
\hline $\begin{array}{l}\text { T. } 156 / 1972 \text {, inumazione in fossa, } \\
\text { seconda metà del III-prima metà } \\
\text { del II sec. a:C. }\end{array}$ & 20728 & ypsilon & fondo interno & ciotola, v.n. locale \\
\hline \multirow{3}{*}{$\begin{array}{l}\text { T. } 183 / 1972 \text {, inumazione in fossa, } \\
\text { seconda metà del III-prima metà } \\
\text { del II sec. a.C. }\end{array}$} & 20730 & $c h i$ & fondo interno & piatto, v.n. locale \\
\hline & 20731 & segno a croce & fondo interno & $\begin{array}{l}\text { coppa, ceramica } \\
\text { grigia }\end{array}$ \\
\hline & 20732 & segno a croce & fondo interno & ciotola, v.n. locale \\
\hline \multirow{15}{*}{$\begin{array}{l}\text { T. } 111 / 1969 \text {, inumazione in fossa, } \\
\text { inizi/primi decenni del II sec. a.C. }\end{array}$} & 20709 & $\begin{array}{l}\text { mura (a), graffito non } \\
\text { det. (b) }\end{array}$ & $\begin{array}{l}\text { fondo interno } \\
\text { (a), fondo } \\
\text { esterno (b) }\end{array}$ & ciotola, v.n. locale \\
\hline & 20710 & due ypsilon opposti & parete esterna & piatto, v.n. locale \\
\hline & 20711 & $\begin{array}{l}\text { segno a croce e graffito } \\
\text { non det. }\end{array}$ & parete esterna & piatto, v.n. locale \\
\hline & 20712 & ypsilon & fondo interno & ciotola, v.n. locale \\
\hline & 20713 & ypsilon (a), chi (b) & $\begin{array}{l}\text { parete esterna } \\
\text { (a), fondo } \\
\text { esterno (b) }\end{array}$ & ciotola, v.n. locale \\
\hline & 20714 & $\begin{array}{l}\text { graffito non det. e } \\
\text { segno a croce (parti } \\
\text { opposte rispetto } \\
\text { all'attacco del piede) }\end{array}$ & parete esterna & piatto, v.n. locale \\
\hline & 20715 & segno a croce & parete esterna & ciotola, v.n. locale \\
\hline & 20716 & $\begin{array}{l}\text { segno a croce (a), } \\
\text { due aste (b), graffito } \\
\text { non det. (c) }\end{array}$ & $\begin{array}{l}\text { parete esterna } \\
\text { (a), parete } \\
\text { esterna (b), } \\
\text { fondo esterno } \\
\text { (c) }\end{array}$ & ciotola, v.n. locale \\
\hline & 20717 & $\begin{array}{l}\text { graffito non det. } \\
\text { lacunoso }\end{array}$ & fondo interno & ciotola, v.n. locale \\
\hline & 20718 & $\begin{array}{l}\text { segno a croce }(a) \\
\text { segno a croce }(b)\end{array}$ & $\begin{array}{l}\text { fondo interno } \\
\text { (a), parete } \\
\text { esterna (b) }\end{array}$ & ciotola, v.n. locale \\
\hline & 20719 & $\begin{array}{l}\text { segno a croce }(a), \\
\text { segno a croce }(b)\end{array}$ & $\begin{array}{l}\text { parete interna } \\
\text { (a), parete } \\
\text { esterna }(\mathrm{b})\end{array}$ & ciotola, v.n. locale \\
\hline & 20720 & $\begin{array}{l}\text { segno a croce }(a), \\
\text { segno a croce }(b)\end{array}$ & $\begin{array}{l}\text { fondo interno } \\
\text { (a), parete } \\
\text { esterna (b) }\end{array}$ & ciotola, v.n. locale \\
\hline & 20721 & segno a croce & parete esterna & ciotola, v.n. locale \\
\hline & 20722 & segno a croce & parete esterna & $\begin{array}{l}\text { grande ciotola con } \\
\text { due scanalature } \\
\text { all'orlo, v.n. locale }\end{array}$ \\
\hline & 20723 & $\begin{array}{l}\text { graffito non det. (a), } \\
\text { due segni a croce (b) }\end{array}$ & $\begin{array}{l}\text { parete interna } \\
\text { (a), parete } \\
\text { esterna }(b)\end{array}$ & ciotola, v.n. locale \\
\hline
\end{tabular}




\begin{tabular}{|c|c|c|c|c|}
\hline Contesto & $C I E$ & TIPO DI EPIGRAFE & Posizione & SUPPORTO \\
\hline \multirow{4}{*}{$\begin{array}{l}\text { T. } 75 / 1969 \text {, inumazione in cassa lignea } \\
\text { probabilmente femminile, } \\
\text { prima metà del II sec. a.C. }\end{array}$} & 20698 & puinia mị & fondo interno & $\begin{array}{l}\text { grande ciotola con } \\
\text { due scanalature } \\
\text { all'orlo, v.n. locale }\end{array}$ \\
\hline & 20699 & latiate & fondo interno & ciotola, v.n. locale \\
\hline & 20700 & $\begin{array}{l}\text { segno a croce }(a), \\
\text { segno a croce }(b) \\
\text { segno a croce }(c)\end{array}$ & $\begin{array}{l}\text { fondo interno } \\
\text { (a), parete } \\
\text { esterna (b), } \\
\text { fondo esterno (c) }\end{array}$ & ciotola, v.n. locale \\
\hline & 20701 & $\begin{array}{l}\text { segno a croce (a), } \\
\text { graffito non det. (b) }\end{array}$ & $\begin{array}{l}\text { parete interna } \\
(\mathrm{a}), \text { parete } \\
\text { esterna }(\mathrm{b})\end{array}$ & ciotola, v.n. locale \\
\hline $\begin{array}{l}\text { T. } 199 / 1972 \text {, inumazione in fossa } \\
\text { forse femminile, } \\
\text { II sec., forse prima metà a.C. }\end{array}$ & 20734 & segno a croce & parete esterna & $\begin{array}{l}\text { grande ciotola con } \\
\text { due scanalature } \\
\text { all'orlo, v.n. locale }\end{array}$ \\
\hline $\begin{array}{l}\text { T. 19/1966, inumazione forse infantile, } \\
\text { seconda metà del II sec. a.C. }\end{array}$ & 20692 & segno a croce & fondo interno & piatto, v.n. locale \\
\hline $\begin{array}{l}\text { 38/1966, inumazione, } \\
\text { seconda metà del II sec. a.C. }\end{array}$ & 20694 & be.me & fondo interno & $\begin{array}{l}\text { ciotola, v.n. } \\
\text { volterrana }\end{array}$ \\
\hline \multirow{2}{*}{$\begin{array}{l}\text { T. } 41 / 1966 \text {, inumazione infantile, } \\
\text { seconda metà del II sec. a.C. }\end{array}$} & 20695 & alpha & fondo interno & ciotola, v.n. locale \\
\hline & 20696 & ypsilon & vasca interna & ciotola, v.n. locale \\
\hline $\begin{array}{l}\text { T. } 43 / 1966 \text {, inumazione, } \\
\text { seconda metà del II sec. a.C. }\end{array}$ & 20697 & kraiku.kaniu. & fondo esterno & $\begin{array}{l}\text { grande ciotola con } \\
\text { due scanalature } \\
\text { all'orlo, v.n. locale }\end{array}$ \\
\hline \multirow{3}{*}{$\begin{array}{l}\text { T. } 105 / 1969 \text {, inumazione bisoma } \\
\text { in fossa, } \\
\text { seconda metà del II sec. a.C. }\end{array}$} & 20702 & graffito non det. & fondo esterno & pisside, v.n. locale \\
\hline & 20703 & graffito non det. & fondo esterno & pisside, v.n. locale \\
\hline & 20704 & $\dot{s u}$ & spalla & anfora greco-italica \\
\hline $\begin{array}{l}\text { T. } 29 / 1966 \text {, inumazione infantile, } \\
\text { II sec. a.C. }\end{array}$ & 20693 & $A$ (lat.) & fondo interno & ciotola, v.n. locale \\
\hline $\begin{array}{l}\text { T. } 145 / 1972 \text {, inumazione, } \\
\text { II sec. a.C. }\end{array}$ & 20726 & segno a croce & fondo esterno & $\begin{array}{l}\text { coppa, ceramica } \\
\text { grigia }\end{array}$ \\
\hline $\begin{array}{l}\text { T. } 108 / 1969 \text {, inumazione, } \\
\text { III-II sec. a.C. }\end{array}$ & 20708 & $\begin{array}{l}\text { graffito non det. } \\
\text { lacunoso }\end{array}$ & parete esterna & ciotola, v.n. riminese \\
\hline
\end{tabular}

L'assenza di uno studio d'insieme dei contesti indagati nel 1969 e nel 1972 preclude la possibilità di una effettiva comparazione tra questi dati e quelli totali dei tre anni di scavo. L'edizione di quelle indagate nel 1966 rivela come su 20 datate anteriormente al I sec. a.C., 8 siano a cremazione ${ }^{106}$, cioè un rapporto di circa 3:2, anomalo per il milieu adriese caratterizzato dalla netta prevalenza del rito inumatorio. In ogni caso, tutte le tombe con documentazione epigrafica qui sono a inumazione. Questo dato, assieme all'alta frequenza delle tombe infantili evidenziata da E. Mangani per il nucleo scavato in questo anno (su 12 inumazioni, 4 sono riconosciute di adulti e 8 infantili ${ }^{107}$ ) porta a ritenere che quest' area avesse una sua specificità di destinazione (Fig. 30). Sempre all'interno del nucleo del 1966, fra le 4 tombe di adulto, due conservano iscrizioni (tombe 38 e 43), mentre tra le infantili si hanno tre tombe $(19,29$ e 41$)$ con graffiti alfabetici e non, ma nessuna con iscrizioni. Purtroppo non è possibile

\footnotetext{
106 Mangani 1982, p. 99.

107 Ibid., p. 100.
} 


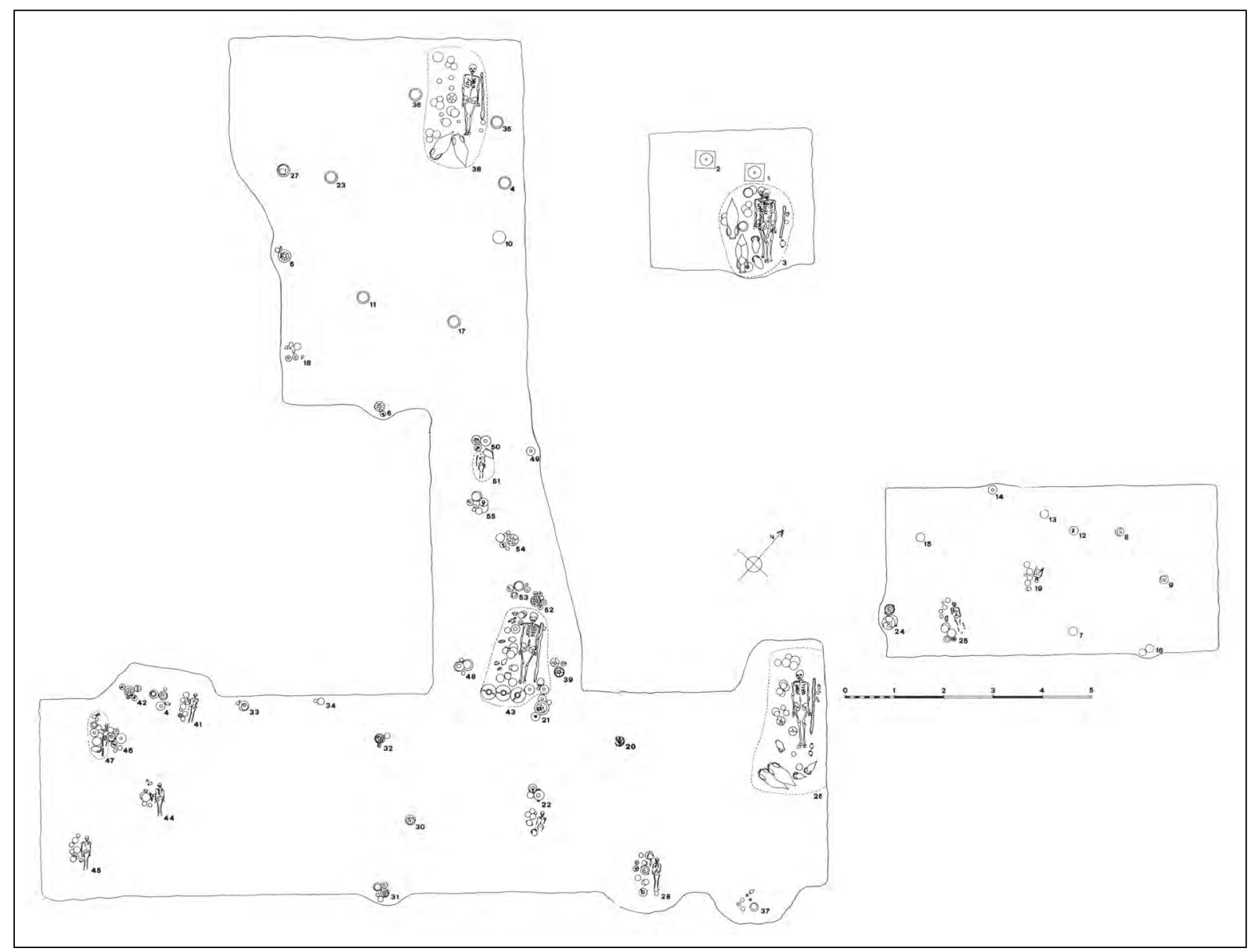

Fig. 30. Planimetria dell'area di Ca’ Garzoni indagata nel 1966 (da Mangani 1982).

mettere a sistema questi dati con quelli delle tombe scavate successivamente, in quanto ancora non studiati nel loro complesso. Si può soltanto osservare che negli anni 1969 e 1972 non si registrano altre tombe infantili con materiale epigrafico. Anche l'analisi spaziale riserva poche informazioni. Si dispone infatti solo dello studio accurato di E. Mangani per lo scavo 1966, dove le 5 tombe con documentazione epigrafica non presentano significative aggregazioni ${ }^{108}$. Nel complesso, è evidente che Ca' Garzoni manifesta caratteristiche che suggeriscono pratiche funerarie speciali, che si auspica possano essere sistematicamente indagate nel futuro.

A est di Canalbianco, a parte una isolata tomba di IV sec. a.C., rinvenuta presso l'attuale via Chieppara $^{109}$ e che ha restituito un vaso con due graffiti alfabetici (CIE 20735), e le 8 sepolture mal documentate nel fondo Baseggio scavato nel $1964^{110}$, di cui una di rito incerto e da cui proviene una coppa in ceramica grigia con un graffito alfabetico (CIE 20738), un numero significativo di testimonianze proviene dall'area funeraria di Piantamelon scavata nel 1996 (Tab. 11 $)^{111}$. Qui, nella terza più grande area funeraria per ordine di grandezza del settore meridionale dopo Canalbianco e Ca' Garzoni, su 110 contesti compresi tra il III sec. a.C. e il I sec. d.C. e distribuiti in tre nuclei distinti (uno nell'area c.d. A e due nell'area c.d. B), 17 registrano testimonianze epigrafiche.

108 Si osserva l'assenza nella planimetria (Fig. 30) della tomba 29.

109 Nella fattispecie, presso il Canapificio "Val di Susa" distrutto durante la Seconda Guerra Mondiale (dove ora sorge il Centro Commerciale "Il Porto"). Si veda sulle vicende storiche di questo luogo Rondina 2014, p. 73 , nota 92.

110 Atria 1989, p. 470, n. 407.

111 Mosca, Puppo 2000, p. 135. 
Tab. 11. Tombe da Piantamelon (scavo 1996) con documenti epigrafici (intesi dopo la cottura se non specificato).

\begin{tabular}{|c|c|c|c|c|}
\hline Contesto & $C I E$ & TIPO DI EPIGRAFE & Posizione & SUPPORTO \\
\hline $\begin{array}{l}\text { T. } 4 \text {, rito incerto, } \\
\text { fine del IV-prima metà del III sec. a.C. }\end{array}$ & 20747 & segno a croce & parete esterna & $\begin{array}{l}\text { piatto da pesce, v.n. locale } \\
\text { o spinetica }\end{array}$ \\
\hline $\begin{array}{l}\text { T. } 7 \text {, inumazione in cassa di legno, } \\
\text { III sec. a.C. }\end{array}$ & 20748 & $\begin{array}{l}\text { segno a croce (inciso a } \\
\text { crudo) }\end{array}$ & fondo esterno & $\begin{array}{l}\text { anfora da tavola, ceramica } \\
\text { depurata }\end{array}$ \\
\hline $\begin{array}{l}\text { T. } 19 \text {, inumazione in fossa, } \\
\text { III sec. a.C. }\end{array}$ & 20754 & graffito non det. & fondo interno & kylix, v.n. di prod. incerta \\
\hline \multirow{2}{*}{$\begin{array}{l}\text { T. } 104 \text {, inumazione infantile in fossa, } \\
\text { III sec. a.C. }\end{array}$} & 20770 & $c h i$ & fondo interno & ciotola, v.n. locale \\
\hline & 20771 & segno a croce & fondo esterno & mortaio, ceramica grigia \\
\hline $\begin{array}{l}\text { T. } 10 \text {, inumazione in fossa, } \\
\text { inizi II sec. a.C. }\end{array}$ & 20749 & $s p$ & fondo interno & ciotola, v.n. locale \\
\hline \multirow{3}{*}{$\begin{array}{l}\text { T. } 80 \text {, inumazione infantile in fossa, } \\
\text { metà del II sec. a.C. }\end{array}$} & 20762 & pruka & fondo interno & ciotola, v.n. locale \\
\hline & 20763 & segno a croce & fondo esterno & ciotola, v.n. locale \\
\hline & 20764 & $\begin{array}{l}\text { graffito non det. } \\
\text { lacunoso }\end{array}$ & fondo interno & piatto, v.n. locale \\
\hline $\begin{array}{l}\text { T. } 1 \text {, inumazione, } \\
\text { II sec. a.C. }\end{array}$ & 20739 & $c h i$ & parete interna & ciotola, v.n. locale \\
\hline \multirow{7}{*}{$\begin{array}{l}\text { T. } 3 \text {, inumazione, } \\
\text { II sec. a.C. }\end{array}$} & 20740 & alpha (?) & parete esterna & piatto, v.n. locale \\
\hline & 20741 & ypsilon e segno a croce & parete esterna & ciotola, v.n. locale \\
\hline & 20742 & segno a croce & fondo interno & ciotola, v.n. locale \\
\hline & 20743 & graffito non det. & fondo interno & ciotola, v.n. locale \\
\hline & 20744 & $\begin{array}{l}\text { graffito non det. } \\
\text { (asterisco?) }\end{array}$ & fondo esterno & ciotola, v.n. locale \\
\hline & 20745 & asterisco (?) & fondo esterno & ciotola, v.n. locale \\
\hline & 20746 & graffito non det. & fondo esterno & ciotola, v.n. locale \\
\hline \multirow{3}{*}{$\begin{array}{l}\text { T. } 17 \text {, inumazione in fossa, } \\
\text { II sec. a.C. }\end{array}$} & 20751 & $\begin{array}{l}\operatorname{nota}(\mathrm{a}) \\
\text { segno a croce }(\mathrm{b})\end{array}$ & $\begin{array}{l}\text { fondo interno } \\
\text { (a), fondo } \\
\text { esterno }(b)\end{array}$ & ciotola, v.n. volterrana \\
\hline & 20752 & segno a croce & fondo interno & ciotola, v.n. locale \\
\hline & 20753 & asterisco & fondo interno & ciotola, v.n. locale \\
\hline \multirow{2}{*}{$\begin{array}{l}\text { T. } 24 \text {, inumazione in fossa forse } \\
\text { femminile, } \\
\text { II sec. a.C. }\end{array}$} & 20755 & $\begin{array}{l}\text { graffito non det. (a), } \\
\text { chi (b) }\end{array}$ & $\begin{array}{l}\text { fondo interno } \\
\text { (a), fondo } \\
\text { esterno (b) }\end{array}$ & ciotola, v.n. locale \\
\hline & 20756 & segno a croce & parete esterna & piatto, v.n. locale \\
\hline $\begin{array}{l}\text { T. } 31 \text {, forse inumazione, } \\
\text { II sec. a.C. }\end{array}$ & 20758 & segno a croce & fondo interno & ciotola, v.n. locale \\
\hline $\begin{array}{l}\text { T. } 50 \text {, inumazione in fossa forse } \\
\text { femminile, } \\
\text { II sec. a.C. }\end{array}$ & 20759 & graffito non det. & fondo interno & piatto, v.n. locale \\
\hline \multirow{2}{*}{$\begin{array}{l}\text { T. } 83 \text {, inumazione in cassone ligneo } \\
\text { probabilmente femminile, } \\
\text { II sec. a.C. }\end{array}$} & 20765 & au (alfabeto leponzio) & parete esterna & piatto, v.n. locale \\
\hline & 20766 & $c h i$ & fondo interno & ciotola, v.n. locale \\
\hline
\end{tabular}




\begin{tabular}{|c|c|c|c|c|}
\hline Contesto & $C I E$ & TIPO DI EPIGRAFE & Posizione & SUPPORTO \\
\hline \multirow{3}{*}{$\begin{array}{l}\text { T. } 85 \text {, inumazione in cassa di legno, } \\
\text { II sec. a.C. }\end{array}$} & 20767 & $c h i$ & parete esterna & piccola ciotola, v.n. locale \\
\hline & 20768 & $\begin{array}{l}\text { segno a croce }(a) \text {, } \\
\text { segno a croce }(b)\end{array}$ & $\begin{array}{l}\text { fondo interno } \\
\text { (a), parete } \\
\text { esterna (b) }\end{array}$ & ciotola, v.n. locale \\
\hline & 20769 & $\begin{array}{l}\text { graffito non det. } \\
\text { (uguale ai graffiti } \\
20760-20761 \text { ) }\end{array}$ & fondo interno & piatto, v.n. locale \\
\hline \multirow{2}{*}{$\begin{array}{l}\text { T. } 74 \text {, inumazione in cassa di legno, } \\
\text { seconda metà del II- inizi del } \\
\text { I sec. a.C. }\end{array}$} & 20760 & $\begin{array}{l}\text { due graffiti non det. } \\
\text { uguali }(a-b)\end{array}$ & $\begin{array}{l}\text { fondo interno } \\
\text { (a), parete } \\
\text { esterna (b) }\end{array}$ & ciotola, v.n. locale \\
\hline & 20761 & $\begin{array}{l}\text { graffito non det. } \\
\text { (uguale a 20760) }\end{array}$ & fondo interno & piatto, v.n. locale \\
\hline $\begin{array}{l}\text { T. 26, inumazione in fossa, } \\
\text { età ellenistica }\end{array}$ & 20757 & $\begin{array}{l}\text { graffito non det. } \\
\text { lacunoso }\end{array}$ & fondo interno & ciotola, v.n. locale \\
\hline $\begin{array}{l}\text { T. } 14 \text {, inumazione in fossa, } \\
\text { età ellenistica }\end{array}$ & 20750 & segno a croce & fondo esterno & coppa, ceramica grigia \\
\hline
\end{tabular}

Non è purtroppo possibile fornire un quadro del sepolcreto mancando anche qui uno studio sistematico, ma solo una stima orientativa basata sulla documentazione di scavo (da usare dunque con molta cautela): nell'area A si avrebbero circa 25 tombe, di cui solo una dubitativamente a cremazione, mentre le restanti a inumazione; nell'area B, circa 25 tombe (per alcune è fornita una datazione tra II e I sec. a.C.), delle quali 7 a cremazione ${ }^{112}$. Ammesso che queste cifre si avvicinino al vero, si avrebbe un rapporto di 2:3 tra tombe con documenti epigrafici e tombe che ne sono prive. Le 17 sepolture con documenti epigrafici sono tutte a inumazione a eccezione di una di rito incerto, e si concentrano nel II sec. a.C. (fine del IV-III sec. a.C.: 4, fra cui quella di rito incerto; II sec. a.C.: 11; 2 sono più genericamente databili al periodo ellenistico). Le tombe con iscrizioni sono solo due di II sec. a.C., dunque una proporzione molto bassa rispetto a Canalbianco, ma che si avvicina a quella dell'altra area periferica, cioè Ca' Garzoni. Sebbene non si abbia un quadro complessivo dei sepolcreti finora noti del settore a sud della città, tuttavia le considerazioni desunte dalle tombe con documentazione epigrafica suggerirebbero un uso più intenso delle aree più periferiche nel II sec. a.C. Tale tendenza non desta sopresa, sebbene possa essere confermata solo da uno studio sistematico di tutti i contesti, e darebbe maggior solidità all'idea di un'unica vasta necropoli che si estenderebbe per circa $2,5 \mathrm{~km}$ in senso est-ovest.

Per quanto riguarda Piantamelon, la consultazione delle planimetrie inedite disponibili presso l'Archivio del Museo Archeologico Nazionale di Adria permettono l'individuazione delle singole tombe con documentazione epigrafica all' interno dei tre nuclei, cioè quello denominato come area A e i due fra loro prossimi dell'area B. Nell'area A, le tombe del nucleo non sembrano disposte secondo un ordine coerente e le 10 sepolture con graffiti non si coagulano, se non per le tombe 19 , $14,17,24,26,10,4$, che si allineano vagamente in senso nord-sud, mentre verso est le tombe 1 e 3 sono fra loro vicine. Nell'area $\mathrm{B}$, il numero più ridotto di sepolture con documenti epigrafici, 7 in totale, si distribuisce fra i due nuclei, quello più numeroso meridionale con le tombe $31,50,74 \mathrm{e}$ 104 , distribuite senza una particolare concentrazione, e nell'altro nucleo più contenuto (14 totali) le tombe 80,83 e 85 di II sec. a.C., fra loro vicine e vagamente allineate secondo un asse est-ovest. Oltre a documentare ognuna più di un vaso graffito, si registra la significativa presenza di una iscri-

112 Si rimanda alla Relazione di G. Valli, Adria (RO) - Canal Bianco - 1996 - Lavori di bonifica archeologica Ponte Amolara, conservata presso l'Archivio del Museo Archeologico Nazionale di Adria. 
zione etrusca in onomastica celtica (pruka; cfr. IV.3.1.B, categoria d) nella tomba 80 infantile e di un digramma in alfabeto leponzio (au) nella vicina tomba 83 di adulto, forse di genere femminile.

Si presenta di seguito una analisi complessiva delle sepolture con documentazione epigrafica del settore meridionale dei sepolcreti urbani, distinguendole per fasi cronologiche: III sec. a.C. (VI.2.1.A), fine III-inizi II sec. a.C. (VI.2.1.B), II sec. a.C. (VI.2.1.C).

\section{VI.2.1.A. III sec. a.C.}

Le sepolture con documenti epigrafici sono 42 (33 da Canalbianco, 5 da Ca' Garzoni, 4 da Piantamelon), a cui si aggiungono 11 contesti da Canalbianco, nei quali i documenti sono di incerta attribuzione. Sono tutte inumazioni, a eccezione di due cremazioni (tombe 131 e 177 di Canalbianco, la prima datata alla fine del secolo e la seconda più genericamente al secolo ${ }^{113}$ ). Nessuna sepoltura presentava un segnacolo al momento del rinvenimento e avevano tutte una profondità compresa tra 2 e $3 \mathrm{~m}$ dal piano di campagna, orientamento OE o NO-SE, con la testa del defunto (quando il dato è registrato) a $\mathrm{O}$ o NO. Il corredo era disposto prevalentemente a destra del defunto e composto perlopiù da ceramica a v.n. di produzione locale, con una presenza molto ridotta di importazioni dal Volterrano, perlopiù concentrate nelle tombe della prima metà-metà del secolo, e da Rimini (circoscritte per quanto noto ad alcune ciotole serie Morel 1552 databili dopo la metà del secolo); inoltre pare evidente una diffusa presenza di ceramica alto-adriatica per tutto il secolo in esame (con una prevalenza di lekanides, ma anche mykai, oinochoai, crateri, anfore e piatti tipici della produzione adriese $^{114}$ ), di contro all'unicità dell'attestazione di ceramica figurata etrusca dalla tomba 327 di Canalbianco ${ }^{115}$. Più in genereale, tra il vasellame a v.n. il numero delle importazioni è molto ridotto, limitandosi a poche forme ${ }^{116}$, di contro a una grande varietà di forme di produzione locale con una netta prevalenza quantitativa di ciotole e solo in misura molto minore piatti, skyphoi, olpai, ollette, askoi. In particolare, nei corredi le ciotole si presentano generalmente in un numero variabile tra le 2 alle 13 unità, comunque non scendendo mai sotto le 5 dopo la metà del secolo. Risultano invece poco rappresentate altre classi quali la ceramica grigia (mortai, coppe, skyphoi, olle, oinochoai), ceramica depurata (mortai, coppe, olle, anfore, lekanides, bacili, situle, pelikai, amphoriskoi, coperchi), ceramica grezza (olle e coppecoperchio).

Le sepolture che conservano iscrizioni etrusche sono 13 (di cui 2 tra quelle di incerta attribuzione). Queste appartengono tutte alla necropoli di Canalbianco e la prevalenza sembra concentrata tra la prima metà-metà del secolo, mentre nella seconda metà si registra un aumento del numero di sepolture con vasi con graffiti alfabetici e non alfabetici (Fig. 31). Nei pochi casi documentati di posizioni certe, i vasi iscritti si collocano sia lungo il fianco destro che ai piedi del defunto.

Nella prima metà del III sec. a.C. la ciotola a v.n. locale (quando definibile la produzione) è il supporto privilegiato per le iscrizioni, con l'unica eccezione della tomba 309, dove è scelta una coppa emisferica in ceramica grigia. Solo nelle sepolture databili entro il pieno III sec. a.C. pare più articolata la scelta dei supporti, in quanto si documentano le uniche kylikes iscritte dell'intera necropoli (tombe 67 e 132), di cui almeno una volterrana, e un piatto (tomba 85; Fig. 35). Un piatto è il supporto anche dell' iscrizione etrusca della tomba 154, purtroppo solo genericamente databile al secolo. Sebbene non appartenente alla cultura e alla lingua etrusca e probabilmente tracciata prima dell'arrivo del contenitore ad Adria, sembra comunque importante segnalare l'eccezionale iscrizione latina CIE 20577

113 La tomba 177 è nota per la patera ombelicata a v.n. con apoteosi di Eracle a rilievo, datata entro la prima metà del III sec. a.C. (da ultimo si veda GAmbaCurTa 2015, p. 81, 2.1.19, con precedente bibliografia). Per il contesto, si veda SANESI Mastrocineue 1982.

114 Su questa classe, si rinvia a RoBIno 2000 per i vasi per versare; EAD. 2003 per crateri, skyphoi e stamnoi.

115 MANGANi 1980; CAMERIN 1996. Si osserva peraltro che quest'ultima classe, già riconosciuta come di prestigio nei contesti adriesi (STEFANI 1996-1997, p. 164), non pare essere attestata oltre gli inizi del secolo seguente.

116 Piatto serie Morel 1314, ciotola serie Morel 1552, kylix serie Morel 3321, 4111, 4115, patera serie Morel 2175, patera ombelicata, kantharos, olpe. 


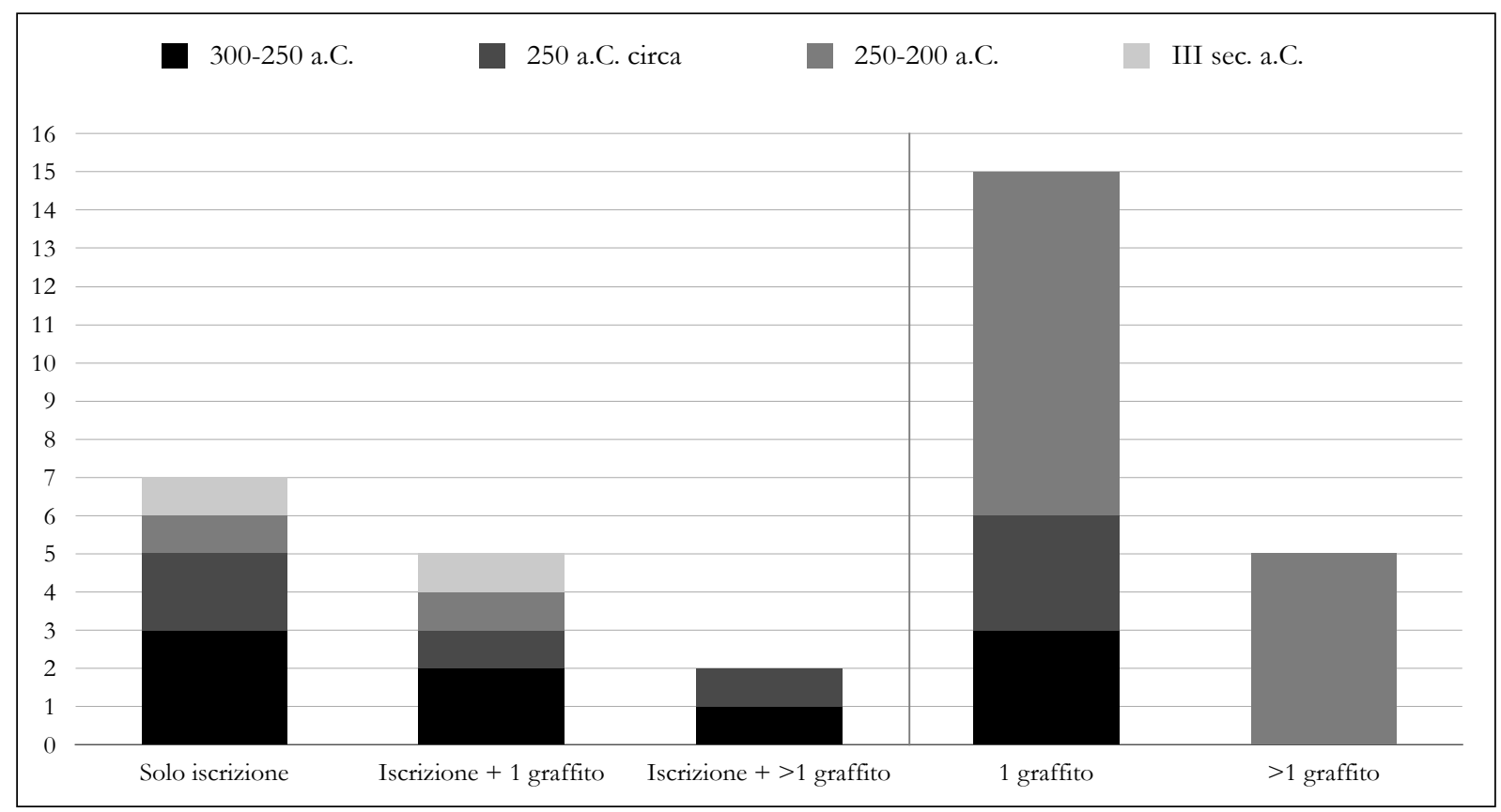

Fig. 31. Quantificazione su base diacronica delle tombe con documentazione epigrafica durante il III sec. a.C. nella necropoli di Canalbianco.

su anfora, attribuita con incertezza alla tomba 250 e che ci documenterebbe un interessante contatto commerciale con intermediari romani probabilmente localizzabili nel medio Adriatico ${ }^{117}$.

La speciale scelta della ceramica grigia e della kylix all'interno delle forme a v.n. necessita alcune considerazioni aggiuntive, di seguito esposte. La coppa in ceramica grigia della tomba 309 , assieme ad altre due iscritte (CIE 20601, per la quale il corredo di attribuzione rimane incerto, e CIE 20464 attribuita ai confusi contesti delle tombe 54-55, collocabili comunque con qualche incertezza entro il III sec. a.C.), rappresentano gli unici casi di iscrizioni in ceramica grigia di tutti i sepolcreti urbani di Adria, a cui si può aggiungere solo quella della tomba 20 del probabile insediamento periferico di Bottrighe, più antica di qualche decennio (per ulteriori considerazioni sulla scelta della ceramica grigia nel IV sec. a.C., cfr. II.1.5). È interessante evidenziare come le tre iscrizioni di Canalbianco manifestino lo stesso lessema helis, isolato (CIE 20601) o all'interno di testi caratterizzati dalla medesima struttura formulare (cfr. IV.3.2, classe V). Proprio le formule suggeriscono la corrispondenza fra il lessema e il supporto (meno probabilmente il contenuto, visto che tutte e tre le iscrizioni sono realizzate nella vasca interna così compromettendone la capacità contenitiva della superficie) nella sua particolare accezione di oggetto funerario, forse quale dono destinato al defunto. Non è tuttavia chiaro quale sia il ruolo di Uvasave e Nalske, (cfr. IV.3.1.B, categoria d), se donatori o i defunti medesimi.

La scelta della kylix, vaso raramente supporto di iscrizione, trova una sostanziale corrispondenza nelle necropoli spinetiche, dove l'analisi proposta per queste evidenze aveva portato a ritenere che il vaso fosse un oggetto che simbolicamente evocava il consumo del vino e perlopiù andasse interpretato come dono funebre ${ }^{118}$. Una kylix con iscrizione è testimoniata anche nella tomba 9/1993 di Ca' Cima (cfr. VI.2.2), dove tuttavia la coerenza di un intero set iscritto e la probabile esplicitazione del nome del defunto marcano un fenomeno che va distinto almeno sul piano formale. Come per Spina, anche in Canalbianco vediamo che in un caso, quello della tomba 132, l'iscrizione è posta nella vasca interna, mentre in quello della tomba 67 nella parete esterna, cioè una parte del vaso piuttosto

\footnotetext{
117 REI LXXIX, p. 348.

118 GaUCCI 2016, pp. 184-186.
} 


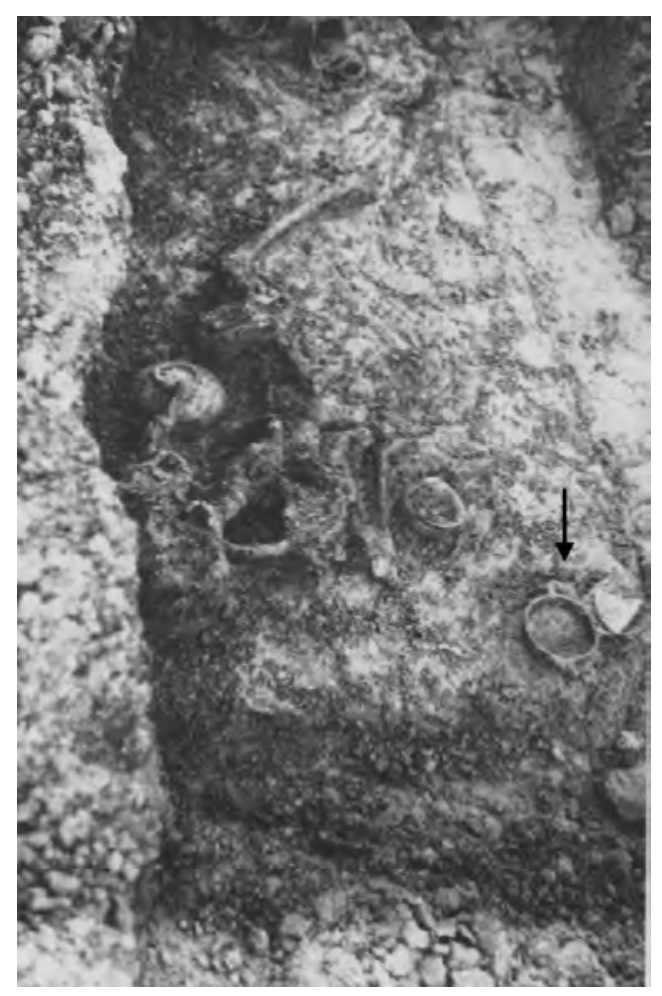

Fig. 32. Fotografia di scavo della tomba 132 di Canalbianco con indicazione della kylix iscritta (dall'Archivio del Museo Archeologico Nazionale di Adria).

eccezionale per la redazione di una iscrizione nel III sec. a.C. (cfr. II.2). Approfondendo l'analisi sulla base della documentazione di scavo dei contesti delle due kylikes di Canalbianco, si osserva che la fotografia della tomba 132 (Fig. 32) permette alcune considerazioni: il vaso era posto con la vasca verso l'alto in posizione defilata rispetto al resto del corredo, a sud-est oltre i piedi del defunto (si notano nella parte alta della fotografia i frammenti dello skyphos a v.n., da supporre a destra della testa), in associazione a un altro vaso frammentario (non confrontabile con alcuno dei conservati) e a sud-est dell'olletta in grezza (inv. 1793), alta poco più di $8 \mathrm{~cm}$ e di cui emerge dal terreno unicamente l'orlo. Una delle due anse doveva essere lacunosa già al momento della deposizione, forse staccata secondo un processo di defunzionalizzazione che trova corrispondenza in alcune evidenze spinetiche. Dunque, la kylix sembra essere dissociata dal nucleo principale del corredo, posto di prassi alla destra del defunto: non è azzardato supporre che sia stata posizionata come dono speciale, forse dopo il completamento della deposizione. Se valida questa lettura, va ribadito che si tratta di una pratica del tutto differente da quella evidenziata dalla tomba 9/1993 di Ca' Cima sopra richiamata. La struttura con pronome e genitivo del nome (cfr. IV.3.2, classe IV), come già evidenziato in sede di analisi delle formule, manifesterebbe anche in questo caso il proprietario, essendo peraltro la medesima formula di quella della tomba di Ca' Cima, ma nella fattispecie non si esclude che in alternativa mi leiuś possa piuttosto indicare un appellativo pertinente alla sfera della morte (es. un generico riferimento al defunto?) piuttosto che un antroponimo (cfr. IV.3.1.B). Purtroppo non si ha un pari livello di informazioni per la tombe 67 , la cui kylix presenta l'eccezionalità dell' iscrizione posta nella parete esterna. Anche qui la formula all'assolutivo chiusa dal pronome $m i$ potrebbe esplicitare il defunto, Laris Tetialus's, e la lacuna di entrambe le anse indica una probabile defunzionalizzazione del vaso. Tale tipo di defunzionalizzazione è ipotizzabile anche per una quarta kylix iscritta di origine incerta ma verosimilmente da necropoli, databile entro il secondo quarto del III sec. a.C. Nella vasca è redatto il gentilizio maschile preznaś (CIE 20819), dunque una formula nella quale si omette il prenome. Quest'ultimo caso, purtroppo privo di contesto, potrebbe indicare il soggetto donatore (soprattutto se si ipotizzasse il genitivo come afunzionale; cfr. IV.3.2, classe III.a), secondo una modalità documentata nella necropoli di Monte Tamburino presso Monte Bibele, dove in almeno 
due sepolture il nome femminile petnei all'assolutivo graffito nella parete esterna di una kylix faceva parte di corredi di defunti maschi ${ }^{119}$.

Ancora in merito ai supporti iscritti della prima metà-pieno III sec. a.C., la tomba 1 dell'Ospedale civile, sebbene non conservi iscrizioni ma solo graffiti alfabetici e non alfabetici, mostra alcune peculiarità nel rapporto tra vasi e documentazione epigrafica su cui conviene soffermarsi. Il corredo richiama aspetti della ritualità funeraria di Spina ${ }^{120}$, a cui punta anche il vasellame a v.n. attribuito dagli editori (in maniera convincente) alla produzione di quest' ultimo centro ${ }^{121}$. Nella vasca interna del piattello su alto piede e del piatto da pesce su alto piede (si ribadisce, forme caratteristiche di Spina) sono graffiti singoli alpha caratterizzati da un breve tratto a prolungamento verso il basso dell'asta verticale (CIE 20665-20666) e un alpha con medesima caratteristica è graffito anche nell'interno della kylix con anse non ripiegate a orecchia attribuita con incertezza a una produzione locale, comunque non etrusco-settentrionale (CIE 20667); anche una ciotola della serie Morel 2536 presenta un graffito nel fondo esterno di incerta interpretazione e solo dubitativamente interpretabile come alpha (CIE 20668), sebben non trovi somiglianza per aspetto con i tre precedenti. La lettera alpha dalla peculiarità del breve tratto sottoavanzante sembra dunque associare almeno tre vasi. L'unico altro caso di reiterazione di alpha in tomba è quello della tomba 349 di Canalbianco e della tomba 11 di Retratto-Donà, dove le lettere sono tutte nell'interno di ciotole a v.n. di produzione locale. Nel nostro caso, si potrebbe vedere nella reiterazione di questa lettera un legame tra kylix, piattello e piatto, secondo uno schema di selezione dei supporti che evoca almeno parzialmente quello delle iscrizioni della tomba 9/1993 di Ca' Cima (cfr. VI.2.2), cronologicamente affine. Da un punto di vista paleografico, colpisce inoltre il confronto con la realizzazione dell'alph a dell' iscrizione fastiu da contesto ignoto e sempre della prima metà del III sec. a.C. (CIE 20776). Se ne potrebbe desumere che il tratto verticale sottostante la lettera sia parte dell'aspetto della medesima, accomunando se non la mano almeno lo stile delle occorrenze trattate.

Passando all'onomastica e alle formule manifestate, si osserva come nella prima metà-pieno III sec. a.C., le iscrizioni, quando non lacunose (quella della tomba 310 conserva solo il pronome mi), presentino formule prevalentemente bimembri, che esplicitano nomi etruschi riferibili a individui maschi e ingenui (Laris Fuliu della tomba 327, Ata Śetina della tomba 243, Laris Tetialuś della tomba 67, Venza VelOuriu della tomba 154), mentre i due soli casi di nomi individuali presentano una onomastica di origine celtica, cioè l'iscrizione atakuś attribuita alla tomba 378 e la formula con il lessema helis (Uvasave). Il diversificato formulario suggerisce per alcuni l'identificazione con il destinatario del dono (IV.3.2, classe II.a), cioè il defunto (Laris Fuliu, Laris Tetialuś), per altri il donatore (Ata Śetina, Venza VelOuriu: classe I.b; Ataku: classe III.b). Tale corrispondenza potrebbe trovare conforto dal contesto della tomba 243, dove la presenza di una collana segnalerebbe come femminile la sepoltura, differenziandosi così dal nome maschile iscritto ripetuta su tre vasi ${ }^{122}$. Questo è forse valido anche per il contesto della tomba 154 (strappato durante lo scavo e subito esposto, ma poi andato perduto a eccezione di questo vaso), sulla base della descrizione dei Mmss. Giornali di Scavo rispettivamente di G.B. Scarpari e dell'assistente Nicolussi. È infatti possibile ricostruire una tomba dubitativamente femminile, come suggerirebbero gli orecchini d'oro indossati dal defunto (e altri tre anelli d'argento del diametro di 5 $\mathrm{cm})$. Si tratterebbe così di una ulteriore conferma dell'ipotesi che la formula all'assolutivo espliciti il donatore dell'offerta funebre. Il vaso, stando alle fotografie della sepoltura, doveva trovarsi in prossi-

119 Sassatelli 2008b, pp. 339-341, nn. 2 e 6. Si vedano le osservazioni in Gaucci 2016, pp. 185-186.

120 GaUcCi 2016, p. 195. Si osserva però che l'uso del piattello (vaso spinetico molto raro nelle deposizioni adriesi del periodo) come coperchio di olla non trova corrispondenza a Spina e soprattutto non sembra presentare sostanza nella documentazione di scavo edita; è pertanto probabile che il piattello e l'olla vadano separati.

121 Bellintani et al. 1995, p. 44.

122 Vale la pena sottolineare che la reiterazione dell'iscrizione da parte forse della medesima mano è una pratica funeraria che si riscontra ancora nel II sec. a.C. in Canalbianco (tomba 52) e nel vicino fondo Donà di Retratto (tomba 15; cfr. infra). 


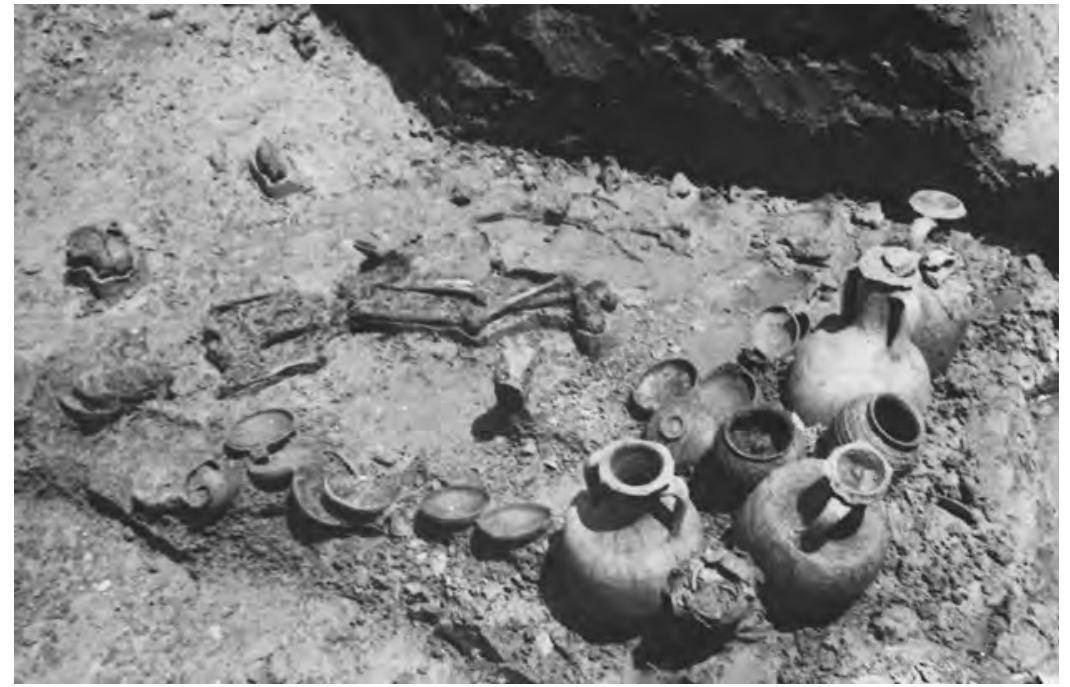

Fig. 33. Fotografia di scavo della tomba 154 di Canalbianco (dall'Archivio del Museo Archeologico Nazionale di Adria).

mità della testa del defunto lungo il lato destro (Fig. 33). Sembra importante puntualizzare che questa era a pochi metri dalla tomba 155 , cioè il currus deposto con i tre cavalli ${ }^{123}$, eccezionale non tanto per la deposizione degli animali (il caso delle tombe 348 e 349 più sopra trattato dimostra che questa pratica non era estranea al milieu adriese) quanto per la presenza del currus che richiama forme rituali proprie del mondo celtico ${ }^{124}$.

Seguendo la ricostruzione proposta, se Laris Fuliu fosse davvero il nome del defunto della tomba 327, allora questa si configurerebbe come una sepoltura maschile sicuramente appartenente all'élite adriese, come suggeriscono oggetti di prestigio e unici quali la kelebe volterrana a f.r. e la kreagra in bronzo $^{125}$. In questo caso, la documentazione d'archivio permette alcune considerazioni sulla collocazione nello spazio tombale delle ciotole iscritte. Secondo il Ms. Giornale di scavo di G.B. Scarpari, la ciotola con segno a croce era situata ai piedi del defunto e nella fotografia da est della tomba (Fig. 34) si distingue bene; la ciotola con iscrizione si doveva invece trovare in prossimità dell'anfora lungo il fianco destro del defunto, pertanto nel nucleo principale del vasellame del corredo, non distinguendosi per posizione.

Sempre a un orizzonte di prima metà del secolo, appartiene l'iscrizione [---]kalu della tomba 160/A, che, sebbene con la cautela dovuta alla lacunosità del testo, potrebbe essere interpretata come una consacrazione all' infero Calu (cfr. V.3) piuttosto che come terminazione di un gentilizio con suffisso -alu. La presenza di probabili indicatori femminili nella tomba (una collana, e anche una statuetta fittile femminile andata dispersa), suggerisce il genere del defunto. Incerta è anche l'interpretazione del lessema tutis (tomba 85, pieno III sec. a.C.), graffito su un piatto a v.n. Questo è da ritenere un appellativo forse riferibile al vaso e afferente alla sfera del sacro piuttosto che un antroponimo (cfr. V.4).

Nella seconda metà del III sec. a.C., il nome individuale Hapile della tomba 301 testimonia forse un donatore dal nome schiettamente etrusco (cfr. IV.3.2, classe I.i). Sembra più interessante l'unico altro caso di contesto funerario con iscrizioni di questo cinquantennio, la tomba 236. Gli scavatori supposero una sepoltura di ragazzo, verosimilmente sulla base delle dimensioni dello scheletro. Qui una delle due ciotole conserva l'antroponimo al genitivo atakuś ripetuto due volte, mentre l'altra la formula laris ațiu all'assolutivo. Se Laris Atiu è forse da intendere come il donatore dell'offerta, speciale e unico è l'altro vaso iscritto, particolare per la ripetizione del nome individuale Ataku di probabile origine celtica e già documentato a Canalbianco non molto tempo prima (tomba 378). Il nome, espresso al

\footnotetext{
123 Fogolari, SCARFì 1970, pp. 73-74, nn. 44, 45, con riferimenti precedenti.

124 Si veda Camerin 1999, p. 44; Lejars 2017, p. 567.

125 Per il corredo, si veda Mangani 1980.
} 

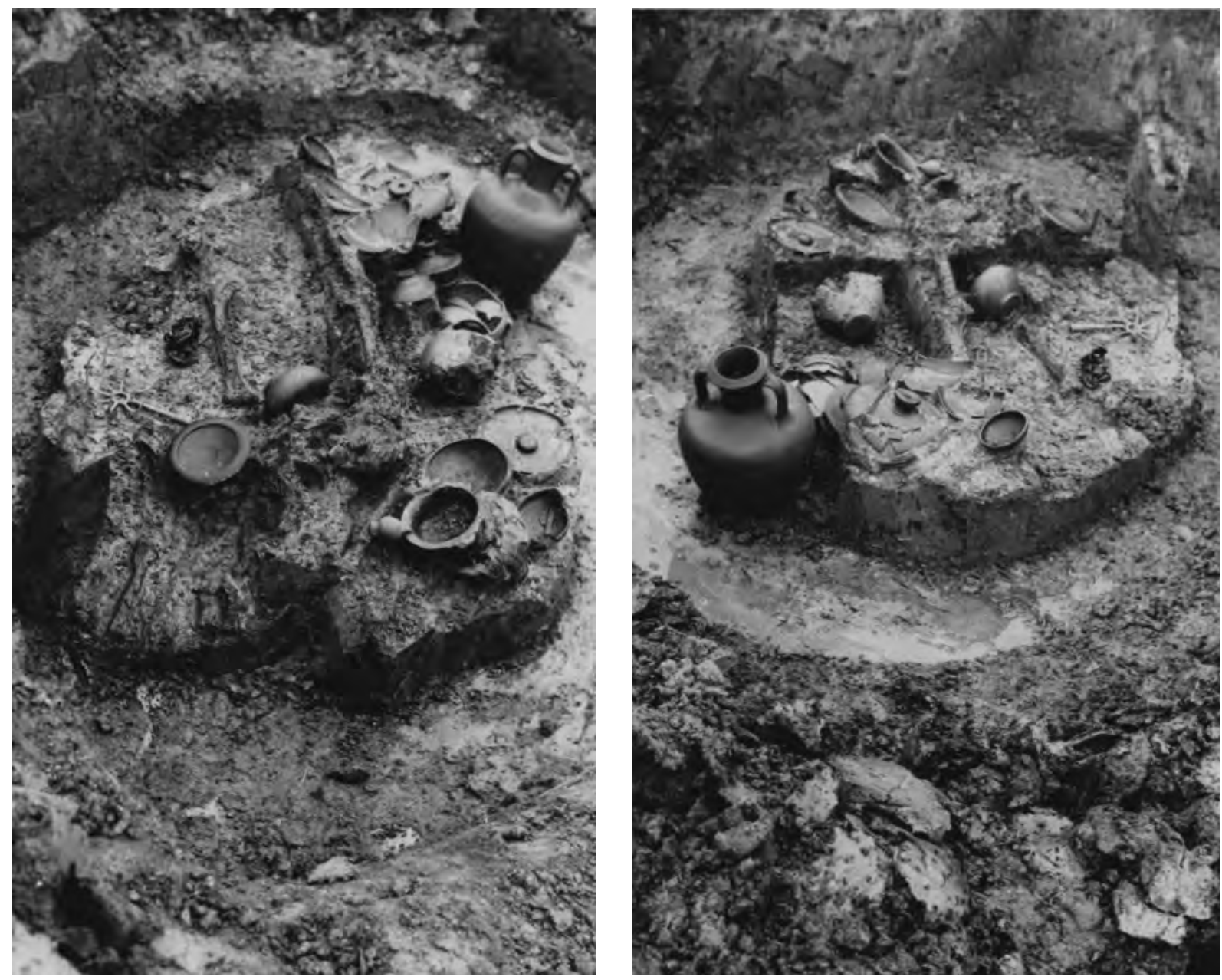

Fig. 34, a-b. Fotografie di scavo della tomba 327 di Canalbianco (dall'Archivio del Museo Archeologico Nazionale di Adria).

genitivo, è redatto entrambe le volte nella fascia di rotellature e probabilmente da due mani distinte. A parte il caso dell' iscrizione lai attribuita alla tomba 326, realizzata con grandi lettere nell' interno di un piatto di II sec. a.C. senza curarsi delle linee incise circoncentriche, e inoltre quello dell' iscrizione latina $N F$ redatta nella fascia rotellata di una ciotola a v.n. di III sec. a.C. (CIE 20835), il nostro è l'unico altro caso dove il testo è tracciato sopra la decorazione al centro del vaso, mentre solitamente l'operazione di graffitura delle iscrizioni (ma più in generale anche dei graffiti) la risparmia (cfr. II.2). La rarità dell' impaginazione fa pensare che questa scelta non sia casuale, come probabilmente non lo è la ripetizione e la disposizione dei due testi, non speculari fra loro ma entrambi leggibili dallo stesso lato. Purtroppo per entrambe le tombe non si possiedono dati relativamente alla collocazione dei vasi iscritti, che avrebbero forse potuto fornire qualche ulteriore spunto all' interpretazione.

La tomba 6, purtroppo di rito incerto dati i scarsi dati d'archivio a disposizione, conserva alpha e chi in legatura, testimoniando così l'unico caso entro il III sec. a.C di una pratica di evocazione della serie alfabetica (dal valore magico-religioso) ben diffusa nel settore padano (cfr. V.1).

A conclusione della rassegna dei contesti di III sec. a.C., conviene citare anche i contesti interpretati come votivi da Canalbianco e quello dell'Ospedale civile. Si osserva che solo due di quelli di Canalbianco (nn. 194 e 296) ospitano iscrizioni. Nel contesto n. 194 l' iscrizione è eccezionalmente realizzata nel fondo esterno del vaso (cfr. II.2) e il testo è ipuz (CIE 20547). Si tratta con ogni probabilità di un nome individuale derivante dall'onomastica greca (cfr. IV.3.1.B, categoria d); l'incerta impaginazione del testo e l'uso dello zeta al posto del sade per indicare il genitivo I, suggerirebbero scarsa dimestichez- 


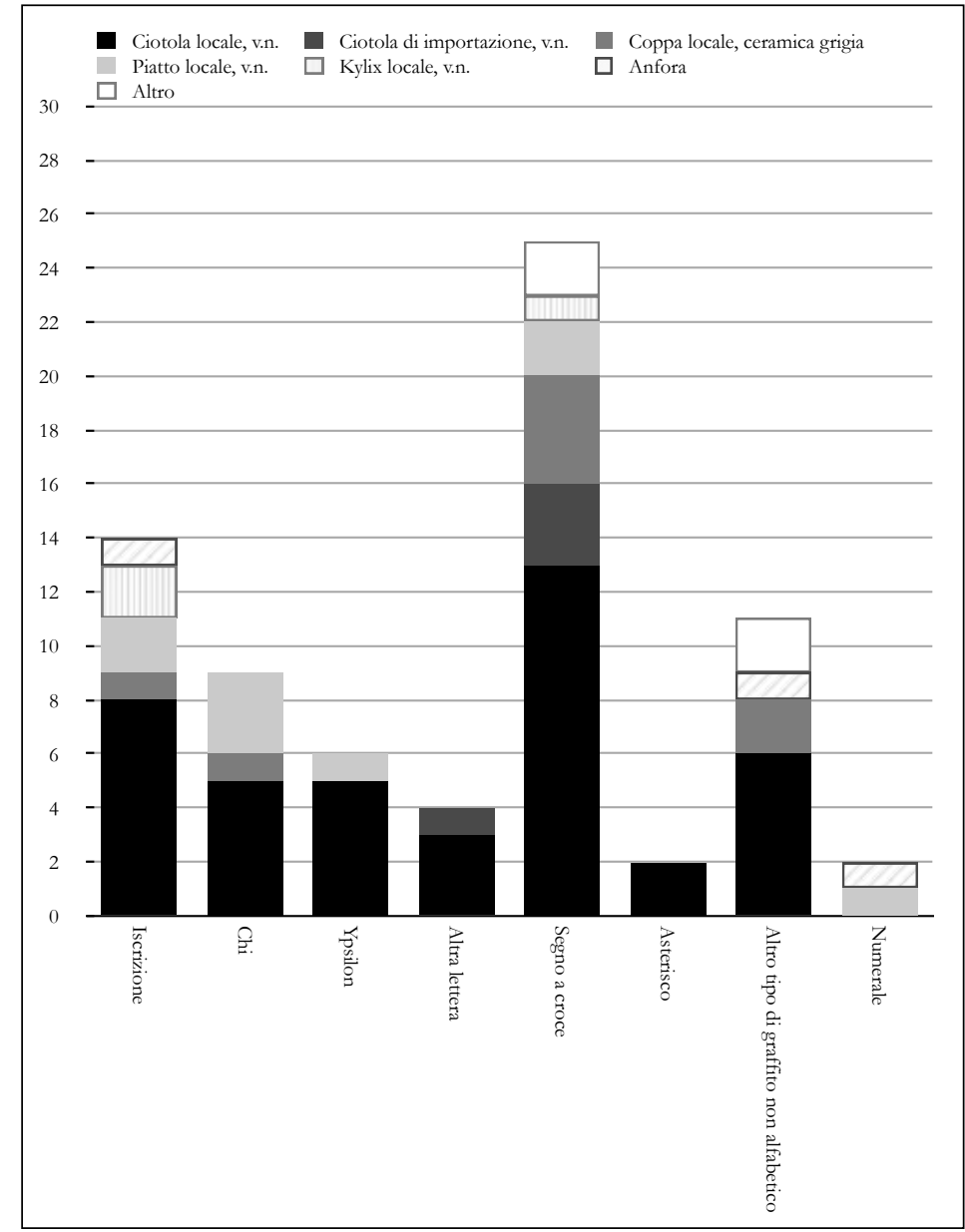

Fig. 35. Quantificazione dei supporti usati per realizzare iscrizioni e graffiti nella necropoli di Canalbianco.

za nell'uso della scrittura etrusca. Nella vasca interna della ciotola a v.n. appartenente al contesto n. 296, oltre a due lettere isolate (un chi e un kappa), si registra il lessema keus (CIE 20590), per il quale si è supposto l'uso dell'alfabeto leponzio, in particolare per la presenza di un sigma a quattro tratti. A questo si associa un testo molto mal conservato su un piatto da pesce a v.n. (CIE 20591), ma nel quale si può con buona verosimiglianza riconoscere il termine $\chi u r$, ben noto nella fase ellenistica di Spina (cfr. V.5). Per il resto, in questi nuclei votivi si individua una maggior preferenza di coppe emisferiche e mortai in ceramica grigia, piatti e skyphoi a v.n. quali supporti per iscrizioni e graffiti, distinguendosi così dalle scelte praticate nelle sepolture. Nel complesso, si osservano alcune differenze fra le iscrizioni da contesti di natura votiva e quelle provenienti da sepolture prima analizzate. Oltre a pratiche legate alla scrittura che parrebbero maggiormente codificate all'interno delle sepolture, prevalentemente riconducibile al dono funebre o al possesso da parte del defunto, le iscrizioni di questo gruppo manifestano onomastica e lessico coerentemente etruschi, sebbene non manchino casi di individui, il cui nome etruschizzato ma di origine alloctona sembra rivelare una condizione di subalternità sociale (libera o servile che sia).

Sicuramente molto rara è la pratica di redigere graffiti sugli skyphoi, come appunto quello del contesto votivo 1 dell' Ospedale civile, che presenta un $c h i$ sulla parete esterna (CIE 20669). Si tratta di una evidenza eccezionale per scelta del supporto, che trova corrispondenza in pochi altri casi a v.n. (CIE 20105, 20637, 20638, 20815, 20938, 20967) e in ceramica grigia (CIE 20902-20903, dove i graffiti sono entrambi incisi a crudo), dei quali solo uno da contesto abitativo (CIE 20105). Tra tutti questi, solo in un frammento di contesto ignoto da Canalbianco con singola $A$ latina (CIE 20638) e uno di provenienza ignota (ma verosimilmente funeraria per la sostanziale integrità del vaso) con segno a croce (CIE 20815) si documentano graffiti nella parete esterna come in quello del 
contesto votivo dell'Ospedale. Si tratta pertanto di una pratica molto circoscritta e purtroppo senza significativi confronti contestuali.

Le manifestazioni scrittorie dei contesti votivi sembrano dunque avere una loro peculiarità, sia per una scelta del supporto meno vincolata, sia perché sembra esserci una maggior libertà anche nella espressione della cultura epigrafica (uso del leponzio).

VI.2.1.B. Il passaggio tra III e II sec. a.C.

Alcune sepolture si datano a cavallo dei due secoli. Tra queste, 3 di Canalbianco e la tomba 15 di Retratto-Donà conservano iscrizioni etrusche.

Le tre di Canalbianco sono la tomba 95, con il probabile gentilizio UniӨiu, la 21 con il lessema $\theta i$, nel quale si riconosce la parola "acqua" (cfr. V.4), e la 348, alla quale è con buona verosimiglianza da riferire la tazzina monoansata con il prenome Ata associato a un probabile alpha e a un chi rovesciato, quest'ultimo da intendere come numerale $(L)$ oppure come lettera allusiva alla fine sequenza alfabetica aperta dall'alpha (cfr. V.1) ${ }^{126}$.

La ciotola della tomba 95 fu rinvenuta lungo il lato destro del defunto in prossimità della testa assieme a molti altri piatti, come mostra la fotografia di scavo (Fig. 36). Per il resto, non emergono indicatori significativi. La ciotola iscritta della tomba 21 , che conserva anche altri vasi con graffiti, si riconosce invece nel gruppo di vasellame ai piedi del defunto (Fig. 37). In questo caso pare singolare la scelta di scrivere la parola che indica l'acqua all'interno della ciotola, ammesso che questo fosse il contenuto del vaso medesimo.

L'eccezionale tomba 348, ancora inedita nel suo complesso ${ }^{127}$, va forse interpretata come femminile sulla base di alcuni indicatori, che potrebbero inoltre suggerire la vicinanza della defunta a culti di tipo ctonio e salvifico legati alla coppia eleusina Demetra e Kore, come in particolare le foglie d'oro sparse sopra il corpo ${ }^{128}$. Sebbene questo contesto specifico meriti un maggior approfondimento che ne valorizzi la complessità (fra le altre cose, fanno parte del corredo una padella in bronzo tipo Montefortino, due spiedi in bronzo simili a quello con iscrizione retica di Ca' de Cavri a Verona ${ }^{129}$, una lucerna a v.n.), se quanto avanzato in questa sede ha qualche fondamento, ciò dimostra ancora una volta la distinzione che deve essere perlopiù fatta tra formula onomastica all'assolutivo da intendere come donatore (Ata maschile) e defunto (femminile). La scelta della tazzina monoansata risulta un caso del tutto eccezionale nel panorama adriese, come è anche peculiare l'associazione tra il prenome e il numerale o l'allusione alla sequenza alfabetica.

La tomba Donà 15 (CIE 20677-20682) a inumazione presenta un corredo quantitativamente ricco databile tra III e II sec. a.C., comunque non oltre la metà del II sec. a.C. ${ }^{130}$. Da questa provengono tre iscrizioni identiche realizzate su ciotole a v.n. di produzione locale (CIE 20677-20678), una iscrizione su piatto da pesce a v.n. sempre di produzione locale (CIE 20680), e infine due graffiti alfabetici su ciotole a v.n., una di produzione riminese (CIE 20681) e una locale (CIE 20682). Le tre iscrizioni identiche ankariu sembrano realizzate da una stessa mano ed evocano i simili casi delle tombe 52 e 243 di Canalbianco (cfr. IV.3.2 e soprattutto VI.2.1.A). Nella quarta iscrizione, arza heturiu, l'aspetto paleografico suggerisce una diversa mano e il testo un diverso gentilizio. Si confermerebbe così la pratica del dono funerario da parte di individui appartenenti a distinti gruppi familiari.

126 L'ipotesi di attribuzione dell' iscrizione al corredo della tomba 348 viene qui formulata per la prima volta. Infatti, l'iscrizione è data come dispersa nella tabella edita in RoвıNo 2016, p. 94 e non è prudenzialmente riferita alla tomba 348 in CIE IV, I, 1. Tuttavia, la trascrizione in lettere greche con punto interrogativo finale nel Ms. Giornale di Scavo di G.B. Scarpari $(\chi p \alpha \nu \alpha$ ?), porta a ritenere plausibile l'identificazione con la nostra $(\chi=\chi ; p=a ; \alpha \nu \alpha=a t a)$.

127 Robino 2016, pp. 93-94, con riferimenti.

128 Si veda al riguardo le considerazioni di G. Morpurgo in Gaucci-Morpurgo-Pizzirani 2018, p. 658, nota 20, e p. 661 per Bologna.

${ }_{129}$ MLR, p. 256, n. 290, con riferimenti.

130 Ibid., pp. 48-55. 


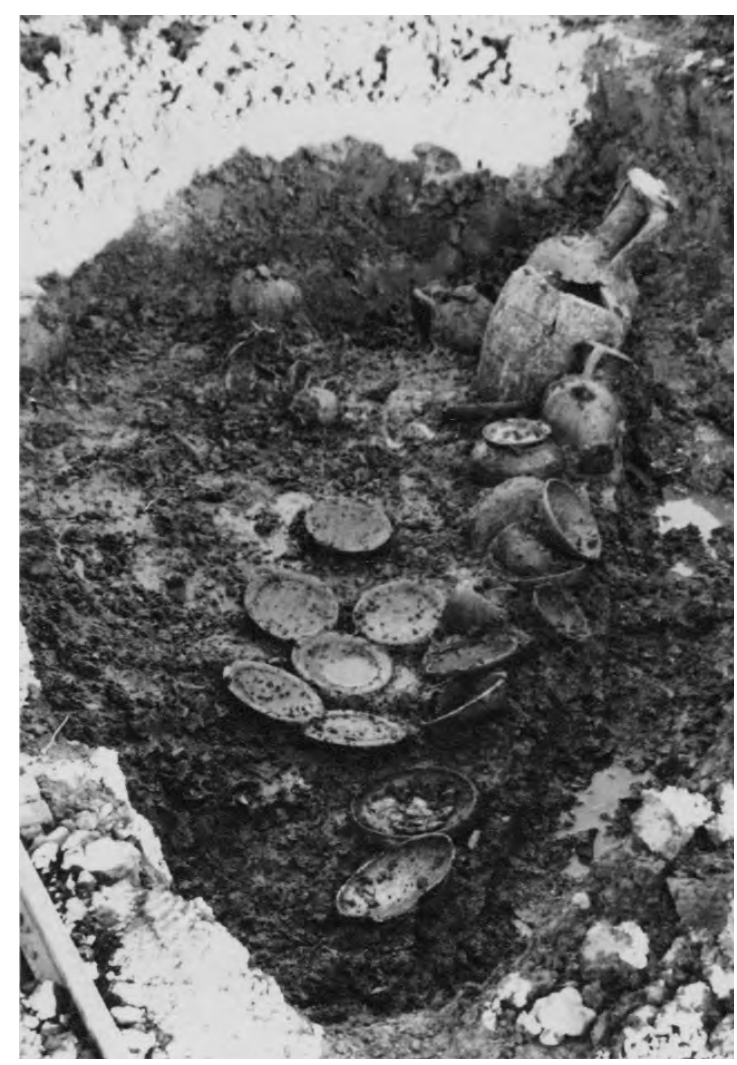

Fig. 36. Fotografia di scavo della tomba 95 di Canalbianco (dall'Archivio del Museo Archeologico Nazionale di Adria).

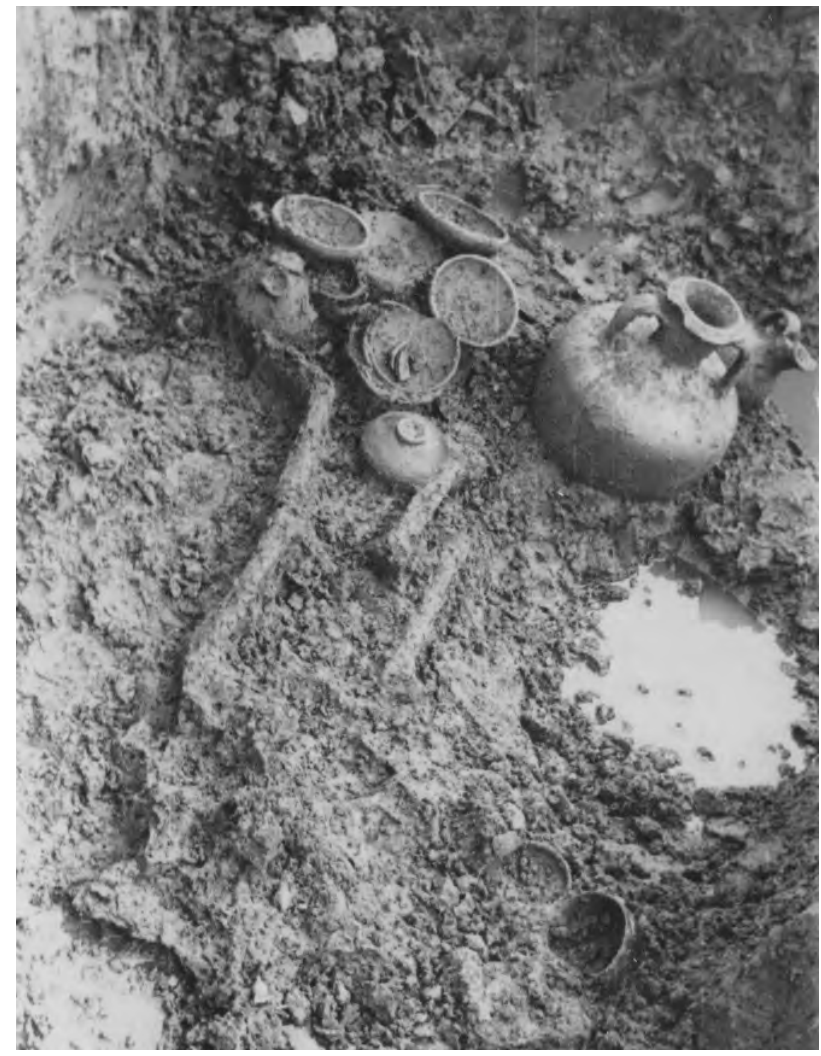

Fig. 37. Fotografia di scavo della tomba 21 di Canalbianco (dall'Archivio del Museo Archeologico Nazionale di Adria).

\section{VI.2.1.C. II sec. a.C.}

Entro il II sec. a.C. si contano 46 sepolture, a cui se ne possono aggiungere 7 tra quelle con corredi di incerta attribuzione da Canalbianco. Una ventina di queste è genericamente databile al secolo. Nelle restanti, si osserva una concentrazione entro la prima metà-metà del secolo $(9 \mathrm{da}$ Canalbianco, $3 \mathrm{da} \mathrm{Ca}$ Garzoni e 2 da Piantamelon, a cui se ne aggiunge una con corredo di incerta attribuzione da Canalbianco), mentre poco inferiore il numero nella seconda metà ( 5 da Canalbianco, 1 da Retratto-Donà, 5 da Ca' Garzoni). Una tomba di Canalbianco si data tra la seconda metà del II e la prima metà del I sec. a.C.

Anche nel II sec. a.C. è evidente la costante presenza di ceramica a v.n. di produzione locale nei corredi, associata almeno fino alla metà del secolo con le importazioni volterrane, mentre nel III sec. a.C. la presenza di queste ultime risultava più saltuaria ${ }^{131}$. La ritualità funeraria adriese, come espresso dallo studio di T. Stefani sulla necropoli di via Spolverin presso Bottrighe e anche dalle analisi successive effettuate da chi scrive, nel passaggio al nuovo secolo si articola ulteriormente, rendendosi più complessa ${ }^{132}$. A parte un aumento quantitativo sostanziale del vasellame di corredo, le sepolture, in prevalenza sempre a inumazione, mostrano la pratica codificata di porre sul lato sinistro del defunto uno spiedo che passa attraverso le anse di kylikes e oinochoai di piccole dimensioni (tendenzialmente di importazione), oltre che l'introduzione di nuove forme in alcuni casi derivanti dall'elaborazione di influenze culturali alloctone, come esemplificato dalla brocca con beccuccio di origine latèniana ${ }^{133}$, ma anche dalle grandi ciotole con due scanalature all'orlo, circoscritte unicamente ad Adria ${ }^{134}$.

131 Cfr. Gaucci 2016, pp. 197, 199, con riferimenti.

132 Stefani 1996-1997; Gaucci 2016, passim.

133 Si vedano le considerazioni in Gaucci 2016, p. 197 e fig. 8.

134 Cfr. Gaucci 2008, p. 102; Gaucci, Pozzi 2009, p. 63. 


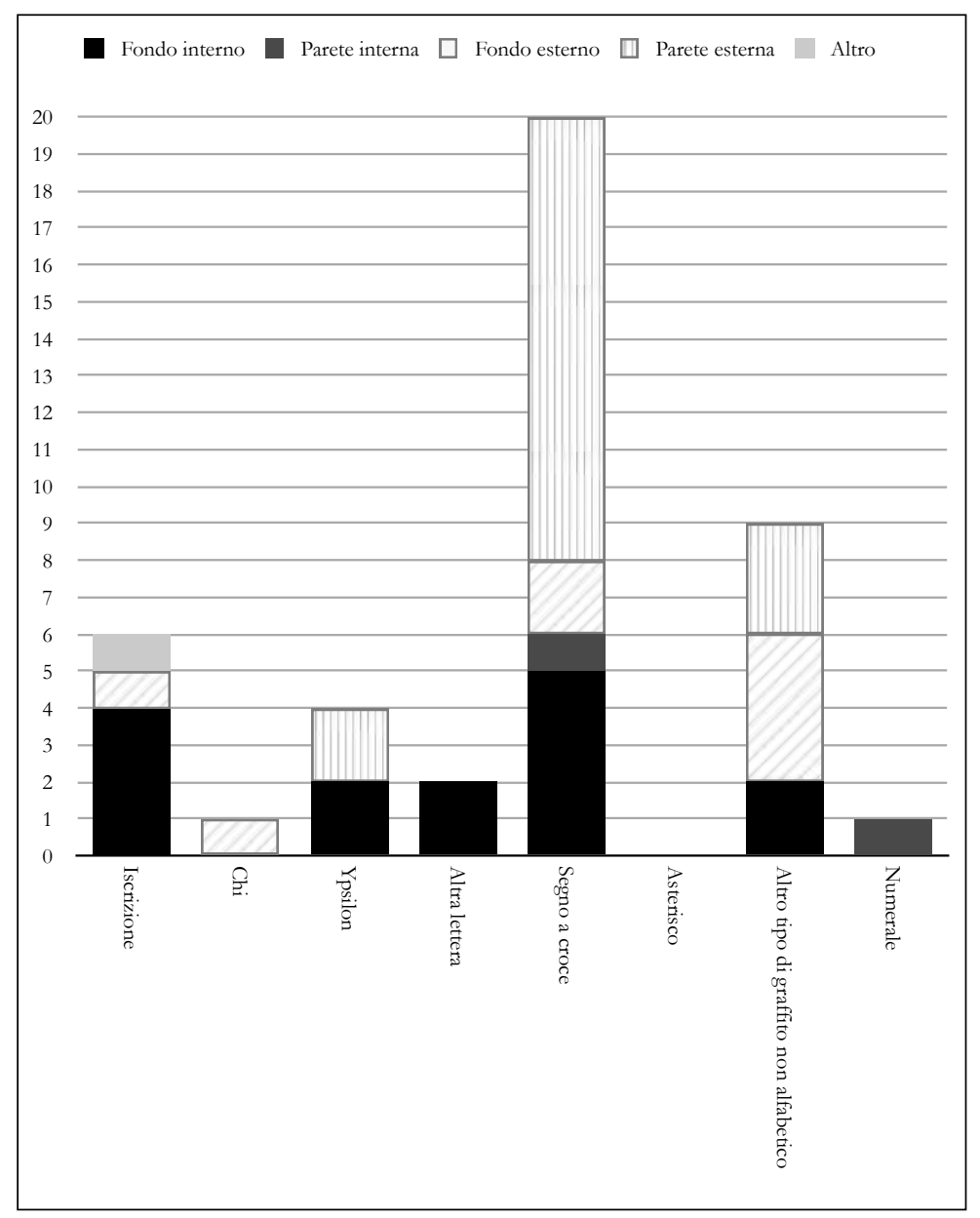

Fig. 38. Rapporto quantitativo tra tipi di documenti epigrafici di II sec. a.C. e parte del vaso su cui furono tracciati nell'area funeraria di Ca' Garzoni.

Come già anticipato, rispetto al III sec. a.C. aumenta il numero di cremazioni con documentazione epigrafica, 6 e sempre tutte concentrate in Canalbianco; fra queste, la tomba 47 degli inizi del secolo, la tomba 18 della seconda metà e la 228 compresa tra la seconda metà del secolo e la prima metà del successivo, conservano iscrizioni etrusche, mentre le restanti solo graffiti. Quali supporti di iscrizioni e graffiti sono privilegiate le ciotole a v.n. locali (24), a seguire i piatti (6), e infine una presenza molto scarsa di coppe in ceramica grigia (un solo esemplare). Per quanto riguarda la posizione di iscrizioni e graffiti sui vasi (Fig. 38), colpisce come anche nel II sec. a.C., coerentemente con il secolo precedente, si preferisca la superficie esterna per quanto riguarda i graffiti, a eccezione dei pochi casi di lettere isolate differenti da ypsilon e $c h i$, nonché delle iscrizione, dove si rileva invece una preferenza dell' interno del vaso. Si conferma così quella distinzione già rilevata in Canalbianco fra la prassi epigrafica delle iscrizioni e quella dei graffiti. Diversamente, la tendenza in Piantamelon, dove le due iscrizioni etrusche sono redatte sul fondo interno di ciotole, così come la prevalenza dei graffiti.

Più rilevante, emergono in questo secolo rari documenti di tradizioni epigrafiche allogene, nella fattispecie celtica e latina, ma ciò sembra riguardare soprattutto necropoli periurbane come quella presso Bottrighe, mentre in quelle urbane è lasciato poco spazio a espressioni scrittorie e lingue che non siano quella etrusca. In tal senso, casi sicuramente significativi sono l'iscrizione șusas attribuita alla tomba 251 di Canalbianco (CIE 20578) e il digramma au della tomba 83 di Piantamelon (CIE 20765), entrambi in alfabeto leponzio. In particolare, il testo della tomba 251 richiama la 257, il cui materiale epigrafico (CIE 20580-20582) presenta cronologie comprese tra il III e il II sec. a.C. Infatti entrambe restituiscono (ammessa la correttezza dell'attribuzione) la medesima iscrizione susas in alfabeto lepon- 
zio e inoltre in entrambi casi su ciotole di II sec. a.C. ${ }^{135}$. Le incerte informazioni sui corredi e queste affinità destano qualche sospetto (appartenenza di entrambe a un unico contesto poi confuso?). In ogni caso, se fosse valida l'attribuzione, queste apparterrebbero a tombe a inumazione con un corredo ricco e complesso, caratterizzato dalla presenza di oggetti di bronzo, in particolare un colatoio nella tomba 257, e anche una lucerna (come nella più antica tomba 348 della fine del III-inizi del II sec. a.C., nella quale alcuni indizi rimanderebbero alla manifestazione di speciali pratiche cultuali; cfr. VI.2.1.B). Indipendentemente dal problema del contesto, colpisce l'emergere di iscrizioni appartenenti a una cultura gallica, che nel III sec. a.C. erano circoscritte ai contesti votivi, e l'eventuale associazione di queste a corredi di impegno, sui quali meriterebbe avviare approfondite riflessioni.

Più puntuali le testimonianze di competenza scrittoria latina su vasi di produzione locale, documentata solo dalla tomba 29/1966 di Ca' Garzoni, ma già attestata in quest'area funeraria nel secolo precedente nella tomba 157/1972 (un caso privo di contesto certo anche da Canalbianco: CIE 20638). Solo nella prima metà del I sec. a.C., in concomitanza con radicali cambiamenti nella ritualità funeraria, la scrittura e la lingua latina furono usate per esprimere una pratica rituale probabilmente ancora radicata nella tradizione etrusca (tomba 104 di Canalbianco).

Passando all'analisi dei testi etruschi, si contano 22 sepolture che ne attestano la presenza (15 di Canalbianco, 5 di Ca' Garzoni, 2 di Piantamelon). Presso Canalbianco, si osserva una concentrazione delle iscrizioni nella prima metà del secolo (7). Il dato non è inficiato dallo scarso numero di sepolture datate genericamente al secolo (tre casi, per i quali lo studio dettagliato del corredo non restituisce comunque dati per una cronologia più puntuale ${ }^{136}$. Si osserva che le tombe che conservano iscrizioni sono perlopiù molto complesse per ritualità e ricche di vasellame. Sebbene nella prima metà del secolo, quando si concentra la maggior parte dei contesti, si manifestino scelte anche diversificate per tipologia di supporti, si conferma la tendenza già colta nel secolo precedente, cioè la netta preferenza di scrivere $\mathrm{i}$ testi nella vasca delle ciotole a v.n. di produzione locale (e in misura nettamente minore dei piatti), deposte assieme al resto del vasellame lungo il fianco destro del defunto. Tale tendenza è confermato anche dal resto della documentazione epigrafica. Infine, rispetto al secolo precedente, nel II sec. a.C. si nota una maggior concentrazione di corredi con iscrizioni e graffiti in numero superiore a uno (Fig. 39), e questo vale in particolare per la prima metà del secolo. Presso Ca' Garzoni, l'incremento del numero di sepolture con documenti epigrafici nel II sec. a.C. permette di osservare speciali pratiche funerarie (cioè la concentrazione di testi nelle tombe di adulti e non in quelle infantili almeno per l'area indagata nel 1966, e per l'ambito epigrafico il rapporto fra tombe con iscrizioni e/o graffiti e tombe con solo graffiti $\left.{ }^{137}\right)$.

In merito alla selezione dei supporti, si osserva che in Canalbianco nella prima metà del secolo, sebbene la ciotola a v.n. locale rimanga la forma privilegiata, la scelta in alcuni casi del piatto (veri della tomba 370 e lai della 326) trova corrispondenza in tombe più antiche (tombe 85 e 154). Colpisce la scelta della pisside per la redazione del gentilizio Spiu nella tomba 175, piccolo contenitore introdotto con la fine del III sec. a.C. e usato come supporto di iscrizioni e graffiti fino alla metà del secolo ${ }^{138}$. Quat-

135 Una terza iscrizione adesso riconosciuta in alfabeto leponzio ( $\mid$ ẹ i s a $\mid$ ), ma precedentemente ricondotta all'epigrafia etrusca (CIE 20581, con riferimenti), è attribuita al corredo della tomba 257.

136 Diversamente, per le tombe che non ospitano iscrizioni ma solo graffiti, la cronologia è piuttosto di massima, non essendo stato affrontato ancora uno studio analitico dei corredi.

137 Similmente che a Canalbianco, si registra una percentuale maggiore di tombe che presentano un solo vaso con un solo graffito rispetto alle tombe con più graffiti fra quelle senza iscrizioni, e invece una percentuale simile tra tombe con solo iscrizioni e tombe con iscrizioni e più di un graffito, secondo una tendenza in questo caso inversa rispetto a Canalbianco.

138 Una pisside con segno a croce graffito si trova già nella tomba 21 della fine III-inizi II sec. a.C. (CIE 20438). Oltre che nella tomba 175 di Canalbianco, si ritrova questo vaso iscritto anche nella 45/1970 di Ca' Cima (digramma śp, CIE 20376), 50 (digramma śp, CIE 20934) e 62 di via Spolverin (digramma se, assieme a un ypsilon e a un segno a croce, CIE 20960), 370 di Canalbianco (segno a croce, CIE 20654), tutte databili tra la fine del III e la prima metà del II sec. a.C. A questo elenco, va aggiunta una pisside da contesto incerto con iscrizione in alfabeto leponzio (a tk, CIE 20804). 


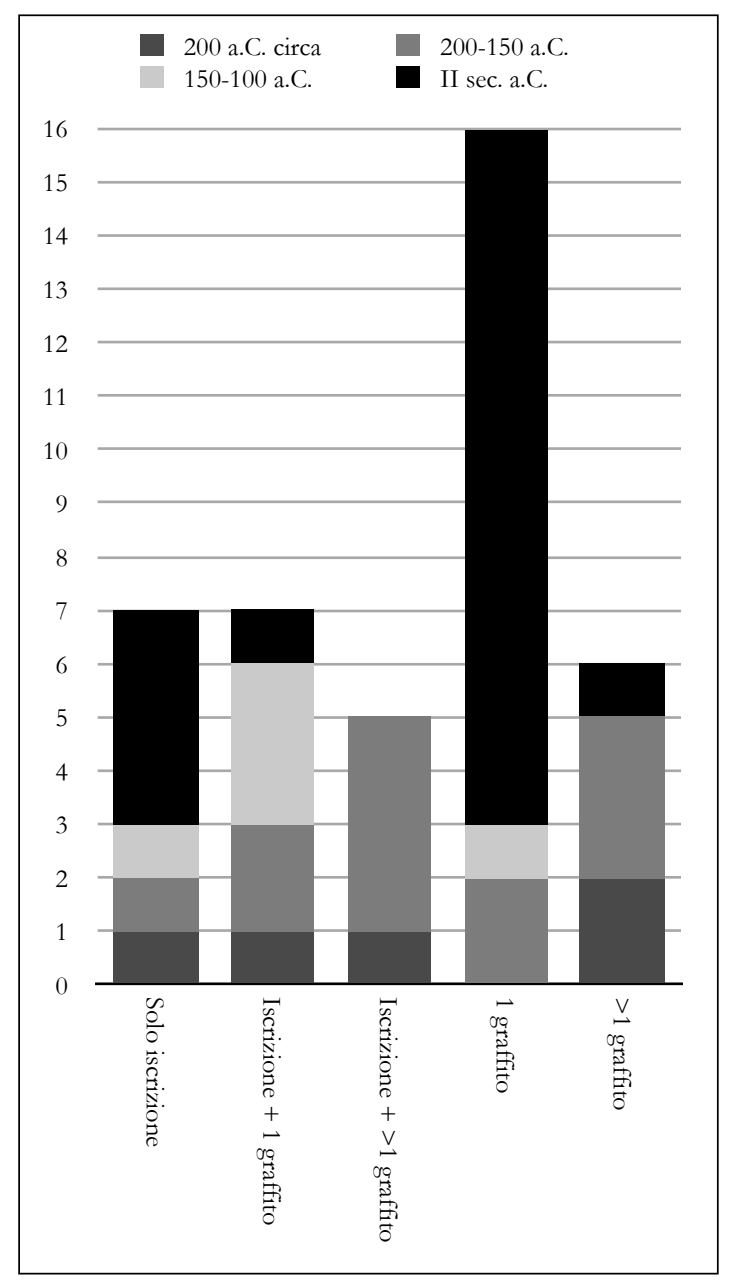

Fig. 39. Quantificazione delle tombe con documentazione epigrafica durante il II sec. a.C. nella necropoli di Canalbianco.

tro sono i casi di grandi ciotole con due scanalature all'orlo. La scelta di questo vaso, che sembrerebbe avere uno speciale valore rituale, accomuna le occorrenze di Ca' Garzoni (2) e Canalbianco (1) a quella isolata della tomba bisoma 45/1970 di Ca' Cima (CIE 20375; cfr. IV.2.2) e quella della tomba 46 di via Spolverin presso Bottrighe (cfr. IV.3). In particolare, l'iscrizione della tomba 362 di Canalbianco può offrire qualche considerazione nel rapporto tra lo speciale formulario e il vaso. Non si conoscono dati relativi alla composizione del corredo (nessun oggetto inventariato sembra pertinente a questa sepoltura), a cui siamo certi che l' iscrizione appartenga grazie alla documentazione di scavo. Si trattava di una inumazione, forse anche dal ricco corredo, come lascerebbe credere l'anello in bronzo rivestito d'oro registrato come oggetto particolare da G.B. Scarpari. L'iscrizione, seppure lacunosa, manifesta un formulario ( ] ś ta mi) altrimenti documentato solo nel III sec. a.C. (cfr. IV.3.2, presumibilmente classe IV.c, ma vicina anche alla classe III.c, da cui si distingue per l'uso del pronome mi). Va aggiunto, sebbene possa risultare una osservazione opinabile, che l'uso del nome al genitivo associato al deittico sembra in effetti privilegiare vasi di grandi dimensioni anche nel III sec. a.C. Purtroppo il ridotto numero di occorrenze della formula (4, tutte da necropoli) non permette di analizzare in maniera efficace il fenomeno, che comunque sembrerebbe avere da una sua coerenza interna e che nel II sec. a.C., data l'unica attestazioni qui discussa, poteva essere evocativo di pratiche ormai altrimenti abbandonate.

Per quanto riguarda la posizione dei vasi iscritti nello spazio tombale, nella prima metà del secolo disponiamo di informazioni per le tombe 175, 324, 334, 370 di Canalbianco ${ }^{139}$. Per la tomba 175, a

139 Nella ricca tomba 313, a fronte della presenza di informazioni d'archivio, purtroppo l'irreperibilità del vaso iscritto, peraltro non menzionato come tale nei Mmss. Giornali di scavo di Scarpari e dell'assistente e non visibile nelle fotografie di scavo, non permette alcuna considerazione. 


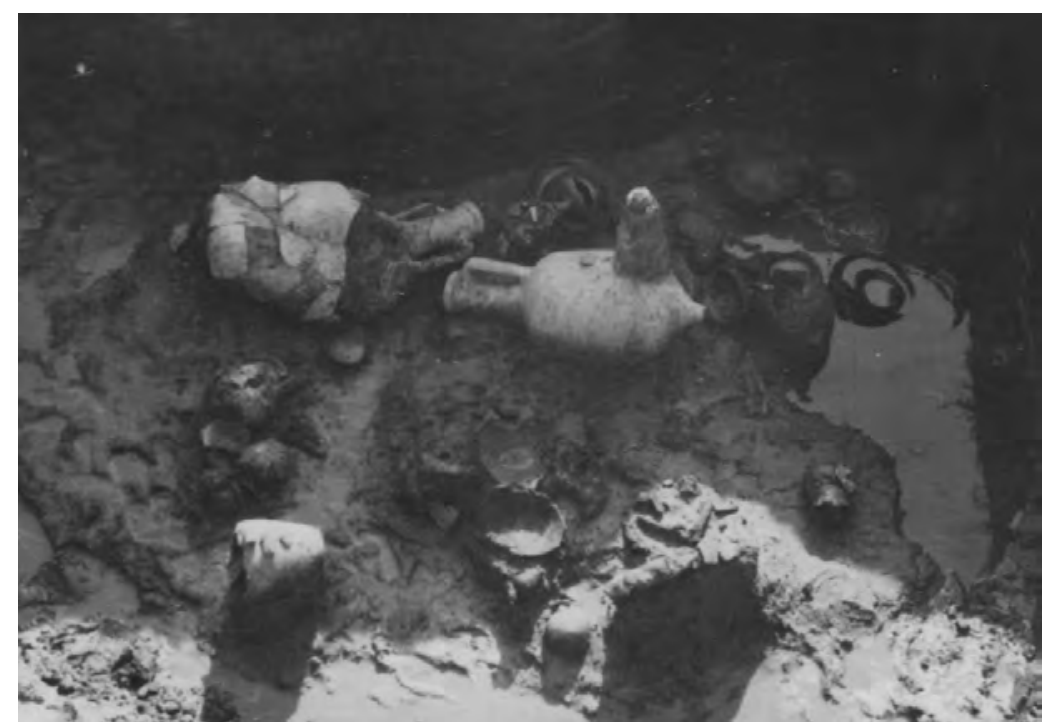

Fig. 40. Fotografia di scavo della tomba 175 di Canalbianco (dall'Archivio del Museo Archeologico Nazionale di Adria).

parte una fotografia di scavo (Fig. 40) e qualche descrizione più accurata (es. la menzione di un vago d'ambra e forse di un candelabro in ferro, non conservati), purtroppo la caotica disposizione del corredo (probabilmente causata da movimenti post-deposizionali) non permette utili considerazioni; si può comunque osservare che la pisside con iscrizione era collocata alla sinistra del defunto. La descrizione del corredo della tomba $324 \mathrm{nel} \mathrm{Ms.} \mathrm{Giornale} \mathrm{di} \mathrm{scavo} \mathrm{di} \mathrm{Scarpari} \mathrm{chiarifica} \mathrm{che} \mathrm{le} \mathrm{ciotole} \mathrm{iscritte}$ si trovavano lungo il fianco destro del defunto assieme a buona parte del vasellame. La medesima posizione è confermata dalla documentazione di scavo anche per le ciotole a v.n. iscritte della tomba 334 (Fig. 41) e della tomba 370 (Fig. 42), dove non vi sono dubbi sulla collocazione del piatto iscritto e della pisside con graffito alla destra del defunto con il resto del vasellame. Infine, sebbene non sia possibile affermare la certa pertinenza dei supporti iscritti della tomba 326, anche questa risulta molto ricca e complessa per composizione del corredo.

Non è invece possibile fornire alcuna valutazione per la seconda metà del secolo. Anche nella tomba 186, dal ricco corredo che però non raggiunge il livello delle sepolture descritte per la prima metà, la documentazione di scavo e in particolare la fotografia (Fig. 43) non consente di individuare la posizione della ciotola iscritta e quindi avanzare utili considerazioni.

Affrontanto il tema dell'onomastica e del formulario delle iscrizioni, si osserva che entro la prima metà del secolo l'onomastica è perlopiù schiettamente etrusca in Canalbianco (il gentilizio Spiu nella tomba 175, Petra nella 313, Larza Mura Pili nella 334, e i femminili Lai nella 326 e Titinai attribuiti alla tomba 65, Titi senza corredo certo; più incerto Veri nella tomba 370), mentre solo Pulu della tomba 47 è un nome individuale verosimilmente di origine greca (probabilmente di condizione servile?). Tra queste iscrizioni, merita sicuramente attenzione la formula trimembre che manifesta l'uso del cognomen (Pili) associato a un gentilizio (Mura), già attivo nel III sec. a.C. e che si ritrova anche in seguito (cfr. infra), e a un prenome di ampia diffusione adriese (Larza); si aggiunge che un'altra ciotola del medesimo corredo mostra un digramma (CIE 20622) probabilmente interpretabile come relitto consonantico del cognomen ( $p l$ piuttosto che $p u$ ). Merita qualche considerazione di dettaglio il contesto della tomba 65 , una inumazione alla quale sono attribuite le due iscrizioni su piatto a v.n. locale che riportano il gentilizio femminile all'assolutivo Titinai. Sebbene nel Ms. Giornale di scavo di Scarpari si parli (significativamente) di "iscrizioni", purtroppo nel puntuale elenco del Nicolussi (una sorta di atlante tipologico delle forme attestate nei contesti) è registrato soltanto un piatto dei due e nessun riferimento è fatto a iscrizioni. Emerge così una discrepanza che in questo caso però sembrerebbe ovviata dalla coerenza dei supporti e del testo iscritto, orientando verso la correttezza dell' informazione di Scarpari. La deposizione, che registra una fibula tardo-latène ad arco sfaccettato, diffusa soprattutto 

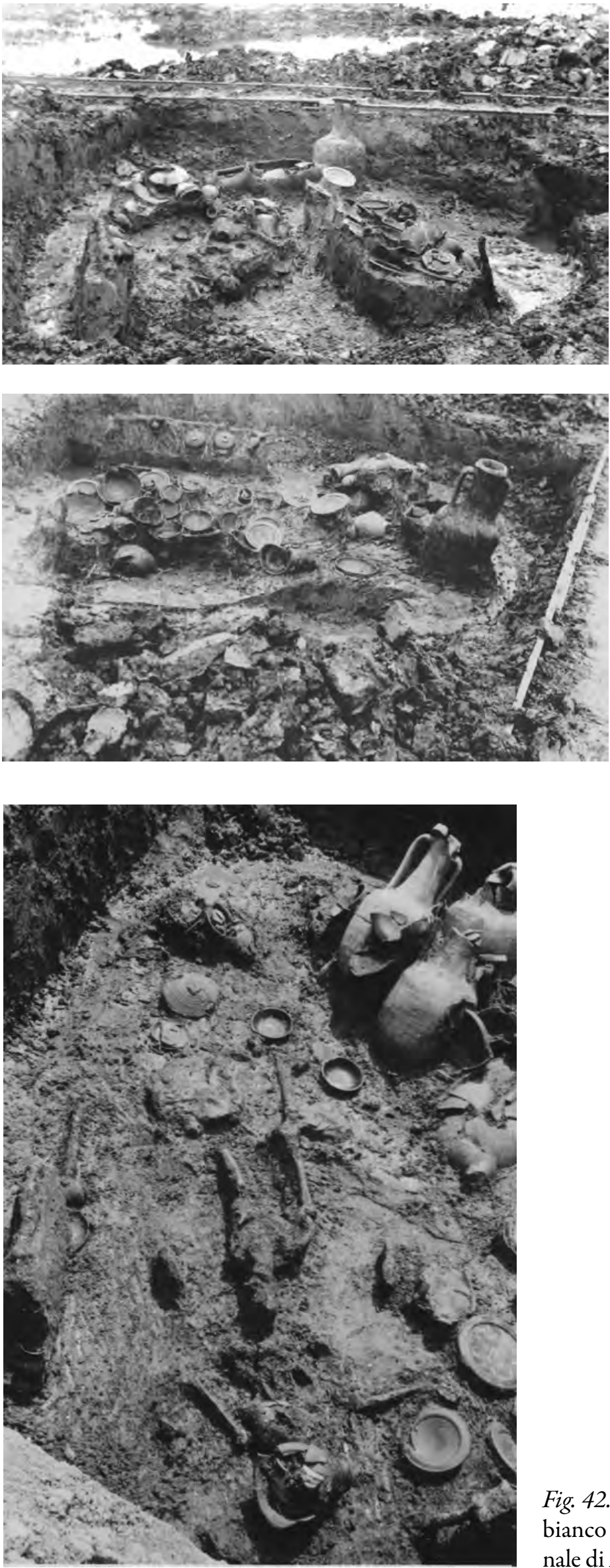

Fig. 41, a-b. Fotografie di scavo della tomba 334 di Canalbianco (dall'Archivio del $\mathrm{Mu}$ seo Archeologico Nazionale di Adria).
Fig. 42. Fotografia di scavo della tomba 370 di Canalbianco (dall'Archivio del Museo Archeologico Nazionale di Adria). 


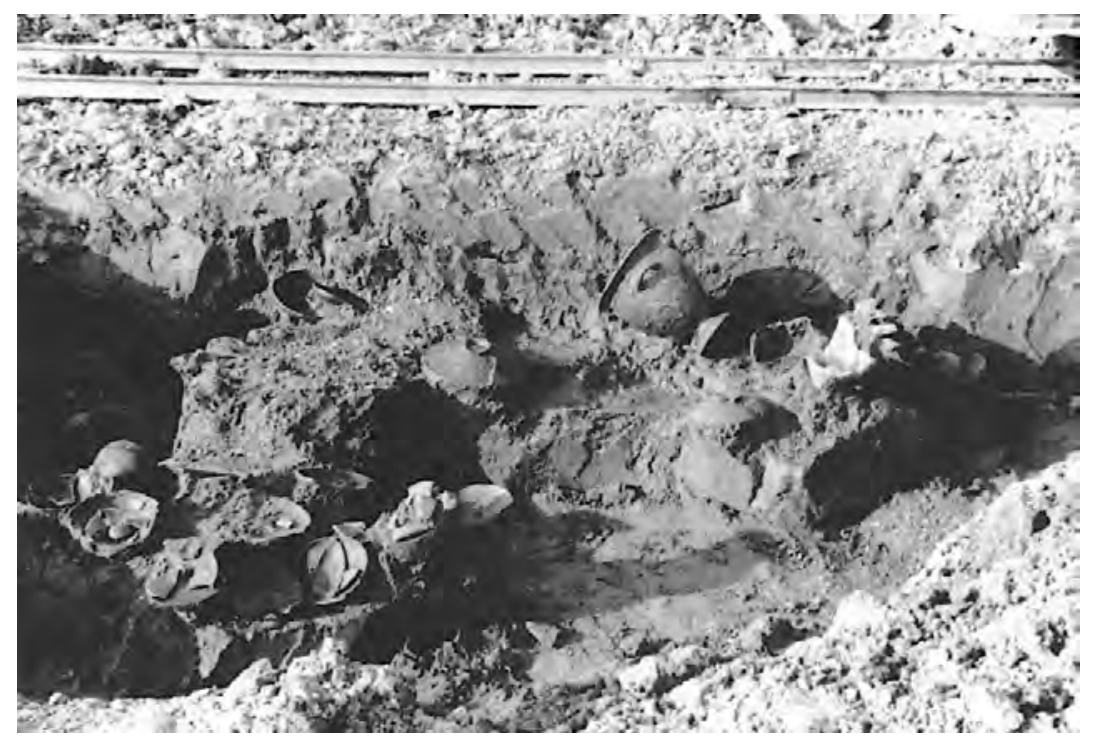

Fig. 43. Fotografia di scavo della tomba 186 di Canalbianco (dall'Archivio del Museo Archeologico Nazionale di Adria).

nel Caput Adriae e nel Veneto (Aquileia, Este, Padova) e in minor misura lungo la valle dell'Adige, una armilla in verga di bronzo a sezione circolare, un probabile ago crinale in osso e un vago di pasta vitrea, suggerirebbe un defunto di genere femminile ${ }^{140}$. La classificazione del formulario (cfr. IV.3.2) porta a ritenere che la convergenza tra genere del gentilizio e del defunto non sia significativa, potendo l'iscrizione identificare sia il donatore (come si ritiene) sia il defunto medesimo. Più nel complesso, tutte le iscrizioni della prima metà del secolo in Canalbianco rientrano nella medesima classe di formule (cfr. IV.3.2, classe I), che manifesterebbe una pratica di dono funebre nella quale è espresso il donatore. Come già osservato, quando desumibile dalla documentazione, questi vasi sono sempre collocati assieme al restante vasellame a lato del defunto, senza una significativa distinzione.

Anche nell'area periferica occidentale di Ca' Garzoni, nel primo cinquantennio del secolo si documenta il gentilizio Mura isolato (tomba 111/1969), testimone della diffusione di tale gens tra l'area di Canalbianco e la più occidentale periferia del medesimo settore meridionale. Sempre in Ca' Garzoni, si ha l'antroponimo di origine alloctona Latiate (CIE 20699). Questo, appartenente alla medesima tomba del gentilizio femminile e schiettamente etrusco Puinia, è noto anche nella tomba 46 di via Spolverin a Bottrighe (cfr. VI.3). Come altrove, anche qui si evidenzia come ancora nella prima metà del II sec. a.C. Latiate è identificabile come nome che sembrerebbe appartenente a un individuo privo di diritti (per ulteriori considerazioni su questo, cfr. VI.3). Inoltre, se guardiamo alle formule, l'iscrizione puinia $m i$, che esprime l'identità come appartenenza dell'oggetto iscritto (dunque forse il nome della defunta; cfr. IV.3.2, classe II), è significativamente parte di un corredo appartenente a un defunto di genere probabilmente femminile, supportando come in altri casi l'ipotesi che questo tipo di formule si riferisca all'individuo sepolto. Anche la presenza dell' iscrizione maschile, nel corredo di una tomba femminile, se in effetti di un donatore (cfr. IV.3.2, classe I), sembra corroborare l'impalcatura classificatoria.

A oriente, nell'area periferica di Piantamelon, con la prima metà del II sec. a.C. l'unica iscrizione etrusca che esprime onomastica testimonia il nome individuale probabilmente maschile Pruka (tomba 80, infantile), di origine celtica (cfr. IV.3.1.B, categoria d). Come per Bottrighe e per la medesima Canalbianco, anche qui nel II sec. a.C. l'uso dell'alfabeto leponzio in un digramma (au) dalla tomba 83 conferma l'emergere di forme di celtizzazione della comunità in questo periodo anche nella pratica epigrafica. Non deve peraltro sfuggire che le due tombe 80 e 83 sono fra loro prossime all'interno di un piccolo settore di necropoli.

140 CAMERIN 1993, p. 165, nota 82 (con elenco del corredo, nel quale non compare il possibile ago crinale in osso), p. 168 , cat. n. 20 , tav. 3. 
In generale, nella prima metà del secolo colpisce la corrispondenza, non necessariamente significativa, fra complessità del corredo e manifestazione di iscrizioni appartenenti a individui ingenui (uomini e donne) che manifestano nelle formule una schietta onomastica etrusca, mentre solo tre tombe, la 47 di Canalbianco, la 75/1969 di Ca' Garzoni e la 80 di Piantamelon testimoniano il probabile dono funebre da parte di individui di origine allogena e privi di gentilizio, tra i quali Pulu verosimilmente di condizione non libera.

Oltre al formulario onomastico, in questo primo cinquantennio il digramma $\theta i$ della tomba 324 , come nella 21 poco più antica (cfr. V.5), manifesta una parola (acqua) e questa volta nella parete esterna, da legare forse a pratiche rituali (nel fondo esterno è graffito invece il digramma $n i$, di difficile interpretazione). Presso Piantamelon il digramma śp potrebbe essere invece dubitativamente abbreviazione di *spura, a indicare forse un dono di natura comunitaria (cfr. III.4 e supra per il supporto, una pisside).

Nel secondo cinquantennio diminuisce sensibilmente il numero di iscrizioni. Canalbianco mostra ancora una significativa concentrazione di antroponimi etruschi e gentilizi quali Mura e Uni $\theta i u$ (tombe 18 e 186 di Canalbianco), che appartengono a gentes già presenti nel III sec. a.C. In particolare, la gens Mura, qui associata al cognomen Afr, doveva essere piuttosto consistente e articolata (si ricorda il Larza Mura Pili del cinquantennio precedente). La triplice ripetizione della formula della tomba 52, probabilmente dalla stessa mano e menzionante la donna ingenua Lentnai (la defunta se si segue la classificazione delle formule, ma il corredo non fornisce lumi al riguardo), testimonia una pratica del tutto simile a quella della più antica tomba 243. Si distingue invece l'iscrizione qaịa mi siliu della tomba 228, databile a cavallo con la prima metà del I sec. a.C., e che assieme al Kraiku Kaniu di Ca' Garzoni e ai Muliu di via Spolverin (cfr. IV.3.1.A), manifesterebbe una formula bimembre con la funzione del prenome espressa da un antroponimo di origine alloctona (probabilmente greca), indizio di un allentamento del sistema onomastico etrusco attivo almeno dal pieno II sec. a.C.

Se valida l'ipotesi che la formula all'assolutivo espliciti il donatore del dono funebre (come sembrerebbe confermato in molti casi nel rapporto tra onomastica e indicatori di genere dei corredi), allora è difficile poter avanzare valutazioni riguardo le motivazioni della concentrazione di onomastica etrusca e di condizione ingenua da Canalbianco piuttosto che nelle aree periferiche, perché in ogni caso quello che è possibile documentare sono solo i pochi individui che scrivevano. Ad esempio, se davvero il Latiate di Ca' Garzoni fosse lo stesso di Bottrighe, si avrebbe un caso piuttosto significativo di un individuo che è in contatto con gruppi familiari eterogenei della società adriese, cioè quello a cui è afferente l'etrusca Puinia e quello dei Muliu, appartenenti a un clan solo recentemente etruschizzatosi e integrato nella società, e che seppelliscono in zone molto distanti. Dall'altra parte, il raggio di azione degli UniӨiu e dei Mura è concentrato soprattutto in Canalbianco. Ciò potrebbe dunque lascia intendere la possibilità che questi fossero i luoghi di sepoltura destinati ai membri delle due gentes.

$\mathrm{Al}$ II sec. a.C. pertengono soltanto due contesti interpretabili come votivi e caratterizzati da documentazione epigrafica, uno con un graffito non alfabetico lacunoso nel fondo esterno di una grande ciotola con due scanalture all'orlo (n. 31), e l'altro (n. 237), contenente l'iscrizione $k$ snalu. Per quest'ultima, l'ipotesi in chiave votiva della formula abbreviata potrebbe ben accordarsi con l'interpretazione del contesto (cfr. IV.3.2.A).

\section{VI.2.2 Il settore orientale}

Risalendo dal limite sud-orientale dell'abitato antico verso nord, si incontra per prima la località Bettola. Questa, assieme alla località Molinterran, doveva essere in periodo etrusco zona di confine tra abitato e necropoli, come confermato dagli scavi condotti negli anni 2000 e $2007^{141}$. Alla Bettola, L. Conton effettuò già indagini nel 1902-1903 all'interno del fondo di Giulio Foster e rinvenne, sotto sepolture romane, materiali attribuibili al periodo ellenistico ${ }^{142}$, andati poi dispersi. Tra questi, il Con-

${ }_{141}$ Riguardo allo scavo del 2007, eventuali reperti iscritti non sono confluiti nel fascicolo adriese del CIE.

${ }_{142}$ Conton 1904, p. 26. 
ton annovera due iscrizioni (CIE 20736-20737), forse pertinenti a una medesima sepoltura ${ }^{143}$. Si tratta dell'unico caso di una formula bimembre femminile (alpi arnai), ripetuta in due supporti distinti. Non è purtroppo possibile proporne una cronologia, se non una generica appartenenza al periodo ellenistico. Altre iscrizioni da quest'area non sono conosciute, sebbene queste due siano citate a titolo esemplificativo di un nucleo più cospicuo.

Alcuni vasi iscritti, rinvenuti fortuitamente durante lavori agricoli nelle località Amolara e Molinterran e quindi donati a F.A. Bocchi, appartengono alle collezioni del Museo Domestico e del Museo Civico ${ }^{144}$, per il loro stato di conservazione sono da ritenere con tutta verosimiglianza provenienti a contesti funerari. I due vasi di Molinterran sono gli unici in ceramica grigia e i più antichi del gruppo qui considerato, datandosi il piatto da pesce entro la seconda metà del IV sec. a.C. (CIE 20424, chi o kappa graffito dopo cottura nel fondo esterno) e l'olla più genericamente tra la seconda metà del medesimo secolo e tutto il seguente (CIE 20423, ypsilon graffito dopo la cottura nella spalla), forse traccia di una o più sepolture di questa fase scarsa di contesti funerari. Presso Amolara, tutti vasi a v.n. iscritti sono di produzione locale e si collocano in prevalenza nel II sec. a.C. (CIE 20410, 20411, 20412, 20414, 20415, 20417, 20420), mentre solo due nel III sec. a.C. (CIE 20413, 20421) e alcuni più genericamente nel III-II sec. a.C. (CIE 20418; 20416 e 20419 per il loro stato frammentario). Si contano qui ben quattro iscrizioni, tutte graffite dopo cottura sul fondo interno: laz su una ciotola serie Morel 2686 (CIE 20411, II sec. a.C.), f]astiu $m[i]$ su una forma aperta purtroppo lacunosa (CIE 20419, III-II sec. a.C.), pulevi $m$ [ $i]$ (eccezionalmente cancellata da linee parallele) su una grande ciotola con due scanalature all'orlo (CIE 20420, II sec. a.C.). Ad eccezione di $l a z$, che si potrebbe ritenere la forma relitta di un prenome come Larza, gli antroponimi Fastiu e Pulevi sono attestati in altre iscrizioni ${ }^{145}$. In tutti i casi, la base onomastica è schiettamente etrusca ed espressione di formule all'assolutivo, secondo quella pratica epigrafica interpretata come possibile dono funerario (cfr. IV.3.2).

Diverse centinaia di metri in direzione nord-est, in località Amolaretta ${ }^{146}, \mathrm{~L}$. Conton assieme alla società degli Archeofili nel fondo di L. Zen nel 1904 scavò alcune sepolture ${ }^{147}$, dalle quali sicuramente proviene una iscrizione conservata al Museo dell'Università di Padova ${ }^{148}$, lausia su un piatto tipo Morel 1441d (CIE 20422, II sec. a.C.). Come per i casi di Amolara, anche questo nome femminile trova confronto altrove, nella fattispecie in una iscrizione su una anfora greco-italica della seconda metà del III-inizi del II sec. a.C. da una tomba non determinabile di Canalbianco (CIE 20568) e in un testo graffito sui due frammenti conservati di una forma aperta di incerta origine (CIE 20829).

Nella periferia più settentrionale dei sepolcreti urbani, la località $\mathrm{Ca}$ ' Cima ha restituito le tombe più antiche, databili a partire dalla metà del VI sec. a.C. e con una continuità di uso fino all'età

143 Ibidem.

144 Dalla località Amolara si documentano dieci vasi, trovati tra il 2-6 (CIE 20410-20413) e il 16 (CIE 20414-20420) settembre 1872 e donati da Ronconi (detto Banchina) a F.A. Bocchi; un vaso (CIE 20421) fu rinvenuto da Giovanni Raulich nel marzo del 1879 nella stessa località, detto provenire da una tomba "romana"; anch'esso fu donato al Bocchi. Nel Museo Domestico della famiglia Bocchi sono inoltre conservati due vasi (CIE 20423-20424) donati a F.A. dall'avvocato G.B. Lupati, rinvenuti in località Molinterran il 7 giugno 1877. L'integrità dei vasi, entrambi in ceramica grigia, suggerisce anche per questi una provenienza da contesto funerario.

145 In particolare, mentre Fastiu richiama il medesimo antroponimo isolato su una ciotola di III sec. a.C. di provenienza ignota (CIE 20776), Pulevi trova confronto in un antroponimo lacunoso su un frammento di coppa in ceramica grigia dal settore NE del Pubblico Giardino (CIE 20190).

146 Gli scavi condotti in via Amolaretta e in via Zaccagnini negli anni 2010-2011 hanno confermato la natura funeraria dell'area (GAmBACURTA et al. 2012, pp. 43-44). Si rimarca che questi scavi non sono stati presi in esame per la redazione del fascicolo adriese del CIE.

147 Tra i rinvenimenti di L. Conton in località Amolaretta, va sicuramente ricordata una tomba databile alla seconda metà del V sec. a.C. (Mambella 1984, pp. 29-30, con riferimenti, tab. 1).

148 Al Museo dell'Università di Padova altri vasi con graffiti sono attribuibili agli scavi del Conton (1904 e 1908), ma sarebbe necessario una più approfondita indagine per accertarne la provenienza. 
ellenistica e quindi romana (II sec. d.C.). Quest'area non è stata indagata in maniera estensiva, ma sempre in relazione a interventi di emergenza che hanno necessariamente vincolato l'ampiezza dei settori di scavo, saggiati prevalentemente per trincee. Inoltre, allo stato attuale non sono disponibili planimetrie di questi settori funerari sui quali impostare una analisi spaziale di distribuzione dei documenti epigrafici.

Le prime indagini in Ca' Cima risalgono al 1970, nella porzione più occidentale in proprietà Catani, dove furono scavate 68 tombe comprese tra il III sec. a.C. e il II sec. d.C., delle quali solo 9 riferibili a un orizzonte di III-II sec. a.C. ${ }^{149}$. I documenti epigrafici qui provengono da 4 distinte sepolture $(45,50,51,56)$, tutte databili al II sec. a.C. ${ }^{150}$. Successivamente, scavi preventivi condotti a più riprese negli anni Novanta hanno consentito il rinvenimento di sepolture di età arcaica ed ellenistica ${ }^{151}$. Anzi tutto lo scavo di condutture funzionali a un metanodotto tra il 1993 e il 1995 nella parte più orientale di Ca' Cima ha portato al rinvenimento di circa settanta sepolture comprese tra l'età arcaica e quella ellenistica, oltre che ulteriori attestazioni di epoca romana; in particolare, l'indagine del 1993, di cui si conoscono in letteratura solo dati preliminari ${ }^{152}$, ha permesso il recupero di 27 tombe di periodo etrusco, divise in due trincee di scavo: i documenti epigrafici provengono da 6 distinti contesti funerari della trincea $\mathrm{A}$, che conta nel complesso un totale di 26 sepolture comprese tra l'età tardo-arcaica e quella ellenistica, oltre a tre depositi votivi. L'indagine condotta nella parte sud-ovest tra il dicembre del 1994 ed il gennaio del 1995 e quindi tra il maggio ed il giugno del 1995 per la costruzione di un supermercato (sigla ACC) ha permesso il recupero di 48 contesti funerari compresi tra il periodo arcaico e quello ellenistico ${ }^{153}$. Tra questi si registrano una tomba di periodo tardo-arcaico e 4 tombe di periodo ellenistico con documentazione epigrafica. Infine, le indagini archeologiche dovute a una lottizzazione di terreno nella parte nord-ovest tra il 1994 e il 1996 (sigla LTZ) hanno messo in luce molte sepolture di età ellenistica e romana; in particolare, nel $1995 \mathrm{si}$ sono rinvenuti 10 contesti sia votivi che funerari (la numerazione di tutti i contesti è consecutiva e quelli votivi si distinguono anche qui per essere introdotti da "n."), databili tra IV e II sec. a.C. ${ }^{154}$ : documenti epigrafici provengono da due sepolture e due contesti votivi ${ }^{155}$. Altre sepolture di età ellenistica e romana sono state indagate grazie a ulteriori scavi preventivi nel 1999 (lottizzazione di terreno) e nel 2010-2011 (metanodotto Snam), ma non è stato possibile visionarle perché ancora in corso di restauro.

Nella fase di fine VI-V sec. a.C. in Ca' Cima sono documentati singoli graffiti alfabetici e non alfabetici in tre sepolture: 3/ACC (fine VI-inizi V sec. a.C.), 14/1993 (V sec. a.C.), 1/1993 (seconda metà del V sec. a.C.). Si tratta di due inumazioni in fossa (3/ACC e 14/1993) e una cremazione in pozzetto $(1 / 1993)^{156}$, tutte con corredi molto contenuti. Le due inumazioni sono attribuibili la prima probabilmente a una donna ${ }^{157}$ e la seconda a un bambino di $5 / 6$ anni $^{158}$. I graffiti sono apposti in due casi su vasi in ceramica depurata etrusco-padana, cioè un piatto su fondo piano (CIE 20391

149 Dallemulle, Marzola 1977, pp. 3-6.

150 I corredi delle tombe 50, 51 e 56 (inedite) conservano singoli vasi iscritti, in un caso con graffito non alfabetico (CIE 20380 dalla t. 56) e in due casi con un graffito non alfabetico e uno alfabetico da ciotole a v.n. (CIE 20378 dalla t. 50, 20379 dalla t. 51); la tomba 45, una sepoltura bisoma già edita (DALlEMULle, MARzola 1977), ha restituito tre iscrizioni su tre differenti vasi a v.n., di cui due di produzione locale (CIE 20375-20376) e uno di produzione volterrana (CIE 20377).

151 Cfr. Bonomi 1994, pp. 509-510; EAD. 1997, pp. 31-34.

152 Cfr. Bonomi 1998, pp. 243-245.

153 Solo una parte dei corredi funerari sono stati visionati, perché i restanti sono ancora in fase di restauro.

154 Inediti se non per alcune anfore: ToNIOLO 2000, pp. 228-229.

155 La tomba 38, inumazione in cassa lignea, inedita e databile approssimativamente al II sec. a.C., presenta all'interno un'anfora con iscrizione verosimilmente latina, $M I$ (CIE 20405).

156 Inedita, ma presente nell'esposizione permanente del Museo, dove è datata alla seconda metà del V sec. a.C.

157 Bonomi-Camerin-TAMASSia 2002a, p. 7.

158 Inedita, ma presente nell'esposizione permanente del Museo, dove è datata al V sec. a.C. 
della tomba 3/ACC: chi) e una coppa emisferica (CIE 20383 della tomba 1/1993: graffito non alfabetico), entrambi realizzati dopo la cottura. Caso diverso è la piccola olla in ceramica grezza della tomba 14/1993 (CIE 20388), sulla quale furono incisi prima della cottura due graffiti, una lettera isolata ( $p i$ presso la superficie esterna dell'orlo) e uno non alfabetico (due aste sul fondo esterno). Va puntualizzato che il $p i$ di aspetto a uncino richiama le più antiche attestazioni del periodo tardo-arcaico (si veda anche l'isolato $p i$ dallo scavo di via San Francesco di più incerta datazione ma paleograficamente coerente con il nostro: CIE 20244; cfr. VI.1), confermando la coerenza formale del grafema fino al V sec. a.C. (cfr. III.2). Non sfugge inoltre la redazione della lettera presso l'orlo, in posizione di grande visibilità (a differenza delle due aste sul fondo esterno) e che dunque difficilmente può essere espressione della catena produttiva. Da questo punto di vista, l'incisione a crudo di prenomi nella medesima posizione in abitato (si veda CIE 20361), potrebbe in effetti suggerire che la nostra lettera possa essere interpretata come una abbreviazione, secondo quanto sottolineato da V. Bellelli per i contesti funerari ${ }^{159}$. In ogni caso, in un panorama di sepolture di VI-V sec. a.C. piuttosto contenuto ${ }^{160}$, la rarefazione delle testimonianze epigrafiche, che peraltro non prevede alcuna iscrizione di contro a quanto documentato per l'abitato, induce ad avanzare l'idea che anche ad Adria una specifica prassi funeraria abbia determinato la generale assenza di manifestazioni scrittorie nei corredi, similmente a quanto già riscontrato per Bologna e Spina nel medesimo periodo ${ }^{161}$.

Nel periodo ellenistico, si registrano documenti epigrafici in 13 sepolture (6 databili tra la fine del IV e il III sec. a.C., una a cavallo di III e II sec. a.C., 6 di II sec. a.C.) ${ }^{162}$ e 3 contesti votivi ( 2 di III e 1 di II sec. a.C.), nei quali il materiale è stato rinvenuto in condizioni frammentarie (Tab. 12).

Tab. 12. Contesti votivi e tombe da Ca' Cima con documentazione epigrafica (tutti realizzati dopo la cottura).

\begin{tabular}{|c|c|c|c|c|}
\hline Contesto & $C I E$ & TIPO DI EPIGRAFE & Posizione & SUPPORTO \\
\hline \multicolumn{5}{|c|}{ Contesti votivi } \\
\hline \multirow{2}{*}{$\begin{array}{l}\text { Contesto votivo US 114/1993, } \\
\text { prima metà del III sec. a.C. }\end{array}$} & 20381 & $c h i$ & fondo interno & ciotola, v.n. locale \\
\hline & 20382 & asterisco & fondo esterno & coppa, ceramica grigia \\
\hline $\begin{array}{l}\text { Contesto votivo n. 1/LTZ, } \\
\text { III sec. a.C. }\end{array}$ & 20403 & segno a croce & fondo esterno & ciotola, v.n. locale \\
\hline $\begin{array}{l}\text { Contesto votivo n. 36/LTZ, } \\
\text { 150-125 a.C. }\end{array}$ & 20404 & $m u[---]$ & parete interna & ciotola, v.n. locale \\
\hline \multicolumn{5}{|c|}{ Tombe } \\
\hline $\begin{array}{l}\text { T. } 39 / \text { LTZ, } \\
\text { cremazione in cassa di legno, } \\
\text { fine IV-prima metà del } \\
\text { III sec. a.C. }\end{array}$ & 20406 & graffito non det. & parete esterna & ciotola, v.n. spinetica o locale \\
\hline
\end{tabular}

159 BELLELLI 2012, p. 384. Sebbene rimanga difficile trovare uno scioglimento convincente, non va a priori scartata l'eventualità di un significato più profondamente religioso di questa lettera e del vaso su cui è stata redatta, similmente alla pratica delle olle iscritte offerte a divinità come Vei/Demetra (BELLELLI 2014, pp. 52-53). Si ricorda inoltre che recentemente E. Govi ha ipotizzato che il digramma $k a$ inciso a crudo sull'orlo di un'olla in ceramica grezza rinvenuto nel temenos del tempio di Uni, possa essere una abbreviazione del teonimo KavaAa (REE LXXX, p. 241 con riferimento al n. 4).

160 Gaucci 2015, pp. 115-118.

161 A. Gaucci in Gaucci, Morpurgo, Pizzirani 2018, pp. 672-674.

${ }^{162}$ Le cronologie proposte risultano più puntuali per i corredi editi (si rimanda ai tituli del CIE IV, I, 1 per riferimenti bibliografici) o per quelli che ospitano iscrizioni in quanto studiati grazie ai lavori di tesi di chi scrive (GAUCCI 2007; ID. 2009). 


\begin{tabular}{|c|c|c|c|c|}
\hline Contesto & $C I E$ & TIPO DI EPIGRAFE & Posizione & SUPPORTO \\
\hline $\begin{array}{l}\text { T. } 2271993 \text {, inumazione, } \\
\text { primo quarto del III sec a.C. }\end{array}$ & 20389 & $c h i$ & parete esterna & ciotola, v.n. locale \\
\hline \multirow{4}{*}{$\begin{array}{l}\text { T. } 9 / 1993 \text {, inumazione, } \\
\text { fine del secondo quarto-metà } \\
\text { del III sec. a.C. }\end{array}$} & 20384 & mi verkantuś & fondo interno & piatto da pesce, v.n. spinetica \\
\hline & 20385 & mi verkantuś & fondo interno & kylix, v.n. volterrana \\
\hline & 20386 & mi verkantuś & fondo interno & ciotola, v.n. spinetica o locale \\
\hline & 20387 & mi verkantus' & fondo interno & ciotola, v.n. spinetica o locale \\
\hline $\begin{array}{l}\text { T. 20/ACC, inumazione, } \\
\text { metà del III sec. a.C. }\end{array}$ & 20392 & segno a croce & fondo interno & ciotola, v.n. locale \\
\hline \multirow{8}{*}{$\begin{array}{l}\text { T. } 30 / \text { ACC, inumazione, } \\
\text { forse femminile, } \\
\text { metà del III sec. a.C. }\end{array}$} & 20394 & kappa & parete esterna & ciotola, v.n. spinetica \\
\hline & 20395 & $\begin{array}{l}c h i(a), k a p p a(b), \\
\text { graffito non det. (c) }\end{array}$ & $\begin{array}{l}\text { fondo interno }(\mathrm{a}), \\
\text { parete esterna }(\mathrm{b}), \\
\text { fondo esterno }(\mathrm{c})\end{array}$ & ciotola, v.n. spinetica \\
\hline & 20396 & $c h i$ & fondo interno & ciotola, v.n. locale \\
\hline & 20397 & $\begin{array}{l}\operatorname{chi}(\mathrm{a}), \text { segno } \\
\text { a croce }(\mathrm{b})\end{array}$ & $\begin{array}{l}\text { parete esterna (a), } \\
\text { fondo esterno (b) }\end{array}$ & piatto, v.n. locale \\
\hline & 20398 & segno a croce & fondo interno & ciotola, v.n. locale \\
\hline & 20399 & segno a croce & fondo interno & ciotola, v.n. spinetica \\
\hline & 20400 & segno a croce & fondo interno & ciotola, v.n. spinetica \\
\hline & 20401 & graffito non det. & fondo interno & ciotola, v.n. locale \\
\hline $\begin{array}{l}\text { T. } 31 / \text { ACC, cremazione, } \\
\text { III sec. a.C. }\end{array}$ & 20402 & graffito non det. & parete interna & ciotola, v.n. locale \\
\hline $\begin{array}{l}\text { T. } 21 / \text { ACC, } \\
\text { inumazione infantile, } \\
\text { 250-150 a.C. }\end{array}$ & 20393 & $\begin{array}{l}\text { segno a croce }(\mathrm{a}) \\
\text { reticolo }(\mathrm{b})\end{array}$ & $\begin{array}{l}\text { cavetto interno }(\mathrm{a}) \\
\text { fondo interno }(\mathrm{b})\end{array}$ & piatto da pesce, v.n. locale \\
\hline \multirow{3}{*}{$\begin{array}{l}\text { T. } 45 / 1970 \text {, inumazione bisoma, } \\
\text { prima metà del II sec. a.C. }\end{array}$} & 20375 & $a k u$ & fondo interno & $\begin{array}{l}\text { grande ciotola con due } \\
\text { scanalature all'orlo, v.n. locale }\end{array}$ \\
\hline & 20376 & $s p$ & parete esterna & pisside, v.n. locale \\
\hline & 20377 & $a \chi$ & parete esterna & ciotola, v.n. volterrana \\
\hline \multirow{3}{*}{$\begin{array}{l}\text { T. } 40 / \mathrm{LTZ} \text {, inumazione } \\
\text { forse femminile, } \\
\text { prima metà del II sec. a.C. }\end{array}$} & 20407 & kusia & fondo interno & ciotola, v.n. locale \\
\hline & 20408 & kemne & spalla & anfora greco-italica \\
\hline & 20409 & $V$ & parete & askòs, ceramica depurata \\
\hline $\begin{array}{l}\text { T. 38/1993, cremazione, } \\
\text { seconda metà del II sec. a.C. }\end{array}$ & 20390 & $k a$ & fondo interno & piatto, v.n. locale \\
\hline $\begin{array}{l}\text { T. 50/1970, inumazione, } \\
\text { II sec. a.C. }\end{array}$ & 20378 & $\begin{array}{l}\text { ypsilon (a), graffito } \\
\text { non det. (b) }\end{array}$ & $\begin{array}{l}\text { fondo interno }(\mathrm{a}), \\
\text { parete interna }(\mathrm{b})\end{array}$ & ciotola, v.n. locale \\
\hline $\begin{array}{l}\text { T. } 51 / 1970 \text {, inumazione, } \\
\text { II sec. a.C. }\end{array}$ & 20379 & $\begin{array}{l}\text { segno a croce }(\mathrm{a}) \\
\operatorname{chi}(\mathrm{b})\end{array}$ & $\begin{array}{l}\text { fondo interno (a), } \\
\text { fondo esterno (b) }\end{array}$ & ciotola, v.n. locale \\
\hline $\begin{array}{l}\text { T. } 56 / 1970 \text {, inumazione, } \\
\text { II sec. a.C. }\end{array}$ & 20380 & asterisco & fondo esterno & ciotola, v.n. locale \\
\hline
\end{tabular}


Nel complesso, durante il periodo ellenistico presso Ca' Cima si ha una assoluta preferenza della ceramica a v.n. come supporto di iscrizioni e graffiti, prevalentemente di produzione locale ma con qualche esempio di vasi di importazione. Altro dato che emerge è la sostanziale assenza di graffiti in tombe con iscrizioni (caso unico è il numerale nella tomba 40/LTZ), a differenza dei sepolcreti a sud dell'abitato che paiono più diversificati da questo punto di vista.

Per la fase di fine IV-III sec. a.C., solo la tomba 9/1993, datata tra la fine del secondo quarto e la metà del III sec. a.C. ${ }^{163}$, conserva iscrizioni. La sepoltura è eccezionale in quanto è l'unica nelle necropoli adriesi di periodo ellenistico che ha restituito un'arma (punta di lancia) e ben 4 iscrizioni identiche realizzate dopo la cottura su vasellame a v.n. Il testo mi verkantuśsi ripete infatti sul fondo interno della kylix, del piatto da pesce e di due ciotole, risparmiando invece il restante vasellame, prevalentemente in ceramica grigia e grezza, classi solo eccezionalmente oggetto di iscrizioni in ambito funerario ${ }^{164}$, ma anche lo skyphos a v.n. Quest'ultimo dettaglio denota una netta distinzione fra i quattro vasi iscritti e lo skyphos, che non è mai oggetto di iscrizione e raramente supporto di altri tipi di graffiti. Si aggiunge che le iscrizioni sul fondo interno della vasca portano a ipotizzare la possibilità di una forma di defunzionalizzazione dei medesimi vasi (ciò potrebbe essere suggerito anche dalla parziale lacuna di un'ansa della kylix), che assieme alla reiterazione della formula, suggeriscono che il set in questione appartenga al medesimo individuo (cfr. IV.3.2). Tale pratica, seppure si possa ipotizzare in altre sepolture adriesi, presenta nella tomba $9 / 1993$ il suo caso più complesso e articolato. Se valida l'idea che Verkantu, portatore di un nome individuale di origine celtica (cfr. IV.3.1.B, categoria d), si identifichi con il defunto (idea corroborata dall'analisi delle diverse tipologie di formule, e non in conflitto con il carattere marcatamente maschile della sepoltura), la scrittura venne qui usata come strumento per marcare il vincolo tra il guerriero sepolto e il suo set da banchetto, indissolubilmente sigillato dai testi graffiti nella vasca interna. Molti elementi della sepoltura di Verkantu (lancia e fibula in particolare) e anche il suo stesso nome rimandano appunto all'ambito celtico. Va peraltro ricordato come le kylikes a v.n., in particolare quelle del tipo con anse non ripiegate a orecchia, siano un elemento frequente nelle sepolture attribuibili ad ambiti culturali celtici in Italia, come già riscontrato variamente dalla critica $^{165}$. Si richiama inotre quanto già rilevato per Spina, dove si è osservato come la pratica di inserimento di queste kylikes nelle sepolture non sia frequente e possa rispondere a logiche di dono funerario (cfr. VI.2.1.A) e comunque in ogni caso sia un vaso da ritenere non funzionale a pratiche funerarie di natura cultuale. Verkantu quindi doveva essere un guerriero celta integrato nella comunità a matrice etrusca di Adria; allo stesso tempo, il rituale funerario, di cui il corredo si fa testimone, non si allinea fedelmente alle tradizioni cittadine e anche il nome individuale sembra tradire la marginalità sociale del guerriero.

Sebbene ci si possa domandare quale sia il rapporto tra questa sepoltura, anomala per molti aspetti, e il resto dell'area funeraria nella quale si inserisce nel III sec. a.C., la risposta non è purtroppo semplice, mancando uno studio sistematico di questo sepolcreto. Limitandoci alle attestazioni epigrafiche, si osserva che per il periodo di fine IV-III sec. a.C. le restanti 5 sepolture ci riservano graffiti, sia alfabetici che non alfabetici, su ciotole a v.n. di produzione locale (con l'unica eccezione di un piatto) ma anche di probabile importazione spinetica. Nella tomba 30/ACC, forse femminile per la presenza di alcune fusaiole, colpisce la presenza di due kappa (CIE 20394, 20395), lettera isolata piuttosto rara che si ritrova in particolare nella tomba 20 di via Spolverin di IV sec. a.C., dove sembra richiamare l'iniziale dell'antroponimo kramuriś graffito su una coppa (cfr. IV.3.2.A).

Nel II sec. a.C., si contano 6 sepolture, delle quali tre con iscrizioni. Fra queste ultime, si ricorda l'inumazione bisoma 45/1970, il cui rito è già stato riconosciuto come eccezionale in ambito

\footnotetext{
163 Camerin 1993, p. 169, n. 31, tav. 2; K. TAmassia, in Akeo 2002, pp. 175-181.

164 Gaucci 2016, pp. 184-186.

165 VitAli 2008, pp. 41-42.
} 
adriese $^{166}$. Questa conserva due ciotole e una pisside a v.n., tutte con iscrizioni etrusche. Si riconosce un antroponimo isolato all'assolutivo, $A k u$ di probabile origine celtica (cfr. IV.3.1.B, categoria d), il digramma a $\chi$ che richiama l'allusione alla sequenza alfabetica (cfr. V.1), e il digramma śp, la cui frequenza nei contesti funerari potrebbe suggerire una pratica trasversale solo ipoteticamente riconoscibile come allusione alla comunità (śp = *spura?; cfr. III.4). Come già osservato in altra sede, l'antroponimo $A k u$ è graffito nel fondo interno di uno delle grandi ciotole con due scanalature che sono un marchio identitario della pratica funeraria di questa città nel II sec. a.C. La ricca tomba 40/ LTZ (forse una donna se si dà peso alla presenza di una fusaiola nel corredo), ci documenta il nome femminile all'assolutivo Kusia di origine etrusco-settentrionale (cfr. IV.3.1.B, categoria c), redatto su una ciotola a v.n., e il lessema kemne su un'anfora greco-italica, che richiama probabilmente pratiche devozionali (cfr. V.3). Infine, la tomba 38/1993, databile approssimativamente alla metàseconda metà del II sec. a.C. (inedita), ci documenta il digramma $k a$ su un piatto a v.n. di produzione locale (CIE 20390), per il quale, data la peculiare reiterazione in più contesti, è stato ipotizzato il valore di deittico isolato oppure l'abbreviazione di un teonimo (es. Calu; cfr. V.3), piuttosto che abbreviazione di nome.

Colpisce la presenza sia nel III che nel II sec. a.C. di antroponimi di origine celtica che parrebbero privi di nome di famiglia e quindi privi di diritti nella società. Sebbene non siano al momento chiare le dinamiche di occupazione di questa vasta area sepolcrale, tuttavia va sottolineato che non sembra unicamente destinata a gruppi marginali e di origine celtica, non solo perché l'iscrizione aku della tomba 45/1970 pare rientrare in una pratica funeraria diffusa e condivisa nel II sec. a.C. alla quale si dà il possibile valore di esplicitazione del donatore (cfr. IV.3.2, classe I) e il digramma śp nella medesima tomba potrebbe suggerire un ruolo della comunità medesima nel rituale funerario del defunto, ma soprattutto questo è confermato dalla tomba $40 / \mathrm{LTZ}$ che sempre nel II sec. a.C. ci testimonia pratiche funerarie che coinvolgono credenze e individui di cultura e origine schiettamente etrusche.

\section{VI.3. Il suburbio}

Dal territorio attorno alla città di Adria provengono alcuni documenti epigrafici, perlopiù rinvenuti nel XIX secolo. Il più importante di questi è l'iscrizione votiva kulśnuteraśśmin $\theta i$ akśke, redatta dopo la cottura nel fondo interno di una ciotola a v.n. locale del pieno III sec. a.C. (CIE 21027). Se coglie nel vero l'idea che i Kulśnuter siano i demoni inferi a cui la ciotola è dedicata (cfr. V.3), questa interpretazione e l'integrità della ciotola medesima portano a ritenere che il vaso fosse stato deposto in una sepoltura, per la quale rimane solo la notizia che si trovava fra Adria ed Este, cioè lungo una direttrice orientata verso nord-ovest ma che non doveva essere molto distante dalla città etrusca. Sempre nel XIX secolo, e in particolare nell'anno 1871, F.A. Bocchi rinvenne nel canale Valdentro, presso Adria, due piatti a v.n. di produzione locale databili tra la seconda metà del III e la prima metà del II sec. a.C. con graffiti alfabetici e non alfabetici. Uno di questi presenta un graffito di difficile interpretazione (CIE 21028), mentre l'altro un alpha in alfabeto leponzio nel fondo interno e un segno a croce nel fondo esterno (CIE 21029). Altri rinvenimenti puntuali si devono a recenti ricognizioni, edite da R. Peretto assieme a M.C. Vallicelli e F. Wiel-Marin nel 2002. Grazie a questa attività, una coppacoperchio in ceramica grezza datata tra $\mathrm{V}$ e inizi del IV sec. a.C. con un graffito non alfabetico redatto nel fondo esterno è stata recuperata a Valle della Croce presso Cicese (CIE 21030). L'irreperibilità del reperto nei magazzini del Museo dei Grandi Fiumi di Rovigo non permette di determinare se tale graffito sia stato realizzato prima o dopo la cottura.

Più consistenti i dati derivati dalle indagini sulle necropoli di via Spolverin in località Bottrighe e della tenuta Campelli, che probabilmente appartenevano a insediamenti periferici sorti a pochi chilometri dal centro urbano lungo direttrici viarie principali ${ }^{167}$.

166 Gaucci, Pozzi 2009, p. 63.

167 Bonomi, Peretto, Tamassia 1993, p. 93. 


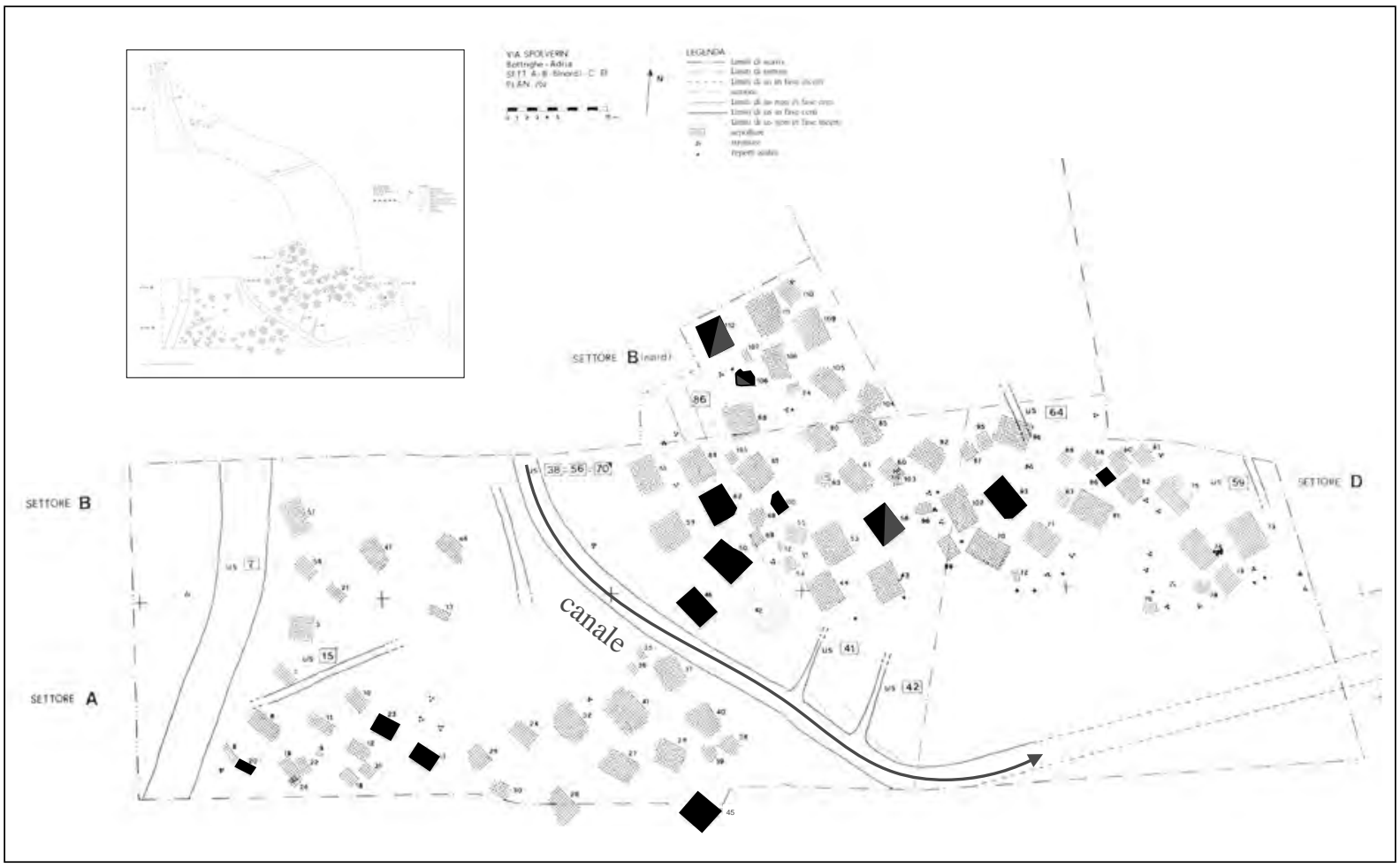

Fig. 44. Dettaglio della planimetria della necropoli di via Spolverin (da Bonomi, Peretto, Tamassia 1993). In nero le sepolture con iscrizioni etrusche, in nero/grigio quelle con iscrizioni etrusche assieme a iscrizioni latine e/o venetiche.

Nella tenuta Campelli, fin dagli inizi del XIX secolo furono rinvenute molte testimonianze di età ellenistica e romana riferibili a contesti funebri ${ }^{168}$. Fra il materiale donato da G. Raulich nel 1872 alla collezione del Museo Domestico della famiglia Bocchi, si enucleano due iscrizioni dette provenienti dallo scolo Valdentro ai Campelli (CIE 21020-21021) ${ }^{169}$. Fra queste, l'iscrizione CIE 21020 è tuttavia da ritenere falsa, sebbene redatta su supporto autentico, mentre l'iscrizione latina CIE 21021 è stata inserita perché su supporto locale di II sec. a.C. Questa manifesta un nome di probabile origine celtica, Casanco o meno probabilmente Vasanco. Nella zona, il più consistente dei rinvenimenti riferibile a età preromana è quello relativo alle undici sepolture scavate nel 19561957 nel podere Stoppa presso la località Passetto e nel podere Belluco in località Tiro a Segno ${ }^{170}$. In località Passetto alcuni graffiti alfabetici e non alfabetici realizzati dopo la cottura in vasi a v.n. di produzione locale appartengono ai corredi della tomba 6 di II sec. a.C (CIE 21024: piatto con graffito un chi nella parete esterna) e della 10 di III sec. a.C. (CIE 21025: kylix con anse defunzionalizzate, con graffito una stella a cinque punte nel fondo interno; CIE 21026, piatto con un graffito non alfabetico a forma di animale nella parete interna). Due vasi con graffito alfabetico (CIE 21022, ciotola a v.n. di produzione locale con graffito ypsilon nel fondo esterno; CIE 21023, piatto a v.n. di produzione locale con alpha graffito nel fondo interno) sono stati erroneamente attribuiti alle tombe 1 e 2 e non è possibile stabilirne il contesto originario.

168 Bolognesi 1998-1999, p. 245.

169 Si ricorda inoltre che tra il materiale edito da G.B. Pellegrini vi è una iscrizione latina di I sec. a.C. acquisita dai Bocchi nel 1872 (ex n. inv. Musei domestici Bocchi D.f. 60, adesso n. inv. Ad 20331), anch'essa proveniente da questa località (Bocchi 1877, p. 198, D.f. 60; Pellegrini, Fogolari 1958, p. 151).

170 Bolognesi 1998-1999, pp. 245-316. 
L'area funeraria di via Spolverin, indagata nel 1990 presso la località Bottrighe, situata a pochi chilometri a sud di Adria, conta 97 deposizioni a inumazione, quasi tutte orientate NO-SE e comprese fra la seconda metà del IV e la fine del II sec. a.C., a cui se ne aggiungono poche altre di successiva fase romana ${ }^{171}$. Il rituale inumatorio delle sepolture prevede sia l'uso della cassa lignea che la semplice fossa rettangolare ${ }^{172}$. Sono stati rinvenuti anche 45 nuclei di oggetti, dislocati ai margini dell'area funeraria o al suo interno, definiti "strutture" e interpretati come depositi votivi connessi a cerimonie commemorative dei defunti ${ }^{173}$.

Come già osservato, l'area iniziò a essere utilizzata solo nel IV sec. a.C., in quanto precedentemente era interessata da depositi alluvionali in un ambiente palustre ${ }^{174}$. Mentre i margini orientale e occidentale sono definiti con certezza dalle indagini archeologiche, rimangono invece incerti quelli settentrionale e soprattutto quello meridionale, dove i limiti di scavo imposti dall'intervento di emergenza non hanno permesso di indagare le sepolture ellenistiche che sicuramente verso sud continuavano $^{175}$ (Fig. 44).

Manca ancora uno studio complessivo dei corredi della necropoli, che potrebbe permette di avanzare una analisi della dinamica di occupazione di quest'area tra il IV sec. a.C. e la romanizzazione; pertanto, a parte alcuni affinamenti cronologici effettuati sulle tombe con iscrizioni, per il resto l'analisi è ancora limitata allo studio preliminare effettuato da T. Stefani, sebbene proprio quest'ultimo lavoro presenti delle proposte cronologiche piuttosto ampie che non sempre collimano con le nuove datazioni derivate da uno studio più puntuale dei corredi.

Qui di seguito si presenta una tabella dei contesti con documenti epigrafici (sepolture e depositi votivi) (Tab. 13).

Tab. 13. Tombe e "strutture" votive di via Spolverin con documenti epigrafci (intesi dopo la cottura se non specificato).

\begin{tabular}{|c|c|c|c|c|}
\hline Contesto & $C I E$ & TIPO DI EPIGRAFE & Posizione & SUPPORTO \\
\hline \multirow{5}{*}{$\begin{array}{l}\text { T. } 20 \text {, inumazione infantile in } \\
\text { fossa, forse femminile, } \\
\text { seconda metà del IV sec. a.C. }\end{array}$} & 20899 & kramuriś & fondo interno & coppa, ceramica grigia \\
\hline & 20900 & kappa (a), kappa (b) & $\begin{array}{l}\text { parete interna } \\
\text { (a), fondo } \\
\text { esterno (b) }\end{array}$ & coppa, ceramica grigia \\
\hline & 20901 & segno a croce & fondo esterno & coppa, ceramica grigia \\
\hline & 20902 & $\begin{array}{l}\text { asterisco } \\
\text { (inciso a crudo) }\end{array}$ & fondo esterno & skyphos, ceramica grigia \\
\hline & 20903 & $\begin{array}{l}\text { asterisco } \\
\text { (inciso a crudo) }\end{array}$ & fondo esterno & skyphos, ceramica grigia \\
\hline \multirow{3}{*}{$\begin{array}{l}\text { T. } 7 \text {, inumazione infantile forse } \\
\text { femminile, } \\
\text { 330-320 a.C. }\end{array}$} & 20887 & alpha & parete esterna & coppa, ceramica grigia \\
\hline & 20888 & chi, fondo interno & fondo interno & $\begin{array}{l}\text { coperchio di lekanis, } \\
\text { ceramica grigia }\end{array}$ \\
\hline & 20889 & digamma (?) & fondo esterno & coppa, ceramica grigia \\
\hline $\begin{array}{l}\text { T. } 47 \text {, inumazione in fossa, } \\
\text { IV sec. a.C. }\end{array}$ & 20931 & alpha, fondo interno & fondo interno & coppa, ceramica grigia \\
\hline
\end{tabular}

171 Bonomi, Peretto, Tamassia 1993, p. 91; Stefani 1996-1997, pp. 159-179; Bonomi 2000, passim.

${ }_{172}$ Un ridotto numero di frammenti di ceramica acroma e a v.n. di produzione locale sporadici dalla necropoli (e privi di inventario) con graffiti alfabetici e non alfabetici, non è stato inserito in CIE IV, I, 1, in quanto ritenuti di scarso valore informativo.

${ }_{173}$ Bonomi, Peretto, Tamassia 1993, p. 92.

174 STEFANi 1996-1997, p. 162.

175 Ibid. 


\begin{tabular}{|c|c|c|c|c|}
\hline Contesto & $C I E$ & TIPO DI EPIGRAFE & Posizione & SUPPORTO \\
\hline \multirow{4}{*}{$\begin{array}{l}\text { T. } 17 \text {, inumazione in fossa, } \\
250-225 \text { a.C. }\end{array}$} & 20895 & malni & parete interna & mortaio, ceramica grigia \\
\hline & 20896 & due $c h i$ contrapposti & parete interna & piatto da pesce, v.n. locale \\
\hline & 20897 & alpha & fondo interno & $\begin{array}{l}\text { ciotola, v.n. probabilmente } \\
\text { riminese }\end{array}$ \\
\hline & 20898 & segno a croce & spalla & anfora greco-italica \\
\hline $\begin{array}{l}\text { T. } 70 \text {, inumazione forse femminile } \\
\text { in cassa di legno, } \\
225-200 \text { a.C. }\end{array}$ & 20983 & alpha & parete esterna & $\begin{array}{l}\text { ciotola, v.n. probabilmente } \\
\text { riminese }\end{array}$ \\
\hline \multirow{2}{*}{$\begin{array}{l}\text { T. } 23 \text {, inumazione probabilmente } \\
\text { femminile in fossa, } \\
\text { seconda metà del III sec. a.C. }\end{array}$} & 20904 & $\begin{array}{l}\text { segno a croce }(\mathrm{a}), \\
\text { venza malni }(\mathrm{b}), \text { due } \\
\text { aste (incise a crudo) (c) }\end{array}$ & $\begin{array}{l}\text { fondo interno } \\
\text { (a), parete } \\
\text { esterna (b), base } \\
\text { d'appoggio del } \\
\text { piede (c) }\end{array}$ & patera, v.n. locale \\
\hline & 20905 & $\begin{array}{l}\text { segno a croce }(\mathrm{a}) \\
\operatorname{chi}(\mathrm{b})\end{array}$ & $\begin{array}{l}\text { fondo interno } \\
\text { (a), parete } \\
\text { esterna (b) }\end{array}$ & ciotola, v.n. locale \\
\hline \multirow{2}{*}{$\begin{array}{l}\text { T. } 27 \text {, inumazione in fossa, } \\
\text { seconda metà del III sec. a.C: }\end{array}$} & 20907 & ypsilon & fondo interno & ciotola, v.n. locale \\
\hline & 20908 & reticolo & fondo interno & ciotola,. v.n. locale \\
\hline $\begin{array}{l}\text { T. } 25 \text {, inumazione in fossa, } \\
\text { seconda metà del III sec. a.C. }\end{array}$ & 20906 & graffito non det. & fondo interno & $\begin{array}{l}\text { ciotola, v.n. probabilmente } \\
\text { riminese }\end{array}$ \\
\hline \multirow{2}{*}{$\begin{array}{l}\text { T. } 60 \text {, inumazione in fossa, } \\
\text { seconda metà del III sec. a.C. }\end{array}$} & 20956 & tau/ypsilon & fondo esterno & coppa, ceramica grigia \\
\hline & 20957 & segno a croce & parete esterna & ciotola, v.n. locale \\
\hline $\begin{array}{l}\text { T. 102, inumazione (forse } \\
\text { infantile) in cassa di legno, } \\
\text { seconda metà del III sec. a.C. }\end{array}$ & 21000 & $\begin{array}{l}P X X X X \text { (lat.) (a), } \\
X X X(\mathrm{~b})\end{array}$ & $\begin{array}{l}\text { collo sotto l'orlo } \\
\text { (a), collo presso } \\
\text { la spalla (b) }\end{array}$ & anfora greco-italica \\
\hline $\begin{array}{l}\text { T. } 108 \text {, inumazione in cassa } \\
\text { di legno, } \\
\text { seconda metà del III sec. a.C. }\end{array}$ & 21006 & segno a croce & parete esterna & ciotola, v.n. locale \\
\hline \multirow{3}{*}{$\begin{array}{l}\text { T. } 6 \text {, inumazione in fossa, } \\
\text { III sec. a.C. }\end{array}$} & 20884 & ypsilon & parete esterna & coppa, ceramica grigia \\
\hline & 20885 & $\begin{array}{l}\text { graffito non det. } \\
\text { lacunoso }\end{array}$ & fondo esterno & coppa, ceramica grigia \\
\hline & 20886 & segno a croce & fondo esterno & ciotola, v.n. locale \\
\hline $\begin{array}{l}\text { T. } 10 \text {, inumazione in fossa, } \\
\text { probabilmente femminile, } \\
\text { III sec. a.C. }\end{array}$ & 20890 & graffito non det. & fondo interno & ciotola, v.n. volterrana \\
\hline \multirow{2}{*}{$\begin{array}{l}\text { T. } 11 \text {, inumazione in fossa, } \\
\text { III sec. a.C. }\end{array}$} & 20891 & $\begin{array}{l}\text { tre } c h i(\mathrm{a}), \text { segno a } \\
\text { croce }(\mathrm{b})\end{array}$ & & coppa, ceramica grigia \\
\hline & 20892 & segno a croce & fondo interno & ciotola, v.n. locale \\
\hline \multirow{2}{*}{$\begin{array}{l}\text { T. } 12 \text {, inumazione in fossa, } \\
\text { III sec. a.C. }\end{array}$} & 20893 & due alpha in legatura & fondo interno & piatto, v.n. locale \\
\hline & 20894 & $\begin{array}{l}\text { serie di aste disposte a } \\
\text { raggiera }\end{array}$ & fondo esterno & ciotola, v.n. locale \\
\hline $\begin{array}{l}\text { T. } 69 \text {, inumazione infantile } \\
\text { in fossa, } \\
\text { III sec. a.C. }\end{array}$ & 20982 & serie di aste & parete esterna & ciotola, v.n. locale \\
\hline $\begin{array}{l}\text { T. } 104 \text {, inumazione infantile } \\
\text { in fossa, } \\
\text { III sec. a.C. }\end{array}$ & 21001 & ypsilon & fondo interno & piatto, v.n. locale \\
\hline
\end{tabular}




\begin{tabular}{|c|c|c|c|c|}
\hline Contesto & $C I E$ & TIPO DI EPIGRAFE & Posizione & SUPPORTO \\
\hline \multirow{2}{*}{$\begin{array}{l}\text { T. } 61 \text {, inumazione in fossa, } \\
\text { fine del III sec. a.C. }\end{array}$} & 20958 & graffito non det. & parete esterna & $\begin{array}{l}\text { ciotola, v.n. probabilmente } \\
\text { riminese }\end{array}$ \\
\hline & 20959 & graffito non det. & parete esterna & $\begin{array}{l}\text { ciotola, v.n. probabilmente } \\
\text { riminese }\end{array}$ \\
\hline \multirow{2}{*}{$\begin{array}{l}\text { T. } 71 \text {, inumazione infantile } \\
\text { (forse femminile) in cassa di legno, } \\
\text { fine del III sec. a.C. }\end{array}$} & 20984 & graffito non det. & parete esterna & ciotola, v.n. locale \\
\hline & 20985 & segno a croce & fondo esterno & ciotola, v.n. locale \\
\hline \multirow{3}{*}{$\begin{array}{l}\text { T. 93, inumazione in cassa di legno, } \\
\text { fine del III sec. a.C. }\end{array}$} & 20993 & $a e$ & fondo esterno & ciotola, v.n. locale \\
\hline & 20994 & $\begin{array}{l}\text { kappa e graffito non } \\
\text { det. }\end{array}$ & fondo interno & piccola ciotola, v.n. locale \\
\hline & 20995 & segno a croce & parete esterna & piatto, v.n. locale \\
\hline \multirow{5}{*}{$\begin{array}{l}\text { T. } 56 \text {, inumazione in cassa di legno, } \\
\text { fine del III-inizi II sec. a.C. }\end{array}$} & 20950 & $A R$ (lat.) & parete esterna & ciotola, v.n. locale \\
\hline & 20951 & la e tau & collo & anfora greco-italica \\
\hline & 20952 & $\widehat{A V}$ o $\widehat{A N}($ lat. $)$ & collo & anfora greco-italica \\
\hline & 20953 & segno a croce & fondo esterno & ciotola, v.n. locale \\
\hline & 20954 & graffito non det. & fondo interno & ciotola, v.n. locale \\
\hline $\begin{array}{l}\text { T. } 86 \text {, inumazione in fossa, } \\
\text { fine del III-inizi del II sec. a.C. }\end{array}$ & 20992 & larza lintialu & fondo interno & ciotola, v.n. locale \\
\hline \multirow{8}{*}{$\begin{array}{l}\text { T. } 62 \text {, inumazione forse femminile } \\
\text { in cassa di legno, } \\
\text { fine del III-prima metà del } \\
\text { I sec. a.C. }\end{array}$} & 20960 & $\begin{array}{l}\text { se, ypsilon e segno a } \\
\text { croce }\end{array}$ & fondo esterno & pisside, v.n. locale \\
\hline & 20961 & $\begin{array}{l}\text { due } c h i(\mathrm{a}) \text {, alpha (lat. } \\
\text { o lep.) }\end{array}$ & $\begin{array}{l}\text { fondo interno } \\
\text { (a), parete } \\
\text { esterna (b) }\end{array}$ & ciotola, v.n. locale \\
\hline & 20962 & $\begin{array}{l}\text { alpha (lat. o lep.) (a), } \\
\text { asterisco (b) }\end{array}$ & $\begin{array}{l}\text { fondo interno } \\
\text { (a), fondo } \\
\text { esterno (b) }\end{array}$ & piatto, v.n. locale \\
\hline & 20963 & $c h i$ & fondo interno & piatto, v.n. locale \\
\hline & 20964 & $\begin{array}{l}\text { segno a croce }(a), \text { segno } \\
\text { a croce }(b)\end{array}$ & $\begin{array}{l}\text { fondo interno } \\
\text { (a), parete } \\
\text { esterna (b) }\end{array}$ & ciotola, v.n. locale \\
\hline & 20965 & segno a croce & fondo esterno & $\begin{array}{l}\text { piattello su alto piede, v.n. } \\
\text { locale }\end{array}$ \\
\hline & 20966 & segno a croce & fondo interno & ciotola, v.n. locale \\
\hline & 20967 & ramo secco & fondo esterno & skyphos, v.n. locale \\
\hline \multirow{5}{*}{$\begin{array}{l}\text { T. } 109 \text {, inumazione di adolescente } \\
\text { in fossa, } \\
\text { seconda metà del III-prima metà } \\
\text { del II sec. a.C. }\end{array}$} & 21007 & alpha & parete esterna & piatto, v.n. locale \\
\hline & 21008 & alpha & fondo interno & ciotola, v.n. locale \\
\hline & 21009 & segno a croce & fondo interno & ciotola, v.n. locale \\
\hline & 21010 & $\begin{array}{l}M P(\text { lat.) o meno } \\
\text { probabilmente } s p\end{array}$ & collo & anfora greco-italica \\
\hline & 21011 & $\begin{array}{l}X X X X V(\mathrm{a}), \text { due aste } \\
\text { (b) }\end{array}$ & $\begin{array}{l}\text { spalla }(\mathrm{a}) \text {, collo } \\
(\mathrm{b})\end{array}$ & anfora greco-italica \\
\hline \multirow{3}{*}{$\begin{array}{l}\text { T. } 42 \text {, rito incerto (forse } \\
\text { inumazione), } \\
\text { prima metà del II sec. a.C. }\end{array}$} & 20915 & $\mid$ ẹisạ| (?) (lep.) & fondo interno & ciotola, v.n. locale \\
\hline & 20916 & chi & parete esterna & ciotola, v.n. locale \\
\hline & 20917 & asterisco & fondo esterno & ciotola, v.n. locale \\
\hline \multirow{2}{*}{$\begin{array}{l}\text { T. } 45 \text {, inumazione in cassa di legno, } \\
\text { prima metà del II sec. a.C. }\end{array}$} & 20921 & skamu & fondo interno & ciotola, v.n. locale \\
\hline & 20922 & segno a croce & parete esterna & ciotola, v.n. locale \\
\hline
\end{tabular}




\begin{tabular}{|c|c|c|c|c|}
\hline Contesto & $C I E$ & TIPO DI EPIGRAFE & Posizione & SUPPORTO \\
\hline \multirow{11}{*}{$\begin{array}{l}\text { T. } 50 \text {, inumazione in cassa di legno, } \\
\text { prima metà del II sec. a.C. }\end{array}$} & 20932 & latiate & parete esterna & ciotola, v.n. locale \\
\hline & 20933 & $\begin{array}{l}\text { papa e pre opposti } \\
\text { rispetto al segno a } \\
\text { croce centrale }\end{array}$ & fondo interno & $\begin{array}{l}\text { grande ciotola con due } \\
\text { scanalature all'orlo, } \\
\text { v.n. locale }\end{array}$ \\
\hline & 20934 & $s p$ & parete esterna & pisside, v.n. locale \\
\hline & 20935 & $\begin{array}{l}\text { graffito non det. (a), } \\
\text { lambda/pi (b) }\end{array}$ & $\begin{array}{l}\text { fondo interno } \\
\text { (a), fondo } \\
\text { esterno (b) }\end{array}$ & ciotola, v.n. locale \\
\hline & 20936 & graffito non det. & collo & anfora greco-italica \\
\hline & 20937 & $\begin{array}{l}\text { segno a croce }(a) \text {, } \\
\text { graffito non det. (b) }\end{array}$ & $\begin{array}{l}\text { fondo interno } \\
\text { (a), parete } \\
\text { esterna (b) }\end{array}$ & ciotola, v.n. locale \\
\hline & 20938 & ypsilon & fondo esterno & skyphos, v.n. locale \\
\hline & 20939 & segno a croce & fondo esterno & ciotola, v.n. locale \\
\hline & 20940 & segno a croce & fondo interno & ciotola, v.n. locale \\
\hline & 20941 & graffito non det. & parete esterna & ciotola, v.n. locale \\
\hline & 20942 & graffito non det. & fondo interno & ciotola, v.n. locale \\
\hline \multirow{8}{*}{$\begin{array}{l}\text { T. } 46 \text {, inumazione in cassa di legno, } \\
\text { metà del II sec. a.C. }\end{array}$} & 20923 & $\begin{array}{l}\text { viza muliu e graffito } \\
\text { non det. }\end{array}$ & parete esterna & $\begin{array}{l}\text { grande ciotola con due } \\
\text { scanalature all'orlo, v.n. } \\
\text { locale }\end{array}$ \\
\hline & 20924 & $\begin{array}{l}\text { viza muliu e chi(a), } \\
\text { lambda/pi (b) }\end{array}$ & $\begin{array}{l}\text { fondo interno } \\
\text { (a), parete } \\
\text { esterna (b) }\end{array}$ & $\begin{array}{l}\text { grande ciotola con due } \\
\text { scanalature all'orlo, v.n. } \\
\text { locale }\end{array}$ \\
\hline & 20925 & $\begin{array}{l}\text { kani } \times[---] \text { e segno a } \\
\text { croce }(a), \text { segno a croce } \\
\text { (b) }\end{array}$ & $\begin{array}{l}\text { fondo interno } \\
\text { (a), parete } \\
\text { esterna (b) }\end{array}$ & ciotola, v.n. locale \\
\hline & 20926 & reiOu & fondo interno & ciotola, v.n. locale \\
\hline & 20927 & $\begin{array}{l}\chi \chi l \text { in legatura e } c h i \text { fra } \\
\text { due aste }\end{array}$ & parete esterna & piatto, v.n. locale \\
\hline & 20928 & my & fondo interno & piatto, v.n. locale \\
\hline & 20929 & my & fondo interno & piatto, v.n. locale \\
\hline & 20930 & asterisco & parete esterna & ciotola, v.n. locale \\
\hline \multirow{3}{*}{$\begin{array}{l}\text { T. 106, inumazione infantile, } \\
\text { metà del II sec. a.C. }\end{array}$} & 21002 & $\operatorname{muliu}(\mathrm{a})$, ais $i \times \times u(\mathrm{~b})$ & $\begin{array}{l}\text { fondo interno } \\
\text { (a), parete } \\
\text { esterna (b) }\end{array}$ & piatto, v.n. locale \\
\hline & 21003 & $\begin{array}{l}\text { Iuni. Sesili (lat.) e } \\
\text { segno a croce }\end{array}$ & fondo interno & ciotola, v.n. locale \\
\hline & 21004 & ypsilon (a), asterisco (b) & $\begin{array}{l}\text { fondo interno } \\
\text { (a), fondo } \\
\text { esterno (b) }\end{array}$ & ciotola, v.n. locale \\
\hline $\begin{array}{l}\text { T.. 32, inumazione in fossa, } \\
\text { seconda metà del II sec. a.C. }\end{array}$ & 20910 & segno a croce & fondo interno & mortaio, ceramica grigia \\
\hline $\begin{array}{l}\text { T. } 99 \text {, rito incerto } \\
\text { (probabilmente defunto } \\
\text { femminile) in cassa di legno, } \\
\text { seconda metà del II sec. a.C. }\end{array}$ & 20996 & $\begin{array}{l}\text { segno a croce }(a), \text { segno } \\
\text { a croce }(b)\end{array}$ & $\begin{array}{l}\text { fondo interno } \\
\text { (a), parete } \\
\text { esterna (b) }\end{array}$ & ciotola, v.n. locale \\
\hline
\end{tabular}




\begin{tabular}{|c|c|c|c|c|}
\hline Contesto & $C I E$ & TIPO DI EPIGRAFE & Posizione & SupPorto \\
\hline \multirow{2}{*}{$\begin{array}{l}\text { T. 112, inumazione infantile } \\
\text { in cassa di legno, } \\
\text { seconda metà del II sec. a.C. }\end{array}$} & 21013 & $\begin{array}{l}\text { apu muliu e segno a } \\
\text { croce }\end{array}$ & collo & anfora greco-italica \\
\hline & 21014 & $\begin{array}{l}\text { ma e kapa (ven.), } \\
\text { graffito non det. (b) }\end{array}$ & $\begin{array}{l}\text { collo (a), ansa } \\
\text { (b) }\end{array}$ & anfora greco-italica \\
\hline \multirow{2}{*}{$\begin{array}{l}\text { T. } 100 \text {, inumazione di adolescente } \\
\text { in fossa, } \\
\text { II sec. a.C. } \\
\text { (probabilmente seconda metà) }\end{array}$} & 20997 & $a t[$ vacat $] n a$ & fondo interno & ciotola, v.n. locale \\
\hline & 20998 & segno a croce & fondo interno & piatto, v.n. locale \\
\hline $\begin{array}{l}\text { T. } 110 \text {, inumazione infantile } \\
\text { in fossa, } \\
\text { II sec. a.C. }\end{array}$ & 21012 & $i u$ o $V I$ & parete esterna & ciotola, v.n. locale \\
\hline $\begin{array}{l}\text { T. 40, inumazione in cassa di legno, } \\
\text { fine III-II sec. a.C. }\end{array}$ & 20914 & segno a croce & fondo interno & ciotola, v.n. locale \\
\hline \multirow{9}{*}{$\begin{array}{l}\text { T. } 66 \text {, inumazione infantile (forse } \\
\text { femminile) in cassa di legno, } \\
\text { fine del III-II sec. a.C. }\end{array}$} & 20972 & segno a croce & parete esterna & $\begin{array}{l}\text { bacino di lekanis, ceramica } \\
\text { depurata }\end{array}$ \\
\hline & 20973 & segno a croce & fondo interno & ciotola, v.n. locale \\
\hline & 20974 & segno a croce & fondo interno & ciotola, v.n. locale \\
\hline & 20975 & segno a croce & fondo interno & piatto, v.n. locale \\
\hline & 20976 & segno a croce & fondo interno & ciotola, v.n. locale \\
\hline & 20977 & segno a croce & fondo interno & piatto, v.n. locale \\
\hline & 20978 & segno a croce & fondo interno & ciotola, v.n. locale \\
\hline & 20979 & segno a croce & fondo interno & ciotola, v.n. locale \\
\hline & 20980 & asterisco & fondo interno & ciotola, v.n. locale \\
\hline \multirow{3}{*}{$\begin{array}{l}\text { T. } 37 \text {, deposizione di rito incerto } \\
\text { ma forse femminile, } \\
\text { II sec. a.C. }\end{array}$} & 20911 & .o.ne (ven.) & parete esterna & $\begin{array}{l}\text { ciotola su alto piede, v.n. } \\
\text { locale }\end{array}$ \\
\hline & 20912 & $\begin{array}{l}\text { quattro aste (incise a } \\
\text { crudo) }\end{array}$ & $\begin{array}{l}\text { base d'appoggio } \\
\text { del piede }\end{array}$ & piatto, v.n. locale \\
\hline & 20913 & $\begin{array}{l}\text { alpha legato a segno a } \\
\text { croce }\end{array}$ & fondo interno & ciotola, v.n. locale \\
\hline \multirow{3}{*}{$\begin{array}{l}\text { T. } 44 \text {, inumazione in cassa di legno, } \\
\text { II sec. a.C. }\end{array}$} & 20918 & segno a croce & fondo interno & ciotola, v.n. locale \\
\hline & 20919 & segno a croce & fondo interno & ciotola, v.n. locale \\
\hline & 20920 & XXIII & collo & anfora greco-italica \\
\hline \multirow{6}{*}{$\begin{array}{l}\text { T. } 51 \text {, inumazione in cassa di legno, } \\
\text { II sec. a.C. }\end{array}$} & 20943 & segno a croce & parete esterna & piatto, v.n. locale \\
\hline & 20944 & segno a croce & parete esterna & piatto, v.n. locale \\
\hline & 20945 & segno a croce & parete esterna & piatto, v.n. locale \\
\hline & 20946 & segno a croce & fondo esterno & ciotola, v.n. locale \\
\hline & 20947 & segno a croce & fondo esterno & ciotola, v.n. locale \\
\hline & 20948 & graffito non det. & fondo esterno & pisside, v.n. locale \\
\hline $\begin{array}{l}\text { T. } 59 \text {, inumazione in cassa di legno, } \\
\text { II sec. a.C. }\end{array}$ & 20955 & graffito non det. & corpo & anfora greco-italica \\
\hline \multirow{4}{*}{$\begin{array}{l}\text { T. } 65 \text {, inumazione in fossa, } \\
\text { II sec. a.C. }\end{array}$} & 20968 & ypsilon & fondo interno & ciotola, v.n. locale \\
\hline & 20969 & ypsilon & fondo esterno & ciotola, v.n. locale \\
\hline & 20970 & $\begin{array}{l}L N(\text { o } \ln ) \text { e } Q(\text { lat., } \\
\text { parti opposte del collo })\end{array}$ & collo & anfora greco-italica \\
\hline & 20971 & segno a croce & parete esterna & piatto, v.n. locale \\
\hline
\end{tabular}




\begin{tabular}{|c|c|c|c|c|}
\hline Contesto & $C I E$ & TIPO DI EPIGRAFE & Posizione & SUPPORTO \\
\hline $\begin{array}{l}\text { T. } 68 \text {, inumazione in cassa di legno, } \\
\text { II sec. a.C. }\end{array}$ & 20981 & $\begin{array}{l}\text { asterisco (a), segno a } \\
\text { croce }(b) \text {, segno a croce } \\
\text { (c) }\end{array}$ & $\begin{array}{l}\text { fondo interno } \\
\text { (a), parete } \\
\text { esterna (b), } \\
\text { fondo esterno } \\
\text { (c) }\end{array}$ & ciotola, v.n. locale \\
\hline $\begin{array}{l}\text { T. } 73 \text {, inumazione in fossa, } \\
\text { II sec. a.C. }\end{array}$ & 20986 & segno a croce & parete esterna & piatto, v.n. locale \\
\hline \multirow{3}{*}{$\begin{array}{l}\text { T. } 76 \text {, inumazione infantile in } \\
\text { fossa, } \\
\text { II sec. a.C. }\end{array}$} & 20987 & $c h i$ & parete esterna & ciotola, v.n. locale \\
\hline & 20988 & segno a croce & parete esterna & coppa, ceramica grigia \\
\hline & 20989 & segno a croce & fondo esterno & piatto, v.n. locale \\
\hline $\begin{array}{l}\text { T. } 78 \text {, inumazione infantile } \\
\text { in fossa, } \\
\text { II sec. a.C. }\end{array}$ & 20990 & segno a croce & fondo esterno & piatto, v.n. locale \\
\hline $\begin{array}{l}\text { T. } 79 \text {, inumazione in fossa, } \\
\text { II sec. a.C. }\end{array}$ & 20991 & segno a croce & fondo esterno & ciotola, v.n. locale \\
\hline $\begin{array}{l}\text { T. } 101 \text {, inumazione infantile } \\
\text { in fossa, } \\
\text { II sec. a.C. }\end{array}$ & 20999 & serie di aste & fondo interno & piatto, v.n. locale \\
\hline $\begin{array}{l}\text { T. } 107 \text {, inumazione infantile } \\
\text { in fossa, } \\
\text { II sec. a.C: }\end{array}$ & 21005 & graffito non det. & fondo interno & ciotola, v.n. locale \\
\hline $\begin{array}{l}\text { T. } 31 \text {, inumazione in fossa } \\
\text { infantile, } \\
\text { III-II sec. a.C. }\end{array}$ & 20909 & segno a croce & fondo esterno & coppa, ceramica grigia \\
\hline $\begin{array}{l}\text { T. } 54 \text {, inumazione infantile } \\
\text { in fossa, } \\
\text { III-II sec. a.C. }\end{array}$ & 20949 & ypsilon & parete esterna & ciotola, v.n. locale \\
\hline \multirow{2}{*}{ Struttura II } & 21015 & alpha & parete esterna & piatto, v.n. locale \\
\hline & 21016 & zeta & parete esterna & piatto, v.n. locale \\
\hline Struttura XX & 21017 & reticolo (?) & fondo esterno & piatto, v.n. locale \\
\hline Struttura XXII & 21018 & $\begin{array}{l}\text { ypsilon e graffito non } \\
\text { det. }\end{array}$ & fondo interno & ciotola, v.n. locale \\
\hline Struttura XXXII & 21019 & $\begin{array}{l}\text { graffito non det. } \\
\text { lacunoso }\end{array}$ & parete interna & forma aperta, v.n. locale \\
\hline
\end{tabular}

Per il IV sec. a.C., T. Stefani, che ha offerto uno studio preliminare dell'intero sepolcreto, individua con certezza 5 sepolture e altre 8 di più incerta datazione, avendo queste ultime elementi che possono scendere al III sec. a.C. Fra le 5 di cronologia sicura, le tombe 7 e 47 presentano alcuni graffiti alfabetici e non alfabetici, mentre fra le tombe del secondo gruppo, la 20, adesso databile alla seconda metà del secolo, conserva una iscrizione. Quest'ultima sepoltura appartiene a un individuo sub-adulto probabilmente femminile per la presenza di due fusaiole e l'impressionante numero di quasi cinquanta vaghi d'ambra ${ }^{176}$, che ne suggeriscono l'alto livello. Una coppa in ceramica grigia conserva nella vasca interna l'iscrizione kramuriś, nome individuale al genitivo di difficile analisi onomastica, e un'altra coppa la ripetizione della lettera kappa (forse l'iniziale del medesimo nome?). Colpisce come in questa fase, caratterizzata da una documentazione molto rarefatta, emerga una preferenza delle coppe in ceramica grigia come supporto di iscrizioni e graffiti a destinazione funeraria, di contro ai secoli seguenti, quan-

\footnotetext{
176 STEFANi 1996-1997, pp. 163-164.
} 
do questa classe, che diventa una eccezione per questa specifica destinazione (cfr. II.2.5), è sostituita dalla ceramica a v.n. locale. Il corredo della tomba 20 accoglie anche due skyphoi in ceramica grigia con una decorazione stampigliata con motivi a $S$ riconducibile a un ambito latèniano ${ }^{177}$, che presentano degli asterischi incisi prima della cottura nel fondo esterno. Lo skyphos, come già più volte sottolineato, è scelto molto raramente come supporto di iscrizioni e nel caso in esame sembra probabile che i due graffiti siano espressione della catena produttiva. La possibilità di avere a disposizione la planimetria della necropoli e lo studio preliminare della Stefani, consente alcune considerazioni riguardo alla disposizione della tomba 20 nello spazio funerario. Questa si inserisce in un significativo allineamento di tombe con indicatori femminili (da sud-ovest a nord-est, tombe 20,6,7,21) nel nucleo più antico della necropoli. Anche la tomba 7 (che conserva alcuni graffiti alfabetici) presenta oggetti di ornamento che rinviano al mondo latèniano (una armilla in pasta vitrea e una fibula di schema La Téne antico ${ }^{178}$ ), mentre la collana della defunta della tomba 21 ha un pendente anforiforme in vetro ${ }^{179}$. Quest'ultimo è un oggetto piuttosto eccezionale nel panorama italiano, come dimostrano i pochi casi documentati, concentrati perlopiù lungo tutta la costa adriatica ${ }^{180}$. Rinviando a un futuro studio dedicato a questo gruppo di donne e i loro indicatori culturali, conviene qui sottolineare solo come questi speciali oggetti non possano essere semplicemente rubricati come appartenenti a circuiti commerciali, ma piuttosto suggeriscono un'origine alloctona delle donne sepolte, verosimilmente lungo una direttrice compresa tra il settore alpino orientale e la Moravia. È significativo che proprio queste donne costituiscano parte del nucleo fondativo della necropoli di via Spolverin attorno agli ultimi decenni del IV sec. a.C.

Nella dinamica di popolamento della necropoli durante il III sec. a.C., che prevede un ampliamento dell'area sfruttata verso est e l'emergere delle sepolture in cassone ligneo ${ }^{181}$, si osserva che le tombe 17 e 23, fra loro vicine e le uniche con iscrizioni, presentano il medesimo gentilizio, Malni. Sebbene si tratti di testi che forse manifestano il nome dei donatori (cfr. IV.3.2, classe I), la loro concentrazione è un possibile indizio di un'area destinata alla sepoltura di individui appartenenti allo stesso gruppo familiare. Inoltre, a ulteriore conferma dell'ipotesi che la formula all'assolutivo priva di pronome indichi il donatore, proprio la tomba 23 , che risulta essere di un individuo femminile, presenta l'iscrizione maschile venza malni, graffita eccezionalmente nella parete esterna di una patera a v.n. Nella tomba 17 , il gentilizio isolato malni è scritto invece nella parete interna di un mortaio in ceramica grigia, accomunandosi per scelta del supporto all' iscrizione meiś ta (CIE 20457) da Canalbianco, che ci documenta però una formula diversa (la proprietà da parte del defunto? cfr. IV.3.2, classe III.c) e un nome forse individuale con base onomastica centro-italica. Altre sepolture in questo settore (tombe 25 e 27 databili alla seconda metà del III sec. a.C., tombe 10-12 più genericamente al secolo e già inserite dalla Stefani nei gruppi di IV-III e III sec. a.C.) presentano graffiti alfabetici (alpha, ypsilon e chi) e non alfabetici in vasi perlopiù a v.n. di produzione locale.

Anche la parte più orientale dell'area durante il medesimo secolo è occupata da sepolture caratterizzate dalla presenza di suppellettili prevalentemente a v.n. di produzione locale con graffiti alfabetici e non alfabetici (tombe 60, 69, 70, 104, 108, inserite da T. Stefani prevalentemente nel gruppo databile a cavallo tra III e II sec. a.C.). Tra queste, la tomba 70 , una delle più eccezionali per quantità di oggetti di corredo e complessità della deposizione ${ }^{182}$, documenta un unico alpha graffito in una ciotola a v.n. forse

177 A. Ruta Serafini, in Gambacurta, Ruta Serafini 2018, p. 68, fig. 49a.

178 CAMERIN 1993, p. 169, nn. 23-24.

179 Camerin 1993, p. 169, n. 22.

180 Vellani 2000, con riferimento ai casi di Adria, Spina e Canosa, a cui è aggiunto quello trovato sporadico nell'abitato della Pianella di Monte Savino a Monte Bibele nell'Appennino emiliano; Tagliamonte, Raccar 2007, p. 216, per Campovalano in territorio pretuzio; M. Natalucci in Natalucci, Zampieri 2019, p. 647, per Numana nel Piceno. Puntuali attestazioni sono registrate anche nel versante tirrenico, in Garfagnana in territorio ligure (loc. Filicaia, CIAMPOLTRINi 1993, p. 49) e a Carsoli in territorio equo (TAGLiAmonte, RACCAR 2007, p. 216, con precedente riferimento).

181 Stefani 1996-1997, p. 162.

182 Bonomi, Peretto, Tamassia 1993, pp. 102-118. 
di produzione riminese. La tomba 102 manifesta invece un fenomeno che si affermerà in maniera più diffusa nella necropoli durante il II sec. a.C., cioè quello delle anfore da trasporto iscritte; queste accolgono numerali e lettere o digrammi perlopiù attribuibili all'alfabeto latino, come nel caso citato. Ciò non stupisce, considerando che l'iscrizione latina su anfora attribuita alla tomba 250 di Canalbianco (CIE 20577) testimonia probabilmente una intermediazione latina nel commercio dell'oggetto e del suo contenuto già dalla prima metà del III sec. a.C. (cfr. VI.2.1).

Con lo scorcio del III sec. a.C. e gli inizi del successivo, si registrano attestazioni epigrafiche nelle tombe 61, 71, 86 (che conserva l'iscrizione forse di dono funebre di larza lintialu), 93. Fra queste, la tomba 93 registra solo un digramma su ciotola a v.n. di produzione locale, interpretabile come inizio della sequeza alfabetica (ae). Si tratta dell'unico caso di questa categoria epigrafica da necropoli (a eccezione forse del medesimo digramma da una ciotola quasi integra della prima metà del III sec. a.C., di origine incerta ma forse ipotizzabile come di destinazione funeraria, CIE 20775; cfr. V.1), suggerendo una pratica evidentemente piuttosto rara, sebbene comunque attestata nel mondo etrusco, dove tuttavia è perlopiù circoscritta all'ambito meridionale, a Vetulonia e ad Aleria ${ }^{183}$.

Sempre in questa fase, si colloca la tomba 56, che risulta invece eccezionale in quanto documenta una iscrizione latina, $A R$, graffita su una ciotola a v.n. di produzione locale, dimostrano così la presenza di scriventi latino già dallo scorcio del III sec. a.C. ${ }^{184}$; anche il digramma latino $N F$ su una ciotola a v.n. sempre di produzione locale del III sec. a.C., ma purtroppo di origine incerta (CIE 20835), conferma una pratica che doveva essere meno rara di quanto si può pensare e sulla quale nel futuro dovranno essere formulate ulteriori riflessioni storiche. Non va peraltro dimenticato che la scrittura latina doveva essere quanto meno conosciuta almeno dalla prima metà del III sec. a.C. (cfr. CIE 20577, prima menzionata). Vale la pena rimarcare che proprio l'insediamento di Bottrighe doveva costituire la proiezione verso l'asse di percorrenza nord-sud che sarà poi ricalcato nella seconda metà del II sec. a.C. dalla via Popilia e che conduceva verso i territori romagnoli e marchigiani già colonizzati o direttamente alleati di Roma.

A cavallo dei due secoli, ma con cronologie più ampie, sono databili anche le tombe 62 e 109, delle quali la prima con un digramma $(s e)$ sul fondo esterno di una pisside a v.n. di produzione locale associato a un ypsilon e a un segno a croce, oltre che due vasi a v.n. entrambi con un alpha isolato non appartenente alla tradizione epigrafica etrusca (forse piuttosto a quella latina o leponzia). Il digramma si presenta di difficile interpretazione, sebbene sia suggestivo richiamare il lessema sesiu rinvenuto in abitato, per il quale si è supposto una pertinenza con il contenuto o il contenitore (cfr. V.4).

Appartengono al II sec. a.C. 24 sepolture che conservano iscrizioni e graffiti; queste mostrano una eccezionale concentrazione di individui sub-adulti, 10 in totale.

Si datano alla prima metà del II sec. a.C. tre sepolture in cassone ligneo (tombe 42, 45, 50). Di queste, la 45 documenta, assieme a un segno a croce, il nome individuale Skamu, da ritenere di estrazione servile e origine greca, probabilmente come donatore dell'offerta funebre. Più complessa la documentazione della tomba 50 , che, oltre a molti graffiti, testimonia diverse pratiche funerarie, come forse quella del dono funebre, qui esercitata da un individuo di nome Latiate, che potremmo ritenere ipoteticamente lo stesso della necropoli di Ca' Garzoni. Se tale ipotesi cogliesse nel vero, questo documenterebbe uno stretto legame fra gruppi che seppelliscono a notevole distanza, addirittura in una probabile comunità satellite di quella urbana quale Bottrighe. Il Latiate in questione, come già sostenuto (cfr. IV.3.1.B, categoria $\mathrm{d}$ ), è peraltro da ritenere persona priva di diritti nella comunità adriese e chiaramente originaria del Lazio, come lascia intendere il nome stesso. È improbabile che questo fosse uno schiavo, ma piuttosto potremmo ritenerlo un prospector romano integrato, sebbene marginalmente, nella società etrusca di Adria ${ }^{185}$. La sepoltura ci fornisce anche una probabile pratica cultuale legata

\footnotetext{
183 Gaucci 2012b, p. 70.

184 Per ulteriori considerazioni su questo, cfr. GaUCCI 2016, p. 208.

185 Come già sostenuto in GAUCCI 2016, p. 208.
} 
a una grande ciotola con due scanalature all'orlo a v.n., forma esclusiva della città e che già in altra sede è stata legata a una speciale pratica funeraria ${ }^{186}$. Al centro del fondo interno è presente un segno a croce e in due degli opposti quadranti delineati dal graffito i lessemi papa e pre, il primo probabile appellativo forse riferibile a una divinità infera (l'assolutivo potrebbe suggerire una consacrazione piuttosto che una dedica; cfr. V.3), mentre il secondo di più difficile interpretazione. La complessa composizione è $\mathrm{da}$ ritenere concepita secondo una precisa organizzazione dello spazio del vaso ${ }^{187}$. Infine, sempre dalla tomba 50 il digramma śp sulla pisside a v.n. di produzione locale potrebbe essere abbreviazione per *spura, come per i casi di Ca' Cima e Piantamelon del medesimo periodo a cui si rinvia (cfr. VI.2.1-2). Si tratta dunque di un contesto molto complesso, nel quale l'epigrafia ha un ruolo importante nel definire distinte azioni rituali connesse con le offerte funebri.

Segnali di un progressivo indebolimento della cultura e della società etrusca di Adria si manifestano chiaramente nel pieno II sec. a.C. con la tomba 46, che assieme alla $106 \mathrm{di}$ sub-adulto, appartiene a questa fase. La prima presenta nelle due grandi ciotole con scanalature all'orlo a v.n. il nome all'assolutivo del probabile donatore, Viza Muliu. La formula, nella sua costruzione identica a quelle costituite da prenomi canonicamente etruschi e gentilizi, e nella presenza del secondo elemento (Muliu) non liquidabile come appositivo, ma esso stesso gentilizio come conferma la sua ripetizione in altre tombe e associato ad altri nomi (cfr. in particolare apu muliu della più tarda tomba 112), è quella di un individuo ingenuo e pienamente inserito nella società. Tuttavia l'uso di un prenome nuovo, non appartenente al tradizionale pool onomastico adriese, dà forse la vera misura del possibile indebolimento dell' identità civica. Le restanti iscrizioni conservate nel corredo, kani $i \times[---]$ e rei $\theta u$, mostrano la gravitazione di altri individui di origine non etrusca attorno a questo gruppo familiare anch'esso di origine alloctona, forse veneta (cfr. IV.3.1.A). Tuttavia, il probabile allentamento delle regole del sistema onomastico degli ingenui non permette di intepretare con certezza questi nomi isolati come appartenenti a individui privi di diritti o non liberi. Infatti l'iscrizione lacunosa CIE 20925, seppure incerta, se valida l'integrazione kani [u, potrebbe richiamare il Kraiku Kaniu di Ca' Garzoni (CIE 20697), anch'egli portatore di un doppio nome probabilmente da intendere come prenome e gentilizio al pari di Viza Muliu. Non pare inoltre peregrino osservare che la ripetizione quasi ossessiva del gentilizio Muliu si palesi anche nell'abbreviazione $m y$ di due piatti a v.n., e in maniera estesa nella tomba 106, dove è associato in un medesimo vaso al lessema aisị $\times \times u$, la cui la radice ais- richiama la sfera divina (cfr. V.3). Ma la tomba 106 è eccezionale anche per la presenza esplicita di un nome bimembre in alfabeto e lingua latina, Iuni . Sesili (CIE 21003; cfr. Fig. 22), che in questo orizzonte cronologico non si può intendere se non come un individuo proveniente dall'esterno, cioè da uno dei centri coloniali che ormai circondavano a sud (da Ariminum sulla costa a Bononia nell'interno) e anche a nord (Aquileia) la città etrusca. Se valida questa ipotesi, si constata che mentre pochi decenni prima uno o più individui di origine latina si esprimevano in etrusco identificandosi con il proprio luogo di origine usato come nome individuale, adesso si esprime il nome ufficiale scritto nella propria lingua, a dichiararne la condizione libera in un contesto che ormai presentava una maglia culturale (quella etrusca caratterisca del centro) fortemente allentata.

Nella seconda metà del II sec. a.C., la tomba 112 conserva due anfore da trasporto iscritte, una con la formula onomastica all'assolutivo di Apu Muliu, da intendere dunque come un altro esponente di questa famiglia, e l'altra con due lessemi (interi o abbreviati) in alfabeto venetico (CIE 21014; Fig. 22). Questa tomba non solo conferma l'estensione di quello che pare a tutti gli effetti un vasto recinto funerario, quello dei $\mathrm{Muliu}^{188}$, ma anche i rapporti culturali intessuti da questa famiglia della più tarda Adria etrusca. Proprio i lessemi in alfabeto venetico in una delle due anfore, assieme all' iscrizione sempre venetica.o.n e dalla tomba 37 di II sec. a.C. (CIE 20911), forse un nome di origine celtica, sono gli

\footnotetext{
186 Gaucci, Pozzi 2009, p. 63.

187 GaUCCI 2020.

188 Si richiamano le osservazioni in GaUcci, Morpurgo, Pizzirani 2018, pp. 674-675.
} 
unici documenti di tale cultura epigrafica ad Adria. Queste più tarde iscrizioni manifestano in maniera chiara come la città medesima fosse frequentata durante la seconda metà del II sec. a.C. in maniera assidua da individui di cultura gallica, latina, veneta, forse retica, a conferma del suo importante ruolo commerciale in questo periodo ${ }^{189}$. Tuttavia, non bisogna pensare con ciò che venisse meno la presenza etrusca. Proprio una delle tombe che potrebbero gravitare nel recinto dei Muliu, la deposizione di un individuo sub-adulto (tomba 100), contiene il gentilizio Atna, di schietta origine etrusca (cfr. IV.3.1.B, categoria $b)$.

L'eccezionalità di questa documentazione epigrafica "mista” è sicuramente almeno in parte da legare all'ambito periferico della necropoli di Bottrighe, sebbene vada ricordato che nella stessa necropoli di Canalbianco si trova un ristretto nucleo di iscrizioni in alfabeto leponzio durante il II sec. a.C. (cfr. VI.3.1), sicuramente più circoscritto e senza la pluralità culturale (e sociale) che i contesti di via Spolverin mostrano.

189 Si veda sul ruolo di Adria in questo periodo, GaUCCi 2016, pp. 196-199. 


\section{CONCLUSIONI Oltre 400 anni di cultura epigrafica e società}

La significativa quantità di documentazione epigrafica che Adria ha restituito durante gli oltre due secoli di scavi, ha permesso di toccare molte problematiche riguardanti una delle grandi città dell'Etruria padana, l'unica assieme a Mantova a mantenere la propria identità culturale etrusca fino al definitivo passaggio sotto l'orbita romana. In particolare, le iscrizioni hanno rappresentato uno strumento sicuramente fondamentale per tentare di impostare uno studio complessivo e diacronico di questo importante centro urbano nel periodo preromano, in quanto non solo grazie a queste è stato possibile approfondire aspetti culturali come la diffusione della scrittura, la paleografia e i modelli in uso, ma anche avviare una riflessione sulla società e sulle pratiche che questa adottava nel confronto con il sacro e con la morte in particolare. Si tratta ovviamente di un percorso che non può ritenersi concluso con le limitate considerazioni scaturite dal presente studio, ma che si dovrà sviluppare nel tempo anche grazie all'apporto e alla messa a sistema di tutte quelle informazioni archeologiche desumibili dai contesti, di cui l'epigrafia costituisce solo una parte, sebbene dal notevole potenziale informativo. È fuori di dubbio che nel panorama degli studi sulla città, caratterizzato da grandi complessi di scavo privi però di analisi d'insieme e poche edizioni spesso di taglio tematico o dedicate a singole sepolture, il ruolo dell'epigrafia appare sicuramente essere molto rilevante. Infatti, la documentazione epigrafica è trasversale a tutti i contesti, costituendo dunque non solo una importante chiave di lettura della società etrusca e delle pratiche scrittorie che questa usava, ma anche uno strumento utile a una più diretta decodifica dei contesti indagati.

Come è stato possibile constatare nel capitolo I, ripercorrendo la storia degli studi sull'epigrafia preromana di Adria, proprio il ruolo di questa disciplina è stato fondamentale nell'orientare la visione storica e culturale della più antica fase di vita del centro polesano. Infatti, se durante l'Ottocento illustri archeologici e linguisti riconoscevano l'etruscità dell'insediamento antico, anche grazie alla conferma che le iscrizioni offrivano rispetto alle informazioni delle fonti scritte, proprio l'epigrafia fu un elemento cardine della virata verso la veneticità del centro, promossa primariamente da G. Ghirardini con gli inizi del XX sec. e poi perseguita negli studi successivi. Solo con un affinamento degli strumenti di analisi, cioè la messa a punto di sistemi paleografici, i progressi nella conoscenza di lingua e lessico, le più puntuali cronologie dei supporti, è stato possibile nel tempo, anche grazie all'apporto di autorevoli 
etruscologi quali G. Colonna, C. de Simone, L. Agostiniani, e A. Maggiani, restituire quell'etruscità linguistica, sociale e culturale che per ragioni di natura preconcetta le era stata tolta.

Tutto ciò, trapelato in filigrana nel fascicolo $C I E \mathrm{IV}, \mathrm{I}, 1$, viene dunque esplicitato nei capitoli che compongono questo lavoro, che si regge necessariamente nell'opera di tutti gli studiosi che su Adria preromana si sono cimentati. Ogni posizione, per quanto condivisibile o da rifiutare oggi possa sembrare, è comunque un valido stimolo nel dibattito scientifico che ha sempre tenuto in vita l'interesse sulle testimonianze più antiche di questa città.

Il presente lavoro, che dunque è solo il più recente tassello di una storia degli studi pluri-secolare, copre un arco cronologico di circa quattro secoli (VI-II sec. a.C.) e prende in considerazione 204 iscrizioni etrusche, comprensive dei digrammi, di cui la maggior parte sono di periodo ellenistico (166).

\section{La formazione della società etrusca nell'Arcaismo}

Già nel periodo compreso tra la fine del VI e il V sec. a.C., Adria e il suo territorio è tra gli insediamenti più significativi del distretto etrusco-padano per numero di iscrizioni (25), subito dopo Spina e Kainua-Marzabotto e prima di Bologna e del Forcello di Bagnolo San Vito (cfr. I.2.1). La notevole limitazione della pratica scrittoria all' interno dei costumi funerari spiega come, a fronte di più articolate indagini nei contesti abitativi di Adria, Spina e Kainua, la ridotta conoscenza dell'abitato di Bologna di V sec. a.C. pesi sicuramente sulla statistica generale. Adria dunque si pone fra i centri etrusco-padani che in maggior quantità hanno restituito testimonianze epigrafiche. Ma la sua eccezionalità allo stato attuale degli studi risiede soprattutto nella conoscenza del territorio tra VI e IV sec. a.C., eleggendola così a osservatorio privilegiato per comprendere le dinamiche sociali e politiche fra centro primario e insediamenti satelliti o complementari, nella fattispecie San Basilio sulla costa, San Cassiano e Le Balone nell' interno.

Uno dei nodi principali nella comprensione storica dell'Adria tardo-arcaica è individuabile sicuramente nella cultura scrittoria del periodo (cfr. III), che negli studi degli ultimi decenni è stata costantemente rinviata a quella etrusco-meridionale e nella fattispecie orvietana. Questo ha sempre distinto in qualche maniera il centro polesano dal resto dell'Etruria padana, dove la norma scrittoria vigente era settentrionale. Adesso si può affermare che ad Adria erano in uso entrambe le norme, meridionale e settentrionale, oltre che possibili casi di testi misti. Inoltre, alla luce delle osservazioni paleografiche, il centro tirrenico che presenta più affinità con Adria sembra essere Vulci (cfr. III.3). Qui era in uso un sistema scrittorio piuttosto fluido, da sempre in dialogo con Vetulonia e il mondo etrusco-settentrionale; un sistema che può aver attecchito ad Adria in quanto efficacemente poteva adattarsi ai molteplici stimoli culturali di questo centro di frontiera, dove peraltro la componente di provenienza vulcente non doveva essere irrilevante.

Da un punto di vista epigrafico, i rapporti scrittori del comprensorio adriese con l'Etruria meridionale sono comunque piuttosto antichi. Infatti, la sporadica manifestazione di San Basilio dubitativamente da attribuire a una pratica scrittoria veiente dal pieno VI sec. a.C. potrebbero confermare un percorso adriatico verso il Delta padano battuto già durante l'Arcaismo da individui e gruppi di provenienza etrusco-meridionale (non è da ignorare da questo punto di vista la kotyle etrusco-corinzia della fine del VII sec. a.C. di San Basilio, riferita da S. Bruni ${ }^{1}$ alla tradizione ceramografica vulcente e arrivata anch'essa tramite un percorso adriatico secondo G. Colonna ${ }^{2}$ ). E proprio tale percorso avrebbe veicolato la cultura epigrafica etrusco-meridionale verso il comparto veneto grazie al ruolo di tramite del comprensorio adriese.

Il sistema scrittorio vulcente arrivò ad Adria forse attraverso questa medesima via, tiberina e poi adriatica, che passava per Orvieto, portato da quei gruppi che evidentemente nella città padana si

\footnotetext{
1 BRUNi 1994.

2 Colonna 2003, in particolare pp. 158-169.
} 
stabilirono. Il modello così veicolato era forse in uso da uno o più gruppi sociali all'interno di una comunità che doveva presentarsi come socialmente e culturalmente complessa. Ammesso che questo sistema scrittorio coerente e codificato - che dunque seguiva nell'uso dell'alfabeto precise regole e relativi insegnamenti -, sia effettivamente esistito ad Adria, non era l'unico e per natura tollerava pratiche epigrafiche diverse, complementari e non rigidamente alternative. Queste considerazioni portano in definitiva a scardinare il principio di uguaglianza tra sito e norma scrittoria vigente, con la volontà di approdare a modelli più complessi che meglio possano rispondere alle problematiche che la documentazione epigrafica pone.

Le osservazioni sulla società adriese del periodo sono piuttosto limitate (cfr. IV.1). Si conoscono alcuni prenomi (Laris e Venel), i gentilizi Uselna, Leceniie, $\operatorname{Amn}(a)$ e forse il lacunoso ]unas. Come più volte sottolineato, questi nomi di famiglia puntano coerentemente all'Etruria meridionale e in particolare a Orvieto. La probabile diversa resa grafica della sibilante nel nome della gens orvietana degli Uśelna potrebbe essere interpretata come il tentativo di una integrazione all'interno di un comparto sociale urbano la cui cultura epigrafica primaria doveva essere appunto settentrionale. Se valida questa osservazione, la scelta di scrivere il genitivo I con il sigma sembra invece suggerire la volontà di una comunicazione scritta destinata a lettori usi alle norme ortografiche meridionali.

Forse afferente a questo medesimo comparto sociale era la donna dal nome celtico Uinia, la cui iscrizione è espressa in norma settentrionale. È possibile che anche Uinia fosse una ingenua, trovando

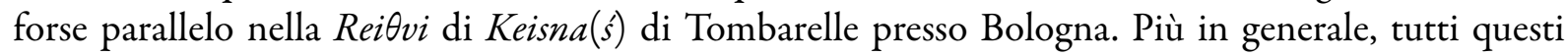
nomi (familiari e non familiari) possono essere considerati indizi di una società composta da distinti gruppi sociali in qualche maniera fra loro intrecciati. Se si considera anche il prenome schiettamente etrusco-settentrionale Pisna (CIE 21041) e il nome dal suffisso padano (e radice celtica) Meslalu (CIE 21040), entrambi da Le Balone, si constata che il territorio adriese era abitato nel complesso da individui di origine etrusco-meridionale, settentrionale e padana, inclusivi verso persone provenienti da altri ambiti culturali (nella fattispecie il mondo di lingua celtica). Ciò è senza ombra di dubbio in linea con le produzioni artigianali che erano commerciate e forse lavorate in città, a partire dalla bronzistica e dalla ceramica figurata, nonché con la rete commerciale proiettata verso le direttrici alpine da un lato e verso il mare Mediterraneo dall'altro. Non va infatti dimenticato che proprio ad Adria sono note iscrizioni che testimoniano persone di cultura greca che offrivano dediche a divinità quali Apollo e Iride nel santuario individuato presso la località La Tomba. Fra questi era anche Soo, eccezionalmente una donna che forse frequentò il porto padano.

Sebbene le poche iscrizioni con elementi onomastici siano perlopiù formule di possesso legate alla vita quotidiana, la cultura epigrafica di periodo tardo-arcaico si esprime ad Adria soprattutto nell'ambito sacro. Si individuano almeno tre principali luoghi dove sono state rinvenute iscrizioni che indiziano la presenza di culti. Il più importante è sicuramente il santuario presso La Tomba. Da qui provengono dediche votive in greco e sicuramente il chous attico a f.n. con graffito nel fondo esterno kai, di interpretazione incerta, ma che potrebbe essere un nome femminile (cfr. IV.1). Molte delle iscrizioni schiettamente etrusche del Museo Domestico della famiglia Bocchi potrebbero ipoteticamente venire proprio da questo luogo. Fra queste, merita sicuramente ricordare il frammento di piede di kylix attica con una iscrizione sulla superficie interna e una su quella esterna, per il quale si è ipotizzato che una delle due (non a caso quella che sembrerebbe in norma settentrionale) fosse stata precedentemente preparata nel santuario e quindi la seconda (in norma meridionale) realizzata dopo l'acquisto per l'offerta, secondo una prassi che si riscontra anche in altri santuari etruschi come Gravisca. Ma anche le due kylikes con digramma $k r$, almeno in un caso nella parete esterna, cioè in un punto di grande visibilità, potrebbero avere una destinazione votiva, se valida l'idea che le due consonanti siano il relitto di kara, sostantivo documentato nello scavo del Cortile Ornati e che probabilmente identifica il vaso, forse nella sua destinazione sacra. Non è peraltro escluso che anche alcune paterae pertusae con graffiti, forse indizio di forme rituali volte al mondo ctonio, siano state rinvenute in questi scavi. 
Dal Cortile Ornati provengono tre documenti molto rilevanti che menzionano doni e offerte, cioè il piatto su fondo piano con iscrizione mi al (cfr. V.2), la coppa con iscrizione mi venelus kar (cfr. IV.1) e il calice in bucchero con la scena complessa graffita nella parete esterna e le lettere in legatura nel fondo esterno (cfr. VI.1.2). Questi vasi, anche per il loro stato di conservazione ancora buono al momento del rinvenimento, sono forse da ritenere parte di un deposito a carattere sacro, che evoca il possibile parallelo con il deposito $\kappa$ del Santuario meridionale di Pyrgi realizzato in corrispondenza di un altare dedicato alla coppia infera Suri-CavaAa.

Anche nel Fondo Lodo alla Bettola i pochi documenti noti orientano l'interpretazione verso un contesto di natura sacra. Infatti si registra l'uso del sostantivo al privo di ulteriori specifiche come nel Cortile Ornati, qui inciso a crudo su una coppacoperchio in ceramica grezza. Sulla base di quanto osservato dalla critica per altri santuari, la scelta del supporto potrebbe alludere a divinità infere; non va infatti dimenticato che la zona in questione era marginale all'abitato e soprattutto in prossimità della cintura funeraria orientale. A rafforzare questa idea, va ricordato che provengono da questo luogo iscrizioni greche forse di natura votiva con antroponimi lacunosi di $\mathrm{V}$ sec. a.C., che dunque potrebbero trovare stretto parallelo con il santuario della Tomba. È inoltre probabile che il Fondo Lodo facesse sistema con il Fondo Calisti, sempre in località Bettola e che (sebbene non localizzato con certezza) doveva essere a questo prossimo. Entrambi hanno restituito iscrizioni che potrebbero indiziare una continuità di uso cultuale delle aree anche in periodo ellenistico.

Se valide le proposte interpretative avanzate per la natura di questi tre luoghi, colpisce l'assenza di esplicite dediche alle divinità, ma solo brevi formule e abbreviazioni focalizzate sull'offerta, oltre alla significativa incidenza di monogrammi composti da due o più lettere in legatura, anch'essi forse funzionali perlopiù a pratiche di offerta, dove l'intreccio di grafemi non aveva solo un valore estetico ma forse anche quello di celare le parole graffite, schermandone il potere magico-religioso.

Molti indizi suggeriscono la natura infera delle divinità venerate. In particolare, nel santuario presso La Tomba la dedica votiva in greca ad Apollo da parte di un certo Tychon potrebbe portare a ritenere che qui fosse venerato dagli Etruschi Śuri, il cui culto era radicato nell'Etruria propria, oppure il suo omologo Man $\theta$ dalla spiccata vocazione poliade, protagonista nella tradizione mitostorica dell'Etruria padana e titolare del santuario meridionale di Pontecagnano, dove era venerato appunto anche con il nome greco di Apollo ${ }^{3}$.

Se questi luoghi sembrano consacrati al culto di divinità a cui si portavano doni e offerte accompagnati da formule epigrafiche spesso compendiarie e allusive, l'unico caso di nome di divinità esplicitato è forse quello di $\Theta a n r$ (cfr. V.1). Sebbene sia incerto il suo luogo di rinvenimento, questo è con buona probabilità da riconoscere nell'area dell'attuale Museo Archeologico Nazionale. Il rimarchevole numero di frammenti con singole lettere e graffiti non alfabetici incisi a crudo, perlopiù su coppe in bucchero, porterebbero a ritenere il luogo come un'area produttiva dove questi graffiti erano usati come marchi nelle fasi di confezione dei vasi. La presenza di paterae pertusae con graffiti e almeno una iscrizione costituita dal solo sostantivo al, aggiungono complessità al contesto, che (anche in questo caso) soltanto con una analisi di tutta la documentazione d'archivio e il materiale archeologico ancora conservato dei molti scavi qui effettuati dall'Ottocento in poi, sarà possibile comprendere nella maniera più opportuna.

\section{Indizi di continuità culturale tra V e IV sec. a.C.}

Molto poco conosciamo per il periodo compreso tra la seconda metà del V e il IV sec. a.C. Non sono purtroppo perspicue le vicende di Adria durante questa fase ed è poco soddisfacente risolvere il problema evocando una contrazione dell'abitato oppure dando acriticamente credito alle notizie delle fonti scritte, che ricordano una colonia siracusana nel IV sec. a.C.

\footnotetext{
3 Per tutto ciò, basti qui citare Colonna 2012, in particolare pp. 210-211.
} 
La società sembra di cultura etrusca, garantendo con tutta evidenza una continuità al sistema scrittorio locale attraverso la ricezione delle novità dal mondo cisappenninico. Infatti, il ridotto numero di testimonianze epigrafiche, assieme a quelle del territorio, documenta nel V sec. a.C. la precoce ricezione del tipo grafico corsivizzante, in sincronia con quanto è documentato per Bologna, Kainua-Marzabotto e il Mantovano (cfr. III.2). È possibile che proprio la ricezione di tale tipo grafico in un orizzonte ancora formativo possa aver portato al mantenimento ad Adria di proprie specificità scrittorie, che si riconoscono alla base della struttura "monolitica" del modello di periodo ellenistico.

Anche sulla società si hanno poche informazioni. Si conosce solo il nome $\operatorname{S} m i n \theta i$, documentato anche nel periodo ellenistico (cfr. IV.3.1.B, categoria c), e Kutvalu(ś), forse un gentilizio di IV sec. a.C. Alla seconda metà del IV sec. a.C. è da attribuire Aia, possibile nome femminile di origine umbra se valida la lettura di una legatura delle lettere. Infine, si data allo scorcio del secolo il nome individuale Kramuri di origine probabilmente non etrusca e conservato nella tomba di una donna appartenente a un gruppo forse originario dell'Europa orientale.

\section{Una società in evoluzione tra III e II sec. a.C.}

Tra il III e il II sec. a.C. la documentazione epigrafica di Adria aumenta in misura notevole, ponendola, come anche Spina, a gareggiare per quantità di attestazioni con alcuni dei più importanti centri dell'Etruria tirrenica. Il quadro padano in questo periodo vede queste due città portuali baluardo assieme a Mantova di una etruscità che è altrimenti presente in sottotraccia nell'entroterra ormai sotto il controllo della componente celtica, come dimostrano le iscrizioni etrusche di Monte Bibele e Bologna. Ma se le testimonianze epigrafiche di questi insediamenti e anche di Spina si limitano al III sec. a.C., solo Adria documenta una persistenza della lingua e della scrittura etrusca fino almeno a tutto il II se non anche nei decenni successivi, cioè fino al compimento del passaggio della città nell'orbita romana tra il 49 con la lex Roscia e il 42 a.C. con la creazione dei municipia. È dunque un osservatorio privilegiato, nella fattispecie dell'esaurirsi della cultura etrusca e della trasformazione della società verso il modello romano. Ma sempre in questo periodo, l'epigrafia ci permette di indagare anche il rapporto fra l'elemento etrusco dominante e i gruppi alloctoni originari dalle popolazioni limitrofe, cioè Celti, Veneti, Reti, e anche il lento inserimento di una compagine di cultura latina, che durante il II sec. a.C. fu verosimilmente causa primaria dell' indebolimento della struttura sociale e culturale etrusca.

Come già osservato, durante il III e il II sec. a.C. Adria mostra una tradizione scrittoria "monolitica" e refrattaria ad accogliere nel proprio sistema stimoli esterni e riforme grafiche provenienti da altri luoghi (cfr. III.2). Non è un caso che tale sistema scrittorio, basato sul tipo corsivizzante, rimase eccezionalmente in uso fino al passaggio alla scrittura latina. La presenza di aspetti paleografici peculiari, quali a esempio l'alpha angolato o i lunghi tratti sottoavanzanti in alcune lettere, è probabilmente dovuta a singole manifestazioni individuali, in alcuni casi da mettere in correlazione con una multicompetenza scrittoria di chi tracciò le iscrizioni. Infatti, già nel III e soprattutto nel II sec. a.C., in un panorama culturale che doveva essere ormai sfilacciato e apertamente incline alle influenze del mondo romano, emergono manifestazioni di cultura epigrafica celtica e latina, nonché rare occorrenze di quella venetica. Non è dunque forse un caso che nel II sec. a.C. alcune rare iscrizioni etrusche abbiano ductus destrorso, da ritenere dovuto all'influenza di culture epigrafiche non etrusche (latina primariamente ma anche venetica), probabilmente possedute da individui detentori di una multi-competenza scrittoria (e linguistica) ma che scelsero di scrivere in etrusco.

Tale processo di progressivo indebolimento della resilienza della cultura etrusca locale ad assorbire spinte culturali esterne è ravvisabile anche nelle trasformazioni della società ricostruibili grazie all'epigrafia. L'assenza di testi che hanno come scopo primario quello dell'autorappresentazione, soprattutto in ambito funerario, è sicuramente un pesante ostacolo. Infatti, coloro che esercitavano la scrittura nelle pratiche legate al rituale funebre, come il dono al defunto o la marcatura di singoli vasi o interi set a questo destinati, sicuramente non miravano a fissare nelle formule onomastiche dei testi graffiti 
nei vasi la propria identità sociale oppure i vincoli di natura personale o familiare che potevano indurli all'atto medesimo. Pertanto districarsi all'interno di un insieme eterogeno di nomi e appellativi scarni e allusivi non è una operazione semplice e sicuramente quanto si è desunto sulla società adriese da questo studio è soltanto una proposta perfettibile, che tuttavia si auspica possa risultare utile per il prosieguo della ricerca.

Anzi tutto emerge un buon numero di gentilizi maschili (cfr. IV.3.1). Molti hanno suffisso derivativo $-n a,-n i$, -ra e mostrano il loro legame con il mondo cisappenninico, con una prevalenza dell'Etruria settentrionale interna (Chiusi, Perugia, Cortona) ma anche meridionale (Vulci, Orvieto). All'Etruria propria possono rimandare anche Kleuste e Tite, sempre di ambito settentrionale,

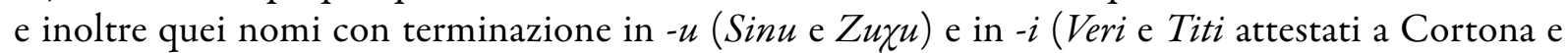
Chiusi). Altri gentilizi di area più strettamente padana per tradizione sono quelli con suffisso -alu (cfr. IV.3.3.A) e forse - va/-vi, mentre più complesso il caso di quei nomi familiari con terminazione $-i u$ (cfr. IV.3.3.B). Si conta anche un buon numero di gentilizi femminili, con suffissi -nai, -ia, - $i$, maggiormente attestati durante il II sec. a.C.

Per quanto riguarda le due terminazioni sicuramente più discusse dalla critica, cioè -alu e -iu, sono possibili alcune considerazioni, che hanno il valore di proposte interpretative da sottoporre al dibattito scientifico. Il suffisso - alu sembra associato a basi onomastiche di origine alloctona o a probabili nomi individuali etruschi. È forse possibile ipotizzare che, indipendentemente dalla sua formazione originaria, almeno nella tarda etruscità padana tale suffisso fosse identificativo di gentes di recente formazione. Per quanto riguarda la terminazione - $i u$, si individuano due gruppi principali di gentilizi, quelli originari dell'Etruria interna e quelli derivati da probabili nomi individuali. Per il primo gruppo, sembra evidente una distinzione tra nomi rimorfologizzati con il suffisso e altri che non subirono tale rimorfologizzazione. L'impressione è che i nomi familiari di questo secondo tipo possano essere formazioni in $-u$ da gentilizi di patroni, secondo un meccanismo già concluso al momento dell'arrivo dei portatori di questi nomi ad Adria. Inoltre, ammessa l'esistenza di percorsi formativi differenziati e interni all'onomastica etrusca, è possibile che almeno ad Adria nel tempo il suffisso -iu si sia sclerotizzato come tale, assumendo pertanto un valore autonomo, tanto da risultare nel tardo II sec. a.C. usato nella formazione di gentilizi da parte di gruppi familiari di origine non etrusca, nella fattispecie veneta e forse anche greca. In questi è forse possibile (ma non necessario) che la somiglianza con il morfema venetico (e greco) -io $(s)$ abbia in qualche maniera influito nella scelta di usare forme appositive in qualità di gentilizi formalmente identici a quelli con terminazione -iu. Infine, sebbene non siano attestati gentilizi maschili e femminili formati su medesime basi onomastiche, si potrebbe avanzare l'idea che il femminile -ia vada a sostituire il maschile -iu, rimorfologizzando anche alcuni gentilizi originari del comparto etrusco-settentrionale (Kusia, Punia) che ci si aspetterebbe in forma diversa (cioè Kusui, Punei).

Una trattazione diacronica rivela in maniera più puntuale come la società di Adria si sia modificata nel tempo in maniera significativa.

Durante il III sec. a.C. si registra un pool onomastico di prenomi maschili etruschi piuttosto ristretto (Arza, Ata, Venza, Venu, Kavi, Larza, Laris). In alcuni casi, l'associazione del metronimico al prenome mostra forme di distinzione basate sul gentilizio materno piuttosto che sulla filiazione, a riprova del ristretto nucleo di prenomi in uso. Oltre a questi metronimici (le madri Krapnai e Lausia), in questo periodo il mondo femminile potrebbe emergere (ma solo dubitativamente) nell' iscrizione devozionale ai Kulśnuter realizzata da $\operatorname{S} m i n \theta i$, nome già in uso nella città nella fase di V-IV sec. a.C. (cfr. IV.2 e IV.3.1.B, categoria c).

I molti gentilizi maschili mostrano tutti basi onomastiche etrusche. Emergono anche alcuni casi di genitivo afunzionale in associazione a suffissi derivativi $(-a l u,-n i)$, lasciando così trapelare una società gentilizia stratificata e complessa. Infatti, si può ritenere che gentes come i Tetialuś e i Śluniś(il cui uso del genitivo afunzionale riusciamo a individuare grazie alle formule all'assolutivo) distinguessero così 
il proprio rango rispetto a gruppi gentilizi di più recente formazione e provenienti da livelli sociali inferiori come Zurtalu, se effettivamente formato sul nome individuale Zurta.

Se dunque l'élite di Adria era di cultura schiettamente etrusca e probabilmente articolata in classi sociali al suo interno, le iscrizioni testimoniano anche una popolazione priva di diritti e di servi molto più variegata. Si contano nomi individuali etruschi come Zurta e Hapile e se Zurta generò davvero gentilizi quali Zurtalu e Zurtiu, ciò sarebbe prova del carattere dinamico e inclusivo della società. Ma la maggior parte dei nomi individuali sono di origine alloctona: Mei e Nalske, di probabile origine italica; Kraiu e Ipu di origine greca; Ataku, Verkantu, Uvasave e forse il femminile Urvi di origine celtica. Nelle iscrizioni non è palesata la condizione di questi individui, anche se è ipotizzabile che almeno alcuni di loro non fossero necessariamente schiavi, ma piuttosto individui privi di diritti come forse il guerriero celta Verkantu.

Significativi cambiamenti avvennero durante il II sec. a.C. Sono noti solo pochi prenomi maschili (Ana, Anta, Larza), associati a famiglie con gentilizi e cognomina, quali i Mura. Con gli inizi del secolo sembrano rarefarsi i gentilizi con suffisso - alu (estinti oppure rimorfologizzati in -iu?). Aumenta il numero di nomi femminili, sempre caratterizzati unicamente dal gentilizio (Kusia, Lausia, Lentnai, Puinia, Titi, Titinai, forse Lai e Pulevi). Questi nomi, che tradiscono perlopiù l'origine da ceppi familiari di ambito tirrenico, sembrerebbero testimoniare per le donne l'abbandono dell'uso del prenome almeno nei testi scritti, forse sulla base di una tendenza culturale propria del mondo romano (che così le privava di una propria individualità) e che colpì nel periodo ellenistico anche le società dell'Etruria propria.

Dopo il pieno II sec. a.C., emergono uomini di origine non etrusca identificati da forme onomastiche bimembri. Si riconoscono nomi di famiglia originali, Kaniu, Muliu, Śiliu, associati a prenomi che non fanno parte della tradizione adriese, ma sono anch'essi di derivazione alloctona, venetica e forse greca (Apu, Viza, Kraiku, Фaia). In una società ben strutturata come quella che sembra definirsi grazie all'onomastica di Adria di periodo ellenistico, pare improbabile che queste formule bimembri siano formate da nomi individuali e appositivi/patronimici usati per simulare un sistema onomastico (e nella fattispecie i gentilizi con medesima terminazione - $i u$ ) che aveva una precisa funzione sociale. Questi uomini non dovevano essere liberti (in quanto è verosimile credere che nel caso avrebbero usato prenomi schiettamente etruschi), ma forse esponenti di quel ceto privo di diritti e di condizione libera che già è stato ipotizzato per il III sec. a.C. e che forse riesce a elevare il proprio livello sociale nel secolo seguente. In tal senso, non sono da sottovalutare gli effetti che il processo di (auto)romanizzazione, avviato sicuramente nell'urbanistica della città già dal pieno II sec. a.C., può aver generato nella società. È possibile che un indebolimento politico dell'élite etrusca abbia favorito l'inserimento nelle sue pieghe di famiglie, sicuramente non prive di mezzi, provenienti dal mondo veneto e forse greco. Queste famiglie sembrano manifestare rapporti molto stretti con i luoghi di origine ma anche con altre realtà culturali fra cui quella romana, come prova appunto il recinto dei Muliu, dove le pratiche funerarie legate alla scrittura testimoniano la gravitazione attorno alla famiglia di individui di origine e cultura latina, celtica, veneta, retica.

Ovviamente, questa situazione più fluida rende ambigui i casi di nomi isolati di origine alloctona almeno dal pieno II sec. a.C. (Pruka, ReiOu), che dunque potrebbero appartenere a ceti subalterni come anche alle nuove famiglie emergenti. Diversamente, nella prima metà del secolo si riconoscono

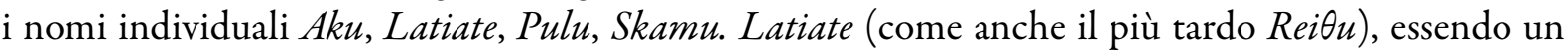
idionimo, sembra riferibile a un individuo solo marginalmente integrato nella società etrusca adriese, mentre per i nomi greci di Pulu e Skamu la condizione servile è verosimilmente la più probabile. È certamente lecito domandarsi cosa distinguesse dopo la metà del secolo da un punto di vista onomastico gli schiavi o gli uomini privi di diritti da taluni che avevano assunto il gentilizio se non proprio quest'ultimo. Va comunque ribadito che le manifestazioni epigrafiche di II sec. a.C. sono in prevalenza sui vasi di corredi funerari e tale pratica non imponeva evidentemente di manifestare la propria condizione sociale. 
In effetti, la cultura epigrafica della tarda società etrusca di Adria sembra perlopiù votata all'ambito funerario. Infatti, le iscrizioni in abitato sono poche e tale rarefazione aumenta nel II sec. a.C. In strutture produttive come quella indagata in via San Francesco, i frammenti graffiti sono tutti in strati di obliterazione o riporto e quindi non direttamente da riferire all'impianto abitativo-artigianale. Nessuno di questi inoltre è da ritenere con buona probabilità funzionale alla catena operativa; l'unico graffito inciso a crudo su una olla in ceramica grezza testimonia un numerale con probabile valore di capacità. In effetti, l'incisione a crudo è una tecnica raramente attestata in questa fase di III-II sec. a.C. (cfr. II.3) e solo sporadicamente si dovette ricorrervi.

A differenza del tardo Arcaismo e a eccezione dell'eclatante dedica ai Kulśnuter, verosimilmente epiclesi compendiaria dei demoni inferi $\operatorname{Xarun}$ e $\operatorname{Van} \theta$, non si registrano iscrizioni correlabili con certezza a pratiche cultuali o di offerta votiva di ambito sacro (cfr. V.3). Solo ipoteticamente la presenza di appellativi come papa e alcune abbreviazioni e monogrammi potrebbe lasciar trapelare forme di devozionalità indirizzate a divinità infere sia in ambito funerario che abitativo (si veda oltre). In particolare, questi indizi potrebbero portare a sostenere una continuità di culto per il probabile luogo sacro in località Bettola. Non è invece affatto chiaro cosa succede nel periodo ellenistico presso La Tomba e il Cortile Ornati. Tornando alla Bettola, qui anche la presenza del sostantivo usti, se coglie nel segno l'interpretazione proposta di appellativo del recipiente (V.4), potrebbe orientare verso aspetti rituali. In tale chiave potrebbero infine essere interpretate anche le due formule onomastiche all'assolutivo di III sec. a.C. (anta $\leq$ $\times$ [ e larza śiluniś), da intendere come esplicitazione del donatore secondo una prassi tipica dell'ambito funerario (cfr. IV.3.1.A e IV.3.2).

Sempre in merito alle aree sacre, non va sottovalutato che l'indagine di fossati ricchi di materiali operata da F.A. Bocchi nell'area dell'attuale Museo ha restituito iscrizioni di varia natura, fra le quali sono riconoscibili formule all'assolutivo prive di pronome personale e lessemi che potrebbero evocare una sfera rituale (sesiu e forse mi leị [ ). Si tratta di documenti databili entro il III sec. a.C. come in località Bettola. Sebbene in giacitura secondaria, è possibile ipotizzare che provenissero da aree sacre limitrofe. Se tutte queste osservazioni possono avere qualche valore, inizia a emergere una topografia del sacro di periodo ellenistico finora ignota. Inoltre, le forme del sacro correlate alla pratica scrittoria sembrano essere strettamente affini a quelle funerarie, sicuramente maggiormente praticate.

Come già detto, l'ambito funerario è sicuramente quello dove la cultura epigrafia sembra essere prevalentemente diretta, peraltro con un notevole incremento nel II sec. a.C. rispetto a quello precedente. Anzi, è possibile affermare che proprio in questo ultimo secolo di vitalità della società etrusca adriese, la scrittura sembra essere una manifestazione meramente funeraria. Va inoltre constatato come, almeno a partire dal III sec. a.C. il supporto privilegiato per le iscrizioni presenti nei corredi e nei contesti votivi sia il vasellame fine da mesa e nella fattispecie la ceramica a v.n., perlopiù di produzione locale, mentre la ceramica grigia è solo eccezionalmente usata, in tre casi su quattro sempre manifestando la presenza del lessema helis, che forse ne motivava la scelta (cfr. V.4), mentre solo una tomba di via Spolverin conserva un gentilizio, Malni, realizzato in un mortaio. Più in generale, sulla base della proposta di classificazione elaborata (cfr. IV.3.2), i testi avevano un formulario codificato in maniera abbastanza rigida. Questi erano graffiti perlopiù nella vasca interna, forse come simbolico atto di defunzionalizzazione del vaso deposto nel corredo del defunto.

La prevalenza delle iscrizioni funerarie si caratterizza per il nominativus pendens, che è probabilmente da intendere come il nome del donatore dell'offerta funebre. Sono invece poco frequenti nomi sempre all'assolutivo ma associati al pronome mi. Quest'ultima formula di sapore arcaico si distingue formalmente dalla precedente e forse è da intendere come proprietà del defunto, come suggerisce l' $\mathrm{i}$ dentità di caso fra il pronome e la formula onomastica. Anche i testi con antroponimi al genitivo, con o senza pronomi $m i$, sono poco frequenti e perlopiù concentrati nel III sec. a.C.; se valida l'idea che almeno quelli con pronome mi possano esprimere destinazione/possesso dell'oggetto, allora si potrebbe pensare che tale formula abbia ceduto nel II sec. a.C. a quella che associava il pronome e l'assolutivo del nome, già raramente usata da due individui (probabilmente di rango? uno di questi è Laris Tetialuś) 
nella prima metà de III sec. a.C. Quest'ultima osservazione conduce il ragionamento verso un altro punto, cioè la possibilità che tale pratica della scrittura effettuata durante il rituale funebre, se effettivamente così articolata, fosse vincolata in qualche maniera alla struttura della società, le cui specificità non sono esplicitate ma trapelano implicitamente dai testi medesimi. Infatti, durante il III sec. a.C. i probabili donatori di offerte funebri sono uomini che si identificano con prenome e gentilizio, oppure prenome e metronimico, mentre sono rari i casi di nomi di famiglia isolati. Tra i possibili nomi di defunti associati al pronome mi, si ricorda Laris Fuliu e Laris Tetialuś nelle formule all'assolutivo. Ma si annoverano anche nomi individuali di origine alloctona (Verkantu, Uvasave e forse Nalske), un prenome (Kavi) e un prenome e gentilizio (Venu Zurtiu) tra quelli con genitivo, tutti uomini e in prevalenza schiavi o privi di diritti, oppure come Venu Zurtiu probabilmente discendente di un liberto, se può essere valida l'idea che all'origine del gentilizio ci sia Zurta. Più difficile inquadrare la formula con onomastica al genitivo senza pronome, dove però il caso del deittico $t a$ associato al nome individuale $M e i$ potrebbe essere in qualche maniera assimilato a quello di Kavi, dove c'è identità fra pronome e deittico (kaviś ta mi). Allora, forse anche i due casi di Ataku espresso al genitivo, nome individuale di origine celtica, e quello di I $u$ anch'esso al genitivo e di probabile origine greca, potrebbero indicare i destinatari dell'offerta, cioè defunti. La seconda iscrizione in particolare appartiene a un probabile deposito votivo di Canalbianco. Proprio in questo ampio settore funerario i depositi evidenziano pratiche scrittorie distinte rispetto a quelle delle sepolture (cfr. VI.2.1). La presenza di nomi individuali come $I p u$, di lessemi di difficile interpretazione ma verosimilmente legati alla sfera del sacro come $\chi u r$, e anche di una iscrizione in alfabeto leponzio (keus), che documenta durante il III sec. a.C. l'eccezionale emergere di forme di cultura epigrafica non etrusca, lasciano trapelare uno scarto culturale e forse sociale tra il rito funebre e i riti devozionali in necropoli almeno in merito alle pratiche epigrafiche, nel primo caso maggiormente codificate all'interno della cultura e della lingua etrusca. Questo vale anche per la scelta dei supporti e la posizione delle iscrizioni, in quanto mentre nelle tombe vi è una significativa preferenza delle ciotole a v.n., seguite dai piatti, dove le iscrizioni sono in prevalenza realizzate nella vasca interna, nei contesti votivi si osserva una predilezione per supporti non altrettanto canonici, come coppe emisferiche e mortai in ceramica grigia, skyphoi a v.n.

Nel II sec. a.C. il nominativus pendens è usato in maniera trasversale da personaggi della gens Mura che manifestano anche di possedere un cognomen (Anta Mura Afr e Larza Mura Pili), da individui ingenui che si rappresentano con prenome e gentilizio (Arza Heturiu, Larza Lintialu) oppure con unicamente il prenome (Kavi, Larza?), da schiavi o uomini privi di diritti (Aku, Ana, Hapile, Latiate, Pruka, Pulu), e da donne ingenue che si identificano con il solo gentilizio (Kusia, Lai, Lausia, Titi, Titinai). Si conta inoltre un numero significativo di nomi familiari isolati, in alcuni casi ripetuti in tombe distinte (Muliu e UniӨiu). A tale riguardo, merita ricordare anche le tre occorrenze del digramma śp, che potrebbe essere spiegato come abbreviazione del termine ${ }^{*}$ spura e quindi dubitativamente riferirsi a una pratica di dono da parte dell' intera comunità (non si ritiene comunque che si tratti di marchi legati alla produzione, data la posizione di visibilità nel vaso; cfr. III.4 e per i contesti VI.2.1-2).

Con la prima metà del II sec. a.C. sembra palesarsi una corrispondenza (non necessariamente significativa) fra complessità del corredo e manifestazione di iscrizioni appartenenti a individui ingenui (uomini e donne, fra cui si ricorda primariamente i Mura, già attestati in un documento erratico di prima metà del III sec. a.C. a Canalbianco) che manifestano nelle formule una schietta onomastica etrusca, mentre solo la tomba 47 documenta il probabile dono funebre da parte un individuo forse in condizione servile (Pulu). Quando è desumibile dalla documentazione, le iscrizioni, tutte in nominativus pendens a evocare il nome del donatore, sono probabilmente testimonianza della pratica del dono funebre e i relativi vasi sono sempre collocati assieme al restante vasellame a lato del defunto, senza una significativa distinzione. In effetti, viene naturale domandarsi quale sia la distinzione fra questi vasi iscritti, in prevalenza ciotole, e quelli privi di iscrizioni. Tale tendenza sembra riscontrabile anche in Ca' Garzoni, dove le due tombe che conservano iscrizioni dall'onomastica etrusca testimoniano il probabile dono funebre esplicitato dal singolo gentilizio Mura (il defunto è forse un esponente della 
gens?) e forse il possesso del vaso da parte di Puinia, che si può identificare con la defunta (la tomba è femminile) se questa può essere l'interpretazione della formula all'assolutivo con pronome mi. Ma proprio la tomba della Puinia conserva anche il nome individuale Latiate, forse un dono funebre da parte di un individuo di origine laziale e che si ritrova anche in una tomba di via Spolverin del medesimo cinquantennio (è la stessa persona?).

È evidente che la pratica del nominativus pendens, circoscritta nel secolo precedente a uomini ingenui, fu estesa a tutti i livelli della società. Non può essere dunque un caso che dopo il pieno II sec. a.C. proprio questa pratica fu usata da gruppi di origine veneta e forse greca che sembrano acquisire quei diritti sanciti dal gentilizio (Kraiku Kaniu, Viza e Apu Muliu). Diversamente, scompare l'uso del genitivo, mentre i nomi all'assolutivo con pronome mi sono usati da donne (Lentnai, Puinia, e forse Pulevi) e ancora da quegli individui con gentilizio ma origine alloctona (Фaia Śiliu).

I pochi dati a disposizione desumibili dai cambiamenti veicolati dalla pratica rituale di queste formule scritte sembrano mostrare come la classe dominante maschile del III sec. a.C. cedette le proprie prerogative di rappresentazione funebre a tutti i livelli sociali, che abbandonarono forme forse considerate in qualche maniera meno prestigiose. Sebbene tutto ciò sia strettamente vincolato al filtro ideologico della ritualità funebre, non pare errato ritenere che questo fenomeno possa essere considerato indizio di un ceto dominante di cultura schiettamente etrusca che si andava indebolendo politicamente e socialmente fino a perdere il controllo sulle classi subalterne. Sempre nel II sec. a.C., nell'area principale di Canalbianco emerge anche una pratica epigrafica di cultura celtica in corredi che sembrerebbero di impegno (ma molti sono i dubbi sulla loro coerenza). Una simile attestazione si ha anche presso Piantamelon e lo stesso vale per la necropoli del probabile insediamento satellite di Bottrighe. Queste occorrenze mostrano così una tendenza diversa rispetto al III sec. a.C. quando queste manifestazioni non etrusche sembrano circoscritte solo ai contesti votivi.

Si ritiene probabile che in ambito funerario si riversino anche forme di devozione dirette a divinità infere. Questo è plausibile per l'iscrizione di dedica ai Kulśnuter, cioè con buona verosimiglianza epiclesi dei demoni dell'Aldilà, realizzata da Śmin $\theta i$ nel III sec. a.C., e per una iscrizione che potrebbe essere una consacrazione all'infero Kalu (cfr. V.3). Ma è forse valido anche per altre iscrizioni dove singoli kappa o digrammi come pe sono associati a nomi individuali nel III sec. a.C. (Zurta) o familiari nel II (Zurtiu, Snalu). Queste iscrizioni potrebbero in effetti essere forme compendiarie di formule più complesse destinate a dèi inferi quali Kalu e $P e \theta n$, secondo una prassi che evidentemente evitava la scrittura di questi teonimi per esteso (anche Kulśnuter fino a prova contraria è riferito alla coppia Van $\theta$ e Xarun, di cui quindi non si scrive direttamente il nome). Se il digramma $k u$ potrebbe essere una abbreviazione dell'epiclesi infera già menzionata, sembra più complesso il caso dei digrammi $k a$, prevalentemente $\mathrm{da}$ contesto abitativo e con una sola occorrenza da necropoli, per i quali dunque è forse più convincente l'interpretazione di particella deittica isolata.

Forme di lessico sacro (cfr. V.3) potrebbero emergere plausibilmente anche da singoli lessemi relativi al concetto di "divino" quali il lacunoso aisi $\times \times$, forse da intendere aisitu (una consacrazione del vaso?), e kemne, probabile locativo di cemna attestato nel $L L$; anche tutis potrebbe essere una forma aggettivale relativo al lessico sacro piuttosto che un antroponimo. Più latamente a una ritualità funeraria (cfr. V.4), oltre a helis, rimanda anche $\theta i$, cioè "acqua", presente su due ciotole a v.n. e forse da intendere come indicazione del contenuto, verosimilmente all'interno di riti (forse di libagione al defunto?). 


\section{BIBLIOGRAFIA}

Per le riviste si sono seguite le abbreviazioni della Archäologische Bibliographie del Deutsches Archäologisches Institut. Quelle non comprese in tale elenco vengono citate per esteso.

\section{Abbreviazioni}

$A C A A=$ Archivio Comunale Antico di Adria.

Agora XII = B.A. Sparkes, L. Talcott, Black and Plain pottery of the 6th, 5th and 4th centuries B.C., The

Athenian Agorà, 12, Princeton 1970.

$C I E=$ Corpus Inscriptionum Etruscarum.

$C I G=$ Corpus Inscriptionum Graecarum.

$C I I=$ A. FABRETTI, Corpus Inscriptionum Italicarum, Augusta Taurinorum 1867.

$C I L=$ Corpus Inscriptionum Latinarum.

$C V A=$ Corpus Vasorum Antiquorum.

$E T^{2}=$ G. Meiser (Hrsg.), Etruskische Texte, Editio Minor, I-II, Hamburg, 2014².

$L G P N$ online $=$ Lexicon of Greek Personal Names (http://clas-lgpn2.classics.ox.ac.uk/).

$L L=$ Liber Linteus.

$L V=$ G.B. Pellegrini, A.L. Prosdocimi, Lingua Venetica, I-II, Padova 1967.

$M L R=$ S. MARCHESINI, Monumenta linguae Raeticae, Roma 2015.

NRIE $=$ M. BUfFA, Nuova raccolta di iscrizioni etrusche, Firenze 1935.

$R E=$ Realencyclopädie der classischen Altertumswissenschaft.

REE $=$ Rivista di Epigrafia Etrusca.

REI = Rivista di Epigrafia Italica.

SECI = J. Kaimio, The South Etruscan Cippus Inscriptions, Roma 2017.

$S T=$ H. RIx, Sabellische Texte. Die Texte des Oskischen, Umbrischen und Südpikenischen (Handbuch der Itali-

schen Dialekte, V. Band), Heidelberg 2002.

$T C=$ Tabula Capuana.

$T C o=$ Tabula Cortonensis.

ThLE $I^{2}=$ E. Benelli (a cura di), Thesaurus linguae Etruscae, I. Indice lessicale, Pisa-Roma $2009^{2}$.

$T L E^{2}=$ M. Pallottino, Testimonia Linguae Etruscae, Firenze $1968^{2}$.

$Z G L E=$ W. SCHULZE, Zur Geschichte lateinischer Eigennamen, Berlin 1933. 
Adiego 2006 = I.-X. Adiego, Etrusco marunuXva cepen, in «StEtr» LXXII, 2006, pp. 199-213.

Agostiniani 1982 = L. Agostiniani, Le Iscrizioni parlanti dell'Italia antica, Firenze 1982.

Agostiniani 1993 = L. Agostiniani, La considerazione tipologica nello studio dell'etrusco, in «Incontri Linguistici»16, 1993, pp. 23-44.

Agostiniani 1997 = L. Agostiniani, Considerazioni linguistiche su alcuni aspetti della terminologia magistratuale etrusca, in R. Ambrosini (a cura di), Scribthair a ainm n-ogaim: scritti in memoria di Enrico Campanile, Pisa 1997, pp. 1-16.

Agostiniani $2011=\mathrm{L}$. Agostiniani, Feluskés $o$ Oeluskés sulla stele di Vetulonia?, in D.F. Maras (a cura di), Corollari. Scritti di antichità etrusche e italiche in omaggio all'opera di Giovanni Colonna, Studia erudita, 14, Pisa-Roma 2011, pp. 177-184.

Agostiniani 2013 = L. Agostiniani, The Etruscan language, in J. MacIntosh Turfa (a cura di), The Etruscan world, London-New York 2013, pp. 457-477.

Agostiniani, Hjordt-Vetlesen 1988 = L. Agostiniani, O. Hjordt-Vetlesen, Lessico etrusco cronologico e topografico: dai materiali del Thesaurus linguae Etruscae, Firenze 1988.

Agostiniani, Nicosia 2000 = L. Agostiniani, F. Nicosia, Tabula Cortonensis, Roma 2000.

Agostiniani, Calderini, Massarelli 2011 = L. Agostiniani, A. Calderini, R. Massarelli, Screhto est. Lingua e scrittura degli antichi Umbri (Catalogo della mostra, Perugia-Gubbio 2011-2012), Perugia 2011.

Agostiniani, Massarelli 2012 = L. Agostiniani, R. Massarelli, Non una ma due: sulla lamina con culśanś al Museo dell'Accademia etrusca e della città di Cortona, in «StEtr» LXXV, 2012, pp. 107-146.

Akeo 2002 = A. MARINETTI (a cura di), Akeo: i tempi della scrittura. Veneti antichi. Alfabeti e documenti (Catalogo della mostra, Montebelluna-Cornuda 2001-2002), Cornuda 2002.

Alfonsi 1908 = A. Alfonsi, Cavarzere. Scoperte di antichità nella tenuta Cuora, in «NSc» s. V, V, 1908, pp. 418-419.

Ambrosini $2000=$ L. Ambrosini, I pesi da telaio con iscrizioni etrusche, in «ScAnt» 10, 2000, pp. 139-162.

Antonelli 2008 = L. Antonelli, Traffici focei di età arcaica. Dalla scoperta dell'Occidente alla battaglia del mare Sardonio, Roma 2008.

Antonetti 2005 = C. Antonetti, I Greci ad Adria fra il VI e il V secolo a.C., in M.G. Angeli Bertinelli, A. Donati (a cura di), Il cittadino, lo straniero, il barbaro fra integrazione ed emarginazione nell'antichità, Atti del I Incontro Internazionale di Storia Antica di Genova, in «Serta antiqua et mediaevalia» VII, Roma, pp. 115-141.

Atria 1989 = Atria. Siti di interesse archeologico in territorio polesano, Rovigo 1989.

BAgnasco Gianni 2008 = G. BAgnasCo GiAnNi, Rappresentazioni dello spazio "sacro" nella documentazione epigrafica etrusca di epoca orientalizzante, in X. Dupré Raventós, S. Ribichini, S. Verger (a cura di), Saturnia tellus. Definizioni dello spazio consacrato in ambiente etrusco, italico, fenicio-punico, iberico e celtico (Atti del Convegno Internazionale, Roma 2004), Roma 2008, pp. 267-281.

Bagnasco Gianni $2014=$ G. Bagnasco Gianni, At First Glance: Remarks on a Group of Sigla from the Sanctuary of the Etruscan Artisans at Cetamura del Chianti, in «EtrSt» 17, 2, 2014, pp. 192-212.

Bagnasco Gianni, Scoccimarro, Gobbi 2015 = G. Bagnasco Gianni, N. Scoccimarro, A. GobBI, Segni eloquenti in necropoli e abitato, in M.-L. HAACK (a cura di), L'écriture et l'espace de la mort. Épigraphie et nécropoles à l'époque préromaine (Atti del Colloquio Internazionale, Roma 2009), Roma 2015, pp. 253-301.

Bailo Modesti 1984 = G. Bailo Modesti, Lo scavo dell'abitato di Pontecagnano e la coppa con iscrizione AMINA [---], in «AIONArch» 6, 1984, pp. 215-246.

Baldassarra 2013 = D. BAldassarra, Dal Saronico all'Adriatico. Iscrizioni greche su ceramica del Museo archeologico nazionale di Adria, Pisa 2013.

Battaglia $1957=$ R. Battaglia, Dal Paleolitico alla civiltà atestina, in Storia di Venezia, I, Venezia 1957, pp. 79-177.

Battaglia 1958-1959= R. Battaglia, Preistoria del Veneto e della Venezia Giulia, «BPI» 67-68, fuori serie, Roma 1958-1959.

BeLfiore 2010 = V. Belfiore, Il Liber linteus di Zagabria: testualitá e contenuto, Pisa-Roma 2010.

Belfiore 2011 = V. Belfiore, Problemi di vocalismo etrusco arcaico. La geminazione di <ii>, in G. vAN HEEMs (a cura di), La variation linguistique dans les langues de l'Italie préromaine (Actes du IVe Séminaire sur les langues de l'Italie préromaine, Lyon 2009), MOM Éditions 45, Lyon 2011, pp. 41-60. 
Belfiore 2012 = V. Belfiore, Problemi di dialettologia etrusca: spie grafiche e questioni «dialettali» non solo perugine, in «MEFRA»124, 2, 2012, pp. 421-437.

BELFIORE 2014a = V. BELFIORE, Il fare etrusco: discussione sulle radici con senso generico di "fare" e conseguenze

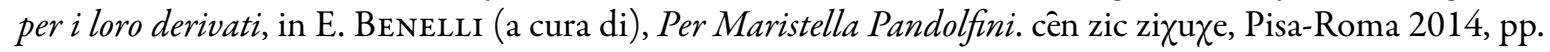
$29-42$.

BeLfiore 2014b $=$ V. Belfiore, La morfologia derivativa in etrusco. Formazioni di parole in - na $e$ in - ra, PisaRoma 2014.

Belfiore 2015 = V. Belfiore, Definizioni locali di spazi tombali, in M.-L. HaACK (a cura di), L'écriture et l'espace de la mort. Épigraphie et nécropoles à l'époque préromaine (Atti del Colloquio Internazionale, Roma 2009), Roma 2015, pp. 105-123.

Belfiore 2017 = V. Belfiore, Per una revisione del testo del Cippo Perugino, in «StEtr» LXXX, 2017, pp. 167-200.

Bellelli 2012 = V. Bellelli, Particolarità d'uso della ceramica comune etrusca, in «MEFRA»124, 2, 2012, pp. 377-392.

BELLELLI $2014=$ V. BeLLELLI, Iscrizioni e graffiti su ceramiche grezze di uso domestico: il caso di Cerveteri, in E.

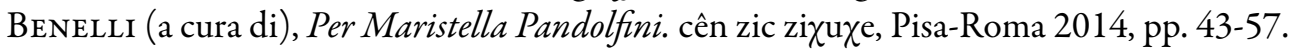

Bellelli, Benelli 2018 = V. Bellelli, E. Benelli, Gli Etruschi. La scrittura, la lingua, la società, Roma 2018.

Bellintani et. al. 1995 = P. Bellintani, S. Bonomi, K. Tamassia, N. Trentin, Adria. Aggiornamento sui rinvenimenti archeologici nell' area dell' azienda ospedaliera, in «Padusa» XXXI, 1995, pp. 41-91.

BENELLI 1994 = E. BENELLI, Le iscrizioni bilingui etrusco-latine, Firenze 1994.

BENELLI 1998 = E. BENELLI, Le iscrizioni funerarie chiusine di età ellenistica, in «StEtr» LXIV, 1998, pp. 225263.

BENELLI $2000=$ E. BeNELli, Alfabeti chiusini di età arcaica, in «AnnFaina» VII, 2000, pp. 205-217.

Benelli 2002a = E. Benelli, L'onomastica etrusca di Perugia: alcune osservazioni, in «AnnFaina» IX, 2002, pp. 517-523.

BENELLI 2002b = E. BENELLI, Le formule onomastiche della Tabula Cortonensis e il valore del metronimico, in La Tabula Cortonensis e il suo contesto storico-archeologico (Atti dell'Incontro di studio, 2001), Roma 2002, pp. 93-100.

BeNELli 2004 = E. BeNELLI, La documentazione epigrafica spinetica, in F. Berti, M. HARARI (a cura di), Storia di Ferrara, II. Spina tra archeologia e storia, Ferrara 2004, pp. 251-270.

Benelli 2011 = E. Benelli, 'Vornamengentilizia'. Anatomia di una chimera, in D.F. Maras (a cura di), Corollari. Scritti di antichità etrusche e italiche in omaggio all'opera di Giovanni Colonna, Pisa-Roma 2011, pp. 193-198.

Benelli 2013 = E. Benelli, Slavery and manumission, in J. MacIntosh Turfa (a cura di), The Etruscan world, London-New York 2013, pp. 447-456.

Benelli 2014 = E. Benelli, Femminili analogici e nomi familiari asuffissati, in E. Benelli (a cura di), Per Maristella Pandolfini. cên zic zi uuxe, Pisa-Roma, pp. 59-72.

BENELLI 2015 = E. BENELLI, Breve in exiguo marmore nomen ero. L'iscrizione funeraria etrusca tra esposizione pubblica e spazio privato, in M.-L. HAACK (a cura di), L'écriture et l'espace de la mort. Épigraphie et nécropoles à l'époque préromaine (Atti del Colloquio Internazionale, Roma 2009), Roma 2015, pp. 401-411.

Benelli, Colivicchi, Zaccagnino 2017 = E. Benelli, F. Colivicchi, C. Zaccagnino, Iscrizioni, sigle e segni non alfabetici dai nuovi scavi nella Vigna Marini di Cerveteri (2012-2016), in «Mediterranea» XIV, 2017, pp. 39-61.

Bernardini 2002 = C. Bernardini 2002, Il Gruppo Spurinas, «Daidalos» 4, 2002.

BERTI 1983 = F. BERTI, Il Museo Archeologico Nazionale di Ferrara, Bologna 1983.

Bоссн1 1877 = F. BocchI, Adria, in «NSc» 1877, pp. 197-201.

Bocchi 1879 = F. Bocchi, Adria, in «NSc»1879, pp. 88-106 e 212-224.

Bocchi 1888 = F.A. BocchI, Scritti vari di argomento storico, Acqui 1888.

Bolognesi 1998-1999 = B. Bolognesi, Le necropoli Campelli-Stoppa e Belluco in località Passetto (Adria), in «Padusa» XXXIV-XXXV, 1998-1999, pp. 245-316.

Boni 1815 = M. Boni, Saggio di studii del p. Luigi Lanzi della Compagnia di Gesù, Venezia 1815. 
Bonomi 1993 = S. Bonomi, Gli scavi di Francesco Antonio Bocchi nell'abitato arcaico di Adria, in A. Lodo (a cura di), Francesco Antonio Bocchi e il suo tempo 1821-1888 (Atti del Convegno, Adria 1990), Rovigo 1993, pp. 75-79.

Bonomi 1994 = S. Bonomi, Adria (Rovigo), in «StEtr» LX, 1994, pp. 509-510.

Bonomi 1997 = S. Bonomi, Adria: la necropoli di Ca' Cima, in Beni culturali e ambientali in Polesine, I, 1997, pp. 31-34.

Bonomi 1998 = S. Bonomi, Adria e Spina, in F. Rebecchi (a cura di), Spina e il delta padano. Riflessioni sul catalogo e sulla mostra ferrarese (Atti del Convegno Internazionale di Studi «Spina: due civiltà a confronto», Ferrara 1993), Roma 1998, pp. 241-246.

Bonomi $2000=$ S. Bonomi, Ceramica attica da corredi tombali del IV sec. a.C. di Adria, in La céramique attique du IV siècle en Méditerranée occidentale (Actes du Colloque International, Arles 1995), Naples 2000, pp. 93-96.

Bonomi 2003a = S. Bonomi, Adrias, Atria, in Luoghi e tradizioni d'Italia, Veneto, II, Roma, 2003, pp. 455463.

Bonomi 2003b = S. Bonomi, Recenti rinvenimenti archeologici nell'Alto Adriatico tra fine VII e IV sec. a.C.: nuovi dati, in F. Lenzi (a cura di), L'Archeologia dell'Adriatico dalla Preistoria al Medioevo (Atti del Convegno, Ravenna 2001), Firenze 2003, pp. 140-145.

Bonomi 2003c = S. Bonomi, La necropoli di Ca' Cima ad Adria (RO), in L. Malnati, M. Gamba (a cura di), I Veneti dai bei cavalli, Canova 2003, pp. 73-74.

Bonomi 2004 = S. Bonomi, ll porto di Adria tra VI e V sec. a.C.: aspetti della documentazione archeologica, in M.A. Guggisberg (a cura di), Die Hydria von Grächwil. Zur Funktion und Rezeption mediterraner Importe in Mitteleuropa im 6. und 5. Jahrbundert v. Chr. (Akten Internationales Kolloquium, Bern 2001), Bern 2004, pp. 65-69.

Bonomi 2011 = S. Bonomi, Corredo funerario tardo-etrusco, in Restituzioni. Tesori d'arte restaurati, Venezia 2011, pp. 62-66.

Bonomi, Peretto, Tamassia 1993 = S. Bonomi, R. Peretto, K. Tamassia, Adria. Appunti preliminari sulla necropoli tardo etrusca e romana di via Spolverin di Bottrighe, in «Padusa» XXIX, 1993, pp. 91-156.

Bonomi, Camerin, Tamassia 2002a = S. Bonomi, N. Camerin, K. Tamassia, Etruschi Adriati. Guida breve all'esposizione, Adria 2002.

Bonomi, Camerin, Tamassia 2000b = S. Bonomi, N. Camerin, K. Tamassia, Adria, via S. Francesco, scavo 1994: materiali dagli strati arcaici, in «Padusa» XXXVIII, 2002, pp. 201-213.

Bonomi, Robino 2007 = S. Bonomi, M.T.A. Robino, Adria fra Etruschi e Romani, in L. Brecciaroli TABORELli (a cura di), Forme e tempi dell'urbanizzazione nella Cisalpina (II secolo a.C.-I. secolo d.C.) (Atti delle Giornate di Studio, Torino 2006), Firenze 2007, pp. 85-90.

Bonomi, Gambacurta 2017 = S. Bonomi, G. Gambacurta, Adria: l'abitato etrusco, in C. Reusser (a cura di), Spina. Neue Perspektiven der archäologischen Erforschung (Tagung, Zürich 2012), Rahden 2017, pp. 69-74.

Bonomi, Vallicelli, Balista 2020 = S. Bonomi, M.C. Vallicelli, C. Balista, Data from the Etruscan Settlement of Adria: New Excavations in Via Ex Riformati (2015-2016), in L. Zamboni, M. FernándeZGötz, C. Metzner-Nebelsick (eds.), Crossing the Alps. Early Urbanism between Northern Italy and Central Europe (900-400 BC), Leiden 2020, pp. 193-205.

BOURDIN 2006 = S. BOURDIN, Fréquentation ou intégration: les présences allogènes dans les emporia étrusques et ligures (VIe-IV s. av. J.-C.), in F. ClÉment, J. Toran, J. Wilgaux (a cura di), Espaces d'échanges en Méditerranée. Antiquité et Moyen Age, Rennes 2006, pp. 19-39.

BraCCESI 2001 = L. BraCCESI, Hellenikòs kolpos. Supplemento a Grecità adriatica, in «Hesperìa» 13, Roma 2001.

BRACCESI 2006 = L. BRACCESI, Rimini prima di Rimini, in F. LENZI (a cura di), Rimini e l'Adriatico nell'età delle guerre puniche (Atti del Convegno, Rimini 2004), Bologna 2006, pp. 95-100.

BRUni 1994 = S. Bruni, L'Etruria tirrenica e il territorio del Delta del Po. Appunto su una kotyle da San Basilio di Ariano Polesine, in «Padusa» XXX, 1994, pp. 187-193.

BRUNi 2001 = S. BRUni, Interessi fiorentini per le antichità di Adria nel XVIII e nel XIX secolo, in Commerci e produzione in età antica nella fascia costiera fra Ravenna ed Adria (Atti della Giornata di Studio, Ferrara 2001), suppl. a «Atti dell’Accademia delle Scienze di Ferrara» LXXVIII, Ferrara 2001, pp. 59-80. 
BRUNI 2008 = S. BRUNI, Volterra e Fiesole nei fenomeni di colonizzazione. Qualche appunto sul caso fiesolano, in «AnnFaina» XV, 2008, pp. 297-339.

Bruni 2011 = S. Bruni, Bronzi veneti da Adria. Nuovi contributi, in Tra protostoria e storia. Studi in onore di Loredana Capuis, Roma 2011, pp. 125-136.

Buchi 1984 = E. BuchI, I quattuorviri iure dicundo di Adria e il culto del dio Nettuno, in «Epigraphica $» 46$, 1984, pp. 65-89.

Buoite, Zamboni 2008 = C. Buoite, L. Zamboni, Ceramica locale, in L. Malnati, D. Neri (a cura di), Gli scavi di Castelfranco Emilia presso il Forte urbano, «Quaderni di archeologia dell'Emilia Romagna» 21, Firenze 2008, pp. 57- 129.

Cagianelli 2002 = M. Cagianelli, Bronzi etruschi a figura umana, in «AnnFaina» IX, pp. 323-341.

Camerin $1993=$ N. Camerin, Testimonianze celtiche da Adria, in «Padusa XXIX, 1993, pp. 157-177.

Camerin 1996 = N. Camerin, Adria, Canal Bianco, tomba 327, in F. Berti, S. Bonomi, M. Landolfi (a cura di), Classico e Anticlassico. Vasi alto-adriatici tra Piceno Spina e Adria (Catalogo della mostra, Comacchio 1996-1997), S. Giovanni in Persiceto 1996, pp. 187-189.

CAMERIN 1999 = N. CAMErin, L'Italia antica: Italia settentrionale, in A. Emiliozzi (a cura di), Carri da guerra eprincipi etruschi (Catalogo della mostra, Viterbo-Roma 1998-1999), Roma 1999, pp. 33-44.

Camerin, Tamassia 1998-1999 = N. Camerin, K. Tamassia, Adria, via San Francesco, scavo 1994: edificio di tipo abitativo-artigianale di III-II sec. a.C., in «Padusa» XXXIV-XXXV, 1998-1999, pp. 209-243.

CAPponi 2018 = F. CAPponi, Velzna. Lo scavo di Campo della Fiera di Orviero. I buccheri, Roma 2018.

Cappuccini, Mohr 2017 = L. Cappuccini, M. Mohr, Strutture a Spina nel IV sec. a.C., in C. Reusser (a cura di), Spina. Neue Perspektiven der archäologischen Erforschung (Tagung, Zürich 2012), Rahden 2017, pp. 21-26.

Capuis, Chieco Bianchi 2006 = L. Capuis, A.M. Chieco Bianchi, Este, II. La necropoli di Villa Benvenuti, Roma 2006.

Capuis, Gambacurta 2015 = L. Capuis, G. Gambacurta, Il Veneto tra il IX e il VI sec. a.C.: dal territorio alla città, G. Leonardi, V. Tiné (a cura di), Preistoria e Protostoria del Veneto, «Studi di Preistoria e Protostoria»2, Firenze 2015, pp. 449-459.

CARLUCCI, MANESCHI 2013 = C. CARLUCCI, L. MANESCHI, La formazione dei depositi rituali nel Santuario Meridionale: analisi delle tipologie e delle modalità attestate, in M.P. BAgLione, M.D. Gentili (a cura di), Riflessioni su Pyrgi. Scavi e ricerche nelle aree del santuario, «ArchCl» suppl., Roma 2013, pp. 41-72.

CerChia 2002 = L. CerChiaI, Il piatto della tomba 65 di Acqua Acetosa Laurentina e i pericoli del mare, in «Ostraka» XI, 2002, pp. 29-36.

Ciampoltrini 1993 = G. Ciampoltrini, Ricerche sugli insediamenti liguri dell'alta valle del Serchio, in «BA»19-20-21, 1993, pp. 39-70.

Cipriano, Mazzocchin 2007 = S. Cipriano, S. Mazzocchin, Produzione e circolazione dei laterizi nel Veneto tra I secolo a.C. e II secolo d.C. Autosufficienza e rapporti con l'area aquileiese, in «Antichità Altoadriatiche» 65, 2007, pp. 633-686.

Colonna 1974a = G. Colonna, I Greci di Adria, in «RStorAnt» 4, 1974, pp. 1-22.

Colonna 1974b = G. Colonna, Ricerche sugli Etruschi e sugli Umbri a Nord degli Appennini, in «StEtr» XLIV, 1974, pp. 3-24.

Colonna 1976 = G. Colonna, Il sistema alfabetico, in Etrusco arcaico (Atti del Colloquio, Firenze 1974), Firenze 1976, pp. 7-24.

Colonna 1983 = G. Colonna, Identità come appartenenza nelle iscrizioni di possesso dell'Italia preromana, in «Epigraphica» 45, 1983, pp. 49-64.

Colonna 1984-1985 = G. Colonna, Novità sui culti di Pyrgi, in «RendPontAc» 57, 1984-1985, pp. 57-88.

Colonna 1985 = G. Colonna (a cura di), Santuari d'Etruria (Catalogo della mostra, Arezzo 1985), Firenze 1985.

Colonna 1989-1990 = G. Colonna, Le iscrizioni votive etrusche, in «ScAnt» 3-4, pp. 875-903.

Colonna 1990a = G. Colonna, L'iscrizione etrusca del Piombo di Linguadoca, in «ScAnt» 2, 1990, pp. 547-555.

Colonna 1990b = G. Colonna, Le iscrizioni etrusche di Fratte, in G. Greco, A. Pontrandolfo (a cura di), Fratte. Un insediamento etrusco-campano, Modena 1990, pp. 301-309. 
Colonna 1993 = G. Colonna, La società spinetica e gli altri ethne, in F. Berti, P.G. Guzzo (a cura di), Spina. Storia di una citta tra Greci ed Etruschi (Catalogo della mostra, Ferrara 1993-1994), Ferrara 1993, pp. 131-143.

Colonna 1995 = G. Colonna, Etruschi a Pitecusa nell'orientalizzante antico, in A. STORCHI Marino (a cura di), L'incidenza dell'antico. Studi in memoria di Ettore Lepore (Atti del Convegno Internazionale, Anacapri 1991), I, Napoli 1995, pp. 325-342.

Colonna 1998 = G. Colonna, Intervento, in F. Rebecchi (a cura di), Spina e il delta padano. Riflessioni sul catalogo e sulla mostra ferrarese (Atti del Convegno Internazionale di Studi «Spina: due civiltà a confronto», Ferrara 1993), Roma 1998, pp. 127-130.

Colonna 2003 = G. ColonNA, L'Adriatico tra VIII e inizio V secolo a.C. con particolare riguardo al ruolo di Adria, in F. Lenzi (a cura di), L'Archeologia dell'Adriatico dalla Preistoria al Medioevo (Atti del Convegno, Ravenna 2001), Firenze 2003, pp. 146-175.

Colonna 2004a = G. Colonna, I Greci di Caere, in «AnnFaina» XI, 2007, pp. 69-94.

Colonna 2004b = G. Colonna, Scrittura e onomastica, in R.C. De Marinis, G. SPadea (a cura di), I Liguri. Un antico popolo europeo tra Alpi e Mediterraneo (Catalogo della mostra, Genova 2004-2005), Milano 2004, pp. 299-307.

Colonna 2005 = G. Colonna, Il santuario di Cupra fra Etruschi, Greci, Umbri e Picenti, in Italia ante romanum imperium. Scritti di antichità etrusche, italiche e romane. 1958-1998, Pisa-Roma 2005, pp. 363-384.

Colonna 2006 = G. Colonna, A proposito della presenza etrusca nella Gallia meridionale, in Gli Etruschi da Genova ad Ampurias (Atti del XXIV Convegno di Studi Etruschi e Italici, Marseille-Lattes 2002), Pisa-Roma 2006, pp. 657-678.

Colonna 2007 = G. Colonna, Un etrusco a Perachora. A proposito della gemma iscritta già Evans col suicidio di Aiace, in «StEtr» LXXIII, 2007, pp. 215-222.

Colonna 2008 = G. Colonna, Etruschi e Umbri in Val Padana, in «AnnFaina » XV, 2008, pp. 39-70.

Colonna 2009 = G. Colonna, Ancora su Śur/Śuri, in «StEtr» LXXV, 2009, pp. 9-32.

Colonna $2012=\mathrm{G}$. Colonna, I santuari comunitari e il culto delle divinità catactonie in Etruria, in «AnnFaina $\gg$ XIX, 2012, pp. 203-226.

Colonna $2014=$ G. Colonna, Firme di artisti in Etruria, in «AnnFaina $»$ XXI, 2014, pp. 45-74.

Colonna 2015 = G. Colonna, Tarquinio il Superbo e la Roma "etrusca": novità dal Campidoglio e dalle pendici nord-orientali del Palatino, in «StEtr» LXXVIII, 2015, pp. 61-75.

Colonna, Gambari 1986 = G. Colonna, G.M. Gambari, Il bicchiere con iscrizione arcaica da Castelletto Ticino e l'adozione della scrittura nell'Italia nord-occidentale, in «StEtr» LIV, 1986, pp. 116-164.

Conton 1904 = L. Conton, Le antiche necropoli di Adria, scoperte dal 16 novembre 1902 al 7 aprile 1904, Adria 1904.

Conton 1908 = L. Conton, Cinquanta tombe di antichi adriesi, in «Ateneo Veneto» 31, 2-3, pp. 41-79.

Conton 1910 = L. Conton, in L. Pigorini, Scavi di Adria, in «BPI» XXXVI, 1910, pp. 196-198.

Cordano, de Simone 1981 = F. Cordano, C. De Simone, Graffiti e iscrizioni provenienti dall'Acqua Acetosa Laurentina, in «PP» 36, 1981, pp. 128-142.

CORDENONS 1912 = F. CORDENONS, Le iscrizioni veneto-euganee decifrate e interpretate, Feltre 1912.

Cristofani 1972 = M. Cristofani, Sull'origine e la diffusione dell'alfabeto etrusco, in H. Temporini (a cura di), Von den Anfangen Roms bis zum Ausgang der Republik. Geschichte und Kultur Roms im Spiegel der Neueren Forschung, II, Berlin-New York 1972, pp. 466-489.

Cristofani 1973-1974 = M. Cristofani, Appunti di epigrafia etrusca arcaica II, in «ArchCl» 25-26, 1973 1974, pp. 151-165.

CRISTOFANI 1976 = M. CRISTOFANI, La leggenda di un tipo monetale etrusco, in L'Italie préromaine et la Rome républicaine. Mélanges offerts à J. Heurgon, 2, Rome 1976, pp. 209-214.

Cristofani $1977=$ M. Cristofani, Appunti di epigrafia etrusca arcaica - III. Le iscrizioni di Chiusi, in «StEtr» XLV, 1977, pp. 193-203.

Cristofani 1991 = M. CRISTOFANI, Etruschi e genti dell'Italia preromana: alcuni esempi di mobilità in età arcaica, in E. CAMPANILE (a cura di), Rapporti linguistici e culturali tra i popoli dell'Italia antica, Pisa 1991, pp. 111-128.

Cristofani 1993 = M. Cristofani, Il testo di Pech-Maho, Aleria e i traffici del V secolo a.C., in «MEFRA» 105, 2, 1993, pp. 833-845. 
Cristofani 1995 = M. Cristofani, Tabula Capuana. Un calendario festivo di etá arcaica, Firenze 1995.

Cupitò, Paltineri $2014=$ M. Cupitò, S. Paltineri, La teoria pigoriniana. Una riconsiderazione critica del problema, in A. Guidi (a cura di), 150 anni di Preistoria e Protostoria in Italia, in «Studi di Preistoria e Protostoria» 1, Firenze 2014, pp. 269-276.

Dallemulle 1977 = U. Dallemulle, Topografia ed urbanistica dell'Adria antica, in «AquilNost» XlVII, 1977, cc. 165-192.

Dallemulle 1993 = U. Dallemulle, Visitatori illustri del Museo Bocchi fra Settecento e Ottocento, in A. Lodo (a cura di), Francesco Antonio Bocchi e il suo tempo 1821-1888 (Atti del Convegno, Adria 1990), Rovigo 1993, pp. 123-161.

Dallemulle, Marzola 1977 = U. Dallemulle, E. Marzola, Una tomba di II sec. a.C. da Adria: la 45 di Ca' Cima, in «Padusa» XIII, 1977, pp. 3-53.

Damiani, Pellegrini, Saltini 1990 = I. Damiani, E. Pellegrini, A. Saltini, San Rigo di Villa Coviolo. Tracce di insediamento, in G. Ambrosetti, R. Macellari, L. Malnati (a cura di), Vestigia Crustunei. Insediamenti etruschi lungo il corso del Crostolo, Reggio Emilia 1990, pp. 169-174.

Da SChio 1853 = G. DA Schio, Sulle iscrizioni ed altri momumenti reto-euganei, Padova 1853.

Delamarre $2003=$ X. Delamarre, Dictionnaire de la langue gauloise, Paris 2003.

DE MARINIS $2007^{2}=$ R.C. DE MARINIS, Le iscrizioni etrusche del Forcello, in R.C. DE MARINIs, M. RAPI (a cura di), L'abitato etrusco del Forcello di Bagnolo S. Vito (Mantova): Le fasi arcaiche, Firenze 20072, pp. 53-69.

De MARINIs, MotTa 1990-1991 = R.C. DE MARINIs, F. MotTA, Una nuova iscrizione lepontica su pietra da Mezzovico (Lugano), in «Sibrium» XXI, 1990-1991, pp. 201-225.

de Marinis, Casini, Rapi 2016 = R.C. De Marinis, S. Casini, M. Rapi, Il basso corso del Mincio nel IVe III secolo a.C., in Govi 2016, pp. 439-467.

Desantis 1995 = P. Desantis, Spina, in F. Berti (a cura di), Uno sguardo sul passato. Archeologia del ferrarese, Catalogo della mostra (Ferrara 1994-1995), Firenze 1995, pp. 14-19.

Desantis 1997 = P. Desantis, Per una carta archeologica del territorio di Ostellato: appunti preliminari, in F. Berti (a cura di), Percorsi di archeologia, Ostellato 1997, pp. 15-31.

De Simone 1970 = C. De Simone, Die griechischen Entlehnungen im Etruskischen, II, Wiesbaden 1970.

DE Simone 1975 = C. DE SimONe, Etruskischer Literaturbericht: neuveröffentlichte Inschriften 1970-1973, in «Glotta» LIII, 1-2, pp. 125-181.

De Simone $1990=$ C. DE Simone, L'epigrafia etrusca arcaica in Orvieto, in «AnnFaina» IV, 1990, pp. 75-79. DE Simone 2001-2002 = C. DE SimONe, Il testo etrusco della Tabula Cortonensis: un primo bilancio critico, in «Ocnus» 9-10, 2001-2002, pp. 69-114.

DE SIMONe 2007 = C. DE SIMONE, Osservazioni generali in margine a CIE II, 1, 5, in «StEtr» LXXIII, 2007, pp. 223-236.

De Simone 2009a = C. De Simone, Etrusco ušil «sole» e il gentilizio latino Aurelius: problemi storico-linguisti$c i$, in «Incidenza dell'antico» 7, 2009, pp. 109-135.

De Simone 2009b = C. De Simone, L'onomastica personale della Tabula Cortonensis, in P. Poccetti (a cura di), L'onomastica dell'Italia antica. Aspetti linguistici, storici, culturali, tipologici e classificatori (Atti del Convegno, Roma 2002), Roma 2009, pp. 535-550.

De Simone 2009c = C. De Simone, La nuova iscrizione tirrenica di Efestia, in A. ArChontidou, C. DE Simone, E. Greco (a cura di), Gli scavi di Efestia e la nuova iscrizione "tirsenica", in «Tripodes» 11, 2009, pp. 3-58.

De Simone 2011 = C. De Simone, Etrusco šutiu "funerario": aporie ermeneutico-testuali della Tabula Cortonensis, in «Oebalus» 11, 2016, pp. 129-177.

De Vit 1888 = V. De Vit, Adria e le sue antiche epigrafi, Firenze 1888.

Di Filippo Balestrazzi 2013 = E. Di Filippo Balestrazzi, La romanizzazione, in M. Gamba et al. 2013, pp. 162-171.

Donati, Parrini 1999 = L. Donati, A. Parrini, Resti di abitazioni di età arcaica ad Adria. Gli scavi di Francesco Antonio Bocchi nel giardino pubblico, in Protostoria e storia del Venetorum angulus (Atti del XX Convegno di Studi Etruschi ed Italici, Portogruaro-Quarto d'Altino-Este, Adria 1996), Pisa-Roma 1999, pp. 567-614.

Ellis Evans 1967 = D. Ellis Evans, Gaulish Personal Names. A Study of some Continental Celtic Formation, Oxford 1967. 
FABretti 1872 = A. Fabretti, Primo supplemento alla raccolta delle antichissime iscrizioni italiche. Con l'aggiunta di alcune osservazioni paleografiche e grammaticali, Roma-Torino-Firenze 1872.

FABRETTI 1878 = A. FABRETTI, Terzo supplemento alla raccolta delle antichissime iscrizioni italiche, RomaTorino-Firenze 1878.

FACCHETTI 2000 = G.M. FACCHETTI, Frammenti di diritto privato etrusco, Firenze 2000.

FACCHETTI 2002a = G.M. FACCHETTI, Appunti di morfologia etrusca. Con una appendice sulla questione delle affinità genetiche dell'etrusco, Firenze 2002.

FACCHETTi 2002b = G.M. FACCheTti, L'appellativo etrusco etera, in «StEtr» LXV-LXVIII, 2002, pp. 225236.

FACCHI 2018 = A. FACCHI, Francesco Antonio Bocchi, primo Ispettore agli Scavi e ai Monumenti del nuovo Regno d'Italia per la città di Adria, in 1866. Adria e il Polesine nel 150 anniversario del Veneto italiano (Atti del Convegno, Adria 2016), Adria 2018, pp. 109-122.

Facchi, Vallicelli, Wiel-Marin c.s. = A. Facchi, M.C. Vallicelli, F. Wiel-Marin, Adria (RO), ceramica greca dagli scavi ottocenteschi (Cortile Ornati, 1878), in Forma e Immagine (Atti del Convegno, Castelfranco Veneto 2019), c.s.

Feruglio 2010-2013 = A.E. Feruglio, Le iscrizioni delle urne della tomba dei cai cutu di Perugia, in «StEtr» LXXVI, 2010-2013, pp. 199-235.

Fogolari 1940 = G. FogolarI, Scavo di una necropoli preromana e romana presso Adria, in «StEtr» XIV, 1940, pp. 431-442.

Fogolari 1955 = G. Fogolari, Atria or Adria, Hatria, Hadria (Venetia, Rovigo), n. 2479, in «FA» X, 1955, p. 196.

Fogolari 1956 = G. Fogolari, Ricerche a cura della Soprintendenza alle Antichità delle Venezie, in «BPI» n.s. X, 65, 2, 1956, pp. 541-542.

Fogolari, SCarfì $1970=$ G. Fogolari, B.M. Scarfì, Adria antica, Venezia 1970.

Forlati TAmaro 1956 = B. Forlati TAmaro, Iscrizioni inedite di Adria, in «Epigraphica» 18, 1956, pp. 50-76.

Fraser, Matthews 1987 = P.M. Fraser, E. Matthews (a cura di), A Lexicon of Greek Personal Names, I. The Aegean Islands, Cyprus, Cyrenaica, Oxford 1987.

Fraser, Matthews $2000=$ P.M. Fraser, E. Matthews (a cura di), A Lexicon of Greek Personal Names, IIIB. Central Greece from the Megarid to Thessaly, Oxford 2000.

Fraser, Matthews $2001^{2}$ = P.M. Fraser, E. Matthews (a cura di), A Lexicon of Greek Personal Names, IIIA. The Peloponnese, Western Greece, Sicily and Magna Graecia, Oxford 20012.

Furlanetto 1847 = G. Furlanetto, Le antiche lapidi patavine illustrate, Padova 1847.

Gamba et al. 2013 = M. Gamba, G. Gambacurta, A. Ruta Serafini, V. Tiné, F. Veronese (a cura di), Venetkens. Viaggio nella terra dei Veneti antichi (Catalogo della mostra, Padova 2013), Venezia 2013.

Gambacurta 2015 = G. Gambacurta, Ceramiche di importazione volterrana dalle necropoli di Adria, in Brixia. Roma e le genti del Po. Un incontro di culture III-I secolo a.C. (Catalogo della mostra, Brescia 20152016), Firenze 2015, p. 81.

Gambacurta, Bacci, Marcassa 2012 = G. Gambacurta, N. Bacci, P. Marcassa, Adria. Lo scavo dell'Ospedale Santa Maria Regina degli Angeli 2010-2011, in «QuadAVen» XXVIII, 2012, pp. 44-50.

Gambacurta et al. 2012 = G. Gambacurta, C. Balista, M. Bertolini, F. Bortolami, A. Del Brusco, F. Fuolega, U. Thun Hohenstein, E. Valli, Adria, via Amolaretta e via Zaccagnini: le indagini 2010-2011. Notizia preliminare, in «NAVe» I, 2012, pp. 35-48.

Gambacurta, Ruta Serafini 2018 = G. Gambacurta, A. Ruta Serafini, I Celti e il Veneto. Storie di culture a confronto, in «AVen» suppl. XL, Padova 2018.

GAMURRIni $1880=$ G.F. GAMURRINI, Appendice al Corpus Inscriptionum Italicarum ed ai suoi supplementi, Firenze 1880.

GaUcCi 2007 = A. GaUCCI, Tombe con iscrizioni etruschi di Adria, Tesi di laurea Specialistica in Archeologia e Culture del Mondo Antico, Università di Bologna, 2007.

Gaucci 2008 = A. GauccI, Adria. Via Spolverin - tombe 46 e 106. Il gentilizio Muliu ad Adria, in «Padusa» XLIV, 2008, pp. 81-115.

GaUCCI 2009 = A. GAucci, Le iscrizioni etrusche di Adria e i loro contesti, Tesi di Specializzazione in Beni Archeologici, Università di Bologna, 2009. 
GAUCCI 2010 = A. GAUCCI, Adria. Iscrizioni etrusche tardo-arcaiche, in «Ocnus» 18, 2010, pp. 35-51.

GAUCCI 2012a = A. GAUCCI, Le iscrizioni etrusche tardo-arcaiche di Adria. Nuove iscrizioni e analisi epigrafica e dei contesti, in «Padusa $\gg$ XLVIII, 2012, pp. 143-179.

GAUCCI 2012b = A. GAUCCI, Alfabetari latini nell'Italia preromana, in «AttiMemPetrarca» LXXII-LXXIII, 2012, pp. 59-83.

GaUCCI 2013 = A. GaUCCI, I porti del delta padano nel IV secolo a.C., in F. Boschi (a cura di), Ravenna e l'Adriatico dalle origini all'età romana, Bologna 2013, pp. 71-90.

GAUCCI 2015 = A. GAUCCI, Organizzazione deglispazi funerari a Spina e in area deltizia con particolare riguardo al periodo tardo-arcaico, in «AnnFaina» XXII, 2015, pp. 113-170.

GaUCCI 2016 = A. GaUCCI, La fine di Adria e Spina etrusche, in Govi 2016, pp. 171-221.

GAUCCI 2017 = A. GAUCCI, Gli Etruschi e l'Adriatico dalla prima Età del ferro alla romanizzazione, in «Iranica et Mediterranea »1, 2017, pp. 63-96.

GAUCCI 2020 = A. GAUCCI, Graffiti dai contesti abitativi e funerari della città etrusca di Adria: il segno a croce, in «Aristonothos. Rivista di Studi sul Mediterraneo antico» 16, 2020, pp. 413-449.

Gaucci c.s.= A. Gaucci, Writing Practice and Society, in E. Govi (ed.), Marzabotto/Kainua, c.s.

Gaucci, Pozzi 2009 = A. GaUCCI, A. Pozzi, L'archeologia funeraria negli empori costieri. Le tombe con iscrizioni etrusche da Spina e Adria, in R. Bonaudo, L. Cerchiai, C. Pellegrino (a cura di), Tra Etruria, Lazio e Magna Grecia: indagini sulle necropoli (Atti dell'Incontro di Studio, Fisciano 2008), Paestum 2009, pp. 51-64.

Gaucci, Vallicelli 2011 = A. Gaucci, M.C. Vallicelli, Chous attico a figure nere, in F. Marzatico, R. Gebhard, P. Gleirscher (a cura di), Le grandi vie delle civiltà. Relazioni e scambi fra Mediterraneo e il Centro Europa dalla preistoria alla romanità (Catalogo della mostra, Trento 2011), Trento 2011, p. 517.

GaUCCI et al. 2017 = A. GaUCCI, V. Minguzzi, G. Gasparotto, E. Zantedeschi, La ceramica etrusca a vernice nera di Valle Trebba: dati archeologici e archeometrici a confronto, in C. REUSSER (a cura di), Spina. Neue Perspektiven der archäologischen Erforschung (Tagung, Zürich 2012), Rahden 2017, pp. 127-138.

Gaucci, Morpurgo, Pizzirani $2018=$ A. Gaucci, G. Morpurgo, C. Pizzirani, Ritualità funeraria in Etruria padana tra VI e III secolo a.C. Progetti di ricerca e questioni di metodo, in «AnnFaina» XXV, 2018, pp. 653-692.

Gaucci, Govi, Sassatelli c.s. = A. Gaucci, E. Govi, G. Sassatelli, Le stele iscritte di Bologna, in c.s.

Gennuso, Marchesini, Vallicelli 2018 = I. Gennuso, M. Marchesini, M.C. Vallicelli, Analisi xilologiche delle strutture e dei manufatti lignei dell'abitato etrusco di Adria (RO): Le indagini di via Ex Riformati, in «West\&East» III, 2018, pp. 126-139.

Gens antiquissima 1990 = F. RonCALli (a cura di), Gens antiquissima Italiae. Antichità dall'Umbria a Leningrado (Catalogo della mostra, Leningrado 1990), Perugia 1990.

Ghirardini 1905 = G. Ghirardini, Il Museo civico di Adria, in «Nuovo Archivio Veneto» IV, IX, 1905, pp. 114-157.

Giangivlio 1984 = M. Giangiulio, Adria, in G. Nenci, G. Vallet (a cura di), Bibliografia Topografica della colonizzazione greca in Italia e nelle isole tirreniche, III. Abaceno-Bari, Pisa-Roma 1984, pp. 38-56.

Govi 1994a = E. Govi, Un nuovo alfabetario etrusco dalla Romagna, in «Ocnus» 2, 1994, pp. 67-77.

Govi 1994b= E. Govi, I graffiti, in SASSATELli 1994, pp. 213-236.

Govi 1999 = E. Govi, Le ceramiche attiche a vernice nera di Bologna, Imola 1999.

Govi 2012 = E. Govi, I vasi etruschi del “Gruppo di Adria”, in «Ocnus» 20, 2012, pp. 107-154.

Govi 2014 = E. Govi, Una nuova iscrizione dal tempio urbano di Tinia a Marzabotto, in «StEtr» LXXVII, 2014, pp. 109-147.

Govi 2016 = E. Govi (a cura di), Il mondo etrusco e il mondo italico di ambito settentrionale prima dell' impatto con Roma (IV-II secolo a.C.) (Atti del Convegno di Studi Etruschi ed Italici - Sezione Etruria padana e Italia settentrionale, Bologna 2013), Roma 2016.

Govi 2017 = E. Govi, Il progetto di ricerca sulla necropoli di Valle Trebba. Qualche spunto di riflessione, in C. Reusser (a cura di), Spina. Neue Perspektiven der archäologischen Erforschung (Tagung, Zürich 2012), Rahden 2017, pp. 99-108.

Govi, Martelli, Sassatelli 2009 = E. Govi, A. Martelli, G. Sassatelli, Le iscrizioni, i segni alfabetici, i segni non alfabetici, in P. GASTALDI (a cura di), Chiusi. Lo scavo del Petriolo (1992-2004), «AIONArch» Quad. 17, Chiusi 2009, pp. 247-283. 
Gras 2010 = M. Gras, Empória ed emporía. Riflessioni sul commercio greco arcaico in Occidente, in E. Govi (a cura di), Dal Mediterraneo all'Europa. Conversazioni adriatiche, «Hesperìa» 25, Roma 2010, pp. 47-56.

Guida 1980 = A.M. Brizzolara et al., Guida al Museo etrusco di Marzabotto, in «EmPrerom» 8, 1980, pp. 97-120.

HAACK 2009 = M.L. HAACK, Grécité réelle et grécité fantasmée à Spina et à Adria, in M.-L. HAACK (a cura di), Écritures, cultures, sociétés dans les nécropoles d'Italie ancienne (Tavola rotonda, Paris 2007), Bordeaux 2009, pp. 45-62.

HAACK 2016 = M.L. HAACK, L'écriture et le sacré à Spina, in Y. LAFOnd, V. MicheL (sous la direction), Espaces sacrés dans la Méditerranée antique (Actes du Colloque, Poitiers 2011), Rennes 2016, pp. 193-201.

Hadas-Lebel 2016 = J. Hadas-Lebel, Les cas locaux en étrusque, Roma 2016.

Harari $2001=$ M. HARARI, Adria da emporion a polis, in Commerci e produzione in età antica nella fascia costiera fra Ravenna ed Adria (Atti della Giornata di Studio, Ferrara 2001), suppl. a «Atti dell'Accademia delle Scienze di Ferrara» LXXVIII, Ferrara, pp. 43-58.

HeichelHeim 1955 = F.M. Heichelheim, Vercana, in RE 15, 2, p. 979.

Heurgon 1973 = J. Heurgon, Les graffites d'Aleria, in J. Jehasse, L. JeHASSE (a cura di), La nécropole préromaine d'Aleria (1960-1968), «Gallia» suppl. XXV, Paris 1973, pp. 547-575.

Hirata 1967 = R. Hirata, L'onomastica falisca e i suoi rapporti con la latina e l'etrusca, Firenze 1967.

Holder 1904 = A. Holder, Alt-celtischer Sprachschatz, II. I-T, Leipzig 1904.

INTRIERI 2010 = M. INTRIERI, Autarkeia. Osservazioni sull'economia corcirese fra V e IV sec. a.C., in C. ANTONETTI (a cura di), Lo spazio ionico e le comunità della Grecia nord-occidentale. Territorio, società, istituzioni (Atti del Convegno Internazionale, Venezia 2010), Pisa 2010, pp. 181-199.

Johnston $1979=$ A.W. Johnston, Trademarks on Greek vases, Warminster 1979.

Johnston 1985 = A.W. Johnston, Etruscans in the Greek Vase Trade?, in Il commercio etrusco arcaico (Atti dell'Incontro di Studio), Roma 1985, pp. 249-255.

Johnston 2006 = A.W. Johnston, Trademarks on Greek vases. Addenda, Oxford 2006.

Komp, Quirino, Rapi = R. Komp, T. Quirino, M. Rapi, The Hidden City: Reconstructing the Urban Structure of the Etruscan Harbour of Forcello di Bagnolo San Vito through Excavations and Non-invasive Methods, in L. Zamboni, M. Fernández-Götz, C. Metzner-Nebelsick (eds.), Crossing the Alps, Early Urbanism between Northern Italy and Central Europe (900-400 BC), Leiden 2020, pp. 227-241.

LAMANNA $2014=$ L. LAMANNA, L'abitato etrusco del Forcello, in «LANX»17, 2014, pp. 53-59.

LANZI 1789 = L. LANZI, Saggio di lingua etrusca e di altre antiche d'Italia, per servire alla storia de' popoli, delle lingue, e delle belle arti, I-III, Roma 1789.

LANZI 1806 = L. LANZI, De’ vasi antichi dipinti volgarmente chiamati etruschi, Firenze 1806.

LANZI 1824-1825 = L. LANZI, Saggio di lingua etrusca e di altre antiche d'Italia per servire alla storia de' popoli, delle lingue e delle belle arti, Firenze 1824-18252.

Lejars 2017 = Th. Lejars, Le armi dei Celti d'Italia, in P. Piana Agostinetti (a cura di), Celti d'Italia. I Celti dell'età di La Tène a sud delle Alpi (Atti del Convegno Internazionale, Roma 2010), Roma 2017, pp. 555-607.

Lejeune $1966=$ M. Lejeune, Venetica, in «Latomus» XXV, 1966, pp. 7-27.

Lejeune 1971 = M. Lejeune, Lepontica, Paris 1971.

Lejeune 1974 = M. Lejeune, Manuel de la langue vénète, Heidelberg 1974.

Liou 1993 = B. Liou, Inscriptionspeintes sur amphoras de Narbonne (Port-la-Nautique), in «Archaeonautica» 11, 1993, pp. 131-148.

Losi 1989 = A. Losi, Cacciola di Sandiano, in G. Ambrosetti, R. Macellari, L. Malnati (a cura di), Rubiera. "Principi" etruschi in val di Secchia (Catalogo della mostra, Reggio Emilia 1989), Reggio Emilia 1989, pp. 143-154.

LUNi $2004=$ M. LUNi, I Greci nel kolpos adriatico, Ankon e Numana, in «Hesperìa 18, 2004, pp. 11-56.

MaCellari 1981-1982 = R. Macellari, Due graffiti vascolari etruschi al Museo di Parma, in «EmPrerom» 9-10, 1981-1982, pp. 278-280.

MaCellari 1994 = R. MACellari, Una nuova iscrizione etrusca da Bologna, in «Ocnus» 2, 1994, pp. 97 105.

Macellari 1997 = R. Macellari, Nuova iscrizione etrusca dalla Romagna al Museo di Reggio Emilia, in «Ocnus» 5, 1997, pp. 115-121. 
Macellari $2004=$ R. Macellari, Gli Etruschi del Po, in «Ocnus» 12, 2004, pp. 145-156.

MACELlari $2008=$ R. MACELlari, Fra Etruschi e Celti. Sorbolo e il suo territorio nel primo millennio a.C., in R. Conversi, R. Macellari (a cura di), Sorbolo e le sue storie. 1806-2006. Una Storia in Comune (Atti della Giornata di Studi, Sorbolo 2006), Parma 2008, pp. 113-128.

Macellari, SQuadrini, Bentini 1990 = R. Macellari, G. SQuadrini, L. Bentini, Casale di Rivalta, in G. Ambrosetti, R. Macellari, L. Malnati (a cura di), Vestigia Crustunei. Insediamenti etruschi lungo il corso del Crostolo, Reggio Emilia 1990, pp. 177-234.

MAggiani 1976 = A. MAGgiani, Contributo alla cronologia delle urne volterrane: $i$ coperchi, in «MemLincei»s. VIII, XIX, 1, 1976, pp. 3-44.

Maggiani 1990 = A. MagGiani, Alfabeti etruschi di età ellenistica, in «AnnFaina »IV, 1990, pp. 177-217.

Maggiani 1992a = A. Maggiani, Documentazione epigrafica, in I. Damiani, A. Maggiani, E. Pellegrini, A.C. Saltini, A. Serges (a cura di), L'Età del Ferro nel Reggiano. I materiali delle collezioni dei Civici Musei di Reggio Emilia, Reggio Emilia 1992, pp. 211-216.

Maggiani 1992b = A. Maggiani, Le iscrizioni di età tardo classica ed ellenistica, in A. Romualdi (a cura di), Populonia in età ellenistica (Atti del Seminario, Firenze 1990), Firenze 1992, pp. 179-190.

MAGgIANI 1994 = A. MAGGIANI, Mantica oracolare in Etruria: litobolia e sortilegio, in «RdA» XVIII, 1994, pp. 68-78.

Maggiani 1997a = A. Maggiani, Modello etico o antenato eroico? Sul motivo di Aiace suicida nelle stele felsinee, in «StEtr» LXIII, 1997, pp. 149-165.

Maggiani 1997b = A. MAGgIANI, Vasi attici figurati con dediche a divinità etrusche, Roma 1997.

Maggiani 1998 = A. Maggiani, Sulla paleografia delle iscrizioni di Spina, in F. Rebecchi (a cura di), Spina e il delta padano. Riflessioni sul catalogo e sulla mostra ferrarese (Atti del Convegno Internazionale di studi «Spina: due civiltà a confronto», Ferrara 1994), Roma 1998, pp. 227-234.

Maggiani 2000a = A. Maggiani, Etruschi nel Veneto in età orientalizzante e arcaica, in «Hesperìa»12, 2000, pp. 89-95.

Maggiani 2000b = A. MAgGIAnI, Tipologia tombale e società: Chiusi in età orientalizzante, in «AnnFaina» VII, 2000, pp. 249-275.

MAgGiani 2002a = A. MAgGiani, L'alfabeto etrusco nel Veneto, in «Akeo» 2002, pp. 55-63.

Maggiani 2002b = A. Maggiani, Nel mondo degli specchi etruschi, in Caelatores. Incisori di specchi e ciste tra Lazio ed Etruria (Atti della Giornata di Studio, Roma 2001), Roma 2002, pp. 7-22.

Maggiani 2003 = A. Maggiani, L'epigrafia di epoca etrusca, in G.M. Della Fina, C. Fratini (a cura di), Storia di Orvieto, I. Antichità, Ponte San Giovanni 2003, pp. 371-384.

Maggiani 2008 = A. Maggiani, Ai margini della colonizzazione. Etruschie Veneti nel VI sec. a.C., in «AnnFaina» XV, 2008, pp. 341-363.

MAGgIANI 2009 = A. MAGGIANI, Une nouvelle attestation du mot sacnioa. Urne inédite de la collection Consortini de Volterra, in M.-L. HAACK (a cura di), Écritures, cultures, sociétés dans les nécropoles d'Italie ancienne (Actes de la Table-Ronde, Paris 2007), Bordeaux 2009, pp. 217-229.

Maggiani 2013a = A. Maggiani, La scrittura nella Volsinii etrusca, in G.M. Della Fina, E. Pellegrini (a cura di), Da Orvieto a Bolsena: un percorso tra Etruschi e Romani (Catalogo della mostra, 2013), Pisa 2013, pp. 167-177.

MAGgiani 2013b = A. MAgGiani, Un'anfora attica con contrassegno commerciale da Vulci, in «Hesperìa 30, 2, pp. $938-954$.

Maggiani 2015 = A. Maggiani, Ostraka iscritti dall'Etruria, in «StEtr» LXXVIII, 2015, pp. 145-155.

Maggiani 2016 = A. Maggiani, La scrittura a Fiesole in età arcaica, in P. Perazzi, G. Poggesi, S. SArti (a cura di), L'ombra degli Etruschi. Simboli di un popolo fra pianura e collina (Catalogo della mostra, Prato 2016), Firenze 2016, pp. 73-81.

MAGGIANI 2017 = A. MAGGIANI, La nascita della scrittura nell'Etruria settentrionale. Una nota, in «StEtr» LXXX, 2017, pp. 133-147.

MaGNIN 2014 = S. MaGnin, Nouvelle lecture de l'inscription vénète dite d'Este, in «Wékwos» 1, pp. 117 130.

Malnati 1989 = L. Malnati, Lo scavo di una fattoria etrusca a Baggiovara - località Case Vandelli, in A. Cardarelli (a cura di), Modena dalle origini all'anno Mille. Studi di archeologia e storia, I, Modena, pp. $262-271$. 
Malnati 2010 = L. Malnati, I graffiti e le iscrizioni, in R. Curina (a cura di), Alla ricerca di Bologna antica e medievale: da Felsina a Bononia negli scavi di via D'Azeglio, «Quaderni di Archeologia dell'Emilia Romagna $\gg 25$, Bologna, pp. 118-120.

Mambella 1984 = R. Mambella, Alcune considerazioni su Adria nel IV-III sec. a.C., in «RdA» 8, 1984, pp. 29-36.

Mambella 1986 = R. Mambella, Osservazioni su alcune iscrizioni preromane di Adria, in «AquilNost» 57, 1986, pp. 265-280.

Mangani 1980= E. Mangani, Materiali volterrani ad Adria in età preromana, in «StEtr» XLVIII, 1980, pp. 121-140.

Mangani 1982 = E. Mangani, Adria, in «NSc»s. VIII, XXXVI, 1982, pp. 5-107.

MARAS 1998 = D.F. MARAs, La dea Thanr e le cerchie divine in Etruria: nuove acquisizioni, in «StEtr» LXIV, 1998, pp. 173-197.

Maras 2002 = D.F. Maras, Appendice II. Le iscrizioni, in G. Colonna (a cura di), Il santuario di Portonaccio a Veio. Gli scavi di Massimo Pallottino nella zona dell'altare (1939-1940), Monumenti Antichi dei Lincei, s. misc., VI, 3, Roma 2002, pp. 261-273.

MARAS 2009a = D.F. MARAS, Interferenze culturali arcaiche etrusco-latine: la scrittura, in «AnnFaina» XVI, 2009, pp. 309-331.

MARAs 2009b = D.F. MARAs, Il dono votivo: gli dei e il sacro nelle iscrizioni etrusche di culto, Pisa-Roma, 2009.

MARAS 2012 = D.F. MARAs, Interferenza e concorrenza di modelli alfabetici e sistemi scrittori nell'Etruria arcai$c a$, in «MEFRA»124, 2, 2012, pp. 331-344.

Maras 2013a = D.F. Maras, Le iscrizioni, in C. Cornelio Cassai, S. Giannini, L. Malnati (a cura di), Spina. Scavi nell'abitato della città etrusca, 2007-2009, Firenze 2013, pp. 156-161.

Maras 2013b = D.F. MARAS, Area Sud: ricerche in corso sulla documentazione epigrafica (contesti, supporti, formulari, teonimi), in M.P. BAglione, M.D. Gentili (a cura di), Riflessioni su Pyrgi. Scavi e ricerche nelle aree del santuario, «ArchCl» suppl., Roma 2013, pp. 195-206.

Maras 2014 = D.F. Maras, Note epigrafiche sulla stele di via Saffi, in «AnnFaina» XXI, 2014, pp. 336-342.

MARAs 2016 = D.F. MARAs, Lettere e sacro. Breve storia della scrittura nel santuario etrusco di Pyrgi, in V. BELLELLI, P. Xella (a cura di), Le lamine di Pyrgi. Nuovi studi sulle iscrizioni in etrusco e in fenicio nel cinquantenario della scoperta, SEL 32-33, 2015-2016, Verona 2016, pp. 89-101.

Marchesini 1994 = S. MARChesini, Etrusco Niritalu: greco Nípitos, in «AIONling» 16, 1994, pp. 273-281.

Marchesini 1997 = S. Marchesini, Studi onomastici e sociolinguistici sull'Etruria arcaica: il caso di Caere, Firenze 1997.

Marchesini 2007 = S. Marchesini, Prosopographia etrusca II, 1 . Studia. Gentium mobilitas, Roma 2007.

MarinetTi 1982 = A. Marinetti, Atta/us: Appius; lat. atta, sabino *appa e sudpiceno apaio-. Sabini a Roma e 'Safini nelle iscrizioni sudpicene, in «Res Publica Litterarum» V, 1, 1982, pp. 169-181.

Marinetti 1994 = A. Marinetti, La documentazione epigrafica, in R. Peretto (a cura di), Balone. Insediamento etrusco presso un ramo del Po, Padova 1994, pp. 81-87.

Marinetti 1998 = A. Marinetti, Il venetico. Bilancio e prospettive, in A. Marinetti, M.T. Vigolo, A. Zamboni (a cura di), Varietà e continuità nella storia linguistica del Veneto (Atti del Convegno della Società italiana di Glottologia, Padova-Venezia 1996), Roma 1998, pp. 49-99.

Marinetti 2002 = A. Marinetti, Caratteri e diffusione dell'alfabeto venetico, in Akeo 2002, pp. 39-54.

MarinetTi 2009 = A. Marinetti, Da Altno- a Giove. La titolarita del santuario. I. La fase preromana, in G. Cresci MARRONe, M. TIRELli (a cura di), ALTNOI. Il santuario altinate: strutture del sacro a confronto $e$ i luoghi di culto lungo la via Annia (Atti del Convegno, Venezia 2006), Roma 2009, pp. 81-127.

Marinetti, Solinas 2014 = A. Marinetti, P. Solinas, I Celti nel Veneto nella documentazione epigrafica locale, in Les Celtes et le Nord de l'Italie (Premier et Second Ages du fer) (Actes du XXXVI' colloque international de l'A.F.E.A.F., Vérone 2012), Dijon 2014, pp. 75-87.

Marinetti, Solinas 2016 = A. Marinetti, P. Solinas, Continuità, aperture, resistenze nelle culture locali: la prospettiva linguistica, in Govi 2016, pp. 31-73.

Marseille 1977-1978 = Le monde etrusque: musée Borély (Catalogo della mostra, Marseille 1977-1978), Marseille 1977-1978.

Martelli $2018=$ M. Martelli, In visita alle tombe principesche ceretane di S. Paolo, in «Ostraka» XXVII, 2018, pp. 57-76. 
Massarelli $2008=$ R. Massarelli, Alcune osservazioni sull'etrusco ${ }^{*}$ tu $\theta$-, in G.M. Facchetti (a cura di), Mla mlakas. Per Luciano Agostiniani, Milano 2008, pp. 195-214.

Massarelli 2014 = R. Massarelli, I testi etruschi su piombo, Pisa-Roma 2014.

Mattioli 2013 = C. Mattioli, Atlante tipologico delle forme ceramiche di produzione locale in Etruria padana, Bologna 2013.

Menotti $2017=$ E.M. Menotti, Nuove ricerche a Mantova, in C. Reusser (a cura di), Spina. Neue Perspektiven der archäologischen Erforschung (Tagung, Zürich 2012), Rahden 2017, pp. 75-79.

Menotti, Maras 2012 = E.M. Menotti, D.F. Maras, Un'area sacra in Mantova etrusca, in N. Negroni Catacchio (a cura di), L'Etruria dal Paleolitico al Primo Ferro. Lo stato delle ricerche (Atti del X Incontro di studi di Preistoria e Protostoria in Etruria, Valentano-Pitigliano 2010), Milano 2012, pp. 875-888.

Mommsen $1853=$ Th. Mommsen, Die nordetruskischen Alphabets auf Inschriften und Münzen, Zürich 1853.

Morandi $1990=$ A. Morandi, Il cippo volterrano «dei Marmini», in «RBelgPhilHist» LVIII, 1, pp. 137148.

Morandi 1999 = A. Morandi, Il cippo di Castelciès nell'epigrafia retica, Roma 1999.

Morandi 2004 = A. Morandi, Celti d'Italia, 2. Epigrafia e lingua dei Celti d'Italia, Roma 2004.

Morandi Tarabella $2004=$ M. Morandi Tarabella, Prosopographia etrusca, I. Corpus, 1. Etruria meridionale, Roma 2004.

Morigi Govi, Colonna 1981 = C. Morigi Govi, G. Colonna, L'anforetta con iscrizione etrusca da Bologna, in «StEtr» XLIX, 1981, pp. 67-94.

Morigi Govi, Sassatelli 1984 = C. Morigi Govi, G. Sassatelli (a cura di), Dalla Stanza delle Antichità al Museo Civico. Storia della formazione del Museo Civico Archeologico di Bologna (Catalogo della mostra, Bologna 1984-1985), Casalecchio di Reno 1984.

Morpurgo 2013 = G. Morpurgo, La ceramica grigia, in Mattioli 2013, pp. 381-494.

Mosca, Puppo 2000 = F. Mosca, P. Puppo, Adria. La tomba 53 della necropoli di Piantamelon, in «Padusa» XXXVI, 2000, pp. 135-144.

MotTA 2000 = F. MотTA, La documentazione epigrafica e linguistica, in R.C. DE MARINIS, S. BIAGgio SIMONA (a cura di), I Leponti tra mito e realtà. Raccolta di saggi in occasione della mostra, II, Locarno 2000, pp. 181-222.

Mотта 2009 = F. Мотта, Tipologie dell'onomastica personale celtica nell'Italia antica, in P. PocCETTI (a cura di), L'onomastica dell'Italia antica. Aspetti linguistici, storici, culturali, tipologici e classificatori (Atti del Convegno, Roma 2002), Roma 2009, pp. 295-318.

Muggia 2004 = A. Muggia, Impronte nella sabbia. Tombe infantili e di adolescenti dalla necropoli di Valle Trebba a Spina, Firenze 2004.

Müller, Deecke 1877 = K.O. Müller, W. Deecke, Die Etrusker, Stuttgard 1877.

Natalucci, Zampieri 2019 = M. Natalucci, E. Zampieri, Numana (AN): nuovi dati dalla necropoli picena Quagliotti-Davanzali, in M. Cipriani, E. Greco, A. Pontrandolfo, M. Scafuro (a cura di), Dialoghi sull'Archeologia della Magna Grecia e del Mediterraneo (Atti del III Convegno Internazionale di Studi, Paestum 2018), Paestum 2019, pp. 643-654.

NijBoer 1998 = A.J. NijBOer, From household production to workshops. Archaeological evidence for economic transformation, pre-monetary exchange and urbanisation in central Italy from 800 to $400 \mathrm{BC}$, Groningen 1998.

Nissen 1902 = H. Nissen, Italische Landeskunde, II. Die Staedte, I-II, Berlin 1902.

Osborne, Byrne 1994 = M.J. Osborne, S.G. Byrne 1994, Lexicon of Greek Personal Names, II. Attica, Oxford 1994.

Paltineri, Robino 2016 = S. Paltineri, M.T.A. Robino, Le ultime fasi del sito di San Cassiano di Crespino e le trasformazioni nell'entroterra di Adria, in Govi 2016, pp. 275-301.

Panciera et al. 1991 = S. PANCIERA et al., Inscriptiones latinae liberae rei pubblicae, in Epigrafia (Actes du colloque international d'épigraphie latine en mémoire de Attilio Degrassi pour le centenaire de sa naissance, Rome 1988), Rome 1991, pp. 241-491.

Pandolfini $1988^{2}$ = M. PANdolfini, Le iscrizioni etrusche del Mantovano, in R.C. De Marinis (a cura di), Gli Etruschi a nord del Po, I, Udine 19882, pp. 116-123.

Pandolfini 1989 = M. Pandolfini, Lo scarico arcaico della Vigna Parrocchiale, in Miscellanea Ceretana 1, in «QuadAEl»17, pp. 69-83. 
Pandolfini $2000=$ M. Pandolfini, Iscrizioni etrusche, in A. Johnston, M. Pandolfini, Santuario di Gravisca. Le iscrizioni, Bari 2000, pp. 69-104.

Pandolfini, Prosdocimi $1990=$ M. Pandolfini, A.L. Prosdocimi, Alfabetari e insegnamento della scrittura in Etruria e nell'Italia antica, Firenze 1990.

Paoli, Parrini 1988 = L. Paoli, A. Parrini, Corredi di età ellenistica dalla necropoli di Spina, Ferrara 1988.

Pappa 2013 = E. PAPpa, Postcolonial Baggage at the End of the Road: How to Put the Genie Back into its Bottle and Where to Go from There, in W.P. vAN PeLt (a cura di), Archaeology and Cultural Mixture, Cambridge 2013, pp. 29-50.

Pastega 2011 = G. Pastega (a cura di), Gli Annali Guarnieri-Bocchi (1745-1848). Un secolo di cronaca e storia adriese, Adria 2011.

Patitucci 1979 = S. Patitucci, Voghiera, un nuovo insediamento etrusco del delta padano, in «StEtr» XLVII, 1979, pp. 93-105.

Pauli 1885 = C. Pauli, Altitalische Forschungen, 1. Die Inschriften nordetruskischen Alphabets, Leipzig 1885.

Pauli 1891 = C. Pauli, Die Veneter und ihre Schriftdenkmäler, Leipzig 1891.

Pellegrini 1956 = G.B. Pellegrini, Corso di Storia comparata delle lingue classiche. Le iscrizioni venetiche, Appendice a Le iscrizioni venetiche, Pisa 1956.

Pellegrini 1957 = G.B. Pellegrini, Iscrizioni nord-etrusche, in Tyrrhenica. Saggi di studi etruschi, Milano 1957, pp. 145-157.

Pellegrini 1959 = G.B. Pellegrini, Gli alfabeti preromani nell'Italia superiore, in Spina e l'Etruria Padana (Atti del Convegno, Ferrara 1957), Firenze 1959, pp. 181-195.

Pellegrini 1965 = G.B. Pellegrini, Nuove iscrizioni etrusche e venetiche di Adria, in Studi in onore di Luisa Banti, Roma, pp. 263-273.

Pellegrini, Fogolari 1958 = G.B. Pellegrini, G. Fogolari, Iscrizioni etrusche e venetiche di Adria, in «StEtr» XXIV, 1958, pp. 103-154.

Pellegrino 2008 = C. Pellegrino, Pontecagnano: la scrittura e l'onomastica in una comunità etrusca di frontiera, in «AnnFaina XV, 2008, pp. 423-463.

Perazzi, Poggesi, Sarti 2016 = P. Perazzi, G. Poggesi, S. Sarti, L'ombra degli Etruschi. Simboli di un popolo tra pianura e collina (Catalogo della mostra, Prato 2016), Firenze 2016.

Peretto, Vallicelli, Wiel-Marin 2002 = R. Peretto, M.C. Vallicelli, F. Wiel-Marin, L'entroterra di Adria: conoscenze archeologiche e paleoambientali, in «Padusa» XXXVIII, pp. 91-114.

PFIFFIg 1961a = A.J. Pfiffig, Osservazioni su nomi veneti nelle iscrizioni etrusche di Spina, in «StEtr» XXIX, 1961, pp. 327-329.

Pfiffig 1961b = A.J. Pfiffig, Untersuchungen zun «Cippus Perusinus», in «StEtr» XXIX, 1961, pp. 111-154.

PFiffig 1968= A.J. Pfiffig, Ein Opfergelübde an die etrusckische Minerva. Studien und Materialen zur Interpretation des Bleistreifens von S. Marinella, Wien 1968.

Pfiffig 1971 = A.J. Pfiffig, Etruskisch apa 'Vater' und Name, in «BzNF» n.s., 6, 1971, pp. 35-39.

Pfiffig 1975 = A.J. Pfiffig, Religio etrusca, Graz 1975.

PizZirani 2005 = C. Pizzirani, Da Odisseo alle Nereidi. Riflessioni sull'iconografia etrusca del mare attraverso i secoli, in «Ocnus»13, 2005, pp. 251- 270.

PocCETTi 1997 = P. PocCetti, Etrusco Feluske = Faliscus? Note sull'iscrizione della stele arcaica di Vetulonia, in «StEtr» LXIII, 1997, pp. 281-290.

Poccetti 2019 = P. Poccetti, Gli Smint(h)ii nella documentazione etrusca e italica, in A. Mele (a cura di), Dalla Troade a Cuma Opicia. Gli Eoli, la Sibilla, Apollo Smintheo, «Quaderni di Oebalus», Roma 2019, pp. 95-139.

Poggi 1879 = V. Poggi, Contribuzioni allo studio dell'epigrafia etrusca, in «Giornale Ligustico» VI, 1879, pp. 245-319.

Pozzi 2011 = A. Pozzi, Le tombe di Spina con iscrizioni etrusche, Tesi di Dottorato in Studio e Conservazione dei Beni Archeologici ed Architettonici (Ciclo XXII), Università di Padova.

Prosdocimi 1988 = A.L. Prosdocimi, La lingua, in G. Fogolari, A.L. Prosdocimi, I Veneti antichi. Lingua e cultura, Padova 1988, pp. 221-420.

Prosdocimi 2009 = A.L. Prosdocimi, Note sull'onomastica di Roma e dell'Italia antica, in P. Poccetti (a cura di), L'onomastica dell'Italia antica. Aspetti linguistici, storici, culturali, tipologici e classificatori (Atti del Convegno, Roma 2002), Roma 2009, pp. 73-151. 
QUIRINO $2017=$ T. QUIRINO, Open architecture RDBMS and GIS as tools for analysing the Etruscan presence in the Po Plain: towards a model of the urban/non urban landscape, in «ACalc» 28, 2, 2017, pp. 253-266.

Ravasio 1995 = T. Ravasio, Due vasi con graffiti dalle collezioni del Museo di Stato della Repubblica di San Marino, in «Ocnus» 3, 1995, pp. 157-162.

Reusser 2016 = C. Reusser, Spina nel IV secolo a.C.: lo scavo dell'Università di Zurigo, in Govi 2016, pp. 111-125.

Rigobianco 2013 = L. Rigobianco, Su numerus, genus $e$ sexus. Elementi per una grammatica dell'etrusco, Roma 2013.

Rigobianco 2017 = L. Rigobianco, Per una grammatica dell'etrusco. Considerazioni morfonologiche sulla derivazione di nomi e aggettivi in etrusco arcaico, in «Mediterranea» XIV, 2017, pp. 185-203.

RIx 1963 = H. Rix, Das etruskische Cognomen. Untersuchungen zu System, Morphologie und Verwendung der Personennamen auf den jüngeren Inschriften Nordetruriens, Wiesbaden 1963.

RIx $1981=$ H. RIx, Rapporti onomastici fra il pantheon etrusco e quello romano, in G. ColonNa (a cura di), Gli Etruschi e Roma (Atti dell'incontro di studio in onore di Massimo Pallottino, Roma 1979), Roma 1981, pp. 104-126.

RIx $1984=$ H. RIx, Etruskisch me $\mathrm{rasnal}=$ lat. res publica, in Studi di antichità in onore di Guglielmo Maetzke, Roma 1984, pp. 455-468.

RIx 1986 = H. RIx, Etruskisch Culs* »Tor« und der Abschnitt VIII 1-2 des Zagreber Liber Linteus, in «VjesMusZagr»s. 3, XIX, 1986, pp. 17-39.

Rix 1987-1988 = H. Rix, Zur Morphostruktur des etruskischen s-Genetivs, in «StEtr» LV, 1987-1988, pp. $169-193$.

Rix 1991 = H. Rıx, Unm, Une, Unu “Te, Tibi, Vos" e le preghiere dei rituali paralleli del Liber Linteus, in Miscellanea etrusco-italica in onore di Massimo Pallottino, in «ArchCl» XLIII, 1991, pp. 665-691.

RIx $1998=$ H. Rix, Teonimi etruschi e teonimi italici, in «AnnFaina» V, 1998, pp. $207-229$.

Rix $2001^{3}=$ H. Rix, La scrittura e la lingua, in M. CRIstofani (a cura di), Gli Etruschi. Una nuova immagine, Prato $2001^{3}$, pp. 199-227.

RobINo 2000 = M.T.A. RobIno, Tipologia e cronologia delle brocche alto-adriatiche nelle necropoli di Adria, in M. Landolfi (a cura di), Adriatico tra IV e III sec. a.C. Vasi alto-adriatici tra Piceno, Spina e Adria (Atti del Convegno di Studi, Ancona 1997), Roma 2000, pp. 71-95.

RoBINo 2003 = M.T.A. RoBINo, Alcune considerazioni morfologiche e stilistiche sulla ceramica alto-adriatica dalle necropoli di Adria: i crateri, gli skyphoi e gli stamnoi, in «Padusa» XXXIX, 2003, pp. 103-141.

RobINo 2004 = M.T.A. RobIno, L'abitato di Adria in età ellenistica e romana, Tesi di Dottorato (XVII Ciclo), Università di Pavia.

Robino 2008 = M.T.A. Robino, Alcune note sulla viabilità di Adria, in L. Quilici, S. Quilici Gigli (a cura di), Edilizia pubblica e privata nelle città romane, in «ATTA»17, 2008, pp. 7-19.

RoBIno 2016 = M.T.A. RobIno, La necropoli del canal bianco di Adria (RO): problemi e prospettive a settant'anni dallo scavo, in P. Rondini, L. Zamboni (a cura di), "Digging up excavations". Processi di ricontestualizzione di "vecchi" scavi archeologici: esperienze, problemi, prospettive (Seminario di Studi, Pavia 2015), Roma 2016, pp. 91-101.

Romagnoli 2014 = S. Romagnoli, Il santuario etrusco di Villa Cassarini a Bologna, Bologna 2014.

Rondina $2014=$ A. Rondina, Il Convento Racconta, Adria 2014.

Salzani, Vitali 2002 = L. Salzani, D. Vitali, Gli scavi archeologici nel podere Forzello a San Basilio di Ariano Polesine, in «Padusa» XXXVIII, 2002, pp. 115-138.

SANesi MAStrocindue 1982 = L. SANESI MAStrocineue, La patera ombelicata con quadrighe del Museo Archeologico di Adria: rapporti con la produzione etrusco-volterrana, in «Padusa» XVIII, 1982, pp. 74-85.

Sanesi Mastrocineue 1994 = L. Sanesi Mastrocineue, Gli Etruschi ad Adria dopo l'età arcaica, in Studi di archeologia della X Regio in ricordo di Michele Tombolani, Roma 1994, pp. 125-133.

SASSATELLI 1981-1982 = G. SASSATELLI, Graffiti alfabetici e contrassegni nel villanoviano bolognese, in «EmPrerom»9-10, 1981-1982, pp. 147-255.

SASSATELli 1984a = G. SASSATELli, Edoardo Brizio e la prima sistemazione storica dell'archeologia bolognese, in Morigi Govi, Sassatelli 1984, pp. 381-400.

SASSATELLi $1984 \mathrm{~b}=$ G. SASSATELLI, I dubbi e le intuizioni di Gherardo Ghirardini, in Morigi Govi, SAsSATELLI 1984, pp. 445-461. 
Sassatelli 1985 = G. SAssatelli, Nuovi dati sulla diffusione dell'alfabeto in Etruria Padana, in La Romagna tra VI e IV sec. a.C. nel quadro della protostoria dell'Italia centrale (Atti del Convegno, Bologna 1982), Bologna 1985, pp. 99-141.

SASSATELli 1988 = G. SASSATELli, Topografia e "sistemazione monumentale" delle necropoli felsinee, in La formazione della città preromana in Emilia-Romagna (Atti del Convegno, Bologna-Marzabotto 1985), Bologna 1988, pp. 197-259.

SASSATELLI 1991 = G. SASSATELLI, Nuovi dati epigrafici nelle iscrizioni di Marzabotto ed il ruolo delle comunità locali nella fondazione delle città, in «ArchCl» XLIII, 1991, pp. 693-715.

SASSATELLI 1993 = G. SASSATELLI, Il bucchero e le ceramiche affini come supporto per iscrizioni e graffiti in area padana, in M. Bonghi Jovino (a cura di), Produzione artigianale ed esportazione nel mondo antico: il bucchero etrusco (Atti del Colloquio Internazionale, Milano 1990), Milano 1985, pp. 195-205.

SASSATELLI 1994 = G. SASSATELli (a cura di), Iscrizioni e graffiti della città etrusca di Marzabotto, Imola 1994.

SAsSatelli 1999 = G. Sassatelli 1999, Spina e gli Etruschi padani, in L. Braccesi, S. Graciotti (a cura di), La Dalmazia e l'altra sponda. Problemi di archaiologhía adriatica (Atti del Convegno, Venezia 1996), Firenze 1999, pp. 71-107.

SAsSATELli 2004 = G. SASSATELli, Gli Etruschi di Spina e la pirateria adriatica, in «Hesperia $» 19,2004$, pp. $21-30$.

SAsSATELli 2008a = G. SASSATElli, Gli Etruschi nella Valle del Po. Riflessioni, problemi eprospettive di ricerca, in «AnnFaina $»$ XV, 2008, pp. 71-114.

SASSATELli 2008b = G. SASSATELli, Celti ed Etruschi nell'Etruria Padana e nell'Italia settentrionale, in D. Vitali, S. Verger (a cura di), Tra mondo celtico e mondo italico. La necropoli di Monte Bibele (Atti della Tavola Rotonda, Roma 1997), Bologna 2008, pp. 323-348.

SAsSATELli 2009 = G. SASSATElli, Riflessioni sulla "stele della nave" di Bologna, in S. BRUni (a cura di), Etruria e Italia preromana. Studi in onore di Giovannangelo Camporeale, II, Pisa-Roma 2009, pp. 833-840.

SAsSatelli 2011 = G. SAsSATElli, Città etrusca di Marzabotto. Una fornace per il tempio di Tina, in D.F. MARAS (a cura di), Corollari. Scritti di antichità etrusche e italiche in omaggio all'opera di Giovanni Colonna, Pisa-Roma 2011, pp. 150-158.

SAsSATELli 2013 = G. SAsSATELli, Etruschi, Veneti e Celti. Relazioni culturali e mobilità individuale, in «AnnFaina $\gg$ XX, 2013, pp. 397-427.

SASSATELLI 2017 = G. SASSATELLI, La città e il sacro in Etruria padana: riti di fondazione, culti e assetti urbanistico-istituzionali, in E. Govi (a cura di), La città etrusca e il sacro. Santuari e istituzioni politiche (Atti del Convegno, Bologna 2016), Bologna 2017, pp. 181-204.

Sassatelli, Govi 2005 = G. Sassatelli, E. Govi, Il tempio di Tina in area urbana, in G. Sassatelli, E. Govi (a cura di), Culti, forma urbana e artigianato a Marzabotto. Nuove prospettive di ricerca (Atti del Convegno di Studi, Bologna 2003), Bologna 2005, pp. 9-62.

Sassatelli, Gaucci 2010 = G. Sassatelli, A. Gaucci, Le iscrizioni e i graffiti, in E. Govi, G. Sassatelli (a cura di), Marzabotto. La Casa 1 della Regio IV-Insula 2, 2. I materiali, Bologna 2010, pp. 313-393.

SCARANi 1971 = R. SCARANI, Dati per una carta archeologica del Polesine, in «Padusa» VII, 1971, pp. 3-38.

SCARFì 1964 = B.M. SCARFì, Adria. Trivellazioni per sondaggio archeologico in quattro zone della città, in «BdA» XLIX, 1964, p. 396.

SCARFì $1968=$ B.M. SCARFì, Adria (Rovigo), in «BdA» s. V, LIII, 1968, pp. 49-50.

SCARPARI 1977 = G.B. SCARPARI, Memorie adriesi, Venezia 1977.

SCHÖne 1878 = R. SCHÖne, Le Antichità del Museo Bocchi di Adria, Roma 1878.

SolIN $1998=$ H. Solin, Analecta Epigraphica. 1970-1997, «Acta Instituti Romani Finlandiae» XXI, Roma 1998.

Solinas 1993-1994 = P. Solinas, Sulla celticità linguistica nell'Italia antica: il Leponzio. Da Biondelli e Mommsen ai nostri giorni II parte, in «AttiVenezia» CLII, IV, 1993-1994, pp. 1237-1335.

SolInas 1998 = P. Solinas, Le iscrizioni in alfabeto leponzio dalla necropoli di Casalindri (Isola Rizza, VR), in L. SAlzani (a cura di), La necropoli gallica di Casalandri a Isola Rizza (Verona), Mantova 1998, pp. 143-148.

STEFANI 1996-1997 = T. STEFANI, Organizzazione sociale e ideologia funeraria in una necropoli tardo-etrusca: il caso di via Spolverin di Adria, in «Padusa» XXXII-XXXIII, 1996-1997, pp. 159-180. 
Stockhammer 2012 = P.W. Stockhammer, Conceptualizing Cultural Hybridization in Archaeology, in P.W. Stockhammer (a cura di), Conceptualizing Cultural Hybridization. A Transdisciplinary Approach, Berlin-Heidelberg 2012, pp. 43-58.

STopponi 1985 = S. STOp poni (a cura di), Case e palazzi d'Etruria (Catalogo della mostra, Siena 1985), Milano 1985.

STOPPoni 1990 = S. STOp poni, Iscrizioni etrusche su ceramiche attiche, in «AnnFaina » IV, 1990, pp. 81-112.

STOpponi 2007 = S. STOpponi, Notizie preliminari dallo scavo di Campo della Fiera, in «AnnFaina» XIV, 2007, pp. 493-530.

Tagliamonte, Raccar 2007 = G. Tagliamonte, M. Raccar, Materiali di tipo e di ascendenza lateniana nel medio e basso Adriatico italiano, in M. Guštin, P. ETtel, M. Buora (a cura di), Piceni ed Europa (Atti del Convegno, Pirano 2006), Udine 2007, pp. 211-220.

Tamassia $1993=$ K. Tamassia, La necropoli preromana di Adria, loc. Retratto-Donà, in «Padusa» XXIX, 1993, pp. 1-90.

Terracini 1931 = B.A. Terracini, Ancora su alcune congruenze fra etrusco e italico, in «StEtr» V, 1931, pp. 317-346.

Tognon 2004 = C. Tognon, Archivio Comunale Antico di Adria. Guida, Adria 2004.

TONGLET 2018 = D. TONGLET, Le kyathos attique de Madame Teithurnai. Échanges artisanaux et interactions culturelles entre Grecs et Étrusques en Méditerranée archä̈que, 1-2, Bruxelles 2018.

Toniolo 2000 = A. Toniolo, Le anfore di Adria (IV-II secolo a.C.), Sottomarina 2000.

Torelli 2017 = M. Torelli, Il 'Marte di Ravenna', i Fasti consolari di Roma e una sodalitas perugina, in «Bullettino dell' Istituto di Diritto Romano “Vittorio Scialoja” » s. IV, VII (CXI), 2017, pp. 123-132.

UGGERI 1978 = G. UGGERI, Primo contributo all'onomastica spinetica, in Studi storico-linguistici in onore di F. Ribezzo, Mesagne 1978, pp. 331-416.

UGgeri 1998 = G. UgGerI, Spina tra Leponzi ed Etruschi. il patronimico -alu, in G. CAPECCHI et al. (a cura di), In memoria di Enrico Paribeni, Roma 1998, pp. 491-503.

Uhlich 2007 = J. Uhlich, More on the linguistic classification of Lepontic, in P.-Y. LAMBert, G.-J. Pinault (a cura di), Gaulois et Celtique continental, Genève 2007, pp. 373-411.

Untermann 1961 = J. Untermann, Die venetischen Personennamen, Wiesbaden 1961.

VAlLICELli 2002 = M.C. VAllicelli, Ceramiche di importazione a figure nere non attiche ad Adria, in «Padusa» XXXVIII, 2002, pp. 191-199.

Vallicelli 2018 = M.C. Vallicelli, Adria e i primi scavi governativi del Nuovo Regno d'Italia, in 1866. Adria e il Polesine nel $150^{\circ}$ anniversario del Veneto italiano (Atti del Convegno, Adria 2016), Adria 2018, pp. 123-138.

VALVASENSE 1758 = P. VAlVASEnSe, Memorie per servire all'istoria letteraria, 11, Venezia 1758.

VAN DER MEER 1985 = L.B. VAN DER MEER, Thematische Symmetrie in der Etruskischen Kunst, in «BABesch» 60, 1985, pp. 72-93.

VAN DeR MeER 2017 = L.B. van Der Meer, Some reflections on the inscription of the Cippus Perusinus (CP), in «StEtr» LXXX, 2017, pp. 201-212.

van Heems 2003 = G. van Heems 2003, $\langle s\rangle /\langle z\rangle$ (á Volsinies), in «StEtr $\gg$ LXIX, 2003, pp. 195-219.

van Heems 2008 = G. van Heems, Diminutifs, sobriquets et hypocoristiques étrusques, in P. Poccetti (ed.), Les prénoms de l'Italie antique (Journée d'études, Lyon 2004), Pisa-Roma 2008, pp. 69-109.

van Heems 2012 = G. van Heems, Dynamiques dialectales en périphérie: le cas d'Aléria, in «MEFRA»124, 2, 2012, pp. 447-460.

Vellani $2000=$ S. Vellani, Perle di vetro anforiformi dalla penisola italiana, in Annales du $14^{e}$ Congrès de l'Association Internationale pour l'bistoire du verre (Venezia-Milano 1998), Lochem 2000, pp. $42-45$.

VITALI 1992 = D. VitALI, Tombe e necropoli galliche di Bologna e del territorio, Bologna 1992.

VITALI 2008 = D. Vitali, La nécropole de Monte Bibele. Préliminaires pour une analyse spatiale et chronologique, in D. Vitali, S. Verger (a cura di), Tra mondo celtico e mondo italico: la necropoli di Monte Bibele (Atti della Tavola Rotonda, Roma 1997), Bologna 2008, pp. 9-52.

Vitali, Kaenel 2000 = D. Vitali, G. Kaenel, Un Helvete chez les Etrusques vers 300 av. J.-C., in Archäologie der Schweiz 23, 3, 2000, pp. 115-122.

Whatmough 1933 = J. Whatmough, The Prae-Italic Dialects of Italy, II. III, The Raetic, Lepontic, Gallic, East-Italic Messapic and Sicel Inscriptions, Cambridge 1933. 
WIEL-MARIN 2005 = F. WIEL-MARIN, La ceramica attica a figure rosse di Adria. La famiglia Bocchi e l'archeologia, Padova 2005.

WYLIN $2000=\mathrm{K}$. WYLIN, Il verbo etrusco. Ricerca morfosintattica delle forme usate in funzione verbale, Roma 2000.

WYLIN $2004=\mathrm{K}$. WyLIN, Un terzo pronome/aggettivo dimostrativo etrusco sa, in «StEtr» LXX, 2004, pp. 213-225.

ZAMBONI 2012 = L. ZAMBONI, Spina. Gli scavi in abitato 1977-1981 e i materiali di età arcaica e classica, I-II, Tesi di Dottorato (XXV Ciclo), Università di Pavia.

ZAMBONI 2016 = L. ZAMBONI, Spina cittá liquida. Gli scavi 1977-1981 nell'abitato e i materiali tardo-arcaici e classici, Rahden 2016.

ZANNONi 1876-1884 = A. ZANnONi, Gli scavi della Certosa, Bologna 1876-1884.

Zerbinati 1993 = E. Zerbinati, Topografia antica, archeologia ed epigrafia latina in Girolamo Silvestri, in Girolamo Silvestri 1728-1788. Cultura e società a Rovigo nel secolo dei lumi (Atti del Convegno, Rovigo 1988), Rovigo 1993, pp. 25-57.

Zerbinati 1994 = E. Zerbinati, Collezionismo archeologico e cultura antiquaria in Polesine tra Cinquecento e Ottocento, in «Archivio Veneto» CXLIII, 1994, pp. 227-249.

Zerbinati 2003 = E. Zerbinati, I tre Silvestri, in Camillo Carlo e Girolamo Silvestri, in Successi delle acque dall'anno 1677 al 1755, Rovigo 2003, pp. XXI-XLV. 


\section{INDICE DEI TITULI DI CIE IV, I, 1}

20001: pp. 38, 48, 62, 137, 143, 144

20002: pp. 34, 137, 144

20003, 20004, 20005: p. 138

20006: pp. 16, 17, 35, 38, 57, 63, 67, 86, 109, 138, 143

20007: pp. 35, 138, 144

20008: pp. 26, 31, 47, 59, 85, 138, 142-143

20009: pp. 138,144

20010: pp. 138,143

20011: pp. 138, 144

20012: pp. $35,38,40,48,59,67,86-87,143$

20013: p. 143

20014, 20015, 20016, 20017, 20018: p. 144

20019: pp. 35, 37-38, 47, 67, 100, 109, 142, 144

20020: pp. 16, 17, 35, 38, 48, 63-64, 67, 73, 86, 112, $115,118,143,144$

20021: p. 144

20022, 20023, 20024, 20025, 20026, 20027: p. 145

20028: pp. 17, 35, 38, 53, 64, 67, 100, 130, 132, 142, $144-145,204,214$

20029: pp. 35, 38, 51, 142, 144

20030: pp. 17, 35, 38, 48, 50-51, 59, 61, 63, 66, 87, 91-92, 110, 115, 142, 144-145, 212

20031: p. 145

20032: pp. 113, 143, 144

20033, 20034: p. 145

20035: pp. 32, 145

20036: pp. 114, 145

20037: pp. 26, 29, 45, 47, 51, 54-55, 62, 66-67, 86,

$107,120-121,142,145,211$
20040: pp. 39, 80, 82, 142, 145

20041: pp. 34, 38, 41, 47, 68, 125, 143, 145

20043: pp. 34, 40, 85, 142, 145, 211

20044: pp. 34, 38-39, 48, 76, 143, 145

20051: pp. 16, 34, 38-39, 48, 66-67, 102, 110, 115,

143,145

20055: p. 40

20079: pp. 34, 38, 53, 58, 87, 143, 145

20083: p. 114

20084: pp. 19, 34, 38, 53, 66, 102, 110, 115, 143, 145,

212

20088: pp. 29, 146

20089: pp. 33, 39, 40, 47, 55, 74, 142, 146

20090: pp. 33, 146

20091: pp. 32, 146

20092: pp. 33, 60, 146

20093: p. 146

20094: pp. 33, 146

20095: p. 146

20100: pp. 143, 145

20105: p. 180

20107: pp. 35, 38, 53, 57, 132, 142, 145

20109: p. 32

20110, 20111: pp. 143, 145

20114: p. 40

20116: pp. 34, 38-39, 61, 67, 76, 142, 145

20120: pp. 34, 38-39, 48, 54-55, 67, 87, 109, 143, 145

20121: pp. 34, 38, 40, 48, 51, 76, 113, 143, 145, 216

20124: pp. 143,145

20125: pp. 29, 123-124, 142, 145 
20126: pp. 29, 142,145

20127: pp. $34,53,85,142,145$

20133: pp. 34, 38, 48, 55-58, 66, 102, 106-107, 109110, 142,145

20134: pp. 32, 141, 148

20135: pp. 142,145

20137: pp. 39, 40,75, 145

20139: pp. 143,145

20143: pp. 31-32, 39, 47, 62-63, 73, 142, 146

20145: p. 34

20146: p. 33

20147: pp. 33, 146

20148: pp. 32, 146

20149, 20150, 20151: p. 146

20152: pp. 32, 146

20153: p. 146

20154: pp. 29, 146

20155: p. 146

20156: pp. 32, 146

20157: pp. 33, 60, 146

20158: pp. 33, 146

20159: pp. 29-30, 39, 43, 47, 49, 56, 58-59, 82-83, 142,146

20160: pp. 29, 39, 47, 49, 126, 141-142, 146

20161: pp. 32, 147

20162: p. 146

20163: p. 34

20164, 20165: pp. 32, 146

20166: pp. 34, 146

20167: pp. 34,146

20168: p. 146

20169: p. 147

20170: pp. 34, 147

20171: p. 147

20172: pp. 33, 147

20173: pp. 33, 60, 147

20174: pp. 32, 147

20176, 20177, 20178, 20179: p. 147

20180: pp. 18, 30, 39, 43, 50-55, 59, 64, 67, 71, 80, $82,142,148,149,209$

20181: pp. $34,38,47,50,53,62,64,66-67,100,110-$ $111,115,119,142,148$

20182: pp. $35,38,52-53,55,59,62-63,86,115,143$, 148

20183: pp. 35, 38, 51, 59, 66, 67, 143, 148-149

20184: pp. $26,31,45,57,58,59,62,85,103,142$, $148-149,211$

20185: pp. 31, 149

20186: pp. 32, 149

20187: p. 149

20188: pp. 35, 38, 51, 53, 55, 59, 64, 66-67, 87-89, 91, $109,115,143-144,212$
20189: pp. $33,39,43,47,49-51,53,55,123-124$, 142,144

20190: pp. 34, 38, 53, 55, 62, 67, 103, 107, 143, 144, $190,213,216$

20191: pp. 35, 38, 65-66, 143-144

20192: pp. 113,144

20193: p. 144

20194: pp. 33, 39-40, 55, 60-61, 74, 142, 144

20195: pp. 31, 144

20196: pp. 19, 35, 38, 47, 50, 66-67, 130, 133, 142, 144

20197: pp. 35, 38, 53, 57, 132, 139

20198: pp. 10, 139

20199, 20200: p. 139

20201: pp. 34, 38, 53, 58, 60-61, 63, 66, 86, 109, 139

20202, 20203: p. 139

20204: pp. 30, 39, 58, 80, 139

20205, 20206: pp. 32, 139

20207, 20208: p. 139

20209: pp. 30, 139

20210, 20211: pp. 33, 139

20212: pp. 29, 30, 39, 47, 58, 80, 126-127, 149, 210

20213: pp. 31, 149

20214: pp. 17, 30, 39, 44, 47, 49, 51-55, 58-59, 63-64, $67,71,74,80,82-84,149,210$

20215: pp. 33, 39-40, 75, 149

20216, 20217: p. 149

20218: pp. 32, 149

20219: p. 149

20220: pp. 29, 149

20221, 20222, 20223: p. 149

20224: pp. 30, 149

20225: pp. 32, 149

20226: pp. 29, 149

20227: p. 149

20228: pp. 34, 149

20229: p. 149

20230: pp. 32, 149

20233, 20234, 20235: p. 150

20236: pp. 17, 34, 38, 51, 53, 61, 63, 67, 102, 110,

$115,118,150$

20237: p. 150

20238, 20239, 20240, 20241: p. 140

20242: pp. $32,40,140$

20243: p. 140

20244: pp. 140, 192

20245, 20246, 20247, 20248, 20249: p. 140

20250, 20251: pp. 32, 140

20252: pp. 33,140

20253: pp. 35, 38, 48, 59, 66, 86, 151, 214

20254, 20255: p. 151

20256: pp. 26, 31, 47, 63, 151

20257: pp. $35,38,48,51,61,113,151-152$ 
20258: pp. 34, 38, 66, 98, 151

20259: pp. 34, 38-39, 47, 59, 60, 147, 151

20260: pp. 109, 151

20261, 20262: p. 151

20263: pp. 34, 38-39, 41, 48, 51, 113, 151-152

20264: pp. 34, 38, 47, 59, 62-63, 67, 87-88, 90-91, 96, $110,115,151,212,214$

20265: pp. 32, 39-40, 46-47, 126, 151

20266, 20267: p. 151

20268: pp. 29, 151

20269: pp. 31, 151

20270: pp. 30, 39-40, 75-76, 151-152

20271: p. 151

20272: pp. 32, 151-152

20273: pp. 19, 35, 38, 56, 65-67, 130, 133, 151-152

20274: p. 151

20275: pp. 35, 151

20276: p. 151

20277: pp. 34, 38, 67, 141

20278: p. 141

20279: pp. 35, 141

20280, 20281: p. 141

20282: pp. $31,39,44,47,51-52,71,80,83-85,98$, 140, 152-153, 209

20283: pp. 31, 152

20284: p. 152

20285, 20286: pp. 31, 152

20287: pp. 31, 39, 51, 62-63, 73-74, 152-153, 209

20288: pp. 31, 152-153

20289: pp. 20, 31-32, 39, 43, 47, 49, 59, 62, 64, 67,

$80,140,152-153,209$

20290: pp. 31, 39, 44, 47, 51, 71, 80, 83-85, 98, 140, 152-153

20291, 20292: pp. 31, 152

20293: pp. 31, 152-153

20294: pp. 31, 152

20295: pp. 31, 39, 44, 47, 51, 71, 80, 83-85, 98, 140, 152-153

20296: pp. 31, 152

20297: p. 152

20298, 20299: pp. 31, 152

20300: pp. 31, 152-153

20301, 20302: pp. 31, 152

20303: pp. 31, 152-153

20304: pp. 31, 152

20305: pp. 31, 39, 51, 62-63, 73-74, 152-153, 209

20306: pp. 31, 152-153

20307: pp. 31, 152

20308: pp. 152,154

20309: pp. 31, 152

20310, 20311: pp. 31, 152-153

20312, 20313, 20314: p. 152

20315: pp. 35, 38, 47, 62, 66-67, 86, 115, 152
20316: pp. $35,38,48,55,152$

20317: p. 152

20318: pp. 35, 152

20319: p. 152

20320: pp. 35, 38, 56, 67-68, 102-103, 109, 115, 152, 212

20321, 20322: p. 152

20323: pp. $35,38,55,63,67,107-108,110,152,154$, 213

20324, 20325: p. 152

20326: pp. 114, 152

20327: p. 152

20328: pp. 35, 38, 55, 57, 76-77, 152, 154

20329: pp. 113,152

20330: pp. 35, 38, 47, 51, 63, 67, 96, 100-101, 110,

$115,118,152,154,213$

20331: pp. 33, 152

20332, 20333: pp. 35, 152

20334: p. 152

20335: pp. 10,35, 152, 154

20336: pp. 35, 152

20337, 20338: p. 152

20339: pp. 10,152

20340, 20341, 20342, 20343: p. 152

20344: pp. 30, 32, 152-153

20345: pp. 32,152-153

20346: p. 152

20347: pp. 31, 147

20348, 20349: p. 147

20350: pp. 33, 147

20351: pp. 32, 147

20352: pp. 34, 147

20353: pp. 32, 39, 46-47, 55, 58, 80, 142, 147

20354: pp. 32, 147

20355: pp. 33, 147

20356: pp. 30, 39, 46-47, 59, 73-74, 142, 147

20357: pp. 34, 147-148

20358: pp. 33, 147

20359: pp. 32, 147

20360: pp. $147-148$

20361: pp. 32, 39, 47, 62-64, 80, 82, 142, 147, 192

20362: pp. 32-33, 141, 147

20363: pp. 33,147

20364: pp. 34, 38, 40, 47, 59, 62, 66, 76, 98, 110-111, $142,147-148$

20365: p. 147

20366: pp. $147-148$

20367: p. 147

20368: pp. 33-34, 39, 43, 47, 49, 53, 58-59, 62, 65-67, 80-83, 141, 209

20369: pp. 34,141

20370: pp. 30, 39, 46-47, 49, 59, 63, 65-67, 127-128, 141 
20371: pp. 30, 39, 40, 43, 46-47, 53, 55, 123-124, 141 20372: pp. 34, 74, 141

20373, 20374: p. 141

20375: pp. 35, 38, 48, 51, 67, 105, 110, 185, 191, 193, 195, 213,215

20376: pp. X, 35, 37, 61-62, 77, 184, 191, 193, 195, 205, 215

20377: pp. 35, 38, 41, 48, 50, 68, 125, 191, 193, 195

20378, 20379, 20380: pp. 191, 193

20381, 20382: p. 192

20383: p. 192

20384, 20385, 20386, 20387: pp. 35, 38, 47, 51, 53,

$55,58-59,62-63,66-67,104-105,110,112,193-$ 194, 213, 215

20388: pp. 32, 192

20389: p. 193

20390: pp. 35, 38, 48, 51, 76, 113, 193, 195, 216

20391: pp. 30, 191

20392: p. 193

20393: p. 193

20394, 20395: pp. 114, 193-194

20396, 20397, 20398, 20399, 20400, 20401, 20402: p. 193

20403: p. 192

20404: pp. 35, 38, 58, 67, 192

20405: pp. 10, 36-37, 62, 191

20406: p. 192

20407: pp. 35, 38, 48, 51, 64, 67, 103-104, 110, 115, $118,193,195,212-213,215$

20408: pp. 36-37, 51, 53, 58, 59, 129, 193, 195, 216

20409: pp. 29, 193

20410: p. 190

20411: pp. 35, 38, 48, 56, 99, 110, 190

20412: p. 190

20413, 20414, 20415, 20416, 20417, 20418: p. 190

20419: pp. 35, 38, 48, 58, 64, 66-67, 87, 109, 190

20420: pp. $35,38,53,55,58,61,67,103,107,109$ $110,190,213,216$

20421: p. 190

20422: pp. 35, 38, 48, 64, 67, 91, 103, 110, 115, 118 , $190,213,215$

20423, 20424: p. 190

20425: p. 157

20426: pp. $35,38,41,48,68,125,157$

20427: p. 157

20428: p. 162

20429: p. 155

20430, 20431: p. 157

20432, 20433: p. 158

20434: pp. $35,38,48,58-59,63,66-68,87,93,96-97$, $110,115,159,189,213,215$

20435: p. 159
20436: pp. 35, 38, 56-57, 73, 76, 130, 132, 158, 181, 189,216

20437: p. 158

20438: pp. 158,184

20439: p. 158

20440, 20441: p. 162

20442, 20443: p. 161

20444: pp. 35, 38, 53, 58, 76, 159

20445: p. 159

20446: p. 162

20447, 20448: p. 159

20449: p. 162

20450, 20451, 20452, 20453, 20454: p. 158

20455, 20456: p. 159

20457: pp. 34, 38, 47, 53, 58, 62, 66, 108, 110, 161, 203, 213, 215

20458: pp. $35,38,61,67,105,110,158,186,189$, 213,215

20459: pp. 36, 158

20460: p. 159

20461, 20462: pp. 19, 35, 37-38, 48, 53, 58-59, 66, $96,103,110-111,115,159,189,213,216$

20463: pp. 35, 37-38, 58, 96, 103, 110, 159

20464: pp. $34,38,48,52-55,57-58,62,64,67,106-$ $107,109-110,130-132,161,175,177,213,215$

20465: pp. 36, 161

20466: p. 161

20467: p. 156

20468: p. 161

20469: p. 157

20470: pp. 36, 162

20471: p. 159

20472, 20473: pp. 35, 38, 66, 103-104, 110, 115, 162, $186,212-213,215$

20474: pp. 35, 38, 48, 59, 66, 96, 103-104, 115, 126, $160,186,213$

20475: pp. 35, 38, 48, 59, 66, 96, 103-104, 115, 160, 186,213

20476: p. 156

20477: pp. 35, 38, 47, 53, 58, 62-67, 87-88, 90-91, 97, $110,115,119,156,176-177,212,214-215$

20478, 20479, 20480: p. 156

20481: p. 161

20482: p. 159

20483: p. 161

20484, 20485, 20486: p. 162

20487: pp. $35,38,45,64,66-67,130,132-133,156$, 178,216

20488: p. 156

20489: pp. $35,38,57,59,67,101,110,115,118,157$, 181,215

20490: pp. 29, 157

20491, 20492, 20493: p. 157 
20494: p. 161

20495: p. 159

20496: p. 162

20497: p. 160

20498: pp. 114,158

20499: p. 161

20500: pp. 10, 160

20501, 20502: p. 161

20503, 20504: p. 158

20505: pp. 29, 161

20506: p. 157

20507: p. 161

20508: p. 160

20509: p. 161

20510: pp. 36, 157

20511: pp. 35, 38, 53-54, 58, 62, 67, 100, 156, 176

20512, 20513: p. 156

20514: pp. $35,38,48,53,56,61,63,66-67,86,89-90$,

$100-101,108,112-113,117,161,213,215-216$

20515, 20516: p. 157

20517: p. 162

20518: p. 161

20519: pp. 35, 38, 47, 50, 53, 55-57, 63, 65-67, 87-89, $110,115,118,157,177$

20520: pp. 29, 156

20521: p. 157

20522: pp. 114,160

20523: pp. 35, 38, 47, 67, 113, 127-128, 156, 178, 216

20524, 20525: p. 156

20526: p. 157

20527, 20528, 20529: p. 156

20530, 20531: p. 158

20532: p. 161

20533: p. 160

20534: pp. 35, 37, 61, 64, 67, 77, 101, 110, 115, 118 , $159,184,186$

20535, 20536, 20537: p. 159

20538: p. 157

20539: p. 159

20540: pp. 36, 162

20541: pp. $35,38,57,59,67,101,110,115,118,157$, $159,189,215$

20542: p. 159

20543, 20544: p. 156

20545, 20546: p. 162

20547: pp. 35, 38, 55-56, 61, 67, 105, 110, 161, 179

20548: p. 162

20549: p. 158

20550: pp. 19, 158

20551, 20552: p. 156

20553: pp. 35, 37-38, 64, 76, 162

20554: p. 161

20555: p. 160
20556: p. 162

20557: p. 158

20558: p. 161

20559: pp. $19,35,38,62,67,101,110,115,118,160$, 213

20560: pp. 19, 35, 37-38, 48, 58, 62, 66-67, 87, 93, 96,

$110,115,118,160,189,213,216$

20561: p. 160

20562: p. 161

20563: pp. 35, 37-38, 47, 50-51, 62, 67, 104-105, 110, $157,178,213,215$

20564: pp. 35, 38, 47, 63-64, 65-67, 87-88, 110, 115, $118,157,178$

20565: p. 157

20566: pp. 35, 38, 48, 51, 59, 64-65, 67, 86, 112-115, $161,189,216$

20567: p. 160

20568: pp. 36-37, 47, 56, 63, 67, 87, 91-93, 103, 110 , $115,118,162,190,212$

20569, 20570: pp. 35, 37-38, 47, 53, 59, 62, 66, 87-

88, 90-91, 110-111, 115, 156, 177

20571: pp. 35, 37-38, 47, 59, 87, 90, 156

20572, 20573: p. 160

20574: pp. 33, 161

20575: p. 162

20576: p. 161

20577: pp. 10, 36-37, 160, 174, 204

20578: pp. 10, 160, 183

20579: p. 161

20580: pp. 10, 161, 183

20581: pp. 10, 52, 161, 184

20582: pp. 161, 183

20583: p. 160

20584: p. 161

20585: pp. 36,160

20586: p. 160

20587: p. 157

20588: p. 161

20589: p. 156

20590: pp. 10, 114, 161, 180, 215

20591: pp. 35, 38, 55, 63, 66-68, 130, 161, 180, 215

20592, 20593: p. 161

20594: pp. $35,38,47,53,55,57,61,108,110,157$, $178,213,215$

20595: pp. 34, 38, 47, 51, 53, 57, 59, 62, 64, 105-106, 109-110, 130-132, 155, 175, 213-215

20596: p. 155

20597: pp. 35, 38, 58, 109, 160

20598, 20599: p. 156

20600: pp. 35, 37, 48, 53-54, 63, 66, 86, 109, 159, 186

20601: pp. 34, 38, 53, 57, 64, 130-132, 162, 175, 214

20602: p. 161 
20603: pp. $35,38,47,51,52,55,58,62,66,98,110$, $136,161,215$

20604: p. 160

20605: p. 161

20606: pp. $35,38,56-57,73,76,130,132,159,188$, 216

20607: pp. 35, 38-39, 59, 76, 159

20608: p. 159

20609: p. 160

20610: pp. 35, 38, 48, 103-104, 110, 115, 160, 179, $184,186,213,215$

20611, 20612: p. 160

20613: pp. 36, 160

20614: pp. 19, 35, 38-39, 47, 58, 63-64, 67, 87-88, 90, $110,115,117-118,155,177-178,215$

20615: p. 155

20616, 20617: p. 162

20618, 20619, 20620: p. 160

20621: pp. $35,38,48,56,58,61,63,67,77,87,93$, 96-97, 110, 115, 159, 186, 189, 213, 215

20622: pp. 35, 38-39, 61, 67, 77, 159, 186

20623, 20624, 20625: p. 159

20626: p. 160

20627: pp. 35, 38, 47, 51, 53, 58, 63, 86, 109, 161

20628: pp. 35, 37, 48, 50, 66, 98, 112, 114, 126, 158

20629, 20630, 20631, 20632, 20633: p. 157

20634: p. 160

20635: pp. 113,162

20636: p. 160

20637: pp. 162,180

20638: pp. $162,180,184$

20639: p. 162

20640: pp. 35, 38, 48, 58, 62, 66, 109, 111-112, 160

20641, 20642, 20643, 20644, 20645, 20646, 20647 , 20648, 20649, 20650, 20651, 20652: p. 158

20653: p. 155

20654: pp. 35, 38, 53, 55, 63, 108, 115, 159, 184, 186, 212

20655: p. 159

20656, 20657: pp. 114, 161

20658, 20659: p. 161

20660: pp. $35,38,47,51,62,66-67,104-105,110$, $160,177-178,213,215$

20661: p. 162

20662: pp. $35,38,53,55-56,58-59,62-63,66-67,87-$ $90,110,115,117-118,162,213,215$

20663: pp. 35, 38, 47, 58, 63, 67, 99, 115, 162

20664: pp. $35,38,48,58,108,162$

20665, 20666, 20667: pp. 50, 168, 177

20668: pp. 168,177

20669: p. 180

20670, 20671, 20672, 20673, 20674, 20675, 20676: p. 167
20677, 20678, 20679: pp. 35, 37-38, 48, 51-52, 59,

$63,67,102,110-111,115,118,168,181$

20680: pp. $18,35,38,48,53-54,57,63,65-67,97$,

$110,115,118,168,181,215$

20681, 20682: pp. 168, 181

20683: pp. 17, 35, 51, 67, 76, 167, 216

20684: p. 167

20685: pp. $35,38,47,56,63,66-67,89-90,100,108$,

$110,115,119,164-165,213$

20686, 20687: pp. 164-165

20688: pp. 113,165

20689, 20690, 20691: p. 165

20692, 20693: p. 170

20694: pp. 35, 38, 53, 57-58, 73, 132, 170

20695, 20696: p. 170

20697: pp. 35, 38-39, 48, 50-52, 63, 67, 73, 87, 93,

95-96, 100, 110, 115, $118170,189,205,213,216$

20698: pp. $35,38,40,48,58-59,61,67,103,110$,

$115,118,170,188-189,213,216$

20699: pp. $35,38,40,48,53,66,105,110,170,188$ -

$189,204,215-216$

20700, 20701, 20702, 20703: p. 170

20704: pp. 36-37, 62, 67, 76, 170

20705, 20706, 20707: p. 168

20708: p. 170

20709: pp. 35, 38-39, 48, 58, 63, 67, 101, 110, 115, $169,188,215-216$

20710, 20711, 20712, 20713, 20714, 20715, 20716,

20717, 20718, 20719, 20720, 20721, 20722,

20723: p. 169

20724, 20725: p. 168

20726: p. 170

20727: p. 168

20728: p. 169

20729: p. 168

20730, 20731, 20732, 20733: p. 169

20734: p. 170

20735: p. 171

20736, 20737: pp. 17, 35, 37, 41, 48, 59, 60-61, 63, $87,96-97,110,111,190$

20738: p. 171

20739, 20740, 20741, 20742, 20743, 20744, 20745, 20746, 20747: p. 172

20748: pp. 29, 40, 172

20749: pp. X, 35, 38, 61-62, 77, 172, 189, 205, 215

20750: p. 173

20751, 20752, 20753, 20754, 20755, 20756: p. 172

20757: p. 173

20758, 20759: p. 172

20760, 20761: p. 173

20762: pp. $35,38,48,51,61,63,67,105,110,172-$

$174,188,213,215$

20763, 20764: p. 172 
20765: pp. 10, 172, 183

20766: p. 172

20767, 20768, 20769: p. 173

20770, 20771: p. 172

20772: pp. 35, 38, 47, 51, 55, 58, 62, 66, 98, 110, 135136,215

20773: pp. 31, 135

20774: p. 135

20775: pp. 35, 38, 40, 47, 53, 123, 135, 204

20776: pp. 16, 35, 38, 47, 50, 64, 66-68, 100-101, $110,115,118,135,177,190$

20777: pp. 35, 38, 48, 51, 56, 98, 110, 135, 215

20778, 20779, 20780, 20781: p. 135

20782: pp. 31, 135

20783, 20784: p. 135

20785: pp. $35-36,38,51,56,63,65-67,86,89,112-$

$115,135,216$

20786, 20787: p. 135

20788: pp. 35, 38, 57, 64, 67, 100, 130, 135

20789, 20790, 20791, 20792, 20793: p. 135

20794: pp. 35-36, 38, 51, 59, 130, 135

20795, 20796, 20797, 20798, 20799: p. 135

20800: pp. 114, 135

20801, 20802, 20803: p. 135

20804: pp. 135, 184

20805, 20806, 20807, 20808, 20809, 20810: p. 135

20811: pp. 40, 135

20812: p. 135

20813: pp. 10, 135

20814: p. 135

20815: pp. 135, 180-181

20816: p. 135

20817: pp. 35-36, 38, 48, 55, 59, 99, 112, 114, 126, 135,150

20818: pp. 35, 135

20819: pp. $35,38,47,53,56,59-63,99-100,110$, $112,115,135,176$

20820: pp. 35, 38-39, 48, 59, 108, 110, 135, 213, 215

20821: pp. 35, 38-39, 48, 55, 62-63, 67-68, 91, 97, $110,115,119,135$

20822: pp. 18, 30, 39, 44, 47, 49, 53, 58-59, 63-64, 67, $71,80-82,87,135,209$

20823: pp. $26,31,58,135$

20824: pp. 35, 58, 135

20825: pp. $35,38-39,135$

20826: pp. 35, 38, 48, 53, 57, 62, 87, 96, 135

20827: pp. $34,38,48,61-63,73,99,135$

20828: pp. 35, 36, 38, 64-65, 67, 101-102, 110, 115, 135,212

20829: pp. $35,38,48,64,67,91,103,110,115,118$, $135,190,213,215$

20830: pp. 34, 38, 61, 66-67, 135

20831: pp. 10, 135
20832: pp. $26,30,58,67,135$

20833: pp. 35, 38, 53, 63, 76, 135

20834: pp. 30, 39-40, 53, 60-61, 74-75, 113, 135

20835: pp. 135, 179, 204

20836, 20837, 20838, 20839: p. 135

20840: pp. 32, 135

20841: pp. 114, 135

20842: pp. 31, 135

20843: pp. 114, 135

20844: p. 135

20845: pp. 33, 60, 135

20846, 20847, 20848, 20849, 20850, 20851, 20852, 20853, 20854, 20855, 20856, 20857, 20858, 20859, 20860, 20861, 20862, 20863, 20864, 20865, 20866, 20867: p. 135

20868: pp. 31, 135

20869, 20870: p. 135

20871: pp. 29, 135

20872: pp. 33, 135

20873, 20874, 20875, 20876, 20877, 20878, 20879: p. 135

20880: pp. 32, 135

20881: p. 135

20882: pp. 33, 135

20883: pp. 32, 135

20884, 20885, 20886: p. 198

20887, 20888: p. 197

20889: pp. 113, 197

20890, 20891, 20892, 20893, 20894: p. 198

20895: pp. 34, 38, 47-48, 58-59, 90, 99, 110, 115, 198, 203, 214

20896, 20897: p. 198

20898: pp. 36, 198

20899: pp. 26, 34, 47, 51, 58, 62-63, 67, 85, 114, 194, $197,202,211$

20900: pp. 114, 197

20901: p. 197

20902, 20903: pp. 180, 197

20904: pp. $35,38-39,47,53,55-56,58-60,87-88$, 90-91, 99, 110, 115, 198, 203

20905, 20906, 20907, 20908: p. 198

20909: p. 202

20910: p. 200

20911: pp. 10, 41, 94, 201, 205

20912: pp. 40, 201

20913, 20914: p. 201

20915: pp. 10, 55, 95, 199

20916, 20917: p. 199

20918, 20919: p. 201

20920: pp. 36, 201

20921: pp. $35,38,48,51-52,58,64-65,67,105,110$, 199, 204, 213

20922: p. 199 
20923, 20924: pp. 35, 38, 40, 48, 55-56, 58, 67, 87, 93-94, 105, 110, 114, 118, 200, 205, 213, 216 20925: pp. 35, 38, 40, 48, 51, 59, 86-87, 109, 200, 205 20926: pp. 35, 38, 40, 53, 57, 63, 67, 105, 110, 200, 205, 213

20927: p. 200

20928, 20929: pp. 114, 200

20930: p. 200

20931: p. 197

20932: pp. $35,38,40,48,53,66,105,110,200,204$, 213, $215-216$

20933: pp. 35, 38, 40, 48, 53, 60-61, 63, 127-128, 200, 205, 214

20934: pp. X, 35, 37, 40, 61, 62, 77, 184, 200, 205, 215

20935: pp. 114, 200

20936: pp. 36, 200

20937: p. 200

20938: pp. 180, 200

20939, 20940, 20941, 20942: p. 200

20943, 20944, 20945, 20946, 20947, 20948: p. 201

20949: p. 202

20950: pp. 10, 40, 65, 95, 199, 204

20951: pp. 36-37, 40, 48, 76, 114, 199

20952: pp. 10, 36, 199

20953, 20954: p. 199

20955: pp. 36, 201

20956, 20957: p. 198

20958, 20959: p. 199

20960: pp. 35, 37, 53, 64, 76, 184, 199

20961, 20962, 20963, 20964, 20965, 20966: p. 199

20967: pp 180, 199

20968, 20969: p. 201

20970: pp. 36, 201

20971: p. 201

20972: pp. 29, 201

20973, 20974, 20975, 20976, 20977, 20978, 20979, 20980: p. 201

20981: p. 202

20982, 20983: p. 198

20984, 20985: p. 199

20986, 20987, 20988, 20989: p. 202

20990, 20991: p. 202

20992: pp. $35,38,48,56,59,63,66-67,87,93,96$, $110,115,119,199,204,215$

20993: pp. 35, 38-39, 48, 53, 123, 126, 199, 204
20994: pp. 114, 199

20995: p. 199

20996: p. 200

20997: pp. 35, 38, 48, 50, 59, 66, 101, 110, 115, 201, 206

20998: p. 201

20999: p. 202

21000: pp. 36,198

21001: p. 198

21002: pp. 35, 37-39, 48, 58, 64, 67, 93, 96, 101, 110,

115, 129-130, 200, 205, 215

21003: pp. 10, 40, 95, 113, 200, 205

21004: p. 200

21005: p. 202

21006: p. 198

21007, 21008, 21009: p. 199

21010: pp. 36, 77,199

21011: pp. 36, 199

21012: p. 201

21013: pp. 36-37, 41, 48, 58, 61, 67, 87, 93-94, 110 , $118,201,205,213,216$

21014: pp. 10, 36, 41, 54, 94-95, 201, 205

21015: p. 202

21016: pp. 113, 202

21017, 21018, 21019: p. 202

21020: p. 196

21021: pp. 10, 16, 196

21022, 21023, 21024, 21025, 21026: p. 196

21027: pp. 14, 16, 21, 35, 37-38, 47-48, 51, 53, 57-59,

$62-63,66-67,76,85,103,109,112-113,127-128$,

$195,212,214,216$

21028, 21029, 21030: p. 195

21031: pp. 10-11, 20, 73, 132-133

21032: pp. 11, 49

21033: p. 11

21034: pp. $11,32,148$

21035, 21036, 21037, 21038, 21039: p. 11

21040: pp. 11, 53-54, 120, 209

21041: pp. 11, 46, 57, 60, 65, 209

21042, 21043, 21044, 21045, 21046, 21047, 21048, 21049, 21050, 21051, 21052, 21053, 21054, 21055, 21056, 21057, 21058, 21059: p. 11

21060: pp. 11, 53-54

21061, 21062, 21063, 21064: p. 11

21065: pp. 11, 46, 49

21066, 21067, 21068, 21069, 21070, 21071: p. 11 
Tavole 


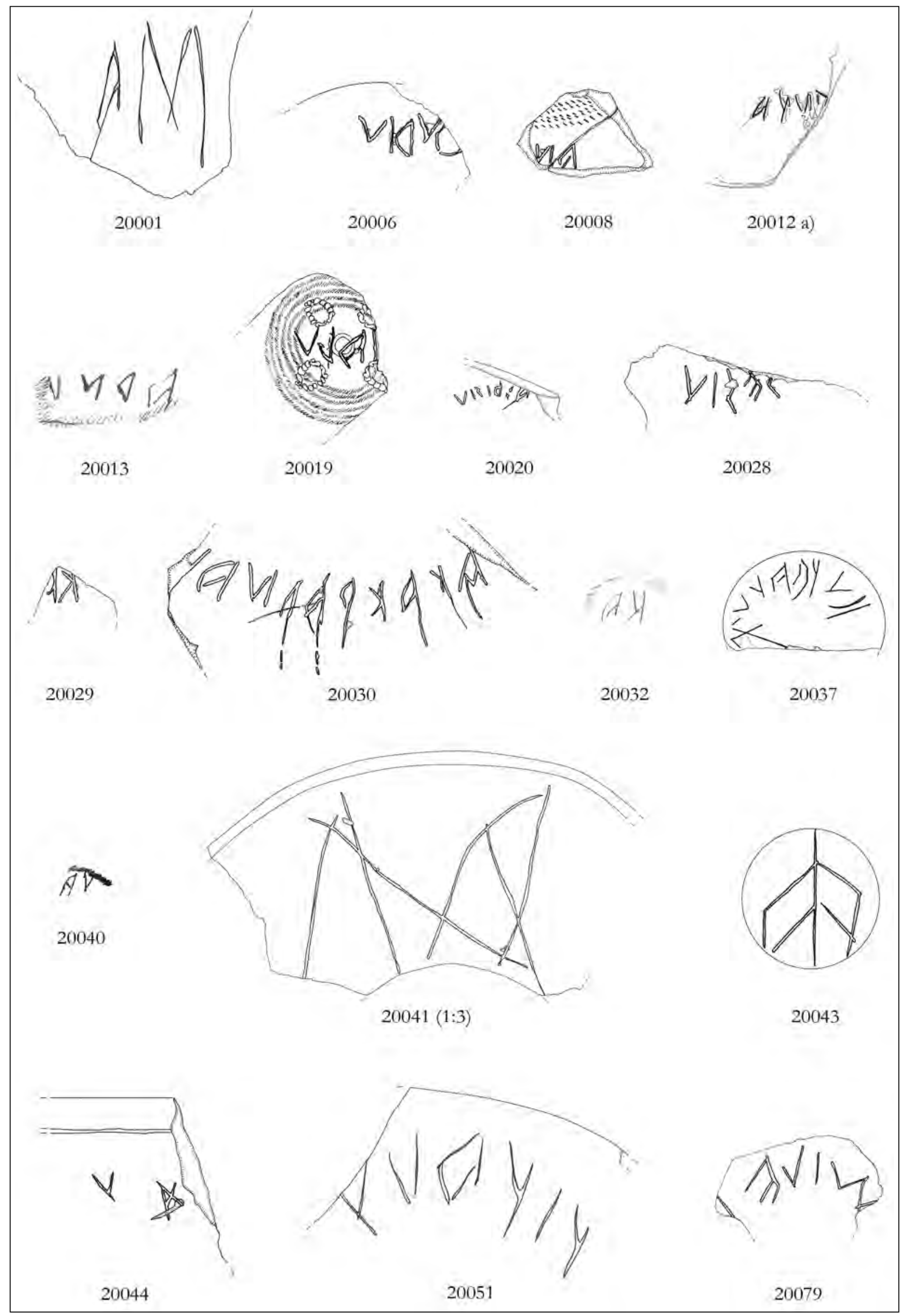

Tav. 1. Apografi delle iscrizioni etrusche, da CIE IV, I, 1 (1:2, dove non diversamente specificato). 


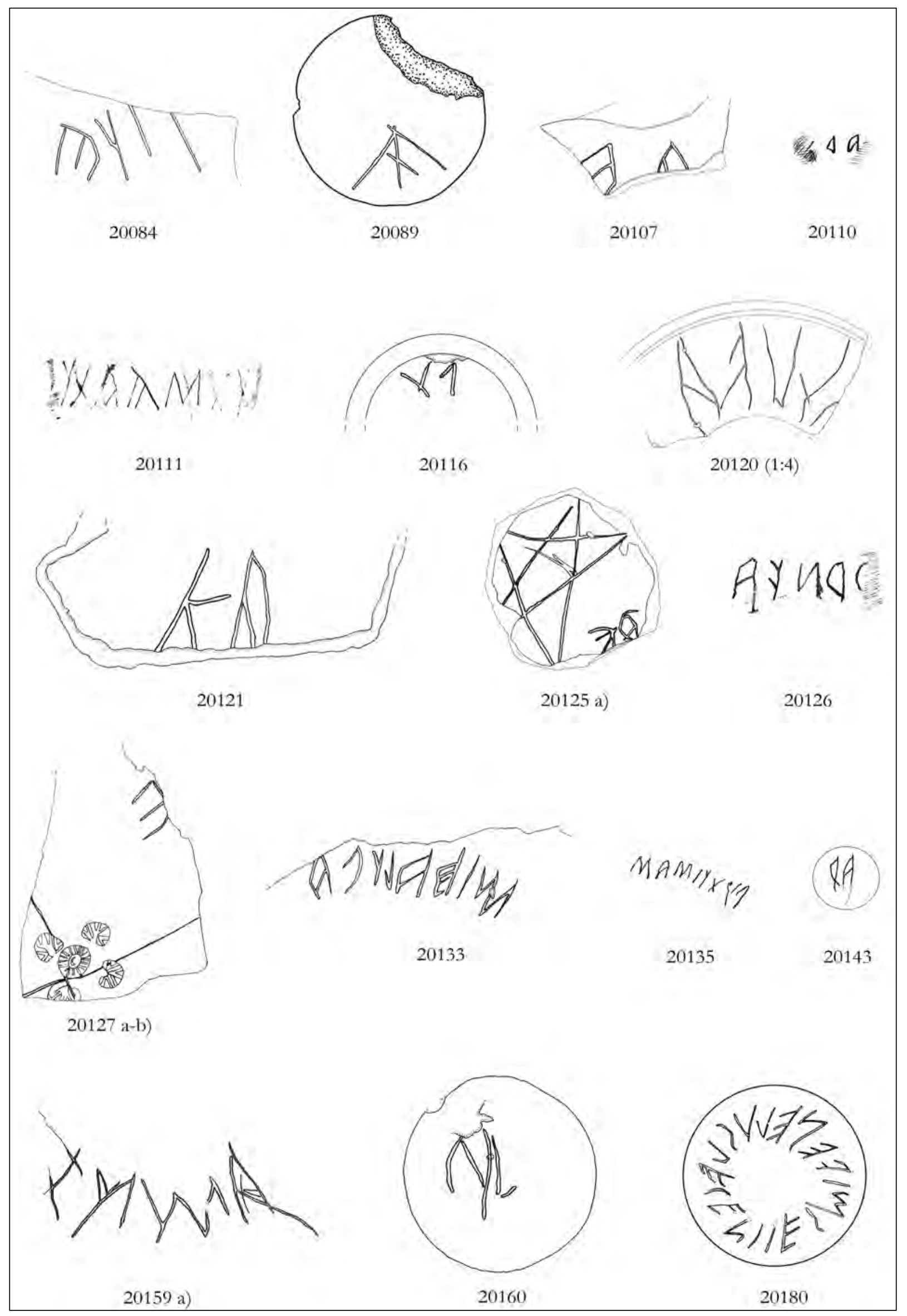

Tav. 2. Apografi delle iscrizioni etrusche, da CIE IV, I, 1 (1:2, dove non diversamente specificato). 


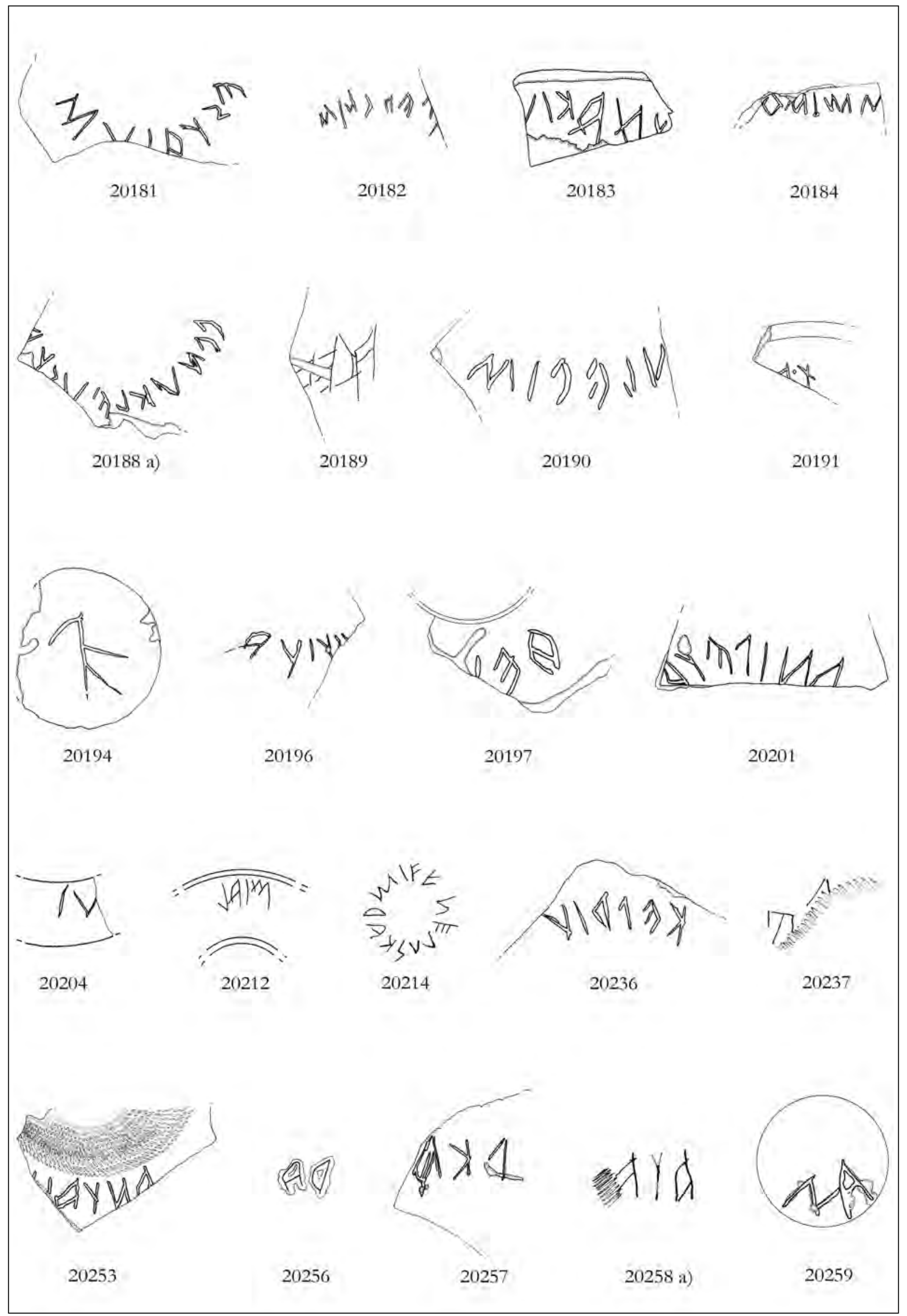

Tav. 3. Apografi delle iscrizioni etrusche, da CIE IV, I, 1 (1:2, dove non diversamente specificato). 


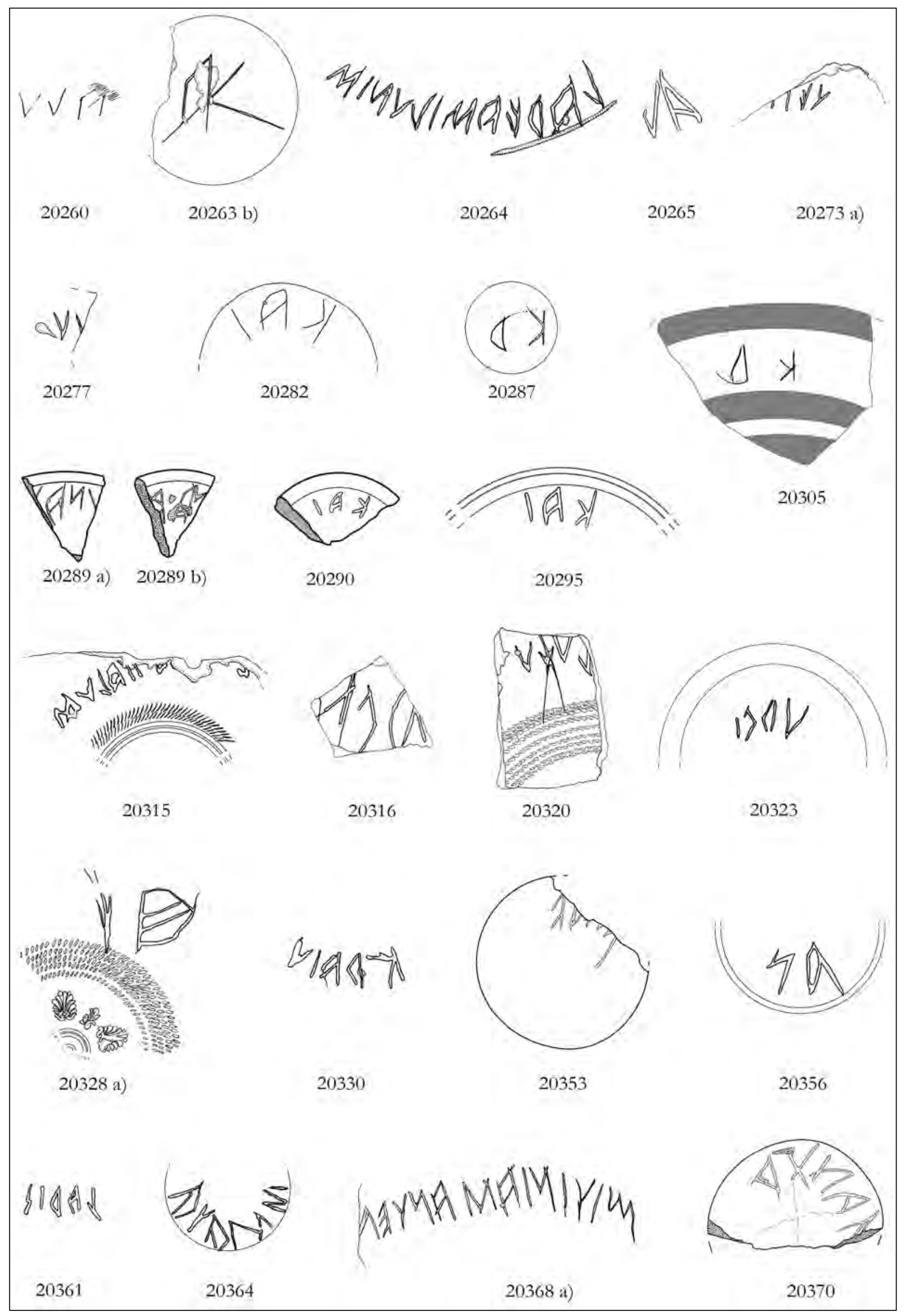

Tav. 4. Apografi delle iscrizioni etrusche, da CIE IV, I, 1 (1:2, dove non diversamente specificato). 


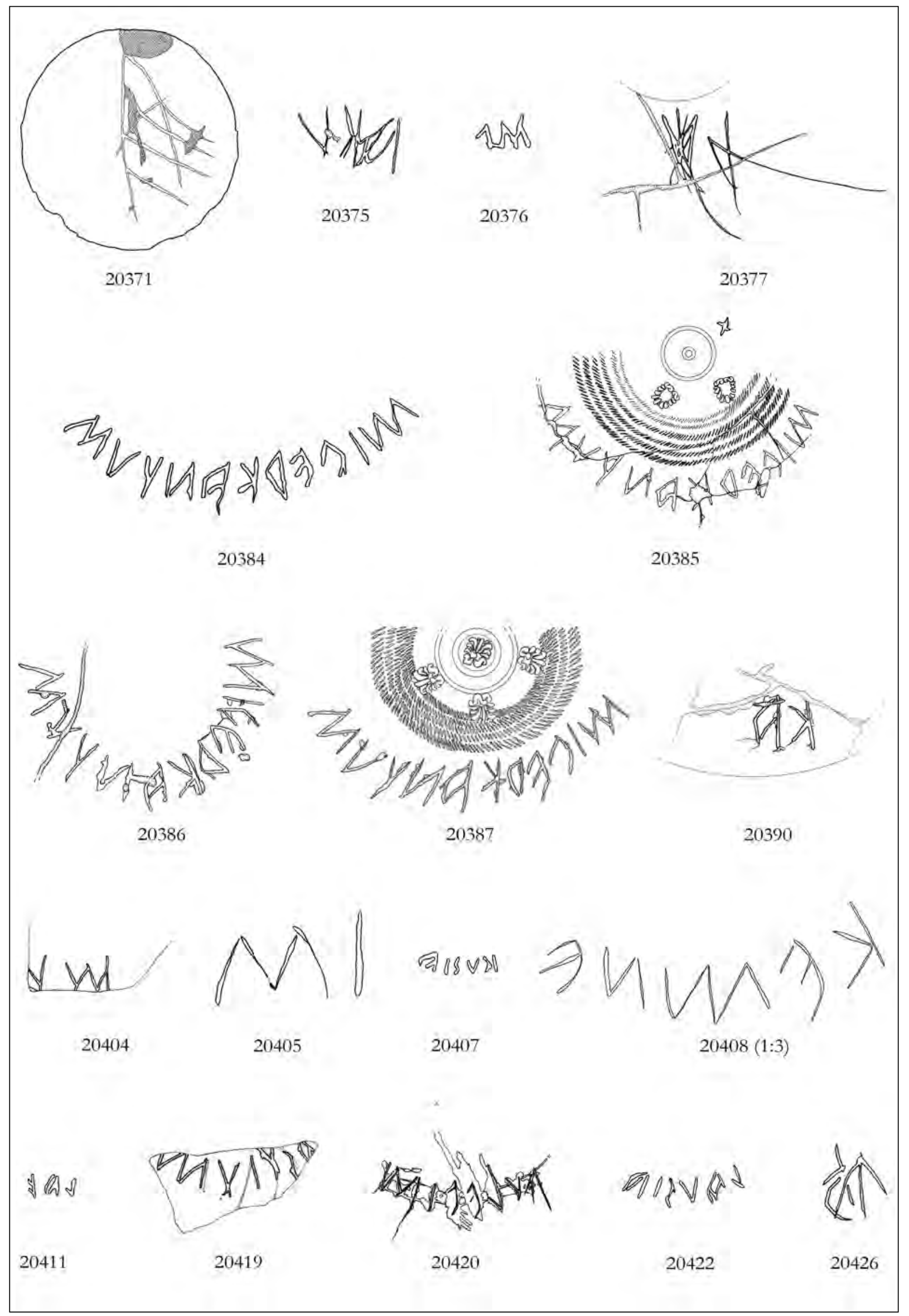

Tav. 5. Apografi delle iscrizioni etrusche, da CIE IV, I, 1 (1:2, dove non diversamente specificato). 


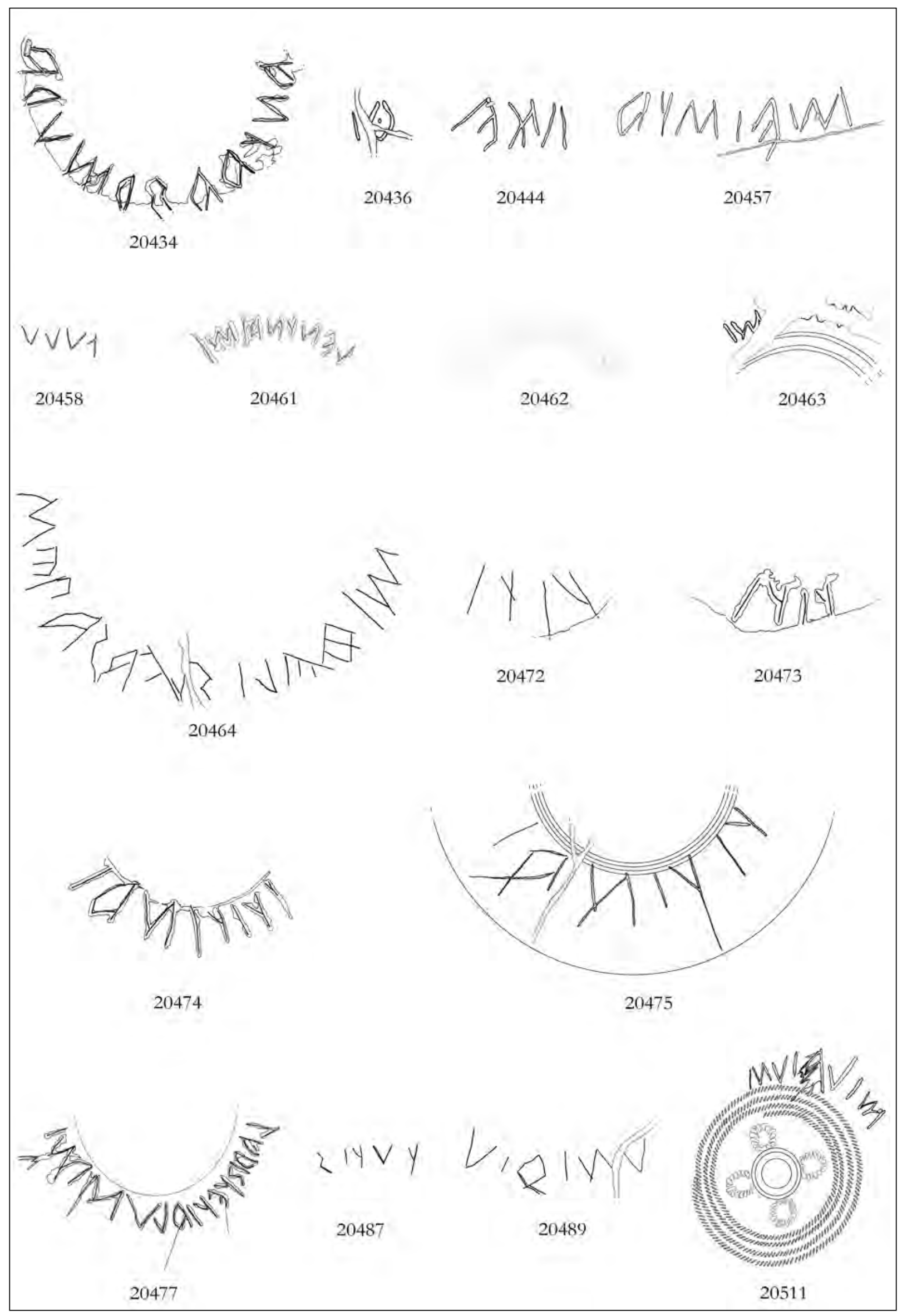

Tav. 6. Apografi delle iscrizioni etrusche, da CIE IV, I, 1 (1:2, dove non diversamente specificato). 


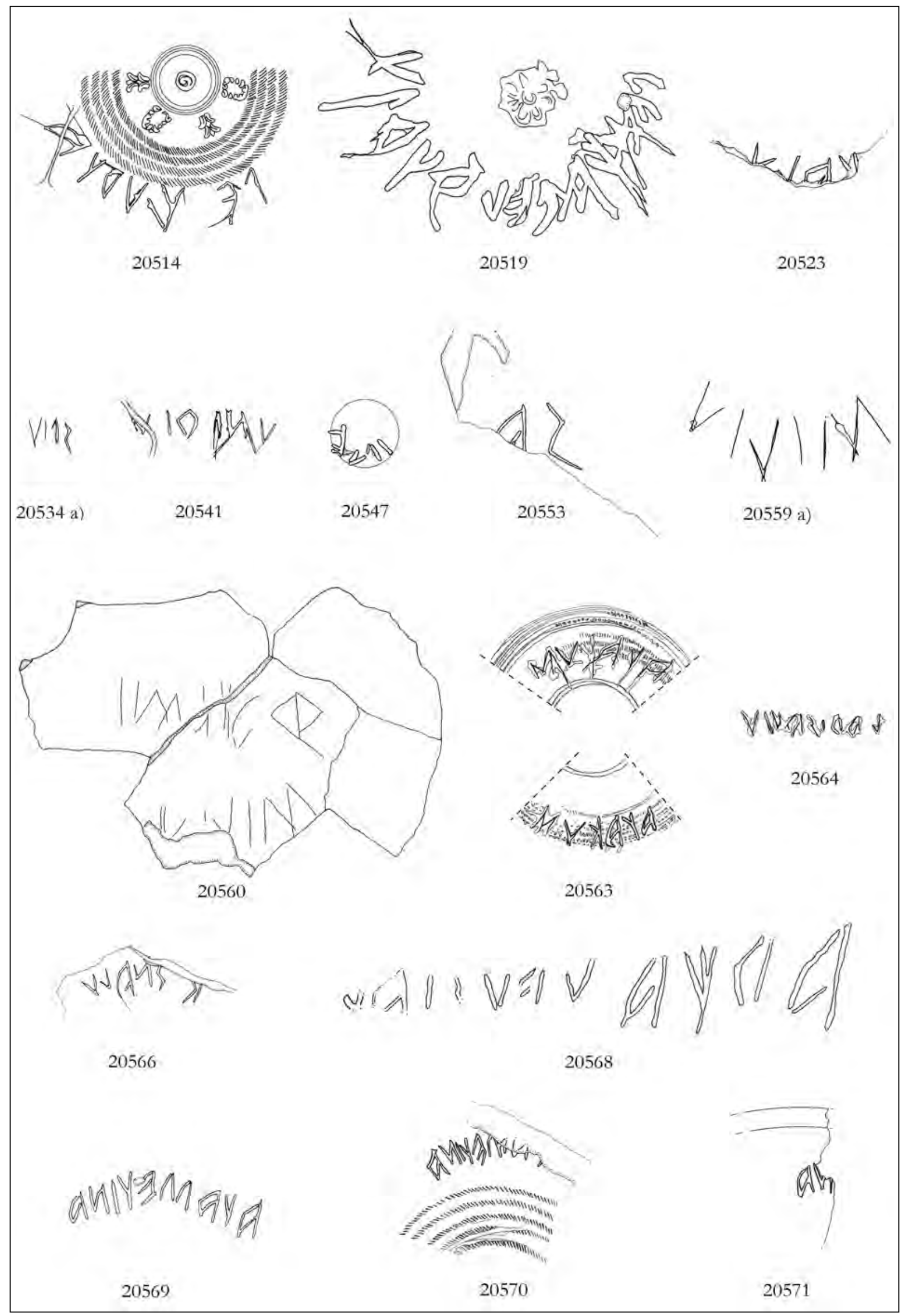

Tav. 7. Apografi delle iscrizioni etrusche, da CIE IV, I, 1 (1:2, dove non diversamente specificato). 


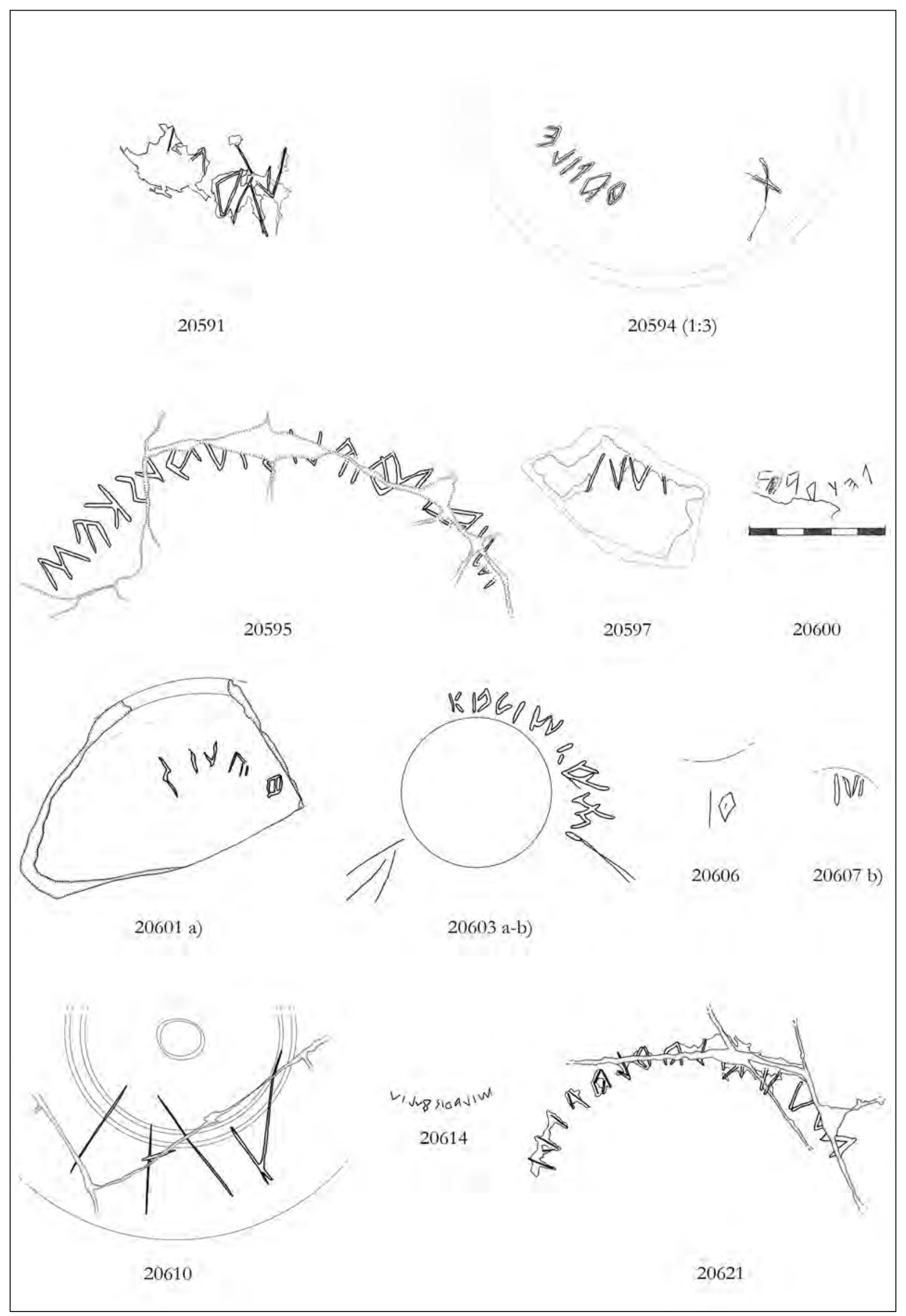

Tav. 8. Apografi delle iscrizioni etrusche, da CIE IV, I, 1 (1:2, dove non diversamente specificato). 


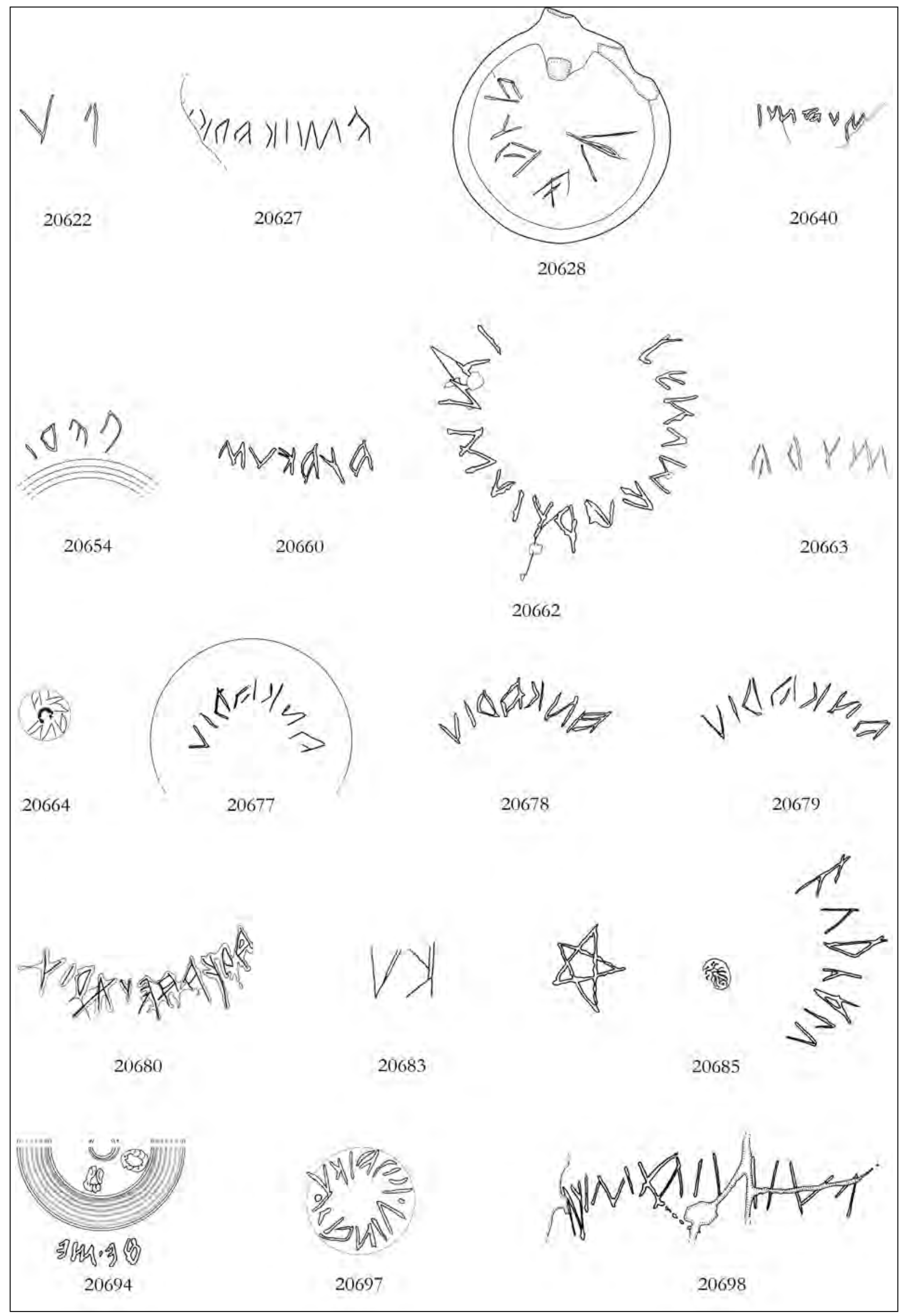

Tav. 9. Apografi delle iscrizioni etrusche, da CIE IV, I, 1 (1:2, dove non diversamente specificato). 


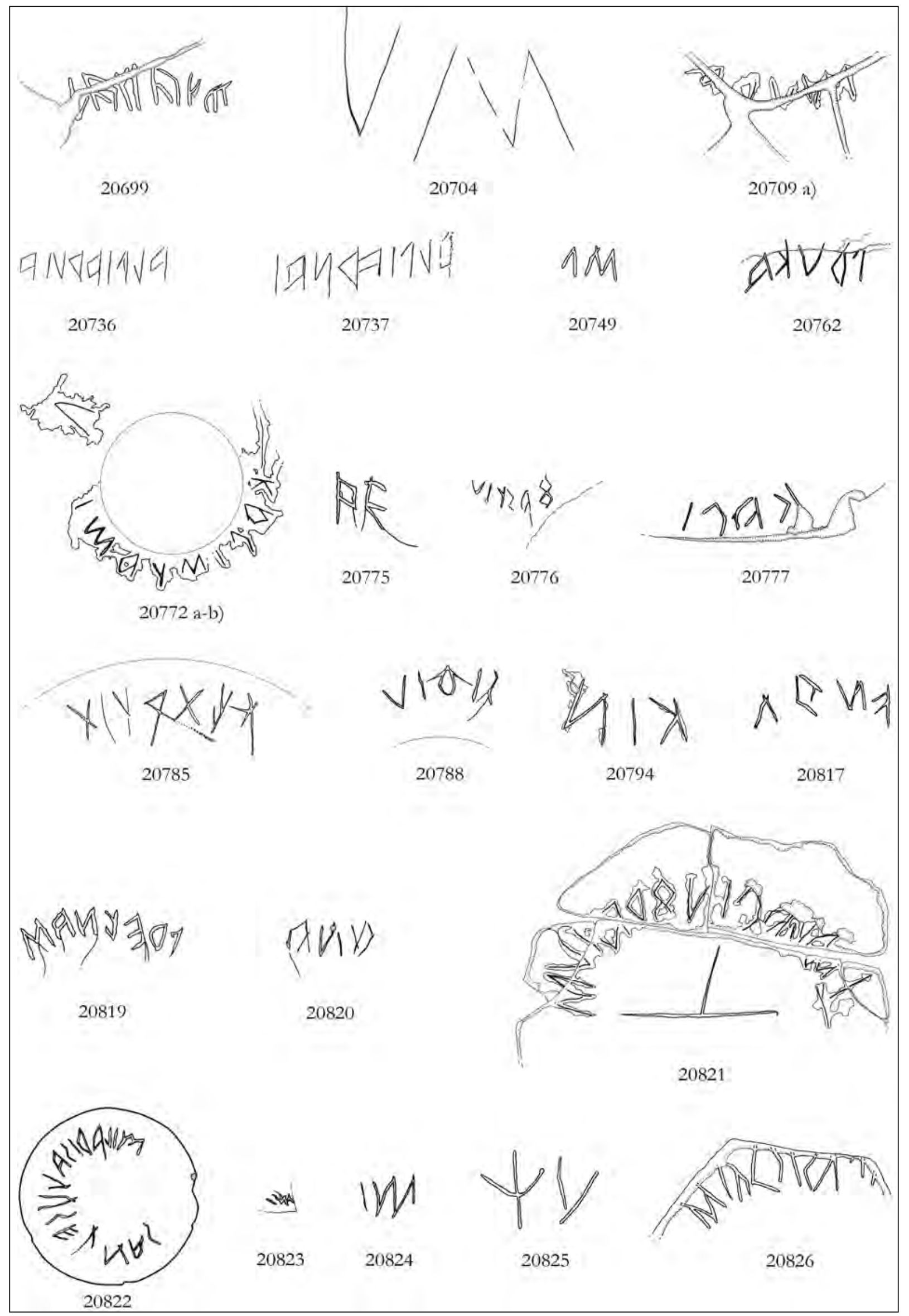

Tav. 10. Apografi delle iscrizioni etrusche, da CIE IV, I, 1 (1:2, dove non diversamente specificato). 


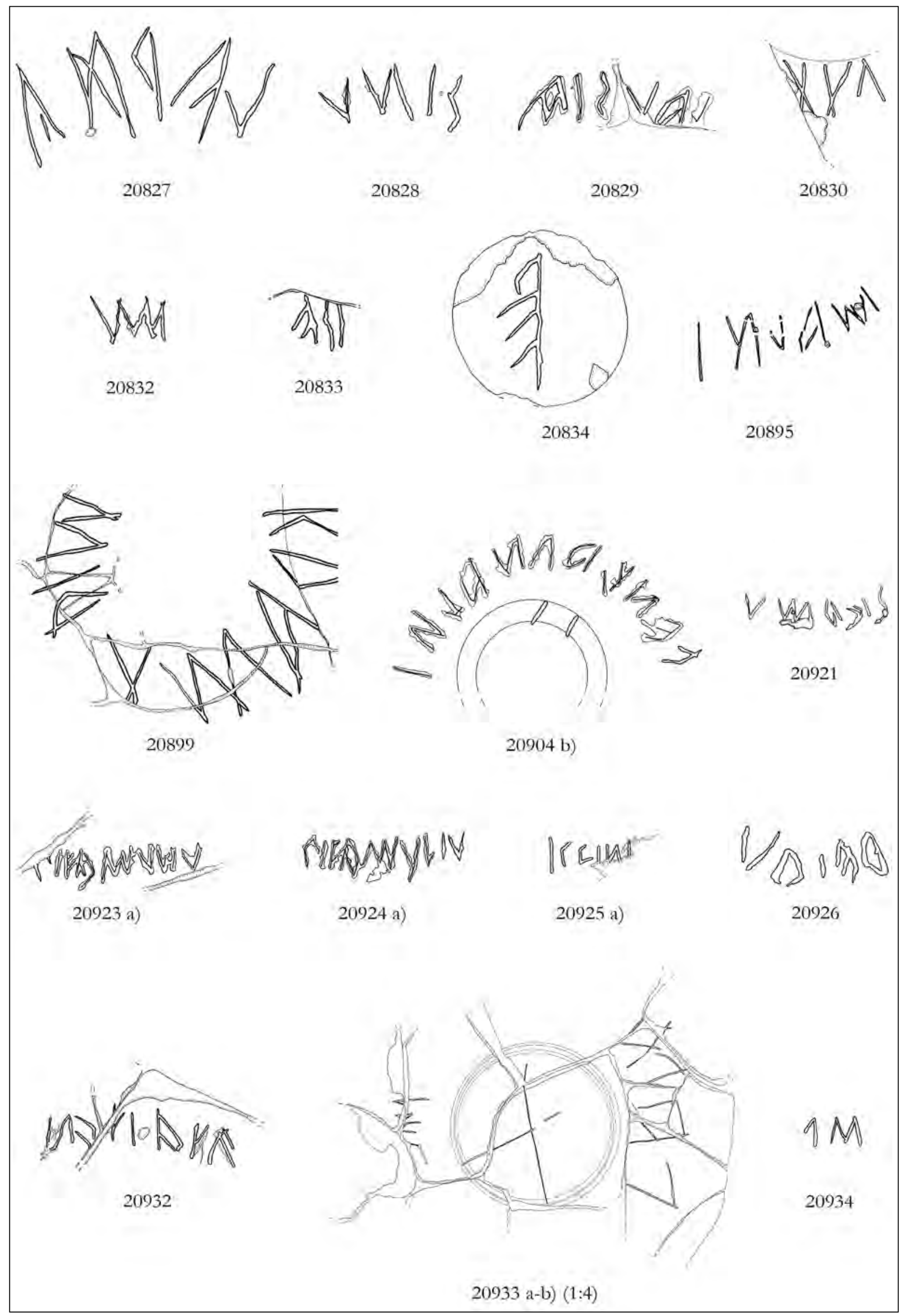

Tav. 11. Apografi delle iscrizioni etrusche, da CIE IV, I, 1 (1:2, dove non diversamente specificato). 

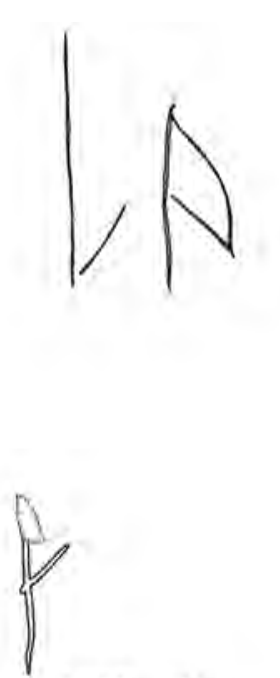

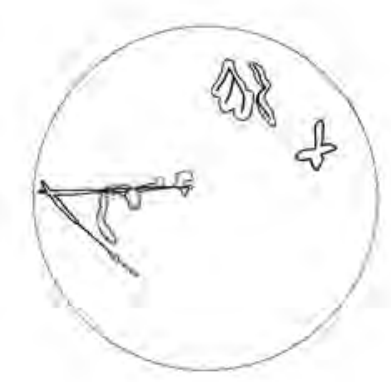

$20960 a-c)$

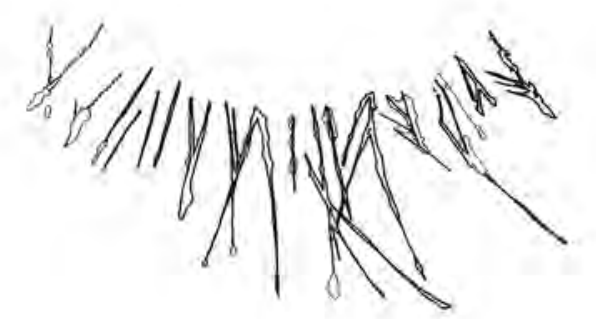

20992

$20951 \mathrm{a}-\mathrm{b})$

Sa

20993

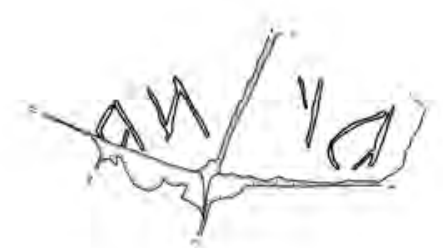

20997
जासे

21002 a)

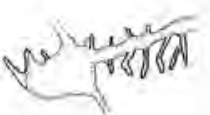

21002 b)

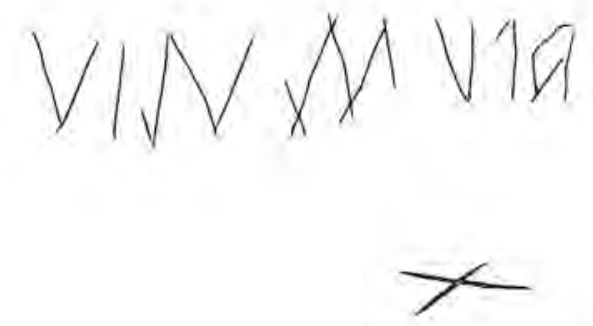

21013

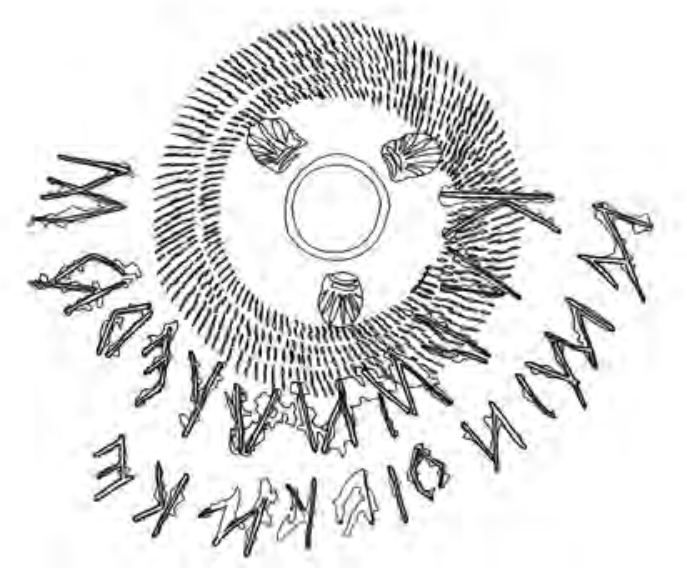

21027

Tav. 12. Apografi delle iscrizioni etrusche, da CIE IV, I, 1 (1:2, dove non diversamente specificato). 



\section{Collana DiSCi Archeologia}

1. Stefano Santocchini Gerg, Incontri Tirrenici. Le relazioni fra Etruschi, Sardi e Fenici in Sardegna (630-480 a.C.), 2014

2. Enrico Giorgi e Paola Buzi, a cura di, Bakchias. Dall'Archeologia alla Storia, 2014

3. Anna Chiara Fariselli, a cura di, Da Tharros a Bitia. Nuove prospettive della ricerca archeologica nella Sardegna fenicia e punica. Atti della Giornata di Studio, Bologna 25 marzo 2013, 2014

4. Silvia Romagnoli, Il santuario etrusco di Villa Cassarini a Bologna, 2014

5. Marco Podini, La decorazione architettonica di età ellenistica e romana nell'Epiro del nord, 2014

6. Isabella Baldini e Monica Livadiotti, a cura di, Archeologia protobizantina a Kos. La città e il complesso episcopale, 2015

7. Enrico Ravaioli, L'insediamento fortificato in Romagna tra fonti scritte e dati archeologici (VIII-XVI sec.). Le province di Forli-Cesena e Ravenna, 2015

8. Giuseppe Sassatelli, Archeologia e Preistoria: alle origini della nostra disciplina. Il Congresso di Bologna del 1871 e i suoi protagonisti, 2015

9. Kevin Ferrari, Ad ostium Liris fluvii. Storia del paesaggio costiero alla foce del Garigliano, 2016

10. Anna Gamberini, Ceramiche fini ellenistiche da Phoinike: forme, produzioni, commerci, 2016

11. Federica Boschi, edited by, Looking to the Future, Caring for the Past. Preventive Archaeology in Theory and Practice, 2016

12. Francesco Belfiori, «Lucum conlucare Romano more». Archeologia e religione del "lucus" Pisaurensis, 2017

13. Michele Silani, Città e territorio: la formazione della città romana nell'ager Gallicus, 2017

14. Sandro De Maria, Celeberrimi loci. Studi sulle strategie della celebrazione nel mondo romano, 2017

15. Elisabetta Govi, a cura di, La città etrusca e il sacro. Santuari e istituzionipolitiche. Atti del Convegno, Bologna 21-23 gennaio 2016, 2017

16. Andrea Augenti, Neil Christie, Jozsef Laszlovsky, Gisela Ripoll, a cura di, La Basilica di San Severo a Classe. Scavi 2006, 2017

17. Carlotta Franceschelli, Pier Luigi Dall'Aglio, Laurent Lamoine, a cura di, Spazi pubblici e dimensione politica nella città romana: funzioni, strutture, utilizzazione. Espaces publics et dimension politique dans la ville romaine: fonctions, aménagements, utilisations. Clermont-Ferrand 30 marzo 2015, Bologna 27 ottobre 2015, 2017

18. Giuseppe Sassatelli, Felsina vocitata tum cum princeps Etruriae esset. Raccolta di studi di Etruscologia e Archeologia italica, 2 tomi, 2017

19. Alessia Morigi, Riccardo Villicich, Scavi nell'area della Villa di Teoderico a Galeata. Le fasi di età romana, 2017

20. Giulia Morpurgo, I sepolcreti etruschi di Bologna nei terreni De Luca e Battistini (fine VI - inizi IV secolo a.C.), 2 tomi, 2018

21. Riccardo Helg, Frontes. Le facciate nell'architettura e nell'urbanistica di Pompei e di Ercolano, 2018 
22. Giuseppe Lepore, Belisa Muka, La necropoli meridionale di Phoinike. Le tombe ellenistiche e romane, 2018

23. Riccardo Villicich, Il teatro di Phoinike, 2018

24. Giulia Marsili, Archeologia del cantiere protobizantino. Cave, maestranze e committenti attraverso i marchi dei marmorari, 2019

25. Elia Rinaldi, Agorai ed edilizia pubblica civile nell'Epiro di età ellenistica, 2020

26. Enrico Giorgi, Filippo Demma, Francesco Belfiori, Il santuario di Monte Rinaldo. La ripresa delle ricerche (2016-2019), 2020

27. Florencia Inés Debandi, Sistemi di gestione economica e alimentazione nelle comunità dell'età del Bronzo con particolare riferimento all'Italia settentrionale, 2021

28. Andrea Gaucci, Iscrizioni della città etrusca di Adria. Testi e contesti tra Arcaismo ed Ellenismo, 2021 

Finito di stampare nel mese di marzo 2021 per i tipi di Bononia University Press 


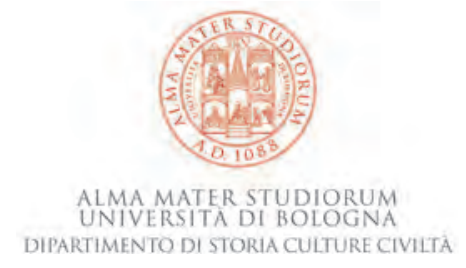

Adria nasce come emporio e almeno dal tardo Arcaismo assume la fisionomia di una città etrusca per cultura e per lingua. Gli scavi condotti in città per oltre due secoli hanno restituito una ricca documentazione epigrafica che conta 212 iscrizioni etrusche e centinaia di graffiti. Nel panorama degli studi su Adria, il ruolo dell'epigrafia appare così assai rilevante. Rilevanza confermata nel quadro dell'Etruria padana, dove il nucleo adriese è al secondo posto per quantità dopo quello di Spina, e compete anche con alcune fra le più importanti città dell'Etruria propria. Ma l'aspetto forse più importante risiede nella continuità, dato che le attestazioni più antiche risalgono al VI sec. a.C. e le più recenti a cavallo di II e I sec. a.C., restituendo così lo spaccato di una comunità sopravvissuta al crollo del sistema padano nel IV sec. a.C.

Questa ricca documentazione è confluita nell'edizione del Corpus Inscriptionum Etruscarum, IV, I, 1. Non consentendo la prassi redazionale dell'opera approfondimenti critici, si è ritenuto opportuno offrire a corredo del fascicolo un testo di analisi epigrafica delle iscrizioni etrusche, valorizzando l'insieme delle informazioni che il singolo oggetto iscritto porta con sé, dunque non solo il testo, ma anche il supporto e il contesto di rinvenimento, essenziali per una soddisfacente interpretazione. Le iscrizioni sono dunque fondamentali per uno studio complessivo e diacronico dell'Adria preromana, in quanto grazie a queste è possibile avviare una riflessione sulla società e sulle pratiche che questa adottava nel confrontarsi con il sacro e con la morte.

Andrea Gaucci è Ricercatore a tempo determinato Senior presso il Dipartimento di Storia Culture Civiltà dell'Università di Bologna, dove insegna Archeologia italica e celtica, Antichità celtiche e Archeologia della Produzione ed è titolare del Laboratorio di Epigrafia etrusca. È inoltre incaricato dell'insegnamento di Etruscologia e antichità italiche presso la Scuola di Specializzazione in Beni Archeologici dell'Università degli Studi di Firenze. Per la Cattedra di Etruscologia, è stato PI del Kainua Project (FIR 2013) ed è responsabile sul campo delle indagini nelle città etrusche di Kainua-Marzabotto e di Spina. Tra i vari filoni di ricerca, l'epigrafia etrusca rappresenta un campo di indagine prediletto, a cui ha destinato molti lavori e anche un progetto di disseminazione multimediale (\#Zich). 